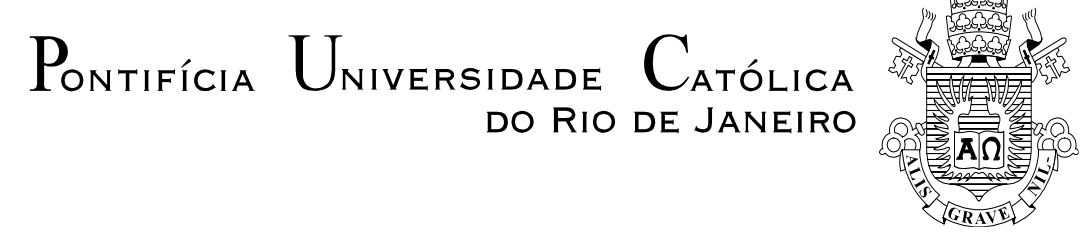

Marcela Cohen Martelotte

Projeto de Filtros para Ajuste Sazonal Robustos a Variações na Sazonalidade

Tese de Doutorado

Tese apresentada como requisito parcial para obtenção do título de Doutor pelo Programa de PósGraduação em Engenharia Elétrica da PUC-Rio.

Orientador: Prof. Reinaldo Castro Souza Co-orientador: Prof. Eduardo Antônio Barros da Silva 


$$
\begin{array}{r}
\text { Pontifícia Universidade Católica } \\
\text { do Rio de Janeiro }
\end{array}
$$

Marcela Cohen Martelotte

\section{Projeto de Filtros para Ajuste Sazonal Robustos a Variações na Sazonalidade}

Tese apresentada como requisito parcial para obtenção do título de Doutor pelo Programa de Pós-Graduação em Engenharia Elétrica da PUC-Rio. Aprovada pela Comissão Examinadora abaixo assinada.

Prof. Reinaldo Castro Souza

Orientador

Departamento de Engenharia Elétrica - PUC-Rio

Prof. Eduardo Antônio Barros da Silva Co-orientador UFRJ

Profa. Karla Tereza Figueiredo Leite Departamento de Engenharia Elétrica - PUC-Rio

Prof. José Francisco Moreira Pessanha UERJ

Prof. Fernando Luiz Cyrino Oliveira Departamento de Engenharia Industrial - PUC-Rio

Prof. Sergio Lima Netto UFRJ

Prof. José Eugenio Leal Coordenador Setorial do Centro Técnico Científico - PUC-Rio

Rio de Janeiro, 26 de agosto de 2014 
Todos os direitos reservados. É proibida a reprodução total ou parcial do trabalho sem autorização da universidade, da autora e do orientador.

\section{Marcela Cohen Martelotte}

Mestre em Engenharia Elétrica pela PUC-Rio e Mestre em Administração Pública e de Empresas pela EBAPE/FGV. Bacharel em Estatística pela Escola Nacional de Ciências Estatísticas do IBGE. É integrante do Programa de Pesquisa em Gestão da Aprendizagem Tecnológica e Inovação Industrial no Brasil.

Ficha Catalográfica

Martelotte, Marcela Cohen

Projeto de filtros para ajuste sazonal robustos a variações na sazonalidade / Marcela Cohen Martelotte ; orientador: Reinaldo Castro Souza ; co-orientador: Eduardo Antônio Barros da Silva. - 2014.

298 f. : il. (color.) ; $30 \mathrm{~cm}$

Tese (doutorado)-Pontifícia Universidade Católica do Rio de Janeiro, Departamento de Engenharia Elétrica, 2014.

Inclui bibliografia

1. Engenharia elétrica - Teses. 2. Séries temporais. 3. Sazonalidade. 4. Sazonalidade móvel. 5. Domínio da frequência. 6. Filtros. 7. X-11. I. Souza, Reinaldo Castro. II. Silva, Eduardo Antônio Barros da. III. Pontifícia Universidade Católica do Rio de Janeiro. Departamento de Engenharia Elétrica. IV. Título. 


\section{Agradecimentos}

Agradeço primeiramente ao professor e orientador Reinaldo Castro Souza, por aceitar me orientar neste doutorado, assim como pelo incentivo e pela confiança em mim depositada.

Ao coorientador Eduardo Silva, pelas inúmeras horas gastas e pelas valiosas contribuições, além de ser fundamental no processo desta tese.

Ao CNPq e à PUC-Rio, pelos auxílios concedidos, sem os quais este trabalho não poderia ter sido realizado.

Aos professores do Departamento de Engenharia Elétrica, pela contribuição na minha formação acadêmica.

Aos membros da banca examinadora pela disponibilidade e atenção concedidas. E aos professores que participaram da defesa da proposta de tese, pelas ricas sugestões dadas ao trabalho.

Agradeço também aos amigos do curso, importantes em toda essa trajetória, e em especial ao Moisés e ao Carlos, por todo o apoio nos momentos de dificuldade. Outros amigos que não são do curso, mas que foram importantes para a elaboração desta tese, são a Sheila e o Luiz. Obrigada pela ajuda!

Por fim, agradeço à minha família pelo constante incentivo. 


\section{Resumo}

Martelotte, Marcela Cohen; Souza, Reinaldo Castro; Silva, Eduardo Antônio Barros da. Projeto de Filtros para Ajuste Sazonal Robustos a Variações na Sazonalidade. Rio de Janeiro, 2014. 298p. Tese de Doutorado Departamento de Engenharia Elétrica, Pontifícia Universidade Católica do Rio de Janeiro.

Quando há mudanças no padrão sazonal de uma série temporal, ao longo do tempo, fica caracterizada a presença de 'sazonalidade móvel'. Existem evidências de séries macroeconômicas que apresentam um grau considerável de sazonalidade móvel. Atualmente, para a realização do ajuste sazonal, o programa utilizado pelo IBGE é o X-12-ARIMA, que implementa o método X-11 de ajuste sazonal. O X11 é um dos métodos mais utilizados no mundo pelos órgãos oficiais de estatística, no entanto, quando existe sazonalidade móvel, ele não consegue tratá-la de forma adequada. Este trabalho propõe dois projetos de filtros de extração da componente sazonal, no domínio da frequência, que são adequados tanto para séries com sazonalidade estável quanto para aquelas que apresentam sazonalidade móvel. O primeiro projeto de filtros, intitulado de 'filtro sazonal-WLS', utiliza critérios baseados em mínimos quadrados. O desempenho do 'filtro sazonal-WLS' é avaliado com base em sinais sazonais artificiais, para séries mensais e trimestrais, baseados nas características das séries macroeconômicas. Os resultados são comparados com o método X-11 e são identificadas as situações nas quais ele é superior ao X-11. Considerando que o 'filtro sazonal-WLS' é tanto superior ao X11 quanto maior for a razão entre a variação da sazonalidade e a intensidade da componente irregular, foi desenvolvido o projeto de um segundo filtro. Este novo filtro combina a abordagem de mínimos quadrados ponderados com as características dos filtros de Chebyshev, minimizando simultaneamente o erro na estimativa da sazonalidade e a influência da componente irregular. A ele intitulouse 'filtro sazonal-WLS-Chebyshev'. Os resultados do 'filtro sazonal-WLSChebyshev' são comparados com o 'filtro sazonal-WLS' onde observam-se algumas melhorias.

\section{Palavras-chave}

Séries temporais; sazonalidade; sazonalidade móvel; domínio da frequência; filtros; X-11. 


\section{Abstract}

Martelotte, Marcela Cohen; Souza, Reinaldo Castro (advisor); Silva, Eduardo Antônio Barros da (co-advisor). Filter Design for the Seasonal Adjustment Robust to Variations in the Seasonal Patterns. Rio de Janeiro, 2014. 298p. Doctoral Thesis - Department of Electrical Engineering, Pontifical Catholic University of Rio de Janeiro.

A time series is said to have 'moving seasonality' when there are changes in the seasonal pattern. There is evidence that macroeconomic series show moving seasonality. Currently, to perform a seasonal adjustment, IBGE uses the program X-12-ARIMA, which implements the seasonal adjustment method X-11. This method is worldwide adopted by official statistical agencies. However, when a time series shows changing seasonal patterns, the X-11 seasonal adjustment method generates unreliable estimates. This thesis proposes two designs of filters to extract seasonal components in the frequency domain, that are suitable for series with stable seasonality and for those with moving seasonality. The first filter, named 'WLS-seasonal filter', uses criteria based on least squares. The performance of this filter is assessed based on artificial seasonal series for monthly and quarterly data, based on the characteristics of real macroeconomic series. The results are compared with the ones of X-11 method, and the situations in which this filter is superior to $\mathrm{X}-11$ are identified. Taking into account the fact that the performance of the 'WLSseasonal filter' improves in relation to the one of X-11 the higher the ratio between the variation of seasonality and irregular intensity, the design of a second filter was developed. This new filter combines the approach of weighted least squares with the Chebyshev filters characteristics, simultaneously minimizing the error in estimating the seasonal component and the influence of the irregular component. It was named 'WLS-Chebyshev-seasonal filter'. The performance of this new filter is compared with the one of the WLS-seasonal filter, and some improvements are observed.

\section{Keywords}

Time series; seasonality; moving seasonality; frequency domain; linear filters; X-11. 


\section{Sumário}

$\begin{array}{ll}1 \text { Introdução } & 37\end{array}$

$\begin{array}{ll}\text { 1.1. Apresentação do tema } & 37\end{array}$

1.2. Objetivo e contribuição da tese 39

1.3. Relevância do tema $\quad 40$

1.4. Delimitação do estudo 42

1.5. Organização da tese 43

2 Ajuste sazonal: conceito, histórico e métodos 44

2.1. Conceitos e breve histórico do desenvolvimento das técnicas de ajuste sazonal 44

2.2. Métodos de ajuste sazonal usados por agências governamentais $\quad 47$

2.2.1. Revisão bibliográfica sobre a comparação entre o X-11 e o SEATS 52

2.3. Procedimento de ajuste sazonal do programa $X-13 A-S$, utilizando o método X-11

2.3.1. Tipos de decomposições de séries temporais no X-13A-S 54

2.3.2. Etapas do ajuste sazonal realizado pelo X-11 56

2.3.3. Outros tópicos relacionados ao ajuste sazonal no X-13A-S 59

2.3.3.1. Filtro de Henderson 59

2.3.3.2. Seleção automática dos filtros sazonais no X-13A-S 60

2.3.3.3. Testes para a presença de sazonalidade no X-13A-S 62

2.3.3.3.1. Teste $F$ para a presença de sazonalidade estável (Fs) 62 
2.3.3.3.3. Teste de Kruskal-Wallis para a presença de sazonalidade estável (KW)

2.3.3.3.4. Teste combinado para a presença de sazonalidade identificável

2.3.3.4. Estatísticas $M$ e Q para qualidade do ajuste 67

2.4. Tópicos gerais relacionados à sazonalidade móvel 69

2.4.1. Uma breve revisão histórica sobre a sazonalidade móvel 69

2.4.2. Motivos para a existência de sazonalidade móvel 70

2.4.3. Alguns testes para a presença de sazonalidade móvel 71

3 Ajuste sazonal no domínio da frequência 73

3.1. Alguns conceitos relacionados à análise no domínio da frequência 73

3.1.1. Sinal 73

3.1.2. Sistemas em tempo discreto 74

3.1.3. Análise de Fourier 76

3.1.3.1. Elementos básicos de um modelo senoidal simples 76

3.1.3.2. Transformada de Fourier 78

$\begin{array}{ll}\text { 3.1.3.2.1. Transformada Z } & 79\end{array}$

3.1.3.3. Espectros e funções de transferência 80

3.1.3.3.1. Espectro de amplitude do sinal 80

3.1.3.3.2. Gráfico do ganho 81

3.1.3.3.3. Espectro de fase 81

3.1.3.3.4. Espectro de potência 82

3.1.3.4. Função Geradora de Autocovariância e sua relação com o espectro 82

3.2. O método $X-11$ no domínio da frequência 84 
3.2.2. O desempenho do X-11 na presença de sazonalidade móvel 87

3.3. Filtros

4 Filtro sazonal-WLS: uma generalização para o ajuste sazonal, na frequência

4.1. O Algoritmo do filtro sazonal-WLS

4.2. Parâmetros do filtro sazonal-WLS

5 Filtro sazonal-WLS: seleção da melhor configuração e resultados

5.1. Componente Sazonal Artificial

5.2. Características das séries utilizadas e definição dos parâmetros do sinal artificial

5.2.1. Séries mensais com decomposição aditiva: parâmetros ' $\mathrm{A} / \mathrm{s}$ ', ' $\mathrm{k}$ ', 'b' e coeficientes da componente de tendência

5.2.2. Séries mensais com decomposição multiplicativa: parâmetros

'A/s', 'k', 'b' e coeficientes da componente de tendência

5.2.3. Séries trimestrais com decomposição aditiva: parâmetros ' $\mathrm{A} / \mathrm{s}$ ', 'k', 'b' e coeficientes da componente de tendência

5.2.4. Séries trimestrais com decomposição multiplicativa: parâmetros 'A/s', ' $k$ ', 'b' e coeficientes da componente de tendência

5.3. Razão Sinal Ruído (SNR)

5.3.1. SNR do filtro equivalente ao método $X-11$ para extração da sazonalidade

5.3.2. SNR do filtro proposto para extração da sazonalidade

5.3.2.1. Cálculo de $\alpha$ com base em $\mathrm{k}$ 
5.4. Seleção da melhor configuração do filtro S-WLS 133

5.4.1. Definição do filtro S-WLS para as séries mensais 135

5.4.2. Definição do filtro S-WLS para as séries trimestrais 140

5.5. Resultados: filtro S-WLS vs X-11 144

5.5.1. Critério de comparação entre o filtro S-WLS e o filtro X-11 144

5.5.2. Resultados: série mensal com decomposição aditiva - filtro

S-WLS vs X-11

5.5.2.1. Espectros da componente irregular: série mensal com decomposição aditiva

5.5.3. Resultados: série mensal com decomposição multiplicativa filtro S-WLS vs X-11

5.5.3.1. Espectros da componente irregular: série mensal com decomposição multiplicativa

5.5.4. Resultados: série trimestral com decomposição aditiva - filtro S-WLS vs X-11

5.5.4.1. Espectros da componente irregular: série trimestral com decomposição aditiva

5.5.5. Resultados: série trimestral com decomposição multiplicativa Filtro S-WLS vs X-11

5.5.5.1. Espectros da componente irregular: série trimestral multiplicativa

6 Filtro sazonal-WLS-Chebyshev: definição, seleção da melhor configuração e resultados

6.1. Filtro sazonal-WLS-Chebyshev: o algoritmo

6.2. Metodologia para seleção do filtro S-WLSC para as séries mensais e trimestrais 
6.3. Série mensal aditiva - filtro S-WLSC 204

6.3.1. Seleção do melhor filtro S-WLSC para cada 'N': filtro mensal 204

6.3.1.1. Resultado: filtro S-WLSC mensal com N $=117$

6.3.1.2. Resultado: filtro S-WLSC mensal com $\mathrm{N}=121$

6.3.1.3. Resultado: filtro S-WLSC mensal com N $=131$

6.3.1.4. Resultado: filtro S-WLSC mensal com $\mathrm{N}=141$

6.3.1.5. Resultado: filtro S-WLSC mensal com N = 145

6.3.1.6. Resultado: filtro S-WLSC mensal com N $=155$

6.3.1.7. Resultado: filtro S-WLSC mensal com $\mathrm{N}=189$

6.3.1.8. Resultado: filtro S-WLSC mensal com N $=193$

6.3.1.9. Resultado: filtro S-WLSC mensal com N $=203$

6.3.2. Parâmetros do filtro S-WLSC: séries mensais 220

6.3.3. Comparação do desempenho do filtro S-WLSC com o filtro

S-WLS, para séries mensais com decomposição aditiva 221

6.4. Série mensal multiplicativa - filtro S-WLSC vs filtro S-WLS 227

6.5. Série trimestral aditiva - filtro S-WLSC 233

6.5.1. Seleção do melhor filtro para cada 'N': filtro trimestral 233

6.5.1.1. Resultado: filtro S-WLSC trimestral com $\mathrm{N}=41$

6.5.1.2. Resultado: filtro S-WLSC trimestral com N $=43$ e 65

6.5.1.3. Resultado: filtro S-WLSC trimestral com $\mathrm{N}=49$

6.5.1.4. Resultado: filtro S-WLSC trimestral com $\mathrm{N}=67$

6.5.1.5. Resultado: filtro S-WLSC trimestral com $\mathrm{N}=51$

6.5.2. Parâmetros do filtro S-WLSC para séries trimestrais 240

6.5.3. Comparação do filtro S-WLSC com o filtro S-WLS, para séries trimestrais com decomposição aditiva

6.6. Série trimestral multiplicativa - filtro S-WLSC vs filtro S-WLS 247 
7 Considerações Finais e Sugestões

$\begin{array}{ll}\text { 7.1. Considerações Finais } & 253\end{array}$

7.2. Sugestões para trabalhos futuros 255

8 Referências bibliográficas

APÊNDICE A - Teste para sazonalidade estável 269

APÊNDICE B - Teste para sazonalidade móvel 270

APÊNDICE C - Cálculo do tamanho do filtro equivalente ao X-11

para séries mensais e trimestrais

271

APÊNDICE D - Dedução das expressões da potência do sinal e da potência do ruído

APÊNDICE E - Séries históricas mensais brasileiras 275

APÊNDICE F - Séries históricas mensais estrangeiras 278

APÊNDICE G - Séries históricas trimestrais estrangeiras 280

APÊNDICE H - Coeficientes dos filtros mensais: S-WLS e X-11 282

APÊNDICE I - Coeficientes dos filtros trimestrais: S-WLS e X-11 283

APÊNDICE J-Coeficientes dos filtros mensais: S-WLS e S-WLSC 284

APÊNDICE K - Programas MATLAB: filtro sazonal-WLS e filtro

sazonal-WLS-Chebyshev

285

(a) Filtro S-WLS mensal 285

(b) Filtro S-WLS trimestral 288

(c) Filtro S-WLSC mensal 291

(d) Filtro S-WLSC trimestral 295 


\section{Lista de figuras}

Figura 2.1 - O procedimento X-12 ARIMA de ajuste sazonal 51

Figura 2.2. - Teste combinado para identificação de sazonalidade $\quad 66$

Figura 4.1 - Esquema detalhado do procedimento de filtragem 95

Figura 5.1 - Espectro, na frequência, de um sinal sazonal artificial 103

Figura 5.2 - Definição dos valores de $\beta_{1}$ e $\beta_{2}$ do filtro X-11 124

Figura 5.3 - Definição dos valores de $\gamma 0$ e $\gamma 1$ do filtro proposto 128

Figura 5.4 - Espectro, na frequência, representando a relação entre $\mathrm{k}$ e $\alpha$, para séries com periodicidade mensal

Figura 5.5 - Etapas do procedimento utilizado para a obtenção dos parâmetros do filtro S-WLS

Figura 5.6 - Configuração do filtro mensal - X-11 e S-WLS segundo a SNR

Figura 5.7 - Configuração do filtro trimestral: X-11 e filtro S-WLS 140

Figura 5.8 - Procedimento utilizado para a obtenção da componente sazonal utilizando o filtro proposto (S-WLS), nas séries multiplicativas

Figura 6.1 - Função de erro típica (linha tracejada) e função envelope (linha contínua) 


\section{Lista de quadros}

Quadro 2.1 - Breve histórico dos principais acontecimentos 46

Quadro 2.2 - Intervalos de MSR 61

Quadro 2.3 - Estatísticas M 68

Quadro 2.4. - Pesos das estatísticas M na composição da estatística Q 68

Quadro 5.1 - Tamanho do filtro de Henderson e média móvel sazonal utilizados pelo $\mathrm{X}-11$ para séries mensais

Quadro 5.2 - Valores da soma dos quadrados dos erros do filtro X-11 para extração da sazonalidade de séries mensais

Quadro 5.3 - Valores da soma dos quadrados dos erros do filtro X-11 para extração da sazonalidade de séries trimestrais

Quadro 5.4 - Valores do tamanho do filtro X-11, para séries mensais 123

Quadro 5.5 - Valores do tamanho do filtro X-11, para séries trimestrais 123

Quadro 5.6 - Valores de $\beta$ quando o tamanho do filtro de Henderson =

9 , para séries mensais

Quadro 5.7 - Valores de $\beta$ quando o tamanho do filtro de Henderson = 13 , para séries mensais

Quadro 5.8 - Valores de $\beta$ quando o tamanho do filtro de Henderson = 23, para séries mensais

Quadro 5.9 - Valores de $\beta$ quando o tamanho do filtro de Henderson =

5 , para séries trimestrais

Quadro 5.10 - Valores de $\beta$ quando o tamanho do filtro de Henderson = 7 , para séries trimestrais

Quadro 5.11 - Mensal Aditivo - Filtro S-WLS $\left(\alpha=1 / 3, \delta=1 / 30\right.$, $\left.\mathrm{w}_{0}=1\right)$ vs X-11: MSE, MAD e SNR. Valores de 'b' a partir do qual o filtro S-WLS supera o X-11, para cada 'k' e 'A/s'. (Eq. 5-1)

Quadro 5.12 - Mensal Multiplicativo - Filtro S-WLS ( $\alpha=1 / 3, \delta=1 / 30$, $W_{o}=1$ ) vs X-11: MSE e MAD. Valores de 'b' a partir do qual o filtro S-WLS supera o X-11, para cada 'k' e 'A/s' (Eq. 5-3). 
Quadro 5.13 - Trimestral Aditivo - Filtro S-WLS vs X-11 (S-WLS: $\alpha=$ $1 / 3, \delta=1 / 30, w_{0}=1$ ): MSE, MAD e SNR. Valores de 'b' a partir do qual o filtro S-WLS supera o X-11, para cada 'k' e 'A/s' (Eq. 5-2).

Quadro 5.14 - Trimestral Multiplicativo - Filtro S-WLS $(\alpha=1 / 3, \delta=1 / 30$, $W_{0}=1$ ) vs X-11: MSE e MAD. Valores de 'b' a partir do qual o filtro $\mathrm{S}$-WLS supera o X-11, para cada ' $\mathrm{k}$ ' e 'A/s'. 


\section{Lista de tabelas}

Tabela 5.1 - Quantidade de séries pesquisadas: total, com sazonalidade, e com sazonalidade móvel

Tabela 5.2 - Séries históricas mensais nacionais com sazonalidade móvel significativa

Tabela 5.3 - Séries históricas mensais internacionais com sazonalidade móvel significativa

Tabela 5.4 - Séries históricas trimestrais internacionais com sazonalidade móvel significativa

Tabela 5.5 - Parâmetros das séries mensais aditivas, com sazonalidade móvel

Tabela 5.6 - Coeficientes da componente de tendência das séries mensais aditivas (Eq. 5-8)

Tabela 5.7 - Parâmetros das séries mensais multiplicativas, com sazonalidade móvel (Eq. 5-3)

Tabela 5.8 - Coeficientes da componente de tendência das séries mensais multiplicativas (Eq. 5-8)

Tabela 5.9 - Parâmetros das séries trimestrais aditivas, com sazonalidade móvel (Eq. 5-2)

Tabela 5.10 - Coeficientes da componente de tendência das séries trimestrais aditivas (Eq. 5-8)

Tabela 5.11 - Parâmetros das séries trimestrais multiplicativas, com sazonalidade móvel (Eq. 5-4)

Tabela 5.12 - Coeficientes da componente de tendência das séries trimestrais multiplicativas (Eq. 5-8)

Tabela 5.13 - Configurações de parâmetros para o filtro S-WLS mensal utilizadas na seleção do melhor filtro

Tabela 5.14 - Valores de $\boldsymbol{S Q}, \boldsymbol{\gamma} \mathbf{0}$ e $|\mathbf{1}-\boldsymbol{\gamma} \mathbf{1}|$ para os nove filtros mensais, considerando $\boldsymbol{k}=72$

Tabela 5.15 - Configurações de parâmetros para o filtro S-WLS trimestral utilizadas na seleção do melhor filtro

Tabela 5.16 - Valores de $\boldsymbol{S Q}, \boldsymbol{\gamma} \mathbf{0}$ e $|\mathbf{1}-\boldsymbol{\gamma} \mathbf{1}|$ para os seis filtros S-WLS trimestrais, considerando $\boldsymbol{k}=24$ 
Tabela 5.17 - MSE, MAD e SNR para valores de ' $b$ ', sendo $\boldsymbol{A} / \boldsymbol{s}=6$, $\boldsymbol{k}=72$ : séries mensais com decomposição aditiva (Eq. $5-1$ )

Tabela 5.18 - MSE, MAD e SNR para valores de ' $\boldsymbol{k}$ ', sendo $\boldsymbol{A} / \boldsymbol{s}=6$, $\boldsymbol{b}=40 \%$ : séries mensais com decomposição aditiva (Eq. 5-1)

Tabela 5.19 - MSE, MAD e SNR para valores de ' $\boldsymbol{A} / \boldsymbol{s}$ ', sendo $\boldsymbol{k}=72$, $\boldsymbol{b}=40 \%$ : séries mensais com decomposição aditiva (Eq. 5-1)

Tabela 5.20 - MSE e MAD para valores de ' $\boldsymbol{b}$ ', sendo $\boldsymbol{A} / \boldsymbol{s}=6, \boldsymbol{k}=72$ : séries mensais com decomposição multiplicativa (Eq. 5-3)

Tabela 5.21 - MSE e MAD para valores de ' $\boldsymbol{k}$ ', sendo $\boldsymbol{A} / \boldsymbol{s}=6, \boldsymbol{b}=$ 40\%: séries mensais com decomposição multiplicativa (Eq. 5-3)

Tabela 5.22 - MSE e MAD para valores de ' $A / \boldsymbol{s}$ ', sendo $\boldsymbol{k}=72, \boldsymbol{b}=$ 40\% (Eq. 5-3): séries mensais com decomposição multiplicativa

Tabela 5.23 - MSE, MAD e SNR para valores de ' $b$ ', sendo $\boldsymbol{A} / \boldsymbol{s}=6$,

$\boldsymbol{k}=24$ (Eq. 5-2): séries trimestrais com decomposição aditiva $(\boldsymbol{N}=43) 176$

Tabela 5.24 - MSE, MAD e SNR para valores de ' $\boldsymbol{k}$ ', sendo $\boldsymbol{A} / \boldsymbol{s}=6$, $\boldsymbol{b}=40 \%$ (Eq. 5-2): séries trimestrais com decomposição aditiva $(\boldsymbol{N}=43) 178$

Tabela 5.25 - MSE, MAD e SNR para valores de ' $A / \boldsymbol{s}$ ', sendo $\boldsymbol{k}=24$, $\boldsymbol{b}=40 \%$ (Eq. 5-2): séries trimestrais com decomposição aditiva $(\boldsymbol{N}=43) 179$

Tabela 5.26 - MSE, MAD e SNR para valores de ' $b$ ', sendo $\boldsymbol{A} / \boldsymbol{s}=6$, $\boldsymbol{k}=24$ - séries trimestrais com decomposição multiplicativa

Tabela 5.27 - MSE, MAD e SNR para valores de ' $\boldsymbol{k}$ ', sendo $\boldsymbol{A} / \boldsymbol{s}=6$, $\boldsymbol{b}=40 \%$ - séries trimestrais com decomposição multiplicativa

Tabela 5.28 - MSE e MAD para valores de ' $A / \boldsymbol{s}$ ', sendo $\boldsymbol{k}=24, \boldsymbol{b}=$ $40 \%$ - séries trimestrais com decomposição multiplicativa

Tabela 6.1 - Filtros S-WLSC mensais para cada N

Tabela 6.2 - Valores de SQ e $\boldsymbol{\gamma} \boldsymbol{s}$ para os filtros S-WLSC mensais, para $\boldsymbol{k}=\mathbf{7 2}$

Tabela 6.3 - SNRs e razão entre as SNRs, para $A / s=6, b=40 \%$ $\boldsymbol{k}=96$ (Eq. 5-1), considerando todos os filtros mensais: comparação entre S-WLSC e S-WLS

Tabela 6.4 - SNR S-WLSC e SNR S-WLS, variando $b$ para $A / s=6$, $\boldsymbol{k}=96$ e $\boldsymbol{N}=121$ e 189

Tabela 6.5 - SNR S-WLSC e SNR S-WLS, variando $k$ para $\boldsymbol{A} / \boldsymbol{s}=6$, $\boldsymbol{b}=40 \%$ e $\boldsymbol{N}=121$ e 189 
Tabela 6.6 - SNR S-WLSC e SNR S-WLS, variando $\boldsymbol{A} / \boldsymbol{s}$, para $\boldsymbol{k}=96$, $b=40 \%$ e $N=121$ e 189

Tabela 6.7 - Valores da componente de tendência, desvio-padrão da irregular e amplitude da componente sazonal

Tabela 6.8 - MSE S-WLSC e MSE S-WLS, $\left(\times 10^{6}\right)$, variando ' $\boldsymbol{b}$ ' para $\boldsymbol{A} / \boldsymbol{s}=6, \boldsymbol{k}=96(\boldsymbol{N}=121$ e 189$)$. Valores baseados no teste $t$, unilateral, de comparação de médias

Tabela 6.9 - MSE do S-WLSC e MSE do S-WLS, $\left(\times 10^{6}\right)$, variando ' $\boldsymbol{k}$ ' para $A / s=6, b=40 \%(N=121$ e 189$)$. Valores baseados no teste $t$, unilateral, de comparação de médias

Tabela 6.10 - MSE S-WLSC e MSE S-WLS, $\left(\times 10^{6}\right)$, variando ' $A / s^{\prime}$ ', para $\boldsymbol{k}=96, \boldsymbol{b}=40 \%(\boldsymbol{N}=121$ e 189). Valores baseados no teste $t$

Tabela 6.11 - Filtros S-WLSC trimestrais para cada N

Tabela 6.12 - Valores de SQ e $\boldsymbol{\gamma} \boldsymbol{s}$ para os filtros S-WLSC trimestrais, para $\boldsymbol{k}=24$

Tabela 6.13 - SNRs e razão entre as SNRs, para $\boldsymbol{A} / \boldsymbol{s}=6, \boldsymbol{b}=40 \% \boldsymbol{k}=$ 32 (Eq. 5-2), considerando todos os filtros trimestrais: comparação entre S-WLSC e S-WLS

Tabela 6.14 - SNR S-WLSC e SNR S-WLS, variando $b$ para $\boldsymbol{A} / \boldsymbol{s}=6$, $\boldsymbol{k}=32$ e $\boldsymbol{N}=43$ e 65

Tabela 6.15 - SNR S-WLSC e SNR S-WLS, variando k para $A / s=6$, $b=40 \%$ e $N=43$ e 65

Tabela 6.16 - SNR S-WLSC e SNR S-WLS, variando $\boldsymbol{A} / \boldsymbol{s}$, para $\boldsymbol{k}=$ $32, b=40 \%$ e $N=43$ e 65

Tabela 6.17 - Valores da componente de tendência, desvio-padrão da irregular e amplitude da componente sazonal

Tabela 6.18 - SNR S-WLSC e SNR S-WLS, $\left(\times 10^{6}\right)$, variando ' $b$ ' para $A / s=6, k=32(N=43$ e 65$)$

Tabela 6.19 - MSE do S-WLSC e MSE do S-WLS, $\left(\times 10^{6}\right)$, variando ' $\boldsymbol{k}$ ' para $A / s=6, b=40 \%(N=43$ e 65$)$

Tabela 6.20 - MSE S-WLSC e MSE S-WLS, $\left(\times 10^{6}\right)$, variando ' $\boldsymbol{A} / \boldsymbol{s}^{\text {', }}$ para $\boldsymbol{k}=32, \boldsymbol{b}=40 \%(\boldsymbol{N}=43$ e 65). Valores baseados no teste $t$, unilateral, de comparação de médias 


\section{Lista de gráficos}

Gráfico 3.1 - Componente sazonal de uma série mensal sem sazonalidade móvel

Gráfico 3.2 - Comparação entre a resposta em magnitude do filtro X-11 (linha tracejada) e o espectro de uma série mensal sem sazonalidade móvel (linha contínua)

Gráfico 3.3 - Componente sazonal de uma série mensal com sazonalidade móvel

Gráfico 3.4 - Comparação entre o espectro de uma série mensal com sazonalidade móvel (setas) e a resposta em magnitude do filtro X-11 (linha tracejada)

Gráfico 3.5 - Componente sazonal artificial e estimativa obtida pelo método $\mathrm{X}-11$

Gráfico 4.1 - Comparação entre a resposta em magnitude do filtro S-WLS (linha contínua) e o espectro de uma série mensal com sazonalidade móvel (setas)

Gráfico 4.2 - Comparação entre a resposta em magnitude do filtro S-WLS, a resposta em magnitude do filtro $X-11$ e o espectro de uma série com sazonalidade móvel (representado pelas setas)

Gráfico 4.3 - Quando a resposta em magnitude do filtro S-WLS se aproxima da resposta em magnitude do filtro $\mathrm{X}-11$

Gráfico 5.1 - Componente sazonal artificial para uma série mensal com sazonalidade móvel, no domínio do tempo

Gráfico 5.2 - Componente Sazonal: Produção Industrial Mensal Indústria Geral do Espírito Santo

Gráfico 5.3 - Histograma de 'b' (Eq. 5-1) das séries mensais aditivas

Gráfico 5.4 - Histograma de ' $A / \boldsymbol{s}^{\prime}$ (Eq. 5-1) das séries mensais aditivas 112

Gráfico 5.5 - Histograma de ' $\boldsymbol{k}$ ' (Eq. 5-1) das séries mensais aditivas 112

Gráfico 5.6 - Histograma de 'b' (Eq. 5-3) das séries mensais multiplicativas

Gráfico 5.7 - Histograma de ' $\boldsymbol{A} / \boldsymbol{s}^{\prime}$ ' (Eq. 5-3) das séries mensais multiplicativas 
Gráfico 5.8 - Histograma de ' $\boldsymbol{k}$ ' (Eq. 5-3) das séries mensais

multiplicativas

Gráfico 5.9 - Histograma de 'b' (Eq. 5-4) das séries trimestrais multiplicativas

Gráfico 5.10 - Histograma de 'A/s' (Eq. 5-4) das séries trimestrais multiplicativas

Gráfico 5.11 - Histograma de ' $k$ ' (Eq. 5-4) das séries trimestrais multiplicativas

Gráfico 5.12 - Respostas em magnitude do filtro X-11 para diferentes MMs: séries mensais. A linha contínua representa o filtro MMs $3 \times 3$; a linha tracejada se refere ao filtro MMs $3 \times 5$; a linha pontilhada indica o filtro MMs $3 \times 9$

Gráfico 5.13 - Valores de SQ, $\boldsymbol{\gamma} \mathbf{0}$ e $|\mathbf{1}-\boldsymbol{\gamma} \mathbf{1}|$ para diversos pesos (wo) 129

Gráfico 5.14 - Resposta em magnitude do filtro S-WLS mensal para $\mathrm{N}=121$

Gráfico 5.15 - Resposta em magnitude do filtro S-WLS mensal para $\mathrm{N}=193$

Gráfico 5.16 - Resposta em magnitude do filtro S-WLS e do filtro X-11 para $\mathrm{N}=121$, série mensal

Gráfico 5.17 - Resposta em magnitude do filtro S-WLS e do filtro X-11 para $\mathrm{N}=193$, série mensal

Gráfico 5.18 - Resposta em magnitude do filtro S-WLS trimestral para $\mathrm{N}=41$

Gráfico 5.19 - Resposta em magnitude do filtro S-WLS trimestral para $\mathrm{N}=67$

Gráfico 5.20 - Resposta em magnitude do filtro S-WLS e do filtro X-11 para $\mathrm{N}=41$, série trimestral

Gráfico 5.21 - Resposta em magnitude do filtro S-WLS e do filtro X-11 para $\mathrm{N}=67$, série trimestral

Gráfico 5.22 - Média da MSE nas simulações com o filtro X-11 e com o filtro S-WLS, para valores de ' $b$ ', considerando $A / s=6$ e $\boldsymbol{k}=72$ (Eq. 5-1)

Gráfico 5.23 - Relação entre ' $b$ ' e a SNR do filtro S-WLS e do filtro $\mathrm{X}-11$, considerando $\boldsymbol{A} / \boldsymbol{s}=6$ e $\boldsymbol{k}=72$ (Eq. 5-1) 
Gráfico 5.24 - Média da MSE nas simulações com o filtro X-11 e com o filtro S-WLS, para valores de ' $k$ ', considerando $A / s=6$ e $b=40 \%$ (Eq. 5-1)

Gráfico 5.25 - Relação entre ' $k$ ' e a SNR do filtro S-WLS e do filtro $\mathrm{X}-11$, considerando $A / s=6$ e $\boldsymbol{b}=40 \%$ (Eq. 5-1)

Gráfico 5.26 - Média da MSE nas simulações com o filtro X-11 e com o filtro S-WLS, para valores de ' $\boldsymbol{A} / \boldsymbol{s}$ ', considerando $\boldsymbol{k}=72$ e $\boldsymbol{b}=40 \%$ (Eq. 5-1)

Gráfico 5.27 - Relação entre ' $A / s$ ' e a SNR do filtro S-WLS e do filtro $\mathrm{X}-11$, considerando $\boldsymbol{k}=72$ e $\boldsymbol{b}=40 \%$ (Eq. 5-1)

Gráfico 5.28 - Valores de ' $b$ ' a partir do qual o filtro S-WLS supera o $\mathrm{X}-11$, na SNR, para vários valores de ' $\boldsymbol{k}$ ', considerando $A / s=6$ (Eq. 5-1)

Gráfico 5.29 - Valores de ' $b$ ' a partir do qual o filtro S-WLS supera o $\mathrm{X}-11$, na SNR, para vários valores de ' $\boldsymbol{A} / \boldsymbol{s}$ ', considerando $\boldsymbol{k}=72$ (Eq. 5-1)

Gráfico 5.30 - Espectro da irregular da série dessazonalizada pelo filtro S-WLS, com: $\boldsymbol{A} / \boldsymbol{s}=6, \boldsymbol{k}=72, \boldsymbol{b}=40 \%$ e $\boldsymbol{N}=121$

Gráfico 5.31 - Espectro da irregular da série dessazonalizada pelo $\mathrm{X}-11$, com: $\boldsymbol{A} / \boldsymbol{s}=6, \boldsymbol{k}=72, \boldsymbol{b}=40 \%$ e $\boldsymbol{N}=121$

Gráfico 5.32 - Espectro da irregular da série dessazonalizada pelo filtro S-WLS, com: $\boldsymbol{A} / \boldsymbol{s}=6, \boldsymbol{k}=72, \boldsymbol{b}=25 \%$ e $\boldsymbol{N}=121$

Gráfico 5.33 - Espectro da irregular da série dessazonalizada pelo $\mathrm{X}-11$, com: $\boldsymbol{A} / \boldsymbol{s}=6, \boldsymbol{k}=72, \boldsymbol{b}=25 \%$ e $\boldsymbol{N}=121$

Gráfico 5.34 - Espectro da irregular da série dessazonalizada pelo filtro S-WLS, com: $\boldsymbol{A} / \boldsymbol{s}=6, \boldsymbol{k}=72, \mathrm{~b}=10 \%$ e $\boldsymbol{N}=121$

Gráfico 5.35 - Espectro da irregular da série dessazonalizada pelo $\mathrm{X}-11$, com: $\boldsymbol{A} / \boldsymbol{s}=6, \boldsymbol{k}=72, \boldsymbol{b}=10 \%$ e $\boldsymbol{N}=121$

Gráfico 5.36 - Espectro da irregular da série dessazonalizada pelo filtro S-WLS, com: $\boldsymbol{A} / \boldsymbol{s}=6, \boldsymbol{k}=96, \boldsymbol{b}=40 \%$ e $\boldsymbol{N}=121$

Gráfico 5.37 - Espectro da irregular da série dessazonalizada pelo $\mathrm{X}-11$, com: $\boldsymbol{A} / \boldsymbol{s}=6, \boldsymbol{k}=96, \boldsymbol{b}=40 \%$ e $\boldsymbol{N}=121$

Gráfico 5.38 - Espectro da irregular da série dessazonalizada pelo filtro S-WLS, com: $\boldsymbol{A} / \boldsymbol{s}=6, \boldsymbol{k}=96, \boldsymbol{b}=25 \%$ e $\boldsymbol{N}=121$

Gráfico 5.39 - Espectro da irregular da série dessazonalizada pelo $\mathrm{X}-11$, com: $\boldsymbol{A} / \boldsymbol{s}=6, \boldsymbol{k}=96, \boldsymbol{b}=25 \%$ e $\boldsymbol{N}=121$ 
Gráfico 5.40 - Espectro da irregular da série dessazonalizada pelo filtro S-WLS, com: $\boldsymbol{A} / \boldsymbol{s}=6, \boldsymbol{k}=96, \boldsymbol{b}=10 \%$ e $\boldsymbol{N}=121$

Gráfico 5.41 - Espectro da irregular da série dessazonalizada pelo $\mathrm{X}-11$, com: $\boldsymbol{A} / \boldsymbol{s}=6, \boldsymbol{k}=96, \boldsymbol{b}=10 \%$ e $\boldsymbol{N}=121$

Gráfico 5.42 - Espectro da irregular da série dessazonalizada pelo filtro S-WLS, com: $\boldsymbol{A} / \boldsymbol{s}=6, \boldsymbol{k}=180, \boldsymbol{b}=40 \%$ e $\boldsymbol{N}=121$

Gráfico 5.43 - Espectro da irregular da série dessazonalizada pelo $\mathrm{X}-11$, com: $\boldsymbol{A} / \boldsymbol{s}=6, \boldsymbol{k}=180, \boldsymbol{b}=40 \%$ e $\boldsymbol{N}=121$

Gráfico 5.44 - Espectro da irregular da série dessazonalizada pelo filtro S-WLS, com: $\boldsymbol{A} / \boldsymbol{s}=6, \boldsymbol{k}=180, \boldsymbol{b}=10 \%$ e $\boldsymbol{N}=121$

Gráfico 5.45 - Espectro da irregular da série dessazonalizada pelo $\mathrm{X}-11$, com: $\boldsymbol{A} / \boldsymbol{s}=6, \boldsymbol{k}=180, \boldsymbol{b}=10 \%$ e $\boldsymbol{N}=121$

Gráfico 5.46 - Média da MSE $\left(\times 10^{6}\right)$ com o filtro X-11 e com o filtro S-WLS, para valores de ' $b$ ', considerando $\boldsymbol{A} / \boldsymbol{s}=6$ e $\boldsymbol{k}=72$ (Eq. 5-3)

Gráfico 5.47 - Média da MSE $\left(\times 10^{6}\right)$ com o filtro X-11 e com o filtro S-WLS, para valores de ' $\boldsymbol{k}$ ', considerando $\boldsymbol{A} / \boldsymbol{s}=6$ e $\boldsymbol{b}=40 \%$ (Eq. 5-3) 164

Gráfico 5.48 - Média da MSE $\left(\times 10^{6}\right)$ com o filtro X-11 e com o filtro S-WLS, para valores de ' $A / \boldsymbol{s}$ ', considerando $\boldsymbol{k}=72$ e $\boldsymbol{b}=40 \%$ (Eq. 5-3)

Gráfico 5.49 - Valores de ' $b$ ' a partir do qual o filtro S-WLS supera o $\mathrm{X}-11$, na MSE, para valores de ' $k$ ', considerando $A / s=6$ (Eq. 5-3)

Gráfico 5.50 - Valores de ' $b$ ' a partir do qual o filtro S-WLS supera o $\mathrm{X}-11$, na MSE, para vários valores de ' $\boldsymbol{A} / \boldsymbol{s}$ ', considerando $\boldsymbol{k}=72$ (Eq. 5-3)

Gráfico 5.51 - Espectro da irregular da série dessazonalizada pelo filtro S-WLS, com: $\boldsymbol{A} / \boldsymbol{s}=6, \boldsymbol{k}=72, \boldsymbol{b}=40 \%$ e $\boldsymbol{N}=121$

Gráfico 5.52 - Espectro da irregular da série dessazonalizada pelo $\mathrm{X}-11$, com: $\boldsymbol{A} / \boldsymbol{s}=6, \boldsymbol{k}=72, \boldsymbol{b}=40 \%$ e $\boldsymbol{N}=121$

Gráfico 5.53 - Espectro da irregular da série dessazonalizada pelo filtro S-WLS, com: $\boldsymbol{A} / \boldsymbol{s}=6, \boldsymbol{k}=72, \boldsymbol{b}=25 \%$ e $\boldsymbol{N}=121$

Gráfico 5.54 - Espectro da irregular da série dessazonalizada pelo $\mathrm{X}-11$, com: $\boldsymbol{A} / \boldsymbol{s}=6, \boldsymbol{k}=72$ e $\boldsymbol{b}=25 \%$ e $\boldsymbol{N}=121$

Gráfico 5.55 - Espectro da irregular da série dessazonalizada pelo filtro S-WLS, com: $\boldsymbol{A} / \boldsymbol{s}=6, \boldsymbol{k}=72, \boldsymbol{b}=10 \%$ e $\boldsymbol{N}=121$ 
Gráfico 5.56 - Espectro da irregular da série dessazonalizada pelo $\mathrm{X}-11$, com: $\boldsymbol{A} / \boldsymbol{s}=6, \boldsymbol{k}=72, \boldsymbol{b}=10 \%$ e $\boldsymbol{N}=121$

Gráfico 5.57 - Espectro da irregular da série dessazonalizada pelo filtro S-WLS, com: $\boldsymbol{A} / \boldsymbol{s}=6, \boldsymbol{k}=96, \boldsymbol{b}=40 \%$ e $\boldsymbol{N}=121$

Gráfico 5.58 - Espectro da irregular da série dessazonalizada pelo $\mathrm{X}-11$, com: $\boldsymbol{A} / \boldsymbol{s}=6, \boldsymbol{k}=96, \boldsymbol{b}=40 \%$ e $\boldsymbol{N}=121$

Gráfico 5.59 - Espectro da irregular da série dessazonalizada pelo filtro S-WLS, com: $\boldsymbol{A} / \boldsymbol{s}=6, \boldsymbol{k}=96, \boldsymbol{b}=25 \%$ e $\boldsymbol{N}=121$

Gráfico 5.60 - Espectro da irregular da série dessazonalizada pelo $\mathrm{X}-11$, com: $\boldsymbol{A} / \boldsymbol{s}=6, \boldsymbol{k}=96, \boldsymbol{b}=25 \%$ e $\boldsymbol{N}=121$

Gráfico 5.61 - Espectro da irregular da série dessazonalizada pelo filtro S-WLS, com: $\boldsymbol{A} / \boldsymbol{s}=6, \boldsymbol{k}=96, \boldsymbol{b}=10 \%$ e $\boldsymbol{N}=121$

Gráfico 5.62 - Espectro da irregular da série dessazonalizada pelo $\mathrm{X}-11$, com: $\boldsymbol{A} / \boldsymbol{s}=6, \boldsymbol{k}=96, \boldsymbol{b}=10 \%$ e $\boldsymbol{N}=121$

Gráfico 5.63 - Espectro da irregular da série dessazonalizada pelo filtro S-WLS, com tendência, e: $\boldsymbol{A} / \boldsymbol{s}=6,5 ; \boldsymbol{k}=72 ; \boldsymbol{b}=40 \%(\mathrm{~N}=121)$

Gráfico 5.64 - Espectro da irregular da série dessazonalizada pelo filtro S-WLS, com tendência, e: $\boldsymbol{A} / \boldsymbol{s}=6,5 ; \boldsymbol{k}=96 ; \boldsymbol{b}=40 \%(\mathrm{~N}=121)$

Gráfico 5.65 - Espectro da irregular da série dessazonalizada pelo filtro S-WLS, com tendência, e: $\boldsymbol{A} / \boldsymbol{s}=6,5 ; \boldsymbol{k}=72 ; \boldsymbol{b}=10 \%(\mathrm{~N}=121)$

Gráfico 5.66 - Espectro da irregular da série dessazonalizada pelo filtro S-WLS, com tendência, e: $\boldsymbol{A} / \boldsymbol{s}=6,5 ; \boldsymbol{k}=96 ; \boldsymbol{b}=10 \%(\mathrm{~N}=121)$

Gráfico 5.67 - Média da MSE nas simulações com o filtro X-11 e com o filtro S-WLS, para valores de ' $b$ ', considerando $A / s=6, k=24$ e $N=$ 43 (Eq. 5-2)

Gráfico 5.68 - Relação entre ' $b$ ' e a SNR do filtro S-WLS e do filtro $\mathrm{X}-11$, considerando $A / s=6, k=24$ e $N=43$ (Eq. 5-2)

Gráfico 5.69 - Média da MSE nas simulações com o filtro X-11 e com o filtro S-WLS, para valores de ' $k$ ', considerando $A / s=6$ e $b=40 \%$ $(N=43)$

Gráfico 5.70 - Relação entre ' $k$ ' e a SNR do filtro S-WLS e do filtro $\mathrm{X}-11$, considerando ' $A / s$ ' $=6$ e $\boldsymbol{b}=40 \%(\boldsymbol{N}=43)$

Gráfico 5.71 - Média da MSE nas simulações com o filtro X-11 e com o filtro S-WLS, para valores de ' $A / s$ ', considerando $k=24$ e $b=40 \%$ $(N=43)$ 
Gráfico 5.72 - Relação entre ' $\boldsymbol{A} / \boldsymbol{s}$ ' e a SNR do filtro S-WLS e do filtro $\mathrm{X}-11$, considerando $\boldsymbol{k}=24$ e $\boldsymbol{b}=40 \%(\boldsymbol{N}=43)$

Gráfico 5.73 - Valores de ' $b$ ' a partir do qual o filtro S-WLS supera o $\mathrm{X}-11$, na SNR, para vários valores de ' $\boldsymbol{k}$ ', considerando $\boldsymbol{A} / \boldsymbol{s}=6 \mathrm{e}$ $N=43$

Gráfico 5.74 - Valores de ' $b$ ' a partir do qual o filtro S-WLS supera o $\mathrm{X}-11$, na SNR, para vários valores de ' $\boldsymbol{A} / \boldsymbol{s}$ ', considerando $\boldsymbol{k}=24 \mathrm{e}$ $N=43$

Gráfico 5.75 - Espectro da irregular da série dessazonalizada pelo filtro S-WLS, com: $\boldsymbol{A} / \boldsymbol{s}=6, \boldsymbol{k}=24, \boldsymbol{b}=40 \%$ e $\boldsymbol{N}=43$

Gráfico 5.76 - Espectro da irregular da série dessazonalizada pelo $\mathrm{X}-11$, com: $\boldsymbol{A} / \boldsymbol{s}=6, \boldsymbol{k}=24, \boldsymbol{b}=40 \%$ e $\boldsymbol{N}=43$

Gráfico 5.77 - Espectro da irregular da série dessazonalizada pelo filtro S-WLS, com: $\boldsymbol{A} / \boldsymbol{s}=6, \boldsymbol{k}=24, \boldsymbol{b}=25 \%$ e $\boldsymbol{N}=43$

Gráfico 5.78 - Espectro da irregular da série dessazonalizada pelo $\mathrm{X}-11$, com: $\boldsymbol{A} / \boldsymbol{s}=6, \boldsymbol{k}=24, \boldsymbol{b}=25 \%$ e $\boldsymbol{N}=43$

Gráfico 5.79 - Espectro da irregular da série dessazonalizada pelo filtro S-WLS, com: $\boldsymbol{A} / \boldsymbol{s}=6, \boldsymbol{k}=24$ e $\boldsymbol{b}=10 \%$ e $\boldsymbol{N}=43$

Gráfico 5.80 - Espectro da irregular da série dessazonalizada pelo $\mathrm{X}-11$, com: $\boldsymbol{A} / \boldsymbol{s}=6, \boldsymbol{k}=24, \boldsymbol{b}=10 \%$ e $\boldsymbol{N}=43$

Gráfico 5.81 - Espectro da irregular da série dessazonalizada pelo filtro S-WLS, com: $\boldsymbol{A} / \boldsymbol{s}=6, \boldsymbol{k}=32, \boldsymbol{b}=40 \%$ e $\boldsymbol{N}=43$

Gráfico 5.82 - Espectro da irregular da série dessazonalizada pelo $\mathrm{X}-11$, com: $\boldsymbol{A} / \boldsymbol{s}=6, \boldsymbol{k}=32, \boldsymbol{b}=40 \%$ e $\boldsymbol{N}=43$

Gráfico 5.83 - Espectro da irregular da série dessazonalizada pelo filtro S-WLS, com: $\boldsymbol{A} / \boldsymbol{s}=6, \boldsymbol{k}=32, \boldsymbol{b}=25 \%$ e $\boldsymbol{N}=43$

Gráfico 5.84 - Espectro da irregular da série dessazonalizada pelo $\mathrm{X}-11$, com: $\boldsymbol{A} / \boldsymbol{s}=6, \boldsymbol{k}=32, \boldsymbol{b}=25 \%$ e $\boldsymbol{N}=43$

Gráfico 5.85 - Espectro da irregular da série dessazonalizada pelo filtro S-WLS, com: $\boldsymbol{A} / \boldsymbol{s}=6, \boldsymbol{k}=32, \boldsymbol{b}=10 \%$ e $\boldsymbol{N}=43$

Gráfico 5.86 - Espectro da irregular da série dessazonalizada pelo $\mathrm{X}-11$, com: $\boldsymbol{A} / \boldsymbol{s}=6, \boldsymbol{k}=32, \boldsymbol{b}=10 \%$ e $\boldsymbol{N}=43$

Gráfico 5.87 - Espectro da irregular da série dessazonalizada pelo filtro S-WLS, com: $\boldsymbol{A} / \boldsymbol{s}=6, \boldsymbol{k}=80, \boldsymbol{b}=40 \%$ e $\boldsymbol{N}=43$ 
Gráfico 5.88 - Espectro da irregular da série dessazonalizada pelo $\mathrm{X}-11$, com: $\boldsymbol{A} / \boldsymbol{s}=6, \boldsymbol{k}=80, \boldsymbol{b}=40 \%$ e $\boldsymbol{N}=43$

Gráfico 5.89 - Espectro da irregular da série dessazonalizada pelo filtro S-WLS, com: $\boldsymbol{A} / \boldsymbol{s}=6, \boldsymbol{k}=80, \boldsymbol{b}=10 \%$ e $\boldsymbol{N}=43$

Gráfico 5.90 - Espectro da irregular da série dessazonalizada pelo $\mathrm{X}-11$, com: $\boldsymbol{A} / \boldsymbol{s}=6, \boldsymbol{k}=80, \boldsymbol{b}=10 \%$ e $\boldsymbol{N}=43$

Gráfico 5.91 - Média da MSE $\left(\times 10^{6}\right)$ com o filtro X-11 e com o filtro S-WLS, para valores de ' $b$ ', considerando $A / s=6$ e $\boldsymbol{k}=24$

Gráfico 5.92 - Média da MSE $\left(\times 10^{6}\right)$ com o filtro X-11 e com o filtro S-WLS, para valores de ' $\boldsymbol{k}$ ', considerando $\boldsymbol{A} / \boldsymbol{s}=6$ e $\boldsymbol{b}=40 \%$

Gráfico 5.93 - Média da MSE $\left(\times 10^{6}\right)$ com o filtro X-11 e com o filtro S-WLS, para valores de ' $A / s$ ', considerando $k=24$ e $\boldsymbol{b}=40 \%$

Gráfico 5.94 - Valores de ' $b$ ' a partir do qual o filtro S-WLS supera o $\mathrm{X}-11$, na MSE, para valores de ' $k$ ', considerando $A / s=6$

Gráfico 5.95 - Valores de ' $b$ ' a partir do qual o filtro S-WLS supera o $\mathrm{X}-11$, na MSE, para vários valores de ' $\boldsymbol{A} / \boldsymbol{s}$ ', considerando $\boldsymbol{k}=24$

Gráfico 5.96 - Espectro da irregular da série dessazonalizada pelo filtro S-WLS, com: $\boldsymbol{A} / \boldsymbol{s}=6, \boldsymbol{k}=24, \boldsymbol{b}=40 \%$ e $\boldsymbol{N}=43$

Gráfico 5.97 - Espectro da irregular da série dessazonalizada pelo $\mathrm{X}-11$, com: $\boldsymbol{A} / \boldsymbol{s}=6, \boldsymbol{k}=24, \boldsymbol{b}=40 \%$ e $\boldsymbol{N}=43$

Gráfico 5.98 - Espectro da irregular da série dessazonalizada pelo filtro S-WLS, com: $\boldsymbol{A} / \boldsymbol{s}=6, \boldsymbol{k}=24, \boldsymbol{b}=25 \%$ e $\boldsymbol{N}=43$

Gráfico 5.99 - Espectro da irregular da série dessazonalizada pelo $\mathrm{X}-11, \mathrm{com}: \boldsymbol{A} / \boldsymbol{s}=6, \boldsymbol{k}=24, \boldsymbol{b}=25 \%$ e $\boldsymbol{N}=43$

Gráfico 5.100 - Espectro da irregular da série dessazonalizada pelo filtro S-WLS, com: $\boldsymbol{A} / \boldsymbol{s}=6, \boldsymbol{k}=24, \boldsymbol{b}=10 \%$ e $\boldsymbol{N}=43$

Gráfico 5.101 - Espectro da irregular da série dessazonalizada pelo $\mathrm{X}-11$, com: $\boldsymbol{A} / \boldsymbol{s}=6, \boldsymbol{k}=24, \boldsymbol{b}=10 \%$ e $\boldsymbol{N}=43$

Gráfico 5.102 - Espectro da irregular da série dessazonalizada pelo filtro S-WLS, com: $\boldsymbol{A} / \boldsymbol{s}=6, \boldsymbol{k}=32, \boldsymbol{b}=40 \%$ e $\boldsymbol{N}=43$

Gráfico 5.103 - Espectro da irregular da série dessazonalizada pelo $\mathrm{X}-11$, com: $\boldsymbol{A} / \boldsymbol{s}=6, \boldsymbol{k}=32, \boldsymbol{b}=40 \%$ e $\boldsymbol{N}=43$

Gráfico 5.104 - Espectro da irregular da série dessazonalizada pelo filtro S-WLS, com: $\boldsymbol{A} / \boldsymbol{s}=6, \boldsymbol{k}=32, \boldsymbol{b}=25 \%$ e $\boldsymbol{N}=43$ 
Gráfico 5.105 - Espectro da irregular da série dessazonalizada pelo $\mathrm{X}-11$, com: $\boldsymbol{A} / \boldsymbol{s}=6, \boldsymbol{k}=32, \boldsymbol{b}=25 \%$ e $\boldsymbol{N}=43$

Gráfico 5.106 - Espectro da irregular da série dessazonalizada pelo filtro S-WLS, com: $\boldsymbol{A} / \boldsymbol{s}=6, \boldsymbol{k}=32, \boldsymbol{b}=10 \%$ e $\boldsymbol{N}=43$

Gráfico 5.107 - Espectro da irregular da série dessazonalizada pelo $\mathrm{X}-11$, com: $\boldsymbol{A} / \boldsymbol{s}=6, \boldsymbol{k}=32, \boldsymbol{b}=10 \%$ e $\boldsymbol{N}=43$

Gráfico 5.108 - Espectro da irregular da série dessazonalizada pelo filtro S-WLS, com tendência, e: $\boldsymbol{A} / \boldsymbol{s}=6,5 ; \boldsymbol{k}=24 ; \boldsymbol{b}=40 \%(\mathrm{~N}=43)$

Gráfico 5.109 - Espectro da irregular da série dessazonalizada pelo filtro S-WLS, com tendência, e: $\boldsymbol{A} / \boldsymbol{s}=6,5 ; \boldsymbol{k}=32 ; \boldsymbol{b}=40 \%(\mathrm{~N}=43)$

Gráfico 5.110 - Espectro da irregular da série dessazonalizada pelo filtro S-WLS, com tendência, e: $\boldsymbol{A} / \boldsymbol{s}=6,5 ; \boldsymbol{k}=24 ; \boldsymbol{b}=10 \%(\mathrm{~N}=43)$

Gráfico 5.111 - Espectro da irregular da série dessazonalizada pelo filtro S-WLS, com tendência, e: $\boldsymbol{A} / \boldsymbol{s}=6,5 ; \boldsymbol{k}=32 ; \boldsymbol{b}=10 \%(\mathrm{~N}=43)$

Gráfico 6.1 - Resposta em magnitude do filtro S-WLSC mensal $(\mathrm{N}=117)$

Gráfico 6.2 - Resposta em magnitude do filtro S-WLSC, do S-WLS e o esperado $(\boldsymbol{N}=117)$

205

Gráfico 6.3 - Banda passante dos filtros S-WLSC e S-WLS ( $N=117)$

206

Gráfico 6.4 - Resposta em magnitude do filtro S-WLSC mensal

$(\mathrm{N}=121)$

207

Gráfico 6.5 - Resposta em magnitude do filtro S-WLSC, do S-WLS e o esperado $(\boldsymbol{N}=121)$

207

Gráfico 6.6 - Banda passante dos filtros S-WLSC e S-WLS ( $N=121)$

208

Gráfico 6.7 - Resposta em magnitude do filtro S-WLSC mensal $(\mathrm{N}=131)$

209

Gráfico 6.8 - Resposta em magnitude do filtro S-WLSC, do S-WLS e o esperado $(\boldsymbol{N}=131)$

Gráfico 6.9 - Banda passante dos filtros S-WLSC e S-WLS ( $N=131)$

Gráfico 6.10 - Resposta em magnitude do filtro S-WLSC mensal $(\mathrm{N}=141)$

Gráfico 6.11 - Resposta em magnitude do filtro S-WLSC, do S-WLS e o esperado $(\boldsymbol{N}=141)$ 
Gráfico 6.12 - Banda passante dos filtros S-WLSC e S-WLS (N=141) 212

Gráfico 6.13 - Resposta em magnitude do filtro S-WLSC mensal

$(\mathrm{N}=145)$

Gráfico 6.14 - Resposta em magnitude do filtro S-WLSC, do S-WLS e o esperado $(\boldsymbol{N}=145)$

Gráfico 6.15 - Banda passante dos filtros S-WLSC e S-WLS (N=145) 214

Gráfico 6.16 - Resposta em magnitude do filtro S-WLSC ( $N=145)$, do S-WLS $(\boldsymbol{N}=155)$ e o esperado

Gráfico 6.17 - Banda passante dos filtros S-WLSC (N=145) e S-WLS $(N=155)$

Gráfico 6.18 - Resposta em magnitude do filtro S-WLSC ( $\mathrm{N}=145)$, do S-WLS $(\boldsymbol{N}=189)$ e o esperado

Gráfico 6.19 - Banda passante dos filtros S-WLSC (N=145) e S-WLS $(N=189)$

Gráfico 6.20 - Resposta em magnitude do filtro S-WLSC mensal $(\mathrm{N}=193)$

Gráfico 6.21 - Resposta em magnitude do filtro S-WLSC, do S-WLS e o esperado $(\boldsymbol{N}=193)$

Gráfico 6.22 - Banda passante dos filtros S-WLSC e S-WLS ( $N=193)$

Gráfico 6.23 - Resposta em magnitude do filtro S-WLSC ( $\mathrm{N}=193)$, do S-WLS $(\boldsymbol{N}=203)$ e o esperado

Gráfico 6.24 - Banda passante dos filtros S-WLSC (N=193) e S-WLS $(N=203)$

Gráfico 6.25 - Razão entre a SNR do S-WLSC e a SNR do S-WLS considerando $\boldsymbol{A} / \boldsymbol{s}=6$ e $\boldsymbol{k}=96$, para diversos valores de $\boldsymbol{b}$ :

comparação entre $\mathrm{N}=121$ e $\mathrm{N}=189$

Gráfico 6.26 - SNR S-WLSC e SNR S-WLS considerando A/s $=6 \mathrm{e}$ $k=96$, para diversos valores de $b(\mathrm{~N}=121)$

Gráfico 6.27 - SNR S-WLSC e SNR S-WLS considerando A/s $=6$ e $k=96$, para diversos valores de $b(N=189)$

Gráfico 6.28 - Razão entre a SNR do S-WLSC e a do SNR S-WLS considerando $\boldsymbol{A} / \boldsymbol{s}=6$ e $\boldsymbol{b}=40 \%$, para diversos valores de $\boldsymbol{k}$ :

comparação entre $\mathrm{N}=121$ e $\mathrm{N}=189$ 
Gráfico 6.29 - SNR S-WLSC e SNR S-WLS considerando $\boldsymbol{A} / \boldsymbol{s}=6 \mathrm{e}$ $\boldsymbol{b}=40 \%$, para diversos valores de $\boldsymbol{k}(\mathrm{N}=121)$

Gráfico 6.30 - SNR S-WLSC e SNR S-WLS considerando $A / s=6$ e $\boldsymbol{b}=40 \%$, para diversos valores de $\boldsymbol{k}(\mathrm{N}=189)$

Gráfico 6.31 - Razão entre a SNR do S-WLSC e a SNR do S-WLS considerando $\boldsymbol{k}=96, \boldsymbol{b}=40 \%$, para diversos valores de $\boldsymbol{A} / \boldsymbol{s}$ : comparação entre $\mathrm{N}=121$ e $\mathrm{N}=189$

Gráfico 6.32 - SNR S-WLSC e SNR S-WLS considerando $\boldsymbol{k}=96$, $\boldsymbol{b}=40 \%$, para diversos valores de $\boldsymbol{A} / \boldsymbol{s}(\mathrm{N}=121)$

Gráfico 6.33 - SNR S-WLSC e SNR S-WLS considerando $\boldsymbol{k}=96$, $\boldsymbol{b}=40 \%$, para diversos valores de $\boldsymbol{A} / \boldsymbol{s}(\mathrm{N}=189)$

Gráfico 6.34 - MSE S-WLSC e MSE S-WLS $\left(\times 10^{6}\right)$ considerando $\boldsymbol{k}=96$, $\boldsymbol{A} / \boldsymbol{s}=6$, para diversos valores de $\boldsymbol{b}: \mathrm{N}=121$

Gráfico 6.35 - MSE S-WLSC e MSE S-WLS $\left(\times 10^{6}\right)$ considerando $\boldsymbol{k}=96$, $\boldsymbol{A} / \boldsymbol{s}=6$, para diversos valores de $\boldsymbol{b}: \mathrm{N}=189$

Gráfico 6.36 - MSE S-WLSC e MSE S-WLS $\left(\times 10^{6}\right)$ considerando $\boldsymbol{A} / \boldsymbol{s}=6$ e $\boldsymbol{b}=40 \%$, para diversos valores de $\boldsymbol{k}(\mathrm{N}=121)$

Gráfico 6.37 - MSE S-WLSC e MSE S-WLS $\left(\times 10^{6}\right)$ considerando $\boldsymbol{A} / \boldsymbol{s}=6$ e $\boldsymbol{b}=40 \%$, para diversos valores de $\boldsymbol{k}(\mathrm{N}=189)$

Gráfico 6.38 - MSE S-WLSC e MSE S-WLS $\left(\times 10^{6}\right)$ considerando $\boldsymbol{k}=96$, $\boldsymbol{b}=40 \%$, para diversos valores de $\boldsymbol{A} / \boldsymbol{s}(\mathrm{N}=121)$

Gráfico 6.39 - MSE S-WLSC e MSE S-WLS $\left(\times 10^{6}\right)$ considerando $\boldsymbol{k}=96$, $\boldsymbol{b}=40 \%$, para diversos valores de $\boldsymbol{A} / \boldsymbol{s}(\mathrm{N}=189)$

Gráfico 6.40 - Espectro da irregular da série dessazonalizada pelo filtro S-WLSC, sem tendência, e: $\boldsymbol{A} / \boldsymbol{s}=6,5 ; \boldsymbol{k}=72 ; \boldsymbol{b}=40 \%(\mathrm{~N}=121)$

Gráfico 6.41 - Espectro da irregular da série dessazonalizada pelo filtro S-WLSC, sem tendência, e: $\boldsymbol{A} / \boldsymbol{s}=6,5 ; \boldsymbol{k}=96 ; \boldsymbol{b}=40 \%(\mathrm{~N}=121)$

Gráfico 6.42 - Espectro da irregular da série dessazonalizada pelo filtro S-WLSC, sem tendência, e: $\boldsymbol{A} / \boldsymbol{s}=6,5 ; \boldsymbol{k}=72 ; \boldsymbol{b}=10 \%(\mathrm{~N}=121)$

Gráfico 6.43 - Espectro da irregular da série dessazonalizada pelo filtro S-WLSC, sem tendência, e: $\boldsymbol{A} / \boldsymbol{s}=6,5 ; \boldsymbol{k}=96 ; \boldsymbol{b}=10 \%(\mathrm{~N}=121)$

Gráfico 6.44 - Espectro da irregular da série dessazonalizada pelo filtro S-WLSC, com tendência, e: $\boldsymbol{A} / \boldsymbol{s}=6,5 ; \boldsymbol{k}=72 ; \boldsymbol{b}=40 \%(\mathrm{~N}=121)$

Gráfico 6.45 - Espectro da irregular da série dessazonalizada pelo filtro S-WLSC, com tendência, e: $\boldsymbol{A} / \boldsymbol{s}=6,5 ; \boldsymbol{k}=96 ; \boldsymbol{b}=40 \%(\mathrm{~N}=121)$ 
Gráfico 6.46 - Espectro da irregular da série dessazonalizada pelo filtro S-WLSC, com tendência, e: $\boldsymbol{A} / \boldsymbol{s}=6,5 ; \boldsymbol{k}=72 ; \boldsymbol{b}=10 \%(\mathrm{~N}=121)$

Gráfico 6.47 - Espectro da irregular da série dessazonalizada pelo filtro S-WLSC, com tendência, e: $\boldsymbol{A} / \boldsymbol{s}=6,5 ; \boldsymbol{k}=96 ; \boldsymbol{b}=10 \%(\mathrm{~N}=121)$ 232

Gráfico 6.48 - Resposta em magnitude do filtro S-WLSC $(N=41)$ 234

Gráfico 6.49 - Resposta em magnitude do filtro S-WLSC, do S-WLS e o esperado $(\boldsymbol{N}=41)$

Gráfico 6.50 - Banda passante dos filtros S-WLSC e S-WLS $(\boldsymbol{N}=41) \quad 234$

Gráfico 6.51 - Resposta em magnitude do filtro S-WLSC (N =43) 235

Gráfico 6.52 - Resposta em magnitude do filtro S-WLSC, do S-WLS e o esperado, para $(\mathrm{N}=43)$

Gráfico 6.53 - Banda passante dos filtros S-WLSC e S-WLS (N=43) 235

Gráfico 6.54 - Resposta em magnitude do filtro S-WLSC (N =65) 236

Gráfico 6.55 - Resposta em magnitude do filtro S-WLSC, do S-WLS e o esperado $(\mathrm{N}=65)$

Gráfico 6.56 - Banda passante dos filtros S-WLSC e S-WLS (N=65) 236

Gráfico 6.57 - Resposta em magnitude do filtro S-WLSC (N =49) 237

Gráfico 6.58 - Resposta em magnitude do filtro S-WLSC, do S-WLS e o esperado $(\mathrm{N}=49)$

Gráfico 6.59 - Banda passante dos filtros S-WLSC e S-WLS (N=49) 237

Gráfico 6.60 - Resposta em magnitude do filtro S-WLSC (N =67) 238

Gráfico 6.61 - Resposta em magnitude do S-WLSC, do S-WLS e esperado $(\mathrm{N}=67)$

Gráfico 6.62 - Banda passante dos filtros S-WLSC e S-WLS (N=67) 238

Gráfico 6.63 - Resposta em magnitude do filtro S-WLSC (N=49) 239

Gráfico 6.64 - Resposta em magnitude do filtro S-WLSC ( $N=49)$, do S-WLS (N=51) e o esperado

Gráfico 6.65 - Banda passante dos filtros S-WLSC e S-WLS (N=51)

Gráfico 6.66 - Espectro da irregular da série dessazonalizada pelo S-WLSC (N=43), com: $\boldsymbol{A} / \boldsymbol{s}=6, \boldsymbol{k}=24$ e $\boldsymbol{b}=40 \%$ (Eq. 5-2) 
Gráfico 6.67 - Espectro da irregular da série dessazonalizada pelo SWLSC ( $N=43$ ), com: $\boldsymbol{A} / \boldsymbol{s}=6, \boldsymbol{k}=32$ e $\boldsymbol{b}=40 \%$ (Eq. 5-2)

Gráfico 6.68 - Razão entre a SNR S-WLSC e a SNR S-WLS considerando $\boldsymbol{A} / \boldsymbol{s}=6$ e $\boldsymbol{k}=32$, para diversos valores de $\boldsymbol{b}$ : comparação entre $\mathrm{N}=43$ e $\mathrm{N}=65$

Gráfico 6.69 - SNR S-WLSC e SNR S-WLS considerando $A / s=6$ e $\boldsymbol{k}=32$, para diversos valores de $\boldsymbol{b}(\mathrm{N}=43)$

Gráfico 6.70 - SNR S-WLSC e SNR S-WLS considerando $A / s=6$ e $\boldsymbol{k}=32$, para diversos valores de $\boldsymbol{b}(\mathrm{N}=65)$

Gráfico 6.71 - Razão entre a SNR do S-WLSC e a SNR S-WLS considerando $\boldsymbol{A} / \boldsymbol{s}=6$ e $\boldsymbol{b}=40 \%$, para diversos valores de $\boldsymbol{k}$ : comparação entre $\mathrm{N}=43$ e $\mathrm{N}=65$

Gráfico 6.72 - SNR S-WLSC e SNR S-WLS considerando $A / s=6$ e $\boldsymbol{b}=40 \%$, para diversos valores de $\boldsymbol{k}(\mathrm{N}=43)$

Gráfico 6.73 - SNR S-WLSC e SNR S-WLS considerando $\boldsymbol{A} / \boldsymbol{s}=6 \mathrm{e}$ $\boldsymbol{b}=40 \%$, para diversos valores de $\boldsymbol{k}(\mathrm{N}=65)$

Gráfico 6.74 - Razão entre a SNR do S-WLSC e a do S-WLS considerando $\boldsymbol{k}=32, \boldsymbol{b}=40 \%$, para diversos valores de $\boldsymbol{A} / \boldsymbol{s}$ : comparação entre $\mathrm{N}=43$ e $\mathrm{N}=65$

Gráfico 6.75 - SNR S-WLSC e SNR S-WLS considerando $\boldsymbol{k}=32$, $\boldsymbol{b}=40 \%$, para diversos valores de $\boldsymbol{A} / \boldsymbol{s}(\mathrm{N}=43)$

Gráfico 6.76 - SNR S-WLSC e SNR S-WLS considerando $\boldsymbol{k}=32$, $\boldsymbol{b}=40 \%$, para diversos valores de $\boldsymbol{A} / \boldsymbol{s}(\mathrm{N}=65)$

Gráfico 6.77 - MSE S-WLSC e MSE S-WLS $\left(\times 10^{6}\right)$ considerando $\boldsymbol{k}=32$, $\boldsymbol{A} / \boldsymbol{s}=6$, para diversos valores de $\boldsymbol{b}: \boldsymbol{N}=43$

Gráfico 6.78 - MSE S-WLSC e MSE S-WLS $\left(\times 10^{6}\right)$ considerando $\boldsymbol{k}=32$, $\boldsymbol{A} / \boldsymbol{s}=6$, para diversos valores de $\boldsymbol{b}: \boldsymbol{N}=65$

Gráfico 6.79 - MSE S-WLSC e MSE S-WLS $\left(\times 10^{6}\right)$ considerando $\boldsymbol{A} / \boldsymbol{s}=6,5$ e $\boldsymbol{b}=40 \%$, para diversos valores de $\boldsymbol{k}(\mathrm{N}=43)$

Gráfico 6.80 - MSE S-WLSC e MSE S-WLS $\left(\times 10^{6}\right)$ considerando $\boldsymbol{A} / \boldsymbol{s}=6,5$ e $\boldsymbol{b}=40 \%$, para diversos valores de $\boldsymbol{k}(\mathrm{N}=65)$

Gráfico 6.81 - MSE S-WLSC e MSE S-WLS $\left(\times 10^{6}\right)$ considerando $\boldsymbol{k}=32$, $\boldsymbol{b}=40 \%$, para diversos valores de $\boldsymbol{A} / \boldsymbol{s}(\mathrm{N}=43)$

Gráfico 6.82 - MSE S-WLSC e MSE S-WLS $\left(\times 10^{6}\right)$ considerando $\boldsymbol{k}=32$, $\boldsymbol{b}=40 \%$, para diversos valores de $\boldsymbol{A} / \boldsymbol{s}(\mathrm{N}=65)$ 
Gráfico 6.83 - Espectro da irregular da série dessazonalizada pelo filtro S-WLSC, com: $A / s=6,5 ; \boldsymbol{k}=24 ; \boldsymbol{b}=40 \%(\boldsymbol{N}=43)$

Gráfico 6.84 - Espectro da irregular da série dessazonalizada pelo filtro S-WLSC, com: $\boldsymbol{A} / \boldsymbol{s}=6,5 ; \boldsymbol{k}=32 ; \boldsymbol{b}=40 \%(\boldsymbol{N}=43)$

Gráfico 6.85 - Espectro da irregular da série dessazonalizada pelo filtro S-WLS, com: $\boldsymbol{A} / \boldsymbol{s}=6,5 ; \boldsymbol{k}=24 ; \boldsymbol{b}=40 \%(\boldsymbol{N}=43)$

Gráfico 6.86 - Espectro da irregular da série dessazonalizada pelo filtro S-WLS, com: $\boldsymbol{A} / \boldsymbol{s}=6,5 ; \boldsymbol{k}=32 ; \boldsymbol{b}=40 \%(\boldsymbol{N}=43)$

Gráfico 6.87 - Espectro da irregular da série dessazonalizada pelo filtro S-WLSC, com tendência, e: $\boldsymbol{A} / \boldsymbol{s}=6,5 ; \boldsymbol{k}=24 ; \boldsymbol{b}=40 \%(\boldsymbol{N}=43)$

Gráfico 6.88 - Espectro da irregular da série dessazonalizada pelo filtro S-WLSC, com tendência, e: $\boldsymbol{A} / \boldsymbol{s}=6,5 ; \boldsymbol{k}=32 ; \boldsymbol{b}=40 \%(\boldsymbol{N}=43) 252$

Gráfico 6.89 - Espectro da irregular da série dessazonalizada pelo filtro S-WLSC, com tendência, e: $\boldsymbol{A} / \boldsymbol{s}=6,5 ; \boldsymbol{k}=24 ; \boldsymbol{b}=10 \%(\boldsymbol{N}=43) 252$ Gráfico 6.90 - Espectro da irregular da série dessazonalizada pelo filtro S-WLSC, com tendência, e: $\boldsymbol{A} / \boldsymbol{s}=6,5 ; \boldsymbol{k}=32 ; \quad \boldsymbol{b}=10 \%(\boldsymbol{N}=43) 252$ 


\section{Lista de abreviaturas e siglas}

$\begin{array}{ll}\text { ARIMA } & \text { Autoregressive Integrated Moving Average } \\ \text { EECCA } & \text { Leste Europeu, Cáucaso e Ásia Central } \\ \text { Eq. } & \text { Equação } \\ \text { FMI } & \text { Fundo Monetário Internacional } \\ \text { IBGE } & \text { Instituto Brasileiro de Geografia e Estatística } \\ & \text { (http://www.ibge.gov.br) } \\ \text { IPEA } & \text { Instituto de Pesquisa Econômica Aplicada } \\ & \text { (http://www.ipeadata.gov.br) } \\ \text { MAD } & \text { Mean Absolute Deviation (Desvio Absoluto Médio) } \\ \text { MMs } & \text { Média móvel sazonal } \\ \text { MSE } & \text { Mean Square Error (Média dos Quadrados dos Erros) } \\ \text { OECD } & \text { Organização para a Cooperação e Desenvolvimento } \\ & \text { Econômico (http://stats.oecd.org) } \\ \text { p } & \text { valor-p } \\ \text { SQ } & \text { Soma dos Quadrados } \\ \text { SQE } & \text { Soma dos Quadrados dos Erros } \\ \text { SNR } & \text { Razão sinal ruído } \\ \text { S-WLS } & \text { Filtro sazonal-WLS } \\ \text { S-WLSC } & \text { Filtro sazonal-WLS-Chebyshev } \\ \text { T-S } & \text { TRAMO-SEATS } \\ \text { WLS } & \text { Mínimos Quadrados Ponderados } \\ \text { X-13A-S } & \text { X-13ARIMA-SEATS } \\ & \end{array}$




\section{Lista de termos técnicos}

Backcast Previsão para trás

Don't care band Banda de transição

Equiripple Ripple de igual amplitude

Filtro sazonal Série de pesos, a ser aplicada na série temporal, para a obtenção da componente sazonal

Forecast Previsão para frente

Passband Banda passante (ou banda de passagem)

$\mathrm{R}^{2} \quad$ Coeficiente de determinação

Ripple Ondulação. Erro na banda passante

Stopband Banda de rejeição

valor-p O menor nível de significância para o qual se rejeita $\mathrm{H}_{0}$ 


\section{Lista de símbolos}

$\begin{array}{ll}Y_{t} \text { ou } Y & \text { Série temporal observada } \\ T_{t} \text { ou } T & \text { Componente de tendência } \\ S_{t} \text { ou } S & \text { Componente sazonal } \\ I_{t} \text { ou } I & \text { Componente irregular } \\ C_{t} & \text { Componente de ciclo } \\ S_{t} \text { ou } S I & \text { Componente Sazonal-Irregular } \\ A_{t} & \text { Série sazonalmente ajustada } \\ S^{\prime} & S-1 \\ I^{\prime} & I-1 \\ \mathrm{t} & \text { Período de tempo (em meses ou trimestres) } \\ \mathrm{FS}_{\mathrm{S}} & \text { Teste } F \text { para a presença de sazonalidade estável } \\ \mathrm{F}_{\mathrm{M}} & \text { Teste } F \text { para a presença de sazonalidade móvel } \\ \mathrm{KW} & \text { Teste de Kruskal-Wallis para a presença de sazonalidade } \\ & \text { estável }\end{array}$

Lista de símbolos relacionados ao sinal sazonal simulado

$A$

$b$

$k$

$S$
Amplitude do sinal

Taxa de variação da sazonalidade

Número de meses (ou trimestres) no qual o padrão de sazonalidade móvel volta a se repetir, no sinal simulado utilizado

Desvio-padrão da componente irregular 


\section{Lista de símbolos relacionados aos filtros propostos}

$\alpha$

$\delta$

$\mathrm{W}_{\mathrm{O}}$

$N$

$\mathrm{N}_{S}$

M

$\beta$

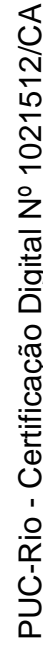

Largura da banda em torno dos harmônicos

Largura da banda de transição

Peso: importância dada às bandas de passagem em torno dos harmônicos

Tamanho do filtro

Periodicidade da série. Se mensal, $\mathrm{N}_{s}=12$; se trimestral, $\mathrm{N}_{s}=4$

Fator de superamostragem

Desvio da resposta da banda passante do filtro $\mathrm{X}-11 \mathrm{em}$ relação à resposta ideal

Resposta na frequência fundamental

Resposta de frequência que mais se desviar do valor 1 , no intervalo equivalente à largura de banda passante Razão entre a atenuação na banda de rejeição e o ripple na banda passante 
The best part is that one inspiration will trigger the next. That's how we all keep moving forward.

Felix Baumgartner 


\section{Introdução}

Este capítulo apresenta um panorama do tema tratado nesta tese, assim como os seus objetivos e sua contribuição. Além disso, o capítulo apresenta a relevância do tema, seguido da delimitação do estudo. E por fim, o capítulo apresenta a estrutura da tese.

\section{1.}

\section{Apresentação do tema}

Uma das funções dos órgãos oficiais de estatística é fornecer aos tomadores de decisão informações sobre a economia do país, disponibilizando séries históricas das variáveis relacionadas aos principais setores. Geralmente, a análise da evolução das variáveis econômicas é realizada com base nas séries sazonalmente ajustadas. Sendo assim, a qualidade do ajuste sazonal é de fundamental importância para os analistas.

O ajuste sazonal é o processo de estimar e remover os efeitos sazonais de uma série temporal. A série resultante de tal processo é denominada série dessazonalizada ou série sazonalmente ajustada.

Embora o conceito de sazonalidade seja familiar, convém apresentar o seu significado. Nesse sentido, adota-se aqui a seguinte definição:

Sazonalidade é o conjunto dos movimentos, com período igual ou inferior a um ano, sistemáticos, mas não necessariamente uniformes, que ocorrem numa série temporal [...]. (Wallis \& Thomas, 1971, p.58).

$\mathrm{Na}$ definição apresentada, o principal aspecto a ser ressaltado é o fato do comportamento da sazonalidade poder apresentar caráter estável ou móvel. Quando a sazonalidade de uma série temporal permanece a mesma ao longo do tempo, tendo variação estocástica constante, ou até mesmo sem variação estocástica, ela é considerada estável (Sutradhart \& Dagum, 1998); enquanto que se houver mudanças graduais na amplitude da componente sazonal, fica caracterizada a presença de sazonalidade móvel (Higginson, 1975). Sendo assim, apesar da 
sazonalidade ser um padrão que tende a se repetir ano após ano, ele não é necessariamente uniforme.

A importância de analisar a sazonalidade móvel foi destacada inicialmente nos trabalhos de Kuznets (1933), que pesquisou a natureza da mudança na amplitude sazonal em 31 séries temporais da indústria. Mais recentemente, Van Dijk et al. (2003) analisaram a sazonalidade móvel nas séries de produção industrial dos países do G7.

Existem hoje disponíveis diversos métodos e programas de ajuste sazonal. No Brasil, o programa utilizado pelo IBGE é o X-12-ARIMA, cujo método de ajuste sazonal implementado é o método X-11. Tal método encontra-se, também, nos programas X-11-ARIMA e X-13ARIMA-SEATS - X-13A-S. Além do Brasil, esse método é adotado por países como Inglaterra, Estados Unidos e Austrália, sendo considerado um dos métodos mais utilizados pelos órgãos oficiais de estatística (International Monetary Fund, 2008).

$\mathrm{Na}$ literatura, há um grande debate comparando os métodos de ajuste sazonal, e principalmente comparando o X-11 aos demais. No geral, os resultados indicam desempenho semelhante quando a série apresenta um padrão estável de sazonalidade. Porém, quando existe sazonalidade móvel, os resultados divergem. E o método X-11, amplamente utilizado, não consegue tratar a sazonalidade móvel de forma adequada (Planas, 1998).

Várias séries temporais da economia brasileira e estrangeira, assim como de outras áreas, apresentam um grau considerável de sazonalidade móvel. Dessa forma, é importante haver um método que apresente um bom desempenho quando utilizado para dados com essa característica. 


\section{2.}

\section{Objetivo e contribuição da tese}

Esta tese objetiva propor dois projetos de filtros de extração da componente sazonal, de forma que eles sejam adequados para as séries que possuem sazonalidade móvel, como também para aquelas nas quais o padrão sazonal é estável. Tais filtros podem ser aplicados diretamente na série temporal, independentemente do padrão sazonal apresentado por ela. Trata-se de um aprimoramento do projeto realizado por Zani (2008), agora estendido a séries com sazonalidade móvel. Sendo assim, essa tese contribui para melhorias nas técnicas de ajustamento sazonal, pois os filtros propostos permitem um ajuste adequado mesmo na presença de variação da amplitude da componente sazonal. Especificamente, essa contribuição é materializada conforme indicado nos parágrafos a seguir.

Inicialmente é proposto o projeto de um filtro extrator de sazonalidade, desenvolvido no domínio da frequência, usando critérios baseados em mínimos quadrados. Esse projeto é intitulado de 'filtro sazonal-WLS' - S-WLS. Para verificar a adequação do filtro S-WLS, são utilizadas séries artificiais baseadas nas características de séries macroeconômicas reais, nacionais e estrangeiras. O desempenho do filtro S-WLS é então comparado com o desempenho do método X11.

A identificação do padrão sazonal das séries macroeconômicas reais é realizada com base em séries mensais e trimestrais. O padrão sazonal, avaliado a partir dessas séries, é utilizado também para a definição de alguns parâmetros do filtro S-WLS.

É importante ressaltar que é apresentada a aplicação desse filtro para séries com modelo aditivo e multiplicativo de decomposição das componentes não observáveis. Porém, nesta tese propõe-se não utilizar a transformação logarítmica na série temporal. Devido ao fato da aplicação do logaritmo introduzir distorções nas componentes senoidais, decidiu-se utilizar os dados originais, sem transformações, no ajuste sazonal. Sendo assim, as características espectrais da sazonalidade são preservadas.

O segundo projeto de filtros aqui proposto combina a abordagem de mínimos quadrados ponderados com as características dos filtros de Chebyshev. A esse 
segundo projeto de filtros, intitulou-se 'filtro sazonal-WLS-Chebyshev' - SWLSC. Vale ressaltar que, ao utilizar um critério que minimiza simultaneamente o erro na estimativa da sazonalidade e a influência da componente irregular, esta tese contribui para a verificação de métodos alternativos de projeto de filtros lineares em técnicas de ajuste sazonal.

\section{3. \\ Relevância do tema}

Em relação à relevância do tema tratado nesta tese, é necessário mencionar a importância do ajuste sazonal para a análise das séries econômicas; a utilização do método X-11 de ajuste sazonal; a consideração da presença de sazonalidade móvel nas séries temporais; e análise no domínio da frequência.

A importância do ajuste sazonal para a análise das séries econômicas reside, principalmente, no fato de que os agentes econômicos tendem a preferir tomar decisões com base nos dados dessazonalizados (Franses, 1996). O motivo de se preferir os dados dessazonalizados, para a análise de fenômenos econômicos, é tratado na literatura por autores como Gould et al. (2008) e Diller (1971), que sugerem que a principal razão para a utilização de dados sazonalmente ajustados seja a de facilitar a identificação da componente cíclica. Além disso, Hillmer e Bell (2002), ao discutirem a importância do ajuste sazonal, relatam que vários autores argumentam que tal ajuste é útil porque a sazonalidade, em uma série temporal, pode obscurecer as relações existentes com outras séries.

Consequentemente, fica nítida a importância que deve ser atribuída ao ajuste sazonal pelas agências governamentais, buscando sempre um aprimoramento dos métodos e programas. E dos programas de ajuste sazonal desenvolvidos pelo US Census Bureau e pelo Statistics Canada, o X-13A-S é o mais recente, sendo adotado aos poucos pelas instituições, como é o caso do Bank of England, que planeja começar a utilizar o programa neste ano (Hussain \& Meader, 2014).

O método X-11 está implementado nos programas X-13A-S, X-12-ARIMA e X-11-ARIMA, sendo utilizado atualmente pelas agências governamentais de diversos países, incluindo o Brasil. Métodos matematicamente mais sofisticados já 
foram desenvolvidos, porém o X-11 continua sendo um dos métodos mais utilizados.

Os motivos da ampla utilização do método X-11 podem estar relacionados à simplicidade de uso, e à praticidade. Pedersen \& Fæste (2006) mencionam que o processo de dessazonalização não deve ser muito demorado e que, portanto, no Banco Central da Dinamarca eles se baseiam nos procedimentos automáticos do X12-ARIMA. Vale destacar também que uma pesquisa realizada em 2009 pelas Nações Unidas, com institutos nacionais de estatística de países da EECCA e dos Balcãs Ocidentais, revelou que $80 \%$ dos países pesquisados indicaram não haver especialistas em ajuste sazonal nas instituições; sendo que a grande maioria deles utiliza o X-12-ARIMA (United Nations, 2009). Com isso, torna-se evidente a simplicidade da sua utilização.

A presença de sazonalidade móvel nas séries temporais foi considerada em diversos trabalhos. Dentre eles estão Cayton \& Bersales (2012), Franses \& Koehler (1998), Wells (1997), Canova \& Hansen (1995), Canova \& Ghysels (1994), Higginson (1975), Burman (1965), Hannan (1964) e Kuznets (1933). Wells (1997) alerta para o fato de que havendo sazonalidade móvel, não é possível remover a sazonalidade de uma forma simples, pois ela não estará limitada aos picos nas frequências sazonais, mas sim ocorrendo em diversas frequências. Vários estudos indicaram que o método X-11 não realiza um ajustamento adequado na presença de sazonalidade móvel (por exemplo, Kaiser \& Maravall (2000), Planas (1998) e Dagum (1978)).

Em relação à análise no domínio da frequência para o tratamento da sazonalidade, destacam-se os trabalhos pioneiros de Hannan (1964), Nerlove (1964) e Nettheim $(1964,1965)$. Segundo Nettheim (1964), a análise no domínio da frequência torna o exame do comportamento da sazonalidade mais preciso. Os desenvolvimentos nessa área possibilitaram novas maneiras de tratar a sazonalidade móvel (ver Haywood \& Wilson (2000), Geweke (1978), Melnick \& Moussourakis (1974)), e de avaliar a qualidade do ajuste sazonal (Cleveland, 1982).

Ainda em relação à análise no domínio da frequência, Nerlove (1964) mencionou o sentido prático de existir um filtro, desenvolvido no domínio da frequência, para o tratamento da sazonalidade móvel. O autor enfatizou a dificuldade na criação de tal filtro, e destacou que não era seu objetivo desenvolvêlo. No ano seguinte, Nettheim (1965) citou mais detalhes sobre como seria o projeto 
desse filtro, e pontuou algumas das dificuldades existentes para o desenvolvimento do mesmo. Desde então, a abordagem do ajuste sazonal no domínio da frequência se desenvolveu, porém os avanços vêm ocorrendo mais no campo da extração de sinal baseada em modelos. As críticas em relação a esse método giram em torno da especificação inadequada do modelo, o que resulta em um ajuste sazonal incorreto. Sendo assim, acredita-se que é relevante a existência de um método desenvolvido com as características descritas por Nettheim (1965), assim como a sua comparação com os demais métodos.

\section{4.}

\section{Delimitação do estudo}

Nesta tese, os filtros propostos são fixos, ou seja, os coeficientes são predeterminados, conhecidos na literatura como ad hoc. Sabe-se que existem críticas em relação aos filtros ad hoc, uma vez que eles não modelam os dados observados, como fazem os modelos econométricos. Estes, apesar de serem mais embasados, não são tão utilizados pelos órgãos oficiais para dessazonalizar um grande volume de séries temporais, pois necessitam de frequentes intervenções do operador. Sendo assim, nesta tese, as comparações do desempenho do filtro são realizadas apenas com o método X-11, pois também utiliza filtros ad hoc.

Como o objetivo é comparar com o X-11, o tamanho dos filtros aqui desenvolvidos têm o mesmo tamanho dos filtros correspondentes ao método X-11. Porém, o projeto de filtros apresentado permite a especificação de qualquer tamanho, desde que seja ímpar, pois tratam-se de filtros simétricos.

Apesar do método X-11, implementado no programa mais recente (X-13AS) permitir a utilização de diversos filtros de média móvel sazonal, nesta tese são utilizados apenas os filtros de média móvel existentes na opção automática do programa.

Por fim, nesta tese são consideradas apenas séries macroeconômicas, pois são as séries utilizadas pelas agências governamentais para o ajuste sazonal. Sendo assim, a periodicidade considerada nas análises é a mensal e a trimestral. 


\section{5 .}

\section{Organização da tese}

Esta tese está estruturada em sete capítulos, incluindo o capítulo introdutório, onde é apresentado o enfoque central.

O Capítulo 2 trata do histórico do estudo da sazonalidade e dos métodos de dessazonalização utilizados pelas agências governamentais. Além disso, são apresentados alguns conceitos relacionados ao tema.

O Capítulo 3 aborda o ajuste sazonal no domínio da frequência, apresentando o arcabouço teórico do método X-11.

No Capítulo 4, é apresentada a proposta de um filtro para o ajuste sazonal, aqui intitulado de 'filtro sazonal-WLS'. Já o Capítulo 5 trata da seleção da melhor configuração de parâmetros do filtro, e dos resultados da sua aplicação.

A proposta do segundo filtro, que combina a abordagem de mínimos quadrados ponderados com as características dos filtros de Chebyshev, intitulado de 'filtro sazonal-WLS-Chebyshev', é apresentada no Capítulo 6, juntamente com a comparação do desempenho em relação ao 'filtro sazonal-WLS'.

Por fim, o Capítulo 7 apresenta as conclusões acerca dos resultados encontrados, bem como contém sugestões para trabalhos futuros nessa área. 


\section{2}

\section{Ajuste sazonal: conceito, histórico e métodos}

Vários métodos de ajuste sazonal foram desenvolvidos ao longo dos anos com o objetivo de estimar a componente sazonal das séries temporais. Neste capítulo são apresentados alguns conceitos e é realizado um breve histórico sobre o tema. O capítulo também apresenta alguns dos métodos utilizados pelas agências governamentais, e detalha o método X-11 de ajuste sazonal. Além disso, o capítulo aborda alguns tópicos relacionados à sazonalidade móvel.

\section{1. Conceitos e breve histórico do desenvolvimento das técnicas de ajuste sazonal}

Em estatística, uma série temporal pode ser definida como um conjunto de observações de uma variável, ordenado segundo o tempo, geralmente em intervalos equidistantes (Souza \& Camargo, 2004).

Os métodos de decomposição assumem que a série temporal $Y_{t}$ é constituída por quatro componentes não observáveis, sendo elas: a componente de tendência $T_{t}$, a componente de ciclo $C_{t}$; a componente sazonal $S_{t}$, e a componente irregular $I_{t}$. Muitas vezes, as componentes de tendência e ciclo são consideradas conjuntamente, formando a componente tendência-ciclo e denominadas apenas por $T_{t}$.

A tendência representa a evolução da série a longo prazo. Já o ciclo, mostra uma sucessão de etapas de crescimento e recessão, representando um movimento suave, periódico, em torno da tendência. A componente sazonal é representada pelas oscilações ocorridas em um determinado período do ano. E a componente irregular, ou componente de erro, é devida a choques aleatórios.

Se a relação existente entre as componentes for aditiva, o modelo de decomposição é escrito da seguinte forma:

$$
Y_{t}=T_{t}+S_{t}+I_{t}
$$


Caso o modelo de decomposição da série, nas várias componentes, for multiplicativo, ele é representado por:

$$
Y_{t}=T_{t} \times S_{t} \times I_{t}
$$

Segundo Grether \& Nerlove (1970), a ideia de que uma série temporal pode ser decomposta em algumas componentes não observáveis, que se somadas ou multiplicadas resultarão nos valores observados da série, surgiu inicialmente na astronomia, no século XVIII. Quando os estudos de meteorologia se tornaram importantes, no início do século XIX, a ideia de componentes não observáveis foi trazida da astronomia e aplicada na análise da variação da temperatura, e variações na pressão barométrica, por pessoas como o meteorologista Buys Ballot, que é frequentemente citado como uma das referências mais antigas sobre sazonalidade (Foldesi et al., 2007).

Foram criadas técnicas similares aplicadas à análise de fenômenos econômicos, em meados do século XIX, mas foi a partir do início do século XX que tiveram início as primeiras grandes contribuições a esse tema. Destaca-se, então, o trabalho de Persons (1919), que desenvolveu o método 'link relative’ para isolar as componentes não observáveis de uma série temporal (European Commission Grant, 2007).

Em 1931, Frederick Macaulay criou a primeira metodologia, completa, de ajuste sazonal, com o desenvolvimento do método 'ratio-to-moving average', no National Bureau of Economic Research - NBER. Essa abordagem ficou conhecida como 'Decomposição Clássica', sendo a base de alguns métodos atuais.

A partir da década de 50 aconteceram os grandes desenvolvimentos nessa área. Um deles foi a introdução das técnicas de alisamento exponencial, que alcançou uma grande popularidade (Gardner, 1985). Além disso, com a chegada dos computadores, os pesquisadores puderam desenvolver métodos mais sofisticados, sendo facilmente testados em um grande número de séries.

A seguir é apresentado um breve histórico dos principais acontecimentos nessa área, a partir de 1960: 


\begin{tabular}{|c|c|}
\hline Ano & Acontecimento \\
\hline 1963 & $\begin{array}{l}\text { Whittle (1963) utilizou o filtro de Wiener-Kolmogorov para estimar uma } \\
\text { componente não observável de uma série temporal, dando origem à } \\
\text { decomposição de componentes no domínio da frequência. }\end{array}$ \\
\hline 1964 & $\begin{array}{l}\text { Nerlove (1964) estudou os efeitos dos procedimentos de ajuste sazonal nas } \\
\text { características das séries, com base na análise espectral. }\end{array}$ \\
\hline 1965 & $\begin{array}{l}\text { Foi desenvolvido o método X-11, no U.S. Census Bureau, sendo considerado o } \\
\text { primeiro método automatizado de dessazonalização. Vale destacar que os } \\
\text { métodos da 'Família X-11' começaram em 1954, com o Método I. No final da } \\
\text { década de } 50 \text { foi desenvolvido o Método II, e algumas versões foram produzidas, } \\
\text { sendo chamadas de 'X-0, X-1, .., X-10', culminando no método X-11. }\end{array}$ \\
\hline 1966 & $\begin{array}{l}\text { Grether \& David (1970) aplicaram alguns resultados de Whittle (1963) a } \\
\text { componentes não observáveis de uma série temporal, utilizando séries } \\
\text { econômicas. }\end{array}$ \\
\hline 1970 & $\begin{array}{l}\text { Desenvolvimento dos métodos de estimação de modelos ARIMA para séries } \\
\text { temporais, proporcionando uma forma de parametrização do espectro da série } \\
\text { temporal, de tal forma que os filtros de extração de sinal poderiam ser derivados } \\
\text { disso. }\end{array}$ \\
\hline 1972 & $\begin{array}{l}\text { Cleveland, na sua tese de doutorado não publicada, aplicou extração de sinal } \\
\text { para o ajustamento sazonal, e sugeriu que quando a série não fosse infinita, } \\
\text { poderiam ser feitas previsões, para auxiliar no processo de filtragem - uma vez } \\
\text { que algumas observações são perdidas. }\end{array}$ \\
\hline 1975 & $\begin{array}{l}\text { Estela Dagum, do Statistics Canada desenvolveu o X-11-ARIMA, no qual a } \\
\text { principal diferença em relação ao método X-11 era a utilização de modelos } \\
\text { ARIMA para realizar o forecast e o backcast, na série temporal, antes de aplicar } \\
\text { os filtros do X-11. }\end{array}$ \\
\hline 1976 & $\begin{array}{l}\text { Cleveland \& Tiao (1976) apresentaram um modelo ARIMA para o qual o } \\
\text { método X-11 é ótimo. }\end{array}$ \\
\hline 1978 & $\begin{array}{l}\text { Box, Hillmer e Tiao (1978) sugeriram que a interação entre a abordagem } \\
\text { empírica e a abordagem baseada em modelos (model based approach) gerava os } \\
\text { melhores resultados. }\end{array}$ \\
\hline 1980 & $\begin{array}{l}\text { Burman, do Banco da Inglaterra, mostrou como um modelo ARIMA poderia ser } \\
\text { usado para gerar um filtro linear infinito para a extração da componente sazonal. }\end{array}$ \\
\hline 1995 & $\begin{array}{l}\text { Gómez e Maravall, juntamente com Burman, Box, Hillmer e Tiao, deram origem } \\
\text { ao SEATS (Signal Extraction in ARIMA Time Series) - um método de ajuste } \\
\text { sazonal que utiliza extração de sinal com base em um modelo ARIMA. }\end{array}$ \\
\hline 2012 & $\begin{array}{l}\text { Os métodos X-11 e SEATS foram incorporados como opções de ajuste sazonal } \\
\text { em um mesmo programa, sendo chamado de X-13ARIMA-SEATS. }\end{array}$ \\
\hline
\end{tabular}

\section{Quadro 2.1 Breve histórico dos principais acontecimentos}

A seguir, na Subseção 2.2, são apresentados os métodos usados por agências governamentais, a partir da década de 60 . 


\section{2. Métodos de ajuste sazonal usados por agências governamentais}

Vários métodos foram criados para o ajuste sazonal, utilizando diferentes abordagens. As agências governamentais vêm adotando alguns desses métodos, uma vez que as séries econômicas estão sujeitas à variação sazonal.

Neste estudo será apresentado detalhadamente o método X-11 de ajuste sazonal, pois se trata do método utilizado pelo órgão oficial de estatística do Brasil, além de ser amplamente utilizado no mundo. Porém, nesta seção, serão descritos brevemente os métodos que foram utilizados por agências governamentais, desde meados do século XX, uma vez que algumas características deles foram consideradas no aprimoramento dos métodos utilizados hoje (Kuiper, 1976).

\section{a) Método X-11 - década de 60}

Em 1954, o U.S. Census Bureau desenvolveu um método de ajuste sazonal chamado 'Método I'. Ele era baseado no método 'ratio-to-moving average', de Macaulay (1931). No final da década de 1950, mais precisamente em 1957, foi criado o Método II, sendo um aprimoramento do Método I. A diferença estava no fato de que, no Método II, a estimativa das componentes de tendência-ciclo, sazonal e irregular utilizava algumas iterações. Além disso, havia um ajuste para variações de dias trabalhados, e para valores extremos. Ambas as versões aditiva e multiplicativa eram disponíveis.

O algoritmo do Método II se tornou estável em 1961. E as diversas variantes desse método foram chamadas de ' $\mathrm{X}$ ' ( $\mathrm{X}-0, \mathrm{X}-1, \mathrm{X}-2, \ldots)$, até o desenvolvimento do método X-11, em 1965. Ou seja, o X-11 resultou de uma modificação do Método II, e esse conjunto de modificações ficou conhecido como 'Família X-11' (descrito em Shiskin et al. (1967)).

Em linhas gerais, o método X-11 é baseado na aplicação sucessiva de filtros de médias móveis.

Vale citar que o método do Bureau of Labour Statistics (BLS), de 1966, era similar ao método X-11. Por esse motivo, na década de 70, o BLS passou a utilizar o X-11. 


\section{b) Método de Burman - década de 60}

Desenvolvido por John Burman, no Bank of England, esse método utilizava uma técnica de extração de sinal.

No método de Burman, após eliminar a tendência, utilizando um filtro de ordem 13 desenvolvido por ele, era realizada uma análise harmônica, de blocos sucessivos de 12 termos, na série SI (Sazonal + Irregular). Nessa análise, as amplitudes eram suavizadas, e combinações lineares dessas amplitudes suavizadas forneciam os fatores sazonais (Burman, 1965).

\section{c) Método da Comunidade Econômica Europeia - década de 60}

O método da Comunidade Econômica Europeia (EEC), também conhecido como método Seabird, foi desenvolvido por Bongard e Mesnage, no Statistical Office of the European Communities. Como uma agência supranacional, a EEC tinha a maioria das suas séries cedidas pelos países membros, sendo assim, era necessário um método universal, ou seja, um método capaz de ajustar uma grande variedade de séries econômicas. E como a utilização do ajuste sazonal era voltada para análises econômicas, o método enfatizava o ajuste dos dados mais recentes.

O procedimento utilizado nesse método consistia em, inicialmente, eliminar os valores extremos; em seguida, era aplicado um filtro de 19 termos aos dados (conhecido como filtro Bongard). O método assumia estabilidade do padrão sazonal.

Na década de 70, a EEC desenvolveu um novo método chamado Dainties.

\section{d) Método de Berlin - década de 60}

Descrito em Godfrey et al. (1964), o método de Berlin, assim como o método da EEC, era usado para análise corrente da série econômica. Criado na década de 60, ele utilizava método de extração de sinal.

A tendência e a sazonalidade eram obtidas usando filtros assimétricos estimados de tal forma que as funções de transferência tinham propriedades espectrais ótimas.

\section{e) DAINTIES - década de 70}

O Dainties foi desenvolvido nos anos 70 para ser o sucessor do método Seabird como método oficial de ajuste sazonal da Comunidade Econômica 
Europeia. O método é baseado em modelos de regressão com janela móvel, tendo a hipótese de que a série e/ou suas componentes não podem ser modeladas em sua total extensão.

O método Dainties funciona relativamente bem, quando os dados apresentam padrões que se aproximam da sazonalidade determinística (OECD, 2005). Atualmente, ele é utilizado pelo ECFIN (Economic and Financial Affairs of the European Commission) e pela República Tcheca.

\section{f) BV4 - década de 70}

Utilizado na Alemanha, o procedimento chamado de 'Berlin Procedure' (BV) teve a primeira versão criada na década de 70. Em 1983, foi desenvolvida a versão BV4; e em 2004, foi substituída pela nova versão - BV4.1. A versão BV4.1 apresenta aprimoramentos no tratamento de outliers e efeitos de calendário, em relação à versão anterior. Essa versão é atualmente utilizada pelo Federal Statistical Office da Alemanha (DESTATIS).

O BV4.1 utiliza modelos de regressão linear, escolhidos de acordo com as características no domínio da frequência (função de ganho, a função de fase) dos filtros lineares utilizados para estimar a componente de tendência-ciclo, e realizar o ajuste sazonal da série (Foldesi et al., 2007).

\section{g) Método X-11-ARIMA - 1980}

O procedimento iterativo, baseado na aplicação de sucessivas médias móveis, utilizado pelo X-11, necessitava de dados de anos adicionais antes dos filtros serem aplicados. Isso tornava necessária a utilização de filtros assimétricos para as últimas observações da série temporal, ocasionando grandes revisões nos valores finais da série ajustada. Foi então que, em 1975, Estela Dagum, do Statistics Canada, apresentou o trabalho 'Seasonal Factor Forecasts from ARIMA Models'.

Com base no trabalho de Dagum (1975), foi introduzida uma modificação no método X-11, que consistia em estender a série temporal, em um ano, com previsões segundo um modelo ARIMA. A esse método foi dado o nome de X-11-ARIMA.

Essa modificação resultou em melhores estimativas dos fatores sazonais, diminuindo as revisões na série ajustada. E esse procedimento é utilizado em todos os programas da Família X-11, desde então. 


\section{h) TRAMO-SEATS - 1997 (OECD)}

TRAMO (Time Series Regression with ARIMA Noise, Missing Observations and Outliers) e SEATS (Signal Extraction in ARIMA Time Series) são programas originalmente desenvolvidos por Victor Gómez e Agustin Maravall, no Banco da Espanha. Esses dois programas foram criados para serem utilizados conjuntamente, de forma automática.

O TRAMO é responsável pelo ajuste prévio da série a ser sazonalmente ajustada, pelo SEATS. O SEATS utiliza a metodologia baseada em modelos ARIMA (AMB), associada com a técnica de extração de sinal, desenvolvida por Burman. Atualmente ele é utilizado pela Espanha, Itália e por alguns outros países da Europa.

\section{i) X-12-ARIMA - 1996 (OECD)}

Desenvolvido pelo U.S. Census Bureau, o X-12-ARIMA, ou X-12RegARIMA, é uma versão atualizada do X-11-ARIMA. Nesse programa foram introduzidas quatro grandes melhorias em relação ao X-11-ARIMA. São elas: (1) extensiva modelagem da série temporal e capacidade de seleção de modelos de regressão linear com erros ARIMA (regARIMA); (2) Novos diagnósticos da qualidade e da estabilidade dos ajustes obtidos; (3) Nova interface ao usuário; (4) Opção de seleção automática de modelo baseada no procedimento do TRAMO.

Foram introduzidas novas opções de ajuste sazonal, com mais possibilidades de filtros de médias móveis, mas o procedimento utilizado para dessazonalização continua sendo o X-11 (Findley et al., 1998). Esse programa é utilizado nos Estados Unidos, Inglaterra, Canadá, Austrália, Nova Zelândia e Brasil, assim como em outros países não citados.

\section{j) Demetra - década de 90}

Trata-se de um software de ajuste sazonal fornecido gratuitamente pelo Eurostat. Ele fornece dois métodos de ajustamento sazonal: X-12-ARIMA e TRAMO/SEATS. O usuário pode optar por um dos métodos e comparar os resultados. Os dados originais podem ser lidos de uma planilha Excel, dentre outros formatos. É um software utilizado por alguns países da Europa, como França, Finlândia, Áustria, Croácia e Portugal (European Commission Grant, 2007). 


\section{k) X-13ARIMA-SEATS - 2012}

O X-13ARIMA-SEATS (X-13A-S) é resultado de uma colaboração entre o U.S. Census Bureau e o Banco da Espanha. Ele combina o X-12-ARIMA com o TRAMO-SEATS. Dessa forma, existem dois métodos de ajuste sazonal disponíveis no X-13A-S: o X-11 e o SEATS. A ilustração a seguir (Figura 2.1) apresenta o procedimento X-12-ARIMA adotado no programa X-13A-S.

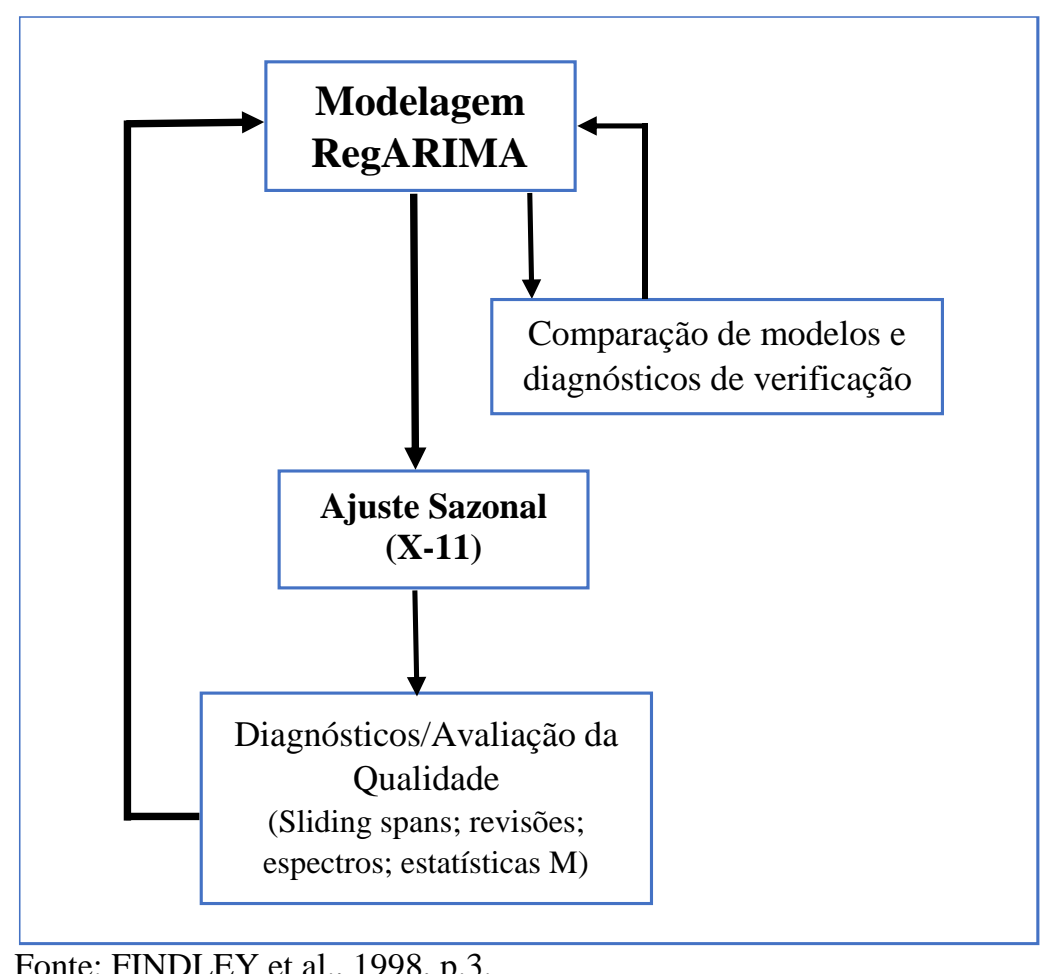

Figura 2.1 O procedimento X-12 ARIMA de ajuste sazonal

Como pode ser observado, atualmente existem diversos métodos e programas de ajustamento sazonal, sendo que aqueles que estão sendo utilizados pelos institutos nacionais de estatística, assim como pelos Bancos Centrais dos países são: X-13A-S, X-12-ARIMA, TRAMO-SEATS, BV4.1, DEMETRA e DAINTIES.

O Eurostat (European Commission), em 2009, recomendou o uso dos programas X-12-ARIMA e TRAMO-SEATS para o ajuste sazonal de dados oficiais (Eurostat, 2009). Vale lembrar que tanto o X-13A-S, quanto o X-12-ARIMA, utilizam o módulo X-11 de ajuste sazonal.

$\mathrm{Na}$ literatura vários autores compararam o método X-11 com os demais métodos. Recentemente, essa comparação vem sendo mais realizada com o SEATS, que é o método de ajuste sazonal do programa TRAMO-SEATS. Na subseção a seguir são apresentadas algumas dessas comparações entre o método X-11 e o SEATS. 


\subsection{1.}

\section{Revisão bibliográfica sobre a comparação entre o X-11 e o SEATS}

O método X-11 é o método de ajuste sazonal utilizado no X-11-ARIMA, X12-ARIMA e X-13A-S. O método SEATS é utilizado no programa TRAMOSEATS - T-S e também faz parte do X-13A-S.

$\mathrm{Na}$ literatura, os principais autores relacionados ao método $\mathrm{X}-11$, e aos programas que adotam este método, são Brian Monsell, David Findley, William Bell e Estela Dagum. Já em relação ao SEATS, os principais nomes são Agustín Maravall, Victor Gómez e Regina Kaiser.

Vários autores compararam tais métodos, como, por exemplo, Gasmi (2013) que comparou os programas de ajuste sazonal T-S e X-12-ARIMA na série mensal de chegada de turistas europeus na Tunísia. Os resultados mostram que o T-S apresentou as melhores previsões.

As revisões nos dados ajustados pelo X-12-ARIMA e pelo SEATS foram comparadas por Monsell et al. (2003) e Hood et al. (2000). Ambos concluíram que em algumas séries as revisões realizadas pelo SEATS foram menores. Ainda sobre as revisões nos dados ajustados, Atuk (2002), ao ajustar as séries de agregados monetários da Turquia, concluiu que o T-S removeu completamente os efeitos sazonais, além de obter revisões menores. Um resultado diferente, em relação às revisões, foi obtido por Hood (2002), que mostra um exemplo de uma série com grandes revisões devido ao modelo escolhido pelo TRAMO.

Os pontos fortes e fracos dos métodos X-11 e SEATS foram comparados por Scott (2007), cuja conclusão sugere o uso combinado dos dois métodos. O autor afirma ainda que o SEATS induz à sazonalidade em algumas séries. Além disso, ele observou que, algumas vezes, é difícil encontrar um modelo bem ajustado aos dados, que gere uma decomposição válida das componentes no SEATS. Outra observação feita por Scott (2007) indica que a grande flexibilidade da seleção dos filtros do SEATS pode ser a causa de frequentes revisões, ou no caso de séries curtas - quando a estimativa dos parâmetros do ARIMA é instável.

Ainda em relação aos filtros de ajuste sazonal, Findley (2005) alerta para o fato de que a maior variedade de filtros de ajustamento sazonal presente no SEATS, em comparação ao X-12-ARIMA, pode levar um usuário amador a obter piores resultados sem ter consciência disso. Findley (2005) também enfatizou a 
importância do desenvolvimento do programa X-13A-S, não apenas pelo conjunto mais completo de diagnóstico de ajuste, mas também porque o usuário sempre pode comparar o ajuste do SEATS com o ajuste do X-11.

Kaiser \& Maravall (2000) compararam o desempenho dos programas X-12ARIMA e T-S no ajuste sazonal da série de volume de negócios do comércio varejista da Alemanha. Ao utilizar o X-12-ARIMA, eles identificaram um problema relacionado aos padrões muito diferentes da componente sazonal.

Na Subseção 2.3, a seguir, é descrito o procedimento de ajuste sazonal do programa X-13A-S, utilizando o módulo X-11 de ajuste. Apesar do X-13A-S conter, também, o método SEATS, este não será tratado aqui, uma vez que o foco deste estudo é o X-11. 


\section{3. Procedimento de ajuste sazonal do programa X-13A-S, utilizando o método X-11}

Como mencionado anteriormente, o método X-11 surgiu em 1965 como um aprimoramento dos Métodos I e II. Inicialmente ele possuía tratamento de outliers, dias trabalhados, e refinamento dos filtros assimétricos. Como ponto negativo, ele apresentava baixa qualidade dos ajustes, devido à utilização de filtros assimétricos nos anos finais da série. Com a chegada do X-11-ARIMA, houve melhoria no ajuste dos anos finais da série, em razão da extensão ARIMA, além de diagnósticos mais refinados, como a introdução das estatísticas M (M1 a M11) e Q.

O módulo de ajuste sazonal foi aprimorado na versão mais recente do programa, chamada de X-13ARIMA-SEATS - X-13A-S. Houve o acréscimo de algumas novas opções, como: os diagnósticos de sliding spans (janelas deslizantes); uma nova rotina do filtro de tendência de Henderson, onde se permite a escolha de qualquer número ímpar para o tamanho do filtro; novas opções para os filtros sazonais; novas opções de detecção de outliers para a componente irregular do ajuste sazonal; e o modo de ajuste sazonal pseudo-aditivo.

A seguir serão apresentados os tipos de decomposições disponíveis no X13A-S.

\subsection{1.}

\section{Tipos de decomposições de séries temporais no X-13A-S}

A decomposição da série temporal $Y_{t}$ em componente de tendência $\left(T_{t}\right)$, componente sazonal $\left(S_{t}\right)$ e componente irregular $\left(I_{t}\right)$ é usualmente realizada sob a forma multiplicativa ou aditiva. Existem, também, a decomposição pseudo-aditiva e a log-aditiva. Elas são representadas da seguinte forma (U.S. CENSUS BUREAU, 2012):

- Multiplicativa (M):

$$
\begin{aligned}
& Y_{t}=T_{t} S_{t} I_{t} \\
& Y_{t}=T_{t}+S_{t}+I_{t} \\
& Y_{t}=T_{t}\left(S_{t}+I_{t}-1\right)=T_{t}\left(S_{t}-1\right)+T_{t} I_{t} \\
& \log \left(\mathrm{Y}_{\mathrm{t}}\right)=T_{t}+S_{t}+I_{t}
\end{aligned}
$$

- Aditiva (A):

- Pseudo-Aditiva (PA):

- Log-Aditiva: 
A decomposição multiplicativa é apropriada para séries com valores positivos, nas quais o tamanho das oscilações sazonais aumenta com o nível da série. Acredita-se que essa é uma característica de muitas séries sazonais macroeconômicas. Sendo assim, a decomposição multiplicativa é usada com mais frequência do que a aditiva.

Já a decomposição pseudo-aditiva é usada quando os valores são muito pequenos, ou zero. $\mathrm{O}$ modelo pseudo-aditivo e o multiplicativo geram resultados semelhantes para séries com sazonalidade multiplicativa, a menos que a amplitude sazonal seja grande (Findley et al., 1998).

A decomposição log-aditiva é usada somente com o objetivo de pesquisa, pois necessita de uma correção de viés para as estimativas de tendência, assim como uma calibragem diferente para a identificação de valores extremos (U.S. CENSUS BUREAU, 2012).

Para realizar a decomposição da série temporal, o usuário do X-13A-S pode definir:

- O tipo de decomposição sazonal;

- As médias móveis sazonais e de tendência (filtros);

- O tipo de ajuste para valor extremo realizado durante o ajuste sazonal.

Existe também a opção automática, na qual o programa seleciona o filtro de tendência e o filtro de médias móveis com base no resultado de algumas estatísticas (apresentadas na Subseção 2.3.3.2). E ainda há a opção de se trabalhar com o modo padrão de ajuste sazonal, chamado de ' $\mathrm{X}-11$ default'.

A seguir é apresentado o algoritmo do método X-11, considerando o ' $\mathrm{X}-11$ default'. 


\subsection{2.}

\section{Etapas do ajuste sazonal realizado pelo X-11}

O algoritmo de dessazonalização do método X-11 é composto de três estágios. No primeiro são realizadas as estimativas iniciais de sazonalidade e tendência; no segundo é definido o fator sazonal; e no terceiro é obtida a componente de tendência. Todos os três estágios estão apresentados a seguir, para uma série temporal mensal $(Y)$, considerando a decomposição multiplicativa $(M)$ e aditiva (A), como descrito em Findley et al. (1998).

\section{Estágio 1 - Estimativas iniciais}

i. Estimativa preliminar da componente de tendência ' $T^{(1)}$ ', aplicando uma média móvel 'centrada em 12 meses' - 13 termos - média móvel 2x12:

$$
\begin{aligned}
T_{t}^{(1)} & =\frac{\frac{Y_{t-6}+Y_{t-5}+\cdots+Y_{t+4}+Y_{t+5}}{12}+\frac{Y_{t-5}+Y_{t-4}+\cdots+Y_{t+5}+Y_{t+6}}{12}}{2} \\
T_{t}^{(1)} & =\frac{1}{24} Y_{t-6}+\frac{1}{12} Y_{t-5}+\cdots+\frac{1}{12} Y_{t}+\cdots+\frac{1}{12} Y_{t+5}+\frac{1}{24} Y_{t+6}
\end{aligned}
$$

ii. Estimativa inicial da componente Sazonal-Irregular - 'SI' inicial:

(M):

$$
\begin{aligned}
& S I_{t}^{(1)}=Y_{t} / T_{t}^{(1)} \\
& S I_{t}^{(1)}=Y_{t}-T_{t}^{(1)}
\end{aligned}
$$

iii. Estimativa preliminar dos fatores sazonais ' $\hat{S}^{(1)}$ ' aplicando uma média móvel sazonal 3x3 - MMs 3x3:

$$
\begin{aligned}
\hat{S}_{t}^{(1)} & =\frac{\frac{S I_{t-24}^{(1)}+S I_{t-12}^{(1)}+S I_{t}^{(1)}}{3}+\frac{S I_{t-12}^{(1)}+S I_{t}^{(1)}+S I_{t+12}^{(1)}}{3}+\frac{S I_{t}^{(1)}+S I_{t+12}^{(1)}+S I_{t+24}^{(1)}}{3}}{3} \\
\hat{S}_{t}^{(1)} & =\frac{1}{9} S I_{t-24}^{(1)}+\frac{2}{9} S I_{t-12}^{(1)}+\frac{3}{9} S I_{t}^{(1)}+\frac{2}{9} S I_{t+12}^{(1)}+\frac{1}{9} S I_{t+24}^{(1)}
\end{aligned}
$$


iv. Fatores sazonais iniciais ' $S$ (1)':

$(\mathrm{M})$ :

$$
S_{t}^{(1)}=\frac{\hat{S}_{t}^{(1)}}{\frac{1}{24} \hat{S}_{t-6}^{(1)}+\frac{1}{12} \hat{S}_{t-5}^{(1)}+\cdots+\frac{1}{12} \hat{S}_{t+5}^{(1)}+\frac{1}{24} \hat{S}_{t+6}^{(1)}}
$$

(A): $\quad S_{t}^{(1)}=\hat{S}_{t}^{(1)}-\left(\frac{1}{24} \hat{S}_{t-6}^{(1)}+\frac{1}{12} \hat{S}_{t-5}^{(1)}+\cdots+\frac{1}{12} \hat{S}_{t+5}^{(1)}+\frac{1}{24} \hat{S}_{t+6}^{(1)}\right)$

v. Ajuste sazonal inicial:

(M):

(A):

$$
\begin{gathered}
A_{t}^{(1)}=\frac{Y_{t}}{S_{t}^{(1)}} \\
A_{t}^{(1)}=Y_{t}-S_{t}^{(1)}
\end{gathered}
$$

Estágio 2 - Fatores sazonais e ajuste sazonal: redefinição das estimativas das componentes

i. Estimativa intermediária da tendência ' $T$ (2)', usando o filtro de Henderson de 13 termos:

$$
T_{t}^{(2)}=\sum_{j=-H}^{H} h_{j}^{(2 H+1)} A_{t+j}^{(1)}
$$

ii. Refinamento da estimativa da componente sazonal-irregular ' $S I^{(2)}$ ':

(M):

$$
\begin{gathered}
S I_{t}^{(2)}=\frac{Y_{t}}{T_{t}^{(2)}} \\
S I_{t}^{(2)}=Y_{t}-T_{t}^{(2)}
\end{gathered}
$$

iii. Refinamento da estimativa da componente sazonal ' $\widehat{S}^{(2),}$ utilizando uma média móvel sazonal 3×5 - MMs 3×5:

$$
\hat{S}_{t}^{(2)}=\frac{1}{15} S I_{t-36}^{(2)}+\frac{2}{15} S I_{t-24}^{(2)}+\frac{3}{15} S I_{t-12}^{(2)}+\frac{3}{15} S I_{t}^{(2)}+\frac{3}{15} S I_{t+12}^{(2)}+\frac{2}{15} S I_{t+24}^{(2)}+\frac{1}{15} S I_{t+36}^{(2)}
$$


iv. Fatores sazonais ' $S{ }^{(2)}$ ':

(M):

$$
S_{t}^{(2)}=\frac{\hat{S}_{t}^{(2)}}{\frac{1}{24} \hat{S}_{t-6}^{(2)}+\frac{1}{12} \hat{S}_{t-5}^{(2)}+\cdots+\frac{1}{12} \hat{S}_{t+5}^{(2)}+\frac{1}{24} \hat{S}_{t+6}^{(2)}}
$$

(A): $\quad S_{t}^{(2)}=\hat{S}_{t}^{(2)}-\left(\frac{1}{24} \hat{S}_{t-6}^{(2)}+\frac{1}{12} \hat{S}_{t-5}^{(2)}+\cdots+\frac{1}{12} \hat{S}_{t+5}^{(2)}+\frac{1}{24} \hat{S}_{t+6}^{(2)}\right)$

v. Série sazonalmente ajustada ' $A{ }^{(2)}$ ':

(M):

(A):

$$
\begin{gathered}
A_{t}^{(2)}=\frac{Y_{t}}{S_{t}^{(2)}} \\
A_{t}^{(2)}=Y_{t}-S_{t}^{(2)}
\end{gathered}
$$

\section{Estágio 3 - Estimativa final das componentes da série}

i. Tendência final ' $T^{(3)}$ ', para um determinado tamanho do filtro de Henderson, possivelmente diferente do que foi determinado no Estágio 2 (i):

$$
T_{t}^{(3)}=\sum_{j=-H}^{H} h_{j}^{(2 H+1)} A_{t+j}^{(2)}
$$

ii. Componente irregular final ' $I^{(3)}$ ':

(M):

(A):

$$
\begin{gathered}
I_{t}^{(3)}=\frac{A_{t}^{(2)}}{T_{t}^{(3)}} \\
I_{t}^{(3)}=A_{t}^{(2)}-T_{t}^{(3)}
\end{gathered}
$$

\section{Decomposição estimada:}

(M):

(A):

$$
\begin{gathered}
Y_{t}=T_{t}^{(3)} S_{t}^{(2)} I_{t}^{(3)} \\
Y_{t}=T_{t}^{(3)}+S_{t}^{(2)}+I_{t}^{(3)}
\end{gathered}
$$




\subsection{3.}

\section{Outros tópicos relacionados ao ajuste sazonal no X-13A-S}

Nesta subseção são apresentados alguns tópicos relacionados ao ajuste sazonal do método X-11, no programa X-13A-S. Dentre eles, está o filtro de Henderson, para extração da tendência; a seleção automática dos filtros sazonais realizada pelo programa; e os testes para a presença de sazonalidade, sazonalidade móvel, e sazonalidade identificável. Além disso, são apresentadas as estatísticas de qualidade do ajuste sazonal desenvolvidas para os programas da Família X-11.

\subsubsection{1.}

\section{Filtro de Henderson}

O método X-11 utiliza o filtro de Henderson para a decomposição da componente de tendência.

Descrito em Macaulay (1931) e citado em Bell \& Monsell (1992), os pesos para o $j$-ésimo termo do filtro de Henderson

$$
H_{n}(B)=\sum_{j} h_{j}^{(n)} B^{j}
$$

são $h_{j}^{(n)}, j=0, \pm 1, \ldots, \pm(n-1) / 2$, e podem ser obtidos pela seguinte fórmula:

$$
h_{j}^{(n)}=\frac{315\left[(m-1)^{2}-j^{2}\right]\left[m^{2}-j^{2}\right]\left[(m+1)^{2}-j^{2}\right]\left[\left(3 m^{2}-16\right)-11 j^{2}\right]}{8 m\left(m^{2}-1\right)\left(4 m^{2}-1\right)\left(4 m^{2}-9\right)\left(4 m^{2}-25\right)}
$$

sendo $m=(n+3) / 2$, e $n=$ tamanho do filtro. Na Eq. (2-26), o termo $B$ é o operador de deslocamento (Backshift operator).

No X-13A-S, o usuário pode escolher qualquer ordem ímpar do filtro de Henderson. Existe também a opção automática, onde o programa seleciona o tamanho do filtro mais adequado para a série. Para séries temporais mensais, esses tamanhos são 9, 13 e 23, e para as trimestrais, os tamanhos são 5 e 7.

A escolha automática do tamanho do filtro de Henderson é dada pela razão $\mathrm{I} / \mathrm{C}$, que equivale à relação entre a variação percentual média da componente irregular e a variação percentual média da componente de tendência (Dagum, 1999). 
Essa razão I/C determinará o filtro a ser aplicado, sendo usado o seguinte critério para séries temporais mensais:

- $\quad$ se $\mathrm{I} / \mathrm{C}=0,99$, usa-se o filtro de Henderson de 9 termos;

- $\quad$ se $\mathrm{I} / \mathrm{C}=3,5$ é aplicado o filtro de Henderson de 13 termos;

- $\quad$ se $\mathrm{I} / \mathrm{C}=7$, Henderson de 23 termos é aplicado.

Ou seja, quanto maior for a variação da irregular em relação à variação da tendência, maior deve ser o tamanho do filtro.

\subsubsection{2.}

\section{Seleção automática dos filtros sazonais no X-13A-S}

Quando é solicitado o ajuste padrão (X-11 default), o programa utiliza as médias móveis sazonais $3 \times 3$ e $3 \times 5$, nos estágios 1 e 2 do procedimento, respectivamente. Porém, dependendo do valor da Razão de Sazonalidade Móvel MSR, outras médias móveis sazonais seriam mais apropriadas (Lothian, 1984).

A MSR é calculada com base na divisão entre a estatística $I$ (relacionada à componente irregular), e a estatística $S$ (relacionada à sazonalidade). As estatísticas $I$ e $S$ são calculadas da seguinte forma:

$$
\begin{gathered}
I=\frac{\sum_{t=2}^{N}\left(I_{t}-I_{t-1}\right)^{2}}{N-1} \\
S=\frac{\sum_{t=2}^{N}\left(S_{t}-S_{t-1}\right)^{2}}{N-1}
\end{gathered}
$$

Os intervalos de valores de MSR foram fornecidos por Lothian (1984) e estão no quadro a seguir: 


\begin{tabular}{|c|c|c|}
\hline Média móvel sazonal & $\begin{array}{c}\text { Intervalo de MSR }(\boldsymbol{I} / \boldsymbol{S}) \text { para } \\
\text { séries menores de } \mathbf{1 5} \text { anos }\end{array}$ & $\begin{array}{c}\text { Intervalo de MSR }(\boldsymbol{I} / \mathbf{S}) \text { para } \\
\text { séries maiores de } \mathbf{1 5} \text { anos }\end{array}$ \\
\hline 3 -termos & $0 \leq I / S \leq 2,3$ & $0 \leq I / S \leq 2,1$ \\
\hline $3 \times 3$ & $2,3<I / S \leq 4,1$ & $2,1<I / S \leq 3,8$ \\
\hline $3 \times 5$ & $4,1<I / S \leq 5,2$ & $3,8<I / S \leq 5,0$ \\
\hline $3 \times 9$ & $5,2<I / S \leq 6,5$ & $5,0<I / S \leq 6,9$ \\
\hline Simples de N-termos & $6,5<I / S \leq 7,1$ & $6,9<I / S \leq 7,1$ \\
\hline
\end{tabular}

Quadro 2.2 Intervalos de MSR

O problema ao incorporar esses intervalos no programa são as revisões desnecessárias em relação aos valores próximos aos limites. Para evitar isso, a seleção automática da média móvel sazonal é feita da seguinte forma:

1) A razão global $I / S$ é calculada até o último ano de dados disponíveis.

2) São usadas 'bandas' ao invés de pontos de corte, onde a seleção é realizada da seguinte forma (Dagum, 1999):

(a) Se a razão global $I / S \leq 2,5$, então a média móvel sazonal 3x3 é utilizada; se $3,5 \leq I / S \leq 5,5$, então a média móvel sazonal $3 \times 5$ é utilizada. E se $I / S \geq 6,5$, então a média móvel sazonal $3 \times 9$ é utilizada.

(b) Se a razão global 2,5 $<I / S<3,5$, ou $5,5<I / S<6,5$, o último ano de dados é suprimido a uma nova razão $I / S$ é calculada. Caso esse novo $I / S$ continue fora dos intervalos especificados no item (a), mais um ano de dados é suprimido. Isso é realizado para os últimos 5 anos, no máximo. Caso $I / S$ continue fora dos intervalos especificados no item (a), a média móvel sazonal 3x5 é utilizada. 


\subsubsection{3.}

\section{Testes para a presença de sazonalidade no X-13A-S}

A componente sazonal é definida como a variação intra-anual que se repete constantemente (sazonalidade estável) ou que vai evoluindo de ano para ano (sazonalidade móvel). Se a alteração nos fatores sazonais, de ano para ano, for muito grande, serão introduzidas distorções na série. Sendo assim, é importante determinar se a sazonalidade é identificável.

Para que a sazonalidade seja identificável, a série deve ser considerada 'sazonal'. Para isso, é utilizado o teste paramétrico para a presença de sazonalidade assumindo estabilidade (teste $F$ ) e o teste não paramétrico para a presença de sazonalidade assumindo estabilidade (Kruskal-Wallis). Além disso, uma vez que a presença da sazonalidade móvel pode provocar distorções, é importante avaliar a sazonalidade móvel juntamente com a sazonalidade estável, a fim de determinar se a sazonalidade é identificável.

O teste para a sazonalidade identificável é realizado combinando-se os testes para sazonalidade estável e móvel. A descrição dos testes, a seguir, baseia-se em Lothian \& Morry (1978) e Higginson (1975). Dagum (1988), fornece detalhes.

\subsubsection{1.}

Teste $F$ para a presença de sazonalidade estável (Fs)

O teste é baseado na ANOVA de um fator da razão SI (ou diferença). O valor da estatística $F$ é a razão entre duas variâncias: (1) 'entre meses', que é devida à componente sazonal; e (2) 'residual', devida à componente irregular.

Uma vez que algumas premissas do teste $F$ são possivelmente violadas, utiliza-se um nível de significância muito pequeno $(0,1 \%)$ para indicar que existe sazonalidade suficiente para justificar a aplicação do método X-11.

Shiskin et al. (1967) alertam para o fato de que a existência de sazonalidade móvel reduz a variação 'entre meses', no teste de sazonalidade estável. Com isso, é possível que o teste conclua pela não evidência de sazonalidade, quando na verdade existe um padrão sazonal na série.

A explicação detalhada deste teste está no Apêndice A. 


\subsubsection{2.}

\section{Teste $\boldsymbol{F}$ para a presença de sazonalidade móvel $\left(F_{M}\right)$}

O teste de sazonalidade móvel é baseado em uma ANOVA de 2 fatores realizada com a razão SI (ou diferença). Tal teste foi desenvolvido por Higginson (1975). Nele é testada a presença de sazonalidade móvel caracterizada por mudanças graduais na amplitude da curva.

Na ANOVA utilizada, os fatores considerados são: mês e ano. A variância entre os 'meses' mede a magnitude da sazonalidade. Já a variância entre os 'anos' mede o movimento (variação) da sazonalidade entre os anos. O teste para a presença de sazonalidade móvel é baseado na razão entre a variância 'entre os anos' e a variância residual. Um valor alto dessa estatística de teste indica a presença de sazonalidade móvel e, com isso, a inadequação do uso do método X-11.

No método X-11, a presença de sazonalidade móvel pode ser entendida como um indicador de sazonalidade residual, uma vez que o programa 'força' a existência de sazonalidade estável.

Este teste está apresentado com mais detalhes no Apêndice B.

\subsubsection{3.}

\section{Teste de Kruskal-Wallis para a presença de sazonalidade estável (KW)}

O teste de Kruskal-Wallis é a alternativa não paramétrica ao teste $F$ da ANOVA de 1 fator. Enquanto que no teste $F$ existe a premissa de normalidade, no teste de Kruskal-Wallis não existem restrições sobre a distribuição das populações analisadas.

No teste de Kruskal-Wallis para a presença de sazonalidade estável de uma série mensal, a estatística de teste segue a distribuição qui-quadrado, com $(12-1)$ graus de liberdade, onde 12 é o número de meses a serem comparados. 


\subsubsection{4.}

\section{Teste combinado para a presença de sazonalidade identificável}

O objetivo deste teste é verificar se a sazonalidade da série é identificável ou não. A ideia é que para uma melhor identificação da sazonalidade, a sazonalidade 'estável' deve ser suficientemente maior do que a quantidade de 'sazonalidade móvel'. Se houver pouca sazonalidade estável e a maior parte do processo for dominada por sazonalidade móvel, a componente sazonal não será bem estimada pelo X-11, pois o método não a identificará apropriadamente (Dagum, 1978).

Em linhas gerais, o teste consiste em combinar os valores da estatística dos testes $F$ obtidas previamente (ver Subseções 2.3.3.3.1 e 2.3.3.3.2), da seguinte forma: sendo $F_{S}$ o valor de $F$ do teste de sazonalidade estável e $F_{M}$ o valor referente ao teste de sazonalidade móvel:

(1) Se a hipótese nula de ausência de sazonalidade estável não for rejeitada ao nível de $0,1 \%$ de significância, então a série é considerada 'não sazonal';

(2) Se a hipótese nula de (1) for rejeitada, então o programa calcula as seguintes estatísticas:

$$
\begin{gathered}
T_{1}=\frac{7}{F_{M}} \\
T_{2}=\frac{3 F_{M}}{F_{S}} \\
T=\frac{\left(T_{1}+T_{2}\right)}{2}
\end{gathered}
$$

(2.1.) Se a hipótese nula de ausência de sazonalidade móvel for rejeitada ao nível de significância de $5 \%$ e $T \geq 1$, a hipótese nula de ausência de sazonalidade identificável não é rejeitada e programa retorna a seguinte mensagem: 'Ausência de Sazonalidade Identificável'.

(2.2.) Se a hipótese nula de ausência de sazonalidade móvel não for rejeitada, mas $T_{1} \geq 1, T_{2} \geq 1$, ou o teste não paramétrico não rejeitar $\mathrm{H}_{0}$ ao nível de $0,1 \%$ de significância, o programa retorna a seguinte mensagem: 'Sazonalidade Identificável Provavelmente Ausente’ 
(2.3.) Se a hipótese nula de ausência de sazonalidade móvel não for rejeitada, e a hipótese nula de ausência de sazonalidade estável for rejeitada no teste $F$ e no teste de Kruskal-Wallis, o programa retorna a seguinte mensagem: 'Sazonalidade Identificável Presente'.

Resumindo, o programa X-13ARIMA-SEATS realiza quatro testes para verificação de sazonalidade. É utilizado o teste $F$ para a presença de sazonalidade estável, e outro para a presença de sazonalidade móvel. Além disso, utiliza-se também o teste não paramétrico de Kruskal-Wallis para a presença de sazonalidade estável. O quarto teste envolve os resultados dos testes anteriores com o objetivo de verificar se existe sazonalidade 'identificável'.

A Figura 2.2, a seguir, ilustra o procedimento completo do teste combinado para identificação de sazonalidade, apresentado nesta subseção. 


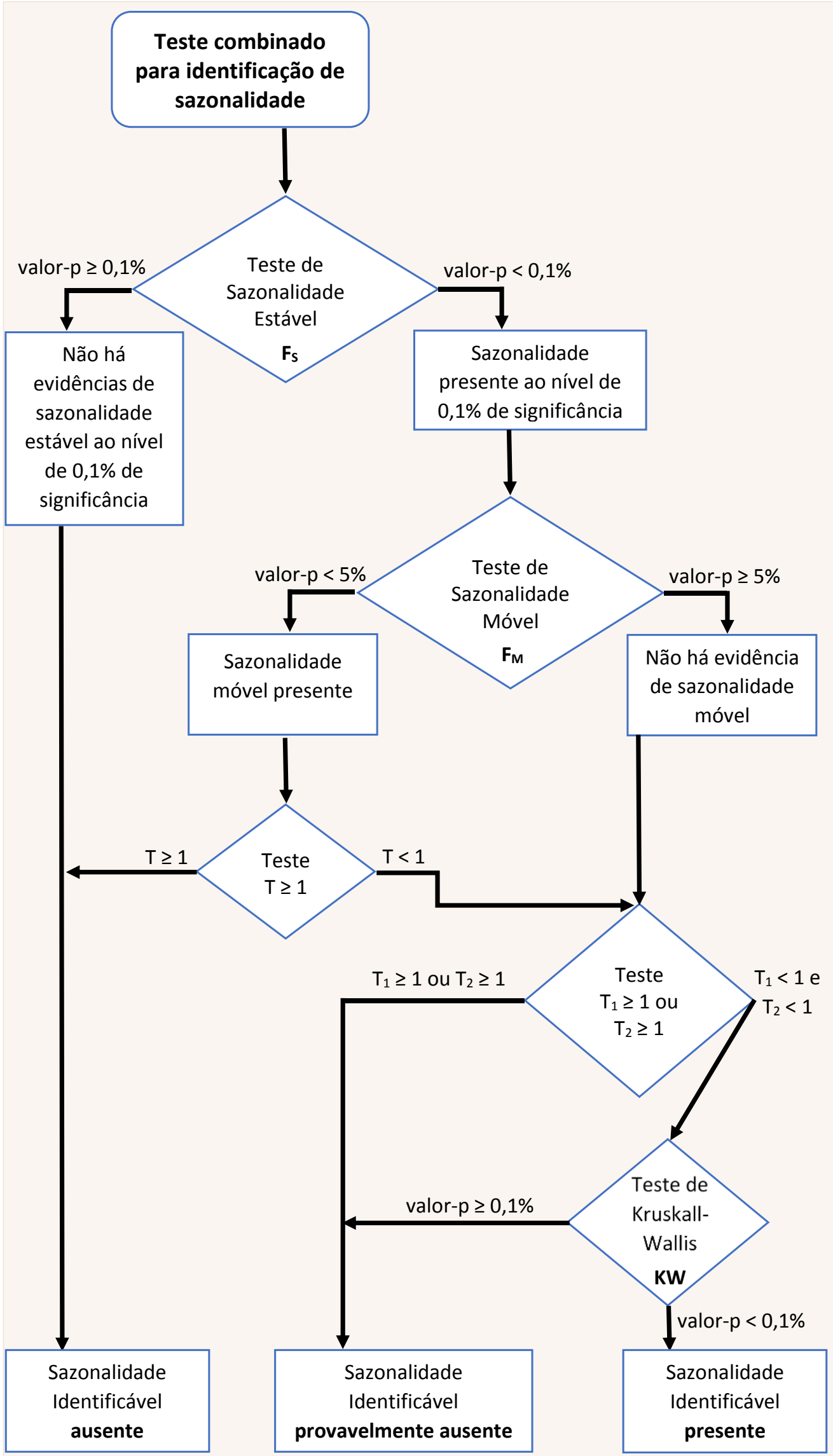

Fonte: SAS. http://support.sas.com/documention/cdl/em/etsug, em 04/06/2013.

Figura 2.2. Teste combinado para identificação de sazonalidade 


\subsubsection{4. \\ Estatísticas M e Q para qualidade do ajuste}

As estatísticas M e Q foram desenvolvidas pelo Statistic Canada para o X-11ARIMA, e estão implementadas no X-12-ARIMA e no X-13A-S. Elas indicam a qualidade do ajuste sazonal realizado. Existem 11 estatísticas M (M1 a M11) e duas estatísticas Q (Q e Q2), sendo que as estatísticas Q são médias ponderadas das estatísticas M (Lothian \& Morry, 1978).

Os valores das estatísticas $\mathrm{M}$ e $\mathrm{Q}$ variam de 0 a 3 , sendo que valores acima de 1 são indícios de problemas potenciais relacionados ao ajuste. Vale ressaltar que algumas estatísticas M são 'mais importantes' do que outras, e não é essencial que todas as estatísticas M sejam inferiores a 1 para o ajuste ser considerado aceitável.

Dentre todas as estatísticas M, a M7 é considerada a mais importante, pois indica se existe muita sazonalidade móvel em relação à sazonalidade estável. Um alto grau de sazonalidade móvel pode causar problemas na estimação das componentes da série, em razão dos filtros de média móvel utilizados no método $\mathrm{X}-11$. Se M7 > 1 significa que o ajuste sazonal realizado pelo $\mathrm{X}-11$ não será apropriado (Pedersen \& Fæste, 2006). A estatística M7 é calculada, com base em uma combinação dos testes $F_{M}$ e $F_{S}$, apresentados anteriormente, da seguinte forma:

$$
\mathrm{M} 7=\sqrt{\frac{1}{2}\left(\frac{7}{F_{S}}+\frac{3 F_{M}}{F_{S}}\right)} .
$$

A descrição de todas as estatísticas M está apresentada no Quadro 2.3, a seguir: 


\begin{tabular}{|c|c|}
\hline Nome & Descrição da Estatística de Diagnóstico \\
\hline M1 & $\begin{array}{l}\text { Mede a contribuição relativa da componente irregular sobre o período de } \\
\text { três meses. }\end{array}$ \\
\hline M2 & $\begin{array}{l}\text { Mede a contribuição relativa da componente irregular à variância da parte } \\
\text { estacionária da série. }\end{array}$ \\
\hline M3 & $\begin{array}{l}\text { Mede a intensidade da mudança mensal da componente irregular comparada } \\
\text { à intensidade da mudança mensal da componente tendência-ciclo. }\end{array}$ \\
\hline M4 & $\begin{array}{l}\text { Mede a intensidade da autocorrelação da componente irregular, descrita pela } \\
\text { duração da média. }\end{array}$ \\
\hline M5 & Mede os períodos de dominação estatística da componente cíclica. \\
\hline M6 & $\begin{array}{l}\text { Mede a intensidade da mudança anual da componente irregular comparada } \\
\text { à intensidade da mudança anual da componente sazonal. }\end{array}$ \\
\hline M7 & $\begin{array}{l}\text { Mede a intensidade da sazonalidade móvel em relação à intensidade da } \\
\text { sazonalidade estável. }\end{array}$ \\
\hline M8 & Mede a dimensão ou a flutuação da componente sazonal na série inteira. \\
\hline M9 & Mede o movimento linear médio da componente sazonal na série inteira. \\
\hline M10 & Mede a dimensão das flutuações da componente sazonal nos anos recentes. \\
\hline M11 & Mede o movimento linear médio da componente sazonal nos anos recentes. \\
\hline
\end{tabular}

\section{Quadro 2.3 Estatísticas M}

A estatística Q é uma medida global do ajuste. O seu cálculo é uma média ponderada das estatísticas M. Os pesos, de cada estatística M, na composição da estatística Q estão apresentados no Quadro 2.4:

\begin{tabular}{|l|c|c|c|c|c|c|c|c|c|c|c|}
\hline & M1 & M2 & M3 & M4 & M5 & M6 & M7 & M8 & M9 & M10 & M11 \\
\hline Peso & 13 & 13 & 10 & 5 & 11 & 10 & $\mathbf{1 6}$ & 7 & 7 & 4 & 4 \\
\hline
\end{tabular}

\section{Quadro 2.4. Pesos das estatísticas M na composição da estatística $Q$}

Como se pode notar, a estatística M7, relacionada à sazonalidade móvel, é a que apresenta o maior peso na composição da estatística $Q$.

No programa X-13A-S existe também a estatística Q2. A diferença entre as estatísticas Q e Q2 está no fato desta não utilizar no cálculo a estatística M2. 


\section{4. Tópicos gerais relacionados à sazonalidade móvel}

Nesta subseção é apresentada uma revisão histórica sobre o tema 'sazonalidade móvel', assim como os motivos de haver essa característica em algumas séries temporais. São apresentados, também, alguns testes desenvolvidos para analisar a existência de sazonalidade móvel.

\subsection{1. \\ Uma breve revisão histórica sobre a sazonalidade móvel}

As pesquisas na área de ajuste sazonal de séries econômicas teve um grande impulso nas décadas de 20 e 30, a partir do trabalho de Persons em 1919. Na década de 30 iniciaram-se os estudos envolvendo sazonalidade móvel. Será apresentado a seguir um breve histórico sobre o tratamento da 'sazonalidade móvel'.

Segundo Hillmer \& Bell (2002), existem registros de que o primeiro a notar essa característica (sazonalidade móvel) foi Gilbart, um banqueiro de meados do século XIX, ao observar a circulação de notas dos bancos do Reino Unido, em 1841. Persons, em 1919, destacou a existência de sazonalidade móvel, ao afirmar que embora existisse uma variação sistemática em algumas séries, não é indicado pensar na variação sazonal como sendo exatamente a mesma ano após ano.

Segundo King (1924), os primeiros a ajustarem sazonalmente os dados considerando a sazonalidade móvel foram Sydenstricker e Britten, em 1922, nos Estados Unidos, utilizando métodos gráficos. King (1924) modificou o método deles, aplicando medianas móveis, e enfatizou a necessidade de se considerar o tratamento da sazonalidade móvel. Alguns outros métodos para lidar com a sazonalidade móvel foram sugeridos no final da década de 20.

Em 1932, Kuznets sugeriu um método para detectar e ajustar mudanças na amplitude sazonal, de ano para ano, assumindo que o padrão sazonal permanecesse constante (Kuznets, 1932). Ele é um dos autores mais citados em relação às suas contribuições pioneiras no estudo da sazonalidade móvel, ao estudar os dados da indústria americana (Kuznets, 1933).

Ainda na década de 30, Mendershausen (1937) analisa as primeiras tentativas de se lidar com a sazonalidade móvel, e diz que a suposição de uma periodicidade regular perfeita no movimento sazonal implica em dizer que, não somente o clima, 
mas também os hábitos de consumo, os métodos de produção e transporte não mudam ao longo do tempo. $\mathrm{O}$ autor afirma que essa suposição não corresponde à realidade, pois o ciclo meteorológico não é perfeito. Além disso, a organização social, as técnicas e os hábitos apresentam instabilidade, não apenas devido a sua evolução, mas também em relação ao ciclo econômico e a outros eventos sociais.

Na década de 90, Canova \& Hansen (1995) citaram casos em que o padrão sazonal de séries reais apresentam mudanças. Os exemplos incluem o consumo de energia estudado por Engle et al. (1989); o consumo japonês examinado por Engle et al. (1993); as séries de produção industrial examinadas por Canova (1993); e algumas séries de PIB analisadas por Hylleberg et al. (1993). Eles apontam para a importância de estudar os diferentes tipos de sazonalidade. Canova é um autor bastante citado em relação a esse assunto.

Mais recentemente, Pezzulli (2005) cita que a variação da sazonalidade não é novidade nas pesquisas climáticas. E Pedersen \& Fæste (2006) defende que com o passar do tempo, o padrão sazonal das séries econômicas será alterado como resultado de mudanças institucionais; e que é importante monitorar esses movimentos uma vez que eles afetam a escolha dos filtros sazonais. Alguns outros pesquisadores notaram que o padrão sazonal de muitas variáveis macroeconômicas mudam ao longo do tempo (ver Bell \& Hillmer, 2002).

\subsection{2. Motivos para a existência de sazonalidade móvel}

A possibilidade de que a sazonalidade é afetada pelos ciclos econômicos vem sendo considerada na literatura. Segundo Ghysels (1990), as mudanças nos padrões sazonais de diversas séries trimestrais americanas, pós-Segunda Guerra Mundial, podem estar relacionadas às etapas do ciclo econômico.

Canova \& Ghysels (1994) estudaram a evolução do padrão sazonal de séries trimestrais americanas, e a relação dessa evolução com o ciclo econômico. $\mathrm{Na}$ análise gráfica realizada, a ideia de que a magnitude da evolução das flutuações sazonais é sensível aos vários estágios do ciclo econômico foi confirmada. Outros estudos que relacionam a existência de sazonalidade móvel com o ciclo econômico são Krane \& Wascher (1999) e Wilcox et al. (1997). 
Porém, os ciclos econômicos não são a única possível razão para as mudanças no padrão sazonal. Van Dijk et al. (2003) estudaram o efeito de mudanças tecnológicas e institucionais nas alterações dos padrões sazonais nas séries de produção industrial dos países do G7, em comparação com os efeitos do ciclo econômico. Eles concluíram que os efeitos das mudanças tecnológicas e institucionais são mais importantes do que os efeitos relacionados ao ciclo econômico.

Na década de 70, Diller (1971) atribuiu um outro possível motivo para a existência de sazonalidade móvel. Para o autor, uma das causas da sazonalidade móvel está na adaptação da economia às mudanças relacionadas a ela.

\subsection{3.}

\section{Alguns testes para a presença de sazonalidade móvel}

$\mathrm{Na}$ literatura foram encontrados testes estatísticos que vêm sendo sugeridos para examinar a estabilidade do padrão sazonal, e com isso a existência de sazonalidade móvel. Dentre eles estão os testes criados por Taylor (2003), Bussetti \& Harvey (2003), Sutradhar \& Dagum (1998), Caner (1998), Franses \& Hobijn (1997), Canova \& Hansen (1995), Sutradhar et al. (1995), Hylleberg et al. (1990), Dickey et al. (1984), e Franzini \& Harvey (1983).

Ghysels et al. (1994) comparam o desempenho dos testes de Dickey et al. (1984) e Hylleberg, Engle et al. (1990), chamado de 'HEGY test'.

Mais especificamente, Sutradhar \& Dagum (1998) compararam os resultados do teste desenvolvido por elas com os resultados do teste utilizado no programa X11-ARIMA, e concluíram que o teste do programa X-11-ARIMA indicou mais séries com sazonalidade móvel do que elas haviam encontrado com base na aplicação do teste criado por elas.

Vale mencionar o teste para a sub/superestimação (under/overadjustment) da sazonalidade proposto por Maravall (2003). A subestimação significa não remover toda a variação sazonal, o que ocorre no método X-11 quando existe sazonalidade móvel; e por superestimação, entende-se remover variação como sendo sazonal, quando na verdade faz parte da componente irregular. Quando existe sazonalidade 
móvel, ocorre o problema da subestimação se o método de ajuste sazonal não for adequado. Portanto, este é um teste indicado nesse caso.

Este capítulo apresentou os conceitos relacionados ao tema de estudo e um breve histórico. Além disso, mostrou os métodos de ajuste sazonal que já foram utilizados pelas agências governamentais. O capítulo também apresentou algumas comparações realizadas, na literatura, entre os métodos X-11 e SEATS, que são hoje os mais usados pelas agências governamentais. Em seguida, o capítulo detalhou as etapas do ajuste sazonal realizado pelo $\mathrm{X}-11$, e as diversas características relacionadas à seleção de filtros pelo método, assim como os testes para sazonalidade. Por fim, o capítulo apresentou alguns tópicos relacionados à sazonalidade móvel. 


\section{3 \\ Ajuste sazonal no domínio da frequência}

Existem dois enfoques principais em análise de séries temporais: análise no domínio do tempo e análise no domínio da frequência. No primeiro enfoque, o interesse está em construir um modelo adequado para explicar o comportamento da série, podendo ser utilizado para a previsão de valores futuros. Já no segundo, existe um maior interesse no estudo das propriedades da série.

A análise no domínio da frequência, ou análise espectral, consiste na decomposição da série em componentes senoidais, de frequências diferentes, estudando a maneira com que a energia total é distribuída entre elas. Algumas componentes de uma série temporal podem ser mais claramente definidas no domínio da frequência, como é o caso da componente sazonal. Já no domínio do tempo, a decomposição da série é incapaz de ter uma definição precisa (Grether \& Nerlove, 1970). Esse fato motivou a construção dos filtros para ajuste sazonal no domínio da frequência, propostos nesta tese.

Neste capítulo são definidos alguns conceitos de análise de séries temporais no domínio da frequência necessários para a compreensão do estudo. Além disso, é apresentado o arcabouço teórico do método X-11 no domínio da frequência. Por fim, é introduzida uma definição de filtros. Os conceitos apresentados neste capítulo baseiam-se principalmente em Diniz et al. (2010) e Pereira et al. (1986).

\section{1.}

\section{Alguns conceitos relacionados à análise no domínio da frequência}

\subsection{1.}

Sinal

Um sinal em tempo discreto é aquele que pode ser representado por uma sequência de valores, onde cada número representa a amplitude do sinal no instante $t$, sendo $t$ um número inteiro. A seguinte notação é utilizada para representar um sinal:

$$
\{x(t), t \in \mathbb{Z}\}
$$


A componente sazonal, de uma série temporal, pode ser interpretada como um sinal em tempo discreto, sendo representada por uma função seno ou cosseno. Considere como exemplo, o seguinte sinal:

$$
x(t)=\cos (\omega t)
$$

onde $\omega$, em rad/amostra, é a frequência angular do sinal, e a frequência linear é $\frac{\omega}{2 \pi}$ ciclos/amostra. Vale ressaltar que o conceito de amostra em processamento de sinais é diferente do conceito utilizado em estatística. No caso de uma série temporal mensal, seria $\frac{\omega}{2 \pi}$ ciclos/meses, uma vez que a unidade de tempo é o mês. Se $\omega=\frac{2 \pi}{12} \mathrm{rad} / \mathrm{amostra}$ significa que se completa um ciclo $(2 \pi$ radianos $)$ a cada 12 meses.

Para converter frequências $(\omega)$ em períodos $(p)$ a seguinte relação pode ser utilizada:

$$
p=\frac{2 \pi}{\omega}
$$

A análise no domínio da frequência trata da extração do sinal. E como uma série temporal pode ser decomposta em 'sinal + ruído', justifica-se a aplicação da análise no domínio da frequência para a extração da componente sazonal. O primeiro registro desse tipo de aplicação data de 1963, com os trabalhos de Whittle.

\subsection{2.}

\section{Sistemas em tempo discreto}

Um sistema de tempo discreto transforma uma série de entrada $x(t)$ em uma série de saída $y(t)$, tal que

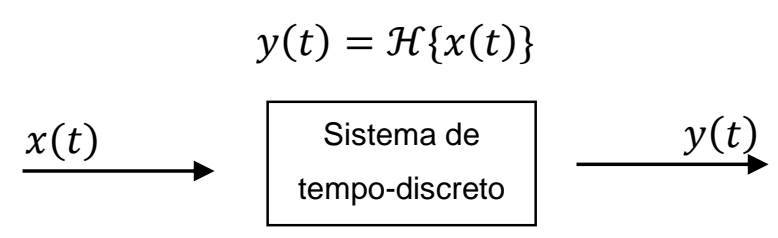

O operador $\mathcal{H}\{\cdot\}$ é a função de transferência, representando o sistema de tempo-discreto. Neste estudo, $\mathcal{H}\{\cdot\}$ terá as seguintes características: linear e invariante no tempo. 
O sinal de entrada $x(t)$ pode ser representado como a soma de impulsos deslocados,

$$
x(t)=\sum_{\mathrm{k}=-\infty}^{\infty} x(k) \delta(t-k)
$$

onde $\delta(t-k)$ é um sinal de impulso unitário em tempo discreto. Pode-se, então, representar a saída $y(t)$, como:

$$
y(t)=\mathcal{H}\left\{\sum_{\mathrm{k}=-\infty}^{\infty} x(k) \delta(t-k)\right\}=\sum_{\mathrm{k}=-\infty}^{\infty} \mathcal{H}\{x(k) \delta(t-k)\}
$$

Como $x(k)$ é uma constante, $y(t)$ pode ser escrito como

$$
y(t)=\sum_{\mathrm{k}=-\infty}^{\infty} x(k) \mathcal{H}\{\delta(t-k)\}=\sum_{\mathrm{k}=-\infty}^{\infty} x(k) h_{k}(t)
$$

onde $h_{k}(t)=\mathcal{H}\{\delta(t-k)\}$ é a resposta do sistema para o impulso em $\mathrm{n}=k$.

E como o sistema é invariante no tempo, define-se

$$
\mathcal{H}\{\delta(t)\}=h(t)
$$

Assim, pode-se reescrever $y(t)$ em termos de $h(t)$ como

$$
y(t)=\sum_{\mathrm{k}=-\infty}^{\infty} x(k) h(t-k)
$$

indicando que o sistema invariante no tempo é completamente caracterizado pela resposta ao impulso $h(t)$. E $y(t)$ pode ser representado como o resultado da convolução entre a série de entrada $x(t)$ e a resposta ao impulso $h(t)$, da seguinte forma:

$$
y(t)=x(t) * h(t)
$$


A resposta em frequência de $\mathcal{H}(\cdot)$ é a medida quantitativa do espectro de um sistema em resposta a um estímulo, e é utilizada para caracterizar a dinâmica do sistema. Trata-se de uma medida da grandeza e da fase da saída, em comparação com a entrada. Em outras palavras, se uma onda senoidal for injetada num sistema linear a uma dada frequência, o sistema irá responder na mesma frequência com uma certa magnitude e ângulo de fase, em relação à entrada. O interesse está em estimar a função de transferência $\mathcal{H}(\cdot)$, conhecendo as séries de entrada $x(t)$ e de saída $\mathrm{y}(\mathrm{t})$; fazer previsões com base na série de entrada e na função de transferência; e controlar a série de saída, trazendo-a o mais próximo possível de um valor desejado.

\subsection{3.}

\section{Análise de Fourier}

A Análise de Fourier é uma forma de análise no domínio da frequência que estuda a aproximação de qualquer série temporal por uma soma de senos e cossenos. Pelo teorema de Fourier, qualquer função $f(x)$, periódica no período $p$, pode ser escrita como uma série de Fourier na forma:

$$
f(x)=\sum_{j=0}^{\infty}\left[a_{j} \cos \left(\frac{2 \pi j}{p} x\right)+b_{j} \operatorname{sen}\left(\frac{2 \pi j}{p} x\right)\right]
$$

onde $a_{0}, b_{0}, a_{1}, b_{1}, \ldots$ são constantes que podem ser determinadas a partir de $f(x)$.

\subsubsection{1.}

\section{Elementos básicos de um modelo senoidal simples}

Uma série, cujos valores variam em torno de uma média e as variações se repetem, pode ser descrita por quatro elementos: período $(p)$, frequência $(f)$, amplitude (A) e fase $(\theta)$.

O período é o tempo necessário para a série temporal se repetir. É medido em unidade de tempo por ciclo. Em geral, uma série é periódica quando $x(t)=$ $x(t+p), \forall t \in \mathbb{R}$. Se $p$ for o menor real positivo possível, ele é chamado de período fundamental. 
A frequência é o inverso do período, fornecendo o número de repetições por unidade de tempo. Um evento que se repete a cada 12 meses possui frequência igual a $1 / 12$ ciclos por mês.

Uma série senoidal $x(t)$ pode ser escrita sob a representação harmônica:

$$
x(t)=\mu+A \cos [2 \pi f(t-\tau)], \quad t=1,2, \ldots, T
$$

onde:

$$
\begin{aligned}
& \mathrm{A}=\text { amplitude } \\
& \theta=2 \pi f \tau=\text { fase }
\end{aligned}
$$

Para expressar a função em termos de frequência angular, medida em radianos por unidade de tempo, temos:

$$
x(t)=\mu+A \cos (\omega t-\phi), \quad t=1,2, \ldots, T
$$

onde:

$$
\begin{aligned}
& \omega=2 \pi f, \quad 0 \leq \omega \leq 2 \pi \mathrm{e} \\
& \phi=2 \pi f \tau
\end{aligned}
$$

Utilizando a regra trigonométrica, reescreve-se $A \cos (\omega t-\phi)$ como:

$$
\begin{aligned}
A \cos (\omega t-\phi) & =A(\cos \omega t \cos \phi+\operatorname{sen} \omega t \operatorname{sen} \phi) \\
= & \alpha \cos \omega t+\beta \operatorname{sen} \omega t
\end{aligned}
$$

onde $\alpha=A \cos \phi$ e $\beta=A \operatorname{sen} \phi$.

Então, pode-se escrever $x(t)$ como:

$$
x(t)=\mu+\alpha \cos \omega t+\beta \operatorname{sen} \omega t, \quad t=1,2, \ldots, T
$$

Como uma série temporal pode ter variações em diferentes frequências, uma generalização da equação acima é dada por:

$$
x(t)=\mu+\sum_{j=1}^{k}\left(\alpha_{j} \cos \omega_{j} t+\beta_{j} \operatorname{sen} \omega_{j} t\right), \quad t=1,2, \ldots, T
$$

onde $\alpha_{j}$ e $\beta_{j}$ são variáveis aleatórias e k é o número de harmônicos. 


\subsubsection{2.}

\section{Transformada de Fourier}

A transformada de Fourier refere-se à representação de Fourier para funções contínuas, e esta por sua vez representa qualquer função integrável $f(x)$ como a soma de exponenciais complexas com frequência angular $\omega$ e amplitude complexa $\mathcal{F}[f(x)]$.

A transformada de Fourier transforma a função $f$, do domínio do tempo, para o domínio da frequência. Ela está apresentada a seguir:

$$
\mathcal{F}(i \omega)=\mathcal{F}[f(x)]=\int_{-\infty}^{\infty} f(x) \mathrm{e}^{-\mathrm{i} \omega x} d x
$$

onde $\omega$ é a frequência angular e $i \equiv \sqrt{-1}$.

A transformada inversa de Fourier faz o caminho contrário, transformando uma função no domínio da frequência, para o domínio do tempo é definida por:

$$
\mathcal{F}^{-1}[\mathcal{F}(\mathrm{i} \omega)]=f(x)=\frac{1}{2 \pi} \int_{-\infty}^{\infty} \mathcal{F}(i \omega) \mathrm{e}^{\mathrm{i} \omega \mathrm{x}} d \omega .
$$

Por meio da transformada de Fourier, uma função matemática - e consequentemente, uma série temporal, pode ser expressa como uma soma de senos e cossenos. Com isso, qualquer conjunto de dados pode ser analisado diretamente por um espectro de frequências.

Uma das vantagens de se trabalhar com a transformada é a facilidade nos cálculos. Por meio da transformada, uma operação de convolução se transforma em uma simples multiplicação.

Na transformada de Fourier, para cada frequência $\omega$, integra-se a função $f(x)$ sobre todos os valores da coordenada $x$. Se o valor da integral for alto para esta frequência, então o sinal tem uma componente significativa nela, ou seja, uma parte significativa deste sinal é composto por esta frequência.

As transformadas de Fourier, direta e inversa, de uma função do tempo discreto $h(t)$, são definidas para $\omega \in \mathbb{R}$, por:

$$
\begin{gathered}
\mathcal{H}\left(e^{i \omega}\right)=\int_{-\infty}^{\infty} h(t) \mathrm{e}^{-\mathrm{i} \omega t} d t \\
h(t)=\frac{1}{2 \pi} \int_{-\pi}^{\pi} \mathcal{H}\left(e^{i \omega}\right) \mathrm{e}^{\mathrm{i} \omega \mathrm{t}} d \omega
\end{gathered}
$$

onde $e^{i \omega t} \equiv \cos (\omega t)+i \operatorname{sen}(\omega t)$. 
Quando é necessário reduzir o domínio a um determinado intervalo para analisar o comportamento do sinal, realiza-se uma discretização da Série de Fourier. Os coeficientes da Transformada Discreta de Fourier (DFT) fornecem uma aproximação para os coeficientes da Série de Fourier.

Dado um sinal do tempo discreto $h(t)$ de comprimento N, a DFT é denotada por $\mathcal{H}_{k}$, definida por:

$$
\mathcal{H}_{k}=\sum_{\mathrm{j}=0}^{\mathrm{N}-1} h(t) \mathrm{e}^{-\mathrm{i} 2 \pi \mathrm{jk} / \mathrm{N}}
$$

Considerando $\mathrm{W}=\mathrm{e}^{-\mathrm{i} 2 \pi / \mathrm{N}}$, tem-se:

sendo

$$
\mathcal{H}_{k}=\sum_{\mathrm{j}=0}^{\mathrm{N}-1} h(t) \mathrm{W}^{\mathrm{jk}}
$$

$$
h(t)=\frac{1}{\mathrm{~N}} \sum_{\mathrm{k}=0}^{\mathrm{N}-1} \mathcal{H}_{k} \mathrm{~W}^{-\mathrm{tk}}
$$

\subsubsection{1. \\ Transformada Z}

A transformada $\mathrm{Z}$ converte um sinal no domínio do tempo ' $x(t)$ ', que é uma sequência de números reais (ou complexos), em uma representação de domínio de frequência ' $X(z)$ ', que é uma expressão algébrica no domínio complexo.

No domínio do tempo: $x(t)=Z^{-1}\{X(z)\}$

No domínio da frequência: $X(z)=Z\{x(t)\}$

A transformada $\mathrm{Z}$ de uma função $x(t)$ é definida como:

$$
X(z)=Z\{x(t)\}=\sum_{t=-\infty}^{\infty} x(t) z^{-t}
$$

onde $z$ é um número complexo.

A transformada de Fourier de uma série discreta é a transformada $\mathrm{Z}$ calculada sobre o círculo unitário no plano complexo. Ou seja, para $z=e^{i \omega}$ 


$$
X\left(e^{i \omega}\right)=\sum_{t=-\infty}^{\infty} x(t) e^{-i \omega t}
$$

onde $t$ é um número inteiro que representa o domínio do tempo discreto, e $\omega$ é um número real em $[-\pi, \pi)$, que representa a frequência angular contínua normalizada (em radianos por amostra).

Sendo assim, a transformada de Fourier de tempo discreto é um caso especial da transformada Z.

Em sistemas de tempo discreto, a relação entre o sinal de entrada $x(t)$ e a saída $y(t)$ é dada por meio da transformada $Z$, e então a função de transferência é escrita como

$$
\mathcal{H}(z)=\frac{Y(\mathrm{z})}{X(\mathrm{z})}
$$

\subsubsection{3. \\ Espectros e funções de transferência}

A transformada de Fourier de $h=h(t)$ é uma função $\mathcal{H}=\mathcal{H}(\omega)$, cuja imagem está no conjunto dos números complexos. Logo, ela pode ser decomposta nas partes real e imaginária, como também pode ser escrita na forma polar. Seja $\mathcal{H}_{\mathrm{r}}=\mathcal{H}_{\mathrm{r}}(\omega)$ e $\mathcal{H}_{\mathrm{I}}=\mathcal{H}_{\mathrm{I}}(\omega)$, as partes real e imaginária, respectivamente, de $\mathcal{H}=$ $\mathcal{H}(\omega)$ e $i \equiv \sqrt{-1}$. Escreve-se $\mathcal{H}$ como:

$$
\mathcal{H}(\omega)=\mathcal{H}_{\mathrm{r}}(\omega)+i \mathcal{H}_{\mathrm{I}}(\omega)=|\mathcal{H}(\omega)| \mathrm{e}^{i \measuredangle \mathcal{H}(\omega)} .
$$

\subsubsection{1.}

\section{Espectro de amplitude do sinal}

A amplitude da transformada de Fourier $\mathcal{H}(\omega)=\mathcal{H}_{\mathrm{r}}(\omega)+i \mathcal{H}_{\mathrm{I}}(\omega)$, ou espectro de amplitude do sinal $h=h(t)$, é representada por:

$$
|\mathcal{H}(\omega)|=\sqrt{\mathcal{H}_{\mathrm{r}}^{2}(\omega)+\mathcal{H}_{\mathrm{I}}^{2}(\omega)}
$$

O espectro discreto de amplitude de um sinal periódico $s=s(t)$ é o gráfico das amplitudes em função das respectivas frequências de $s=s(t)$. 


\subsubsection{2.}

\section{Gráfico do ganho}

O gráfico do ganho mostra como a amplitude de uma senóide é modificada em função de sua frequência por um sistema linear.

No estado-estacionário, o ganho é a razão entre a variação da saída e a variação da entrada. Ou seja, ele pode ser analisado como o coeficiente da regressão do processo de saída sobre o processo de entrada, na frequência $\omega$.

Se um sinal $x(t)$, com uma componente senoidal de amplitude $|X|$, frequência angular $\omega$ e fase $\phi$, representado por

$$
X(\omega)=|X(\omega)| e^{i(\omega t+\phi)}
$$

for a entrada de um sistema, então a saída será

$$
Y(\omega)=|Y(\omega)| e^{i(\omega t+\theta)}
$$

e a resposta em frequência $\mathcal{H}(\mathrm{i} \omega)$ descreverá a mudança para cada frequência $\omega$ em termos do ganho $\mathrm{G}(\omega)$ dado por:

$$
\mathrm{G}(\omega)=\frac{|Y(\omega)|}{|X(\omega)|}=|\mathcal{H}(\mathrm{i} \omega)|
$$

Se o sinal é real, o ganho é simétrico sobre as frequências de $-\pi$ a $\pi$. Por esta razão, o gráfico do ganho é tipicamente representado no intervalo de 0 a $\pi$.

\subsubsection{3.}

\section{Espectro de fase}

O ângulo de fase da transformadas de Fourier $\mathcal{H}$, também conhecido como o espectro de fase do sinal $h=h(t)$ é definido por

$$
\measuredangle \mathcal{H}(\omega)=\measuredangle Y(\omega)-\measuredangle X(\omega)=\arctan \left(\frac{\mathcal{H}_{\mathrm{I}}(\omega)}{\mathcal{H}_{\mathrm{r}}(\omega)}\right)
$$

O espectro de fase é o gráfico das fases em função das respectivas frequências de $x=x(t)$.

É importante que a fase seja linear, para defasar as observações no mesmo período de tempo. Caso não seja linear, haverá uma distorção no sinal. 


\subsubsection{4.}

\section{Espectro de potência}

O espectro de potência de um sinal aleatório exibe a importância de cada frequência na série. As frequências mais relevantes apresentarão o chamado 'pico espectral'. Ele é utilizado para analisar somente séries temporais estacionárias. Se a série é não-estacionária, recomenda-se tornar a série estacionária, por meio de uma transformação, para depois obter o espectro.

O espectro de potência do sinal determinístico $h=h(t)$ é definido como

$$
\mathrm{P}(\omega)=|\mathcal{H}(\omega)|^{2}=\mathcal{H}_{\mathrm{r}}^{2}(\omega)+\mathcal{H}_{\mathrm{I}}^{2}(\omega) .
$$

Nerlove (1964, p. 262) define sazonalidade como "a característica de uma série temporal que dá origem a picos espectrais em frequências sazonais". Dessa forma, dado que o espectro de potência mostra as frequências que compõem a série, e uma vez que uma série é formada por algumas frequências, o ajuste sazonal equivale a remover a frequência sazonal.

O espectro de um processo estacionário é a transformada de Fourier da função de autocovariância do processo. Sendo assim, ele complementa o estudo realizado no domínio do tempo através da função de autocorrelação, auxiliando na captura da frequência do processo estocástico e identificando periodicidades na série.

\subsubsection{4.}

\section{Função Geradora de Autocovariância e sua relação com o espectro}

Se $Y_{t}$ é um processo estacionário de segunda ordem, com média $E\left(Y_{t}\right)=\mu$, variância $\sigma^{2}$ e j-ésima autocovariância

$$
\gamma_{j}=E\left[\left(Y_{t}-\mu\right)\left(Y_{t+j}-\mu\right)\right] .
$$

Assumindo que essas autocovariâncias são somáveis, a função geradora de autocovariância (AGF) é dada por

$$
g_{Y}(z)=\sum_{j=-\infty}^{\infty} \gamma_{j} z^{j}
$$

onde: $j \in \mathbb{Z} ; z=\cos (\omega)-i \operatorname{sen}(\omega)=\mathrm{e}^{-\mathrm{i} \omega} ; i \equiv \sqrt{-1} \mathrm{e} \omega$ é o ângulo em radianos que $z$ faz com o eixo dos reais. 
Essa função é construída tomando-se a j-ésima autocovariância e multiplicando-a por um escalar complexo, elevado à $j$-ésima potência, e depois somando todos os possíveis valores de $j$.

Se a função geradora de autocovariância é avaliada em $z=\mathrm{e}^{-\mathrm{i} \omega}$ e dividida por $2 \pi$, a função resultante de $\omega$ é

$$
s_{Y}(\omega)=\frac{1}{2 \pi} g_{Y}\left(e^{-i \omega}\right)=\frac{1}{2 \pi} \sum_{j=-\infty}^{\infty} \gamma_{j} e^{-i \omega j}
$$

que também pode ser escrita como:

$$
s_{Y}(\omega)=\frac{1}{2 \pi}\left\{\gamma_{0}+2 \sum_{j=1}^{\infty} \gamma_{j} \cos (\omega j)\right\}
$$

Tal função é periódica em $\omega$, e chamada de densidade espectral de potência de $Y$.

Isto significa que uma vez conhecida a função geradora de autocovariância de um processo, pode-se chegar ao espectro do processo.

O inverso também é verdadeiro, ou seja, se o espectro do processo for conhecido (o valor de $s_{Y}(\omega)$ para qualquer $\omega \in[0, \pi]$ ), pode-se calcular o valor da $j$-ésima autocovariância, para qualquer $j$. Isso é realizado calculando-se a transformada de Fourier inversa, definida como:

$$
\gamma_{j}=\int_{-\pi}^{\pi} s_{Y}(\omega) e^{-i \omega j} d \omega
$$

Ou de forma equivalente:

$$
\gamma_{j}=\int_{-\pi}^{\pi} s_{Y}(\omega) \cos (\omega j) d \omega
$$

Se $j=0$, tem-se $\gamma_{0}=\int_{-\pi}^{\pi} s_{Y}(\omega) d \omega$, ou seja, a variância de $Y_{t}$ como a área sob o espectro entre $-\pi$ e $\pi$.

Isto mostra que o espectro $s_{Y}(\omega)$ pode ser interpretado como a decomposição do processo de variância. Um pico no espectro indica uma importante contribuição à variância por parte das componentes com frequências no correspondente intervalo. As baixas frequências correspondem essencialmente às componentes que evoluem lentamente, como a tendência e o ciclo de uma série temporal. As altas frequências correspondem às componentes que evoluem mais rapidamente. Já a sazonalidade representa o pico nas frequências sazonais e nos seus múltiplos. 


\section{2.}

\section{O método X-11 no domínio da frequência}

Como esta proposta trata da construção de um filtro no domínio da frequência para o ajuste sazonal, que terá o seu desempenho comparado ao método X-11, é interessante apresentar o algoritmo do método X-11 no domínio da frequência.

\subsection{1.}

\section{$O$ algoritmo base do $X-11$}

Antes de apresentar o algoritmo do método X-11, serão definidas algumas notações relacionadas aos filtros de médias móveis utilizados pelo X-11. Inicialmente, define-se a média móvel simples de $P$ termos, utilizando ' $d$ ' observações para frente e ' $P-d-1$ ' observações após a observação atual, como um filtro, dado pela seguinte transformada $\mathrm{Z}$ :

$$
M A_{P}(z)=\frac{z^{d}}{P}\left[\begin{array}{lllll}
1 & 1 & \ldots & 1 & 1
\end{array}\right]_{P \times 1} \mathbf{E}_{P}(z)
$$

Onde

$$
\mathbf{E}_{P}(z)=\left[\begin{array}{lllll}
1 & z^{-1} & z^{-2} & \ldots & z^{-(P-1)}
\end{array}\right]^{T} .
$$

Uma média móvel $P$ x Q é baseada em uma média móvel de $P$ termos e uma média móvel de $Q$ termos. Se $P+Q$ for par, esta média móvel será centrada, e será dada por:

$M A_{P X Q}(z)=\frac{z^{\frac{P+Q-2}{2}}}{P Q}\left[\begin{array}{lllllllllllll}1 & 2 & \ldots & P-1 & P & P & \ldots & P & P-1 & \ldots & 2 & 1\end{array}\right] \mathbf{E}_{P+Q-1}(z)$

Como apresentado em Dagum (1988), os passos do algoritmo padrão do X11 são listados a seguir. Para isso, foi considerada uma série mensal com decomposição aditiva:

$$
y_{t}=t_{t}+s_{t}+i_{t}
$$

Onde $y_{t}$ é a série original, e $t_{t}, s_{t}, i_{t}$ são as componentes não observáveis de tendência, sazonalidade e a componente irregular, respectivamente.

As operações de filtragem serão apresentadas no domínio da frequência, por meio da transformada $\mathrm{Z}$. 


\section{Estágio 1 - Estimativas iniciais}

i. Estimativa preliminar da componente de tendência $\mathrm{T}^{(1)}(z)$ usando uma média móvel $M A_{2 X 12}(z)$ :

$$
\mathrm{T}^{(1)}(z)=\mathrm{Y}(z) M A_{2 X 12}(z)
$$

ii. Estimativa inicial da componente Sazonal-Irregular - 'SI' inicial

Tendo a primeira estimativa da tendência $\mathrm{T}^{(1)}(z)$, a componente sazonalirregular é estimada fazendo-se:

$$
\mathrm{SI}^{(1)}(z)=\mathrm{Y}(\mathrm{z})-\mathrm{T}^{(1)}(z)
$$

iii. Estimativa preliminar dos fatores sazonais $-\widehat{S}^{(1)}(z)$ - aplicando uma média móvel sazonal 3x3, $M A_{3 \times 3}\left(z^{12}\right)$ :

$$
\widehat{\mathrm{S}}^{(1)}(z)=\mathrm{SI}^{(1)}(z) M A_{3 X 3}\left(z^{12}\right)
$$

iv. Fatores sazonais iniciais:

É importante que a componente sazonal tenha média zero no período de 12 meses, então, é realizada uma média móvel $2 \times 12$, resultando na seguinte estimativa da sazonalidade:

$$
\mathrm{S}^{(1)}(z)=\widehat{\mathrm{S}}^{(1)}(z)-\widehat{\mathrm{S}}^{(1)}(z) M A_{2 X 12}(z)
$$

v. Ajuste sazonal inicial:

$$
\mathrm{A}^{(1)}(z)=\mathrm{Y}(z)-\mathrm{S}^{(1)}(z)
$$

Estágio 2 - fatores sazonais e ajuste sazonal: redefinição das estimativas das componentes

i. Estimativa intermediária da tendência, usando o filtro de Henderson de 13 termos, $H_{13}(z)$ :

$$
\mathrm{T}^{(2)}(z)=\mathrm{A}^{(1)}(z) H_{13}(z)
$$

ii. Refinamento da estimativa da componente sazonal-irregular:

$$
\mathrm{SI}^{(2)}(z)=\mathrm{Y}(z)-\mathrm{T}^{(2)}(z)
$$


iii. Refinamento da estimativa da componente sazonal utilizando uma média móvel sazonal 3x5. Nessa etapa, o procedimento automático do programa pode selecionar uma média móvel sazonal diferente, podendo ser: MMs 3x3, $3 \times 5$ ou $3 \times 9$.

$$
\widehat{\mathrm{S}}^{(2)}(z)=\mathrm{SI}^{(2)}(z) M A_{3 X 5}\left(z^{12}\right)
$$

iv. Fatores sazonais:

$$
\mathrm{S}^{(2)}(z)=\widehat{\mathrm{S}}^{(2)}(z)-\widehat{\mathrm{S}}^{(2)}(z) M A_{2 X 12}(z)
$$

v. Série sazonalmente ajustada:

$$
\mathrm{A}^{(2)}(z)=\mathrm{Y}(z)-\mathrm{S}^{(2)}(z)
$$

Como as operações apresentadas nos estágios 1 e 2 do algoritmo são lineares, é possível representá-las como um filtro equivalente ao método X-11 para o ajuste sazonal. Neste trabalho, será utilizado o termo 'filtro X-11' para designar tal filtro equivalente.

Vale ressaltar que as expressões apresentadas nos estágios 1 e 2 representam uma forma conveniente de se calcular os pesos e as funções de transferência de vários filtros resultantes da seleção padrão ou das diversas opções de médias móveis sazonais. Além disso, facilitam o estudo das propriedades do filtro X-11. 


\subsection{2.}

\section{O desempenho do X-11 na presença de sazonalidade móvel}

Em muitas situações relacionadas ao comportamento sazonal, o X-11 é um método adequado. Entretanto, em outros casos pode ocorrer um subajustamento da sazonalidade. Essas situações serão ilustradas no domínio da frequência. Segundo Nettheim (1964), o espectro de uma série com sazonalidade apresenta picos nas frequências sazonais, ou próximo a elas. Dessa forma, torna-se mais preciso analisar o comportamento da sazonalidade da série com base no espectro.

Nerlove (1964) ressalta que 'quanto mais estreitos estiverem os picos, nas frequências sazonais, mais forte e regular será a sazonalidade'. Essa é uma situação na qual o método X-11 apresenta um bom desempenho.

Isso pode ser ilustrado com base nos dois gráficos a seguir. O Gráfico 3.1 apresenta a componente sazonal de uma série sem sazonalidade móvel. E o Gráfico 3.2 mostra uma comparação entre a resposta em magnitude do filtro $\mathrm{X}-11$, e o espectro de uma série sem sazonalidade móvel. Nesse gráfico, a linha contínua representa o espectro da componente sazonal da série 'sem' sazonalidade móvel, e a linha tracejada representa a resposta em magnitude do filtro X-11.

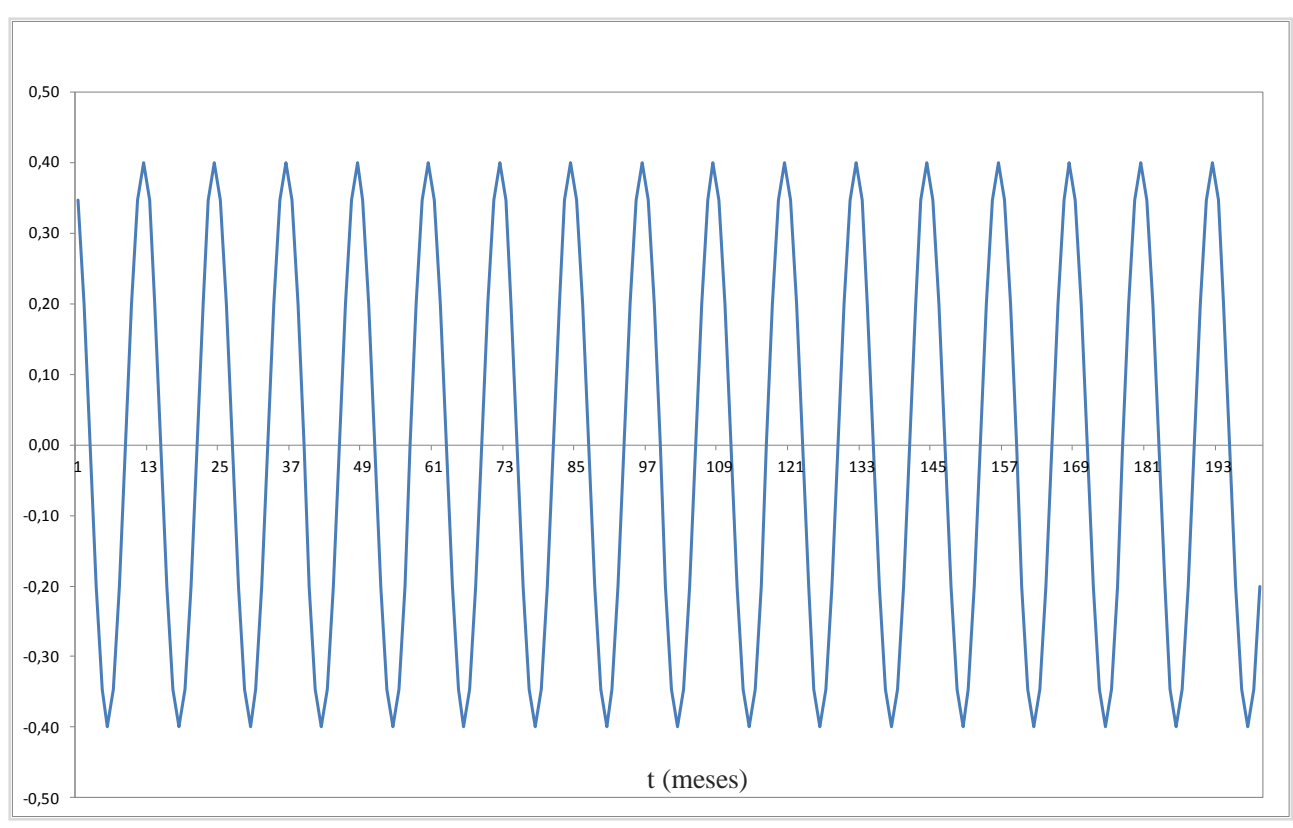

Gráfico 3.1 Componente sazonal de uma série mensal sem sazonalidade móvel 


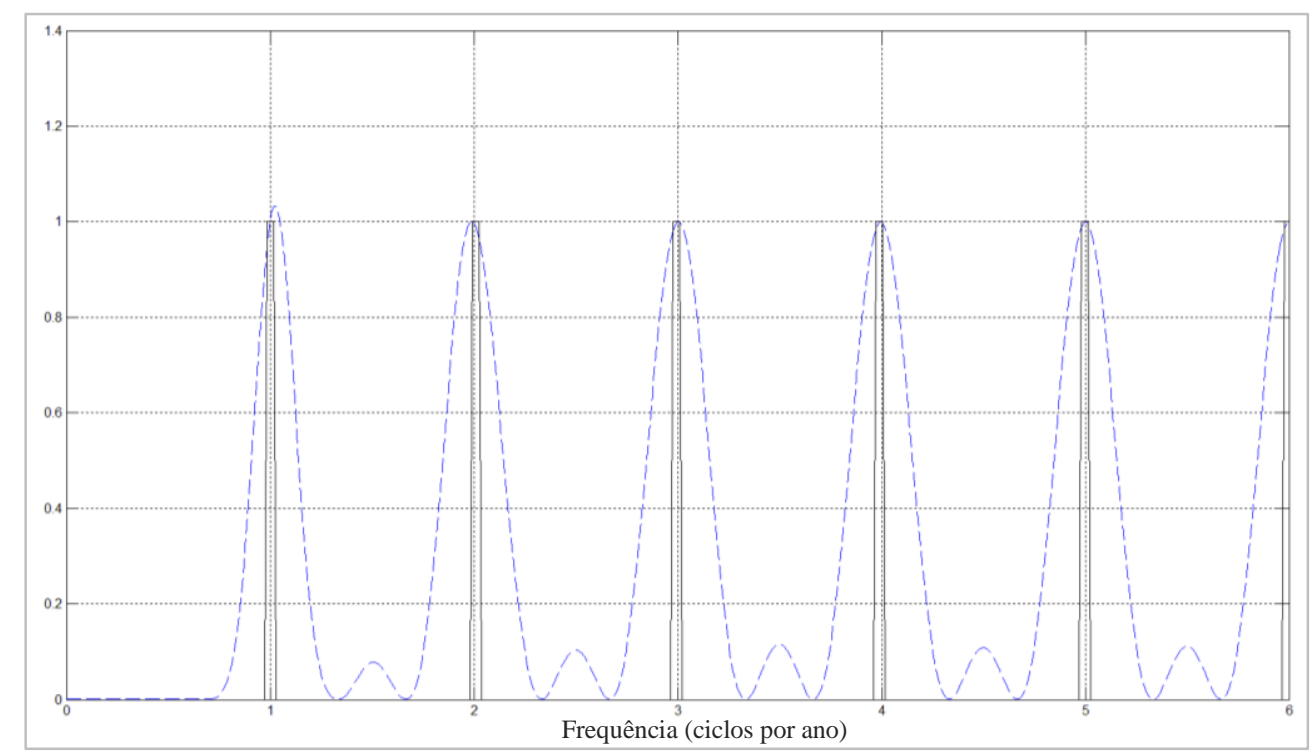

\section{Gráfico 3.2 Comparação entre a resposta em magnitude do filtro X-11 (linha tracejada) e o espectro de uma série mensal sem sazonalidade móvel (linha contínua)}

No Gráfico 3.2, pode-se notar que o X-11 consegue acompanhar o padrão da sazonalidade de forma adequada, apresentando um bom desempenho quando existe sazonalidade estável. Porém, quando se trata de sazonalidade móvel, o resultado é diferente.

Quanto à sazonalidade móvel, Nettheim (1964) menciona que se os picos estiverem espalhados ao redor das frequências sazonais, de forma relevante, então pode-se descrever a situação como um com um padrão sazonal de alteração lenta. Em outras palavras, a largura desses picos depende essencialmente da regularidade do padrão sazonal.

A seguir é apresentado o desempenho do X-11, na presença de sazonalidade móvel, com base na análise do espectro. Mesmo utilizando o filtro de média móvel sazonal 3x3, que é o mais adequado para a sazonalidade instável, o X-11 não é capaz de lidar corretamente com isso. Planas (1998) apontou esse problema, que pode ser visualizado no gráfico 3.4 , onde a linha tracejada representa a resposta em magnitude do filtro $\mathrm{X}-11$ e as setas representam o espectro de uma série com sazonalidade móvel. Antes, no Gráfico 3.3, é apresentada a componente sazonal de uma série com sazonalidade móvel no domínio do tempo. 


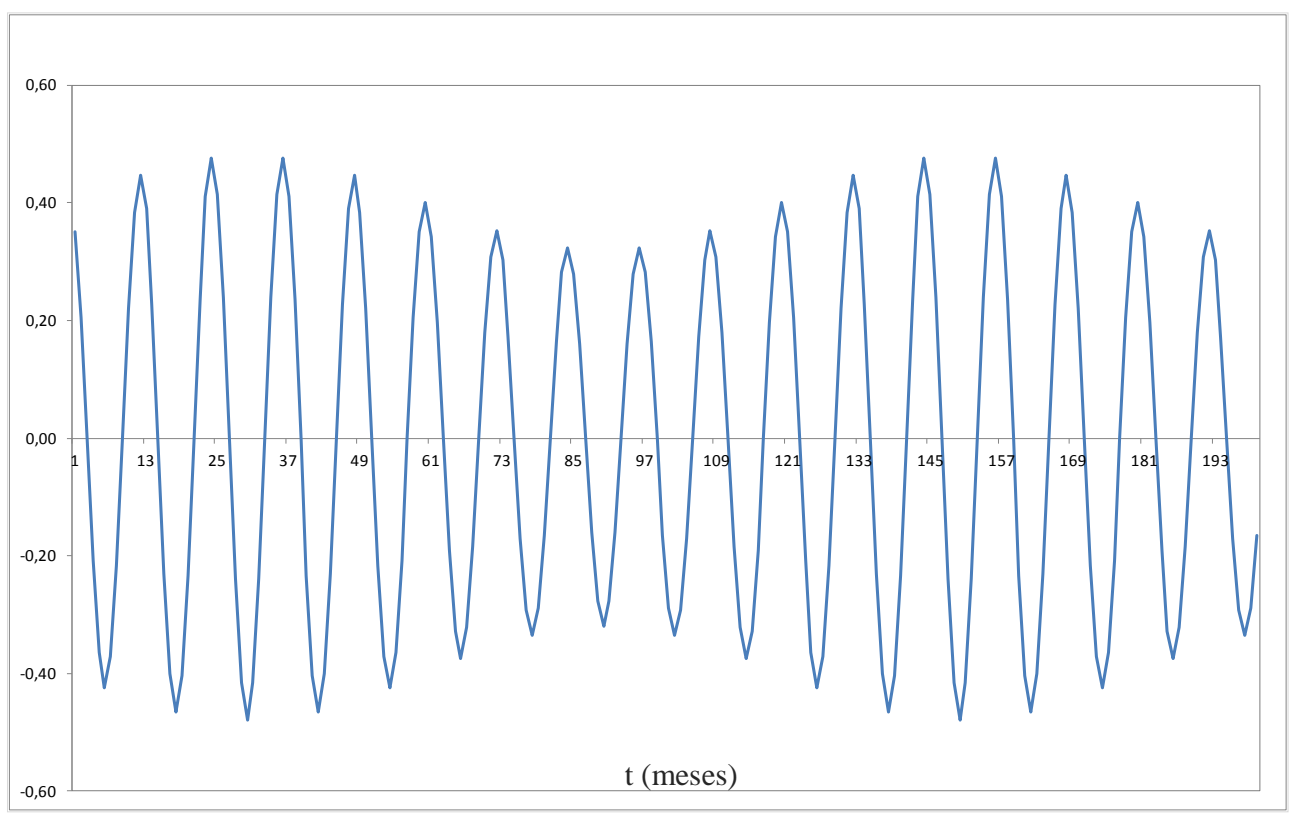

Gráfico 3.3 Componente sazonal de uma série mensal com sazonalidade móvel

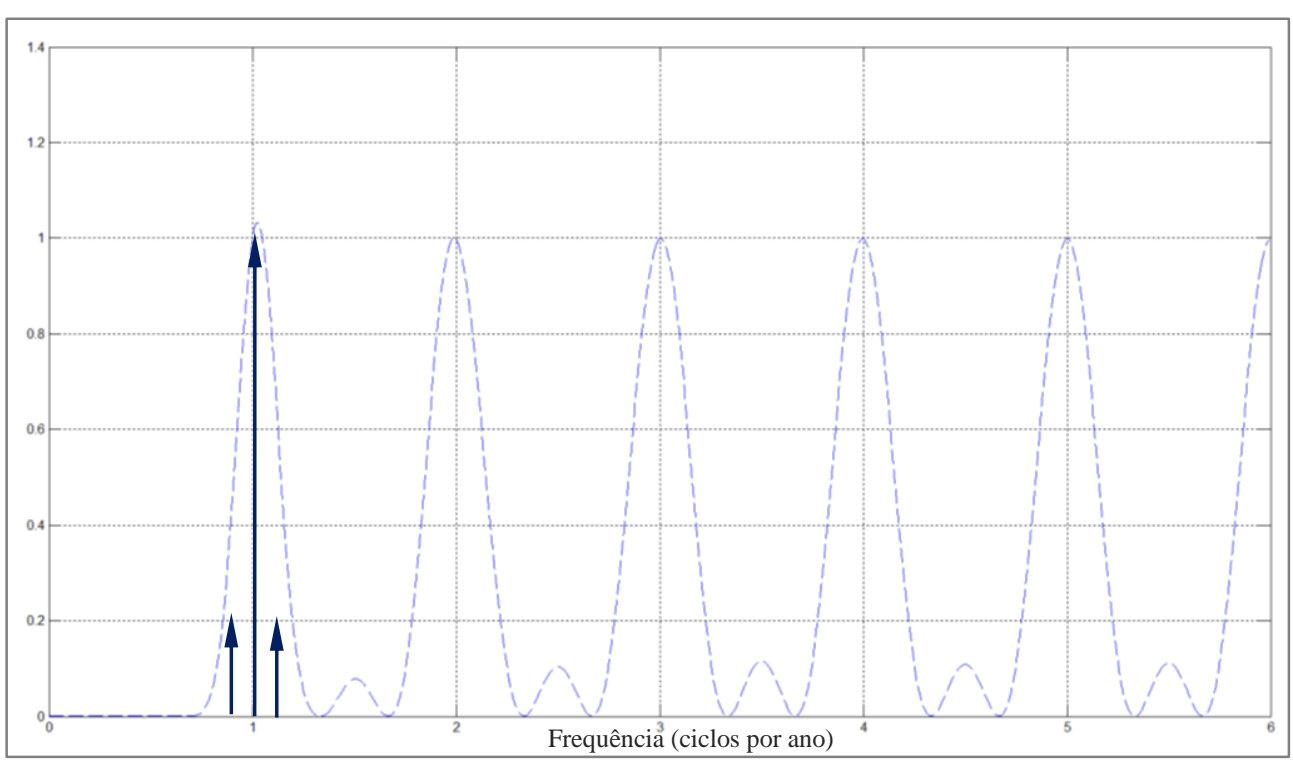

Gráfico 3.4 Comparação entre o espectro de uma série mensal com sazonalidade móvel (setas) e a resposta em magnitude do filtro X-11 (linha tracejada)

Como se pode observar, no Gráfico 3.4, o filtro X-11 faz com que as raias laterais tenham resposta em frequência menor do que 1, que seria o valor ideal. Com isso, a variação na amplitude da componente sazonal será reduzida, fazendo com que o filtro X-11 não responda de forma adequada à variação na sazonalidade. Isso pode ser visualizado no Gráfico 3.5, a seguir, onde é apresentada a componente 
sazonal artificial e a extração obtida pelo método X-11. Observa-se que o X-11 não consegue capturar adequadamente as variações existentes na amplitude da componente sazonal.

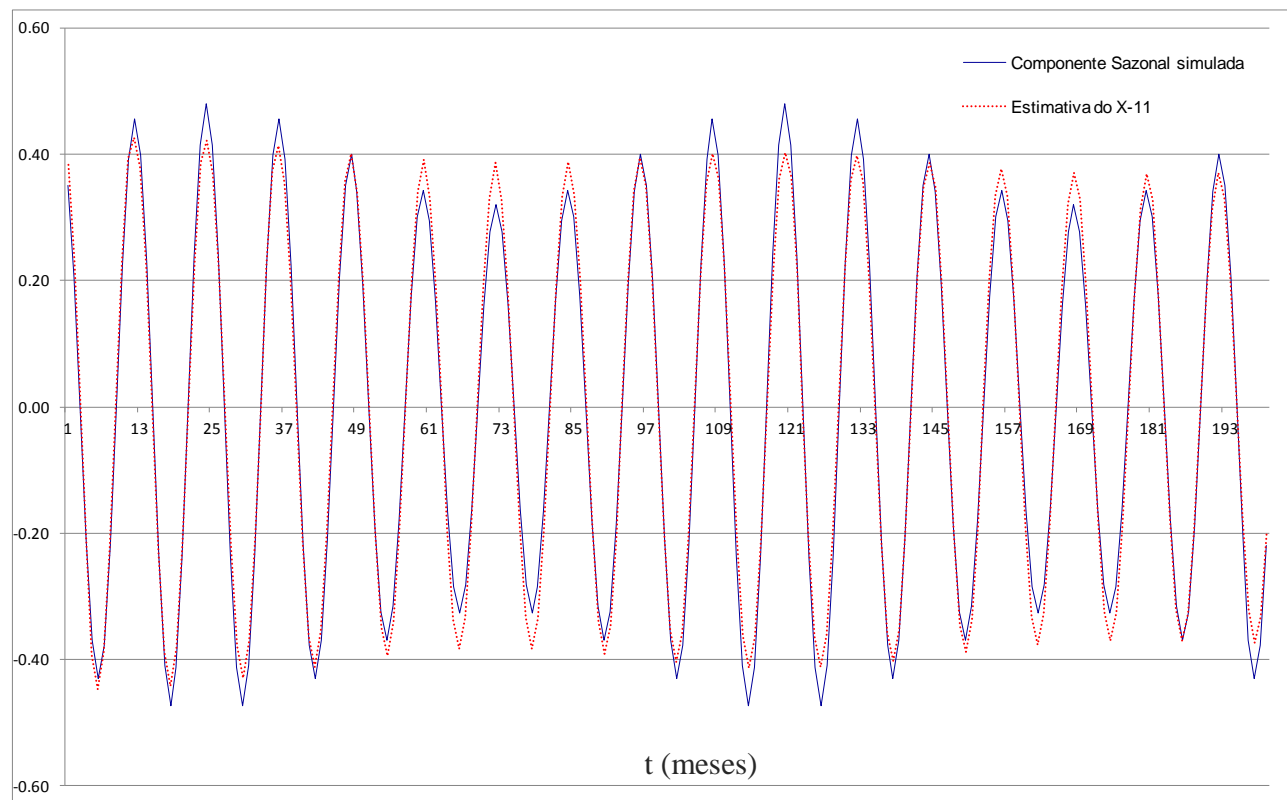

\section{Gráfico 3.5 Componente sazonal artificial e estimativa obtida pelo método X-11}

Maravall, no trabalho intitulado 'Short-term analysis of macroeconomic time series', de 1999, mostra que o X-11 subestima a sazonalidade quando a largura dos picos sazonais, no espectro, são maiores do que os capturados pelo filtro X-11. O autor menciona ainda que o subajustamento é refletido nos picos que permanecem na vizinhança de cada frequência sazonal, indicando que o X-11 não removeu toda a variação sazonal da série. Esses picos na vizinhança das frequências sazonais, mencionados por Maravall (1999) podem ser observados no Gráfico 3.4. 


\section{3.}

\section{Filtros}

Os filtros são processos que têm por finalidade eliminar as frequências não desejadas de um sinal de entrada, ou selecionar uma determinada frequência do sinal. São exemplos de filtros: passa-baixa, passa-banda, passa-alta e filtros de múltiplas bandas.

Um filtro do tipo passa-baixa permite que as baixas frequências passem, porém elimina os valores relacionados às altas frequências. Como exemplo, têm-se os filtros de tendência-ciclo.

Há também os filtros de múltiplas bandas, que deixam passar os valores correspondentes a determinadas faixas de frequência, eliminando os demais valores. É o caso dos filtros de sazonalidade, que deixam passar as frequências em torno dos harmônicos.

Em relação aos filtros de sazonalidade, vale mencionar que Nettheim (1965) citou como uma das possibilidades de tratamento da sazonalidade, na presença de mudança do padrão sazonal, a elaboração de um filtro no qual a função resposta fosse pequena, exceto na vizinhança dos pontos onde há o pico sazonal, e que essa função resposta fosse retangular tanto quanto possível. A aplicação de tal filtro, na série temporal, resultaria na sazonalidade estimada. Porém, o autor informou que não há como saber quão largos os picos deveriam ser, e que não seria fácil a construção desse filtro sem a perda de um grande número de observações nas extremidades da série.

Este capítulo apresentou os conceitos de análise de séries temporais no domínio da frequência. No Capítulo 4, a seguir, é apresentado o projeto de um filtro para a extração da sazonalidade, construído segundo as especificações mencionadas por Nettheim (1965). 


\section{4}

\section{Filtro sazonal-WLS: uma generalização para o ajuste sazonal, na frequência}

No contexto de ajuste sazonal com base em extração de sinal, deseja-se que o filtro tenha largura adequada de bandas passantes para permitir a correta dessazonalização quando a componente sazonal for instável. Além disso, ele deve apresentar bom desempenho, também, quando a componente sazonal for estável.

O filtro aqui proposto, projetado no domínio da frequência, é indicado para ser aplicado a uma classe de séries que apresentem até um determinado grau de sazonalidade móvel. Ele pode ser aplicado diretamente, independente da distribuição da série, permitindo a existência de tendência. Dessa forma, a série a ser sazonalmente ajustada passará por dois processos de filtragem: processo de eliminação da tendência e o processo de extração da sazonalidade. A esse filtro, intitulou-se 'filtro sazonal-WLS' - S-WLS.

Neste capítulo é descrito o algoritmo do filtro S-WLS, e realizada uma comparação entre a resposta em magnitude dele e a resposta em magnitude do filtro X-11. A ideia é que o uso sucessivo de filtros de médias móveis, no X-11, distorce o sinal a ser extraído. Sendo assim, o objetivo aqui é contornar esse problema, projetando um único filtro equivalente ao processo completo de extração da sazonalidade. Além disso, o capítulo apresenta os parâmetros do filtro S-WLS. Os conceitos apresentados neste capítulo baseiam-se em Diniz et al. (2010).

\section{1. \\ O Algoritmo do filtro sazonal-WLS}

Considerando que a tendência pode ser modelada como um polinômio de ordem $k$, o filtro para anular a tendência e extrair a sazonalidade é representado pela seguinte função:

$$
\mathrm{P}(z)=\left(1-z^{-1}\right)^{k+1} \mathrm{G}(z)
$$

onde $k$ é a ordem do polinômio de tendência e $z$ é um número complexo. O termo $\left(1-z^{-1}\right)^{k+1}$ garante que todos os polinômios de ordem $k$, na entrada do filtro, 
geram saída nula, garantindo assim a eliminação da tendência polinomial de até ordem $k$.

O termo $\mathrm{G}(z)$ é definido como

$$
\mathrm{G}(z)=\sum_{t=-p}^{L-p-1} \mathrm{~g}(t) z^{-t}
$$

onde $p$ representa um deslocamento, $L$ é o tamanho do filtro, representado pelo número de coeficientes, e $t$ é o tempo $\in \mathbb{Z}$. Os coeficientes $g(t) \in \mathbb{R}$.

Quando $z^{t}$ é uma senóide complexa com frequência $\omega$, utiliza-se a representação $z=e^{i \omega}$.

Sendo assim, $\mathrm{P}(z)$ pode ser reescrito como

$$
\mathrm{P}\left(e^{i \omega}\right)=\left(1-e^{-i \omega}\right)^{k+1} \mathrm{G}\left(e^{i \omega}\right)
$$

Pode-se representar $\mathrm{P}\left(e^{i \omega}\right)$ por

$$
\mathrm{P}\left(e^{i \omega}\right)=P(\omega)=e^{-i\left(\frac{\omega}{2}\right)(k+1)}\left(2 i \operatorname{sen} \frac{\omega}{2}\right)^{k+1} \mathrm{G}\left(e^{i \omega}\right)
$$

A função $G(z)$ pode ser escrita como

$$
\mathrm{G}\left(e^{i \omega}\right)=e^{-i \omega(p)} E^{T}(\omega) \mathrm{g}
$$

onde $E(\omega)$ e g são vetores de tamanho L, definidos como

$$
\begin{gathered}
E(\omega)=\left[\begin{array}{llll}
1 & e^{-i \omega} & \cdots & e^{-i \omega(L-1)}
\end{array}\right]^{T} \\
g=\left[\begin{array}{llll}
g(-p) & g(-p+1) & \cdots & g(L-p-1)
\end{array}\right]^{T}
\end{gathered}
$$

Substituindo os valores, tem-se:

$$
\begin{gathered}
\mathrm{P}\left(e^{i \omega}\right)=e^{-i\left(\frac{\omega}{2}\right)(k+1)-i \omega(p)}\left(2 i \operatorname{sen} \frac{\omega}{2}\right)^{k+1} E^{T}(\omega) \mathrm{g} \\
\mathrm{P}\left(e^{i \omega}\right)=e^{-i \omega\left[\left(\frac{k+1}{2}\right)+p\right]}\left(2 i \operatorname{sen} \frac{\omega}{2}\right)^{k+1} E^{T}(\omega) \mathrm{g}
\end{gathered}
$$


Fazendo

$$
s(\omega, k, p)=e^{-i \omega\left[\left(\frac{k+1}{2}\right)+p\right]}\left(2 i \operatorname{sen} \frac{\omega}{2}\right)^{k+1}
$$

escreve-se $\mathrm{P}\left(e^{i \omega}\right)$ como

$$
\mathrm{P}\left(e^{i \omega}\right)=s(\omega, k, p) E^{T}(\omega) \mathrm{g}
$$

Finalmente escolhem-se frequências $\omega_{1}, \omega_{2}, \ldots \omega_{n}$ dentro das faixas em torno dos harmônicos (bandas passantes) e nas bandas de rejeição, e define-se P como o vetor

$$
\mathrm{P}=\left[\begin{array}{llll}
\mathrm{P}\left(e^{i \omega_{1}}\right) & \mathrm{P}\left(e^{i \omega_{2}}\right) & \cdots & \mathrm{P}\left(e^{i \omega_{n}}\right)
\end{array}\right]^{T}
$$

E a matriz U como

$$
\mathrm{U}=\left[\begin{array}{c}
E^{T}\left(\omega_{1}\right) s\left(\omega_{1}, k, p\right) \\
E^{T}\left(\omega_{2}\right) s\left(\omega_{2}, k, p\right) \\
\cdots \\
E^{T}\left(\omega_{n}\right) s\left(\omega_{n}, k, p\right)
\end{array}\right]_{n \times L}
$$

P pode ser escrito como:

$$
\mathrm{P}=\mathrm{U} \text { g. }
$$

Como o termo $\left(1-e^{-i \omega}\right)^{k+1}$ na equação que define $\mathrm{P}\left(e^{i \omega}\right)$ garante que as tendências polinomiais de ordem $k$ sejam eliminadas, pode-se variar os coeficientes de $\mathrm{G}(z)$ para obter o desempenho desejado pelo filtro. Assim sendo, para que o filtro seja robusto a variações na sazonalidade, os coeficientes de $G(z)$ devem ser otimizados de modo a deixar passar a sazonalidade em torno dos harmônicos numa faixa de frequência de largura correspondente a uma porcentagem $(\alpha)$ da frequência sazonal. Além disso, deve-se eliminar o máximo possível da componente irregular. Dessa forma, as bandas passantes terão resposta desejada igual a um, as bandas de rejeição terão resposta desejada igual a zero, e as bandas de transição (don't care band) apenas facilitarão o processo de otimização, não possuindo um valor desejado específico. Essas características podem se melhor visualizadas na Figura 4.1 a seguir. 


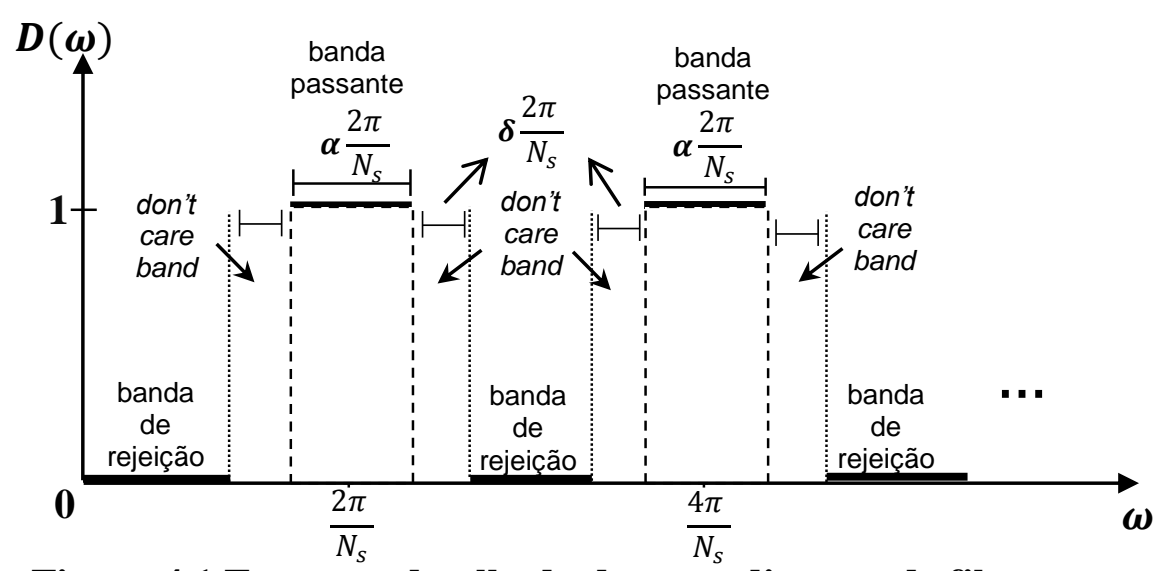

Figura 4.1 Esquema detalhado do procedimento de filtragem

Vale ressaltar que por deixar passar a sazonalidade considerando uma certa banda em torno dos harmônicos (como observado na Figura 4.1), o filtro permite que a sazonalidade móvel de até um determinado grau seja contemplada na análise.

Considerando $P(\omega)$ a resposta do filtro e $D(\omega)$ a resposta desejada, na frequência $\omega$, deseja-se que a soma dos quadrados da diferença entre a resposta do filtro $(\mathrm{P}(\omega))$ e o desejado $(\mathrm{D}(\omega))$ seja a menor possível. Isso equivale a minimizar o quadrado dos erros. Além disso, no processo de otimização será utilizado um vetor de pesos, $W(\omega)$, que estabelecerá a importância relativa da resposta em cada frequência $\omega$, durante a otimização, conforme será especificado a seguir.

Pode-se representar o erro da seguinte forma:

$$
\operatorname{Erro}(\omega)=[P(\omega)-D(\omega)] w(\omega)
$$

Considerando os vetores para cada frequência, tem-se:

$$
\text { erro }=\mathrm{W}[\mathrm{P}-\mathrm{D}]
$$

ou ainda,

$$
\text { erro }=\mathrm{W}[\mathrm{Ug}-\mathrm{D}]
$$

sendo $\mathrm{W}$ igual a

$$
\mathrm{W}=\left[\begin{array}{cccc}
\mathrm{w}_{1}\left(\omega_{1}\right) & 0 & 0 & 0 \\
0 & \mathrm{w}_{2}\left(\omega_{2}\right) & 0 & 0 \\
\vdots & \vdots & \ddots & \vdots \\
0 & 0 & \cdots & \mathrm{w}_{n}\left(\omega_{n}\right)
\end{array}\right]
$$

Como mencionado anteriormente, deseja-se minimizar a soma dos quadrados dos erros:

$$
\varepsilon=\| \operatorname{erro}^{\mathrm{T}} \text { erro } \|=(U \mathrm{~g}-D)^{*}{ }^{\mathrm{T}} \mathrm{W}^{* \mathrm{~T}} \mathrm{~W}(U \mathrm{~g}-D)
$$


$E$ como $W=W^{T}$, e W, D e $g \in \mathbb{R}$, reescreve-se a expressão acima da seguinte forma:

$$
\begin{gathered}
\varepsilon=\left(\mathrm{g}^{T} U^{* T}-D^{T}\right) \mathrm{W}^{2}(U \mathrm{~g}-D) \\
\varepsilon=\mathrm{g}^{T} U^{*^{T}} \mathrm{~W}^{2} U \mathrm{~g}-D^{T} \mathrm{~W}^{2}\left(U+U^{*}\right) \mathrm{g}-D^{T} \mathrm{~W}^{2} D
\end{gathered}
$$

Derivando a equação acima em relação à g, e igualando a zero, encontra-se o vetor com os coeficientes do filtro extrator da sazonalidade

$$
\mathrm{g}=\left(U^{* T} \mathrm{~W}_{\mathrm{s}}^{2} U+U^{T} \mathrm{~W}_{\mathrm{s}}^{2} U^{*}\right)^{-1}\left(U^{T}+U^{* T}\right) \mathrm{W}_{\mathrm{s}}^{2} D
$$

Convoluindo este vetor de coeficientes g com os coeficientes do polinômio $\left(1-z^{-1}\right)^{k+1}$, obtém-se o vetor com os coeficientes de $\mathrm{P}(z)$. E finalmente, a convolução do vetor de coeficientes de $\mathrm{P}(z)$ com a série temporal resulta nos fatores sazonais.

\section{2. \\ Parâmetros do filtro sazonal-WLS}

A principal diferença entre o filtro aqui proposto, e os filtros do método X11, está no fato daquele permitir a existência de sazonalidade móvel, uma vez que a banda passante será bem mais larga do que a do filtro X-11. Isso é possível devido aos parâmetros especificados no filtro S-WLS. São eles: alpha $(\alpha)$, delta $(\delta)$, peso $\left(\mathrm{w}_{\mathrm{O}}\right)$, fator de superamostragem $(\mathrm{M})$, e ordem do filtro $(\mathrm{N})$.

Inicialmente considera-se um modelo aditivo de decomposição da série temporal $Y_{t}$ :

$$
Y_{t}=T_{t}+S_{t}+I_{t}
$$

onde:

$T_{t}$ é a componente de Tendência

$S_{t}$ é a componente Sazonal

$I_{t}$ é a componente Irregular 
Considerando que a série $Y_{t}$ é periódica, de período $N_{s}$, deseja-se obter respostas próximas (ou iguais) a 1, nas bandas em torno das frequências sazonais $\left(\frac{2 \pi}{N_{S}}, \frac{4 \pi}{N_{S}}, \ldots, \frac{12 \pi}{N_{S}}\right)$, e zero, ou próximas a zero, nas demais frequências.

Nesse procedimento, alpha $(\boldsymbol{\alpha})$ corresponde à largura da banda em torno dos harmônicos. Trata-se de um dado de projeto e está relacionado ao conteúdo de frequência da variação da sazonalidade. Ele pode assumir qualquer valor entre $[1 / 200 ; 1 / 2]$. Neste filtro, será utilizado $\alpha=1 / 3$. Essa escolha baseou-se nas características das séries reais, que serão abordadas no Capítulo 5.

O delta $(\boldsymbol{\delta})$ representa a largura da banda de transição (don't care band). Ele auxilia no processo de otimização. A Figura 4.1 ilustra o conceito desse parâmetro, assim como o $\alpha$.

$\mathrm{O}$ peso $\left(\mathbf{w}_{\mathbf{o}}\right)$ indica a importância dada às bandas de passagem, em torno dos harmônicos. Especificamente, neste projeto, um peso $\mathrm{w}_{\mathrm{o}}$ significa que $\mathrm{w}(\omega)=1$ nas frequências das bandas de rejeição e $w(\omega)=w_{0}$ nas frequências das bandas de passagem. Valores altos do peso $\left(\mathrm{w}_{\mathrm{o}}\right)$ geram ganho nos harmônicos próximo de 1 , mas a atenuação fora dos harmônicos diminui.

O número de pontos utilizados durante a otimização equivale ao fator de superamostragem multiplicado pelo tamanho do filtro. A restrição é que o fator de superamostragem seja um número ímpar. No filtro sazonal-WLS foi utilizado fator de superamostragem igual a 401, pois esse valor respondeu adequadamente aos propósitos do filtro.

O último parâmetro a ser definido é a ordem do filtro (N), ou seja, o tamanho do filtro representado pelo número de coeficientes que ele possui. Como o propósito inicial é compará-lo ao X-11, primeiro será definido o tamanho do filtro equivalente ao X-11 a ser utilizado na extração da sazonalidade, e então este mesmo tamanho será utilizado no filtro S-WLS.

O Gráfico 4.1 ilustra um caso onde a série apresenta sazonalidade móvel, tendo o espectro representado pelos impulsos (setas). A resposta em magnitude do filtro S-WLS mostra a adequação do filtro em relação ao ajuste. Nos Gráficos 4.1 e 4.2 foi utilizada a seguinte configuração dos parâmetros do filtro S-WLS: N=145, $\alpha=1 / 3, \delta=1 / 10$ e $\mathrm{w}_{\mathrm{o}}=10$. 


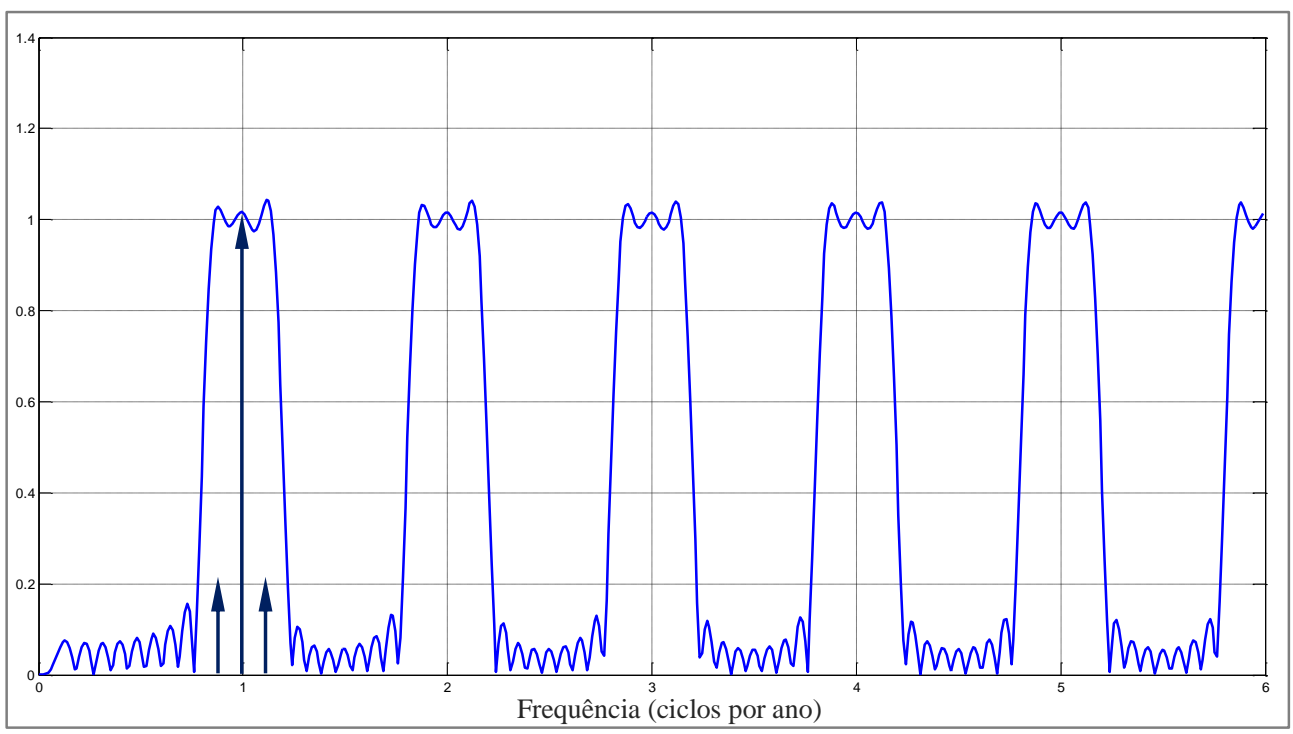

Gráfico 4.1 Comparação entre a resposta em magnitude do filtro S-WLS (linha contínua) e o espectro de uma série mensal com sazonalidade móvel (setas)

No Gráfico 4.2, a seguir, é apresentada a resposta em magnitude do filtro X11 considerando média móvel sazonal 3x3 (linha tracejada), juntamente com a resposta em magnitude do filtro S-WLS (linha contínua), no caso em que existe um grau relevante de sazonalidade móvel na série. O espectro da série com sazonalidade móvel está representado pelos impulsos (setas).

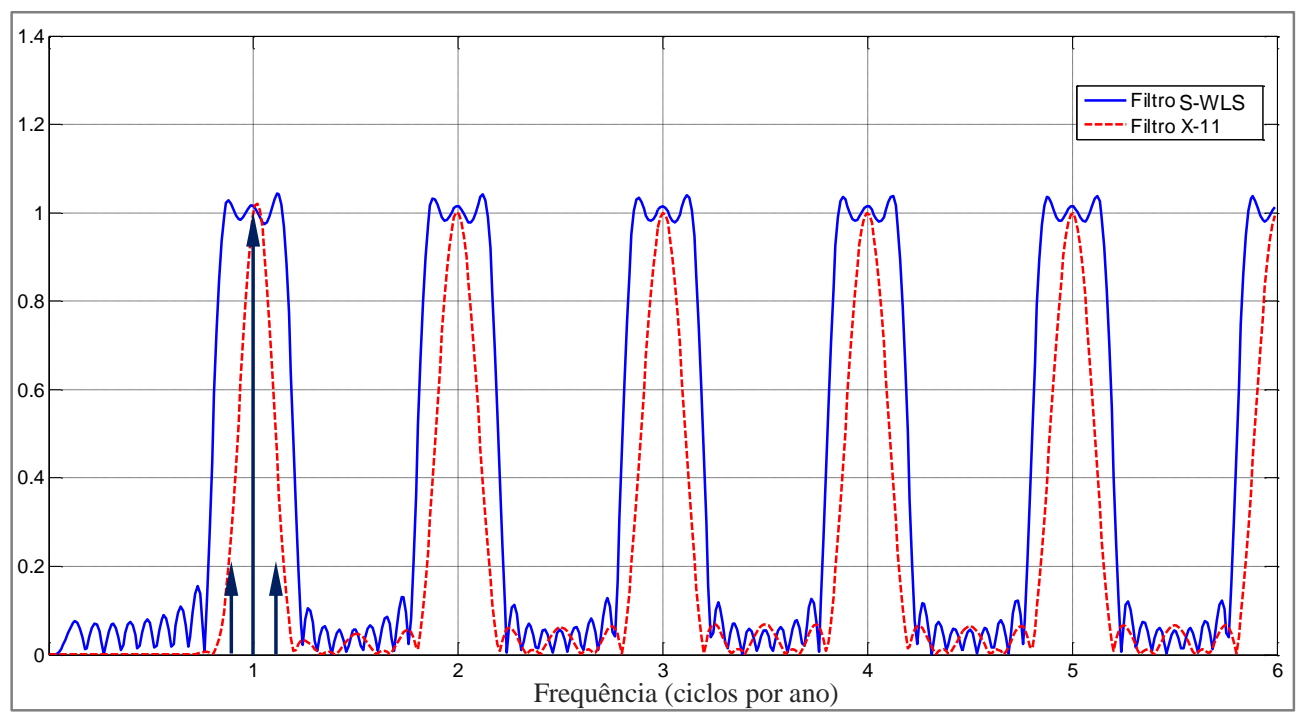

Gráfico 4.2 Comparação entre a resposta em magnitude do filtro S-WLS, a resposta em magnitude do filtro $X-11$ e o espectro de uma série com sazonalidade móvel (representado pelas setas) 
Como pode ser observado, no Gráfico 4.2, mesmo a média móvel sazonal 3x3 não é suficiente para captar as oscilações da componente sazonal, enquanto que o filtro S-WLS, por apresentar bandas passantes mais largas, não altera as amplitudes das raias espectrais e, portanto, capta a variação da amplitude da série.

É interessante notar que algumas configurações dos parâmetros do filtro SWLS o deixam parecido com o X-11, em termos de largura da banda passante. A comparação pode ser analisada pelo espectro, apresentado no Gráfico 4.3, a seguir, onde a curva contínua representa o espectro do filtro S-WLS, e a curva pontilhada representa o espectro do filtro X-11. Esses resultados foram encontrados utilizandose tamanho do filtro de Henderson igual a 13 e média móvel sazonal 3x3; já no filtro S-WLS foram adotados os seguintes parâmetros: $\mathrm{N}=145, \alpha=\frac{1}{35}, \delta=\frac{1}{4} \mathrm{e} \mathrm{w}_{\mathrm{o}}=$ 0,02, como definidos na seção 4.2.

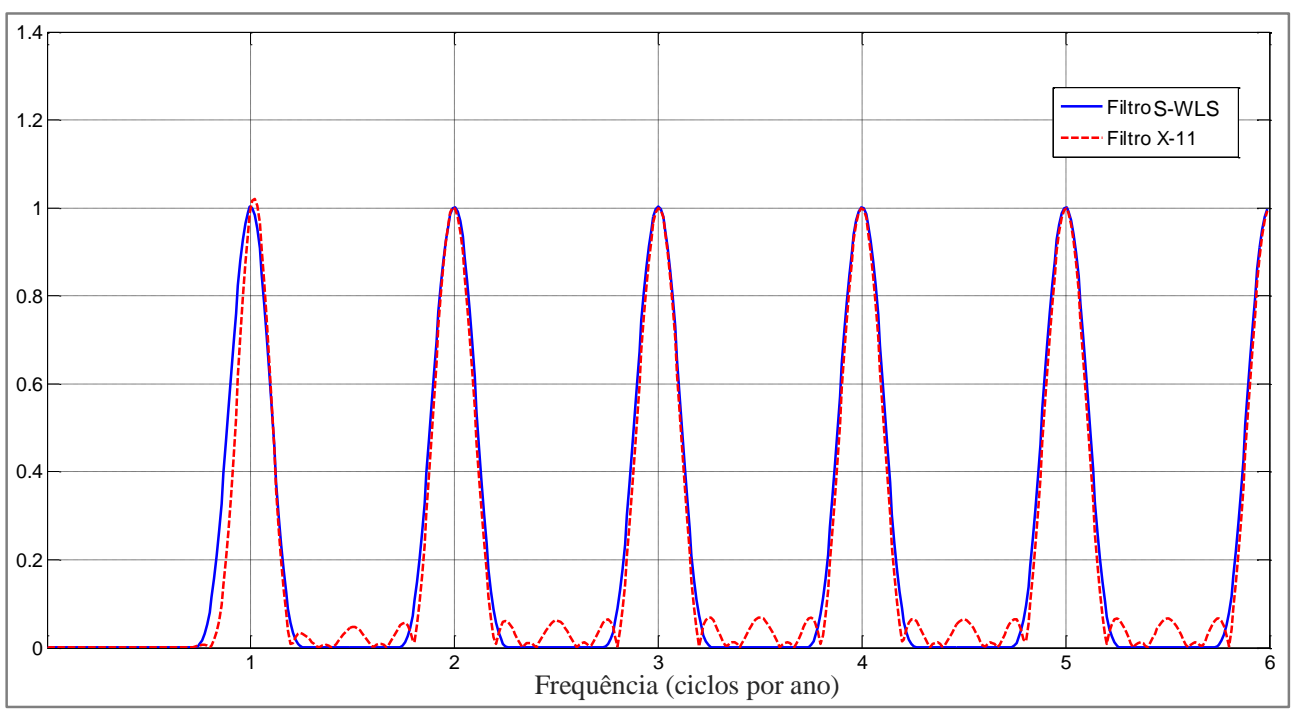

\section{Gráfico 4.3 Quando a resposta em magnitude do filtro S-WLS se aproxima} da resposta em magnitude do filtro $\mathrm{X}-11$

Vale ressaltar que não é o objetivo trabalhar, no filtro S-WLS, com um valor baixo para o parâmetro $\alpha$, uma vez que pretende-se permitir a presença de sazonalidade móvel. A utilização do parâmetro $\alpha$ igual a $\frac{1}{35}$ foi meramente para comparar as respostas em magnitude dos filtros. 
Capítulo 4. Filtro sazonal-WLS: uma generalização para o ajuste sazonal, na frequência

Este capítulo apresentou o algoritmo do filtro S-WLS e seus parâmetros. Além disso, foi realizada uma comparação, no domínio da frequência, com o filtro equivalente ao método $\mathrm{X}-11$, ilustrando por meio da resposta em magnitude a inadequação do X-11 ao ajustar séries com sazonalidade móvel. Contudo, é necessário especificar os valores dos parâmetros do filtro, o que será realizado no Capítulo 5, a seguir. 


\section{5 \\ Filtro sazonal-WLS: seleção da melhor configuração e resultados}

Neste Capítulo 5 é apresentado o procedimento utilizado na seleção dos parâmetros do filtro sazonal-WLS - S-WLS, para as séries com periodicidade mensal e trimestral. Além disso, são expostos os resultados do desempenho do filtro S-WLS, em comparação com o X-11, no ajuste de séries que apresentam algum grau de sazonalidade móvel. Dado que foi utilizado uma componente sazonal artificial na seleção dos parâmetros do filtro e na análise do desempenho do mesmo, é apresentada - inicialmente - a estrutura da componente sazonal artificial utilizada, assim como o processo de seleção dos parâmetros.

O Capítulo 5 está organizado da seguinte forma: na Subseção 5.1 é apresentada a estrutura da componente sazonal artificial; na Seção 5.2 são apresentadas as características das séries reais utilizadas na análise, incluindo os valores dos parâmetros do sinal sazonal, da componente de tendência e da componente irregular. A Subseção 5.3 apresenta a razão sinal ruído (SNR) de cada filtro. A seleção da melhor configuração do filtro S-WLS para as séries mensais e trimestrais é abordada na Subseção 5.4. E, finalmente, os resultados da comparação entre o filtro S-WLS e o filtro X-11 são apresentados na Subseção 5.5 .

\section{1.}

\section{Componente Sazonal Artificial}

Para escolher a melhor configuração de parâmetros para o filtro S-WLS, foi utilizado uma componente sazonal artificial utilizando a combinação de senos e cossenos, e algum grau de sazonalidade móvel, ou seja, amplitude variável. Esta componente, para uma série temporal mensal, está apresentada na Equação (5-1), a seguir:

$$
\begin{gathered}
A\left[1+b \operatorname{sen}\left(2 \pi \frac{t}{k}\right)\right]\left[\cos \left(2 \pi \frac{t}{12}\right)\right]= \\
A \cos \left(2 \pi \frac{t}{12}\right)+\frac{A b}{2} \operatorname{sen} 2 \pi t\left(\frac{1}{k}+\frac{1}{12}\right)+\frac{A b}{2} \operatorname{sen} 2 \pi t\left(\frac{1}{k}-\frac{1}{12}\right)
\end{gathered}
$$


onde:

$A=$ amplitude do sinal. $A \in \mathbb{R}^{*}+$

$b=$ taxa de variação da sazonalidade. $b \in(0,1)$;

$k=$ número de meses no qual o padrão de sazonalidade móvel volta a se repetir. $\mathrm{Na}$ análise foi utilizado $k \geq 48$;

$t=$ período que está sendo analisado. $t=1,2, \ldots, 500$.

No caso de uma série com periodicidade trimestral, a componente sazonal artificial é representada da seguinte forma:

$$
\begin{gathered}
A\left[1+b \operatorname{sen}\left(2 \pi \frac{t}{k}\right)\right]\left[\cos \left(2 \pi \frac{t}{4}\right)\right]= \\
A \cos \left(2 \pi \frac{t}{4}\right)+\frac{A b}{2} \operatorname{sen} 2 \pi t\left(\frac{1}{k}+\frac{1}{4}\right)+\frac{A b}{2} \operatorname{sen} 2 \pi t\left(\frac{1}{k}-\frac{1}{4}\right)
\end{gathered}
$$

onde:

$A=$ amplitude do sinal. $A \in \mathbb{R}^{*}+$

$b=$ taxa de variação da sazonalidade. $b \in(0,1)$;

$k=$ número de trimestres no qual o padrão de sazonalidade móvel volta a se repetir.

$\mathrm{Na}$ análise foi utilizado $k \geq 16$;

$t=$ período que está sendo analisado. $t=1,2, \ldots, 500$.

Como ilustração, o Gráfico 5.1 apresenta uma componente sazonal artificial, para uma série temporal mensal, utilizando: $A=0,4, b=20 \%$ e $k=96$. Observando o padrão da sazonalidade móvel, nota-se que ele volta a se repetir depois de 8 anos (96 observações).

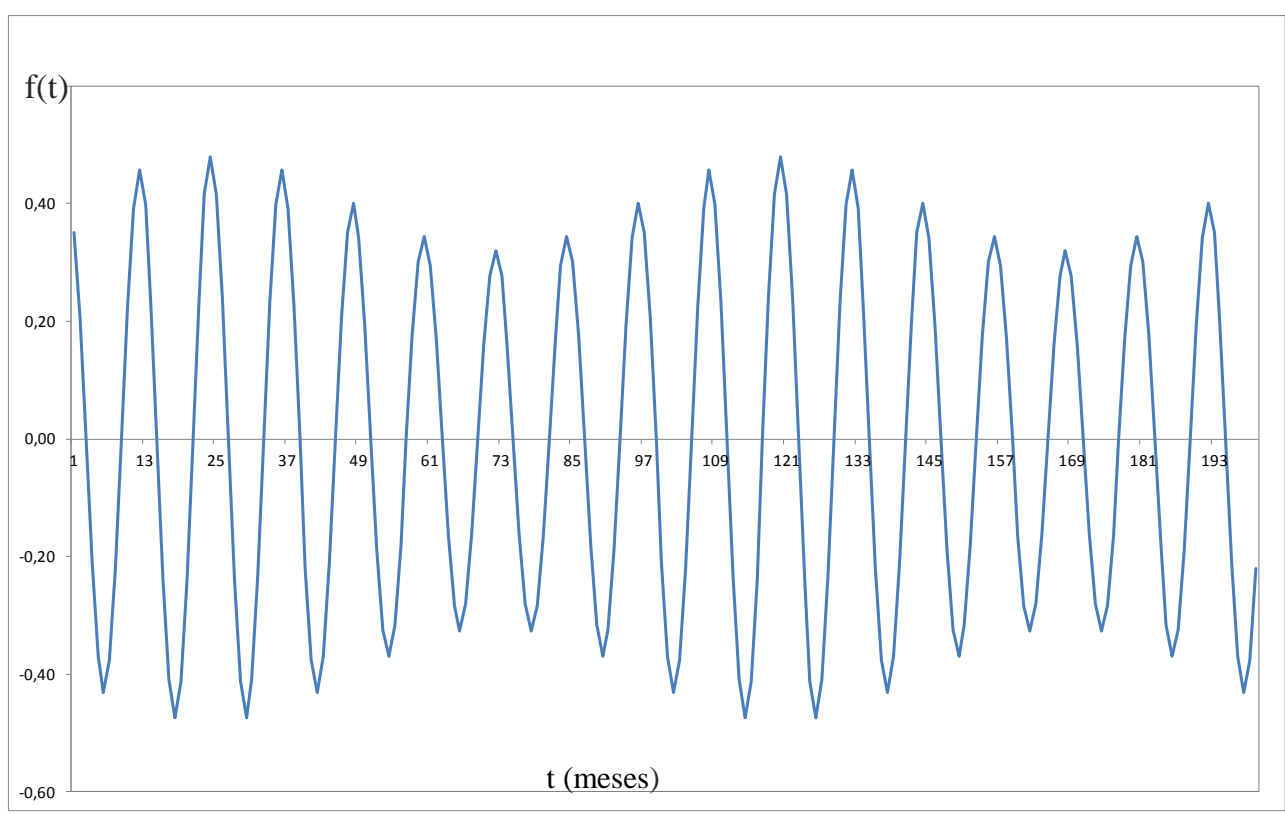

Gráfico 5.1 Componente sazonal artificial para uma série mensal com sazonalidade móvel, no domínio do tempo 
A Figura 5.1 apresenta o espectro, na frequência, do sinal equivalente ao apresentado no Gráfico 5.1. De acordo com essa figura, pode-se visualizar o impacto da amplitude $(A)$, da taxa de variação da sazonalidade $(b)$ e do parâmetro $k$, na frequência angular. $\mathrm{O}$ parâmetro $\mathrm{N}_{\mathrm{s}}$ corresponde à periodicidade da série. Em uma série mensal, $N_{S}=12$, e na série trimestral, $N_{S}=4$.

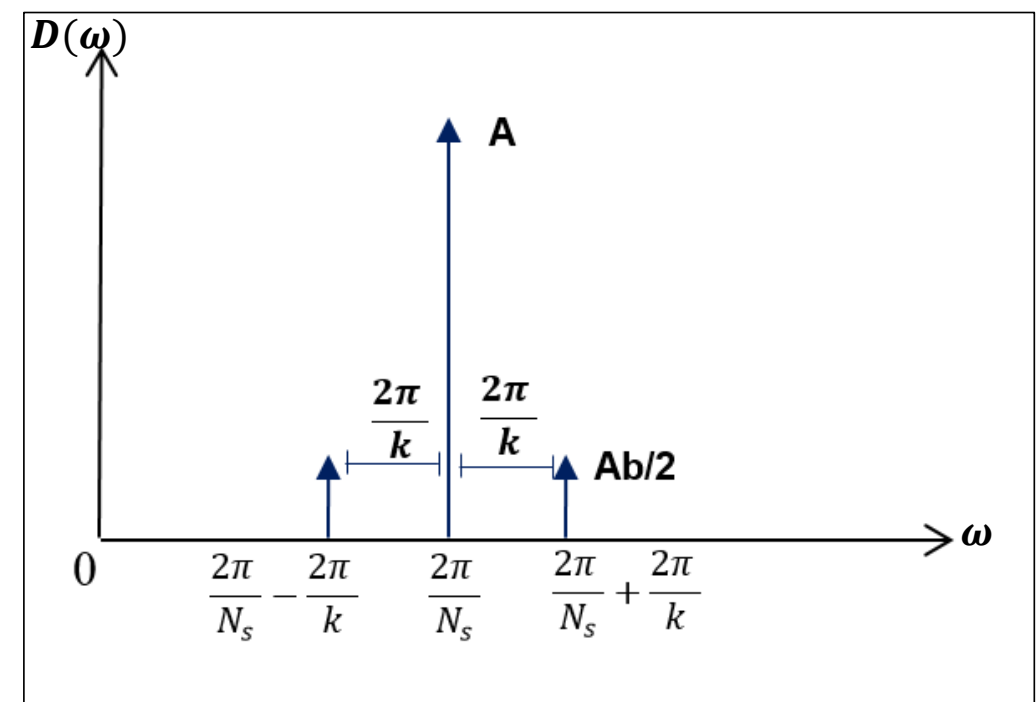

Figura 5.1 Espectro, na frequência, de um sinal sazonal artificial

Os sinais definidos nas Equações (5-1) e (5-2) se referem às séries com modelo aditivo de decomposição (ver Eq. 2-1). Para as séries com modelo multiplicativo de decomposição (Eq. 2-2), o sinal sazonal é representado da seguinte forma:

- Sinal sazonal para as séries mensais:

$$
1+\left\{A\left[1+b \operatorname{sen}\left(2 \pi \frac{t}{k}\right)\right]\left[\cos \left(2 \pi \frac{t}{12}\right)\right]\right\}
$$

Nota: os parâmetros estão definidos na Eq. (5-1).

- Sinal sazonal as séries trimestrais:

$$
1+\left\{A\left[1+b \operatorname{sen}\left(2 \pi \frac{t}{k}\right)\right]\left[\cos \left(2 \pi \frac{t}{4}\right)\right]\right\}
$$

Nota: os parâmetros estão definidos na Eq. (5-2). 
Com base nas Equações (5-3) e (5-4), é possível reescrever o modelo multiplicativo de decomposição: $Y=T \times S \times I$. Considerando que a componente sazonal $S$ tem média 1 , assim como a componente irregular $I, S$ será representada por $\left(S^{\prime}+1\right)$ e $I$ por $\left(I^{\prime}+1\right)$, onde $S^{\prime}$ e $I^{\prime}$ têm média zero. Com isso, a decomposição da série temporal $Y$ ficará assim definida:

$$
\begin{gathered}
Y=T \times\left(S^{\prime}+1\right) \times\left(I^{\prime}+1\right) \\
Y=\left(T S^{\prime}+T\right) \times\left(I^{\prime}+1\right) \\
Y=T+T S^{\prime}+T I^{\prime}+T S^{\prime} I^{\prime}
\end{gathered}
$$

Analisando a Equação (5-7) no domínio da frequência, é possível afirmar que como a tendência é um sinal passa baixas de banda muito estreita, o termo $\left(T S^{\prime}\right)$, que na frequência é a convolução do espectro da sazonalidade com o da tendência, possui um espectro semelhante ao do $S^{\prime}$, com as bandas em torno dos harmônicos ligeiramente aumentadas. Já o espectro do termo $\left(T I^{\prime}+T S^{\prime} I^{\prime}\right)$ é a convolução do espectro de $\left(T+T S^{\prime}\right)$ com o espectro de $I^{\prime}$, que é plano (pois $I^{\prime}$ é descorrelatado), equivalente a um espectro de irregular. Assim, a Equação (5-7) equivale a $Y=T+$ $S^{\prime \prime}+I^{\prime \prime}$, o que implica que $T$ e $S^{\prime \prime}$ podem ser estimadas da mesma maneira do que no modelo aditivo, bastando fazer $S=S^{\prime}+1=\frac{S^{\prime \prime}}{T}+1$.

Neste estudo, as séries construídas com base nas Equações (5-1), (5-2), (5-3) e (5-4) tiveram 400 observações. Escolheu-se trabalhar com um tamanho grande para a série temporal, pois como se trata de um filtro simétrico, algumas observações da série são perdidas. É importante citar que este estudo não teve como objetivo estender a série - com forecasts e backcasts. Porém, para o filtro ser utilizado em séries reais, as mesmas devem ser estendidas, preferencialmente com base em modelos ARIMA (Dagum, 1978).

$\mathrm{Na}$ Subseção 5.2, a seguir, serão apresentadas as características das séries temporais reais utilizadas neste estudo, que influenciaram na construção das séries artificiais. 


\section{2. Características das séries utilizadas e definição dos parâmetros do sinal artificial}

Para a definição dos parâmetros a serem usados nos sinais sazonais artificiais, foram utilizadas como referência séries reais brasileiras e estrangeiras. Já em relação à periodicidade das séries temporais, trabalhou-se com séries mensais e trimestrais.

Foram coletados, inicialmente, dados de 78 séries mensais brasileiras, obtidos nos sites do IPEA e do IBGE no dia 26/6/2013. O período de análise considerado teve início em jan/1998, na maioria das séries. Dessas séries, o dado mais recente foi de abril de 2013. Além disso, foram obtidas 66 séries históricas mensais estrangeiras, no site da OECD (Organização para a Cooperação e Desenvolvimento Econômico) e do U.S. Census Bureau, no dia 20/11/2013. O período de análise considerado, para essas séries, foi de jan/1993 a set/2013. As séries mensais brasileiras e estrangeiras estão especificadas nos Apêndices E e F, respectivamente.

Como as séries econômicas brasileiras disponíveis são, na grande maioria, mensais, optou-se por trabalhar com séries históricas trimestrais apenas estrangeiras. Sendo assim, foram obtidas 106 séries históricas trimestrais estrangeiras, no site da OECD e do U.S. Census Bureau, no dia 20/11/2013. O período de análise considerado foi, na maioria das séries, do $1^{\circ}$ trimestre de 1995 ao $2^{\circ}$ trimestre de 2013. Tais séries estão especificadas no Apêndice G.

Todas as séries foram submetidas ao programa X-13A-S, onde foi testado se havia sazonalidade, e também se havia 'sazonalidade móvel'. Os resultados dos testes mostraram que das 250 séries pesquisadas, 235 apresentaram sazonalidade. Em relação à sazonalidade móvel, 105 séries apresentaram essa característica. A Tabela 5.1, a seguir, apresenta a quantidade de séries analisadas, segundo o nível de significância dos testes de sazonalidade realizados (ver Subseção 2.3.3.3). Os níveis de significância utilizados nos testes foram $0,1 \%, 1 \%$ e $5 \%$. 


\begin{tabular}{c||c||ccc||ccc}
\hline \multicolumn{7}{c}{ Séries Temporais Mensais } \\
\hline \multicolumn{1}{c||}{ Séries pesquisadas } & \multicolumn{3}{c}{ Sazonalidade } & \multicolumn{3}{c}{ Sazonalidade Móvel } \\
\hline Origem & Total & $\mathrm{p}<0,1 \%$ & $\mathrm{p}<1 \%$ & $\mathrm{p}<5 \%$ & $\mathrm{p}<0,1 \%$ & $\mathrm{p}<1 \%$ & $\mathrm{p}<5 \%$ \\
\hline Nacional & 78 & 72 & 0 & 0 & 10 & 11 & 10 \\
Internacional & 66 & 45 & 12 & 2 & 30 & 12 & 3 \\
\hline \multicolumn{7}{c}{ Séries Temporais Trimestrais } \\
\hline Internacional & 106 & 99 & 4 & 1 & 11 & 10 & 8 \\
\hline Nota: $\mathrm{p}$ = valor-p.
\end{tabular}

Tabela 5.1 Quantidade de séries pesquisadas: total, com sazonalidade, e com sazonalidade móvel

Como se pode observar na Tabela 5.1, das 72 séries nacionais com sazonalidade, 31 apresentam sazonalidade móvel, ou seja, 43\% delas possuem sazonalidade móvel. Em relação às séries internacionais, $76 \%$ das mensais, com sazonalidade, apresentam sazonalidade móvel, e $28 \%$ das séries sazonais trimestrais possuem sazonalidade móvel. Sendo assim, nota-se que a característica de mudança no padrão de sazonalidade está presente em grande parte das séries reais, tanto nacionais quanto internacionais.

Das séries nacionais pesquisadas, aquelas que apresentaram sazonalidade móvel significativa a um nível de significância menor do que 5\%, e que por isso foram consideradas na definição dos parâmetros do sinal artificial, estão listadas na Tabela 5.2, segundo o grau de sazonalidade móvel, identificado pelo valor-p. Vale citar que das séries nacionais com sazonalidade móvel significativa, 52\% apresentaram modelo aditivo de decomposição das componentes não observáveis. 


\begin{tabular}{|c|c|c|c|}
\hline Rank & ID & Séries mensais nacionais & valor-p \\
\hline $1^{\circ}$ & X64 & Vendas nominais - varejo - materiais de construção & 0,000 \\
\hline$\underline{2}^{\circ}$ & $\underline{\mathrm{X55}}$ & Venda - fertilizantes - qde. - Tonelada & 0,000 \\
\hline $3^{\circ}$ & $\mathrm{X} 68$ & Produção industrial - bens de consumo duráveis - quantum & 0,000 \\
\hline $4^{\circ}$ & $\mathrm{X} 77$ & Pessoal empregado - indústria - índice (média 2006 = 100) & 0,000 \\
\hline $5^{\circ}$ & $\mathrm{X} 31$ & Cheques sem fundo - $(\%)$ & 0,000 \\
\hline $6^{\circ}$ & $\mathrm{X} 76$ & Utilização da capacidade instalada - indústria - (\%) & 0,000 \\
\hline $7^{\circ}$ & $\mathrm{X} 53$ & SPC - número de registros recebidos - Unidade & 0,000 \\
\hline $8^{\circ}$ & $\mathrm{X} 41$ & Consumo - energia elétrica - comércio - qde. - GWh & 0,000 \\
\hline $9^{\circ}$ & $\mathrm{X} 43$ & Consumo - energia elétrica - residência - qde. - GWh & 0,000 \\
\hline $10^{\circ}$ & X6 & Produção física industrial. Indústria geral Espírito Santo & 0,001 \\
\hline $11^{\circ}$ & X67 & Produção industrial - bens de consumo - quantum & 0,001 \\
\hline$\underline{12^{\circ}}$ & $\underline{\mathrm{X} 36}$ & Consumo - energia elétrica - Região Sudeste (SE) - qde. - GWh & 0,001 \\
\hline $13^{\circ}$ & $\mathrm{X} 73$ & Produção industrial - bebidas - quantum - índice (média 2002 = 100) & 0,002 \\
\hline$\underline{14^{\circ}}$ & $\underline{\mathrm{X} 47}$ & Consumo - energia elétrica - residência - tarifa média por MWh - R\$ & 0,002 \\
\hline $15^{\circ}$ & $\mathrm{X} 7$ & Produção física industrial. Indústria geral Rio de Janeiro & 0,002 \\
\hline $16^{\circ}$ & X69 & Produção industrial - bens de consumo não duráveis - quantum & 0,003 \\
\hline $17^{\circ}$ & $\mathrm{X} 10$ & Produção física industrial. Indústria geral Santa Catarina & 0,003 \\
\hline $18^{\circ}$ & $\mathrm{X} 1$ & Produção física industrial. Indústria geral Brasil & 0,003 \\
\hline $19^{\circ}$ & $\mathrm{X} 38$ & Consumo aparente - gasolina - média - qde./dia - Barril (mil) & 0,008 \\
\hline $20^{\circ}$ & $\mathrm{X} 45$ & Consumo - energia elétrica - comércio - tarifa média por MWh - R\$ & 0,009 \\
\hline $21^{\circ}$ & $\mathrm{X} 75$ & Horas trabalhadas - indústria - índice (média $2006=100$ ) & 0,009 \\
\hline $22^{\circ}$ & $\mathrm{X} 58$ & Vendas nominais - varejo - índice (média $2011=100)$ & 0,013 \\
\hline $23^{\circ}$ & $\mathrm{X} 46$ & Consumo - energia elétrica - indústria - tarifa média por MWh - R\$ & 0,017 \\
\hline $24^{\circ}$ & $\mathrm{X} 71$ & Produção industrial - indústria de transformação - quantum & 0,020 \\
\hline $25^{\circ}$ & $\mathrm{X} 11$ & Produção física industrial. Indústria geral Rio Grande do Sul & 0,020 \\
\hline $26^{\circ}$ & $\mathrm{X} 74$ & Produção industrial - têxtil - quantum - índice (média 2002 = 100) & 0,023 \\
\hline $27^{\circ}$ & $\mathrm{X} 8$ & Produção física industrial. Indústria geral São Paulo & 0,024 \\
\hline $28^{\circ}$ & $\mathrm{X} 15$ & Comércio - cine, foto, som e ótica - faturamento - RMSP & 0,032 \\
\hline $29^{\circ}$ & $\mathrm{X} 42$ & Consumo - energia elétrica - indústria - qde. - GWh & 0,037 \\
\hline $30^{\circ}$ & X63 & Vendas nominais - varejo - veículos, motos, partes e peças & 0,038 \\
\hline $31^{\circ}$ & $\mathrm{X} 12$ & Comércio - automotivo e construção - faturamento - RMSP & 0,042 \\
\hline
\end{tabular}

Tabela 5.2 Séries históricas mensais nacionais com sazonalidade móvel significativa

As séries internacionais mensais com sazonalidade móvel significativa, a um nível de significância menor do que 5\%, estão listadas na Tabela 5.3. Dessas séries, $36 \%$ apresentaram modelo aditivo de decomposição, e 64\% apresentaram modelo multiplicativo. Com isso, observa-se uma diferença no perfil das séries nacionais e internacionais. A indicação do tipo de decomposição de cada série mensal será apresentada nas Subseções 5.2.1 e 5.2.2. 


\begin{tabular}{|c|c|c|c|}
\hline Rank & ID & Séries mensais internacionais & valor-p \\
\hline $1^{\circ}$ & Y122 & Employment Level; USA; Number in thousands & 0,000 \\
\hline $2^{\circ}$ & Y128 & General Merchandise Stores; USA; millions of dollars & 0,000 \\
\hline $3^{\circ}$ & Y132 & Gasoline Stations; USA; millions of dollars & 0,000 \\
\hline $4^{\circ}$ & Y133 & Clothing and Clothing Access. Stores; USA; millions of dollars & 0,000 \\
\hline $5^{\circ}$ & Y138 & Nonmetallic Mineral Products; U.S.; millions of dollars & 0,000 \\
\hline $6^{\circ}$ & Y154 & Dairy Product Manufacturing; U.S.; millions of dollars & 0,000 \\
\hline $7^{\circ}$ & Y140 & Construction Machinery Manufacturing; U.S.; millions of dollars & 0,000 \\
\hline $8^{\circ}$ & Y145 & Plastics and Rubber Products; U.S.; millions of dollars & 0,000 \\
\hline $9^{\circ}$ & Y146 & Construction Materials and Supplies; U.S.; millions of dollars & 0,000 \\
\hline $10^{\circ}$ & Y149 & Food Products; U.S.; millions of dollars & 0,000 \\
\hline $11^{\circ}$ & Y156 & Beverage and Tobacco Products; U.S.; millions of dollars & 0,000 \\
\hline $12^{\circ}$ & Y166 & Relative consumer price indices; Iceland & 0,000 \\
\hline $13^{\circ}$ & Y170 & Relative consumer price indices; Korea & 0,000 \\
\hline $14^{\circ}$ & Y162 & Relative consumer price indices; Finland & 0,000 \\
\hline $15^{\circ}$ & Y180 & Relative consumer price indices; Turkey & 0,000 \\
\hline $16^{\circ}$ & Y181 & Relative consumer price indices; United Kingdom & 0,000 \\
\hline $17^{\circ}$ & Y187 & Relative consumer price indices; Indonesia & 0,000 \\
\hline $18^{\circ}$ & Y169 & Relative consumer price indices; Japan & 0,000 \\
\hline $19^{\circ}$ & Y175 & Relative consumer price indices; Norway & 0,000 \\
\hline $20^{\circ}$ & Y124 & Retail Trade and Food Services, ex Auto; USA; millions of dollars & 0,000 \\
\hline $21^{\circ}$ & Y178 & Relative consumer price indices; Sweden & 0,000 \\
\hline $22^{\circ}$ & Y127 & Grocery Stores; USA; millions of dollars & 0,000 \\
\hline $23^{\circ}$ & Y182 & Relative consumer price indices; United States & 0,000 \\
\hline $24^{\circ}$ & Y150 & Consumer Goods; U.S.; millions of dollars & 0,000 \\
\hline $25^{\circ}$ & Y165 & Relative consumer price indices; Greece & 0,000 \\
\hline $26^{\circ}$ & Y153 & Grain and Oilseed Milling; U.S.; millions of dollars & 0,001 \\
\hline $27^{\circ}$ & Y179 & Relative consumer price indices; Switzerland & 0,001 \\
\hline $28^{\circ}$ & Y168 & Relative consumer price indices; Italy & 0,001 \\
\hline $29^{\circ}$ & Y152 & Consumer Nondurable Goods; U.S.; millions of dollars & 0,001 \\
\hline $30^{\circ}$ & Y137 & Wood Products; U.S.; millions of dollars & 0,001 \\
\hline $31^{\circ}$ & Y160 & Relative consumer price indices; Canada & 0,001 \\
\hline $32^{\circ}$ & Y130 & Food and Beverage Stores; USA; millions of dollars & 0,002 \\
\hline $33^{\circ}$ & Y164 & Relative consumer price indices; Germany & 0,002 \\
\hline $34^{\circ}$ & Y167 & Relative consumer price indices; Ireland & 0,002 \\
\hline $35^{\circ}$ & Y174 & Relative consumer price indices; New Zealand & 0,003 \\
\hline $36^{\circ}$ & Y184 & Relative consumer price indices; Brazil & 0,004 \\
\hline $37^{\circ}$ & Y173 & Relative consumer price indices; Netherlands & 0,004 \\
\hline $38^{\circ}$ & Y172 & Relative consumer price indices; Mexico & 0,005 \\
\hline $39^{\circ}$ & Y143 & Audio and Video Equipment; U.S.; millions of dollars & 0,005 \\
\hline $40^{\circ}$ & Y183 & Relative consumer price indices; Euro area (17 countries) & 0,006 \\
\hline $41^{\circ}$ & Y185 & Relative consumer price indices; China & 0,007 \\
\hline $42^{\circ}$ & Y159 & Relative consumer price indices; Belgium & 0,008 \\
\hline $43^{\circ}$ & Y157 & Relative consumer price indices; Australia & 0,012 \\
\hline $44^{\circ}$ & Y158 & Relative consumer price indices; Austria & 0,018 \\
\hline $45^{\circ}$ & Y163 & Relative consumer price indices; France & 0,022 \\
\hline
\end{tabular}

Tabela 5.3 Séries históricas mensais internacionais com sazonalidade móvel significativa 
Em relação às séries internacionais trimestrais, das 29 que apresentaram sazonalidade móvel, apenas 4 têm decomposição aditiva, enquanto que 25 têm decomposição multiplicativa. Ou seja, $86 \%$ das séries estrangeiras trimestrais, com sazonalidade móvel, têm decomposição multiplicativa. Essas séries estão apresentadas na Tabela 5.4.

\begin{tabular}{|c|c|c|c|}
\hline Rank & ID & Séries trimestrais internacionais & valor-p \\
\hline $1^{\mathrm{o}}$ & Y3 & Gross domestic product; Belgium & 0,000 \\
\hline $2^{\circ}$ & Y52 & Exports of goods and services; Euro area (17 countries) & 0,000 \\
\hline $3^{\circ}$ & Y53 & Exports of goods and services; European Union (27 countries) & 0,000 \\
\hline $4^{\circ}$ & Y95 & Imports of goods and services; European Union (27 countries) & 0,000 \\
\hline $5^{\circ}$ & Y112 & Total gross debt; Luxembourg & 0,000 \\
\hline $6^{\circ}$ & Y74 & Imports of goods and services; Czech Republic & 0,000 \\
\hline $7^{\circ}$ & Y37 & Exports of goods and services; Germany & 0,000 \\
\hline $8^{\circ}$ & Y84 & Imports of goods and services; Luxembourg & 0,000 \\
\hline $9^{\circ}$ & Y13 & Gross domestic product - expenditure approach; Japan & 0,000 \\
\hline $10^{\circ}$ & Y91 & Imports of goods and services; Slovenia & 0,000 \\
\hline $11^{\circ}$ & Y12 & Gross domestic product - expenditure approach; Italy & 0,001 \\
\hline $12^{\circ}$ & Y47 & Exports of goods and services; Poland & 0,001 \\
\hline $13^{\circ}$ & Y94 & Imports of goods and services; Euro area (17 countries) & 0,001 \\
\hline $14^{\circ}$ & Y120 & Retail Sales; Total; (millions of dollars) & 0,002 \\
\hline $15^{\circ}$ & Y35 & Exports of goods and services; Finland & 0,003 \\
\hline $16^{\circ}$ & Y38 & Exports of goods and services; Hungary & 0,005 \\
\hline $17^{\circ}$ & Y98 & Total gross debt; Australia & 0,005 \\
\hline $18^{\circ}$ & Y25 & Gross domestic product; Euro area (17 countries) & 0,007 \\
\hline $19^{\circ}$ & Y99 & Total gross debt; Austria & 0,007 \\
\hline $20^{\circ}$ & Y121 & Retail Sales; E-commerce; (millions of dollars) & 0,008 \\
\hline $21^{\circ}$ & Y20 & Gross domestic product - expenditure approach; Poland & 0,010 \\
\hline $22^{\circ}$ & Y77 & Imports of goods and services; Finland & 0,013 \\
\hline $23^{\circ}$ & Y10 & Gross domestic product - expenditure approach; Greece & 0,019 \\
\hline $24^{\circ}$ & Y73 & Imports of goods and services; Belgium & 0,022 \\
\hline $25^{\circ}$ & Y89 & Imports of goods and services; Poland & 0,023 \\
\hline $26^{\circ}$ & Y45 & Exports of goods and services; New Zealand & 0,024 \\
\hline $27^{\circ}$ & Y11 & Gross domestic product - expenditure approach; Hungary & 0,029 \\
\hline $28^{\circ}$ & Y81 & Imports of goods and services; Italy & 0,035 \\
\hline $29^{\circ}$ & Y41 & Exports of goods and services; Korea & 0,042 \\
\hline
\end{tabular}

Tabela 5.4 Séries históricas trimestrais internacionais com sazonalidade móvel significativa

A indicação do modelo de decomposição das séries trimestrais será apresentada nas Subseções 5.2.3 e 5.2.4. 
Como ilustração da sazonalidade móvel presente nas séries reais, é apresentado o Gráfico 5.2 da componente sazonal da série X6 (Produção Física Industrial: Espírito Santo), extraída pelo programa X-13A-S. Nesse gráfico é possível notar a variação da amplitude da sazonalidade ao longo dos meses.

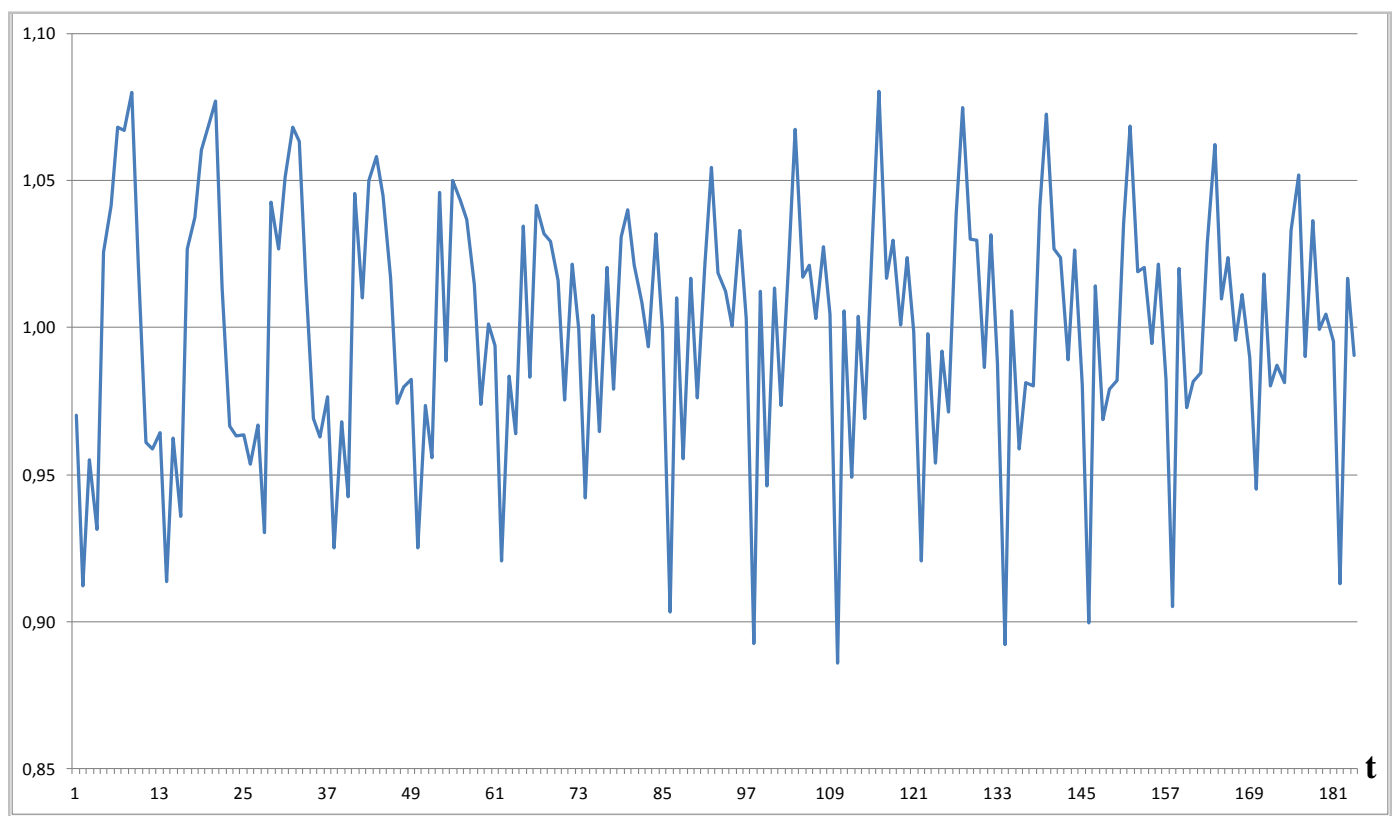

\section{Gráfico 5.2 Componente Sazonal: Produção Industrial Mensal - Indústria Geral do Espírito Santo}

Após a seleção das séries temporais com sazonalidade móvel significativa, foi analisado o gráfico da componente sazonal de cada série, a fim de observar os valores dos parâmetros ' $A$ ', ' $k$ ' e ' $b$ ', definidos na Equação (5-1). Tais valores devem ser utilizados nas séries artificiais, para que estas tenham um mesmo nível de variação da sazonalidade. Porém, em algumas séries não foi possível essa observação. As séries analisadas, para a obtenção dos valores dos parâmetros ' $A$ ', ' $k$ ' e ' $b$ ', serão apresentadas nas subseções a seguir, segundo a periodicidade (mensal e trimestral) e o modelo de decomposição (aditivo e multiplicativo). 


\subsection{1.}

\section{Séries mensais com decomposição aditiva: parâmetros ' $A / s$ ', ' $k$ ', ' $b$ ' e coeficientes da componente de tendência}

As séries mensais reais, com sazonalidade móvel e decomposição aditiva, foram analisadas para a obtenção do valor da razão ' $A / S$ ', onde ' $A$ ' é a amplitude do sinal sazonal e ' $s$ ' é o desvio-padrão da componente irregular. Além disso, foram identificados os valores dos parâmetros ' $b$ ' e ' $k$ '. A identificação dos valores de ' $A$ ', ' $b$ ' e ' $k$ ' foi feita com base no gráfico da componente sazonal, obtida pelo X13A-S, e no gráfico do sinal sazonal artificial (Eq. 5-1); onde variaram-se os valores dos parâmetros ' $A$ ', ' $b$ ' e ' $k$ ' até obter a combinação que melhor se ajustava à componente sazonal da série. Quanto ao valor de ' $s$ ', foi calculado o desvio-padrão da componente irregular, gerada pelo X-13A-S, sem os outliers. Os valores encontrados estão apresentados na Tabela 5.5.

\begin{tabular}{|c|c|c|c|c|c|c|}
\hline Série & $\boldsymbol{k}$ & $b$ & $A$ & $s$ & $A / s$ & Origem \\
\hline X12 & 96 & $15 \%$ & 10 & 2,8 & 3,6 & \multirow{11}{*}{ nacional } \\
\hline X31 & 72 & $13 \%$ & 2,5 & 0,4 & 6,3 & \\
\hline X36 & 96 & $32 \%$ & 0,6 & 0,3 & 2,0 & \\
\hline $\mathrm{X} 41$ & 144 & $24 \%$ & 0,4 & 0,2 & 2,0 & \\
\hline $\mathrm{X} 43$ & 120 & $52 \%$ & 0,4 & 0,2 & 2,0 & \\
\hline X53 & 60 & $33 \%$ & 60 & 9,1 & 6,6 & \\
\hline X67 & 84 & $19 \%$ & 13 & 1,1 & 11,8 & \\
\hline X69 & 84 & $20 \%$ & 12 & 1,3 & 9,2 & \\
\hline X73 & 96 & $10 \%$ & 30 & 2,6 & 11,5 & \\
\hline X74 & 84 & $11 \%$ & 20 & 1,3 & 15,4 & \\
\hline $\mathrm{X} 76$ & 84 & $22 \%$ & 1,6 & 0,2 & 8,0 & \\
\hline Y122 & 132 & $30 \%$ & 1250 & 103 & 12,1 & \multirow{7}{*}{ internacional } \\
\hline Y137 & 120 & $47 \%$ & 300 & 47 & 6,4 & \\
\hline Y166 & 144 & $60 \%$ & 1,8 & 0,53 & 3,4 & \\
\hline Y168 & 216 & $70 \%$ & 0,5 & 0,34 & 1,5 & \\
\hline Y180 & 156 & $50 \%$ & 2 & 0,61 & 3,3 & \\
\hline Y181 & 120 & $40 \%$ & 1,5 & 0,57 & 2,6 & \\
\hline Y183 & 144 & $50 \%$ & 0,9 & 0,41 & 2,2 & \\
\hline
\end{tabular}

Tabela 5.5 Parâmetros das séries mensais aditivas, com sazonalidade móvel

Analisando a Tabela 5.5, nota-se que, para as séries mensais aditivas, os valores de ' $k$ ' variam, em sua maioria, entre 84 e 132. Os valores de ' $b$ ' estão mais concentrados entre $10 \%$ e $30 \%$, sendo que $25 \%$ se concentram entre $30 \%$ e $60 \%$. Já a razão ' $A / s$ ' mostra uma concentração nos valores inferiores a 7. A distribuição dos parâmetros citados pode ser melhor visualizada nos histogramas a seguir. 


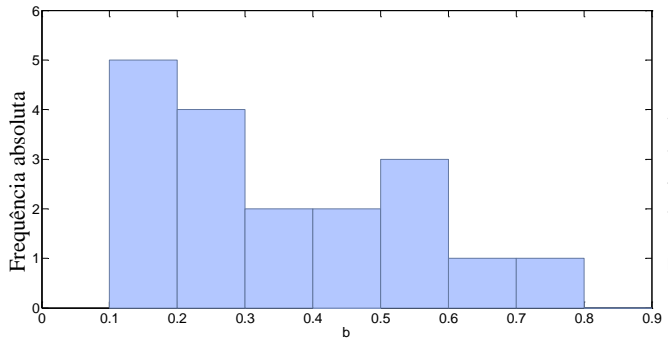

Gráfico 5.3 Histograma de ' $b$ ' (Eq. 5-1) das séries mensais aditivas

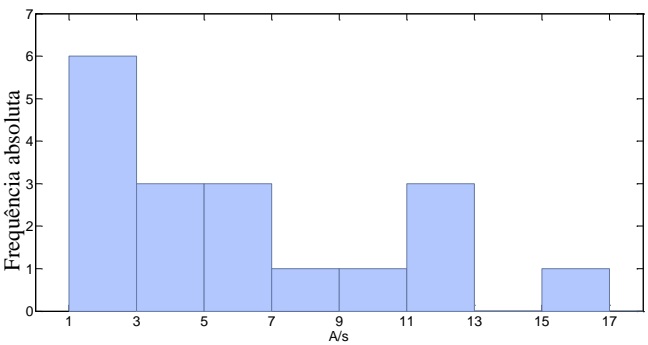

Gráfico 5.4 Histograma de ' $A / s$ '

(Eq. 5-1) das séries mensais aditivas

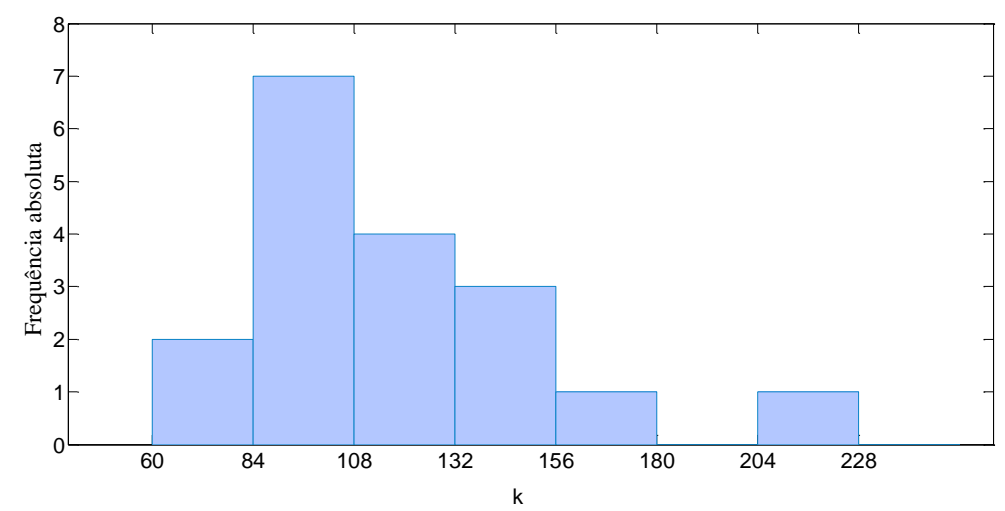

Gráfico 5.5 Histograma de ' $k$ ' (Eq. 5-1) das séries mensais aditivas

Para obter os coeficientes da componente de tendência, verificou-se o grau do polinômio que melhor se ajustava a essa componente, estimada pelo X-13A-S. Foram ajustados desde polinômios de ordem 2 até os de ordem 5. O polinômio de ordem 5 está apresentado a seguir:

$$
T=b_{0}+b_{1} t+b_{2} t^{2}+b_{3} t^{3}+b_{4} t^{4}+b_{5} t^{5}
$$

onde $t=$ período que está sendo analisado. $t=1,2, \ldots$

Os coeficientes encontrados, assim como o $\mathrm{R}^{2}$ obtido no ajuste, estão apresentados na Tabela 5.6. 


\begin{tabular}{c|r|r|r|r|r|r|c}
\hline Série & \multicolumn{1}{c|}{$\mathbf{b}_{\mathbf{0}}$} & \multicolumn{1}{c|}{$\mathbf{b}_{\mathbf{1}}$} & \multicolumn{1}{c|}{$\mathbf{b}_{\mathbf{2}}$} & \multicolumn{1}{c}{$\mathbf{b}_{\mathbf{3}}$} & $\mathbf{b}_{\mathbf{4}}$ & $\mathbf{b}_{\mathbf{5}}$ & $\mathbf{R}^{\mathbf{2}}$ \\
\hline $\mathrm{X} 12$ & 107,45 & $-1,9738$ & 0,0424 & $-3 \mathrm{E}-04$ & $7 \mathrm{E}-07$ & & $65 \%$ \\
\hline $\mathrm{X} 31$ & 9,93 & $-0,1387$ & 0,0065 & $-6 \mathrm{E}-05$ & $1 \mathrm{E}-07$ & & $93 \%$ \\
\hline X36 & 14,280 & $-0,0781$ & 0,0012 & $-4 \mathrm{E}-06$ & & & $90 \%$ \\
\hline X41 & 3,13 & $-0,0016$ & 0,0010 & & & & $94 \%$ \\
\hline X43 & 6,10 & 0,0098 & $-0,0006$ & $7 \mathrm{E}-06$ & $-2 \mathrm{E}-08$ & & $93 \%$ \\
\hline X53 & 389,78 & $-4,7035$ & 0,0635 & $-2 \mathrm{E}-04$ & & & $94 \%$ \\
\hline X67 & 98,27 & $-0,2740$ & 0,0065 & $-2 \mathrm{E}-05$ & & & $95 \%$ \\
\hline X73 & 100,94 & $-0,2596$ & 0,0046 & $-1 \mathrm{E}-05$ & & & $94 \%$ \\
\hline X74 & 106,63 & 0,4666 & $-0,0191$ & $2 \mathrm{E}-04$ & $-6 \mathrm{E}-07$ & & $92 \%$ \\
\hline X76 & 90,79 & 1,2709 & $-0,0461$ & $7 \mathrm{E}-04$ & $-4 \mathrm{E}-06$ & $8 \mathrm{E}-09$ & $79 \%$ \\
\hline Y122 & 118,03 & 0,0574 & $-0,0002$ & & & & $63 \%$ \\
\hline Y137 & $6.500,00$ & 186,5900 & $-4,8966$ & 0,0562 & $-3 \mathrm{E}-04$ & $5 \mathrm{E}-07$ & $75 \%$ \\
\hline Y166 & 124,68 & 0,5505 & $-0,0298$ & $4 \mathrm{E}-04$ & $-2 \mathrm{E}-06$ & $4 \mathrm{E}-09$ & $67 \%$ \\
\hline Y168 & 93,950 & $-0,1077$ & 0,0020 & $-6 \mathrm{E}-06$ & & & $67 \%$ \\
\hline Y180 & 66,69 & $-0,2551$ & 0,0045 & $-1 \mathrm{E}-05$ & & & $81 \%$ \\
\hline Y181 & 107,93 & $-0,1974$ & 0,0112 & $-9 \mathrm{E}-05$ & $2 \mathrm{E}-07$ & & $80 \%$ \\
\hline Y183 & 98,41 & 1,0174 & $-0,0346$ & $4 \mathrm{E}-04$ & $-2 \mathrm{E}-06$ & $3 \mathrm{E}-09$ & $65 \%$ \\
\hline
\end{tabular}

Tabela 5.6 Coeficientes da componente de tendência das séries mensais aditivas (Eq. 5-8)

Os valores encontrados com base nessas séries temporais serão utilizados na criação de séries artificiais. Os resultados serão apresentados na Subseção 5.5.

\subsection{2.}

Séries mensais com decomposição multiplicativa: parâmetros ' $A / s$ ', ' $k$ ', ' $b$ ' e coeficientes da componente de tendência

Nesta subseção são apresentados os parâmetros da componente sazonal das séries mensais, com modelo multiplicativo, assim como o desvio-padrão da componente irregular das séries. Também são apresentados os coeficientes da componente de tendência dessas séries.

A Tabela 5.7 apresenta os parâmetros da componente sazonal das séries mensais - nacionais e internacionais - com decomposição multiplicativa, juntamente com o desvio-padrão da irregular. Para a identificação dos valores foi utilizado o mesmo procedimento descrito na Subseção 5.2.1. 


\begin{tabular}{|c|c|c|c|c|c|c|}
\hline Série & $k$ & $b$ & $A$ & $s$ & $A / s$ & Origem \\
\hline $\mathrm{X} 6$ & 108 & $30 \%$ & 0,060 & 0,02500 & 2,4 & \multirow{8}{*}{ nacional } \\
\hline $\mathrm{X} 10$ & 72 & $25 \%$ & 0,080 & 0,01462 & 5,5 & \\
\hline X38 & 60 & $40 \%$ & 0,100 & 0,01850 & 5,4 & \\
\hline $\mathrm{X} 58$ & 84 & $20 \%$ & 0,400 & 0,00774 & 51,7 & \\
\hline X63 & 84 & $20 \%$ & 0,150 & 0,03477 & 4,3 & \\
\hline X64 & 84 & $24 \%$ & 0,100 & 0,00824 & 12,1 & \\
\hline X71 & 72 & $22 \%$ & 0,110 & 0,00853 & 12,9 & \\
\hline X77 & 84 & $28 \%$ & 0,012 & 0,00115 & 10,5 & \\
\hline Y124 & 60 & $15 \%$ & 0,150 & 0,00418 & 35,9 & \multirow{24}{*}{ internacional } \\
\hline Y127 & 48 & $20 \%$ & 0,080 & 0,00427 & 18,7 & \\
\hline Y130 & 72 & $21 \%$ & 0,110 & 0,00377 & 29,1 & \\
\hline Y140 & 120 & $20 \%$ & 0,030 & 0,00712 & 4,2 & \\
\hline Y143 & 96 & $18 \%$ & 0,140 & 0,02159 & 6,5 & \\
\hline Y145 & 72 & $25 \%$ & 0,025 & 0,00408 & 6,1 & \\
\hline Y146 & 120 & $22 \%$ & 0,025 & 0,00284 & 8,8 & \\
\hline Y150 & 120 & $30 \%$ & 0,030 & 0,00444 & 6,8 & \\
\hline Y152 & 120 & $40 \%$ & 0,025 & 0,00394 & 6,3 & \\
\hline Y153 & 120 & $18 \%$ & 0,075 & 0,01961 & 3,8 & \\
\hline Y157 & 96 & $34 \%$ & 0,015 & 0,01275 & 1,2 & \\
\hline Y159 & 84 & $30 \%$ & 0,005 & 0,00390 & 1,3 & \\
\hline Y160 & 144 & $38 \%$ & 0,010 & 0,00658 & 1,5 & \\
\hline Y162 & 120 & $40 \%$ & 0,008 & 0,00467 & 1,7 & \\
\hline Y163 & 120 & $32 \%$ & 0,005 & 0,00364 & 1,4 & \\
\hline Y164 & 180 & $25 \%$ & 0,008 & 0,00301 & 2,7 & \\
\hline Y167 & 156 & $25 \%$ & 0,006 & 0,00500 & 1,2 & \\
\hline Y169 & 84 & $42 \%$ & 0,016 & 0,01257 & 1,3 & \\
\hline Y174 & 96 & $35 \%$ & 0,016 & 0,00817 & 2,0 & \\
\hline Y175 & 96 & $26 \%$ & 0,008 & 0,00585 & 1,4 & \\
\hline Y178 & 84 & $30 \%$ & 0,010 & 0,00659 & 1,5 & \\
\hline Y179 & 120 & $25 \%$ & 0,011 & 0,00637 & 1,7 & \\
\hline Y182 & 120 & $22 \%$ & 0,010 & 0,00584 & 1,7 & \\
\hline Y185 & 120 & $26 \%$ & 0,010 & 0,00562 & 1,8 & \\
\hline
\end{tabular}

Tabela 5.7 Parâmetros das séries mensais multiplicativas, com sazonalidade móvel (Eq. 5-3)

Os histogramas a seguir foram construídos para melhor visualização da concentração de valores dos parâmetros ' $b$ ', ' $A / s$ ' e ' $k$ '; onde ' $b$ ' representa a taxa de variação da sazonalidade; ' $A / s$ ' indica a amplitude do sinal em relação ao desvio-padrão da irregular; e ' $k$ ' é o número de meses no qual o padrão de sazonalidade móvel volta a se repetir, segundo o sinal artificial (ver Eq. 5-3).

Analisando os histogramas de cada parâmetro (Gráficos 5.6 a 5.8), nota-se que a taxa ' $b$ ' apresenta a maior parte dos valores entre $20 \%$ e $30 \%$, sendo que $20 \%$ está entre $35 \%$ e $45 \%$. 
Sobre a razão $A / s$, a grande maioria das observações está nos valores abaixo de 7

Em relação ao ' $k$ ', percebe-se uma concentração entre 72 e 96 . E, com base na Tabela 5.7, observa-se uma grande quantidade de observações no valor 120 .

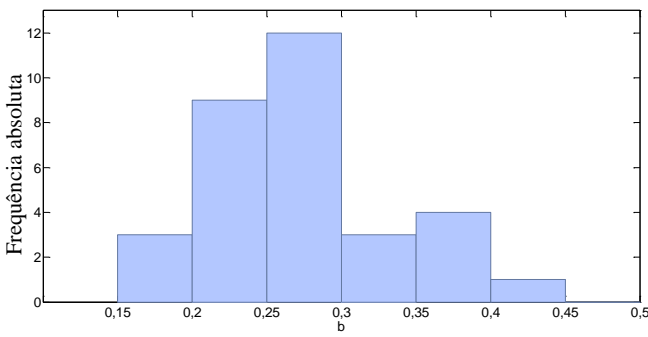

Gráfico 5.6 Histograma de ' $b$ ' (Eq. 53) das séries mensais multiplicativas

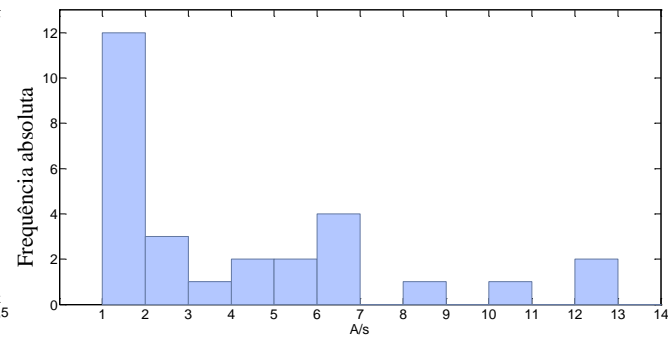

Gráfico 5.7 Histograma de ' $A / s$ ' (Eq. 5-3) das séries mensais multiplicativas

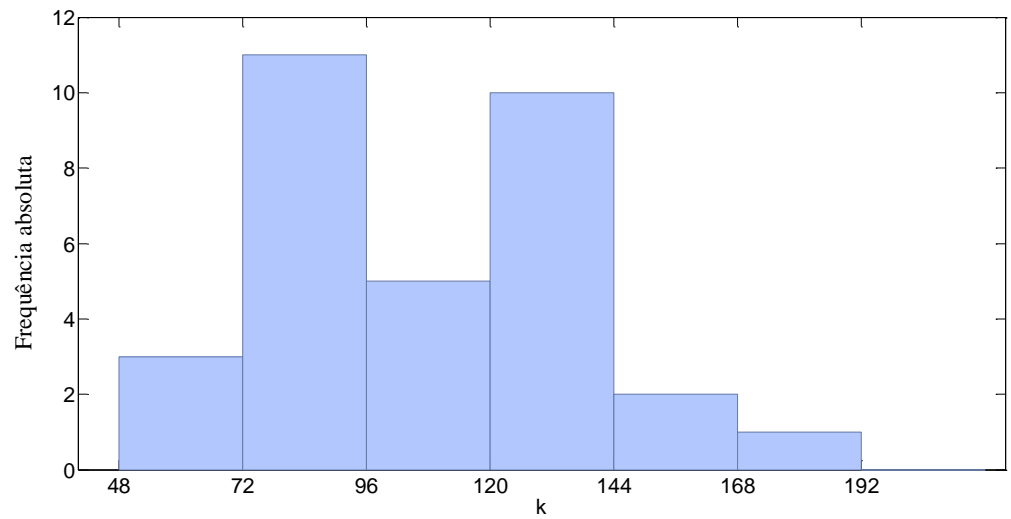

Gráfico 5.8 Histograma de ' $k$ ' (Eq. 5-3) das séries mensais multiplicativas 
Em se tratando da componente de tendência, gerada pelo X-13A-S, os coeficientes estimados estão apresentados na Tabela 5.8. Analisando tal tabela, nota-se que, na maioria dos casos, o polinômio de grau 3 é o que melhor se ajusta à componente.

\begin{tabular}{|c|c|c|c|c|c|c|c|}
\hline Série & bo & $b_{1}$ & $\mathbf{b}_{2}$ & $\mathbf{b}_{3}$ & $\mathbf{b}_{4}$ & b5 & $\mathbf{R}^{2}$ \\
\hline $\mathrm{X} 10$ & 93,90 & 1,0797 & $-0,0387$ & 0,001 & $-3 \mathrm{E}-06$ & 7E-09 & $38 \%$ \\
\hline X38 & 300,43 & 0,8901 & $-0,0246$ & $2 \mathrm{E}-04$ & & & $95 \%$ \\
\hline $\mathrm{X} 58$ & 32,45 & $-0,0860$ & 0,0031 & & & & $99,8 \%$ \\
\hline X6 & 76,30 & 0,3114 & 0,0028 & $1 \mathrm{E}-05$ & & & $87 \%$ \\
\hline X63 & 76,94 & $-1,6111$ & 0,0197 & $-6 E-05$ & & & $98 \%$ \\
\hline X64 & 44,33 & $-0,3087$ & 0,0039 & & & & $99 \%$ \\
\hline X71 & 94,620 & $-0,0849$ & 0,0045 & $-2 \mathrm{E}-05$ & & & $91 \%$ \\
\hline X77 & 96,90 & $-0,3640$ & 0,0057 & $-2 \mathrm{E}-05$ & & & $97 \%$ \\
\hline Y124 & $129.109,00$ & 860,4300 & & & & & $98 \%$ \\
\hline Y127 & $28.263,00$ & $42.543,0000$ & 0,1576 & & & & $99,5 \%$ \\
\hline $\mathrm{Y} 130$ & $30.915,00$ & 49,3660 & 0,1818 & & & & $99,6 \%$ \\
\hline Y140 & $2.491,30$ & 45,3900 & $-0,6734$ & 3,9E-03 & $-7 \mathrm{E}-06$ & & $69 \%$ \\
\hline Y143 & 675,47 & 33,4190 & $-0,8471$ & $8,5 \mathrm{E}-03$ & $-4 \mathrm{E}-05$ & $6 \mathrm{E}-08$ & $61 \%$ \\
\hline $\mathrm{Y} 145$ & $12.768,00$ & 65,1050 & $-0,3083$ & 8E-04 & & & $81 \%$ \\
\hline Y146 & $37.689,00$ & 125,3800 & $-0,1360$ & & & & $80 \%$ \\
\hline Y150 & $107.588,00$ & 65,2320 & 1,1238 & & & & $92 \%$ \\
\hline Y152 & $85.836,00$ & $-5,1194$ & 1,3846 & & & & $94 \%$ \\
\hline Y153 & $2.874,90$ & 67,8540 & $-1,3886$ & 9,4E-03 & $-2 \mathrm{E}-05$ & & $83 \%$ \\
\hline Y157 & 72,73 & $-0,0736$ & 0,0009 & & & & $84 \%$ \\
\hline Y159 & 91,30 & 0,4744 & $-0,0149$ & $2 \mathrm{E}-04$ & $-7 \mathrm{E}-07$ & 1E-09 & $86 \%$ \\
\hline Y160 & 91,62 & $-0,5864$ & 0,0057 & $-1 \mathrm{E}-05$ & & & $88 \%$ \\
\hline Y162 & 93,46 & 0,8361 & $-0,0220$ & 0,000 & $-9 \mathrm{E}-07$ & 1E-09 & $48 \%$ \\
\hline Y163 & 101,53 & 0,5890 & $-0,0193$ & 2E-04 & $-9 \mathrm{E}-07$ & 1E-09 & $71 \%$ \\
\hline Y164 & 107,28 & 0,7533 & $-0,0246$ & $3 \mathrm{E}-04$ & $-1 \mathrm{E}-06$ & 2E-09 & $83 \%$ \\
\hline Y167 & 80,22 & 0,8233 & $-0,0260$ & $3 \mathrm{E}-04$ & $-1 \mathrm{E}-06$ & 2E-09 & $91 \%$ \\
\hline Y169 & 125,40 & $-0,2329$ & 0,0004 & & & & $54 \%$ \\
\hline Y174 & 65,15 & 2,3818 & $-0,0635$ & $7 \mathrm{E}-04$ & $-3 E-06$ & 4E-09 & $69 \%$ \\
\hline Y175 & 91,97 & $-0,0941$ & 0,0014 & $-4 \mathrm{E}-06$ & & & $65 \%$ \\
\hline Y178 & 117,04 & 0,0047 & $-0,0012$ & 4E-06 & & & $56 \%$ \\
\hline Y179 & 98,220 & 0,0580 & $-0,0014$ & $5 \mathrm{E}-06$ & & & $43 \%$ \\
\hline Y182 & 103,28 & $-0,8914$ & 0,0304 & $-3 \mathrm{E}-04$ & $1 \mathrm{E}-06$ & $-2 \mathrm{E}-09$ & $88 \%$ \\
\hline Y185 & 144,47 & $-5,5528$ & 0,1651 & $-2,1 \mathrm{E}-03$ & $1 \mathrm{E}-05$ & $-4 \mathrm{E}-08$ & $81 \%$ \\
\hline
\end{tabular}

Tabela 5.8 Coeficientes da componente de tendência das séries mensais multiplicativas (Eq. 5-8)

Nas duas próximas subseções serão apresentados os valores dos parâmetros das séries reais trimestrais: aditivas e multiplicativas. 


\subsection{3.}

Séries trimestrais com decomposição aditiva: parâmetros ' $A / s$ ', ' $k$ ', ' $b$ ' e coeficientes da componente de tendência

Apenas três séries trimestrais apresentaram modelo aditivo de decomposição. Vale ressaltar que todas as séries temporais trimestrais analisadas são internacionais, e as séries internacionais são conhecidas por ter uma prevalência de padrão multiplicativo de decomposição. Isso se confirmou nesse conjunto de séries.

A Tabela 5.9 mostra as características dos sinais sazonais e do desvio-padrão da irregular (s). Percebe-se uma homogeneidade em relação aos valores dos parâmetros ' $k$ ' e ' $b$ '. Nota-se que ' $b$ ' apresenta valores altos, indicando um grau elevado de variação da sazonalidade móvel.

Vale ressaltar que para a identificação dos valores foi utilizado o mesmo procedimento descrito na Subseção 5.2.1.

\begin{tabular}{c|r|r|r|r|r|c}
\hline Série & \multicolumn{1}{c|}{$\boldsymbol{k}$} & \multicolumn{1}{c|}{$\boldsymbol{b}$} & \multicolumn{1}{c|}{$\boldsymbol{A}$} & \multicolumn{1}{c|}{$\boldsymbol{s}$} & \multicolumn{1}{c|}{$\boldsymbol{A} / \boldsymbol{s}$} & Origem \\
\hline Y47 & 44 & $50 \%$ & 2000 & 370 & 5,4 & \multirow{2}{*}{ internacional } \\
\cline { 1 - 6 } Y81 & 48 & $40 \%$ & 1500 & 360 & 4,2 & \\
\cline { 1 - 6 } Y89 & 48 & $40 \%$ & 4000 & 400 & 10,0 & \\
\hline
\end{tabular}

Tabela 5.9 Parâmetros das séries trimestrais aditivas, com sazonalidade móvel (Eq. 5-2)

Os coeficientes estimados da componente de tendência gerada pelo X-13AS, para as séries trimestrais com modo de decomposição aditivo, estão apresentados na Tabela 5.10. De acordo com essa tabela, constata-se que em duas séries o polinômio do $2^{\circ}$ grau se ajusta quase que perfeitamente à componente de tendência.

\begin{tabular}{c|c|r|r|r|r}
\hline Série & $\mathbf{b}_{\mathbf{0}}$ & \multicolumn{1}{c|}{$\mathbf{b}_{\mathbf{1}}$} & \multicolumn{1}{c|}{$\mathbf{b}_{\mathbf{2}}$} & $\mathbf{b}_{\mathbf{3}}$ & $\mathbf{R}^{\mathbf{2}}$ \\
\hline $\mathrm{y} 47$ & $18.003,00$ & 857,51 & 20,9860 & & $99 \%$ \\
\hline $\mathrm{y} 81$ & $48.631,00$ & 532,07 & 26,3780 & $-0,2983$ & $92 \%$ \\
\hline $\mathrm{y} 89$ & $17.834,00$ & $1.339,30$ & 13,9220 & & $98 \%$ \\
\hline
\end{tabular}

Tabela 5.10 Coeficientes da componente de tendência das séries trimestrais aditivas (Eq. 5-8)

Na Subseção 5.2.4, a seguir, serão apresentados os valores dos parâmetros das séries reais trimestrais multiplicativas. 


\subsection{4.}

Séries trimestrais com decomposição multiplicativa: parâmetros ' $A / s$ ', ' $k$ ', ' $b$ ' e coeficientes da componente de tendência

Para as séries trimestrais multiplicativas, os valores dos parâmetros do sinal sazonal e do desvio-padrão da componente irregular (s) estão apresentados na Tabela 5.11. Assim como nos casos anteriores, para a identificação dos valores foi utilizado o mesmo procedimento descrito na Subseção 5.2.1.

\begin{tabular}{|c|c|c|c|c|c|c|}
\hline Série & $\boldsymbol{k}$ & $b$ & $A$ & $s$ & $A / s$ & Origem \\
\hline Y10 & 20 & $25 \%$ & 0,050 & 0,00461 & 10,9 & \multirow{14}{*}{ internaciona } \\
\hline Y11 & 20 & $25 \%$ & 0,060 & 0,00459 & 13,1 & \\
\hline Y20 & 28 & $20 \%$ & 0,060 & 0,00305 & 19,7 & \\
\hline Y25 & 24 & $23 \%$ & 0,025 & 0,00190 & 13,2 & \\
\hline Y37 & 24 & $30 \%$ & 0,032 & 0,00506 & 6,3 & \\
\hline Y73 & 16 & $10 \%$ & 0,045 & 0,00456 & 9,9 & \\
\hline Y74 & 32 & $20 \%$ & 0,042 & 0,00630 & 6,7 & \\
\hline Y77 & 28 & $18 \%$ & 0,038 & 0,00594 & 6,4 & \\
\hline Y84 & 24 & $15 \%$ & 0,036 & 0,00685 & 5,3 & \\
\hline Y91 & 48 & $34 \%$ & 0,036 & 0,00843 & 4,3 & \\
\hline Y98 & 40 & $40 \%$ & 0,013 & 0,00401 & 3,2 & \\
\hline Y99 & 40 & $45 \%$ & 0,030 & 0,00460 & 6,5 & \\
\hline Y112 & 44 & $48 \%$ & 0,012 & 0,00777 & 1,5 & \\
\hline Y120 & 28 & $22 \%$ & 0,050 & 0,00337 & 14,8 & \\
\hline
\end{tabular}

Tabela 5.11 Parâmetros das séries trimestrais multiplicativas, com sazonalidade móvel (Eq. 5-4)

Os histogramas mostram os valores de ' $b$ ' variando entre $10 \%$ e $50 \%$, e mais concentrados entre $20 \%$ e $30 \%$. Já os valores da razão ' $A / s$ ' estão concentrados entre 4 e 7 . E, em relação ao parâmetro ' $k$ ', nota-se uma frequência absoluta alta entre 24 e 32, indicando que as séries levam de 6 a 8 anos para repetir o padrão sazonal.

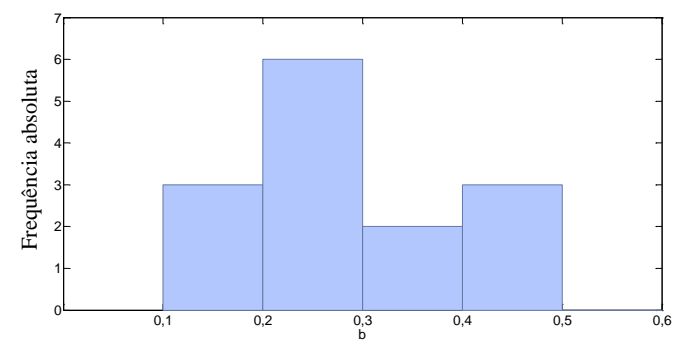

Gráfico 5.9 Histograma de ' $b$ ' (Eq. 5-4) das séries trimestrais multiplicativas

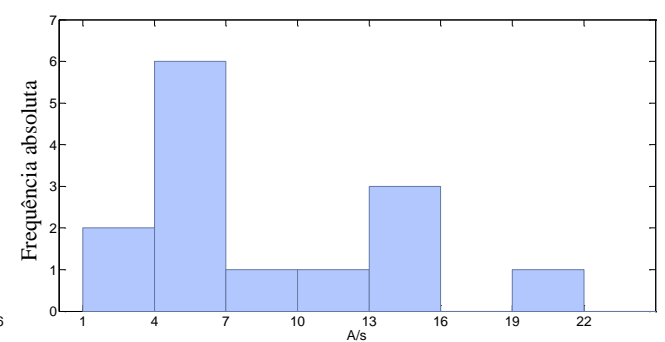

Gráfico 5.10 Histograma de ' $A / s$ ' (Eq. 54) das séries trimestrais multiplicativas 


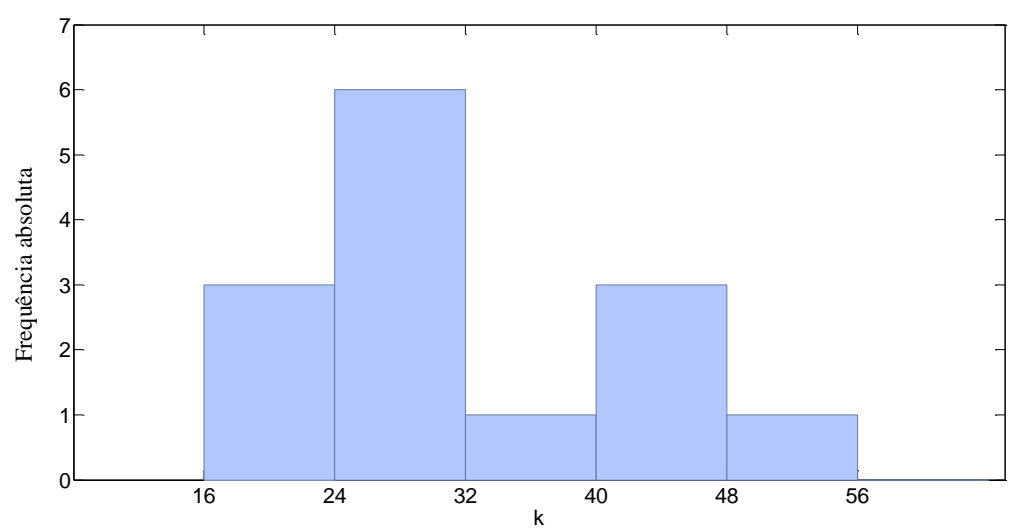

Gráfico 5.11 Histograma de ' $k$ ' (Eq. 5-4) das séries trimestrais multiplicativas

Em relação aos coeficientes estimados da componente de tendência gerada pelo X-13A-S, para as séries trimestrais com decomposição multiplicativa, os valores obtidos a partir do ajuste do polinômio de melhor adequação estão apresentados na Tabela 5.12. Observa-se que na grande maioria das séries foi necessário apenas um polinômio de grau 2 para se ajustar à componente de tendência.

\begin{tabular}{c|r|r|r|r|r|c}
\hline Série & \multicolumn{1}{c|}{$\mathbf{b}_{\mathbf{0}}$} & \multicolumn{1}{c|}{$\mathbf{b}_{\mathbf{1}}$} & \multicolumn{1}{c|}{$\mathbf{b}_{\mathbf{2}}$} & \multicolumn{1}{c}{$\mathbf{b}_{\mathbf{3}}$} & $\mathbf{b}_{\mathbf{4}}$ & $\mathbf{R}^{\mathbf{2}}$ \\
\hline Y10 & $20.936,00$ & $-92,14$ & 35,7710 & $-0,408$ & & $99 \%$ \\
\hline Y11 & $839.026,00$ & $133.447,00$ & $-616,6700$ & & & $99 \%$ \\
\hline Y112 & $1.906,70$ & $-153,40$ & 5,7311 & & & $95 \%$ \\
\hline Y120 & $778.814,00$ & $-17.339,00$ & $2.375,9000$ & $-74,852$ & 0,7308 & $93 \%$ \\
\hline Y20 & $88.897,00$ & $3.338,00$ & 14,3480 & & & $99 \%$ \\
\hline Y25 & $1.000 .000,00$ & $6.859,00$ & 389,6500 & $-4,091$ & & $99,2 \%$ \\
\hline Y37 & $99.058,00$ & $2.772,90$ & 9,0691 & & & $96 \%$ \\
\hline Y73 & $29.975,00$ & 574,30 & 1,4187 & & & $94 \%$ \\
\hline Y74 & $172.291,00$ & $7.755,90$ & $-9,1250$ & & & $96 \%$ \\
\hline Y77 & $5.864,10$ & 221,30 & $-0,3204$ & & & $91 \%$ \\
\hline Y84 & $2.590,30$ & 159,70 & 0,4119 & & & $95 \%$ \\
\hline Y91 & $1.503,80$ & $-23,41$ & 3,8097 & $-0,036$ & & $95 \%$ \\
\hline Y98 & $152.207,00$ & $3.653,60$ & $-302,0700$ & 7,263 & & $99 \%$ \\
\hline Y99 & $131.646,00$ & 457,56 & 19,9080 & & & $98 \%$ \\
\hline
\end{tabular}

Tabela 5.12 Coeficientes da componente de tendência das séries trimestrais multiplicativas (Eq. 5-8) 
Nesta Subseção 5.2 foram apresentados os valores dos parâmetros relacionados às componentes sazonal, de tendência e irregular, das séries nacionais e internacionais. A partir da definição dos valores desses parâmetros, foram geradas as séries simuladas, $Y_{t}$, para serem submetidas ao filtro S-WLS.

Com a finalidade de avaliar o desempenho do filtro S-WLS, o ajuste sazonal realizado por ele será comparado com o ajuste sazonal realizado pelo método X-11. Para essa comparação, serão utilizadas algumas medidas de erro e a razão sinal ruído (SNR) dos filtros. Sendo assim, a SNR dos filtros - proposto e X-11 - será apresentada na Subseção 5.3, a seguir. 


\section{3.}

\section{Razão Sinal Ruído (SNR)}

A razão sinal ruído (SNR) é dada pela relação entre o valor quadrático médio da amplitude do sinal e o valor quadrático médio da amplitude do erro de estimativa. Ou, em outras palavras, a razão entre a potência do sinal e a potência do erro de estimativa, como apresentada abaixo:

$$
S N R=\frac{P_{\text {sinal }}}{P_{\text {erro de estimativa }}}=\left(\frac{A_{\text {sinal }}}{A_{\text {erro de estimativa }}}\right)^{2}
$$

onde $P=$ potência e $A=$ amplitude.

A potência é definida como o valor quadrático médio da amplitude.

Devido à estrutura do filtro proposto, a métrica de desempenho adequada para comparações é a razão sinal ruído. Nesse caso, o filtro será superior a outro, quando a razão sinal ruído dele for maior do que a razão sinal ruído do outro.

A seguir, são definidas a SNR do filtro X-11 e a SNR do filtro proposto.

\subsection{1. \\ SNR do filtro equivalente ao método X-11 para extração da sazonalidade}

Para calcular a razão sinal ruído do filtro X-11, foi calculada a resposta de frequência desse filtro para cada combinação de tamanho do filtro Henderson e tamanho da média móvel sazonal, utilizados pelo programa X-13A-S. Com base nos resultados, foram obtidos a soma dos quadrados dos coeficientes do filtro, e os valores - aqui chamados de $\beta_{1}$ e $\beta_{2}$ (futuramente ilustrados na Figura 5.2) equivalentes à interseção entre a resposta em magnitude do filtro e a largura ideal da banda passante, para determinado nível de sazonalidade móvel.

Os valores utilizados referentes ao tamanho do filtro de Henderson e ao tamanho da média móvel sazonal, para séries mensais, foram:

\begin{tabular}{|c||c|}
\hline Henderson & $\begin{array}{c}\text { Média Móvel } \\
\text { Sazonal }\end{array}$ \\
\hline 9 & $3 \times 3$ \\
\hline 13 & $3 \times 5$ \\
\hline 23 & $3 \times 9$ \\
\hline
\end{tabular}

Quadro 5.1 Tamanho do filtro de Henderson e média móvel sazonal utilizados pelo $\mathrm{X}-11$ para séries mensais 
Com isso, foram realizadas nove combinações no total. Cada combinação apresentou um resultado para a soma dos quadrados dos erros (SQE) (ver Apêndice D), que juntamente com a variância da componente irregular, compõem o erro de estimativa do filtro. O Quadro 5.2 apresenta os valores encontrados para as séries mensais.

\begin{tabular}{|c|c|c|c|c|}
\hline \multirow{2}{*}{ SQE X-11 } & \multicolumn{3}{|c|}{ Média Móvel Sazonal } \\
\cline { 2 - 5 } & $\mathbf{3 x 3}$ & $\mathbf{3 x 5}$ & $\mathbf{3 x 9}$ \\
\hline \multirow{2}{*}{} & $\mathbf{9}$ & 0,199 & 0,144 & 0,090 \\
\cline { 2 - 5 } & $\mathbf{1 3}$ & 0,205 & 0,147 & 0,0906 \\
\cline { 2 - 5 } & $\mathbf{2 3}$ & 0,211 & 0,149 & 0,0912 \\
\hline
\end{tabular}

Quadro 5.2 Valores da soma dos quadrados dos erros do filtro X-11 para extração da sazonalidade de séries mensais

No caso das séries trimestrais, são usados filtro de Henderson de tamanhos 5 ou 7. O tamanho da média móvel sazonal é o mesmo usado para as séries mensais: $3 \times 3,3 \times 5$ ou $3 \times 9$. Sendo assim, existem seis combinações entre filtro de Henderson e filtro de média móvel sazonal.

Para as séries trimestrais, os valores da soma dos quadrados dos erros do filtro X-11, para a extração da sazonalidade, estão expostos no Quadro 5.3:

\begin{tabular}{|c|c|c|c|c|}
\hline \multirow{2}{*}{\multicolumn{2}{|c|}{ SQE X-11 }} & \multicolumn{3}{|c|}{ Média Móvel Sazonal } \\
\hline & & $3 \times 3$ & $3 \times 5$ & $3 \times 9$ \\
\hline \multirow{2}{*}{ 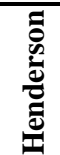 } & 5 & 0,1549 & 0,1145 & 0,0723 \\
\hline & 7 & 0,1631 & 0,1178 & 0,0734 \\
\hline
\end{tabular}

Quadro 5.3 Valores da soma dos quadrados dos erros do filtro X-11 para extração da sazonalidade de séries trimestrais

Nota-se que quanto maior for o tamanho do filtro de Henderson e menor for o tamanho da média móvel sazonal, maior é a SQE. Nota-se também que para os tamanhos da média móvel sazonal 3x5 e 3x9, a SQE sofre pouca alteração com o aumento do tamanho do filtro de Henderson - nas séries mensais e trimestrais. Os valores da SQE são utilizados no cálculo da SNR (Eq. 5-9), assim como o tamanho do filtro $(N)$. 
O tamanho do filtro X-11 é calculado com base no tamanho do filtro de Henderson, na média móvel sazonal, e no tamanho de outras médias móveis que o $\mathrm{X}-11$ utiliza. Intitulou-se 'N' para o tamanho do filtro $\mathrm{X}-11$. O valor de 'N', para cada combinação de tamanho do filtro de Henderson e de média móvel sazonal, está apresentado a seguir, para séries mensais e trimestrais, nos Quadros 5.4 e 5.5 respectivamente. A explicação sobre o cálculo do tamanho do filtro está no Apêndice C.

\begin{tabular}{|c|c|c|c|c|}
\hline & & \multicolumn{3}{|c|}{ Média Móvel Sazonal } \\
\hline & & $3 \times 3$ & $3 \times 5$ & $3 \times 9$ \\
\hline \multirow{3}{*}{ 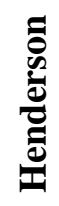 } & 9 & 117 & 141 & 189 \\
\hline & 13 & 121 & 145 & 193 \\
\hline & 23 & 131 & 155 & 203 \\
\hline
\end{tabular}

Quadro 5.4 Valores do tamanho do filtro X-11, para séries mensais

\begin{tabular}{|c|c|c|c|c|}
\hline \multirow{2}{*}{\multicolumn{2}{|c|}{$\mathbf{N}$}} & \multicolumn{3}{|c|}{ Média Móvel Sazonal } \\
\hline & & $3 \times 3$ & $3 \times 5$ & $3 \times 9$ \\
\hline \multirow{2}{*}{ 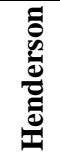 } & 5 & 41 & 49 & 65 \\
\hline & 7 & 43 & 51 & 67 \\
\hline
\end{tabular}

\section{Quadro 5.5 Valores do tamanho do filtro X-11, para séries trimestrais}

A seguir, no Gráfico 5.12, estão apresentadas as respostas em magnitude do filtro X-11 para os três tipos de médias móveis sazonais (MMs), considerando filtro de Henderson de 13 termos, e periodicidade mensal. Em relação à equação que define a resposta em magnitude, ver Subseção 3.2.1.

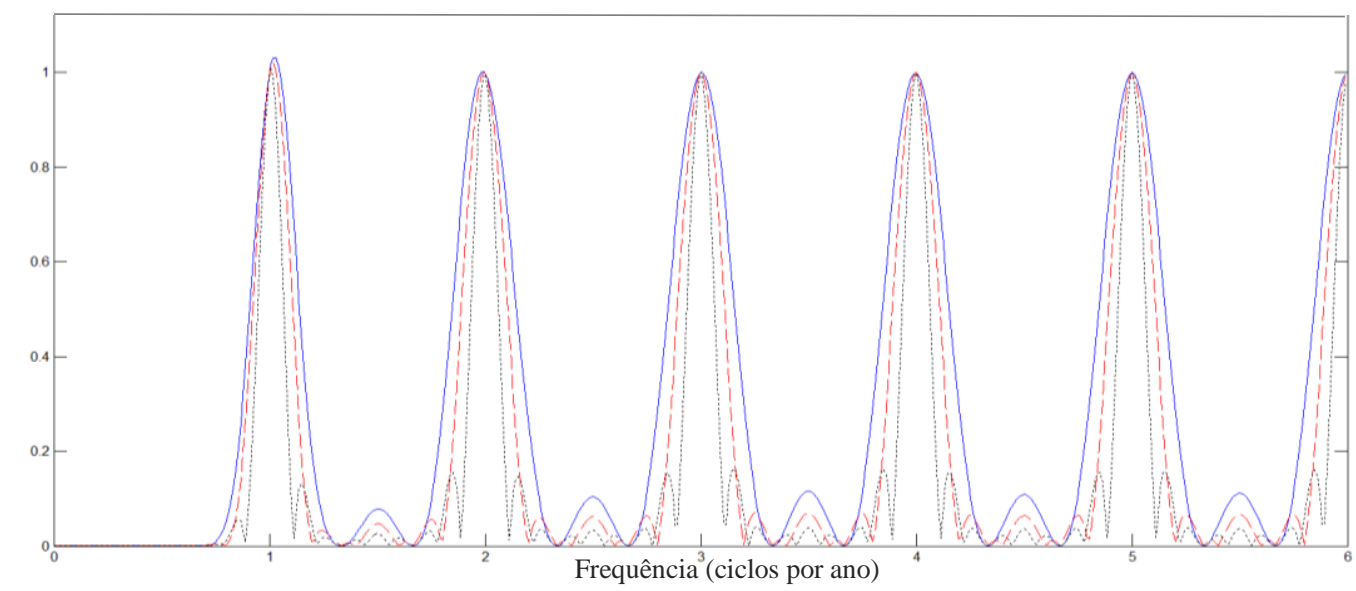

Gráfico 5.12 Respostas em magnitude do filtro X-11 para diferentes MMs: séries mensais. A linha contínua representa o filtro MMs 3x3; a linha tracejada se refere ao filtro MMs 3x5; a linha pontilhada indica o filtro MMs 3x9 
O Gráfico 5.12 ilustra o fato de que quanto menor for o tamanho do filtro de média móvel sazonal, maior será a largura de banda passante, ou seja, mais ela é indicada para sazonalidade móvel.

Como o grau de sazonalidade móvel é representado pela largura da banda passante, para calcular a SNR do filtro é necessário encontrar o valor da resposta em frequência, do filtro, para um grau de sazonalidade móvel determinado.

A Figura 5.2, a seguir, ilustra os valores da resposta em frequência do filtro, para um dado grau de sazonalidade móvel. Chama-se de $(1-\beta)$ o desvio da resposta da banda passante do filtro em relação à resposta ideal (no caso igual a 1). Nessa figura, a linha tracejada representa o espectro do filtro equivalente ao X-11, e a linha contínua representa a magnitude do espectro do filtro ideal para um determinado grau de sazonalidade móvel, dado pelo valor de $\alpha$ :

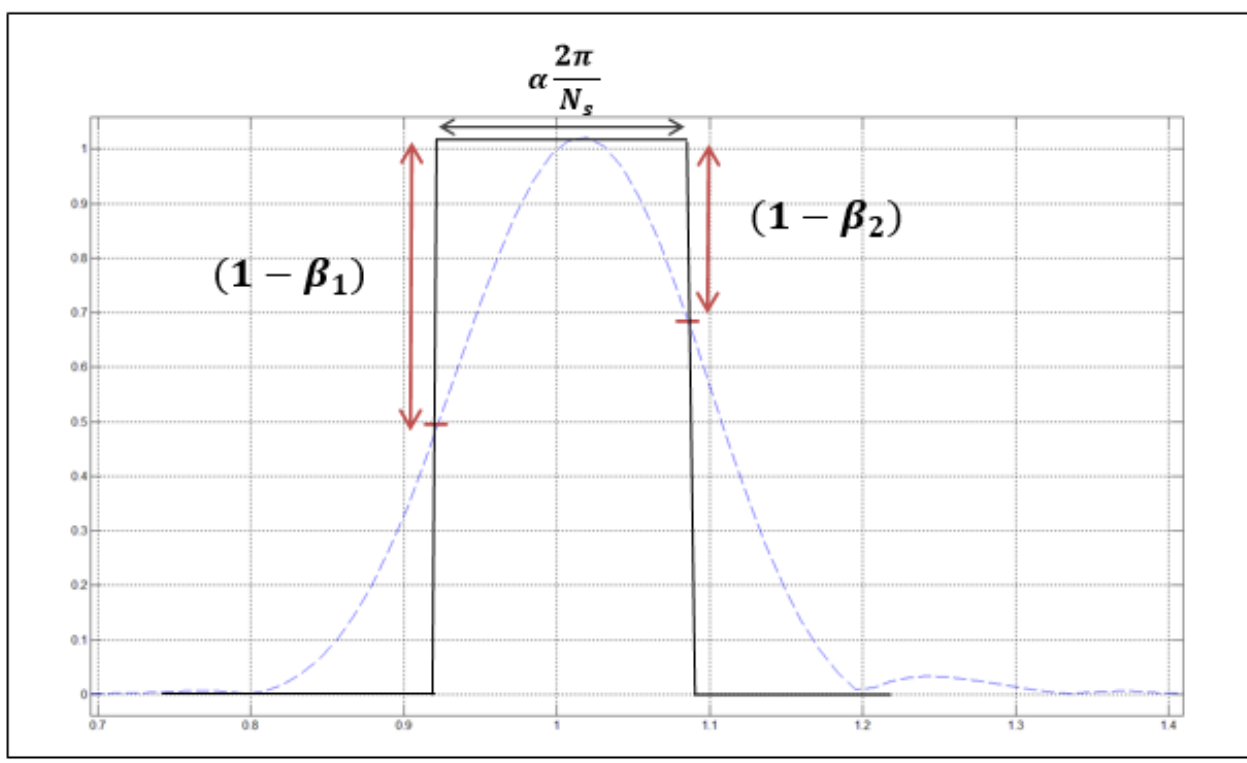

Figura 5.2 Definição dos valores de $\beta_{1}$ e $\beta_{2}$ do filtro $X-11$

O espectro possui a banda de transição (don't care band), a banda de rejeição (stopband) e a banda passante (passband). O ideal é que a resposta em frequência da banda de rejeição seja zero; e a da banda passante seja um. Como a largura da banda passante ideal está definida com base no valor de ' $k$ ' (ver Figura 5.1), calculam-se os $\beta_{s}$ para cada ' $k$ ', e nota-se que quanto mais acentuada for a sazonalidade móvel (ou seja, valores baixos de ' $k$ '), mais longe da ideal será a resposta do filtro $\mathrm{X}-11$. 
A seguir são apresentados os valores de $\beta$ para diferentes valores de ' $k$ ', de um mesmo filtro $\mathrm{X}-11$, para as séries mensais. Os valores de $\beta$ dependem da largura da banda passante, assim como do tamanho dos filtros de Henderson e de média móvel sazonal. Sendo assim, o Quadro 5.6 apresenta os valores de $\beta$ para o filtro de Henderson de tamanho 9; o Quadro 5.7 apresenta os valores de $\beta$ para o filtro de Henderson de tamanho 13; e o Quadro 5.8, para o Henderson de tamanho 23.

\begin{tabular}{|c|c|c|c|c|c|c|}
\hline \multirow{3}{*}{$\boldsymbol{k}$} & \multicolumn{6}{|c|}{ Henderson 9} \\
\hline & \multicolumn{2}{|c|}{ MMs $3 \times 3$} & \multicolumn{2}{|c|}{ MMs $3 \times 5$} & \multicolumn{2}{|c|}{ MMs $3 \times 9$} \\
\hline & $\beta_{1}$ & $\boldsymbol{\beta}_{2}$ & $\beta_{1}$ & $\boldsymbol{\beta}_{2}$ & $\boldsymbol{\beta}_{1}$ & $\boldsymbol{\beta}_{2}$ \\
\hline 60 & 0,047 & 0,1447 & 0,0029 & 0,0082 & 0,0109 & 0,0289 \\
\hline 72 & 0,1262 & 0,3098 & 0,0434 & 0,0958 & 0,0411 & 0,1020 \\
\hline 84 & 0,2137 & 0,4563 & 0,11 & 0,22 & 0,0501 & 0,1183 \\
\hline 96 & 0,27 & 0,58 & 0,16 & 0,35 & 0,04 & 0,07 \\
\hline 120 & 0,40 & 0,78 & 0,29 & 0,59 & 0,04 & 0,14 \\
\hline 144 & 0,55 & 0,85 & 0,46 & 0,69 & 0,21 & 0,28 \\
\hline 168 & 0,63 & 0,91 & 0,55 & 0,79 & 0,33 & 0,43 \\
\hline 192 & 0,67 & 0,96 & 0,60 & 0,87 & 0,40 & 0,59 \\
\hline 216 & 0,71 & 0,96 & 0,65 & 0,87 & 0,47 & 0,59 \\
\hline 240 & 0,78 & 1 & 0,74 & 0,94 & 0,61 & 0,74 \\
\hline 264 & 0,78 & 1 & 0,74 & 0,94 & 0,61 & 0,74 \\
\hline 288 & 0,78 & 1,03 & 0,74 & 0,99 & 0,61 & 0,86 \\
\hline 300 a 450 & 0,85 & 1,03 & 0,83 & 0,99 & 0,74 & 0,86 \\
\hline$\geq 450$ & 0,92 & 1,03 & 0,90 & 1,02 & 0,86 & 0,95 \\
\hline
\end{tabular}

Quadro 5.6 Valores de $\beta$ quando o tamanho do filtro de Henderson = 9, para séries mensais

\begin{tabular}{|c|c|c|c|c|c|c|}
\hline \multirow{3}{*}{$\boldsymbol{k}$} & \multicolumn{6}{|c|}{ Henderson 13} \\
\hline & \multicolumn{2}{|c|}{ MMs $3 \times 3$} & \multicolumn{2}{|c|}{ MMs $3 \times 5$} & \multicolumn{2}{|c|}{ MMs $3 \times 9$} \\
\hline & $\beta_{1}$ & $\boldsymbol{\beta}_{2}$ & $\beta_{1}$ & $\boldsymbol{\beta}_{2}$ & $\beta_{1}$ & $\boldsymbol{\beta}_{2}$ \\
\hline 60 & 0,0570 & 0,1821 & 0,0035 & 0,0104 & 0,0131 & 0,0362 \\
\hline 72 & 0,1418 & 0,3461 & 0,0487 & 0,1068 & 0,0462 & 0,1139 \\
\hline 84 & 0,2324 & 0,4860 & 0,1195 & 0,2360 & 0,0546 & 0,1262 \\
\hline 96 & 0,29 & 0,60 & 0,17 & 0,36 & 0,04 & 0,07 \\
\hline 120 & 0,42 & 0,79 & 0,30 & 0,60 & 0,04 & 0,14 \\
\hline 144 & 0,57 & 0,86 & 0,48 & 0,70 & 0,22 & 0,28 \\
\hline 168 & 0,65 & 0,91 & 0,57 & 0,79 & 0,34 & 0,43 \\
\hline 192 & 0,69 & 0,96 & 0,62 & 0,87 & 0,41 & 0,59 \\
\hline 216 & 0,72 & 0,96 & 0,66 & 0,87 & 0,47 & 0,59 \\
\hline 240 & 0,80 & 1 & 0,75 & 0,94 & 0,62 & 0,74 \\
\hline 264 & 0,80 & 1 & 0,75 & 0,94 & 0,62 & 0,74 \\
\hline 288 & 0,80 & 1,02 & 0,75 & 0,99 & 0,62 & 0,86 \\
\hline 300 a 450 & 0,86 & 1,02 & 0,84 & 0,99 & 0,75 & 0,90 \\
\hline$\geq 450$ & 0,92 & 1,03 & 0,91 & 1,01 & 0,87 & 0,95 \\
\hline
\end{tabular}

Quadro 5.7 Valores de $\beta$ quando o tamanho do filtro de Henderson = 13, para séries mensais 


\begin{tabular}{|c|c|c|c|c|c|c|}
\hline \multirow{3}{*}{$\boldsymbol{k}$} & \multicolumn{6}{|c|}{ Henderson 23} \\
\hline & \multicolumn{2}{|c|}{ MMs $3 \times 3$} & \multicolumn{2}{|c|}{ MMs $3 \times 5$} & \multicolumn{2}{|c|}{ MMs $3 \times 9$} \\
\hline & $\beta_{1}$ & $\boldsymbol{\beta}_{2}$ & $\beta_{1}$ & $\boldsymbol{\beta}_{2}$ & $\beta_{1}$ & $\boldsymbol{\beta}_{2}$ \\
\hline 60 & 0,1163 & 0,3066 & 0,007 & 0,0176 & 0,0264 & 0,0607 \\
\hline 72 & 0,2325 & 0,4750 & 0,0791 & 0,1460 & 0,0768 & 0,1563 \\
\hline 84 & 0,3384 & 0,5960 & 0,1735 & 0,2887 & 0,0799 & 0,1553 \\
\hline 96 & 0,40 & 0,69 & 0,24 & 0,41 & 0,08 & 0,07 \\
\hline 120 & 0,53 & 0,83 & 0,38 & 0,63 & 0,05 & 0,15 \\
\hline 144 & 0,67 & 0,88 & 0,56 & 0,72 & 0,25 & 0,29 \\
\hline 168 & 0,73 & 0,92 & 0,64 & 0,80 & 0,38 & 0,44 \\
\hline 192 & 0,76 & 0,96 & 0,69 & 0,87 & 0,45 & 0,59 \\
\hline 216 & 0,80 & 0,96 & 0,73 & 0,87 & 0,52 & 0,59 \\
\hline 240 & 0,85 & 0,99 & 0,81 & 0,92 & 0,66 & 0,73 \\
\hline 264 & 0,85 & 0,99 & 0,81 & 0,92 & 0,66 & 0,73 \\
\hline 288 & 0,85 & 1,01 & 0,81 & 0,97 & 0,66 & 0,85 \\
\hline 312 a 384 & 0,90 & 1,01 & 0,88 & 0,98 & 0,79 & 0,85 \\
\hline 408 a 432 & 0,90 & 1,02 & 0,88 & 1 & 0,79 & 0,94 \\
\hline$\geq 450$ & 0,95 & 1,02 & 0,93 & 1 & 0,89 & 0,94 \\
\hline
\end{tabular}

Quadro 5.8 Valores de $\boldsymbol{\beta}$ quando o tamanho do filtro de Henderson = 23, para séries mensais

Em se tratando das séries com periodicidade trimestral, os valores de $\beta_{1}$ e $\beta_{2}$, para o filtro de Henderson de tamanhos 5 e 7, estão apresentados nos Quadros 5.9 e 5.10, respectivamente.

\begin{tabular}{|c|c|c|c|c|c|c|}
\hline \multirow{3}{*}{$\boldsymbol{k}$} & \multicolumn{6}{|c|}{ Henderson 5} \\
\hline & \multicolumn{2}{|c|}{ MMs $3 \times 3$} & \multicolumn{2}{|c|}{ MMs $3 \times 5$} & \multicolumn{2}{|c|}{ MMs $3 \times 9$} \\
\hline & $\boldsymbol{\beta}_{1}$ & $\boldsymbol{\beta}_{2}$ & $\boldsymbol{\beta}_{1}$ & $\boldsymbol{\beta}_{2}$ & $\boldsymbol{\beta}_{1}$ & $\boldsymbol{\beta}_{2}$ \\
\hline 16 & 0,014 & 0,078 & 0,008 & 0,045 & 0,004 & 0,025 \\
\hline 20 & 0,068 & 0,215 & 0,004 & 0,012 & 0,014 & 0,043 \\
\hline 24 & 0,160 & 0,370 & 0,050 & 0,114 & 0,053 & 0,121 \\
\hline 28 & 0,255 & 0,496 & 0,124 & 0,241 & 0,066 & 0,128 \\
\hline 32 & 0,344 & 0,599 & 0,208 & 0,361 & 0,043 & 0,077 \\
\hline 36 & 0,422 & 0,680 & 0,288 & 0,464 & 0,027 & 0,043 \\
\hline 40 & 0,493 & 0,747 & 0,367 & 0,556 & 0,069 & 0,103 \\
\hline 44 & 0,542 & 0,791 & 0,424 & 0,618 & 0,127 & 0,184 \\
\hline 48 & 0,592 & 0,832 & 0,484 & 0,680 & 0,196 & 0,273 \\
\hline 52 & 0,642 & 0,870 & 0,545 & 0,739 & 0,274 & 0,370 \\
\hline
\end{tabular}

Quadro 5.9 Valores de $\beta$ quando o tamanho do filtro de Henderson = 5, para séries trimestrais 


\begin{tabular}{|c|c|c|c|c|c|c|}
\hline \multirow{3}{*}{$\boldsymbol{k}$} & \multicolumn{6}{|c|}{ Henderson 7} \\
\hline & \multicolumn{2}{|c|}{ MMs $3 \times 3$} & \multicolumn{2}{|c|}{ MMs $3 \times 5$} & \multicolumn{2}{|c|}{ MMs $3 \times 9$} \\
\hline & $\beta_{1}$ & $\beta_{2}$ & $\beta_{1}$ & $\beta_{2}$ & $\beta_{1}$ & $\beta_{2}$ \\
\hline 16 & 0,032 & 0,126 & 0,018 & 0,073 & 0,010 & 0,040 \\
\hline 20 & 0,117 & 0,309 & 0,007 & 0,018 & 0,024 & 0,061 \\
\hline 24 & 0,236 & 0,473 & 0,073 & 0,146 & 0,077 & 0,155 \\
\hline 28 & 0,343 & 0,590 & 0,167 & 0,286 & 0,088 & 0,153 \\
\hline 32 & 0,436 & 0,677 & 0,263 & 0,407 & 0,055 & 0,087 \\
\hline 36 & 0,513 & 0,743 & 0,350 & 0,506 & 0,033 & 0,047 \\
\hline 40 & 0,581 & 0,796 & 0,433 & 0,592 & 0,081 & 0,109 \\
\hline 44 & 0,626 & 0,830 & 0,490 & 0,649 & 0,146 & 0,192 \\
\hline 48 & 0,671 & 0,862 & 0,549 & 0,704 & 0,221 & 0,282 \\
\hline 52 & 0,715 & 0,891 & 0,607 & 0,756 & 0,305 & 0,378 \\
\hline
\end{tabular}

\section{Quadro 5.10 Valores de $\beta$ quando o tamanho do filtro de Henderson = 7, para séries trimestrais}

Sendo assim, observando os Quadros 5.6 a 5.10, percebe-se que os valores dos $\beta_{s}$ dependem da largura da banda, enquanto que a SQE depende apenas do tamanho do filtro de Henderson e do filtro de média móvel sazonal (MMs) (ver Quadros 5.2 e 5.3).

Após calcular SQE $\beta_{1}$ e $\beta_{2}$, pode-se calcular a razão sinal ruído do filtro X11 para diversas combinações de ' $A$ ', ' $b$ ' e ' $k$ ' (descritos na Eq. 5-1). A SNR do filtro X-11 é assim definida:

$$
\begin{aligned}
S N R_{X-11}= & \frac{\frac{A^{2}}{2}+\frac{A^{2} b^{2}}{4}}{\frac{A^{2} b^{2}}{4} \frac{\left[\left(1-\beta_{1}\right)^{2}+\left(1-\beta_{2}\right)^{2}\right]}{2}+S N_{0}} \\
= & \frac{\frac{A^{2}}{2}\left(1+\frac{b^{2}}{2}\right)}{\frac{A^{2} b^{2}}{8}\left[\left(1-\beta_{1}\right)^{2}+\left(1-\beta_{2}\right)^{2}\right]+S N_{0}}
\end{aligned}
$$

Onde:

$\mathrm{S}=$ Soma dos Quadrados dos coeficientes do filtro X-11 (SQE)

$N_{0}=$ variância da componente irregular $\left(\sigma_{\varepsilon}^{2}\right)$. 


\subsection{2.}

\section{SNR do filtro proposto para extração da sazonalidade}

O cálculo da SNR do filtro proposto faz uso do somatório dos coeficientes do filtro ao quadrado, e dos valores referentes à resposta em frequência na banda passante. Tais valores serão chamados de gama zero $\left(\gamma_{0}\right)$, quando se referir à resposta na frequência equivalente a $1 / 12$, e gama $1\left(\gamma_{1}\right)$, que será a resposta de frequência que mais se desviar do valor 1 , no intervalo equivalente à largura de banda. Os valores de $\gamma_{0}$ e $\gamma_{1}$, na resposta em magnitude do filtro proposto, estão ilustrados na Figura 5.3.

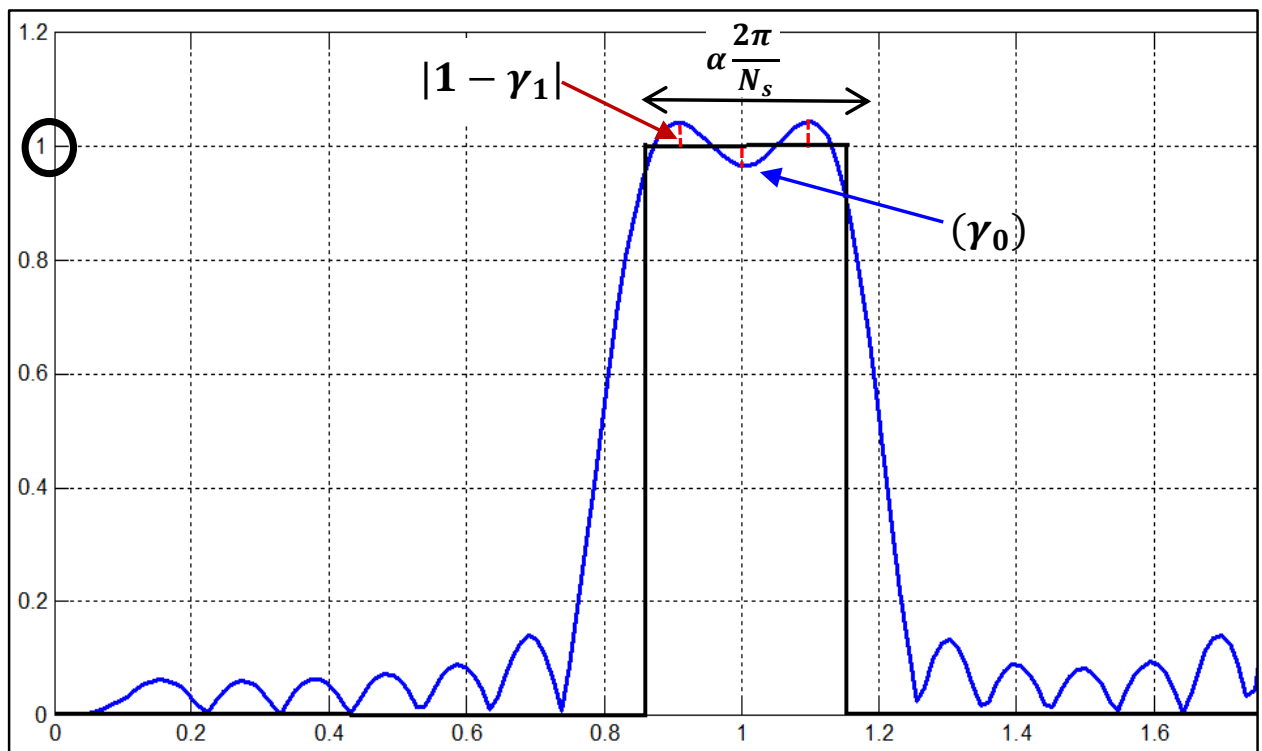

Figura 5.3 Definição dos valores de $\gamma_{0}$ e $\gamma_{1}$ do filtro proposto

Os parâmetros necessários para o projeto do filtro S-WLS, em uma série mensal, são: alpha $(\alpha)$, delta $(\delta)$ e peso $\left(\mathrm{w}_{\mathrm{o}}\right)$, além do tamanho do filtro $(N)$ e do fator de superamostragem (definidos na Subseção 4.2). Para obter os valores de $\gamma_{0}$, $\gamma_{1}$, e da soma dos quadrados dos coeficientes do filtro $(S Q)$, a magnitude da resposta em magnitude do filtro S-WLS foi calculada para diversas combinações desses parâmetros. Vale lembrar que o parâmetro $\alpha$ guarda uma relação com o valor $k$. Essa relação será mostrada na próxima subseção.

Para cada combinação de $\alpha, \delta, \mathrm{w}_{\mathrm{o}}$ e $N$, existem $S Q$ diferentes e $\gamma_{S}$ diferentes. O Gráfico 5.13, a seguir, mostra a relação entre o peso $\left(\mathrm{w}_{\mathrm{o}}\right)$ e: a $S Q$, o $\gamma_{0}$, e o valor absoluto de $\left(1-\gamma_{1}\right)$; considerando $N=145, k=72, \alpha=\frac{1}{3}$ e $\delta=\frac{1}{30}$ de uma série mensal. Vale lembrar que neste projeto será utilizado o fator de superamostragem (M) igual a 401, tanto para o filtro mensal quanto para o trimestral. 


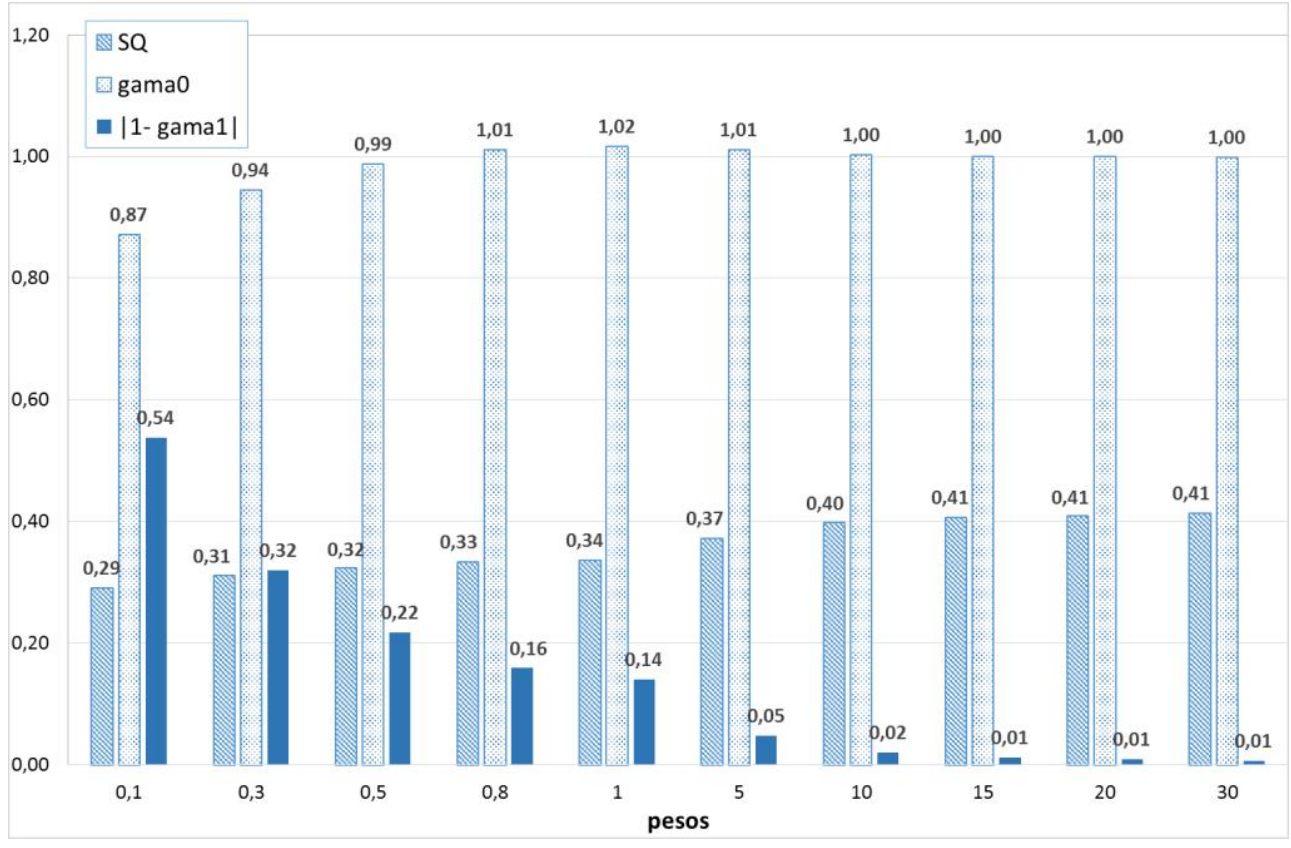

Gráfico 5.13 Valores de SQ, $\gamma_{0}$ e $\left|1-\gamma_{1}\right|$ para diversos pesos $\left(w_{0}\right)$

Nota-se que à medida que o peso $\left(\mathrm{w}_{\mathrm{o}}\right)$ aumenta, o $\gamma_{0}$ se aproxima do valor 1 , e o $\left(1-\gamma_{1}\right)$ se aproxima de zero, indicando uma melhor adequação da banda passante. Por outro lado, a $S Q$ cresce com o aumento do peso. Sendo assim, é necessário encontrar um equilíbrio para a obtenção de um filtro com resultados satisfatórios.

Quanto ao $\gamma_{1}$, ele pode ser maior ou menor do que 1 , sendo que o valor 1 é o valor ideal. Para os cálculos, o que é considerado é o valor absoluto da diferença entre o $\gamma_{1}$ e o valor 1 .

A relação entre os valores de $\gamma_{0}, \gamma_{1}$ e $S Q$ é analisada na SNR do filtro proposto, que é dada por:

$$
S N R_{\text {Filtro Proposto }}=\frac{\frac{A^{2}}{2}+\frac{A^{2} b^{2}}{4}}{N_{0} S Q+\left(1-\gamma_{0}\right)^{2} \frac{A^{2}}{2}+\left(1-\gamma_{1}\right)^{2} \frac{A^{2} b^{2}}{4}}
$$

onde:

$\mathrm{N}_{0}$ = variância da componente irregular

$S Q=$ soma dos quadrados dos coeficientes do filtro

$\left(S Q \cdot \mathrm{N}_{0}+\left(1-\gamma_{0}\right)^{2} \frac{A^{2}}{2}+\left(\gamma_{1}-1\right)^{2} \frac{A^{2} b^{2}}{4}\right)$ é a potência (completa) do ruído do filtro proposto.

Vale mencionar que essa SNR é válida para os dois filtros propostos nesta tese: o S-WLS e o S-WLSC (a ser apresentado no Capítulo 6). 


\subsubsection{1.}

\section{Cálculo de $\alpha$ com base em $k$}

Define-se a largura da banda passante como $\Delta \mathrm{f}$. E,

$$
\Delta \mathrm{f}=\alpha \text {. frequência fundamental }
$$

Em uma série temporal mensal, a frequência fundamental é igual a 1/12. Logo,

$$
\Delta f=\alpha \frac{1}{12}
$$

Como as raias espectrais têm que estar dentro da banda passante,

$$
\alpha \geq 2\left[\frac{1}{k} / \frac{1}{12}\right]
$$

onde $k=$ número de meses com que o padrão sazonal volta a se repetir, no sinal artificial (Eq. 5-1).

Com isso, $\alpha \geq \frac{24}{k}$.

Essa relação entre $k$ e $\alpha$ pode ser melhor entendida na Figura 5.4, a seguir:

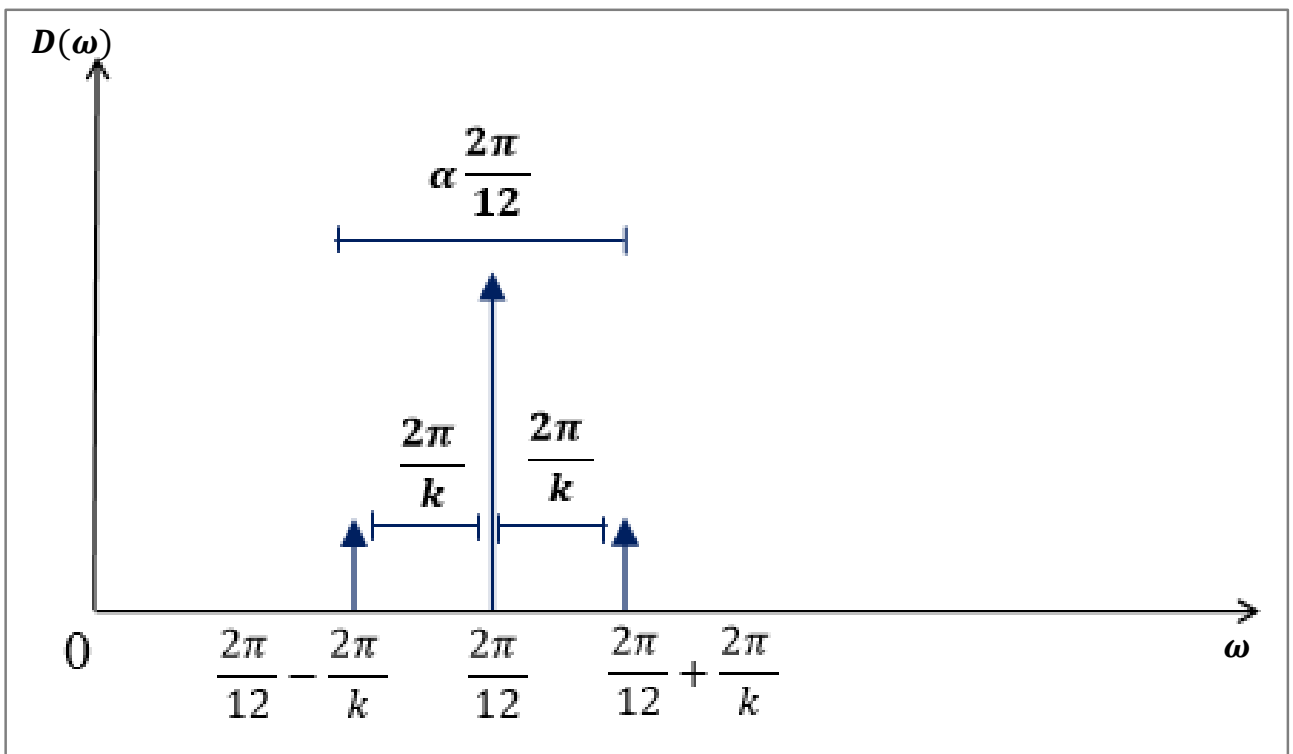

Figura 5.4 Espectro, na frequência, representando a relação entre $k$ e $\alpha$, para séries com periodicidade mensal 
Para as séries temporais trimestrais, a frequência fundamental é igual a 1/4. Sendo assim,

$$
\Delta f=\alpha \frac{1}{4}
$$

Como as raias espectrais têm que estar dentro da banda passante,

$$
\alpha \geq 2\left[\frac{1}{k} / \frac{1}{4}\right]
$$

onde $k=$ número de trimestres com que o padrão sazonal volta a se repetir.

Com isso, $\alpha \geq \frac{8}{k}$, para as séries trimestrais. 


\subsection{3.}

\section{Razão entre a SNR do filtro proposto e a SNR do filtro X-11}

A comparação entre a SNR do filtro proposto e a SNR do filtro X-11 indica quando o primeiro supera o segundo. Isso ocorre quando $\frac{S_{N R_{F} \text { Proposto }}}{S_{N} R_{X-11}}>1$. Essa razão entre as SNRs é válida para ambos os filtros propostos: S-WLS e S-WLSC (a ser apresentado no Capítulo 6).

$$
\begin{aligned}
& \frac{S N R_{F_{-} \text {Proposto }}}{S N R_{X-11}}= \\
& =\frac{\frac{A^{2}}{2}+\frac{A^{2} b^{2}}{4}}{N_{0} S Q+\left(1-\gamma_{0}\right)^{2} \frac{A^{2}}{2}+\left(1-\gamma_{1}\right)^{2} \frac{A^{2} b^{2}}{4} /} / \frac{\frac{A^{2}}{2}+\frac{A^{2} b^{2}}{4}}{\frac{A^{2} b^{2}}{8}\left[\left(1-\beta_{1}\right)^{2}+\left(1-\beta_{2}\right)^{2}\right]+S N_{0}} \\
& \frac{S N R_{F_{-} \text {Proposto }}}{S N R_{X-11}}=\frac{\frac{A^{2} b^{2}}{8}\left[\left(1-\beta_{1}\right)^{2}+\left(1-\beta_{2}\right)^{2}\right]+S N_{0}}{N_{0} S Q+\left(1-\gamma_{0}\right)^{2} \frac{A^{2}}{2}+\left(1-\gamma_{1}\right)^{2} \frac{A^{2} b^{2}}{4}}
\end{aligned}
$$

Onde:

$\gamma_{0}$ e $\gamma_{1}=$ valores associados ao espectro de potência do filtro proposto

$\beta_{1} e \beta_{2}=$ valores associados ao espectro de potência do filtro X-11

$S Q=$ Soma dos Quadrados dos coeficientes do filtro proposto

$S=$ Soma dos Quadrados dos coeficientes do filtro X-11

$N_{0}=$ variância da componente irregular $\left(\sigma^{2}\right)$

$b=$ taxa de variação da sazonalidade móvel

$A=$ amplitude do sinal

Em se tratando do filtro S-WLS, o desafio é encontrar os parâmetros desse filtro que fazem com que o resultado da razão, entre a SNR dele e a SNR do filtro $\mathrm{X}-11$, seja a maior possível. Para isso, foram testadas várias combinações dos parâmetros, a fim de identificar qual dessas combinações deixa o filtro S-WLS superior ao X-11. Esse procedimento foi realizado no software MATLAB, onde o filtro foi desenvolvido (ver Apêndice K). A Subseção 5.4, a seguir, apresenta o procedimento utilizado na escolha dos parâmetros do filtro S-WLS, assim como o filtro escolhido para as séries mensais e para as séries trimestrais. 


\section{4 . Seleção da melhor configuração do filtro S-WLS}

Após a obtenção dos valores de ' $A / s$ ', ' $b$ ' e ' $k$ ', com base nas séries reais (apresentadas na Subseção 5.2), buscou-se qual a combinação, entre $\delta$ e peso $\left(\mathrm{w}_{\mathrm{o}}\right)$, tornava o filtro S-WLS mais competitivo frente ao X-11. Em relação ao parâmetro $\alpha$, relacionado à largura da banda passante, foi analisado o comportamento sazonal das séries reais, mensais e trimestrais (ver Subseções 5.2.1 a 5.2.4). Foi constatado que a maioria das séries reais mensais com sazonalidade móvel apresentou valor de $k>72$; e as trimestrais apresentaram $k>24$. Dado que $k$ guarda uma relação com $\alpha$, como apresentado nas Equações (5-13) e (5-15), definiu-se o valor $\alpha=\frac{1}{3}$ a ser utilizado no filtro.

O procedimento utilizado para a seleção dos valores dos parâmetros do filtro proposto (S-WLS), mensal e trimestral, pode ser resumido em três etapas, da seguinte forma:

(1) Construção da base de dados

(1.a) Filtro sazonal-WLS (S-WLS)

(1.b) Filtro X-11

(2) $1^{\text {a }}$ seleção do melhor X-11 e filtro S-WLS - com base na SNR

A partir dos sinais sazonais típicos, obteve-se o melhor X-11, e em seguida, o melhor S-WLS

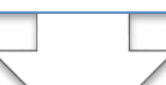

(3) $2^{\mathrm{a}}$ seleção do melhor X-11 e filtro S-WLS - com base na MSE

(3.a) $k$ aleatorizado entre dois valores típicos (3.b) Identificação do $X-11$ com menor MSE (3.c) Com base em (3.b), identificação do S-WLS com menor MSE

Figura 5.5 Etapas do procedimento utilizado para a obtenção dos parâmetros do filtro S-WLS

A seguir é apresentada a explicação das etapas presentes na Figura 5.5: 


\section{Etapa (1)}

(1a) A magnitude da resposta em frequência do filtro S-WLS foi calculada para um conjunto de valores dos parâmetros $\alpha, \delta, \mathrm{w}_{o}$ e $N$. Esses passos estão detalhados nas Subseções 5.4.1 e 5.4.2, para as séries mensais e trimestrais, respectivamente.

(1b) Além disso, foi calculada a magnitude da resposta em frequência de um filtro equivalente ao $\mathrm{X}-11$, a partir da sua resposta ao impulso. Isso foi feito para cada tamanho do filtro de Henderson e de média móvel sazonal (MMs) usada pelo X13A-S. Como resultado, encontrou-se o valor de $S Q, \beta_{1}$ e $\beta_{2}$ (apresentados na Subseção 5.3.1), necessários para o cálculo da SNR.

\section{Etapa (2)}

Foi criada uma planilha onde era possível atribuir valores para ' $A$ ', ' $s$ ', ' $b$ ' e ' $k$ ', assim como para os coeficientes de tendência ' $b_{0}$ ', ' $b_{1}$ ', ' $b_{2}$ ' e ' $b_{3}$ '. Esses valores foram baseados nos parâmetros das séries reais, obtidos na Subseção 5.2.

A base de dados gerada em (1a) e (1b) foi incorporada nesse arquivo e, para cada conjunto de valores de ' $A$ ', ' $s$ ', ' $b$ ' e ' $k$ ', foi identificada a melhor configuração do filtro X-11 (em termos de filtro de Henderson e MMs), ou seja, a configuração que gerou a maior SNR do X-11. Com isso, encontrou-se o tamanho do filtro: ' $N$ '.

Após ter definido o tamanho do filtro, buscou-se a configuração do filtro S-WLS com a maior SNR. Porém, como foram testados vários valores de ' $A$ ', ' $s$ ', ' $b$ ' e ' $k$ ', mais de uma combinação de parâmetros do filtro foi obtida. Ou seja, não houve uma única combinação que fosse a melhor em todas as situações. Com isso, passouse para a Etapa (3), usando as melhores combinação de parâmetros obtidas nesta Etapa (2).

\section{Etapa (3)}

Na Etapa (2) foram identificados os melhores conjuntos de parâmetros do filtro SWLS, mas era necessário escolher apenas um. Sendo assim, foi gerada uma série com sazonalidade móvel e tendência, seguindo um polinômio de $3^{\mathrm{a}}$ ordem. Em seguida, foi identificada qual configuração do filtro X-11 gerava o menor MSE para a estimativa da componente sazonal dessa série. A partir de então, usando o tamanho do filtro definido pelo X-11, foram gerados 100 valores aleatórios para o 
' $k$ ', seguindo uma distribuição uniforme entre [72 e 120] para as séries mensais, e [24 e 40] para as séries trimestrais. Todas as melhores configurações do filtro SWLS encontradas na Etapa (2) foram testadas, e tiveram o MSE comparado com o MSE do X-11. A configuração que obteve o melhor resultado (menor MSE), em comparação do com o X-11, foi a selecionada.

A seguir, nas Subseções 5.4.1 e 5.4.2, é apresentada a escolha do filtro SWLS para as séries mensais e trimestrais, respectivamente.

\subsection{1. Definição do filtro S-WLS para as séries mensais}

Com o objetivo de encontrar a combinação de parâmetros do filtro proposto (S-WLS) que gerasse SNR superior à SNR do filtro X-11, procedeu-se às etapas descritas na Figura 5.5.

$\mathrm{Na}$ Etapa (1.a), foram rodadas 8.748 possibilidades de variação nos parâmetros do filtro. Os seguintes valores foram utilizados:

- Denominador de $\boldsymbol{\alpha}:[2,3,4,5,6,7,8,9,10]$;

- Denominador de $\boldsymbol{\delta}$ : $[4,5,10,15,20,30,40,50,60]$;

- Peso da banda passante $\left(\mathbf{w}_{\mathbf{0}}\right)$ :[0.02 0.05 0.1 0.3 0.5 0.815101520 25];

- Tamanho do filtro $(\boldsymbol{N})$ : [117, 121, 131, 141, 145, 155, 189, 193, 203];

Ao se calcular a resposta em magnitude do filtro, também se calculou o valor da $S Q$ (soma dos quadrados dos coeficientes), do $\gamma_{0}$, que representa a resposta ao impulso na frequência $\frac{1}{12}$, e do valor absoluto da diferença máxima entre os pontos da banda passante e o valor 1 , que será chamado de $\left|1-\gamma_{1}\right|$. Tais valores permitem a realização do cálculo da SNR do filtro S-WLS, como apresentado na Equação (5$11)$.

Também foram obtidos os valores de $\beta_{1}, \beta_{2}$ e da soma dos quadrados dos coeficientes do filtro equivalente ao método $\mathrm{X}-11$, necessários para o cálculo da SNR do mesmo (Equação 5-10).

Na Etapa (2), foi obtido o valor da maior SNR do X-11 para as combinações de valores testados dos parâmetros ' $A$ ', ' $s$ ', ' $b$ ' e ' $k$ ', definidos na Equação (5-1). Também foi obtida a SNR do filtro S-WLS. Essa etapa está ilustrada na Figura 5.6. 


\begin{tabular}{|c|c|c|c|c|c|c|c|c|c|c|}
\hline$\Delta$ & A & B & C & D & $E$ & $\mathrm{~F}$ & $\mathrm{H}$ & I & $J$ & K \\
\hline 1 & A & b & s & & & & & & & \\
\hline 2 & 6 & $40 \%$ & 1 & & & & & & & \\
\hline 3 & k & $\mathbf{N}$ & Henderson & MMs & SNR $X-11$ & \begin{tabular}{|c|} 
SNR \\
Proposto
\end{tabular} & Dalpha & D_delta & Peso & \begin{tabular}{|c|} 
RAZKO \\
das SNRs
\end{tabular} \\
\hline 4 & 48 & 121 & 13 & 3 & 13,5 & 36,6 & 2,0 & 4 & 0,02 & 2,704 \\
\hline 5 & 60 & 121 & 13 & 3 & 13,5 & 36,6 & 2,5 & 4 & 0,02 & 2,704 \\
\hline 6 & 72 & 145 & 13 & 5 & 23,3 & 53,1 & 3.0 & 4 & 0,8 & 2,274 \\
\hline 7 & 84 & 145 & 13 & 5 & 23,3 & 53,1 & 3,5 & 4 & 0,8 & 2,274 \\
\hline 8 & 96 & 145 & 13 & 5 & 35,9 & 65,3 & 4,0 & 5 & 0,02 & 1,821 \\
\hline 9 & 108 & 145 & 13 & 5 & 35,9 & 65,3 & 4,5 & 5 & 0,02 & 1,821 \\
\hline 10 & 120 & 145 & 13 & 5 & 49,7 & 77,8 & 5,0 & 10 & 0,1 & 1,564 \\
\hline 11 & 132 & 145 & 13 & 5 & 49,7 & 77,8 & 5,5 & 10 & 0,1 & 1,564 \\
\hline 12 & 144 & 131 & 23 & 3 & 64,5 & 88,1 & 6.0 & 10 & 0,1 & 1,367 \\
\hline 13 & 156 & 131 & 23 & 3 & 64,5 & 88,1 & 6,5 & 10 & 0,1 & 1,367 \\
\hline 14 & 168 & 155 & 23 & 5 & 72,9 & 105,8 & 7,0 & 10 & 0,1 & 1,451 \\
\hline 15 & 180 & 155 & 23 & 5 & 72,9 & 105,8 & 7.5 & 10 & 0,1 & 1,451 \\
\hline 16 & 192 & 155 & 23 & 5 & 83,5 & 110,1 & 8,0 & 10 & 0,1 & 1,319 \\
\hline 17 & 204 & 155 & 23 & 5 & 83,5 & 110,1 & 8,5 & 10 & 0,1 & 1,319 \\
\hline 18 & 216 & 203 & 23 & 9 & 90,5 & 132,2 & 9,0 & 20 & 0,8 & 1,460 \\
\hline 19 & 228 & 203 & 23 & 9 & 90,5 & 132,2 & 9,5 & 20 & 0,8 & 1,460 \\
\hline 20 & 240 & 203 & 23 & 9 & 108,2 & 141,4 & 10,0 & 60 & 25 & 1,307 \\
\hline
\end{tabular}

Figura 5.6 Configuração do filtro mensal - X-11 e S-WLS - segundo a SNR

Com base nas SNRs, foram identificadas as oito melhores combinações de parâmetros do filtro S-WLS. Esses valores estão apresentados na Tabela 5.13. Porém, era necessária uma única melhor configuração.

\begin{tabular}{c|c|c|c}
\hline Grupo & $\boldsymbol{\alpha}$ & $\boldsymbol{\delta}$ & $\mathbf{w}_{\mathbf{0}}$ \\
\hline 1 & $1 / 3$ & $1 / 10$ & 1 \\
\hline 2 & $1 / 3$ & $1 / 10$ & 5 \\
\hline 3 & $1 / 3$ & $1 / 10$ & 10 \\
\hline 4 & $1 / 3$ & $1 / 4$ & 0,8 \\
\hline 5 & $1 / 3$ & $1 / 30$ & 1 \\
\hline 6 & $1 / 3$ & $1 / 30$ & 10 \\
\hline 7 & $1 / 3$ & $1 / 60$ & 10 \\
\hline 8 & $1 / 3$ & $1 / 60$ & 25 \\
\hline
\end{tabular}

Tabela 5.13 Configurações de parâmetros para o filtro S-WLS mensal utilizadas na seleção do melhor filtro

Para a obtenção de uma única configuração de parâmetros, foram realizadas - na Etapa (3) - simulações, nas quais foram gerados 100 valores aleatórios para ' $k$ ' no intervalo uniforme $[72,120]$, utilizando o MATLAB. Quanto à componente irregular, foram geradas 30 séries seguindo uma distribuição $\mathrm{N}\left(0, \sigma^{2}\right)$, utilizando a função randn do MATLAB. Nessas simulações, foi usado: $A / s=6$ e $b=40 \%$.

Em relação ao valor de $\alpha$, determinou-se que seria utilizado $\alpha=\mathbf{1} / \mathbf{3}$, pois equivale a uma largura da banda passante de pelo menos 1 ciclo a cada 72 meses (ver Seção 5.4). Ou seja, para $k \geq 72$, o sinal estará dentro da banda passante. É importante ressaltar que, neste projeto, existe a opção de trabalhar com diferentes 
larguras da banda de transição, pois é permitido que as bandas (passante e de transição) sejam definidas conforme desejado.

Com base na comparação entre a MSE (Eq. 5-18) dos filtros testados, foi encontrado o melhor filtro S-WLS. Tal filtro apresenta a seguinte configuração:

$$
\alpha=1 / 3, \delta=1 / 30 \text { e } w_{0}=1 .
$$

A comparação entre os coeficientes do filtro S-WLS com o X-11 está apresentada no Apêndice $\mathrm{H}$.

Os Gráficos 5.14 e 5.15 apresentam a magnitude da resposta em frequência do filtro de tamanho 121 e do filtro de tamanho 193, respectivamente. Como pode ser observado nos gráficos, para cada tamanho $(N)$ (ver Quadro 5.4), a magnitude da resposta em frequência do filtro apresenta aspectos, relacionados à banda passante e à banda de rejeição, diferentes.

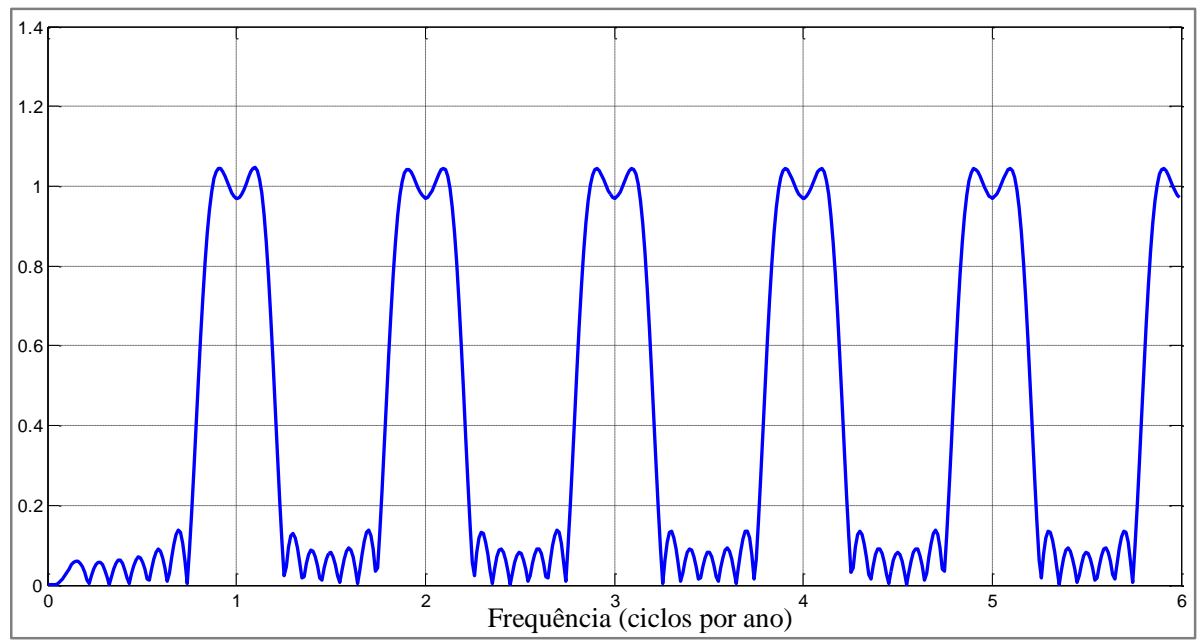

Gráfico 5.14 Resposta em magnitude do filtro S-WLS mensal para N = 121 


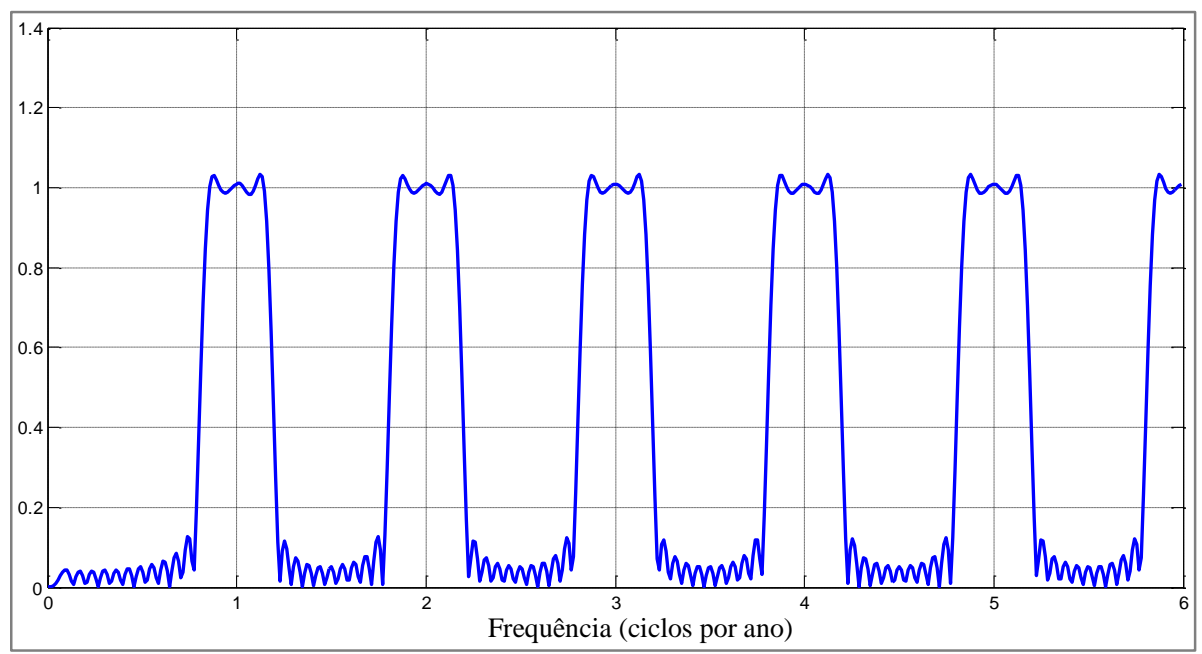

Gráfico 5.15 Resposta em magnitude do filtro S-WLS mensal para N = 193

Foram obtidos os valores de $S Q, \gamma_{0}$ e $\left|1-\gamma_{1}\right|$, para cada tamanho do filtro $(N)$. Eles serão utilizados no cálculo da SNR e estão apresentados na Tabela 5.14. Analisando os valores de $S Q$ e $\gamma_{0}$, não foi observado um padrão em relação ao tamanho do filtro. Porém, para $\left|1-\gamma_{1}\right|$, nota-se que o valor diminui consideravelmente nos filtros maiores.

\begin{tabular}{c|c|c|c}
\hline $\mathbf{N}$ & $\boldsymbol{S Q}$ & $\boldsymbol{\gamma}_{\mathbf{0}}$ & $\left|\mathbf{1}-\boldsymbol{\gamma}_{\mathbf{1}}\right|$ \\
\hline $\mathbf{1 1 7}$ & 0,335 & 0,946 & 0,205 \\
\hline $\mathbf{1 2 1}$ & 0,342 & 0,969 & 0,165 \\
\hline $\mathbf{1 3 1}$ & 0,342 & 0,969 & 0,167 \\
\hline $\mathbf{1 4 1}$ & 0,342 & 0,978 & 0,163 \\
\hline $\mathbf{1 4 5}$ & 0,337 & 1,017 & 0,141 \\
\hline $\mathbf{1 5 5}$ & 0,336 & 1,014 & 0,138 \\
\hline $\mathbf{1 8 9}$ & 0,331 & 1,030 & 0,158 \\
\hline $\mathbf{1 9 3}$ & 0,335 & 1,009 & 0,118 \\
\hline $\mathbf{2 0 3}$ & 0,335 & 1,010 & 0,119 \\
\hline
\end{tabular}

Tabela 5.14 Valores de $S Q, \gamma_{0}$ e $\left|1-\gamma_{1}\right|$ para os nove filtros mensais, considerando $k=72$

A fim de comparar a resposta em magnitude do filtro S-WLS com a resposta em magnitude do X-11, foram gerados os Gráficos 5.16 e 5.17. O Gráfico 5.16 apresenta as respostas em magnitude dos filtros de tamanho 121. Esse filtros têm média móvel sazonal 3x3, que é o tamanho de média móvel sazonal mais indicada para a sazonalidade móvel no X-11. Mesmo assim, observa-se que o filtro S-WLS apresenta banda passante mais larga, garantindo um melhor ajuste. 


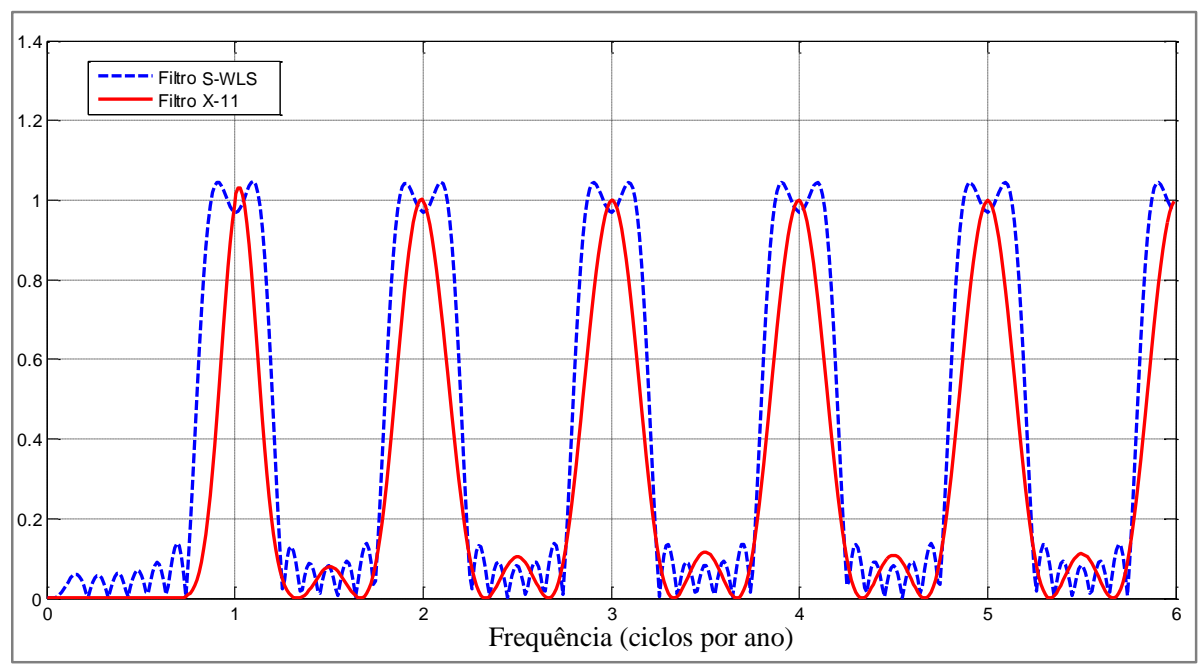

\section{Gráfico 5.16 Resposta em magnitude do filtro S-WLS e do filtro X-11 para $\mathrm{N}=121$, série mensal}

Já o Gráfico 5.17 mostra as respostas em magnitude dos filtros de tamanho 193. No X-11, isso equivale a uma média móvel sazonal 3x9. Observa-se que a resposta em magnitude do filtro X-11 tem largura de banda estreita, o que resultará em erros grandes na estimativa da componente sazonal, caso haja instabilidade no seu padrão.

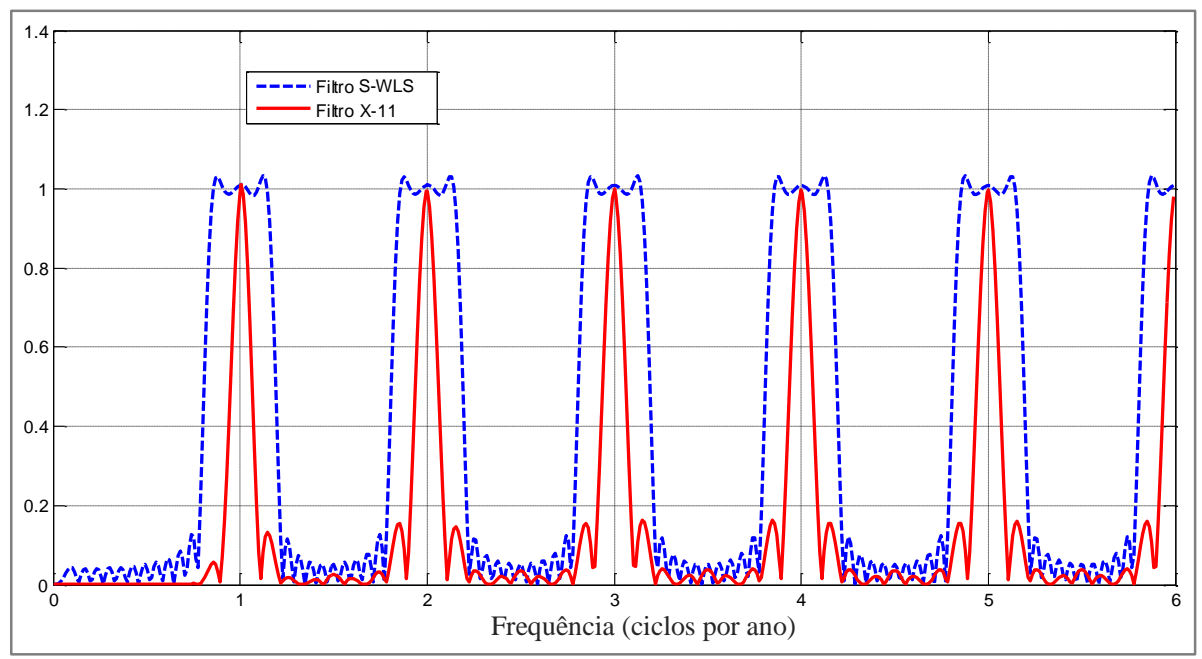

Gráfico 5.17 Resposta em magnitude do filtro S-WLS e do filtro X-11 para $\mathrm{N}=193$, série mensal

Esta subseção 5.4.1 apresentou a definição do filtro S-WLS para as séries mensais. Na próxima subseção será apresentada essa definição para as séries trimestrais. 


\subsection{2.}

\section{Definição do filtro S-WLS para as séries trimestrais}

O procedimento de seleção do melhor filtro trimestral foi realizado segundo as etapas descritas na Figura 5.5, apresentada na Subseção 5.4.

Inicialmente, na Etapa 1, foram geradas possibilidades de variação nos parâmetros do filtro: $\boldsymbol{\alpha}, \boldsymbol{\delta}$ e $\boldsymbol{w}_{\mathbf{0}}$, para cada tamanho $(\boldsymbol{N})$. Os seguintes valores foram utilizados, totalizando 4.536 possibilidades de combinação de parâmetros:

- Denominador de $\boldsymbol{\alpha}:[2,3,4,5,6,7,8]$;

- Denominador de $\boldsymbol{\delta}$ : $[4,5,10,15,20,30,40,50,60]$;

- Peso da banda passante ( $\left.\mathbf{w}_{\mathbf{0}}\right)$ : [0,02 0,05 0,1 0,3 0,5 0,8 15101520 25];

- Tamanho do filtro (N): [41, 43, 49, 51, 65, 67];

Foram registrados, em cada combinação de parâmetros, o $\gamma_{0}$ (que representa a resposta ao impulso na frequência $\frac{\mathbf{1}}{\mathbf{4}}$ ), o $\left|\mathbf{1}-\boldsymbol{\gamma}_{\mathbf{1}}\right|$ (valor absoluto da diferença máxima entre os pontos da banda passante e o valor 1) e a $\mathbf{S Q}$. A partir deles, foi criado um banco de dados, juntamente com o banco de dados do filtro X-11, com os valores de $\mathbf{S} \mathbf{Q}, \boldsymbol{\beta}_{\mathbf{0}}$ e $\boldsymbol{\beta}_{\mathbf{1}}$. Vale citar que, no filtro X-11 para séries trimestrais, são usados filtros de Henderson de tamanho 5 e 7.

Após a criação do banco de dados, foi identificada qual configuração do X11 gerava a maior SNR para um determinado padrão da série (Etapa 2). Em seguida, com base no tamanho do filtro definido pelo X-11, foi encontrada qual combinação de parâmetros do filtro S-WLS gerava a maior SNR. O procedimento utilizado para a primeira seleção do filtro está ilustrado na Figura 5.7.

\begin{tabular}{|c|c|c|c|c|c|c|c|c|c|c|}
\hline$\Delta$ & A & B & C & D & $E$ & $\mathrm{~F}$ & $\mathrm{H}$ & I & $J$ & K \\
\hline 1 & A & b & $s$ & & & & & & & \\
\hline 2 & 6 & $18 \%$ & 1 & & & & & & & \\
\hline 3 & k & $N$ & Henderson & MMs & SNR X-11 & $\begin{array}{c}\text { SNR } \\
\text { Proposto }\end{array}$ & Dalpha & D_delta & Peso & $\begin{array}{c}\text { RAZÃO das } \\
\text { SNRs }\end{array}$ \\
\hline 4 & 16 & 49 & 5 & 5 & 52,1 & 45,4 & 2,0 & 60 & 10 & 0,870 \\
\hline 5 & 20 & 49 & 5 & 5 & 52,1 & 45,4 & 2,5 & 60 & 10 & 0,870 \\
\hline 6 & 24 & 49 & 5 & 5 & 63,3 & 65,2 & 3,0 & 60 & 10 & 1,030 \\
\hline 7 & 28 & 49 & 5 & 5 & 63,3 & 65,2 & 3,5 & 60 & 10 & 1,030 \\
\hline 8 & 32 & 67 & 7 & 9 & 81,4 & 86,5 & 4,0 & 60 & 25 & 1,062 \\
\hline 9 & 36 & 67 & 7 & 9 & 81,4 & 86,5 & 4,5 & 60 & 25 & 1,062 \\
\hline 10 & 40 & 67 & 7 & 9 & 96,8 & 97,4 & 5,0 & 60 & 25 & 1,006 \\
\hline 11 & 44 & 67 & 7 & 9 & 96,8 & 97,4 & 5,5 & 60 & 25 & 1,006 \\
\hline 12 & 48 & 67 & 7 & 9 & 114,1 & 116,2 & 6,0 & 60 & 25 & 1,018 \\
\hline 13 & 52 & 67 & 7 & 9 & 114,1 & 116,2 & 6,5 & 60 & 25 & 1,018 \\
\hline
\end{tabular}

Figura 5.7 Configuração do filtro trimestral: X-11 e filtro S-WLS 
Nessa $1^{a}$ seleção, foram testados vários valores de ' $A$ ', ' $s$ ', ' $b$ ' e ' $k$ '. Os três melhores conjuntos de parâmetros encontrados para o filtro S-WLS trimestral estão apresentados na Tabela 5.15, abaixo:

\begin{tabular}{c|c|c|c}
\hline Grupo & $\boldsymbol{\alpha}$ & $\boldsymbol{\delta}$ & $\boldsymbol{w}_{\mathbf{0}}$ \\
\hline 1 & $1 / 3$ & $1 / 10$ & 1 \\
\hline 2 & $1 / 3$ & $1 / 30$ & 1 \\
\hline 3 & $1 / 3$ & $1 / 60$ & 10 \\
\hline
\end{tabular}

\section{Tabela 5.15 Configurações de parâmetros para o filtro S-WLS trimestral} utilizadas na seleção do melhor filtro

$\mathrm{Na}$ etapa seguinte da escolha do filtro (Etapa 3), os resultados das três melhores configurações de parâmetros foram comparados com os resultados do X11 em relação às medidas de erro: MSE e MAD. Para isso, no MATLAB, foram construídos sinais sazonais, nos quais foram gerados 100 valores aleatórios para ' $k$ ' no intervalo uniforme $[24,40]$. Quanto à componente irregular, foram geradas 30 séries seguindo uma distribuição $\mathrm{N}\left(0, \sigma^{2}\right)$, utilizando a função randn do MATLAB. Nessas simulações, foi usado $A / s=6$; e em relação à taxa de variação da sazonalidade ' $\boldsymbol{b}$ ', foi usado o valor de $40 \%$.

Quanto ao valor de $\boldsymbol{\alpha}$, foi determinado anteriormente (ver Seção 5.4) que seria utilizado $\alpha=\mathbf{1} / \mathbf{3}$, pois equivale a uma largura da banda passante de pelo menos 1 ciclo a cada 24 trimestres $(k=24)$. Ou seja, para $k \geq 24$, o sinal estará dentro da banda passante. Dessa forma, as séries que tiverem um comportamento de sazonalidade móvel forte ( $k$ pequeno) serão contempladas, assim como aquelas com padrão mais suave. Porém, se $k<24$, a utilização do filtro S-WLS não é indicada.

Os resultados do filtro S-WLS, para as séries trimestrais, foram melhores do que os do X-11 quando o filtro apresentava os seguintes parâmetros: $\alpha=\mathbf{1} / \mathbf{3}, \delta=$ $\mathbf{1 / 3 0}$ e $\boldsymbol{w}_{\mathbf{0}}=\mathbf{1}$, assim como nas séries mensais. A comparação entre os coeficientes do filtro S-WLS com o X-11 está apresentada no Apêndice I.

As respostas em magnitude, para o menor e o maior tamanho ' $\boldsymbol{N}$ ' do filtro, podem ser verificadas nos Gráficos 5.18 e 5.19 . 


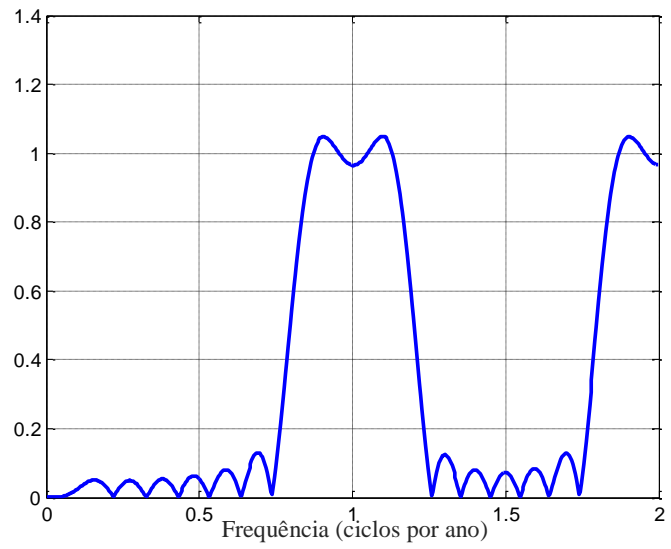

Gráfico 5.18 Resposta em magnitude do filtro $S$-WLS trimestral para $N=41$

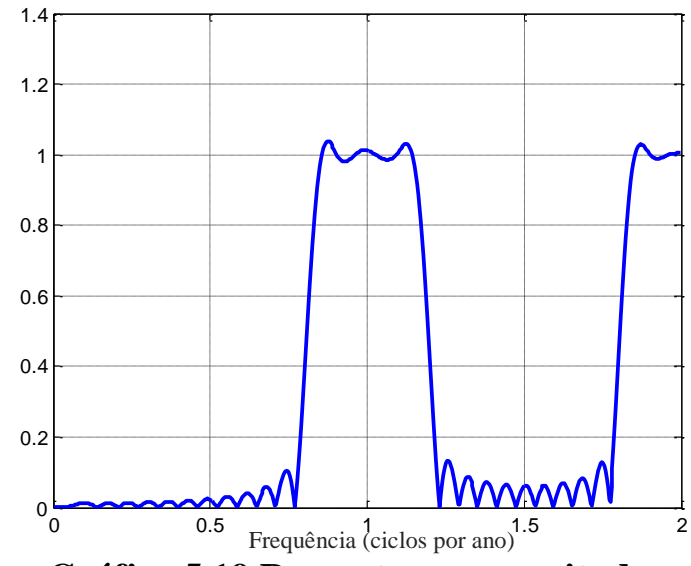

Gráfico 5.19 Resposta em magnitude do filtro S-WLS trimestral para N = 67

Para cada tamanho do filtro $(N)$, foram obtidos os valores de $S Q, \gamma_{0}$ e $\left|1-\gamma_{1}\right|$, a serem utilizados no cálculo da SNR, definida na Equação (5-11). Os valores de $S Q, \quad \gamma_{0}$ e $\left|1-\gamma_{1}\right|$ estão apresentados na Tabela 5.16. Para $\left|1-\gamma_{1}\right|$, nota-se que o valor diminui à medida que o tamanho do filtro aumenta, contribuindo para uma SNR maior.

\begin{tabular}{c|c|c|c}
\hline $\mathbf{N}$ & $\boldsymbol{S Q}$ & $\boldsymbol{\gamma}_{\mathbf{0}}$ & $\left|\mathbf{1}-\boldsymbol{\gamma}_{\mathbf{1}}\right|$ \\
\hline $\mathbf{4 1}$ & 0,2790 & 0,9650 & 0,1716 \\
\hline $\mathbf{4 3}$ & 0,2798 & 0,9656 & 0,1774 \\
\hline $\mathbf{4 9}$ & 0,2761 & 1,0120 & 0,1478 \\
\hline $\mathbf{5 1}$ & 0,2754 & 1,0123 & 0,1447 \\
\hline $\mathbf{6 5}$ & 0,2736 & 1,0120 & 0,1228 \\
\hline $\mathbf{6 7}$ & 0,2737 & 1,0121 & 0,1271 \\
\hline
\end{tabular}

Tabela 5.16 Valores de $S Q, \gamma_{0}$ e $\left|1-\gamma_{1}\right|$ para os seis filtros $S$ WLS trimestrais, considerando $k=24$ 
Os Gráficos 5.20 e 5.21 apresentam a comparação entre a resposta em magnitude do filtro S-WLS e a resposta em magnitude do X-11, para dois tamanhos de filtro $(N=41$ e $N=67)$. O Gráfico 5.20 apresenta as respostas em magnitude dos filtros de tamanho 41 - nesse caso, o X-11 utiliza média móvel sazonal 3x3, que é o tipo de média móvel sazonal mais indicada para a sazonalidade móvel. Já no Gráfico 5.21, estão as respostas em magnitude dos filtros de tamanho 67, no qual o X-11 utiliza média móvel sazonal 3x9. Em ambos os casos observa-se que o filtro S-WLS apresenta banda passante mais larga do que o X-11, garantindo uma melhor extração da componente sazonal na presença de sazonalidade móvel.

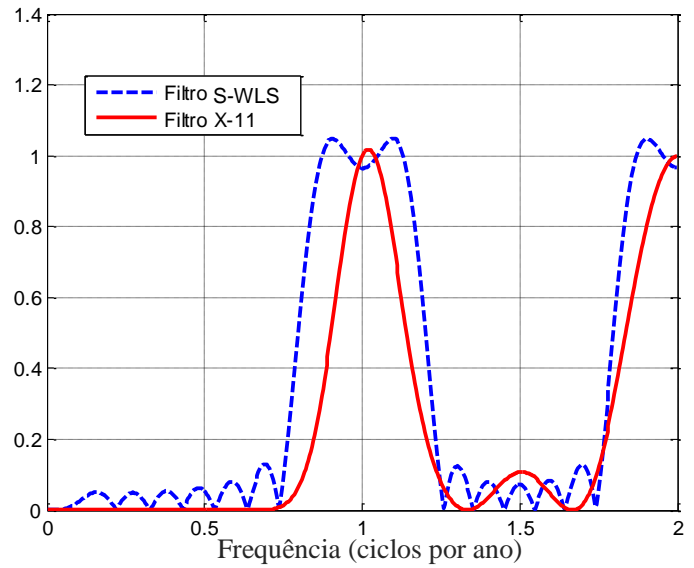

Gráfico 5.20 Resposta em magnitude do filtro S-WLS e do filtro X-11 para $\mathrm{N}=41$, série trimestral

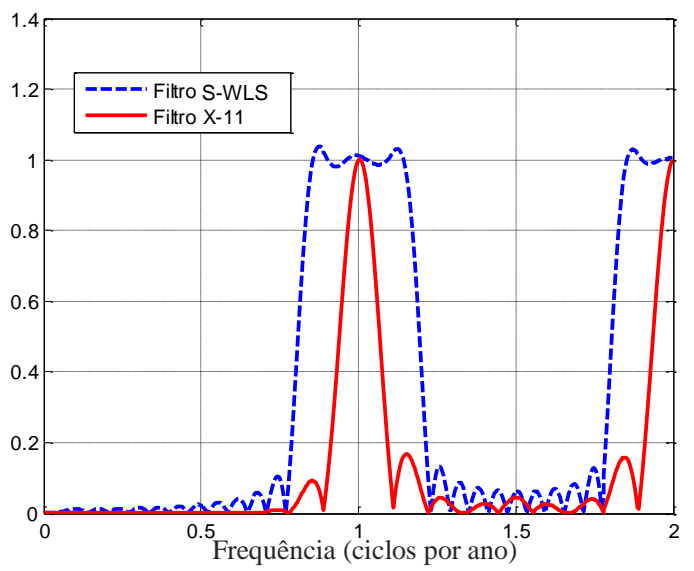

Gráfico 5.21 Resposta em magnitude do filtro S-WLS e do filtro X-11 para $\mathrm{N}=67$, série trimestral

Na Subseção 5.5, a seguir, serão apresentados os resultados da comparação entre o filtro S-WLS, mensal e trimestral, e o filtro X-11, em relação à SNR e às estatísticas de erro: MSE e MAD. 


\section{5.}

\section{Resultados: filtro S-WLS vs X-11}

Após a escolha do filtro S-WLS para as séries mensais e trimestrais nas Subseções 5.4.1 e 5.4.2, foram geradas séries artificiais com sazonalidade móvel, seguindo os padrões das séries reais (ver Subseção 5.2), e procedeu-se à dessazonalização delas, utilizando o filtro S-WLS e o filtro X-11. Os resultados encontrados serão apresentados nas subseções a seguir. Inicialmente, na Subseção 5.5.1, é descrito o critério usado para a comparação entre o filtro S-WLS e o X-11.

\subsection{1.}

\section{Critério de comparação entre o filtro S-WLS e o filtro X-11}

Para avaliar o desempenho do filtro sazonal-WLS, em relação ao filtro X-11, foram geradas séries artificiais. A decisão de se trabalhar com séries artificiais foi tomada uma vez que, como a componente sazonal é não observável, a forma adequada de avaliar o desempenho do filtro é a partir de séries onde se conheça o valor desejado.

Foram geradas séries cujos parâmetros estão descritos na Subseção 5.1. A definição desses parâmetros foi baseada nas características de séries reais, com sazonalidade móvel, apresentadas na Subseção 5.2. Dessa forma, as séries artificiais se assemelham aos dados originais.

Nas comparações, inicialmente encontrou-se qual combinação entre o filtro de Henderson e o tamanho do filtro de média móvel sazonal (MMs), do X-11, gerava a menor MSE. A partir da definição desses valores, foi determinado o mesmo tamanho para o filtro S-WLS - para cada um dos sinais artificiais. Isso foi feito tanto para as séries mensais, quanto para as trimestrais.

A comparação dos resultados entre o filtro S-WLS e o X-11, realizada para as séries com modo de decomposição aditivo, foi baseada na razão entre as SNRs (Eq. 5-17), e nas estatísticas MSE (Mean Square Error) e MAD (Mean Absolute Deviation). Essas estatísticas estão apresentadas nas Equações (5-18) e (5-19), respectivamente.

$$
\begin{gathered}
M S E=\frac{1}{n} \sum_{i=1}^{n}(\text { Sazonalidade Original }- \text { Sazonalidade Ajustada })^{2} \\
M A D=\frac{1}{n} \sum_{i=1}^{n} \mid \text { Sazonalidade Original }- \text { Sazonalidade Ajustada } \mid
\end{gathered}
$$


Para as séries com modo de decomposição multiplicativo, foram usadas - nas comparações entre o filtro S-WLS e o X-11 - as estatísticas MSE e MAD, mas não a SNR, pois não é possível construir a resposta em magnitude dos filtros.

O teste $t$, de igualdade de médias $(\mu)$ de duas populações com variâncias equivalentes e desconhecidas, foi realizado para a comparação das estatísticas MSE e MAD dos filtros, separadamente. Nesses testes, utilizou-se nível de significância de 5\%. As hipóteses testadas foram as seguintes:

$$
\left\{\begin{array}{l}
H_{0}: \mu_{\mathrm{S}-\mathrm{WLS}}=\mu_{\mathrm{X}-11} \\
H_{1}: \mu_{\mathrm{S}-\mathrm{WLS}}<\mu_{\mathrm{X}-11}
\end{array}\right.
$$

A estatística de teste é dada por:

$$
t_{\text {calculado }}=\frac{\left(\bar{X}_{\mathrm{S}-\mathrm{WLS}}-\bar{X}_{\mathrm{X}-11}\right)-\left(\mu_{\mathrm{S}-\mathrm{WLS}}-\mu_{\mathrm{X}-11}\right)}{\sqrt{\left(\frac{1}{n_{\mathrm{S}-\mathrm{WLS}}}+\frac{1}{n_{\mathrm{X}-11}}\right) \hat{\sigma}^{2}}} \sim t\left(n_{\mathrm{S}-\mathrm{WLS}}+n_{\mathrm{X}-11}-2\right)
$$

sendo:

$$
\hat{\sigma}^{2}=\frac{\left(n_{\mathrm{S}-\mathrm{WLS}}-1\right) s_{\mathrm{S}-\mathrm{WLS}}^{2}+\left(n_{\mathrm{X}-11}-1\right) s_{\mathrm{X}-11}^{2}}{n_{\mathrm{S}-\mathrm{WLS}}+n_{\mathrm{X}-11}-2}
$$

Onde:

$\bar{X}_{\mathrm{S}-\mathrm{WLS}}=$ média da medida de erro (MSE e MAD) do filtro S-WLS

$\bar{X}_{\mathrm{X}-11}=$ média da medida de erro (MSE e MAD) do filtro X-11

$s_{\mathrm{S}-\mathrm{WLS}}^{2}=$ variância da medida de erro (MSE e MAD) do filtro S-WLS

$s_{\mathrm{X}-11}^{2}=$ variância da medida de erro (MSE e MAD) do filtro X-11

$n_{\mathrm{S}-\mathrm{WLS}}=$ quantidade de valores referentes ao desvio-padrão da irregular

aleatorizados para o cálculo da média da MSE, e da média da MAD, no filtro

S-WLS. Nesse caso, $n_{\mathrm{S}-\mathrm{WLS}}=100$

$n_{\mathrm{X}-11}=$ quantidade de valores referentes ao desvio-padrão da irregular

aleatorizados para o cálculo da média da MSE, e da média da MAD, no filtro

$\mathrm{X}-11$. Nesse caso, $n_{\mathrm{X}-11}=100$

A apresentação dos resultados, a partir da Subseção 5.5.2, será realizada inicialmente para as séries mensais aditivas, e em seguida para as séries multiplicativas. Após isso, serão apresentados os resultados das séries trimestrais. 


\subsection{2. Resultados: série mensal com decomposição aditiva - filtro S-WLS vs $\mathrm{X}-11$}

Nesta Subseção 5.5.2, serão apresentados os resultados das comparações entre o desempenho do filtro S-WLS e o desempenho do método X-11, nas séries mensais com decomposição aditiva, sob diversos aspectos relacionados aos parâmetros do sinal artificial. Nessas comparações, foi utilizado apenas o 'melhor

filtro', definido na Subseção 5.4.1, cuja configuração é a seguinte: $\alpha=\frac{1}{3}, \delta=\frac{1}{30}$, $\mathrm{w}_{0}=1$ e $\mathrm{M}=401$.

Para comparar os resultados do filtro proposto (S-WLS) com o filtro X-11, foram geradas séries com e sem componente de tendência. Nas séries com componente de tendência, foram usados os coeficientes: $b_{0}=100, b_{1}=-0,2596, b_{2}$ $=0,0046$ e $b_{3}=-1 E-5$, baseados na série real X69.

Na elaboração da componente sazonal artificial, foram utilizados os seguintes valores dos parâmetros $A / s, b$ e $k$ :

- $A / s=[1,2,3,4,6,8,10]$

- $b=[10 \%, 15 \%, 20 \%, 25 \%, 30 \%, 40 \%, 50 \%, 60 \%, 70 \%, 80 \%]$

- $k=[72,84,96,108,120,132,144,156,180]$

Em relação à componente irregular, foram geradas 100 séries, com distribuição $\mathrm{N}\left(0, \sigma^{2}\right)$, utilizando a função randn do MATLAB, para cada combinação de parâmetros do sinal artificial.

A fim de avaliar as condições que fazem o filtro S-WLS superar o X-11, foi analisada a razão sinal ruído (SNR), a média quadrática dos resíduos (MSE), e a média absoluta dos erros (MAD), dos resíduos gerados pelos filtros. Isso foi feito variando os parâmetros do sinal artificial, mencionados na Equação (5-1).

A análise dos resultados obtidos está apresentada da seguinte forma: no item (i) é apresentado o resultado obtido com a variação de ' $b$ '; o item (ii) expõe o resultado da variação de ' $k$ '; no item (iii) são apresentadas as variações de ' $A / s$ ' e, por fim, no item (iv) são mostradas as condições nas quais o filtro S-WLS supera o $\mathrm{X}-11$, analisando os valores de ' $b$ ', ' $k$ ' e ' $A / s$ ', simultaneamente. 


\section{(i) Resultados encontrados com base na variação de ' $b$ ': série mensal com decomposição aditiva}

O parâmetro ' $b$ ' representa a taxa de variação da sazonalidade, assumindo valores no intervalo $(0,1)$. Nas séries reais mensais, com modelo aditivo de decomposição, foi observada uma concentração dessas taxas entre os valores $10 \%$ e $30 \%$; e também entre $50 \%$ e $60 \%$. O valor máximo encontrado, nessas séries, foi $b=70 \%$.

A seguir, os Gráficos 5.22 e 5.23 ilustram a comparação dos valores da MSE e da SNR, respectivamente, entre o filtro S-WLS e o X-11. Nas comparações, foi utilizado $A / s=6$ e $k=72$.

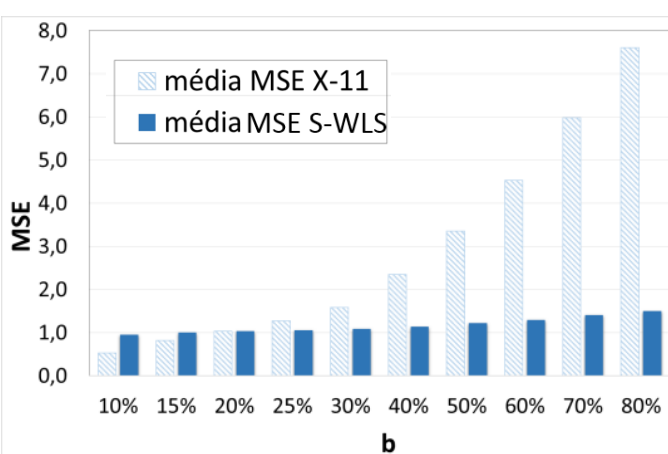

Gráfico 5.22 Média da MSE nas simulações com o filtro $X-11$ e com o filtro S-WLS, para valores de ' $b$ ', considerando $A / s=6$ e $k=72($ Eq. 5-1)

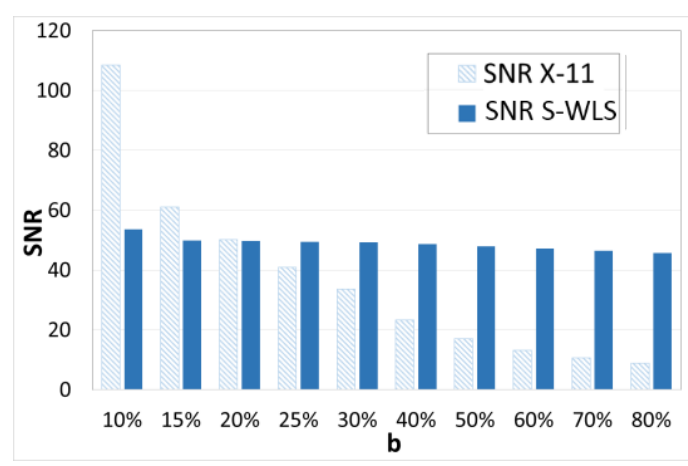

Gráfico 5.23 Relação entre ' $b$ ' e a SNR do filtro S-WLS e do filtro $X-11$, considerando $A / s=6$ e $k=72$ (Eq. 5-1)

A Tabela 5.17 mostra os dados utilizados na elaboração dos gráficos. Além disso, é apresentado o valor-p, do teste $t$ de comparação de médias, realizado entre o filtro S-WLS e o X-11.

\begin{tabular}{c|ccc|ccc|ccc}
\hline $\boldsymbol{b}$ & MSE & MSE & valor-p & $\begin{array}{c}\text { MAD } \\
\text { S-WLS }\end{array}$ & $\begin{array}{c}\text { MAD } \\
\text { X-11 }\end{array}$ & valor-p & $\begin{array}{c}\text { SNR S- } \\
\text { WLS }\end{array}$ & $\begin{array}{c}\text { SNR } \\
\text { X-11 }\end{array}$ & $\begin{array}{c}\text { SNR } \\
\text { S-WLS } / \\
\text { SNR X-11 }\end{array}$ \\
\hline $\mathbf{1 0 \%}$ & 0,96 & 0,53 & 1,000 & 0,78 & 0,59 & 1,000 & 53,6 & 108,4 & 0,5 \\
$\mathbf{1 5 \%}$ & 1,00 & 0,82 & 1,000 & 0,80 & 0,73 & 1,000 & 49,8 & 61,0 & 0,8 \\
$\mathbf{2 0 \%}$ & 1,04 & 1,03 & 0,722 & 0,81 & 0,81 & 0,375 & 49,7 & 50,1 & 1,0 \\
$\mathbf{2 5 \%}$ & 1,05 & 1,28 & 0,000 & 0,82 & 0,91 & 0,000 & 49,5 & 40,9 & 1,2 \\
$\mathbf{3 0 \%}$ & 1,08 & 1,58 & 0,000 & 0,83 & 1,02 & 0,000 & 49,2 & 33,5 & 1,5 \\
$\mathbf{4 0 \%}$ & 1,14 & 2,35 & 0,000 & 0,85 & 1,25 & 0,000 & 48,6 & 23,3 & 2,1 \\
$\mathbf{5 0 \%}$ & 1,22 & 3,35 & 0,000 & 0,88 & 1,50 & 0,000 & 48,0 & 17,1 & 2,8 \\
$\mathbf{6 0 \%}$ & 1,29 & 4,53 & 0,000 & 0,90 & 1,75 & 0,000 & 47,2 & 13,2 & 3,6 \\
$\mathbf{7 0 \%}$ & 1,41 & 5,98 & 0,000 & 0,95 & 2,01 & 0,000 & 46,5 & 10,6 & 4,4 \\
$\mathbf{8 0 \%}$ & 1,50 & 7,60 & 0,000 & 0,98 & 2,27 & 0,000 & 45,7 & 8,8 & 5,2 \\
\hline
\end{tabular}

Tabela 5.17 MSE, MAD e SNR para valores de ' $b$ ', sendo $A / s=6, k=72$ : séries mensais com decomposição aditiva (Eq. 5-1) 
Analisando os resultados expostos nos Gráficos 5.22 e 5.23, e na Tabela 5.17, constata-se que apenas para $b \geq 25 \%$, o ajuste realizado pelo filtro S-WLS se mostra mais adequado do que o ajuste do X-11. Isso ocorre tanto para a SNR, quanto para a MSE e a MAD, significando que o X-11 é superior quando a taxa de variação da sazonalidade é pequena.

Por outro lado, quando a taxa de variação (b) é igual a 40\%, a SNR do filtro S-WLS é maior do que o dobro da SNR do filtro X-11. E essa diferença aumenta à medida que os valores de ' $b$ ' crescem, atingindo a razão ( $\left.\frac{\text { SNR S-WLS }}{\text { SNR X-11 }}\right)$ igual a 4,4 quando $b=70 \%$ (ou seja, a SNR do S-WLS é 4,4 vezes maior do que a SNR do X11 se a taxa de variação da sazonalidade é igual a 70\%). Vale citar que esse valor foi encontrado nas séries reais.

Observa-se também que, tanto em relação à SNR, quanto em relação ao MSE e à MAD, os valores apresentados, referentes aos resultados do filtro S-WLS se mantêm num mesmo patamar, enquanto que os valores referentes aos resultados do $\mathrm{X}-11$ variam exponencialmente. Isso indica que o filtro S-WLS está realizando uma estimação correta da sazonalidade, uma vez que não ocorrem grandes variações quanto o valor de ' $b$ ' é alterado. 
(ii) Resultados encontrados com base na variação de ' $k$ ': série mensal com decomposição aditiva

Para analisar o desempenho do filtro S-WLS, em comparação com o X-11, com base na variação ' $k$ ', foi fixado o valor de $b=40 \%$ e razão $A / s=6$.

Como o parâmetro ' $k$ ' representa o número de meses no qual o padrão de sazonalidade móvel volta a se repetir, quanto menor for o ' $k$ ', mais instável será a sazonalidade. Nas séries reais observou-se grande concentração de valores de ' $k$ ' entre 84 e 108.

Os Gráficos 5.24 e 5.25 e a Tabela 5.18 apresentam os resultados obtidos nas comparações entre o desempenho do filtro S-WLS, e o do X-11, para variações nos valores de ' $k$ '. Vale lembrar que o parâmetro ' $\alpha$ ' do filtro S-WLS é igual a $\frac{1}{3}$, e com base na relação entre ' $k$ ' e ' $\alpha$ ', o menor valor de ' $k$ ' tolerável para $\alpha=\frac{1}{3}$ é 72. Isso pode ser notado pela maior relação entre a SNR do filtro S-WLS e a SNR do X-11 quando $k=72$. Nota-se, no entanto, que o filtro S-WLS mantém um nível satisfatório de SNR para os demais valores de ' $k$ '.

Observa-se, na Tabela 5.18 e nos Gráficos 5.24 e 5.25, que a partir de $k \geq$ 132 , o filtro X-11 se mostra mais adequado para o ajuste sazonal. Isso porque $k \geq$ 132 equivale a um período de 11 anos necessários para o padrão de sazonalidade móvel voltar a se repetir. Com isso, mesmo considerando $b=40 \%$, a sazonalidade móvel não se mostra muito forte. Por outro lado, para valores de ' $k$ ' entre 72 e 120, o filtro S-WLS apresenta um desempenho superior ao X-11.

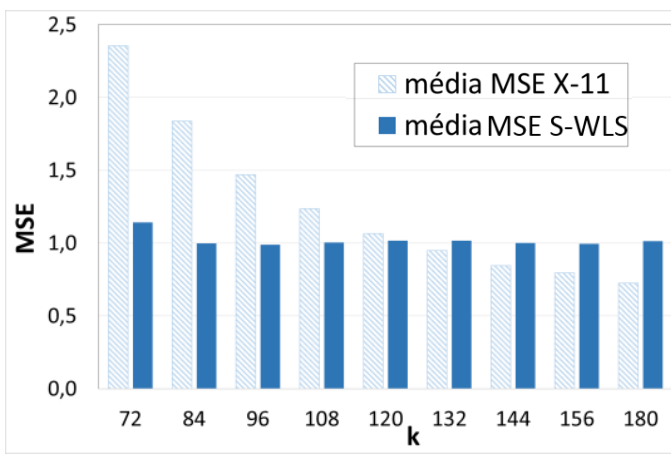

Gráfico 5.24 Média da MSE nas simulações com o filtro $X-11$ e com o filtro S-WLS, para valores de ' $k$ ', considerando $A / s=6$ e $b=40 \%($ Eq. 5-1)

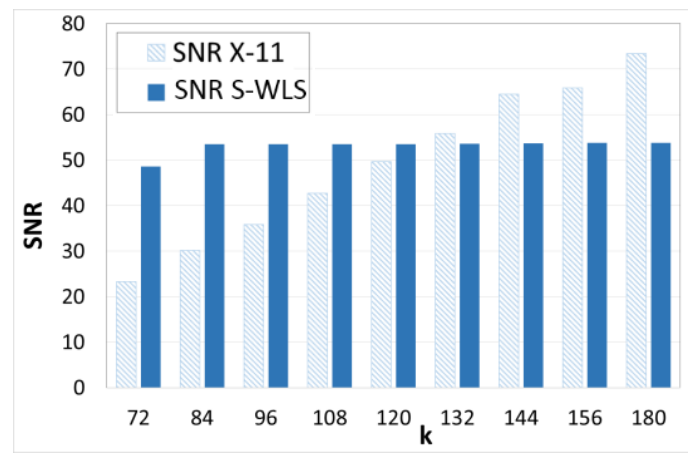

Gráfico 5.25 Relação entre ' $k$ ' e a SNR do filtro S-WLS e do filtro $X-11$, considerando $A / s=6$ e $b=40 \%$ (Eq. 5-1) 
Vale ressaltar que, com a variação dos valores de ' $k$ ', tanto a SNR, quanto a MSE e a MAD dos resultados do filtro S-WLS se mantêm num mesmo patamar, ao passo que os valores referentes aos resultados do $\mathrm{X}-11$ variam linearmente.

\begin{tabular}{c|ccc|ccc|ccc}
\hline $\boldsymbol{k}$ & $\begin{array}{c}\text { MSE } \\
\text { S-WLS }\end{array}$ & $\begin{array}{c}\text { MSE } \\
\text { X-11 }\end{array}$ & valor-p & $\begin{array}{c}\text { MAD } \\
\text { S-WLS }\end{array}$ & $\begin{array}{c}\text { MAD } \\
\text { X-11 }\end{array}$ & valor-p & $\begin{array}{c}\text { SNR S- } \\
\text { WLS }\end{array}$ & $\begin{array}{c}\text { SNR } \\
\text { X-11 }\end{array}$ & $\begin{array}{c}\text { SNR } \\
\text { S-WLS / } \\
\text { SNR X-11 }\end{array}$ \\
\hline $\mathbf{7 2}$ & 1,14 & 2,35 & 0,000 & 0,85 & 1,25 & 0,000 & 48,6 & 23,3 & 2,1 \\
$\mathbf{8 4}$ & 1,00 & 1,84 & 0,000 & 0,80 & 1,11 & 0,000 & 53,5 & 30,2 & 1,8 \\
$\mathbf{9 6}$ & 0,99 & 1,47 & 0,000 & 0,79 & 0,99 & 0,000 & 53,5 & 35,9 & 1,5 \\
$\mathbf{1 0 8}$ & 1,00 & 1,24 & 0,000 & 0,80 & 0,90 & 0,000 & 53,5 & 42,7 & 1,3 \\
$\mathbf{1 2 0}$ & 1,02 & 1,06 & 0,001 & 0,80 & 0,83 & 0,000 & 53,5 & 49,7 & 1,1 \\
$\mathbf{1 3 2}$ & 1,02 & 0,95 & 1,000 & 0,81 & 0,79 & 0,999 & 53,6 & 55,8 & 1,0 \\
$\mathbf{1 4 4}$ & 1,00 & 0,84 & 1,000 & 0,80 & 0,74 & 1,000 & 53,7 & 64,5 & 0,8 \\
$\mathbf{1 5 6}$ & 1,00 & 0,80 & 1,000 & 0,80 & 0,71 & 1,000 & 53,8 & 65,8 & 0,8 \\
$\mathbf{1 8 0}$ & 1,01 & 0,73 & 1,000 & 0,80 & 0,68 & 1,000 & 53,8 & 73,4 & 0,7 \\
\hline
\end{tabular}

Tabela 5.18 MSE, MAD e SNR para valores de ' $k$ ', sendo $A / s=6, b=40 \%$ : séries mensais com decomposição aditiva (Eq. 5-1) 
(iii) Resultados encontrados com base na variação de ' $A / s$ ': série mensal com decomposição aditiva

A razão entre a amplitude do sinal, dada por ' $A$ ', e o desvio-padrão da componente irregular, dado por ' $s$ ', apresenta uma relação interessante com a SNR dos filtros, como pode ser observado no Gráfico 5.27. Enquanto a SNR do filtro SWLS cresce exponencialmente, com o aumento de ' $A / s$ ', a SNR do X-11 cresce a uma taxa de $24 \%$, sendo bem menos acentuada.

Analisando a MSE, a MAD e a SNR (Tabela 5.19), do filtro S-WLS e do X11, para vários valores da razão ' $A / S$ ', nota-se que o filtro $S$-WLS já apresenta resultados significativamente melhores do que o X-11 para valores baixos de ' $A / s$ ' $(A / s=3)$. E essa superioridade se intensifica conforme aumentam os valor de ' $A / s$ ', ou seja, quanto maior for a amplitude do sinal em relação ao ruído.

Além disso, é interessante observar que, diferentemente dos casos anteriores, em que havia variação dos parâmetros (' $k$ ' e ' $b$ ') e as medidas do filtro S-WLS se mantinham num mesmo patamar, as variações nos valores de ' $A / s$ ' alteram, fortemente, as medidas do filtro S-WLS.

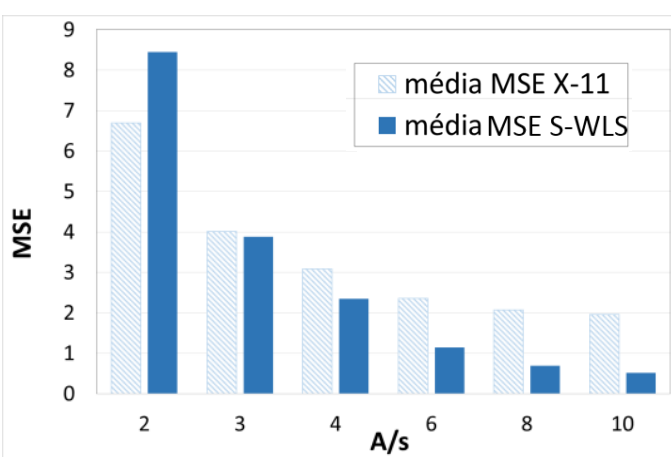

Gráfico 5.26 Média da MSE nas simulações com o filtro $X-11$ e com o filtro $S$-WLS, para valores de ' $A / s$ ', considerando $k=72$ e $b=40 \%$ (Eq. 5-1)

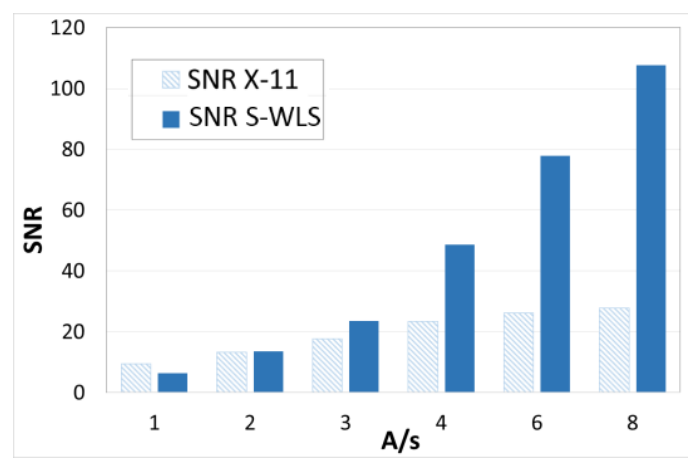

Gráfico 5.27 Relação entre ' $A / s$ ' e a SNR do filtro $S$-WLS e do filtro $X-11$, considerando $k=72$ e $b=40 \%$ (Eq. 5-1)

\begin{tabular}{c|ccc|ccc|ccc}
\hline $\boldsymbol{A} / \boldsymbol{s}$ & $\begin{array}{c}\text { MSE } \\
\text { S-WLS }\end{array}$ & $\begin{array}{c}\text { MSE } \\
\text { X-11 }\end{array}$ & valor-p & $\begin{array}{c}\text { MAD } \\
\text { S-WLS }\end{array}$ & $\begin{array}{c}\text { MAD } \\
\text { X-11 }\end{array}$ & valor-p & $\begin{array}{c}\text { SNR } \\
\text { S-WLS }\end{array}$ & $\begin{array}{c}\text { SNR } \\
\text { X-11 }\end{array}$ & $\begin{array}{c}\text { SNR } \\
\text { S-WLS / } \\
\text { SNR X-11 }\end{array}$ \\
\hline $\mathbf{2}$ & 8,45 & 6,69 & 1,000 & 2,08 & 2,31 & 1,000 & 6,4 & 9,5 & 0,7 \\
$\mathbf{3}$ & 3,88 & 4,01 & 0,008 & 1,61 & 1,58 & 0,001 & 13,6 & 13,3 & 1,0 \\
$\mathbf{4}$ & 2,34 & 3,09 & 0,000 & 1,42 & 1,22 & 0,000 & 23,5 & 17,7 & 1,3 \\
$\mathbf{6}$ & 1,14 & 2,35 & 0,000 & 1,25 & 0,85 & 0,000 & 48,6 & 23,3 & 2,1 \\
$\mathbf{8}$ & 0,69 & 2,07 & 0,000 & 1,18 & 0,66 & 0,000 & 77,8 & 26,2 & 3,0 \\
$\mathbf{1 0}$ & 0,51 & 1,96 & 0,000 & 1,15 & 0,57 & 0,000 & 107,7 & 27,8 & 3,9 \\
\hline
\end{tabular}

Tabela 5.19 MSE, MAD e SNR para valores de ' $A / s$ ', sendo $k=72, b=40 \%$ : séries mensais com decomposição aditiva (Eq. 5-1) 
(iv) Condições nas quais as estatísticas do filtro S-WLS superam o filtro X11 , segundo valores de ' $A / s$ ', ' $b$ ' e ' $k$ ': série mensal com decomposição aditiva

A fim de avaliar as condições que fazem o filtro S-WLS superar o X-11, foram comparadas as estatísticas dos filtros, para alguns parâmetros do sinal artificial. Isso foi realizado com base na variação de ' $b$ ', ' $k$ ' e da razão ' $A / s$ '.

Para analisar os resultados, buscou-se identificar a partir de qual valor de ' $\boldsymbol{b}$ ' (taxa de variação da sazonalidade), o filtro S-WLS começa a superar o X-11, para cada valor de ' $\boldsymbol{k}$ ' (número de meses no qual o padrão de sazonalidade móvel volta a se repetir) e ' $\boldsymbol{A} / \boldsymbol{s}$ ' (amplitude do sinal em relação ao desvio-padrão da irregular). Os resultados foram analisados com base nas estatísticas MSE, MAD e SNR.

O Quadro 5.11 apresenta os resultados para as séries sem tendência (Não) e com tendência ( $\mathrm{Sim}$ ). O valor em cada célula, no corpo da tabela, indica a partir de qual ' $\boldsymbol{b}$ ' o filtro S-WLS supera o X-11. Apenas os resultados dos testes cujo valor$\mathrm{p}<5 \%$ foram considerados significativamente melhores. No quadro, todos os valores-p não apresentados são menores do que $1 \%$. 


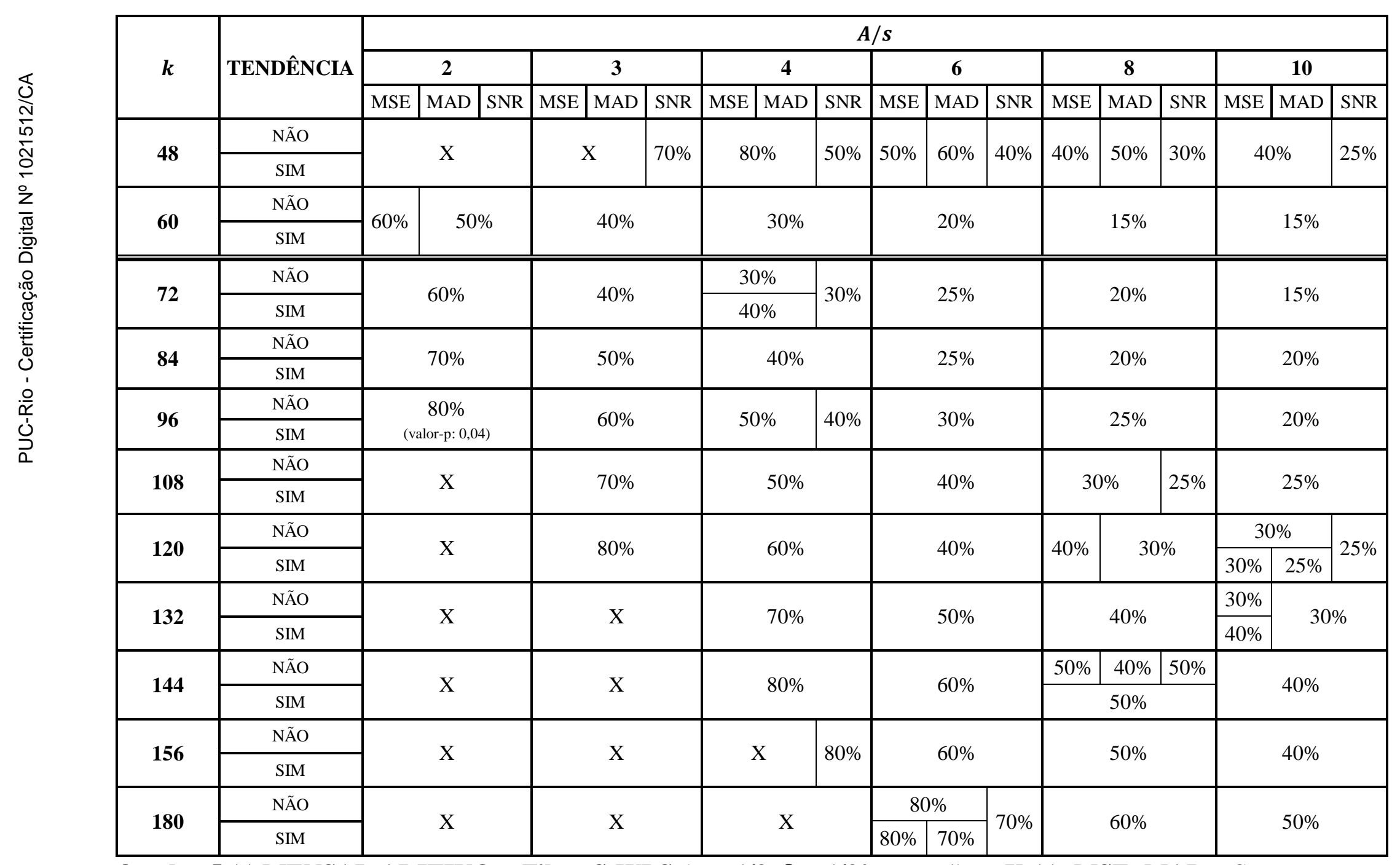

Quadro 5.11 MENSAL ADITIVO - Filtro S-WLS $\left(\alpha=1 / 3, \delta=1 / 30, w_{0}=1\right)$ vs X-11: MSE, MAD e SNR. Valores de ' $b$ ' a partir do qual o filtro S-WLS supera o X-11, para cada ' $k$ ' e ' $A / s$ '. Séries com e sem tendência (Eq. 5-1) 
Analisando o Quadro 5.11, nota-se que quanto maior for a razão ' $A / s$ ', e menor o valor de ' $k$ ', o filtro S-WLS supera o X-11 a partir de valores baixos de ' $b$ ', como 15\%. Nota-se, também, uma consistência nos resultados obtidos da MSE, MAD e SNR. Em poucos casos eles diferem, e ainda assim o resultado é próximo.

Em relação ao ajuste realizado pelo filtro S-WLS na presença de componente de tendência, destaca-se a sua robustez, com base nos resultados obtidos para as séries temporais com e sem tendência. Em apenas dois casos eles diferem em relação ao MSE.

Quanto ao parâmetro ' $k$ ', vale ressaltar que para os valores 48 e 60, o filtro S-WLS não é indicado, pois $\alpha=1 / 3$ contempla apenas $k \geq 72$. Com isso, notamse valores de ' $b$ ' altos para $k=48$. Porém, para $k=60$, os resultados são satisfatórios, levando a concluir que, ao ser comparado com o X-11, o filtro S-WLS apresenta melhor desempenho. Isso ocorre devido à resposta em magnitude do filtro S-WLS, pois esta apresenta bandas de transição caindo suavemente, o que pode gerar bons resultados para séries com $k<72$.

Para ilustrar a razão entre a SNR do filtro S-WLS e a SNR do X-11, e os parâmetros ' $k$ ' e ' $A / s$ ', foram construídos os Gráficos 5.28 e 5.29. Quanto ao Gráfico 5.28, observa-se que à medida que o valor de ' $k$ ' aumenta, a taxa de variação da sazonalidade $(b)$ precisa ser cada vez maior para o filtro S-WLS superar o X-11. Já no Gráfico 5.29, nota-se que para $A / s=6$ é necessário apenas $b=25 \%$ para o filtro S-WLS apresentar desempenho superior ao X-11.

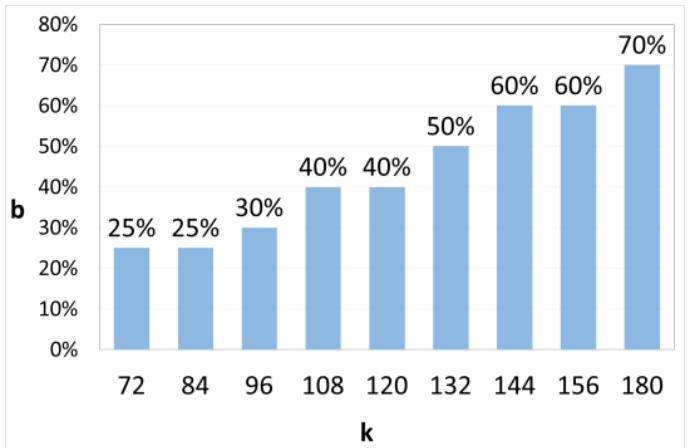

Gráfico 5.28 Valores de ' $b$ ' a partir do qual o filtro S-WLS supera o X-11, na SNR, para vários valores de ' $k$ ', considerando $A / s=6$ (Eq. 5-1)

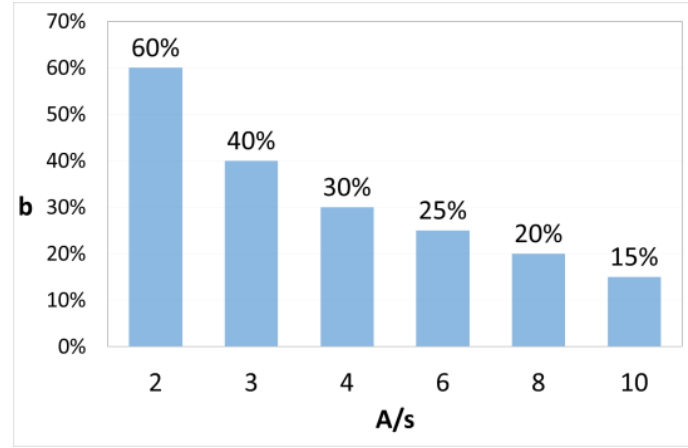

Gráfico 5.29 Valores de ' $b$ ' a partir do qual o filtro $S$-WLS supera o $X-11$, na SNR, para vários valores de ' $A / s$, considerando $k=72$ (Eq. 5-1) 


\subsubsection{1. \\ Espectros da componente irregular: série mensal com decomposição aditiva}

Uma outra forma de comparar a qualidade da extração da componente sazonal realizada pelo filtro S-WLS, em relação ao X-11, é elaborar o espectro da componente irregular, da série dessazonalizada (Hannan, 1964). Tais espectros estão apresentados nos Gráficos 5.30 a 5.45, e foram construídos para diferentes valores de ' $k$ ' e ' $b$ '. Quanto à razão ' $A / s$ ', foi utilizado o valor 6.

O motivo de analisar o espectro da irregular está no fato de que se o filtro extrair a sazonalidade de forma adequada (ou seja, remover todo o efeito sazonal), o espectro da irregular não apresentará picos nas frequências sazonais, ou próximos a elas.

Os espectros foram elaborados considerando sinais sazonais com $b=40 \%$, $25 \%$ e $10 \%$; e $k=72$ e 96 . Quanto maior o valor de ' $b$ ', e menor o valor de ' $k$ ', mais forte é a sazonalidade móvel. Vale mencionar que as séries artificiais não apresentam componente de tendência.

Os gráficos 5.30 a 5.35 apresentam os espectros da irregular para séries com $k=72$, o que indica um grau de sazonalidade móvel forte, e tamanho de filtro $(N)$ igual a 121. Tais filtros são adequados para o X-11, na dessazonalização de séries com sazonalidade móvel, pois utilizam média móvel sazonal 3x3. Os gráficos estão apresentados em pares, sendo o primeiro gráfico referente ao ajuste realizado pelo filtro S-WLS, e o segundo referente ao ajuste realizado pelo método X-11. 


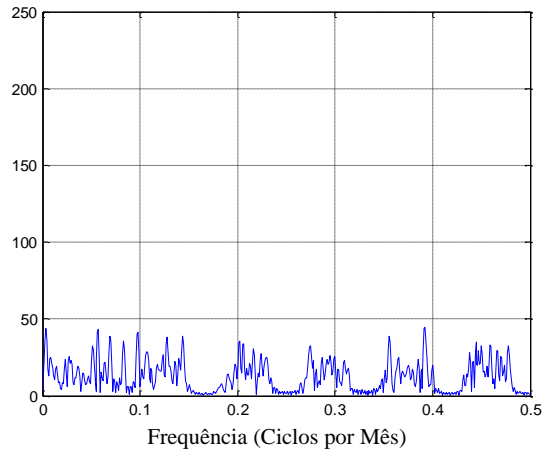

Gráfico 5.30 Espectro da irregular da série dessazonalizada pelo filtro S-WLS, com: $A / s=6, k=72, b=40 \%$ e $N=121$

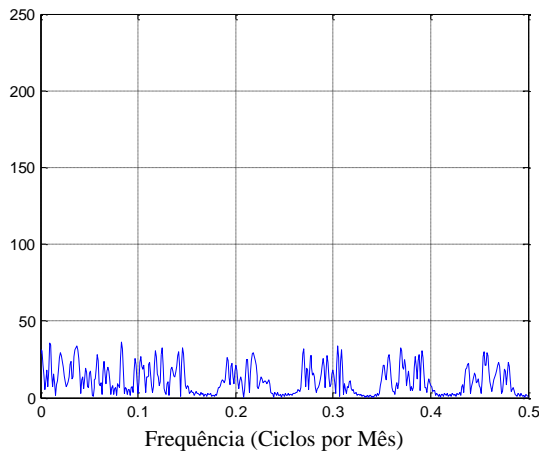

Gráfico 5.32 Espectro da irregular da série dessazonalizada pelo filtro $S$ WLS, com: $A / s=6, k=72, b=25 \% \mathrm{e}$ $N=121$

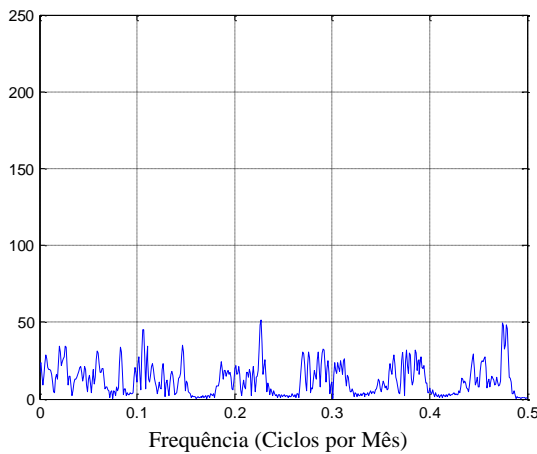

Gráfico 5.34 Espectro da irregular da série dessazonalizada pelo filtro S-WLS, com: $A / s=6, k=72, b=10 \%$ e $N=121$

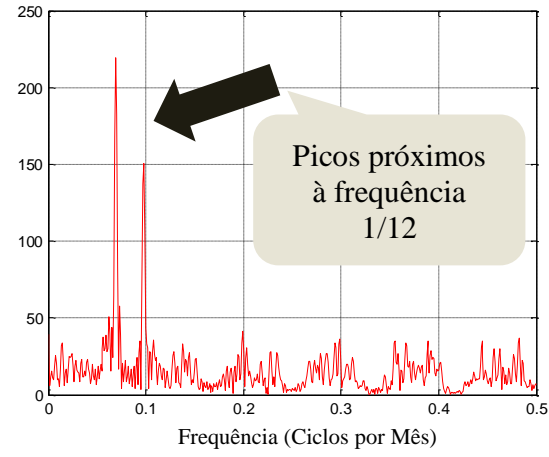

Gráfico 5.31 Espectro da irregular da série dessazonalizada pelo $X-11$, com: $A / s=6, k=72, b=40 \%$ e $N$ $=121$

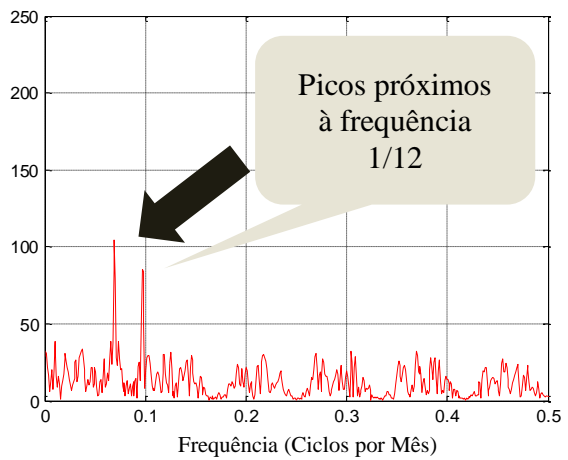

Gráfico 5.33 Espectro da irregular da série dessazonalizada pelo $\mathrm{X}-11$, com: $A / s=6, k=72, b=25 \%$ e $N$ $=121$

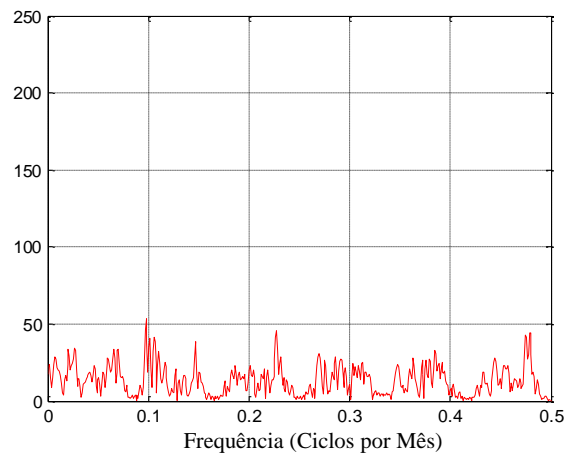

Gráfico 5.35 Espectro da irregular da série dessazonalizada pelo $X-11$, com: $A / s=6, k=72, b=10 \%$ e $N=121$

Analisando os Gráficos 5.30 a 5.35, nota-se que os espectros da irregular cuja série original foi dessazonalizada pelo X-11 apresentam picos próximos à frequência $\frac{1}{12}$, exceto para o caso em que $b=10 \%$, que representa uma taxa pequena de variação da amplitude sazonal. Já os espectros da irregular das séries dessazonalizadas pelo filtro S-WLS não apresentam picos na frequência sazonal, ou próximos a ela, indicando um ajuste sazonal adequado. 
Os gráficos 5.36 a 5.41 apresentam os espectros da irregular para séries com $k=96$, indicando que o padrão sazonal se repete em 8 anos. Os pares de espectros (S-WLS e X-11) mostram os resultados para $b=40 \%, 25 \%$ e $10 \%$, respectivamente.

Analisando os gráficos, quando $k=96$, os picos nos espectros do $\mathrm{X}-11$ reduzem, porém, continuam presentes - exceto para o caso em que $b=10 \%$.

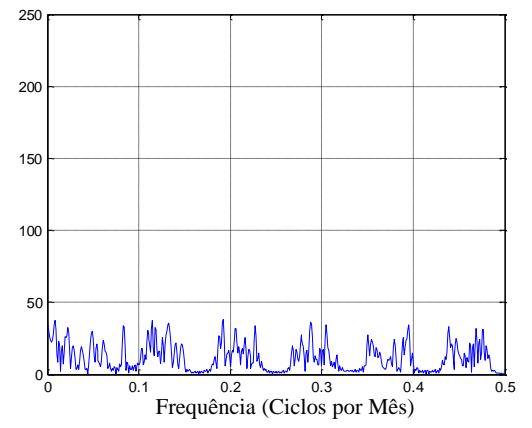

Gráfico 5.36 Espectro da irregular da série dessazonalizada pelo filtro $S$-WLS, com: $A / s=6, k=96, b=40 \%$ e $N=121$

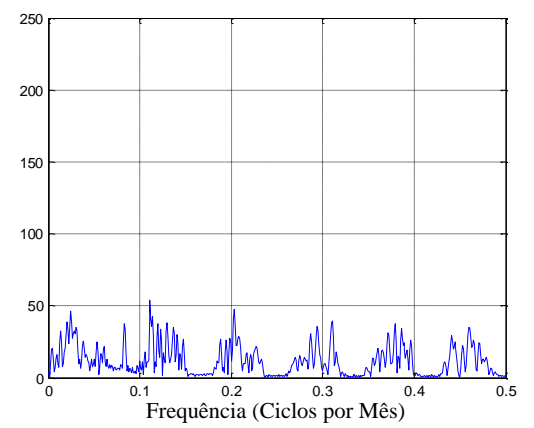

Gráfico 5.38 Espectro da irregular da série dessazonalizada pelo filtro $S$ WLS, com: $A / s=6, k=96, b=25 \%$ e $N$ $=121$

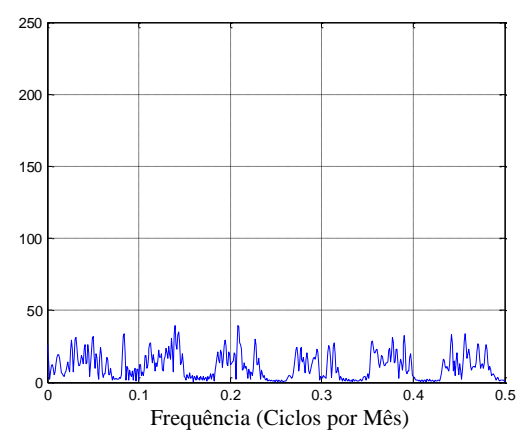

Gráfico 5.40 Espectro da irregular da série dessazonalizada pelo filtro S-WLS, com: $A / s=6, k=96, b=10 \%$ e $N=121$

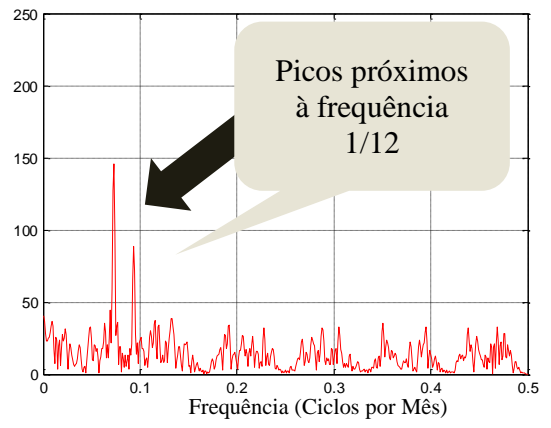

Gráfico 5.37 Espectro da irregular da série dessazonalizada pelo $X-11$, com: $A / s=6, k=96, b=40 \%$ e $N=121$

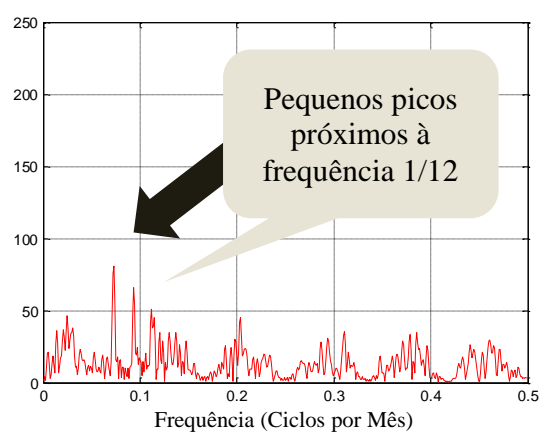

Gráfico 5.39 Espectro da irregular da série dessazonalizada pelo $X-11$, com: $A / s=6, k=96, b=$ $25 \%$ e $N=121$

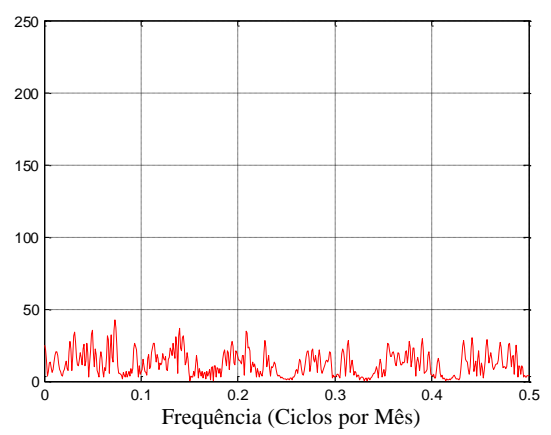

Gráfico 5.41 Espectro da irregular da série dessazonalizada pelo $X-11$, com: $A / s=6, k=96, b=$ $10 \%$ e $N=121$ 
Por fim, os Gráficos 5.42 a 5.45 apresentam os espectros da irregular para séries com $k=180$, que representa um grau baixo de sazonalidade móvel. Nesse caso, apenas o espectro do X-11 referente a taxa de variação sazonal ' $b$ ' igual a $40 \%$ apresenta um pequeno pico próximo à frequência $\frac{1}{12}$.

Mais uma vez, os espectros relacionados à irregular do filtro S-WLS não apresentam picos.

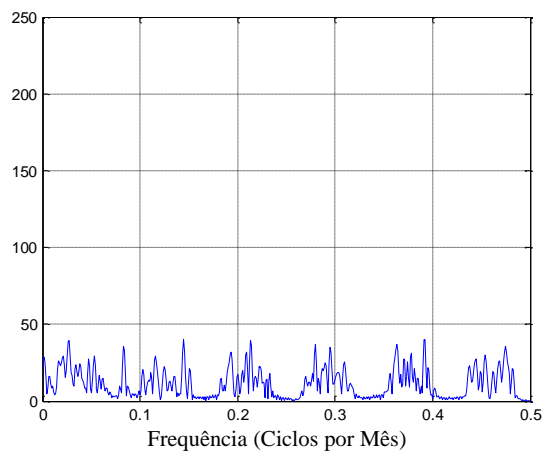

Gráfico 5.42 Espectro da irregular da série dessazonalizada pelo filtro S-WLS, com: $A / s=6, k=180, b=40 \%$ e $N=121$

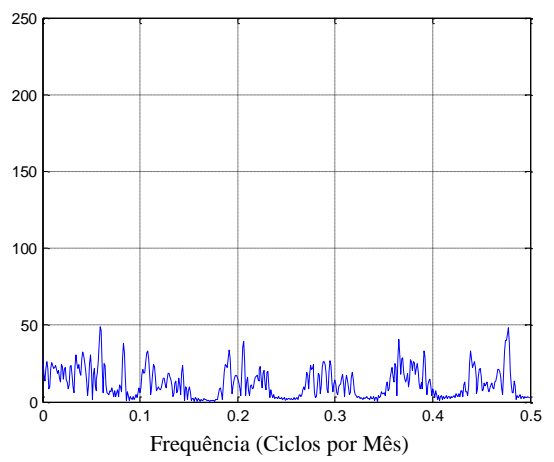

Gráfico 5.44 Espectro da irregular da série dessazonalizada pelo filtro $S$-WLS, com: $A / s=6, k=180, b=10 \%$ e $N=121$

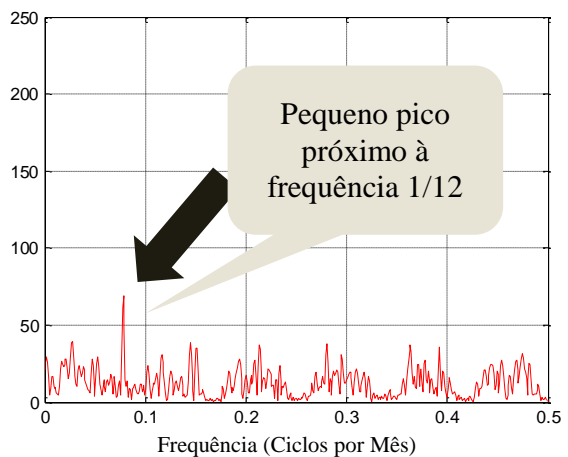

Gráfico 5.43 Espectro da irregular da série dessazonalizada pelo $X-11$, com: $A / s=6, k=180, b$ $=40 \%$ e $N=121$

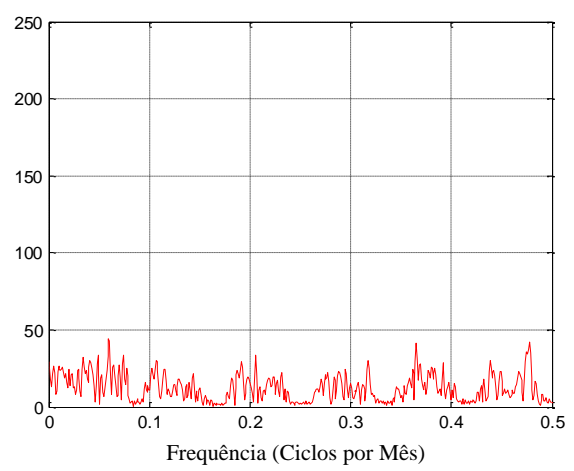

Gráfico 5.45 Espectro da irregular da série dessazonalizada pelo $X-11$, com: $A / s=6, k=180, b=$ $10 \%$ e $N=121$ 


\subsection{3.}

\section{Resultados: série mensal com decomposição multiplicativa - filtro S-WLS vs $\mathrm{X}-11$}

No caso das séries mensais com modelo multiplicativo de decomposição, pode-se optar por trabalhar com o logaritmo da mesma e utilizar o modelo aditivo de decomposição; ou não transformar a série e seguir como descrito em Findley et al. (1998). Optou-se aqui pela utilização do procedimento indicado em Findley et al. (1998), uma vez que utilizar a transformação logarítmica, equivale a trabalhar com o modelo aditivo de decomposição, que já foi tratado na Subseção 5.5.2. Além disso, como ressaltado por Franses et al. (1998), no caso de sazonalidade multiplicativa a transformação logarítmica altera a série de várias formas, ao contrário de simplesmente remover a variação não-constante.

Recorda-se que em se tratando do modelo multiplicativo de decomposição $(Y=T \times S \times I)$, a série temporal $(Y)$ pode ser reescrita da seguinte forma (ver Eq. $5-5$ a $5-7)$ :

$$
Y=T+T S^{\prime}+T I^{\prime}+T S^{\prime} I^{\prime},
$$

onde $S^{\prime}=(S-1)$ e $I^{\prime}=(I-1)$. Com isso, $S^{\prime}$ e $I^{\prime}$ têm média zero; enquanto $S$ e $I$ têm média igual a um. Vale ressaltar que $T I^{\prime}$ e $T S^{\prime} I^{\prime}$ têm espectro de irregular, podendo ser tratadas como tal.

De acordo com a discussão relacionada à Eq. (5-7), a utilização do filtro proposto para a extração da componente sazonal, no caso de decomposição multiplicativa, foi realizada segundo o procedimento descrito a seguir.

(i) Aplica-se o filtro proposto na série original, para a extração da componente sazonal. Porém, como a decomposição é multiplicativa, ele traz a tendência junto com a sazonalidade, resultando em $T S^{\prime}$;

(ii) Subtrai-se, da série original $(Y)$, o resultado $T S^{\prime}$, obtido em (i), donde $Y=T \times S \times I=T \times\left(S^{\prime}+1\right) \times\left(I^{\prime}+1\right)$, resultando em:

$$
\begin{gathered}
Y-T S^{\prime}=T S I-T S^{\prime}= \\
T\left(S I-S^{\prime}\right)
\end{gathered}
$$

(iii) Com o resultado de (ii), aplica-se o filtro de Henderson. Consequentemente, tem-se a componente de tendência $(T)$;

(iv) Por fim, divide-se o resultado de (i) pelo resultado obtido em (iii), e somase uma unidade: $\left[\frac{T S^{\prime}}{T}+1\right]$, obtendo-se a componente sazonal $S$.

A Figura 5.8 ilustra, mais detalhadamente, esse procedimento. 


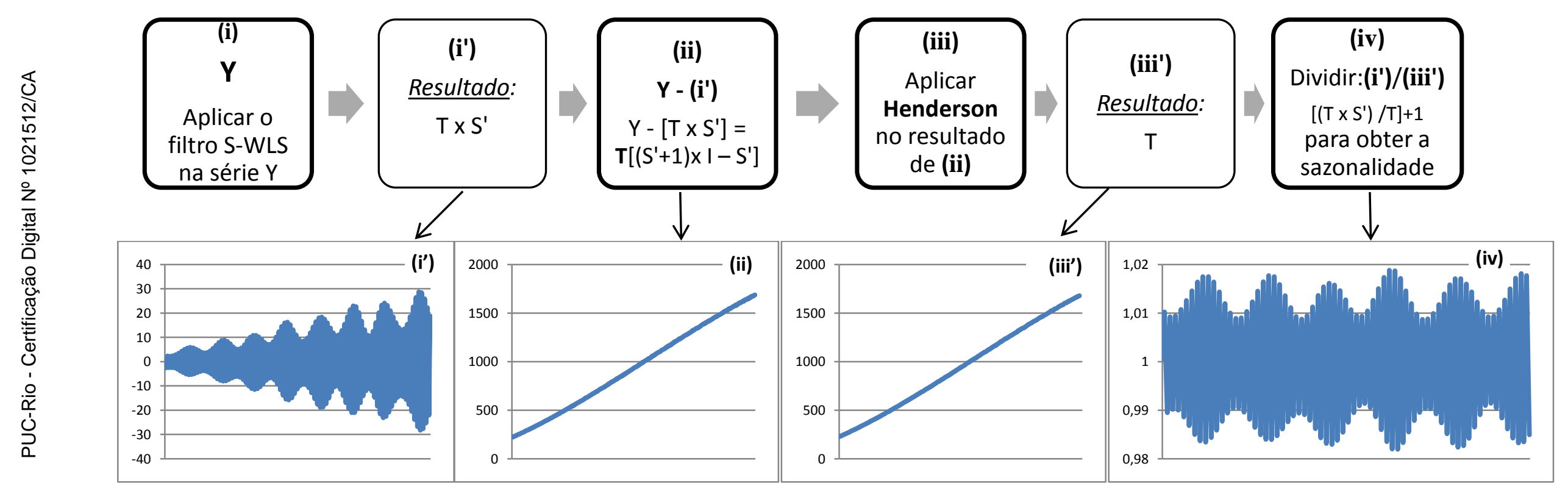

Figura 5.8 Procedimento utilizado para a obtenção da componente sazonal utilizando o filtro proposto (S-WLS), nas séries multiplicativas

O procedimento apresentado na Figura 5.8 pode ser detalhado da seguinte forma:

(i) Inicialmente aplica-se o filtro proposto na série original $Y$.

(i') A série resultante será $T S^{\prime}$, ou seja, Tendência x (Sazonalidade - 1), apresentada no gráfico (i').

(ii) Subtrai-se $T S^{\prime}$ de $Y$. O resultado é $T\left[\left(S^{\prime}+1\right) I-S^{\prime}\right]$, apresentado no gráfico (ii).

(iii) Aplica-se o filtro de Henderson em $T\left[\left(S^{\prime}+1\right) I-S^{\prime}\right]$.

(iii') O resultado de (iii) é a componente de tendência $T$, apresentada no gráfico (iii').

(iv) Por fim, faz-se $\left[\frac{T S^{\prime}}{T}+1\right]$, e com isso tem-se a componente sazonal $S$. 
Ao utilizar esse procedimento de extração da componente sazonal, não é possível obter os valores de $S Q$, assim como calcular os $\gamma_{s}$, pois a resposta em magnitude do filtro não pode ser reproduzida. Sendo assim, optou-se por trabalhar com as medidas de erro: MSE (Eq. 5-18) e MAD (Eq. 5-19), para a comparação dos resultados obtidos pelo filtro proposto e pelo filtro X-11, com base em séries artificiais.

$\mathrm{Na}$ comparação dos resultados dos filtros, foram utilizados os seguintes valores para o sinal sazonal artificial:

- $A / s=[1,2,3,4,6,8,10]$

- $b=[15 \%, 20 \%, 25 \%, 30 \%, 40 \%, 50 \%, 60 \%, 70 \%, 80 \%]$

- $k=[72,84,96,108,120,132,144,156,180]$

Foram geradas séries com, e sem, componente de tendência. Nas séries com componente de tendência, foram usados os coeficientes: $b_{0}=98,2, b_{1}=0,058$, $b_{2}=-0,0014$ e $b_{3}=0,000005$, obtidos na série Y179.

Em relação à componente irregular, foram geradas 100 séries, com distribuição $\mathrm{N}\left(0, \sigma^{2}\right)$, utilizando a função randn do MATLAB, para cada combinação de parâmetros do sinal simulado. Com isso, calculou-se a média dos valores da MSE e MAD, para as diversas combinações de parâmetros testadas.

Para a comparação das médias das medidas de erro (MSE e MAD) de cada filtro, foi realizado o teste $t$, de igualdade de médias de duas populações com variâncias desconhecidas e equivalentes (Eq. 5-20). No teste, utilizou-se $\alpha=5 \%$.

Nos itens a seguir são apresentados os resultados das comparações entre o desempenho do filtro S-WLS e o desempenho do X-11, sob diversos aspectos relacionados aos parâmetros do sinal artificial. Os resultados estão apresentados da seguinte forma: o item (i) apresenta o resultado obtido com a variação de ' $b$ '; no item (ii) é exibido o resultado da variação de ' $k$ '; o item (iii) compara o desempenho dos filtros segundo as variações de ' $A / s$ '; e o item (iv) mostra as condições nas quais o filtro S-WLS supera o X-11, para diversos valores de ' $b$ ', ' $k$ ' e ' $A / s$ ', simultaneamente. 
(i) Resultados encontrados com base na variação de ' $b$ ': série mensal com decomposição multiplicativa

Os resultados encontrados, a partir da variação do parâmetro ' $b$ ', estão apresentados no Gráfico 5.46. Para a confecção do gráfico, os valores da MSE foram multiplicados por $10^{6}$.

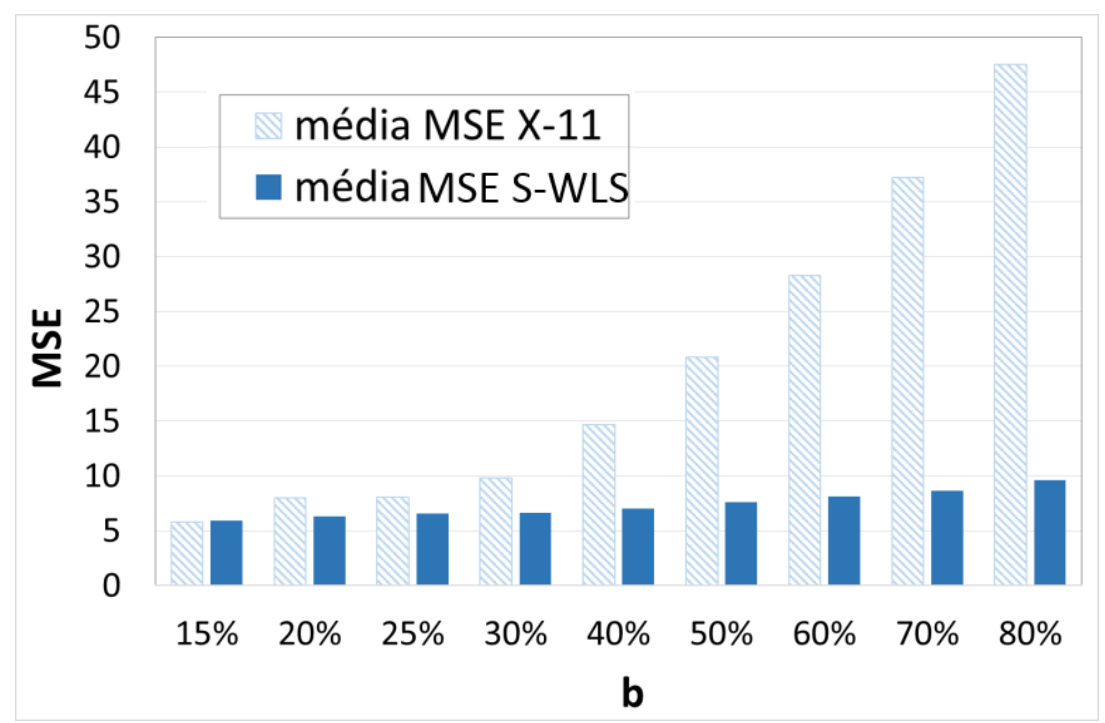

Gráfico 5.46 Média da MSE $\left(\mathrm{x}^{10}{ }^{6}\right)$ com o filtro X-11 e com o filtro S-WLS, para valores de ' $b$ ', considerando $A / s=6$ e $k=72(\mathrm{Eq} .5-3)$

Analisando o Gráfico 5.46, nota-se um padrão quase que constante nos resultados da MSE do filtro S-WLS, para os diversos valores de ' $b$ '. Já a MSE do filtro X-11 cresce exponencialmente à medida que aumenta a taxa de variação da amplitude sazonal.

Vale citar que, a partir de $b=20 \%$, o ajuste sazonal realizado pelo filtro SWLS obtém MSE significativamente menor quando comparado ao X-11. Além disso, para $b=40 \%$, a MSE do X-11 passa a ser maior do que o dobro da MSE do filtro S-WLS. E, ao analisar as séries reais mensais com decomposição multiplicativa, foi constatado uma grande quantidade delas com ' $b$ ' entre $20 \%$ e $40 \%$. 
A Tabela 5.20 apresenta os dados originais que serviram para a construção dos gráficos. Os valores se referem ao MSE e à MAD, do filtro S-WLS e do X-11, para diversos valores de ' $b$ '. Nessa análise, considerou-se $A / s=6$ e $k=72$. A tabela apresenta, também, o valor-p do teste $t$ de comparação de médias.

\begin{tabular}{c|ccc|ccc}
\hline $\boldsymbol{b}$ & MSE S-WLS & MSE X-11 & valor-p & MAD S-WLS & MAD X-11 & valor-p \\
\hline $\mathbf{1 5 \%}$ & $6,0 \mathrm{E}-06$ & $5,8 \mathrm{E}-06$ & 0,938 & $1,94 \mathrm{E}-03$ & $2,0 \mathrm{E}-03$ & 0,288 \\
$\mathbf{2 0 \%}$ & $6,3 \mathrm{E}-06$ & $8,0 \mathrm{E}-06$ & 0,000 & $2,01 \mathrm{E}-03$ & $2,3 \mathrm{E}-03$ & 0,000 \\
$\mathbf{2 5 \%}$ & $6,6 \mathrm{E}-06$ & $8,0 \mathrm{E}-06$ & 0,000 & $2,05 \mathrm{E}-03$ & $2,3 \mathrm{E}-03$ & 0,000 \\
$\mathbf{3 0 \%}$ & $6,6 \mathrm{E}-06$ & $9,8 \mathrm{E}-06$ & 0,000 & $2,05 \mathrm{E}-03$ & $2,5 \mathrm{E}-03$ & 0,000 \\
$\mathbf{4 0 \%}$ & $7,0 \mathrm{E}-06$ & $1,5 \mathrm{E}-05$ & 0,000 & $2,12 \mathrm{E}-03$ & $3,1 \mathrm{E}-03$ & 0,000 \\
$\mathbf{5 0 \%}$ & $7,6 \mathrm{E}-06$ & $2,1 \mathrm{E}-05$ & 0,000 & $2,20 \mathrm{E}-03$ & $3,7 \mathrm{E}-03$ & 0,000 \\
$\mathbf{6 0 \%}$ & $8,1 \mathrm{E}-06$ & $2,8 \mathrm{E}-05$ & 0,000 & $2,27 \mathrm{E}-03$ & $4,4 \mathrm{E}-03$ & 0,000 \\
$\mathbf{7 0 \%}$ & $8,6 \mathrm{E}-06$ & $3,7 \mathrm{E}-05$ & 0,000 & $2,35 \mathrm{E}-03$ & $5,0 \mathrm{E}-03$ & 0,000 \\
$\mathbf{8 0 \%}$ & $9,6 \mathrm{E}-06$ & $4,8 \mathrm{E}-05$ & 0,000 & $2,57 \mathrm{E}-03$ & $5,7 \mathrm{E}-03$ & 0,000 \\
\hline
\end{tabular}

Tabela 5.20 MSE e MAD para valores de ' $b$ ', sendo $A / s=6, k=72$ : séries mensais com decomposição multiplicativa (Eq. 5-3) 
(ii) Resultados encontrados com base na variação de ' $k$ ': série mensal com decomposição multiplicativa

O Gráfico 5.47 apresenta a média da MSE de cada filtro, para diversos valores de ' $k$ ', mantendo constantes a taxa ' $b$ ' e a razão ' $A / s$ '.

Recorda-se que o parâmetro ' $k$ ' indica o número de meses que o padrão de sazonalidade móvel volta a se repetir. Nas séries reais, ' $k$ ' aparece com mais frequência entre 72 e 96, seguido de 120 a 144.

Novamente, percebe-se a MSE do filtro S-WLS mantendo-se num mesmo nível para os diversos valores de ' $k$ ', enquanto que a MSE do X-11 aumenta à medida que o valor de ' $k$ ' diminui. Isso mostra que os erros de ajuste do filtro $\mathrm{X}$ 11 ficam maiores quando o grau de sazonalidade móvel aumenta.

Nota-se que para valores de $k \geq 132$, o filtro X-11 começa a apresentar MSE menor do que o filtro S-WLS. Porém, destaca-se que a diferença entre as MSEs dos filtros, para $k \geq 132$, não são tão grandes se comparadas à diferença em relação aos valores baixos de ' $k$ '. Por exemplo, para $k=156$, a MSE do filtro S-WLS é $26 \%$ maior do que a MSE do X-11, mas para $k=84$, a MSE do X-11 é 72\% maior do que a MSE do filtro S-WLS. Isso mostra que quando o X-11 é melhor, ele é um pouco melhor; mas quando é pior, ele é muito pior.

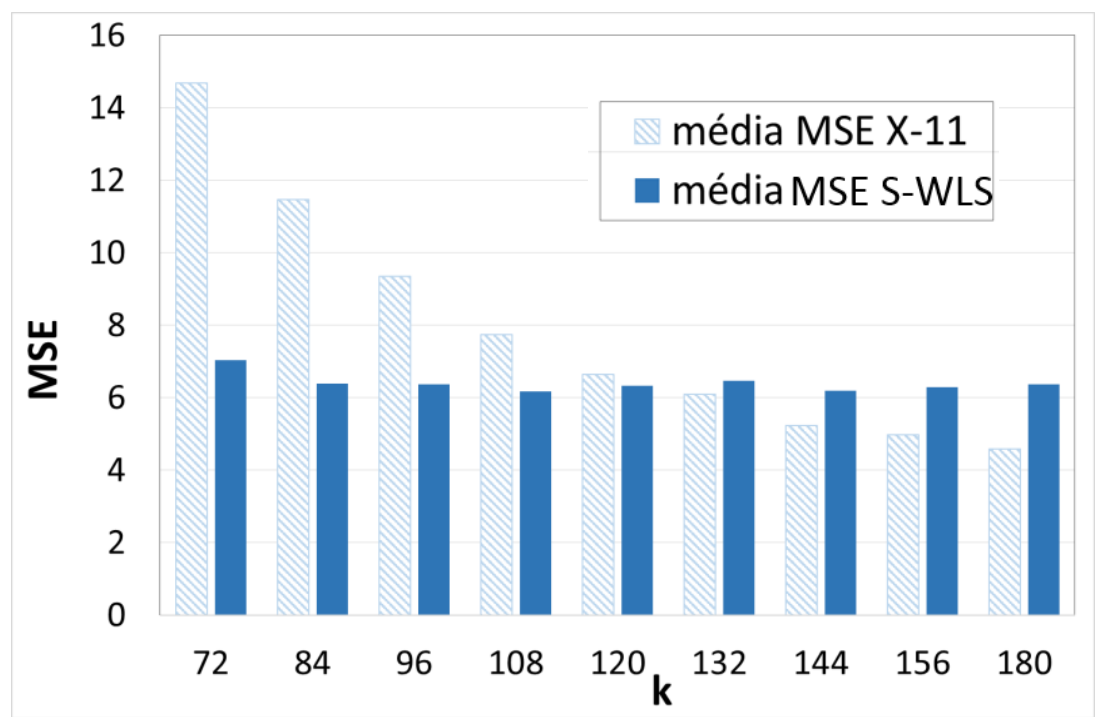

Gráfico 5.47 Média da MSE $\left(\mathrm{x}^{10^{6}}\right)$ com o filtro X-11 e com o filtro S-WLS, para valores de ' $k$ ', considerando $A / s=6$ e $b=40 \%(E q . ~ 5-3)$ 
Os dados utilizados na construção do Gráfico 5.47 estão apresentados na Tabela 5.21, a seguir. A tabela contém os resultados da MSE e MAD, do filtro SWLS e do X-11, para diversos valores de ' $k$, considerando $A / s=6$ e $b=40 \%$. Além disso, a tabela apresenta o valor-p do teste de comparação de médias.

\begin{tabular}{c|ccc|ccc}
\hline $\boldsymbol{k}$ & MSE S-WLS & MSE X-11 & valor-p & MAD S-WLS & MAD X-11 & valor-p \\
\hline $\mathbf{7 2}$ & $7,0 \mathrm{E}-06$ & $1,5 \mathrm{E}-05$ & 0,000 & $2,12 \mathrm{E}-03$ & $3,1 \mathrm{E}-03$ & 0,000 \\
$\mathbf{8 4}$ & $6,4 \mathrm{E}-06$ & $1,1 \mathrm{E}-05$ & 0,000 & $2,02 \mathrm{E}-03$ & $2,8 \mathrm{E}-03$ & 0,000 \\
$\mathbf{9 6}$ & $6,4 \mathrm{E}-06$ & $9,3 \mathrm{E}-06$ & 0,000 & $2,01 \mathrm{E}-03$ & $2,5 \mathrm{E}-03$ & 0,000 \\
$\mathbf{1 0 8}$ & $6,2 \mathrm{E}-06$ & $7,7 \mathrm{E}-06$ & 0,000 & $1,98 \mathrm{E}-03$ & $2,3 \mathrm{E}-03$ & 0,000 \\
$\mathbf{1 2 0}$ & $6,3 \mathrm{E}-06$ & $6,6 \mathrm{E}-06$ & 0,003 & $2,01 \mathrm{E}-03$ & $2,1 \mathrm{E}-03$ & 0,000 \\
$\mathbf{1 3 2}$ & $6,5 \mathrm{E}-06$ & $6,1 \mathrm{E}-06$ & 1,000 & $2,03 \mathrm{E}-03$ & $2,0 \mathrm{E}-03$ & 0,994 \\
$\mathbf{1 4 4}$ & $6,2 \mathrm{E}-06$ & $5,2 \mathrm{E}-06$ & 1,000 & $1,98 \mathrm{E}-03$ & $1,8 \mathrm{E}-03$ & 1,000 \\
$\mathbf{1 5 6}$ & $6,3 \mathrm{E}-06$ & $5,0 \mathrm{E}-06$ & 1,000 & $2,00 \mathrm{E}-03$ & $1,8 \mathrm{E}-03$ & 1,000 \\
$\mathbf{1 8 0}$ & $6,4 \mathrm{E}-06$ & $4,6 \mathrm{E}-06$ & 1,000 & $2,01 \mathrm{E}-03$ & $1,7 \mathrm{E}-03$ & 1,000 \\
\hline
\end{tabular}

Tabela 5.21 MSE e MAD para valores de ' $k$ ', sendo $A / s=6, b=40 \%$ : séries mensais com decomposição multiplicativa (Eq. 5-3) 
(iii) Resultados encontrados com base na variação de ' $A / s$ ': série mensal com decomposição multiplicativa

Os resultados obtidos com base na razão entre a amplitude do sinal, dada por ' $A$ ', e o desvio-padrão da componente irregular, dado por ' $s$ ', estão ilustrados no Gráfico 5.48. Nessa análise, utilizou-se $k=72$ e $b=40 \%$.

Assim como observado no caso das séries mensais com decomposição aditiva, o MSE do filtro S-WLS apresenta redução mais acentuada, do que o MSE do $\mathrm{X}-11$, à medida que a razão ' $A / s$ ' aumenta.

Percebe-se também que para $A / s>3$, o resultado do filtro S-WLS já supera, de forma significativa, o filtro X-11.

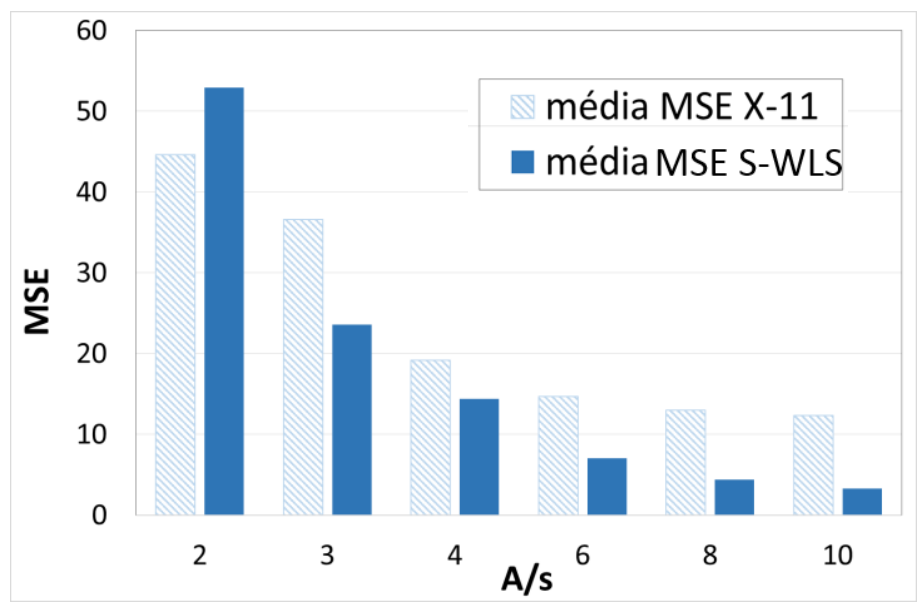

Gráfico 5.48 Média da MSE ( $\left(\mathrm{10}^{6}\right)$ com o filtro X-11 e com o filtro S-WLS, para valores de ' $A / s$ ', considerando $k=72$ e $b=40 \%$ (Eq. 5-3)

Na Tabela 5.22, estão expostos os dados referentes ao Gráfico 5.48. Os valores se referem à MSE e à MAD, do filtro S-WLS e do X-11, para diversos valores de ' $A / s$ ', considerando a taxa de variação da sazonalidade $(b)$ igual a $40 \%$, e o número de meses, no qual o padrão de sazonalidade móvel volta a se repetir $(k)$, igual a 72 .

\begin{tabular}{c|ccc|ccc}
\hline $\boldsymbol{A} / \boldsymbol{s}$ & MSE S-WLS & MSE X-11 & valor-p & MAD S-WLS & MAD X-11 & valor-p \\
\hline $\mathbf{2}$ & $5 \mathrm{E}-05$ & $4 \mathrm{E}-05$ & 1,000 & $6 \mathrm{E}-03$ & $5 \mathrm{E}-03$ & 1,000 \\
$\mathbf{3}$ & $2 \mathrm{E}-05$ & $4 \mathrm{E}-05$ & 0,000 & $4 \mathrm{E}-03$ & $5 \mathrm{E}-03$ & 0,000 \\
$\mathbf{4}$ & $1 \mathrm{E}-05$ & $2 \mathrm{E}-05$ & 0,000 & $3 \mathrm{E}-03$ & $4 \mathrm{E}-03$ & 0,000 \\
$\mathbf{6}$ & $7 \mathrm{E}-06$ & $1 \mathrm{E}-05$ & 0,000 & $2 \mathrm{E}-03$ & $3 \mathrm{E}-03$ & 0,000 \\
$\mathbf{8}$ & $4 \mathrm{E}-06$ & $1 \mathrm{E}-05$ & 0,000 & 2E-03 & $3 \mathrm{E}-03$ & 0,000 \\
$\mathbf{1 0}$ & $3 \mathrm{E}-06$ & $1 \mathrm{E}-05$ & 0,000 & $1 \mathrm{E}-03$ & $3 \mathrm{E}-03$ & 0,000 \\
\hline
\end{tabular}

Tabela 5.22 MSE e MAD para valores de ' $A / s$ ', sendo $k=72, b=40 \%$ (Eq. 5-3): séries mensais com decomposição multiplicativa 
(iv) Condições nas quais a MSE e a MAD do filtro S-WLS são melhores do que o $\mathrm{X}-11$, segundo valores de ' $A / s^{\prime}$, ' $b$ ' e ' $k$ ': série mensal com decomposição multiplicativa

O Quadro 5.12 apresenta o resultado dos testes para as séries sem tendência (Não) e com tendência (Sim). O valor em cada célula, no corpo da tabela, indica a partir de qual ' $\boldsymbol{b}$ ' o filtro S-WLS supera o X-11, em relação à MSE e à MAD - com base na variação de diversos valores de ' $k$ ' e ' $A / s$ '.

Nos testes realizados, apenas os resultados com valor-p $<5 \%$ foram considerados significativamente melhores. No Quadro 5.12, todos os valores-p encontrados foram menores do que $1 \%$.

\begin{tabular}{|c|c|c|c|c|c|c|c|c|c|c|c|c|c|}
\hline \multirow{3}{*}{$\boldsymbol{k}$} & \multirow{3}{*}{ TENDÊNCIA } & \multicolumn{12}{|c|}{$A / s$} \\
\hline & & \multicolumn{2}{|c|}{2} & \multicolumn{2}{|c|}{3} & \multicolumn{2}{|r|}{4} & \multicolumn{2}{|c|}{6} & \multicolumn{2}{|c|}{8} & \multicolumn{2}{|c|}{10} \\
\hline & & MSE & MAD & MSE & MAD & $\overline{M S E}$ & MAD & MSE & MAD & MSE & MAD & MSE & MAD \\
\hline \multirow{2}{*}{48} & $\mathrm{NÃO}$ & \multirow{2}{*}{\multicolumn{2}{|c|}{$\mathrm{X}$}} & \multirow{2}{*}{\multicolumn{2}{|c|}{$\mathrm{X}$}} & \multirow{2}{*}{\multicolumn{2}{|c|}{$80 \%$}} & \multirow{2}{*}{$60 \%$} & \multirow{2}{*}{$50 \%$} & $40 \%$ & $50 \%$ & \multirow{2}{*}{\multicolumn{2}{|c|}{$40 \%$}} \\
\hline & SIM & & & & & & & & & \multicolumn{2}{|c|}{$50 \%$} & & \\
\hline \multirow{2}{*}{60} & NÃO & \multirow{2}{*}{\multicolumn{2}{|c|}{$60 \%$}} & \multirow{2}{*}{\multicolumn{2}{|c|}{$40 \%$}} & \multirow{2}{*}{\multicolumn{2}{|c|}{$30 \%$}} & \multirow{2}{*}{\multicolumn{2}{|c|}{$20 \%$}} & 15 & & \multirow{2}{*}{\multicolumn{2}{|c|}{$15 \%$}} \\
\hline & SIM & & & & & & & & & 15 & & & \\
\hline \multirow{2}{*}{72} & $\mathrm{NA \tilde {O }}$ & \multirow{2}{*}{\multicolumn{2}{|c|}{$50 \%$}} & \multirow{2}{*}{\multicolumn{2}{|c|}{$40 \%$}} & \multirow{2}{*}{\multicolumn{2}{|c|}{$25 \%$}} & \multirow{2}{*}{\multicolumn{2}{|c|}{$20 \%$}} & \multirow{2}{*}{\multicolumn{2}{|c|}{$15 \%$}} & \multirow{2}{*}{\multicolumn{2}{|c|}{$15 \%$}} \\
\hline & SIM & & & & & & & & & & & & \\
\hline 81 & $\mathrm{NÃO}$ & & hor & $40 \%$ & $30 \%$ & & $50 \%$ & $20 \%$ & $15 \%$ & 15 & & & $O$ \\
\hline 84 & SIM & & 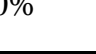 & $40 \%$ & $30 \%$ & & $5 \%$ & $20 \%$ & $20 \%$ & 15 & & & $\%$ \\
\hline o6 & NÃO & $\mathrm{Y}$ & $80 \%$ & & 01 & & $0 \%$ & & 07 & 25 & & & $10 \%$ \\
\hline 90 & SIM & $\lambda$ & $\mathrm{X}$ & & $\%$ & & $0 \%$ & & $\%$ & 25 & & & $\%$ \\
\hline & NÃO & & & & & & & & & & & & \\
\hline 108 & SIM & & X & & $\%$ & & $0 \%$ & 40 & $\%$ & 30 & $\%$ & 25 & $\%$ \\
\hline 120 & NÃO & & $X$ & & $\%$ & & $0 \%$ & 40 & 07 & $40 \%$ & $30 \%$ & $30 \%$ & $25 \%$ \\
\hline 120 & SIM & & A & & & & 70 & & & 4070 & 3070 & 30 & $\%$ \\
\hline & NÃO & & & & & & & & & & & 30 & $\%$ \\
\hline 132 & SIM & & X & & $x$ & & $0 \%$ & & $\%$ & 40 & & $40 \%$ & $30 \%$ \\
\hline & $\mathrm{NÃO}$ & & & & & & & & & $50 \%$ & $40 \%$ & & \\
\hline 144 & SIM & & X & & $x$ & & $0 \%$ & 60 & $\%$ & 50 & $\%$ & 40 & $\%$ \\
\hline 156 & NÃO & & $X$ & & $x$ & & $X$ & 60 & $\%$ & 50 & & 40 & $\%$ \\
\hline 150 & SIM & & $\mathbf{x}$ & & 1 & & $\Lambda$ & $70 \%$ & $60 \%$ & 30 & & & 10 \\
\hline & $\mathrm{NÃO}$ & & & & & & & 80 & $\%$ & & & & \\
\hline 180 & SIM & & X & & $x$ & & X & $80 \%$ & $70 \%$ & 60 & & 50 & $\%$ \\
\hline
\end{tabular}

Quadro 5.12 MENSAL MULTIPLICATIVO - Filtro S-WLS $\left(\alpha=1 / 3, \delta=1 / 30, w_{0}=1\right)$ vs X-11: MSE e MAD. Valores de ' $b$ ' a partir do qual o filtro S-WLS supera o X-11, para cada ' $k$ ' e ' $A / s$ ' (Eq. 5-3). Séries com e sem tendência 
Analisando o Quadro 5.12, constata-se que para $60 \leq k \leq 120$, o filtro SWLS se mostra superior ao X-11, para valores de ' $b$ ' razoáveis, como $40 \%$ (quando $A / s=6$ ). Além disso, há sempre uma possibilidade do desempenho do ajuste sazonal realizado pelo filtro S-WLS ser melhor do que o X-11, se $A / s \geq 2$.

Considerando os valores de ' $k$ ' acima de 120, o filtro S-WLS também apresenta resultados melhores do que o X-11, mas isso ocorre somente quando ' $b$ ' e ' $A / s$ ' são altos. Ressalta-se que quanto menor o ' $k$ ', melhor é o desempenho do filtro S-WLS em relação ao X-11. A exceção é $k=48$ e $k=60$, pois a banda passante do filtro S-WLS não é suficiente para ajustar sazonalidades com essa variação. Porém, o filtro S-WLS se mostrou adequado no caso de $k=60$, devido ao fato da banda de transição não ter um caimento retilíneo.

Os Gráficos 5.49 e 5.50 foram elaborados com base no Quadro 5.12. Neles, é possível notar o valor mínimo de ' $\boldsymbol{b}$ ' a partir do qual o MSE do filtro S-WLS passa a ser inferior ao MSE do X-11.

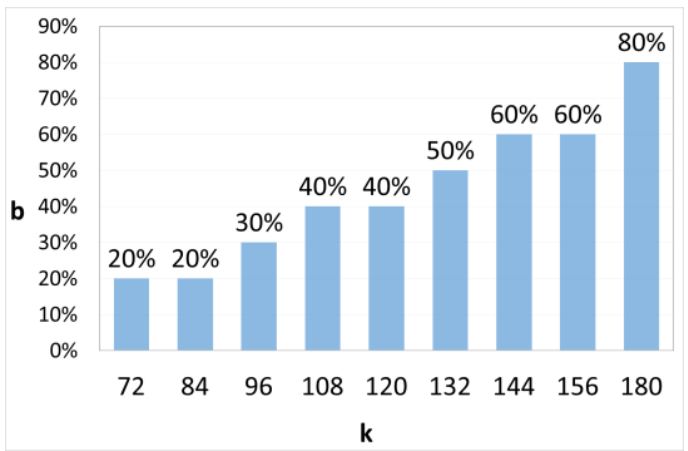

Gráfico 5.49 Valores de ' $b$ ' a partir do qual o filtro S-WLS supera o $X-11$, na MSE, para valores de ' $k$ ', considerando $A / s=6($ Eq. 5-3)

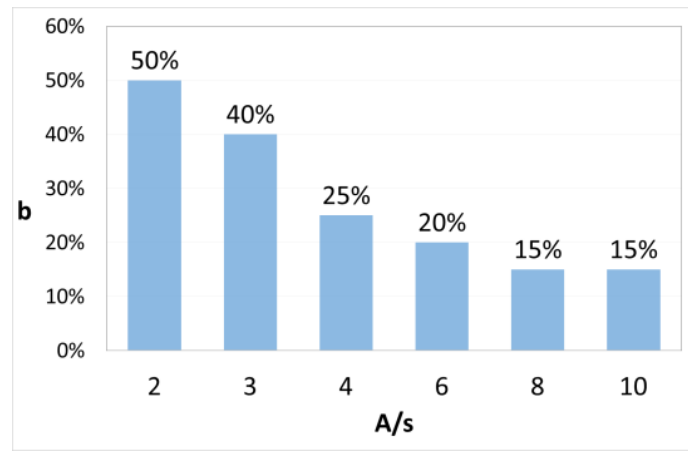

Gráfico 5.50 Valores de ' $b$ ' a partir do qual o filtro S-WLS supera o X-11, na MSE, para vários valores de ' $A / s$ ', considerando $k=72($ Eq. 5-3)

No Gráfico 5.49, nota-se que considerando $A / s=6$, e $k=72$ ou $k=84$, basta a taxa de variação da sazonalide $(b)$ ser igual a $20 \%$ para o desempenho do filtro $S$ WLS superar o X-11. Recorda-se que nas séries reais, analisadas na Subseção 5.2.2, constatou-se um grande número de séries com tais valores de ' $k$ '.

Já em relação ao Gráfico 5.50, a análise é realizada por meio da variação dos valores de ' $A / s$ ', mantendo fixo o valor de ' $k$ ' igual a 72 . Observa-se que, para $A / s$ $\geq 4$, o filtro S-WLS necessita apenas de $b=25 \%$ para superar o X-11. E, nas séries reais, foi encontrada uma grande concentração de valores de ' $b$ ' entre $25 \%$ e $30 \%$. 
Portanto, existem situações reais plausíveis para o filtro S-WLS superar o X11 em termos da qualidade do ajuste sazonal nas séries com algum grau de sazonalidade móvel.

\subsubsection{1. \\ Espectros da componente irregular: série mensal com decomposição multiplicativa}

Para analisar a qualidade da extração da componente sazonal realizada pelo filtro S-WLS, em relação ao X-11, foram elaborados os espectros da componente irregular da série ' $\mathrm{Y}$ ' dessazonalizada pelos filtros. Os espectros estão apresentados nos Gráficos 5.51 a 5.62. Eles são apresentados em pares, sendo o primeiro gráfico referente ao ajuste realizado pelo filtro S-WLS, e o segundo gráfico referente ao ajuste realizado pelo $\mathrm{X}-11$.

Os espectros foram gerados considerando a dessazonalização de séries artificiais com $b=40 \%, 25 \%$ e $10 \%$; e $k=72$ e 96 . A razão ' $A / s$ ' utilizada, nessas séries, foi igual a 6 . Vale citar que na construção dos espectros, foi usado o logaritmo neperiano da série dessazonalizada, multiplicado por 10 (U.S. Census Bureau, 2012).

Os Gráficos 5.51 a 5.56 apresentam os espectros para séries com $k=72$, significando que o padrão de sazonalidade móvel leva 6 anos para se repetir; e tamanho de filtro $(N)$ igual a 121 . Essas séries foram geradas sem a componente de tendência.

Nota-se que, quando ' $b$ ' é igual a $40 \%$ ou $25 \%$, o espectro da irregular da série dessazonalizada pelo X-11 apresenta picos próximos à frequência $\frac{1}{12}$ (Gráficos 5.52 e 5.54). Isso evidencia que na presença de sazonalidade móvel, quando a taxa de variação da sazonalidade é maior do que $25 \%$, o filtro X-11 realiza um subajustamento (underfitting) da sazonalidade. Ou seja, ele não tem capacidade de realizar uma extração adequada da componente sazonal, deixando parte dela migrar para a irregular.

Já nos Gráficos 5.51 e 5.53 - que equivalem ao ajuste sazonal realizado pelo filtro S-WLS, não se observam picos próximos à frequência fundamental. Esse fato 
comprova que o filtro S-WLS realiza um ajuste sazonal correto quando a série apresenta sazonalidade móvel.

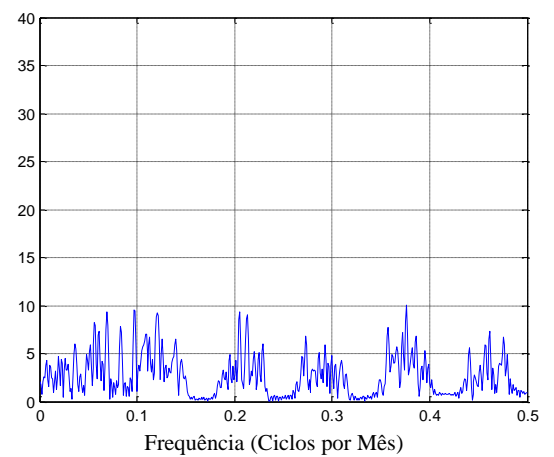

Gráfico 5.51 Espectro da irregular da série dessazonalizada pelo filtro $S$ WLS, com: $A / s=6, k=72, b=40 \% \mathrm{e}$ $N=121$

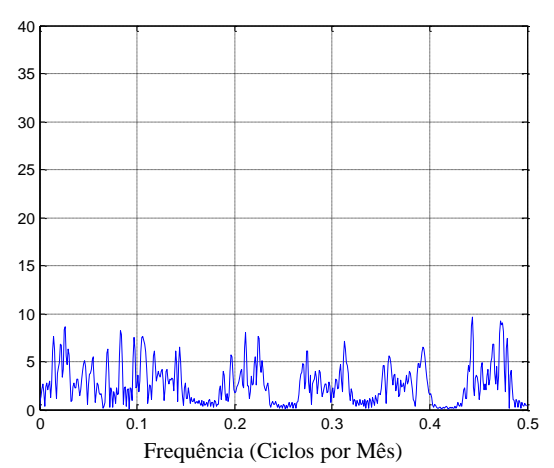

Gráfico 5.53 Espectro da irregular da série dessazonalizada pelo filtro $S$ WLS, com: $A / s=6, k=72, b=25 \% \mathrm{e}$ $N=121$

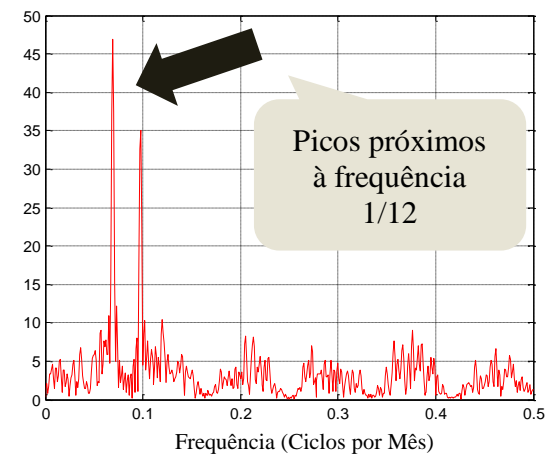

Gráfico 5.52 Espectro da irregular da série dessazonalizada pelo $X-11$, com: $A / s=6, k=72, b=40 \%$ e $N$ $=121$

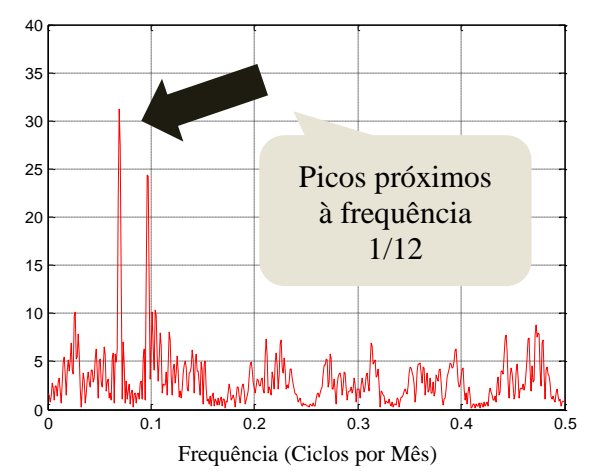

Gráfico 5.54 Espectro da irregular da série dessazonalizada pelo $\mathrm{X}-11$, com: $A / s=6, k=72$ e $b=25 \%$ e $N$ $=121$

Os Gráficos 5.55 e 5.56, mostram o espectro da irregular das séries dessazonalizadas pelo filtro S-WLS e pelo X-11, respectivamente, quando $b=10 \%$. Nesse caso, o filtro X-11 não apresenta picos relevantes no espectro, assim como o filtro S-WLS, indicando uma correta dessazonalização, dos dois métodos, quando a taxa de variação da sazonalidade é baixa.

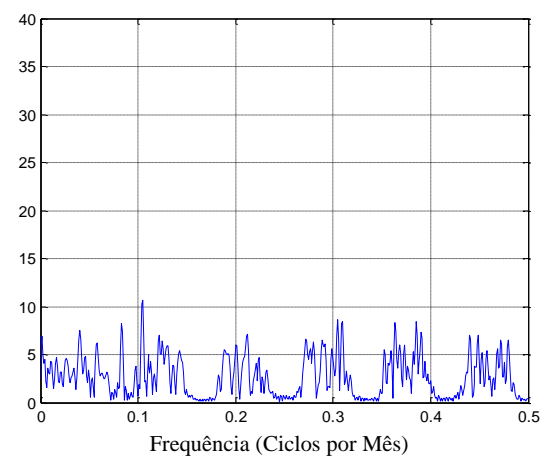

Gráfico 5.55 Espectro da irregular da série dessazonalizada pelo filtro $S$ WLS, com: $A / s=6, k=72, b=10 \% \mathrm{e}$ $N=121$

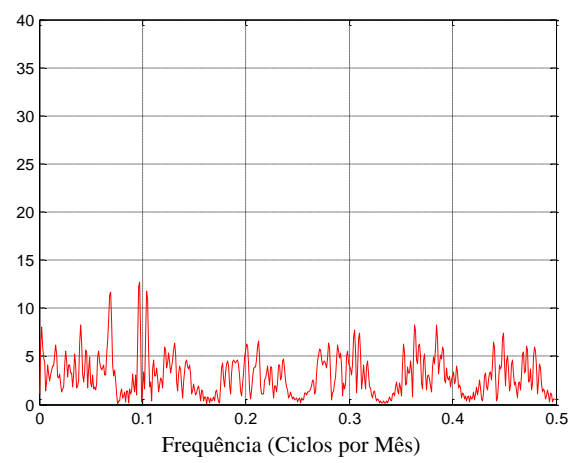

Gráfico 5.56 Espectro da irregular da série dessazonalizada pelo $X-11$, com: $A / s=6, k=72, b=10 \%$ e $N$ $=121$ 
Considerando séries artificiais com $k=96$, o que significa que o padrão de sazonalidade móvel leva 8 anos para se repetir, foram construídos os espectros da irregular das séries dessazonalizadas pelo filtro S-WLS e pelo X-11. Eles estão apresentados nos Gráficos 5.57 a 5.62 .

Assim como no caso anterior, foram geradas séries com $b=40 \%, 25 \%$ e $10 \%$. A razão ' $A / s$ ' utilizada foi igual a 6 . E, para a dessazonalização, foi usado o tamanho de filtro igual a 121, sem componente de tendência.

Os gráficos 5.58 e 5.60, apresentam os espectro relacionados às séries dessazonalizadas pelo X-11 (com $b=40 \%$ e $25 \%$, respectivamente), e mostram grandes picos próximo à frequência fundamental $\frac{1}{12}$. Nota-se que esses picos têm largura menor do que os anteriormente apresentados (quando $k=72$ : Gráficos 5.52 e 5.54).

Quando $b=10 \%$, o que indica uma taxa baixa de variação sazonal, o espectro do X-11 apresenta um único pequeno pico próximo à frequência $\frac{1}{12}$. Com isso, conclui-se que o X-11, já consegue realizar um ajuste adequado, para valores baixos de ' $b$ '.

Nos espectros da irregular das séries dessazonalizadas pelo filtro S-WLS, não são observados picos na frequência fundamental $\frac{1}{12}$, ou próximos a ela. Sendo assim, conclui-se que para os diversos valores testados de ' $b$ ' e ' $k$ ', o filtro S-WLS apresentou um bom desempenho no que tange ao ajuste sazonal, enquanto que o X11 realiza um ajuste adequado apenas para valores pequenos de ' $b$ '. 


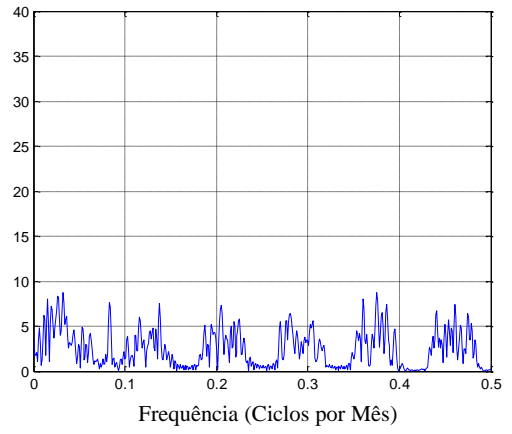

Gráfico 5.57 Espectro da irregular da série dessazonalizada pelo filtro S-WLS, com: $A / s=6, k=96, b=$ $40 \%$ e $N=121$

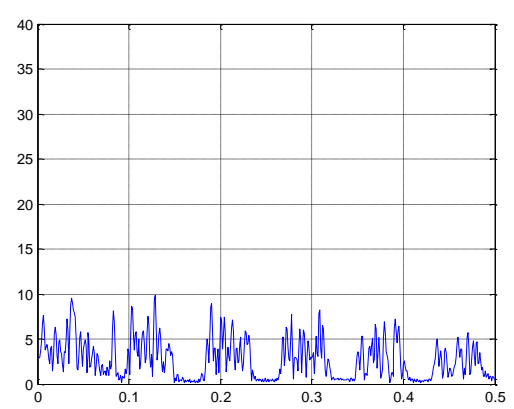

Frequência (Ciclos por Mês)

Gráfico 5.59 Espectro da irregular da série dessazonalizada pelo filtro S-WLS, com: $A / s=6, k=96, b=$ $25 \%$ e $N=121$

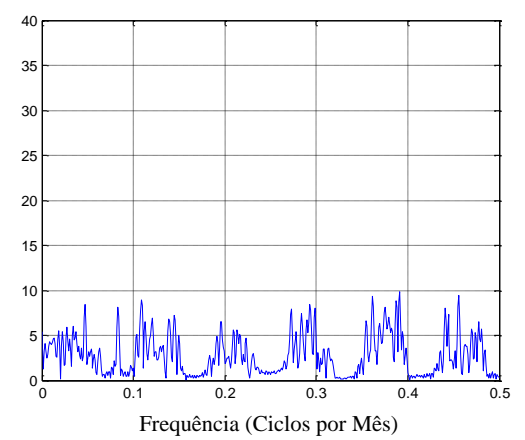

Gráfico 5.61 Espectro da irregular da série dessazonalizada pelo filtro S-WLS, com: $A / s=6, k=96, b=10 \%$ e $N=121$

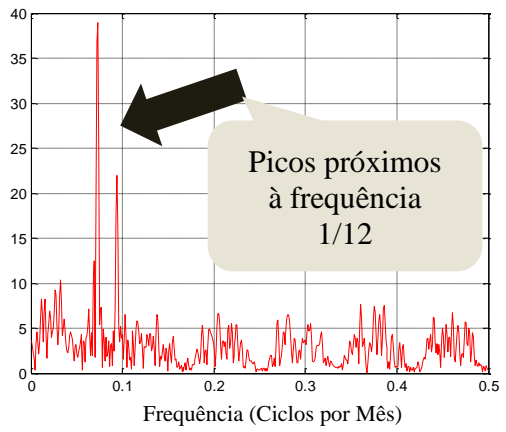

Gráfico 5.58 Espectro da irregular da série dessazonalizada pelo $X-11$, com: $A / s=6, k=96, b=40 \%$ e $N$ $=121$

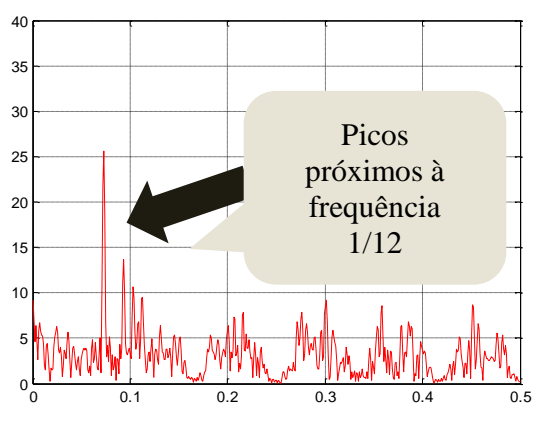

Gráfico 5.60 Espectro da irregular da série dessazonalizada pelo X-11, com: $A / s=6, k=96, b=$ $25 \%$ e $N=121$

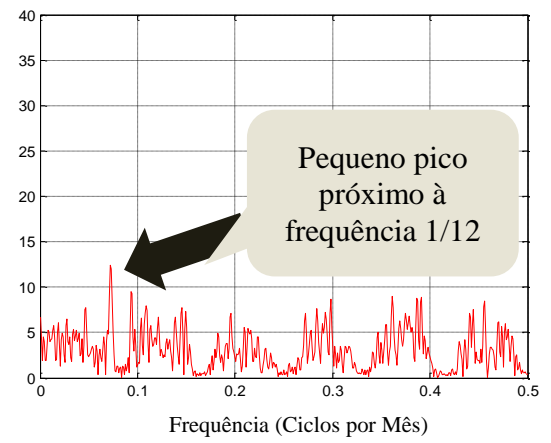

Gráfico 5.62 Espectro da irregular da série dessazonalizada pelo $X-11$, com: $A / s=6, k=96, b=10 \%$ e $N$ $=121$ 
Caso houvesse a componente de tendência na série (baseados em y149), e fosse utilizado o filtro S-WLS, os espectros da irregular gerados seriam os seguintes:

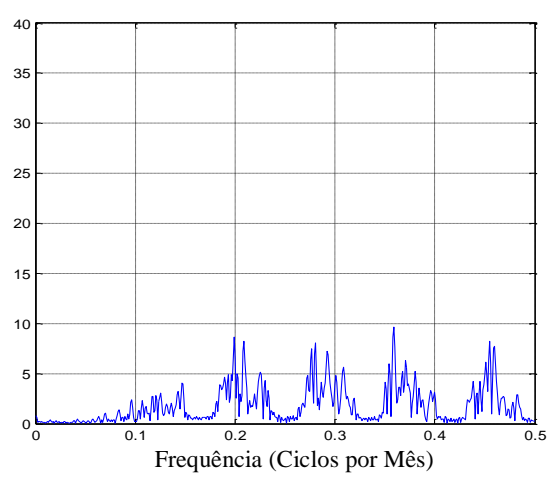

Gráfico 5.63 Espectro da irregular da série dessazonalizada pelo filtro S-WLS, com tendência, e: $A / s$ $=6,5 ; k=72 ; b=40 \%(\mathrm{~N}=121)$

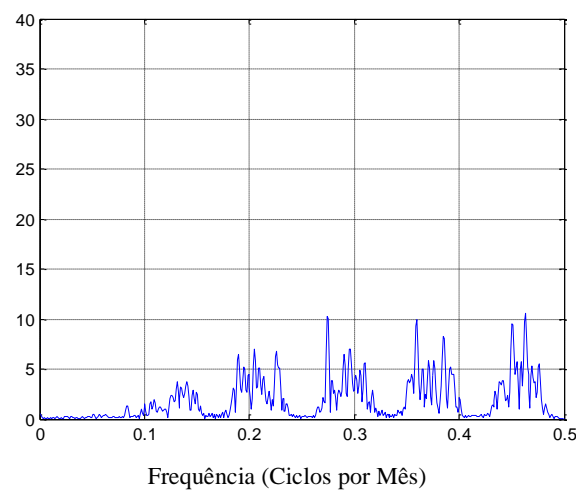

Gráfico 5.65 Espectro da irregular da série dessazonalizada pelo filtro S-WLS, com tendência, e: $A / s$ $=6,5 ; k=72 ; b=10 \%(\mathrm{~N}=121)$

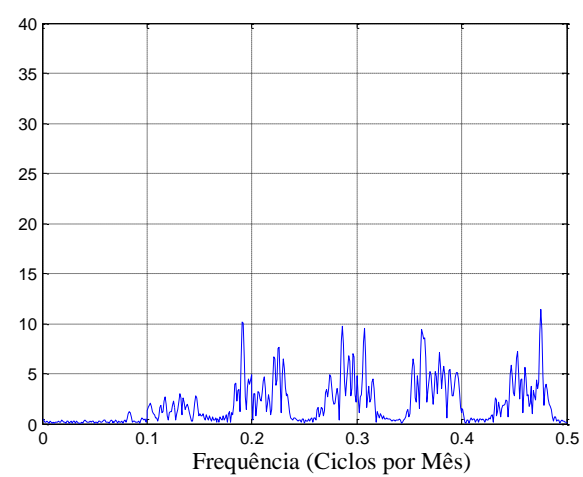

Gráfico 5.64 Espectro da irregular da série dessazonalizada pelo filtro S-WLS, com tendência, e: $A / s$ $=6,5 ; k=96 ; b=40 \%(\mathrm{~N}=121)$

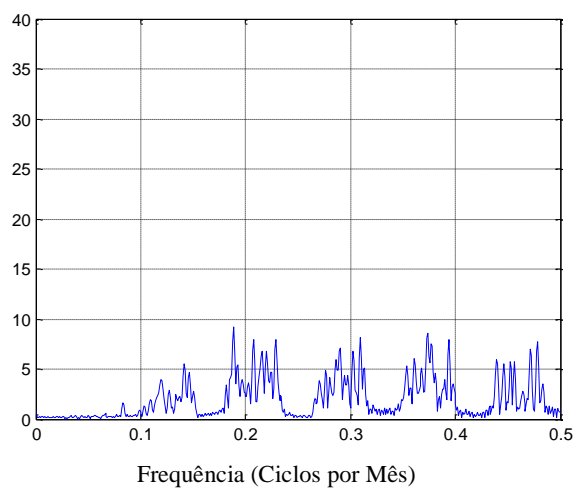

Gráfico 5.66 Espectro da irregular da série dessazonalizada pelo filtro S-WLS, com tendência, e: $A / s=6,5 ; k=96 ; b=10 \%(\mathrm{~N}=121)$

Com base na análise dos Gráficos 5.63 a 5.66, onde foram considerados valores de ' $k$ ' igual a 72 e 96 , e valores de ' $b$ ' igual a $10 \%$ e $40 \%$, percebe-se o correto ajuste sazonal realizado pelo filtro S-WLS, também quando há tendência na série. 


\subsection{4.}

Resultados: série trimestral com decomposição aditiva - filtro S-WLS vs $\mathrm{X}-11$

Para comparar os resultados do ajuste sazonal realizado pelos filtros (S-WLS e X-11), nas séries trimestrais aditivas, foram utilizados os seguintes valores para o sinal sazonal artificial:

- $A / s=[1,2,3,4,6,8,10]$

- $b=[5 \%, 10 \%, 15 \%, 20 \%, 25 \%, 30 \%, 40 \%, 50 \%, 60 \%, 70 \%, 80 \%]$

- $\quad k=[16,20,24,28,32,36,40,44,48,80]$

Foram geradas séries com e sem tendência. Nas séries com componente de tendência, foram usados os seguintes valores para os coeficientes do polinômio: $\mathrm{b}_{0}=48.631, \mathrm{~b}_{1}=532,07, \mathrm{~b}_{2}=26,378$ e $\mathrm{b}_{3}=-0.2983$, baseados na série $\mathrm{y} 81$.

Em relação à componente irregular, geraram-se 100 séries, com distribuição $\mathrm{N}\left(0, \sigma^{2}\right)$, com base no desvio-padrão da irregular das séries reais. E, assim como nos casos anteriores, o teste $t$ de igualdade de médias de duas populações, com variâncias desconhecidas e equivalentes, foi realizado para o MSE e o MAD dos filtros. Nesse teste, utilizou-se nível de significância de 5\%. Além disso, foi calculada a razão entre a SNR do filtro S-WLS e a SNR do X-11.

Como mencionado na Subseção 5.4.2, a configuração do filtro utilizado foi a seguinte: $\alpha=\mathbf{1 / 3}, \delta=1 / 30$ e $w_{0}=1$.

A seguir serão apresentados, nos itens (i), (ii), (iii) e (iv), os resultados das situações em que o filtro aqui proposto (S-WLS) apresenta desempenho superior ao $\mathrm{X}-11$. Essa análise foi realizada com base na variação dos parâmetros do sinal artificial (Eq. 5-2). 


\section{(i) Resultados encontrados com base na variação de ' $b$ ': série trimestral} aditiva

Como mencionado anteriormente, o parâmetro ' $b$ ' representa a taxa de variação da sazonalidade, assumindo valores no intervalo $(0,1)$. Analisando o comportamento desse parâmetro, nas séries reais, foram encontrados valores entre $40 \%$ e $50 \%$. Sendo assim, mesmo realizando os testes para os demais valores de ' $b$ ', a análise será direcionada para $b<50 \%$.

O Gráfico 5.67, a seguir, mostra a relação entre o valor de ' $b$ ' e a MSE resultante da aplicação do filtro S-WLS e do filtro X-11. Analisando o gráfico, notase que a MSE do filtro S-WLS não se altera de forma relevante com o aumento ' $b$ ', enquanto que a MSE do X-11 apresenta um crescimento acentuado. Além disso, destaca-se que quando $b=50 \%$, a MSE do X-11 é 3 vezes maior do que a MSE do filtro S-WLS, indicando uma melhor adequação deste.

Quanto ao Gráfico 5.68, observa-se que quanto maior for o valor de ' $b$ ', maior é a SNR do filtro S-WLS em relação à SNR do X-11. Nota-se, também, que a SNR do filtro S-WLS sofre pouca alteração com a variação de ' $b$ ', enquanto que a SNR do X-11 apresenta uma forte queda. Ainda em relação à SNR, a partir de $b=20 \%$, a SNR do filtro S-WLS supera o X-11. Vale lembrar que esse resultado está relacionado ao valor de ' $k$ ', além da razão ' $A / s$ ' que serão analisados nos próximos itens.

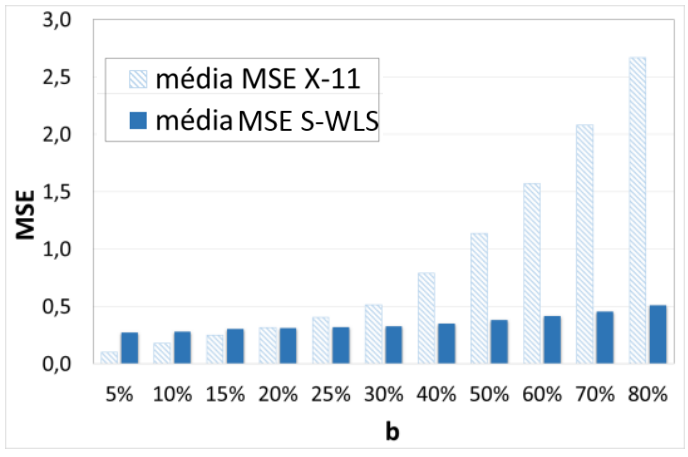

Gráfico 5.67 Média da MSE nas simulações com o filtro $X-11$ e com o filtro $S-W L S$, para valores de ' $b$ ', considerando $A / s=6, k=24$ e $N=43$ (Eq. 5-2)

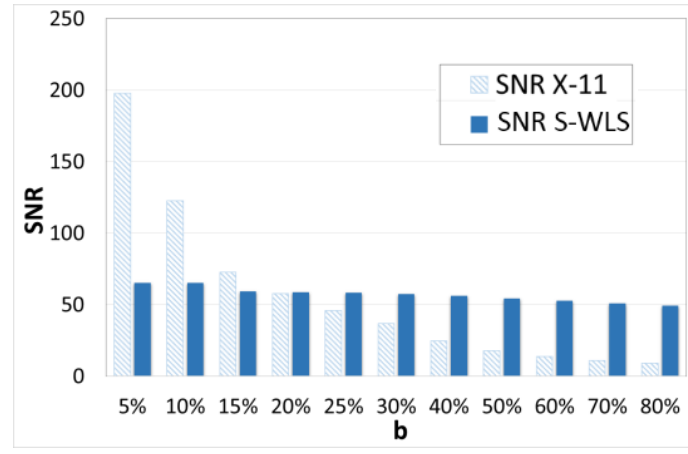

Gráfico 5.68 Relação entre ' $b$ ' e a SNR do filtro $S$-WLS e do filtro $X-11$, considerando $A / s=6, k=24 \mathrm{e} N=43$ (Eq. 5-2) 
A tabela 5.23, apresenta os dados referentes aos Gráficos 5.67 e 5.68. Além disso, são apresentados os valores referentes ao MAD, dos filtros. Constata-se que a partir de $b=25 \%$, a MSE e a MAD do filtro S-WLS passam a ser significativamente menores do que essas medidas do X-11 - considerando $A / s=6$ e $k=24$.

\begin{tabular}{c|ccc|ccc|ccc}
\hline $\boldsymbol{b}$ & MSE & MSE & valor-p & $\begin{array}{c}\text { MAD } \\
\text { S-WLS }\end{array}$ & $\begin{array}{c}\text { MAD } \\
\text { X-11 }\end{array}$ & valor-p & $\begin{array}{c}\text { SNR S- } \\
\text { WLS }\end{array}$ & $\begin{array}{c}\text { SNR } \\
\text { X-11 }\end{array}$ & $\begin{array}{c}\text { SNR S- } \\
\text { WLS } / \\
\text { SNR X-11 }\end{array}$ \\
\hline $\mathbf{1 0 \%}$ & 0,28 & 0,18 & 1,000 & 0,42 & 0,33 & 1,000 & 65,2 & 122,7 & 0,53 \\
$\mathbf{1 5 \%}$ & 0,31 & 0,25 & 1,000 & 0,45 & 0,40 & 1,000 & 59,2 & 72,7 & 0,81 \\
$\mathbf{2 0 \%}$ & 0,32 & 0,32 & 0,225 & 0,45 & 0,45 & 0,216 & 58,8 & 57,7 & 1,02 \\
$\mathbf{2 5 \%}$ & 0,32 & 0,41 & 0,000 & 0,45 & 0,51 & 0,000 & 58,2 & 45,8 & 1,27 \\
$\mathbf{3 0 \%}$ & 0,34 & 0,52 & 0,000 & 0,46 & 0,57 & 0,000 & 57,6 & 36,7 & 1,57 \\
$\mathbf{4 0 \%}$ & 0,35 & 0,78 & 0,000 & 0,47 & 0,69 & 0,000 & 56,1 & 24,8 & 2,26 \\
$\mathbf{5 0 \%}$ & 0,38 & 1,14 & 0,000 & 0,49 & 0,82 & 0,000 & 54,4 & 17,9 & 3,05 \\
$\mathbf{6 0 \%}$ & 0,41 & 1,56 & 0,000 & 0,51 & 0,95 & 0,000 & 52,7 & 13,6 & 3,87 \\
$\mathbf{7 0 \%}$ & 0,45 & 2,07 & 0,000 & 0,53 & 1,09 & 0,000 & 50,9 & 10,9 & 4,69 \\
$\mathbf{8 0 \%}$ & 0,51 & 2,66 & 0,000 & 0,56 & 1,23 & 0,000 & 49,2 & 9,0 & 5,48 \\
\hline
\end{tabular}

Tabela 5.23 MSE, MAD e SNR para valores de ' $b$ ', sendo $A / s=6, k=24$ (Eq. 5-2): séries trimestrais com decomposição aditiva $(N=43)$ 


\section{(ii) Resultados encontrados com base na variação de ' $k$ ': série trimestral com decomposição aditiva}

Ao comparar o desempenho do filtro S-WLS, com o X-11, variando os valores do parâmetro ' $k$ ', foram mantidos constantes os parâmetros ' $b$ ' e ' $A / s$ '. O tamanho do filtro utilizado foi 43 . O filtro de tamanho 43, no método X-11, utiliza média móvel sazonal 3x3, que é a mais indicada no caso de sazonalidade móvel.

O Gráfico 5.69 apresenta a média do MSE de cada filtro, para vários valores de ' $k$ ', onde nota-se que a MSE do filtro S-WLS sofre pouca alteração com a variação de ' $k$ '. Vale destacar que, quando $k=24$, a MSE do filtro S-WLS é um pouco maior do que as demais MSEs, relacionadas aos outros valores de ' $k$ '. Isso ocorre porque o filtro utiliza $\alpha=\frac{1}{3}$, que corresponde a $k=24$. Sendo assim, a resposta de frequência do filtro não está totalmente adequada devido à proximidade do sinal nas bordas da banda passante. Mesmo assim, o desempenho do filtro SWLS, para $k=24$, é superior ao do filtro X-11.

O filtro X-11 apresenta desempenho melhor do que o filtro S-WLS para $k \geq$ 44; tanto em relação à SNR (Gráfico 5.70) quanto em relação à MSE (Gráfico 5.69). O valor de $k=44$ equivale a 11 anos necessários para o padrão de sazonalidade móvel voltar a se repetir, o que representa um grau baixo de sazonalidade móvel.

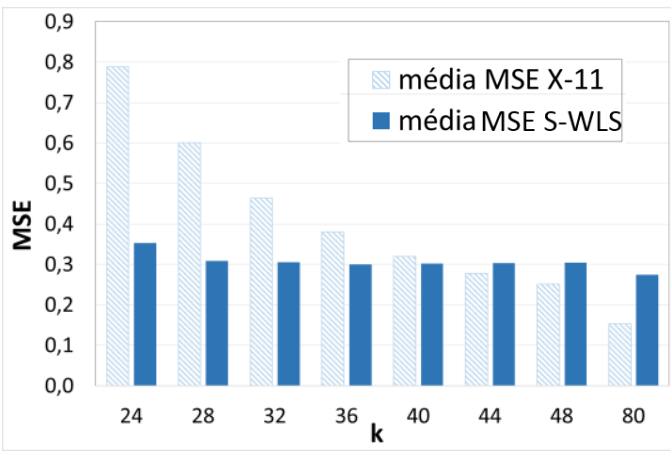

Gráfico 5.69 Média da MSE nas simulações com o filtro $X-11$ e com o filtro S-WLS, para valores de ' $k$ ', considerando $A / s=6$ e $b=40 \%(N=43)$

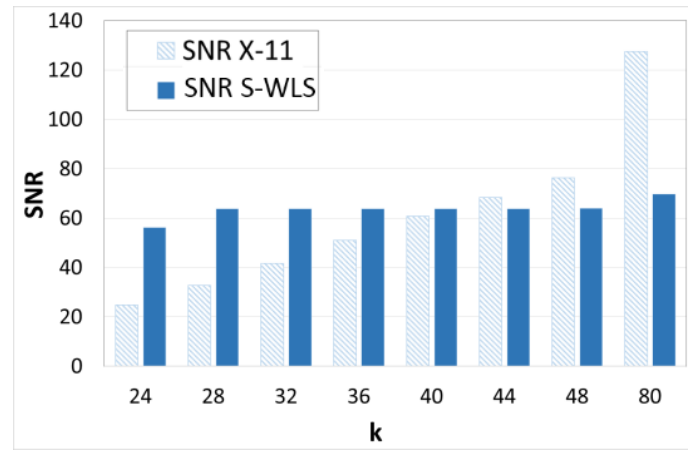

Gráfico 5.70 Relação entre ' $k$ ' e a SNR do filtro $S-W L S$ e do filtro $X-11$, considerando $' A / s$ ' $=6$ e $b=40 \%(N=43)$ 
A Tabela 5.24 mostra os valores utilizados nos Gráficos 5.69 e 5.70. Além disso, são apresentados os resultados referentes à estatística MAD. Nessa tabela, observa-se que o comportamento do MAD está consistente com o comportamento da MSE e da SNR.

Com base nos resultados, conclui-se que mesmo o X-11 sendo melhor em alguns casos, o filtro S-WLS mantém sempre um bom desempenho.

\begin{tabular}{c|ccc|ccc|ccc}
\hline $\boldsymbol{k}$ & MSE & MSE & valor-p & $\begin{array}{c}\text { MAD } \\
\text { S-WLS }\end{array}$ & $\begin{array}{c}\text { MAD } \\
\text { X-11 }\end{array}$ & valor-p & $\begin{array}{c}\text { SNR S- } \\
\text { WLS }\end{array}$ & $\begin{array}{c}\text { SNR } \\
\text { X-11 }\end{array}$ & $\begin{array}{c}\text { SNR S- } \\
\text { WLS / } \\
\text { SNR X-11 }\end{array}$ \\
\hline $\mathbf{2 4}$ & 0,35 & 0,78 & 0,000 & 0,47 & 0,69 & 0,000 & 56,1 & 24,8 & 2,26 \\
$\mathbf{2 8}$ & 0,31 & 0,60 & 0,000 & 0,44 & 0,61 & 0,000 & 63,7 & 32,7 & 1,95 \\
$\mathbf{3 2}$ & 0,30 & 0,47 & 0,000 & 0,44 & 0,55 & 0,000 & 63,7 & 41,6 & 1,53 \\
$\mathbf{3 6}$ & 0,30 & 0,38 & 0,000 & 0,43 & 0,49 & 0,000 & 63,7 & 50,9 & 1,25 \\
$\mathbf{4 0}$ & 0,30 & 0,32 & 0,002 & 0,44 & 0,45 & 0,000 & 63,7 & 60,8 & 1,05 \\
$\mathbf{4 4}$ & 0,31 & 0,28 & 1,000 & 0,44 & 0,42 & 1,000 & 63,8 & 68,3 & 0,93 \\
$\mathbf{4 8}$ & 0,31 & 0,25 & 1,000 & 0,44 & 0,40 & 1,000 & 64,0 & 76,3 & 0,84 \\
$\mathbf{8 0}$ & 0,28 & 0,16 & 1,000 & 0,42 & 0,32 & 1,000 & 69,7 & 127,5 & 0,55 \\
\hline
\end{tabular}

Tabela 5.24 MSE, MAD e SNR para valores de ' $k$ ', sendo $A / s=6, b=40 \%$ (Eq. 5-2): séries trimestrais com decomposição aditiva $(N=43)$ 
(iii) Resultados encontrados com base na variação de ' $A / s$ ': série trimestral com decomposição aditiva

Os resultados referentes à variação da razão ' $A / s$ ' estão ilustrados nos Gráficos 5.71, 5.72 e na Tabela 5.25. Nessa análise, foi mantido ' $b$ ' igual a 40\% e ' $k$ ' igual a 24. Essa combinação de valores dos parâmetros ' $b$ ' e ' $k$ ', indicam um grau elevado de sazonalidade móvel.

Com base nos resultados, percebe-se que a partir de ' $A / S$ ' igual a 3 , o filtro S-WLS já mostra um desempenho significativamente melhor do que o X-11. Isso ocorre, possivelmente, devido ao alto grau de sazonalidade móvel que está sendo considerado.

É interessante notar, no Gráfico 5.72, que com o aumento da razão ' $A / s$ ', ou seja, com o aumento da amplitude do sinal sazonal em relação ao desvio-padrão da componente irregular, ambos os filtros mostram um crescimento no valor da SNR. No entanto, o crescimento da SNR do filtro S-WLS é muito mais acentuado do que o crescimento da SNR do X-11.

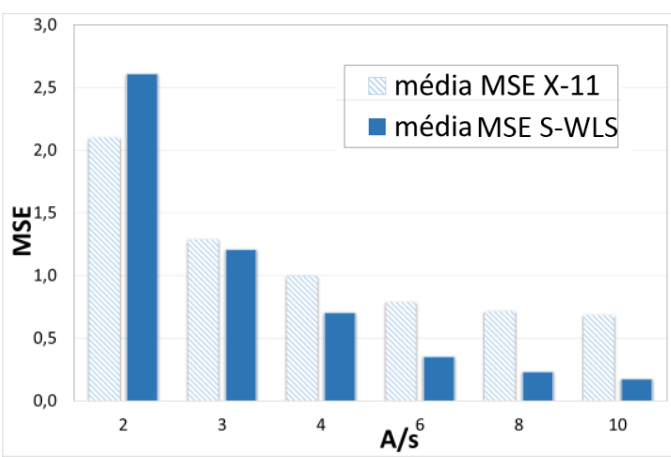

Gráfico 5.71 Média da MSE nas simulações com o filtro $X-11$ e com o filtro S-WLS, para valores de ' $A / s$ ', considerando $k=24$ e $b=40 \%(N=43)$

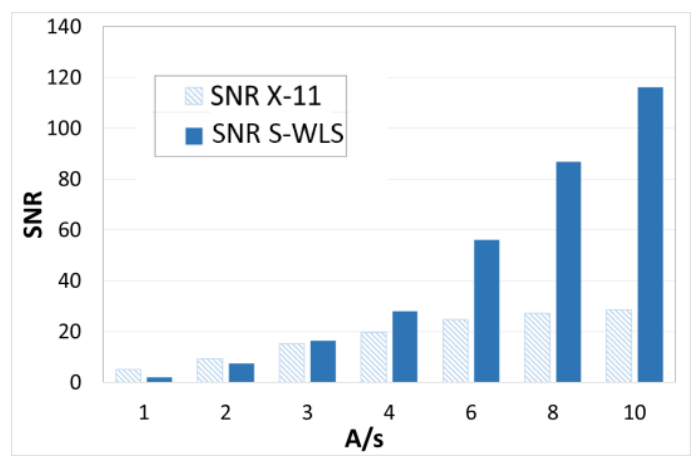

Gráfico 5.72 Relação entre ' $A / s$ ' e a SNR do filtro S-WLS e do filtro $X-11$, considerando $k=24$ e $b=40 \%(N=43)$

\begin{tabular}{c|ccc|ccc|ccc}
\hline \multirow{2}{*}{$/ s$} & $\begin{array}{c}\text { MSE } \\
\text { S-WLS }\end{array}$ & $\begin{array}{c}\text { MSE } \\
\text { X-11 }\end{array}$ & valor-p & $\begin{array}{c}\text { MAD } \\
\text { S-WLS }\end{array}$ & $\begin{array}{c}\text { MAD } \\
\text { X-11 }\end{array}$ & valor-p & $\begin{array}{c}\text { SNR S- } \\
\text { WLS }\end{array}$ & $\begin{array}{c}\text { SNR } \\
\text { X-11 }\end{array}$ & $\begin{array}{c}\text { SNR S- } \\
\text { WLS / } \\
\text { SNR X-11 }\end{array}$ \\
\hline $\mathbf{2}$ & 2,61 & 2,10 & 1,000 & 1,29 & 1,16 & 1,000 & 7,5 & 9,3 & 0,81 \\
$\mathbf{3}$ & 1,21 & 1,29 & 0,000 & 0,88 & 0,90 & 0,000 & 16,4 & 15,3 & 1,07 \\
$\mathbf{4}$ & 0,70 & 1,00 & 0,000 & 0,67 & 0,79 & 0,000 & 27,9 & 19,7 & 1,42 \\
$\mathbf{6}$ & 0,35 & 0,79 & 0,000 & 0,47 & 0,69 & 0,000 & 56,1 & 24,8 & 2,26 \\
$\mathbf{8}$ & 0,23 & 0,72 & 0,000 & 0,38 & 0,65 & 0,000 & 86,8 & 27,3 & 3,18 \\
$\mathbf{1 0}$ & 0,17 & 0,68 & 0,000 & 0,33 & 0,63 & 0,000 & 116,1 & 28,6 & 4,06 \\
\hline
\end{tabular}

Tabela 5.25 MSE, MAD e SNR para valores de ' $A / s$ ', sendo $k=24, b=40 \%$ (Eq. 5-2): séries trimestrais com decomposição aditiva $(N=43)$ 
(iv) Condições nas quais as estatísticas do filtro S-WLS superam o filtro X11 , segundo valores de ' $A / s^{\prime}$ ', ' $b$ ' e ' $k$ ': série trimestral com decomposição aditiva

Com o propósito de avaliar as condições que deixam o desempenho do filtro S-WLS melhor do que o desempenho do X-11, para diversas combinações de valores dos parâmetros $b$ ', ' $k$ ' e ' $A / s$ ', foram comparadas a SNR, MSE e MAD dos resultados obtidos com a aplicação dos filtros.

Nessa análise, buscou-se identificar a partir de qual valor da taxa de variação da sazonalidade ' $\boldsymbol{b}$ ', o filtro S-WLS passa a superar o X-11, para cada valor de ' $\boldsymbol{k}$ ' (número de trimestres no qual o padrão de sazonalidade móvel volta a se repetir) e ' $\boldsymbol{A} / \boldsymbol{s}$ ' (amplitude do sinal em relação ao desvio-padrão da irregular). Esses parâmetros estão descritos na Equação (5-2).

O Quadro 5.13 apresenta os resultados dessa análise para as séries sem tendência (Não) e com tendência (Sim). Os valores das células, no corpo da tabela, indicam o valor mínimo de ' $\boldsymbol{b}$ ' a partir do qual o filtro S-WLS supera o X-11. Apenas os resultados dos testes cujo valor-p $<5 \%$ foram considerados como significativamente melhores. No quadro, todos os valores-p não apresentados são menores do que 0,001 . 


\begin{tabular}{|c|c|c|c|c|c|c|c|c|c|c|c|c|c|c|c|c|c|c|c|}
\hline \multirow{3}{*}{$\boldsymbol{k}$} & \multirow{3}{*}{ TENDÊNCIA } & \multicolumn{18}{|c|}{$A / s$} \\
\hline & & \multicolumn{3}{|c|}{2} & \multicolumn{3}{|c|}{3} & \multicolumn{3}{|c|}{4} & \multicolumn{3}{|c|}{6} & \multicolumn{3}{|c|}{8} & \multicolumn{3}{|c|}{10} \\
\hline & & MSE & MAD & SNR & MSE & MAD & SNR & MSE & MAD & SNR & MSE & MAD & SNR & MSE & MAD & SNR & MSE & MAD & SNR \\
\hline \multirow{2}{*}{16} & NÃo & \multirow{2}{*}{\multicolumn{3}{|c|}{$X$}} & \multirow{2}{*}{\multicolumn{3}{|c|}{$X$}} & \multirow{2}{*}{\multicolumn{2}{|c|}{$\mathrm{X}$}} & \multirow{2}{*}{$70 \%$} & \multirow{2}{*}{\multicolumn{2}{|c|}{$60 \%$}} & \multirow{2}{*}{$50 \%$} & \multirow{2}{*}{\multicolumn{2}{|c|}{$50 \%$}} & \multirow{2}{*}{$40 \%$} & \multirow{2}{*}{\multicolumn{2}{|c|}{$40 \%$}} & \multirow{2}{*}{$35 \%$} \\
\hline & SIM & & & & & & & & & & & & & & & & & & \\
\hline \multirow{2}{*}{20} & NÃO & \multirow{2}{*}{\multicolumn{3}{|c|}{$60 \%$}} & \multirow{2}{*}{\multicolumn{3}{|c|}{$40 \%$}} & \multirow{2}{*}{\multicolumn{3}{|c|}{$30 \%$}} & \multirow{2}{*}{\multicolumn{3}{|c|}{$20 \%$}} & \multirow{2}{*}{\multicolumn{3}{|c|}{$15 \%$}} & \multirow{2}{*}{\multicolumn{3}{|c|}{$15 \%$}} \\
\hline & SIM & & & & & & & & & & & & & & & & & & \\
\hline \multirow{2}{*}{24} & NÃO & & $60 \%$ & & & $40 \%$ & & & $30 \%$ & & & $\%$ & $20 \%$ & & $20 \%$ & & & $15 \%$ & \\
\hline & SIM & & $00 \%$ & & & $40 \%$ & & & $50 \%$ & & & & & & 2010 & & & 10 & \\
\hline & NÃO & & & & & & & & & & & & & & & & & & \\
\hline 28 & SIM & & $10 \%$ & & & $50 \%$ & & & $40 \%$ & & & $25 \%$ & & & $20 \%$ & & & $20 \%$ & \\
\hline & NÃO & & & & & & & & & & & & & & & & & & \\
\hline 32 & SIM & & $80 \%$ & & & $60 \%$ & & & alor-p: $0, \mathrm{c}$ & & & $30 \%$ & & & $25 \%$ & & & $20 \%$ & \\
\hline 36 & NÃO & & & & & $70 \%$ & & & & & & & & & & & & & \\
\hline 30 & SIM & & $x$ & & & $10 \%$ & & & $50 \%$ & & & $40 \%$ & & & $30 \%$ & & & $25 \%$ & \\
\hline & NÃO & & & & & & & & & & & & & & & & & & \\
\hline 40 & $\begin{array}{l}\text { SIM } \\
\end{array}$ & & $x$ & & & $80 \%$ & & & $60 \%$ & & & lor-p: 0 & & & $40 \%$ & & & $30 \%$ & \\
\hline 44 & NÃO & & & & & & & & & & & & & & & & & & \\
\hline 44 & SIM & & $x$ & & & $x$ & & & $10 \%$ & & & $50 \%$ & & & $40 \%$ & & 40 & $\%$ & $30 \%$ \\
\hline 48 & NÃO & & $\mathrm{X}$ & & & $\mathrm{x}$ & & & $80 \%$ & & & & $50 \%$ & & & $40 \%$ & & $40 \%$ & \\
\hline 48 & SIM & & $\lambda$ & & & $\lambda$ & & & $80 \%$ & & & $\%$ & $50 \%$ & & $\%$ & $40 \%$ & & $40 \%$ & \\
\hline 80 & NÃo & & $\mathrm{X}$ & & & $\mathrm{X}$ & & & $\mathrm{X}$ & & & $\mathrm{X}$ & & & $\mathrm{X}$ & & $X$ & $80 \%$ & $\mathrm{X}$ \\
\hline 80 & SIM & & $\lambda$ & & & $\lambda$ & & & $X$ & & & $X$ & & & $X$ & & 80 & $\%$ & $x$ \\
\hline
\end{tabular}

Quadro 5.13 TRIMESTRAL ADITIVO - Filtro S-WLS vs X-11 (S-WLS: $\alpha=1 / 3, \delta=1 / 30$, wo $=1$ ): MSE, MAD e SNR. Valores de ' $b$ ' a partir do qual o filtro S-WLS supera o X-11, para cada ' $k$ ' e ' $A / s$ ' (Eq. 5-2). Séries com e sem tendência 
Os gráficos 5.73 e 5.74 ilustram alguns dos resultados obtidos no Quadro 5.13 .

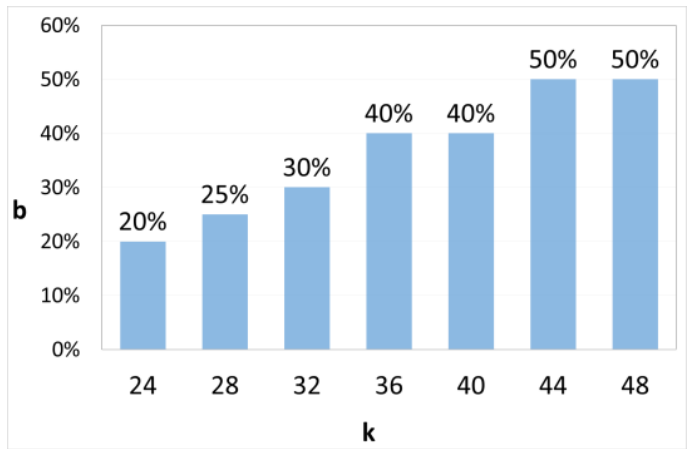

Gráfico 5.73 Valores de ' $b$ ' a partir do qual o filtro $S$-WLS supera o X-11, na SNR, para vários valores de ' $k$ ', considerando $A / s=6$ e $N=43$

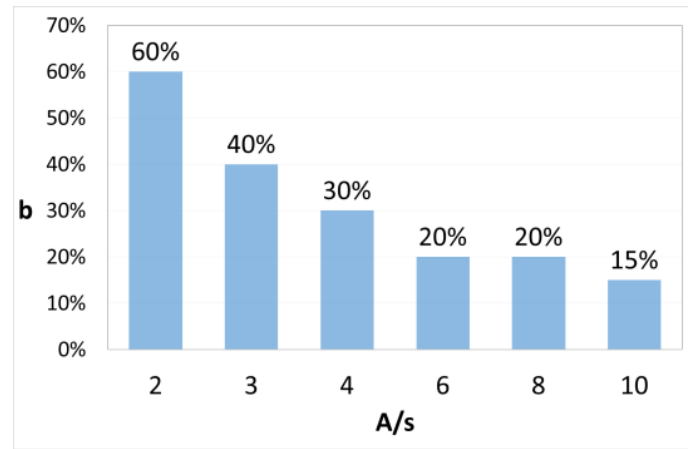

Gráfico 5.74 Valores de ' $b$ ' a partir do qual o filtro $S$-WLS supera o $X-11$, na $\mathrm{SNR}$, para vários valores de ' $A / s$ ', considerando $k=24$ e $N=43$

Analisando o Gráfico 5.73, nota-se que a partir de $20 \%$ no valor ' $b$ ', a MSE do filtro S-WLS passa a ser inferior à MSE do X11, quando ' $k$ ' é igual a 24 . Esse valor apresenta uma taxa baixa de variação da amplitude sazonal.

Em relação à ' $A / s$ ', no Gráfico 5.74 , tem-se que para valores a partir de 6,0 filtro S-WLS apresenta melhores resultados, em relação ao X-11, se ' $b$ ' for maior ou igual $20 \%$.

Essas constatações indicam que o filtro S-WLS se mostra superior ao X-11 mesmo quando o grau de sazonalidade móvel não é elevado. 


\subsubsection{1. \\ Espectros da componente irregular: série trimestral com decomposição aditiva}

Foram elaborados os espectros da componente irregular da série ' $\mathrm{Y}$ ' dessazonalizada pelos filtros S-WLS e X-11. Eles estão apresentados nos Gráficos 5.75 a 5.90 .

Os espectros foram gerados considerando a dessazonalização de séries artificiais com $b=40 \%, 25 \%$ e 10\%; para valores de ' $k$ ' iguais a 24,32 e 80 . Recorda-se que quanto maior for a taxa de variação sazonal ' $b$ ', e menor for o valor de ' $k$ ', mais forte é o grau de sazonalidade móvel. Nessa análise foi utilizada a razão $A / s=6$, e não foi utilizada a componente de tendência.

No processo de dessazonalização foi utilizado o filtro de tamanho 43. Tal filtro é o mais indicado para o X-11, quando as séries apresentam sazonalidade móvel.

Os gráficos são apresentados em pares, sendo o primeiro referente ao ajuste realizado pelo filtro S-WLS, e o segundo referente ao ajuste realizado pelo método $\mathrm{X}-11$.

Inicialmente, os Gráficos 5.75 a 5.80 apresentam os espectros da irregular para séries com $k=24$, indicando que o padrão de sazonalidade móvel leva 6 anos para se repetir. Esse valor de ' $k$ ' é o que representa a maior largura da banda passante, dentre as testadas. Nos Gráficos 5.76 e 5.78, nota-se que o espectro do X11 apresenta picos próximos à frequência $\frac{1}{4}$, quando ' $b$ ' é igual a $40 \%$ e $25 \%$. Esses valores de ' $b$ ' representam as maiores taxas de variação sazonal. Com os picos, fica evidente que, na presença de sazonalidade móvel e com a taxa de variação sazonal acima de $25 \%$, o filtro X-11 realiza um sub-ajustamento da sazonalidade.

Quando $b=10 \%$, ou seja, quando a taxa de variação sazonal é baixa, o filtro X-11 já não apresenta picos relevantes no espectro (Gráfico 5.80), indicando que ele consegue realizar um bom ajuste sazonal na série.

Nos espectros da irregular das séries dessazonalizadas pelo filtro S-WLS (Gráficos 5.75, 5.77 e 5.79), não se observam picos fortes na frequência sazonal, ou próximos a ela, indicando um ajuste sazonal adequado. 


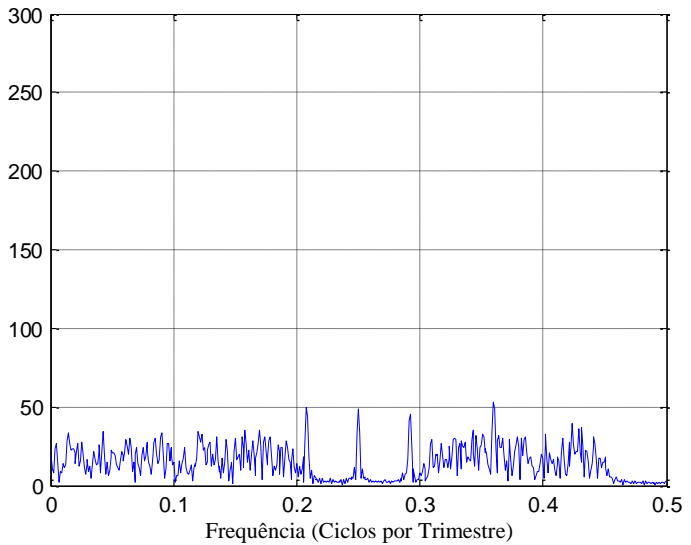

Gráfico 5.75 Espectro da irregular da série dessazonalizada pelo filtro S-WLS, com: $A / s=6, k=24, b=40 \%$ e $N=43$

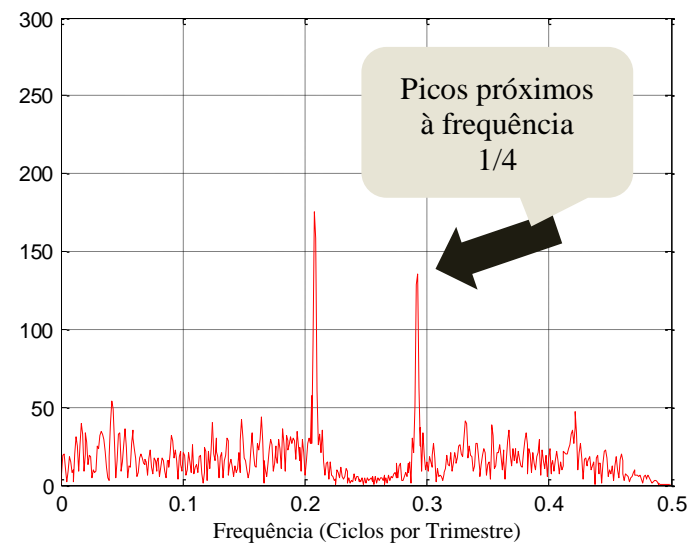

Gráfico 5.76 Espectro da irregular da série dessazonalizada pelo $X-11$, com: $A / s=6, k=24, b=40 \%$ e $N$ $=43$

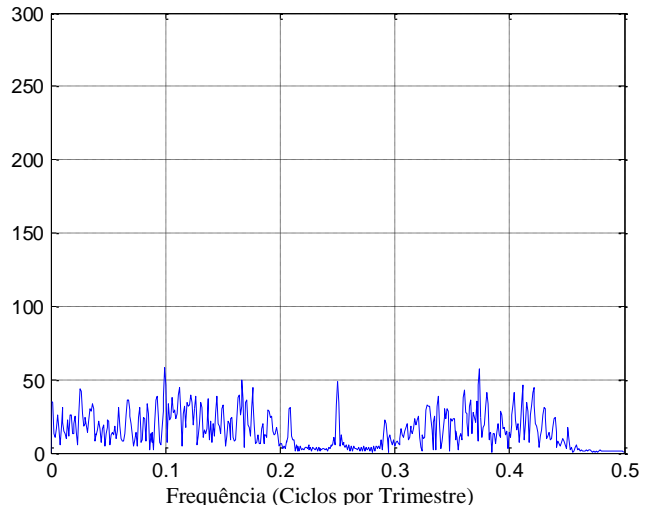

Gráfico 5.77 Espectro da irregular da série dessazonalizada pelo filtro S-WLS, com: $A / s=6$, $k=24, b=25 \%$ e $N=43$

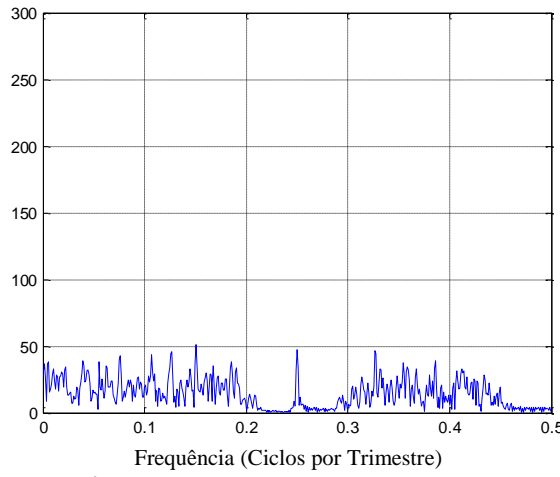

Gráfico 5.79 Espectro da irregular da série dessazonalizada pelo filtro $S$ WLS, com: $A / s=6, k=24$ e $b=10 \%$ e $N=43$

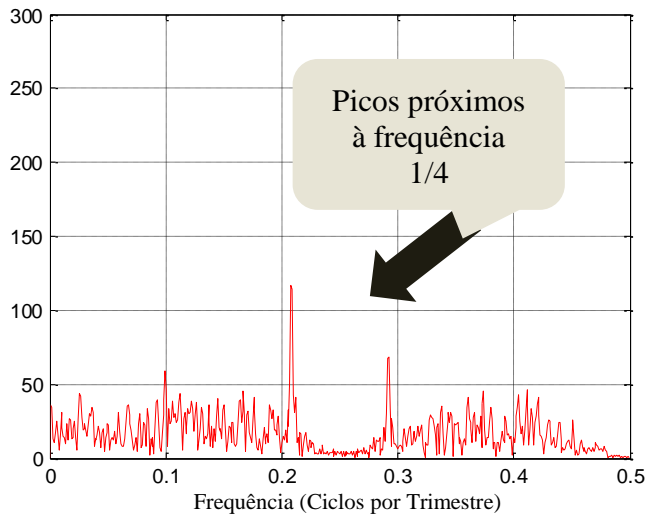

Gráfico 5.78 Espectro da irregular da série dessazonalizada pelo X-11, com: $A / s=6, k=24, b=$ $25 \%$ e $N=43$

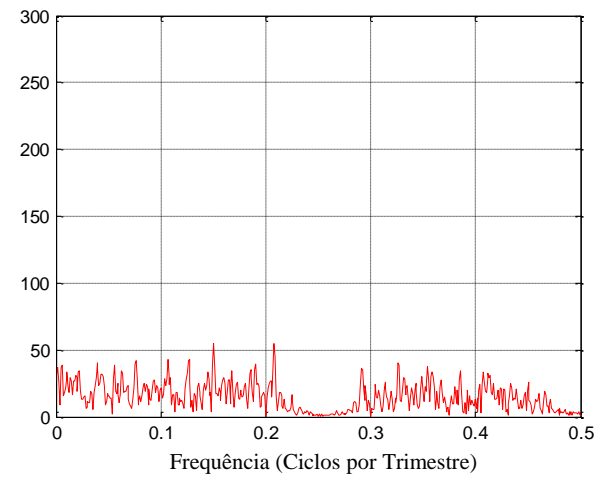

Gráfico 5.80 Espectro da irregular da série dessazonalizada pelo $\mathrm{X}-11$, com: $A / s=6, k=24, b=10 \%$ e $N$ $=43$ 
Os Gráficos 5.81 a 5.86 apresentam os espectros da irregular para séries com $k=32$. Isso significa que o padrão de sazonalidade móvel leva 8 anos para se repetir. Nos Gráficos 5.82 e 5.84, observa-se que os picos próximos à frequência $1 / 4$ são menores, se comparados aos picos quando $k=24$. Porém, eles ainda são relevantes.

Novamente, quando $b=10 \%$, ou seja, quando a taxa de variação sazonal é baixa, o filtro X-11 não apresenta picos relevantes no espectro (Gráfico 5.86), indicando que ele consegue realizar um bom ajuste sazonal.

Como ocorrido anteriormente (quando $k=24$ ), os espectros da irregular das séries dessazonalizadas pelo filtro S-WLS não possuem picos fortes na frequência sazonal, ou próximos a ela, indicando que o filtro S-WLS realizou um ajuste sazonal correto. Esses espectros estão apresentados nos Gráficos 5.81, 5.83 e 5.85.

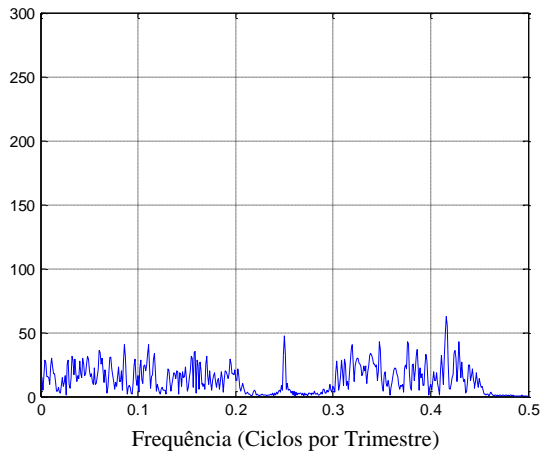

Gráfico 5.81 Espectro da irregular da série dessazonalizada pelo filtro S-WLS, com: $A / s=6, k=32, b=$ $40 \%$ e $N=43$

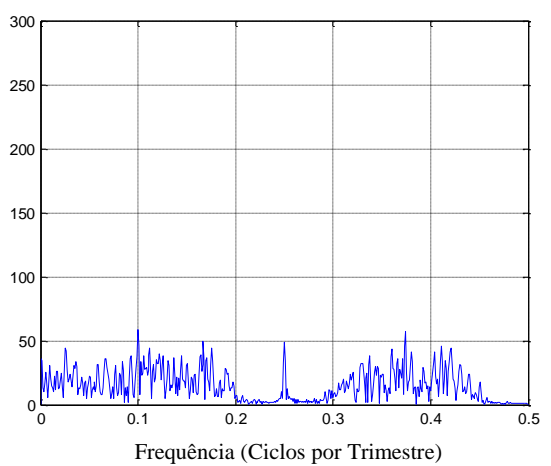

Gráfico 5.83 Espectro da irregular da série dessazonalizada pelo filtro S-WLS, com: $A / s=6$, $k=32, b=25 \%$ e $N=43$

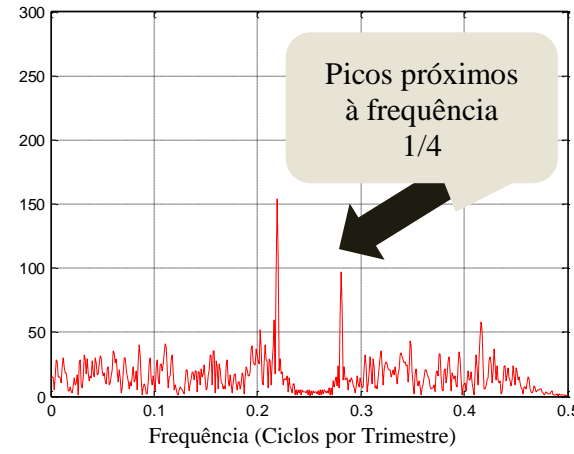

Gráfico 5.82 Espectro da irregular da série dessazonalizada pelo $\mathrm{X}-11$, com: $A / s=6, k=32, b=$ $40 \%$ e $N=43$

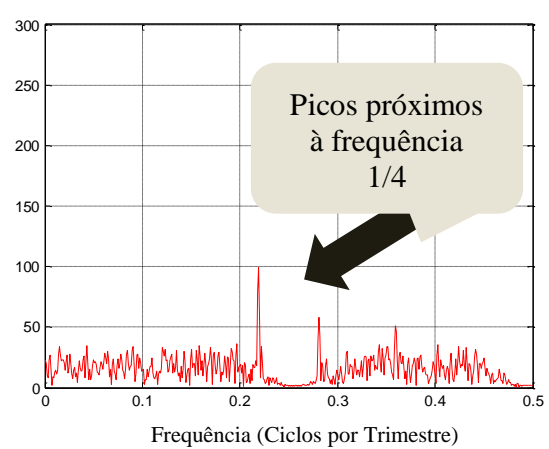

Gráfico 5.84 Espectro da irregular da série dessazonalizada pelo $X-11$, com: $A / s=6, k=32, b=$ $25 \%$ e $N=43$ 


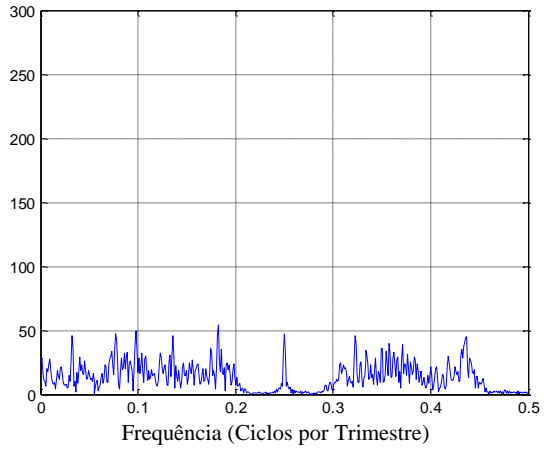

Gráfico 5.85 Espectro da irregular da série dessazonalizada pelo filtro S-WLS, com: $A / s=6, k=32, b=10 \%$ e $N=43$

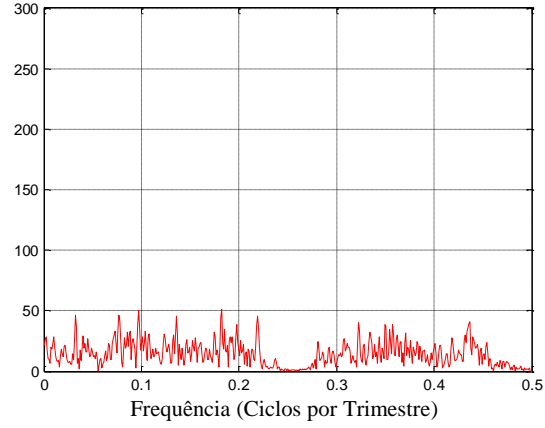

Gráfico 5.86 Espectro da irregular da série dessazonalizada pelo $\mathrm{X}-11$, com: $A / s=6, k=32, b=10 \%$ e $N=43$

Os espectros da irregular das séries que possuem $k=80$ (Gráficos 5.87 a 5.90) não apresentam quaisquer picos na frequência fundamental, ou próximos a ela. $\mathrm{O}$ valor $k=80$, indica que o padrão de sazonalidade móvel se repete em 20 anos. Com isso, mesmo para $b=40 \%$, o grau de sazonalidade móvel é baixíssimo. E, sendo assim, o filtro X-11 apresenta um bom desempenho. O filtro S-WLS, já apresentava bom desempenho para valores menores de ' $k$ ', e continua apresentando para $k=$ 80.

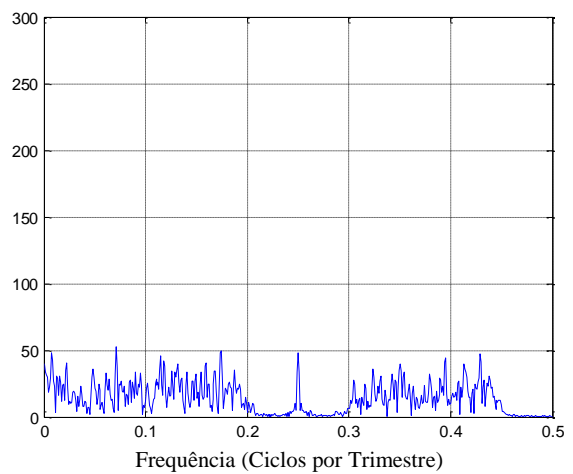

Gráfico 5.87 Espectro da irregular da série dessazonalizada pelo filtro S-WLS, com: $A / s=6, k=80, b=40 \%$ e $N=43$

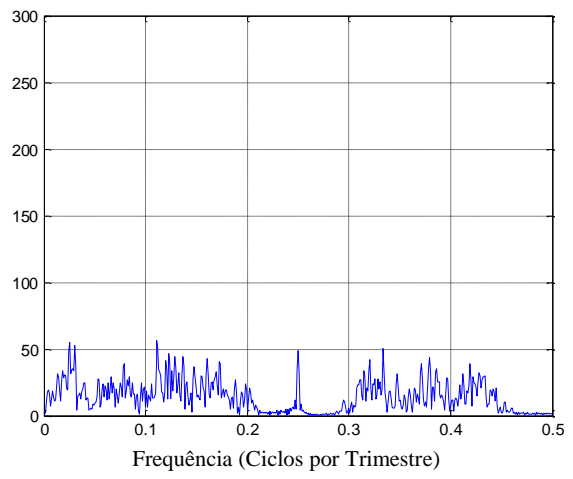

Gráfico 5.89 Espectro da irregular da série dessazonalizada pelo filtro $S-W L S$, com: $A / s=6, k=80, b=10 \%$ e $N=43$

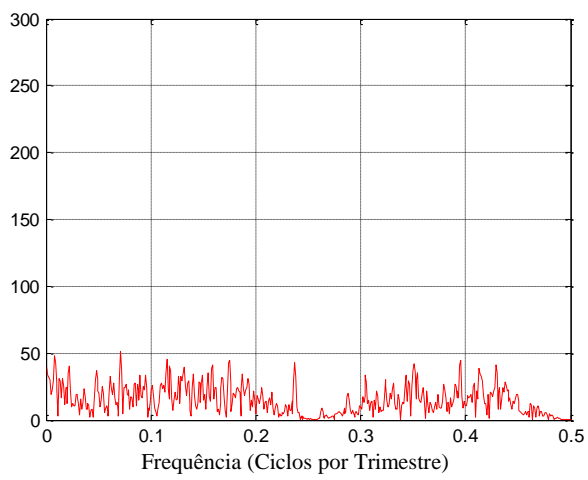

Gráfico 5.88 Espectro da irregular da série dessazonalizada pelo $X-11$, com: $A / s=6, k=80, b=40 \%$ e $N=43$

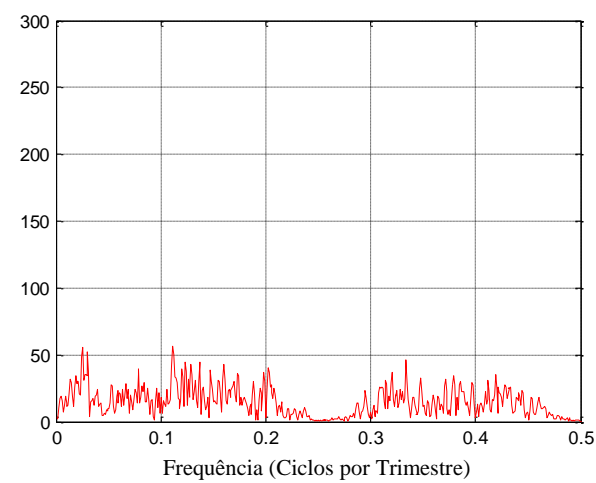

Gráfico 5.90 Espectro da irregular da série dessazonalizada pelo $X-11$, com: $A / s=6, k=80, b=10 \%$ e $N=43$ 


\subsection{5.}

\section{Resultados: série trimestral com decomposição multiplicativa - Filtro S-WLS vs $\mathrm{X}-11$}

Para as comparações envolvendo o filtro S-WLS e o X-11 no caso nas séries trimestrais com decomposição multiplicativa, foi utilizado o mesmo procedimento utilizado nas séries mensais, descrito na Figura 5.8 da Subseção 5.5.3. A configuração do filtro utilizado foi a seguinte: $\alpha=\mathbf{1 / 3}, \delta=\mathbf{1 / 3 0}$ e $\boldsymbol{w}_{\mathbf{0}}=\mathbf{1}$.

Em relação aos valores dos sinais artificiais, foram utilizados:

- $A / s=[1,2,3,4,6,8,10]$

- $b=[5 \%, 10 \%, 15 \%, 20 \%, 25 \%, 30 \%, 40 \%, 50 \%, 60 \%, 70 \%, 80 \%]$

- $k=[16,20,24,28,32,36,40,44,48,80]$

Foram geradas séries com, e sem, tendência. Nas séries com componente de tendência, foram usados os seguintes valores para os coeficientes do polinômio: $b_{0}=1.503,8 ; b_{1}=-23.415 ; b_{2}=3,8097$ e $b_{3}=-0,00357$, baseados na série Y91.

Quanto à componente irregular, geraram-se 100 séries, com distribuição $\mathrm{N}\left(0, \sigma^{2}\right)$, com base no desvio-padrão da irregular das séries reais. E, assim como nos casos anteriores, o teste $t$ de igualdade de médias de duas populações, com variâncias desconhecidas e equivalentes, foi realizado para o MSE e o MAD dos filtros. Nesse teste, utilizou-se nível de significância de 5\%.

Nos itens (i), (ii), (iii) e (iv) são apresentados os resultados das comparações entre o filtro S-WLS e o X-11. As análises foram realizadas com base na variação dos parâmetros ' $b$ ', ' $k$ ' e ' $A / s$ ', do sinal artificial, apresentados na Equação (5-4). 


\section{(i) Resultados encontrados com base na variação de ' $b$ ': série trimestral multiplicativa}

Nas séries reais, os valores do parâmetro ' $b$ ' variam entre $10 \%$ e $50 \%$. Recorda-se que ' $b$ ' representa o número de trimestres com que o padrão de sazonalidade móvel volta a se repetir.

O Gráfico 5.91, a seguir, mostra a comparação entre a MSE do filtro S-WLS e a MSE do X-11, segundo diferentes valores de ' $b$ '. Mais uma vez, nota-se pouca variação na MSE do filtro S-WLS, enquanto que a MSE do X-11 cresce exponencialmente à medida que o valor de ' $b$ ' aumenta.

Analisando a Tabela 5.26, que apresenta os valores utilizados no Gráfico 5.91, e o valor-p do teste de comparação de médias, percebe-se que a partir de $b=$ $25 \%$ o filtro S-WLS apresenta desempenho melhor do que o X-11, tanto em relação à MSE quanto em relação à MAD.

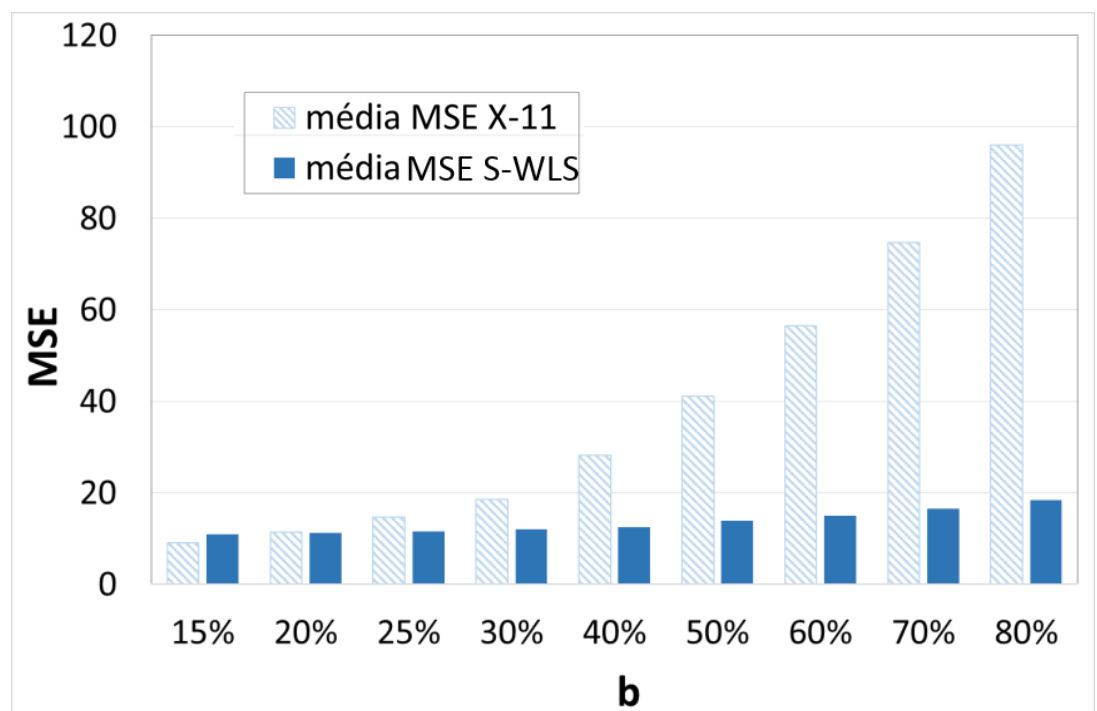

Gráfico 5.91 Média da MSE $\left(\mathrm{x}^{10}{ }^{6}\right)$ com o filtro X-11 e com o filtro S-WLS, para valores de ' $b$ ', considerando $A / s=6$ e $k=24$

\begin{tabular}{c|ccc|ccc}
\hline $\boldsymbol{b}$ & MSE & MSE X-11 & valor-p & $\begin{array}{c}\text { MAD } \\
\text { S-WLS }\end{array}$ & MAD X-11 & valor-p \\
\hline $\mathbf{1 5 \%}$ & $1,10 \mathrm{E}-05$ & $9,01 \mathrm{E}-06$ & 1,000 & $2,65 \mathrm{E}-03$ & $2,40 \mathrm{E}-03$ & 1,000 \\
$\mathbf{2 0 \%}$ & $1,12 \mathrm{E}-05$ & $1,14 \mathrm{E}-05$ & 0,090 & $2,67 \mathrm{E}-03$ & $2,69 \mathrm{E}-03$ & 0,203 \\
$\mathbf{2 5 \%}$ & $1,16 \mathrm{E}-05$ & $1,47 \mathrm{E}-05$ & 0,000 & $2,71 \mathrm{E}-03$ & $3,03 \mathrm{E}-03$ & 0,000 \\
$\mathbf{3 0 \%}$ & $1,20 \mathrm{E}-05$ & $1,86 \mathrm{E}-05$ & 0,000 & $2,76 \mathrm{E}-03$ & $3,41 \mathrm{E}-03$ & 0,000 \\
$\mathbf{4 0 \%}$ & $1,24 \mathrm{E}-05$ & $2,82 \mathrm{E}-05$ & 0,000 & $2,81 \mathrm{E}-03$ & $4,14 \mathrm{E}-03$ & 0,000 \\
$\mathbf{5 0 \%}$ & $1,38 \mathrm{E}-05$ & $4,11 \mathrm{E}-05$ & 0,000 & $2,96 \mathrm{E}-03$ & $4,95 \mathrm{E}-03$ & 0,000 \\
$\mathbf{6 0 \%}$ & $1,50 \mathrm{E}-05$ & $5,64 \mathrm{E}-05$ & 0,000 & $3,08 \mathrm{E}-03$ & $5,74 \mathrm{E}-03$ & 0,000 \\
$\mathbf{7 0 \%}$ & $1,65 \mathrm{E}-05$ & $7,47 \mathrm{E}-05$ & 0,000 & $3,22 \mathrm{E}-03$ & $6,55 \mathrm{E}-03$ & 0,000 \\
$\mathbf{8 0 \%}$ & $1,84 \mathrm{E}-05$ & $9,61 \mathrm{E}-05$ & 0,000 & $3,38 \mathrm{E}-03$ & $7,38 \mathrm{E}-03$ & 0,000 \\
\hline
\end{tabular}

Tabela 5.26 MSE, MAD e SNR para valores de ' $b$ ', sendo $A / s=6, k=24$ séries trimestrais com decomposição multiplicativa 
(ii) Resultados encontrados com base na variação de ' $k$ ': série trimestral multiplicativa

Em relação ao parâmetro ' $k$ ', há nas séries reais uma concentração entre os valores 24 e 32, indicando que é necessário de 6 a 8 anos para que o padrão de sazonalidade móvel volte a se repetir.

No Gráfico 5.92 são apresentadas a MSE do X-11 e do filtro S-WLS para diferentes valores de ' $k$ '. Nota-se que a MSE do X-11 é muito alta para valores baixos de ' $k$ '. Até $k=40$, o filtro S-WLS apresenta resultados melhores do que o $\mathrm{X}-11$, tanto na MSE quanto na MAD. Isso pode ser observado nos valores-p, da Tabela 5.27.

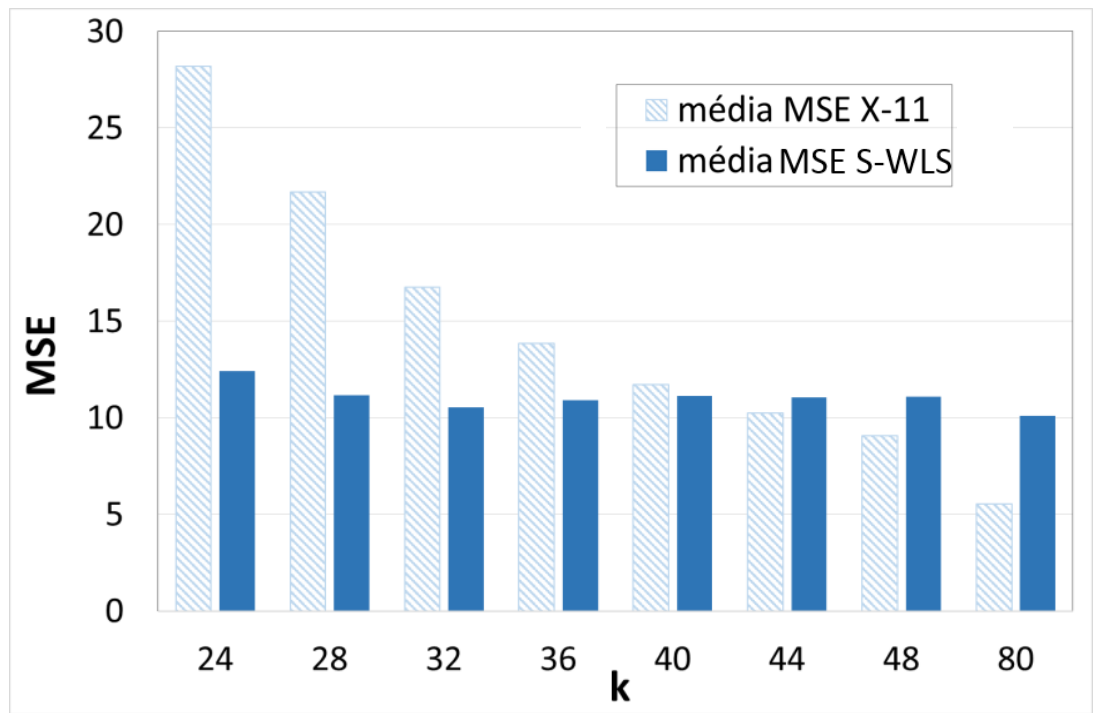

Gráfico 5.92 Média da MSE $\left(\mathrm{x}^{10^{6}}\right)$ com o filtro X-11 e com o filtro S-WLS, para valores de ' $k$ ', considerando $A / s=6$ e $b=40 \%$

\begin{tabular}{|c|c|c|c|c|c|c|}
\hline $\boldsymbol{k}$ & $\begin{array}{c}\text { MSE } \\
\text { S-WLS } \\
\end{array}$ & MSE X-11 & valor-p & $\begin{array}{c}\text { MAD } \\
\text { S-WLS } \\
\end{array}$ & MAD X-11 & valor-p \\
\hline 20 & $2,25 \mathrm{E}-05$ & $3,82 \mathrm{E}-05$ & 0,000 & 3,73E-03 & 4,71E-03 & 0,000 \\
\hline 24 & $1,24 \mathrm{E}-05$ & $2,82 \mathrm{E}-05$ & 0,000 & $2,81 \mathrm{E}-03$ & 4,14E-03 & 0,000 \\
\hline 28 & $1,12 \mathrm{E}-05$ & $2,17 \mathrm{E}-05$ & 0,000 & $2,67 \mathrm{E}-03$ & $3,69 \mathrm{E}-03$ & 0,000 \\
\hline 32 & $1,05 \mathrm{E}-05$ & $1,67 \mathrm{E}-05$ & 0,000 & $2,60 \mathrm{E}-03$ & $3,27 \mathrm{E}-03$ & 0,000 \\
\hline 36 & $1,09 \mathrm{E}-05$ & $1,39 \mathrm{E}-05$ & 0,000 & $2,63 \mathrm{E}-03$ & 2,98E-03 & 0,000 \\
\hline 40 & $1,11 \mathrm{E}-05$ & $1,17 \mathrm{E}-05$ & 0,001 & $2,66 \mathrm{E}-03$ & 2,74E-03 & 0,000 \\
\hline 44 & $1,11 \mathrm{E}-05$ & $1,02 \mathrm{E}-05$ & 1,000 & $2,65 \mathrm{E}-03$ & $2,57 \mathrm{E}-03$ & 1,000 \\
\hline 48 & $1,11 \mathrm{E}-05$ & $9,08 \mathrm{E}-06$ & 1,000 & $2,66 \mathrm{E}-03$ & $2,41 \mathrm{E}-03$ & 1,000 \\
\hline 80 & $1,01 \mathrm{E}-05$ & $5,55 \mathrm{E}-06$ & 1,000 & $2,53 \mathrm{E}-03$ & $1,88 \mathrm{E}-03$ & 1,000 \\
\hline
\end{tabular}

Tabela 5.27 MSE, MAD e SNR para valores de ' $k$ ', sendo $A / s=6, b=40 \%$ séries trimestrais com decomposição multiplicativa 
(iii) Resultados encontrados com base na variação de ' $A / s^{\prime}$ ': série trimestral multiplicativa

No que tange à razão entre a amplitude do sinal sazonal e o desvio-padrão da componente irregular $(A / S)$, observa-se no Gráfico 5.93 que a MSE de ambos os filtros diminuem à medida que ' $A / s$ ' aumenta. Porém, assim como no caso aditivo, a redução da MSE do filtro S-WLS é mais acentuada do que a do X-11.

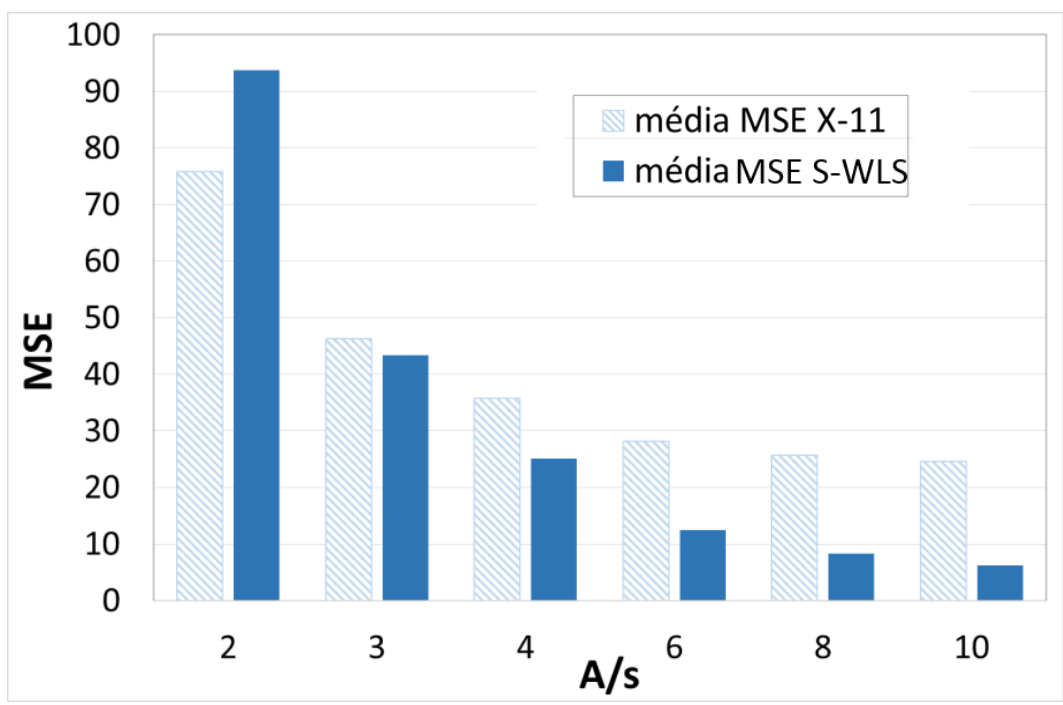

Gráfico 5.93 Média da MSE ( $\left.\mathrm{x}^{10}{ }^{6}\right)$ com o filtro X-11 e com o filtro S-WLS, para valores de ' $A / s$ ', considerando $k=24$ e $b=40 \%$

A Tabela 5.28, a seguir, mostra o valor-p da comparação entre o filtro S-WLS e o X-11, para MSE e MAD. Em ambos os casos, o filtro S-WLS começa a superar o X-11 quando $A / s=3$. Vale mencionar que, nas séries reais, os valores da razão ' $A / s$ ' estão concentrados entre 4 e 7 . Portanto, $A / s \geq 3$ é um resultado satisfatório para o filtro S-WLS, com base nas séries reais.

\begin{tabular}{c|ccc|ccc}
\hline $\boldsymbol{A} / \boldsymbol{s}$ & MSE & MSE X-11 & valor-p & $\begin{array}{c}\text { MAD } \\
\text { S-WLS }\end{array}$ & MAD X-11 & valor-p \\
\hline $\mathbf{2}$ & $9,4 \mathrm{E}-05$ & $7,6 \mathrm{E}-05$ & 1,000 & $7,7 \mathrm{E}-03$ & $6,9 \mathrm{E}-03$ & 1,000 \\
$\mathbf{3}$ & $4,3 \mathrm{E}-05$ & $4,6 \mathrm{E}-05$ & 0,000 & $5,3 \mathrm{E}-03$ & $5,4 \mathrm{E}-03$ & 0,000 \\
$\mathbf{4}$ & $2,5 \mathrm{E}-05$ & $3,6 \mathrm{E}-05$ & 0,000 & $4,0 \mathrm{E}-03$ & $4,7 \mathrm{E}-03$ & 0,000 \\
$\mathbf{6}$ & $1,2 \mathrm{E}-05$ & $2,8 \mathrm{E}-05$ & 0,000 & $2,8 \mathrm{E}-03$ & $4,1 \mathrm{E}-03$ & 0,000 \\
$\mathbf{8}$ & $8,3 \mathrm{E}-06$ & $2,6 \mathrm{E}-05$ & 0,000 & $2,3 \mathrm{E}-03$ & $3,9 \mathrm{E}-03$ & 0,000 \\
$\mathbf{1 0}$ & $6,2 \mathrm{E}-06$ & $2,5 \mathrm{E}-05$ & 0,000 & $1,9 \mathrm{E}-03$ & $3,8 \mathrm{E}-03$ & 0,000 \\
\hline
\end{tabular}

Tabela 5.28 MSE e MAD para valores de ' $A / s$ ', sendo $k=24, b=40 \%$ - séries trimestrais com decomposição multiplicativa 
(iv) Condições nas quais as estatísticas MSE e MAD do filtro S-WLS são melhores do que as do $\mathrm{X}-11$, segundo valores de ' $A / s$ ', ' $b$ ' e ' $k$ ': série trimestral com decomposição multiplicativa

O resultado das comparações entre o filtro S-WLS e o X-11 para as séries trimestrais multiplicativas, encontra-se no Quadro 5.14, a seguir. O filtro S-WLS utilizado foi: $\alpha=1 / 3, \delta=1 / 30$ e $\mathrm{w}_{0}=1$.

No Quadro 5.14, os valores das células indicam o valor mínimo de ' $\boldsymbol{b}$ ' a partir do qual o filtro S-WLS supera o X-11. Apenas os resultados dos testes cujo valor$\mathrm{p}$ foram menores do que $5 \%$ foram considerados como significativamente melhores. No quadro, todos os valores-p não apresentados são menores do que $1 \%$.

\begin{tabular}{|c|c|c|c|c|c|c|c|c|c|c|c|c|}
\hline \multirow{3}{*}{$\boldsymbol{k}$} & \multirow{3}{*}{ Tendência } & \multicolumn{11}{|c|}{$A / s$} \\
\hline & & \multicolumn{2}{|c|}{2} & \multicolumn{2}{|c|}{3} & \multicolumn{2}{|c|}{4} & \multicolumn{2}{|c|}{6} & 8 & \multicolumn{2}{|c|}{10} \\
\hline & & MSE & MAD & MSE & MAD & MSE & MAD & MSE & MAD & MSE MAD & MSE & MAD \\
\hline \multirow{2}{*}{16} & NÃO & \multirow{2}{*}{\multicolumn{2}{|c|}{$60 \%$}} & \multirow{2}{*}{\multicolumn{2}{|c|}{$40 \%$}} & \multirow{2}{*}{\multicolumn{2}{|c|}{$30 \%$}} & \multirow{2}{*}{\multicolumn{2}{|c|}{$25 \%$}} & \multirow{2}{*}{$20 \%$} & \multirow{2}{*}{\multicolumn{2}{|c|}{$15 \%$}} \\
\hline & SIM & & & & & & & & & & & \\
\hline \multirow{2}{*}{20} & NÃO & \multirow{2}{*}{\multicolumn{2}{|c|}{$60 \%$}} & \multirow{2}{*}{\multicolumn{2}{|c|}{$40 \%$}} & \multirow{2}{*}{\multicolumn{2}{|c|}{$30 \%$}} & \multirow{2}{*}{\multicolumn{2}{|c|}{$25 \%$}} & \multirow{2}{*}{$20 \%$} & \multirow{2}{*}{\multicolumn{2}{|c|}{$15 \%$}} \\
\hline & SIM & & & & & & & & & & & \\
\hline \multirow{2}{*}{24} & NÃO & \multirow{2}{*}{\multicolumn{2}{|c|}{$60 \%$}} & \multirow{2}{*}{\multicolumn{2}{|c|}{$40 \%$}} & \multirow{2}{*}{\multicolumn{2}{|c|}{$30 \%$}} & \multirow{2}{*}{\multicolumn{2}{|c|}{$25 \%$}} & \multirow{2}{*}{$20 \%$} & \multirow{2}{*}{\multicolumn{2}{|c|}{$15 \%$}} \\
\hline & SIM & & & & & & & & & & & \\
\hline \multirow{2}{*}{28} & NÃO & & & & & & & & & 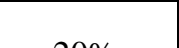 & & \\
\hline & $\overline{\text { SIM }}$ & $10 \%$ & & & & & & & & $20 \%$ & & $\%$ \\
\hline$\Omega$ & NÃO & 000 & & & & & $\%$ & & & 250 & & 07 \\
\hline 32 & SIM & $80 \%$ & & & & $50 \%$ & $40 \%$ & & & $25 \%$ & & J\% \\
\hline & NÃO & & & & & & & & & $20 \Omega$ & & -07 \\
\hline 36 & SIM & $X$ & & & & & $1 \%$ & & & $30 \%$ & & $\%$ \\
\hline 10 & NÃO & $\mathbf{V}$ & & & & & & & & & & \\
\hline 40 & SIM & $X$ & & & & & $\%$ & & & $40 \%$ & & $\%$ \\
\hline & NÃo & & & & & & & & & 100 & & \\
\hline 44 & SIM & $X$ & & & & & $\%$ & & $\%$ & $40 \%$ & & $\%$ \\
\hline 10 & NÃO & $\mathrm{Y}$ & & & & & & & & & & \\
\hline 48 & $\overline{\text { SIM }}$ & $X$ & & & & & $1 \%$ & & $1 \%$ & $50 \%$ & & 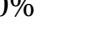 \\
\hline 80 & NÃO & $\mathrm{Y}$ & & & & & 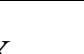 & & 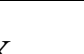 & $\mathrm{Y}$ & & $\%$ \\
\hline 00 & SIM & $\Lambda$ & & & & & 1 & & 1 & $\Lambda$ & (valor-1 & p: 0,031$)$ \\
\hline
\end{tabular}

Quadro 5.14 TRIMESTRAL MULTIPLICATIVO - Filtro S-WLS ( $\alpha=1 / 3$, $\left.\delta=1 / 30, w_{0}=1\right)$ vs $X-11$ : MSE e MAD. Valores de ' $b$ ' a partir do qual o filtro SWLS supera o X-11, para cada ' $k$ ' e ' $A / s$ '. Séries com e sem tendência 
Comparando os resultados do Quadro 5.14, com os resultados do Quadro 5.13 (referentes às séries com decomposição aditiva), percebe-se valores muito semelhantes, indicando uma robustez do filtro quanto ao ajuste dos séries com diferentes modelos de decomposição.

A consistência dos resultados também está presente em relação à tendência. Os resultados, apresentados no Quadro 5.14, mostram que em apenas um caso houve diferença em relação à tendência.

Os Gráficos 5.94 e 5.95 foram elaborados com base no Quadro 5.14. Nesses gráficos é possível notar o valor mínimo ' $\boldsymbol{b}$ ' a partir do qual a MSE do filtro S-WLS passa a ser inferior à MSE do X11. Observa-se que para ' $k$ ' entre 24 e 28, é necessário apenas que ' $b$ ' seja pelo menos $25 \%$ para o filtro S-WLS superar o X11. Já em relação à ' $A / s$ ', para valores a partir de 6 , o filtro $S$-WLS apresenta melhores resultados, em relação ao X-11, se ' $b$ ' maior ou igual $25 \%$.

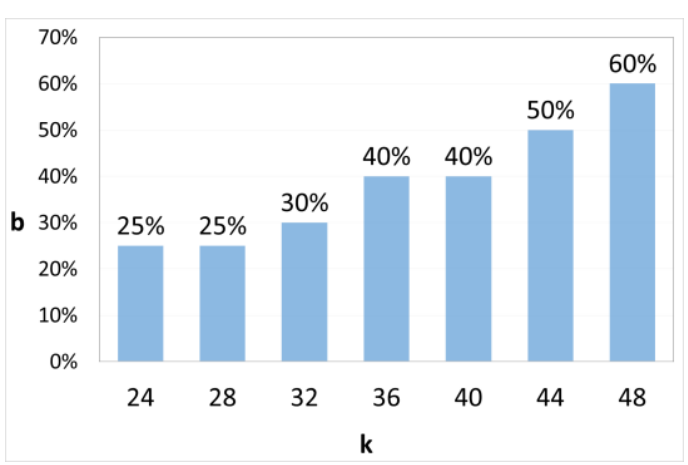

Gráfico 5.94 Valores de ' $b$ ' a partir do qual o filtro S-WLS supera o X-11, na MSE, para valores de ' $k$ ', considerando $A / s=6$

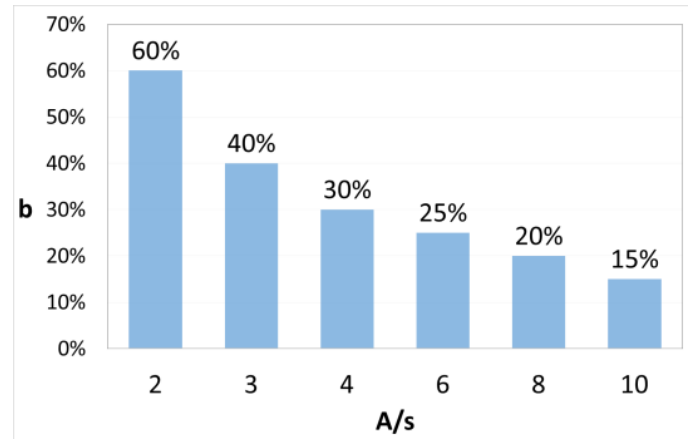

Gráfico 5.95 Valores de ' $b$ ' a partir do qual o filtro S-WLS supera o $X-11$, na MSE, para vários valores de ' $A / s$ ', considerando $k=24$ 


\subsubsection{1. Espectros da componente irregular: série trimestral multiplicativa}

Assim como nos casos anteriores, foram elaborados os espectros da irregular da série ' $Y$ ' dessazonalizada pelo filtro S-WLS e pelo X-11. Eles estão apresentados nos Gráficos 5.96 a 5.107. Vale lembrar que o espectro da irregular fornece uma indicação visual do sucesso na remoção da sazonalidade.

Os espectros foram gerados considerando a dessazonalização de séries artificiais com $b=40 \%, 25 \%$ e $10 \%$; para valores de ' $k$ ' iguais a 24 e 32 . Foi adotada a razão $A / s=6$. Na construção dos espectros, foi usado o logaritmo neperiano da série dessazonalizada, multiplicado por 10. Além disso, não foi utilizada a componente de tendência nas séries artificiais.

O filtro utilizado no processo de dessazonalização teve tamanho 43. Como já mencionado, tal tamanho de filtro é indicado para o X-11 quando as séries apresentam sazonalidade móvel, pois a média móvel utilizada é a 3x3.

Os gráficos são apresentados em pares, sendo o primeiro referente ao ajuste realizado pelo filtro S-WLS, e o segundo referente ao ajuste realizado pelo método X-11. Inicialmente, os Gráficos 5.96 a 5.101 apresentam os espectros para séries com $k=24$, indicando que o padrão de sazonalidade móvel leva 6 anos para se repetir. Esse valor de ' $k$ ' é o que representa a maior largura da banda passante, dentre as testadas.

Nos Gráficos 5.97 e 5.99, notam-se grandes picos sazonais residuais no espectro do X-11, indicando que a sazonalidade não foi removida de forma adequada, quando a taxa de variação da amplitude sazonal (b) é igual a $40 \%$ ou $25 \%$.

Nos demais gráficos (Gráficos 5.96, 5.98, 5.100 e 5.101), notam-se pequenos picos na frequência $\frac{1}{4}$ ou próximos a ele. Eles não são considerados relevantes, pois a magnitude deles é a mesma dos resíduos, porém, fica evidente que eles estão relacionados à componente sazonal. 


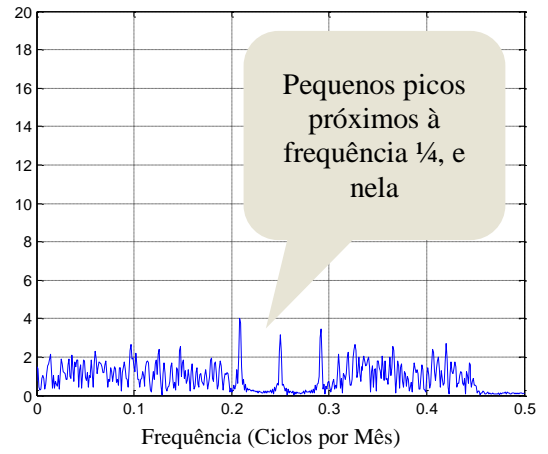

Gráfico 5.96 Espectro da irregular da série dessazonalizada pelo filtro $S$-WLS, com: $A / s=6, k=24, b=40 \%$ e $N=43$

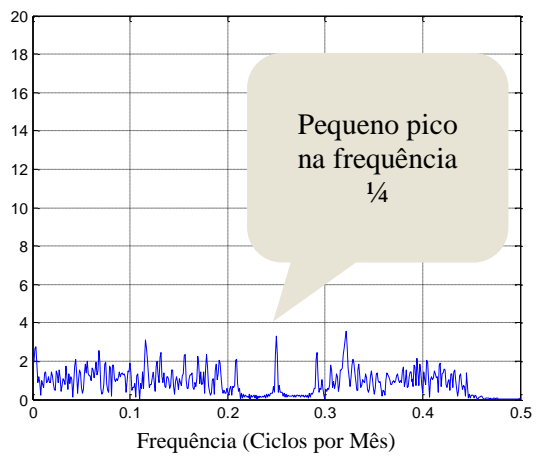

Gráfico 5.98 Espectro da irregular da série dessazonalizada pelo filtro S-WLS, com: $A / s=6, k=24, b=$ $25 \%$ e $N=43$

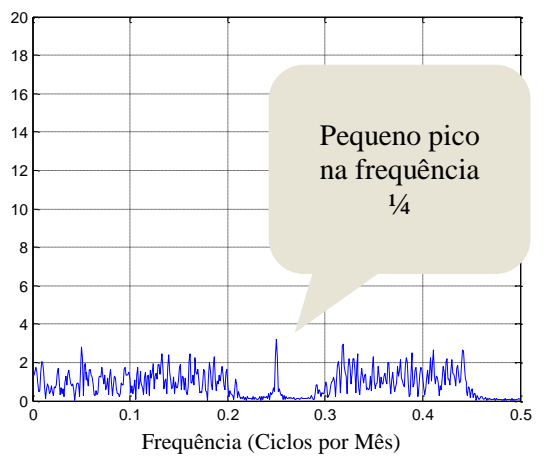

Gráfico 5.100 Espectro da irregular da série dessazonalizada pelo filtro $S$ WLS, com: $A / s=6, k=24, b=10 \% \mathrm{e}$ $N=43$

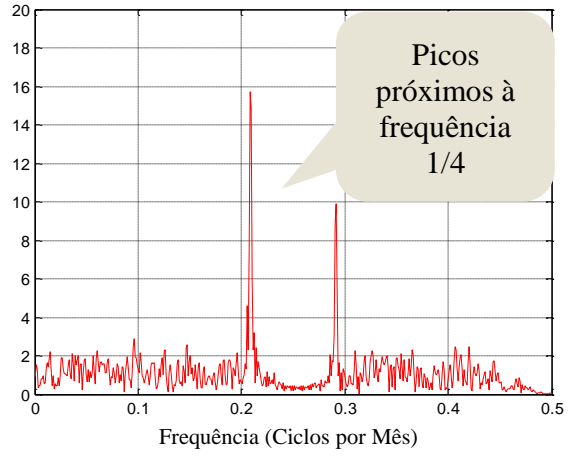

Gráfico 5.97 Espectro da irregular da série dessazonalizada pelo $X-11$, com: $A / s=6, k=24, b=40 \%$ e $N=43$

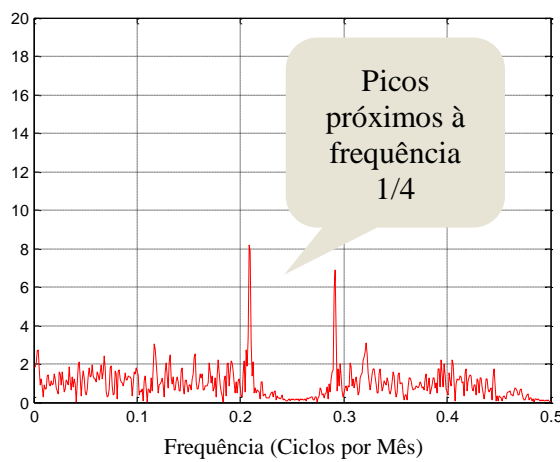

Gráfico 5.99 Espectro da irregular da série dessazonalizada pelo $X-11$, com: $A / s=6, k=24, b=25 \%$ e $N=43$

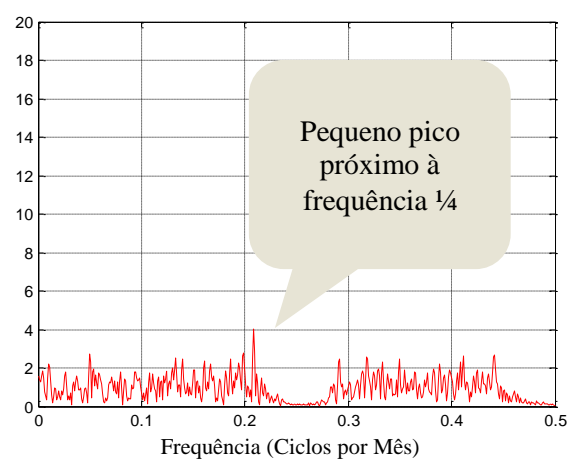

Gráfico 5.101 Espectro da irregular da série dessazonalizada pelo $X-11$, com: $A / s=6, k=24, b=$ $10 \%$ e $N=43$ 
Os Gráficos 5.102 a 5.107 apresentam os espectros da irregular para séries com $k=32$. Isso significa que o padrão de sazonalidade móvel leva 8 anos para se repetir, com base no sinal artificial (Eq. 5-4). Nos Gráficos 5.103 e 5.105, observase que os picos próximos à frequência $1 / 4$ são inferiores aos picos quando $k=24$. Porém, eles ainda são relevantes.

Quando $b=10 \%$ (Gráfico 5.107), ou seja, quando a taxa de variação sazonal é muito baixa, o espectro do filtro X-11 não apresenta picos, indicando que o X-11 consegue realizar um ajuste sazonal adequado.

Os espectros relacionados ao filtro S-WLS (Gráfico 5.102, 5.104 e 5.106) mostram um pequeno pico na frequência $\frac{1}{4}$. Como citado anteriormente, este pico está no mesmo nível dos resíduos, não se mostrando relevante. 


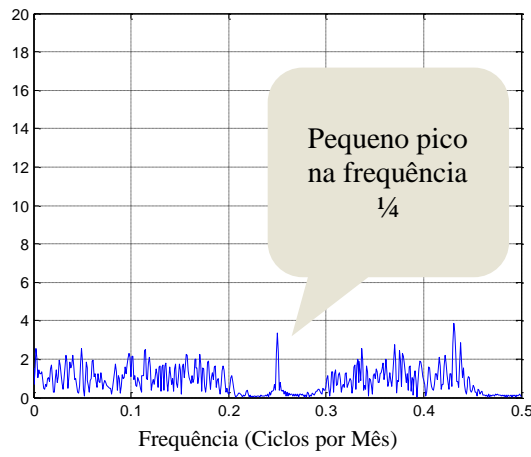

Gráfico 5.102 Espectro da irregular da série dessazonalizada pelo filtro $S$ WLS, com: $A / s=6, k=32, b=40 \%$ e $N$ $=43$

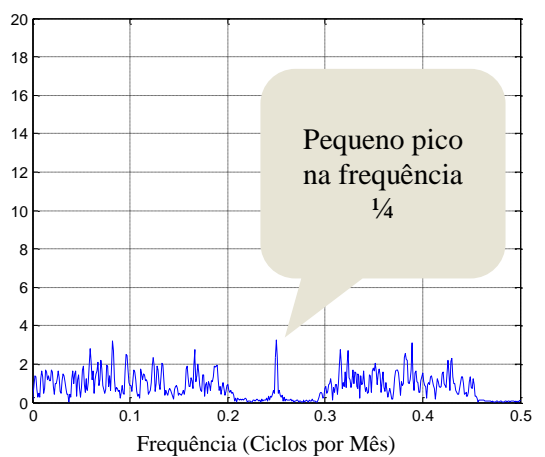

Gráfico 5.104 Espectro da irregular da série dessazonalizada pelo filtro $S$ WLS, com: $A / s=6, k=32, b=25 \% \mathrm{e}$ $N=43$

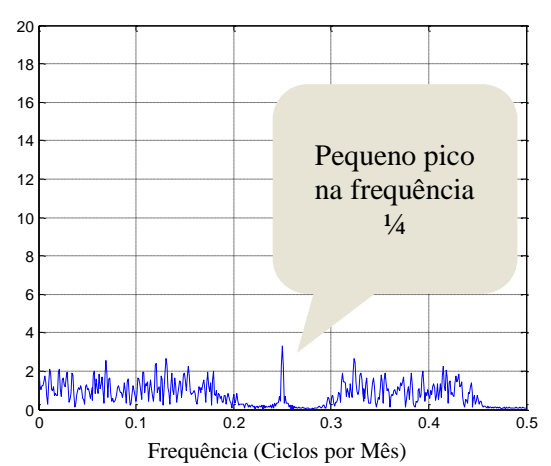

Gráfico 5.106 Espectro da irregular da série dessazonalizada pelo filtro $\mathrm{S}$ WLS, com: $A / s=6, k=32, b=10 \% \mathrm{e}$ $N=43$

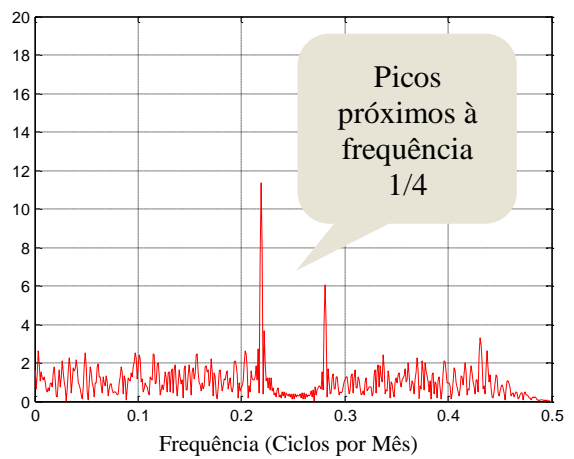

Gráfico 5.103 Espectro da irregular da série dessazonalizada pelo X-11, com: $A / s=6, k=32, b=$ $40 \%$ e $N=43$

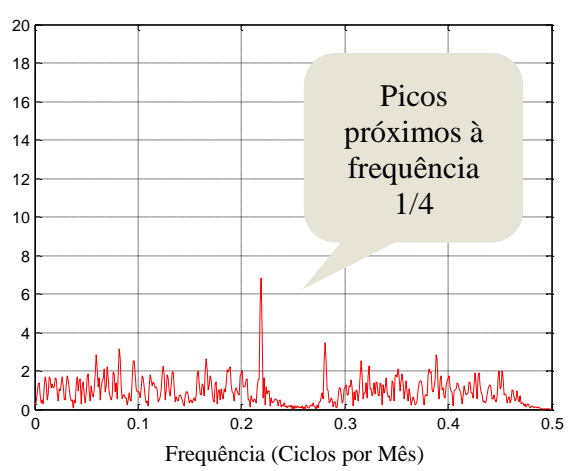

Gráfico 5.105 Espectro da irregular da série dessazonalizada pelo X-11, com: $A / s=6, k=32, b=$ $25 \%$ e $N=43$

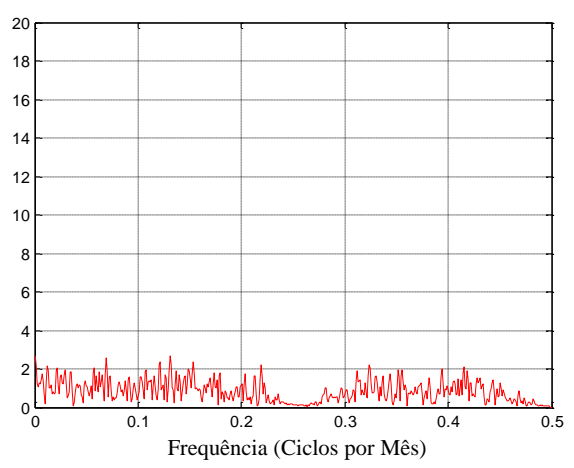

Gráfico 5.107 Espectro da irregular da série dessazonalizada pelo $X-11$, com: $A / s=6, k=32, b=10 \%$ e $N=43$ 
Considerando a existência de componente de tendência na série (com os coeficientes do polinômio da série Y91), os espectros da componente irregular (para $k=24$ e 32 , e $b=40 \%$ e $10 \%$ ) das séries dessazonalizadas pelo S-WLS seriam os seguintes:

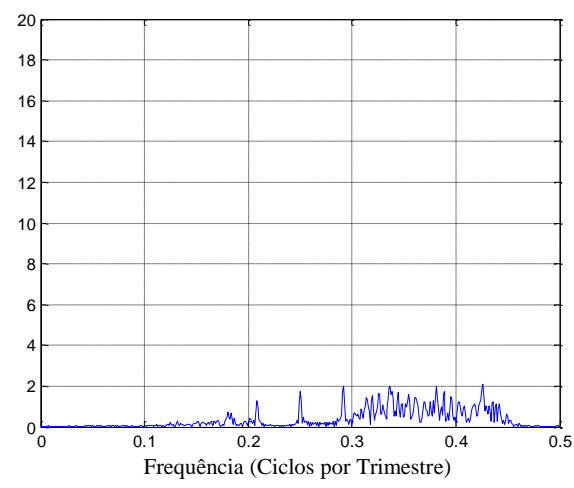

Gráfico 5.108 Espectro da irregular da série dessazonalizada pelo filtro $S$ WLS, com tendência, e: $A / s=6,5 ; k=$ $24 ; b=40 \%(N=43)$

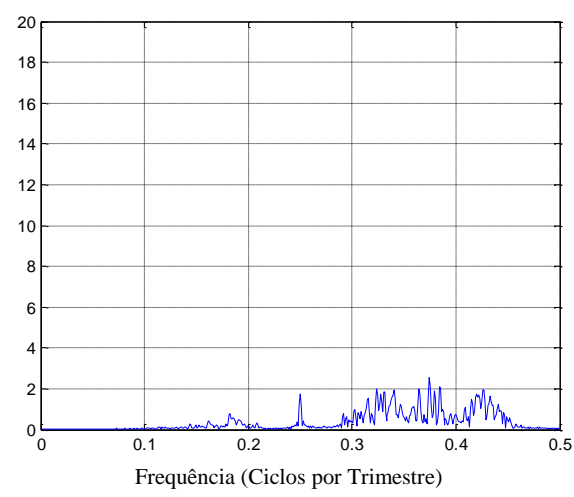

Gráfico 5.110 Espectro da irregular da série dessazonalizada pelo filtro S-WLS, com tendência, e: $A / s$ $=6,5 ; k=24 ; b=10 \%(\mathrm{~N}=43)$

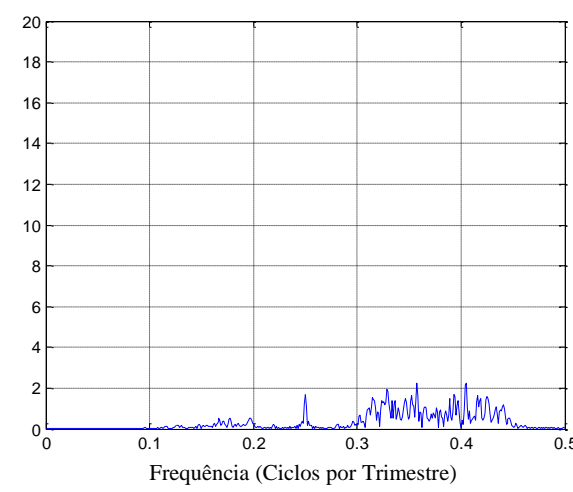

Gráfico 5.109 Espectro da irregular da série dessazonalizada pelo filtro S-WLS, com tendência, e: $A / s$ $=6,5 ; k=32 ; b=40 \%(\mathrm{~N}=43)$

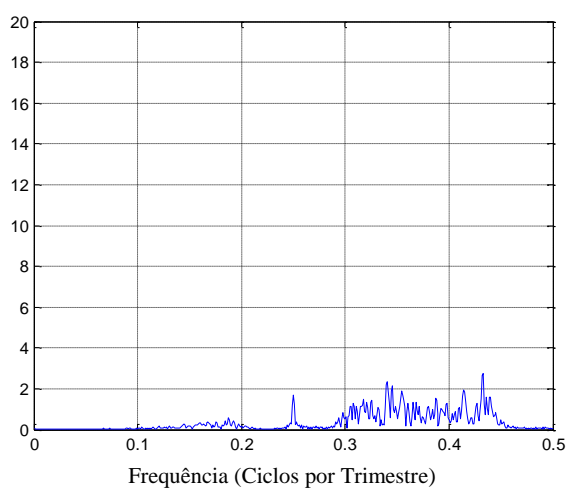

Gráfico 5.111 Espectro da irregular da série dessazonalizada pelo filtro S-WLS, com tendência, e: $A / s$ $=6,5 ; k=32 ; b=10 \%(\mathrm{~N}=43)$

Esses espectros mostram que o filtro S-WLS é adequado, também, para séries com tendência. 
Neste capítulo foi encontrada a melhor configuração de parâmetros para o filtro S-WLS mensal e trimestral. Além disso, o desempenho do filtro S-WLS foi comparado com o X-11. Tal comparação foi realizada com base em séries artificiais, inspiradas no comportamento das séries reais com sazonalidade móvel. Nessas análises foram consideradas séries com modelo de decomposição aditivo e multiplicativo.

Os resultados obtidos foram apresentados para os diversos tipos de séries, separadamente. A ordem de apresentação foi a seguinte: séries mensais aditivas; séries mensais multiplicativas; séries trimestrais aditivas; séries trimestrais multiplicativas. 


\section{6 \\ Filtro sazonal-WLS-Chebyshev: definição, seleção da melhor configuração e resultados}

Este capítulo apresenta a proposta do segundo filtro sazonal, aqui intitulado de 'filtro sazonal-WLS-Chebyshev' - S-WLSC. Este filtro combina a abordagem de Mínimos Quadrados Ponderados (WLS) com as características dos filtros de Chebyshev. Adicionalmente o capítulo realizará comparações do desempenho do filtro S-WLSC, com o filtro proposto anteriormente: S-WLS. Os conceitos apresentados neste capítulo baseiam-se em Diniz et al. (2010).

\section{1. \\ Filtro sazonal-WLS-Chebyshev: o algoritmo}

Conforme visto nos Capítulos 4 e 5, um bom filtro extrator da sazonalidade deve tender a minimizar o erro na sua estimativa. No caso do filtro S-WLS, este erro possui os seguintes elementos:

1. O erro na amplitude da sazonalidade devido à banda passante não plana $\left(\gamma_{1}\right.$, na Figura 5.3);

2. O erro causado pela componente irregular. Este erro possui dois elementos:

a. A componente irregular que vaza devido a uma atenuação finita na banda de rejeição;

b. A componente irregular que está misturada à componente sazonal na banda passante.

A componente (2.b), descrita acima, está relacionada ao compromisso entre a robustez às variações de sazonalidade e a quantidade de componente irregular que passa pela banda passante. É importante ressaltar que ela estaria presente mesmo se a resposta do filtro projetado fosse a 'ideal'. Entretanto, os efeitos dos elementos (1) e (2.a) podem ser reduzidos com um projeto adequado.

Analisando o projeto do filtro S-WLS, nota-se que o seu objetivo é minimizar o erro quadrático médio tanto na banda passante quanto na banda de rejeição. 
Porém, minimizar o erro médio quadrático na banda de rejeição tende a maximizar a atenuação na banda de rejeição (elemento 2.a). No entanto, o elemento (1), do erro, está relacionado com o máximo erro na banda passante, e não com o erro quadrático médio.

Sendo assim, o projeto ideal de filtro seria aquele que minimizasse, simultaneamente, o erro máximo na banda passante e a energia na banda de rejeição. Os filtros que minimizam o erro máximo de aproximação são os chamados 'filtros de Chebyshev' (Diniz et al., 2010). Tal erro é minimizado quando ele possui a característica equiripple, isto é, ele é oscilatório com uma amplitude constante. Já os filtros que combinam a característica de minimizar a energia na banda de rejeição (usando WLS) com a característica equiripple na banda passante, são comumente denominados de 'filtros WLS-Chebyshev' (Diniz et al., 2010).

Dessa forma, neste capítulo são projetados filtros de extração da sazonalidade com a característica de um filtro WLS-Chebyshev. Ao filtro aqui projetado, intitulou-se 'filtro sazonal-WLS-Chebyshev' - S-WLSC.

A diferença em relação ao método utilizado no filtro S-WLS, apresentado anteriormente, está no fato deste novo filtro (S-WLSC) atualizar o vetor de pesos a cada iteração de um projeto WLS. Com isso, permite-se um controle do erro de aproximação, podendo-se obter um projeto equiripple.

A atualização do vetor de pesos do filtro S-WLSC ocorre somente nas faixas de frequência onde se deseja um erro de aproximação equiripple. Nessas faixas de frequência, a atualização é realizada com base na envoltória do erro de aproximação. Onde a envoltória do erro é pequena, os pesos diminuem, e a tendência é que o erro aumente na próxima iteração. Onde a envoltória é grande, os pesos aumentam, e a tendência é que o erro diminua na próxima iteração. Assim, na convergência, o erro de aproximação se torna equiripple. E esse é o comportamento desejado na banda passante, onde se deseja minimizar o erro máximo de estimativa da sazonalidade.

Em relação às faixas de frequência em que o vetor de pesos 'não é' atualizado, a característica WLS, de minimizar o erro quadrático médio, é mantida. Este tipo de característica é importante na banda de rejeição, onde o desvio-padrão da componente irregular deve ser minimizado. 
No algoritmo de projeto do filtro S-WLSC, os novos pesos são calculados com base nos erros gerados pela aplicação dos pesos anteriores, e o processo continua até a sua convergência - que ocorre quando a diferença entre os resultados é inferior a um valor previamente determinado $(\epsilon)$. O algoritmo está descrito em Diniz et al. (2010). Porém, neste trabalho, ele sofreu algumas modificações.

A figura, a seguir, ilustra a função de erro típica, $B(\omega)$, representada pela linha tracejada, e a envoltória do erro, $B^{e}(\omega)$, representada pela linha contínua.

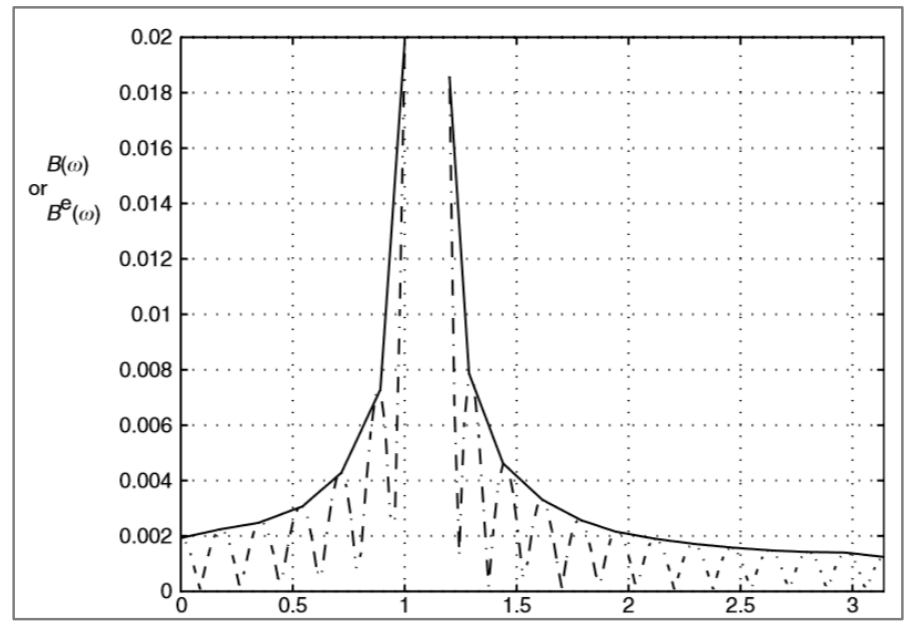

Fonte: Diniz et al. (2010, p. 329)

\section{Figura 6.1 Função de erro típica (linha tracejada) e função envelope (linha contínua)}

A implementação do algoritmo WLS-Chebyshev ocorre da seguinte forma:

(i) Estimar a ordem ' $\mathrm{M}$ ' (fator de superamostragem); selecionar o número máximo de iterações $\left(k_{\text {máx }}\right)$; definir os limites de frequência das bandas passantes e das bandas de rejeição, onde as respostas devem ser equiripple nas bandas passantes e WLS nas bandas de rejeição. Especificar um valor de tolerância para o erro. Arbitrar um valor para $\Gamma$, responsável pela atualização de pesos da banda de rejeição e pela relação entre os erros nas bandas passantes e de rejeição. Foi utilizado nesse projeto $M=401$ e $\Gamma \geq 0,005$;

(ii) Criar uma frequência grid de $k \mathrm{M}$ pontos $[0, \pi]$;

(iii) Definir $k=0$ e calcular $\mathbf{W}_{q}, \mathbf{d}$ e $\mathbf{U}$, como definido no algoritmo WLS;

(iv) Definir $k=k+1$, e determinar $\mathbf{p}^{*}(k)$;

(v) Calcular o vetor de erro $\boldsymbol{e}(k)$; 
(vi) Verificar se $k_{\text {máx }}>k$ ou se a convergência foi atingida. A convergência é atingida quando $\epsilon<0,0001$;

(vii) Calcular $\left\{\mathbf{B}_{\boldsymbol{k}}\right\}_{\boldsymbol{j}}=\left|\{\mathbf{e}(k)\}_{j}\right|$, para $j=0,1, \ldots, k \mathrm{M}$.

(viii) Encontrar o maior valor da banda de rejeição de $\mathbf{B}_{k}^{e}$;

(ix) Multiplicar os valores de $B_{k}^{e}(\omega)$ na banda de rejeição por $\Gamma$;

(x) Atualizar o vetor de pesos $\mathbf{W}_{q}^{2}$ e voltar para o passo (iv), sendo $W_{k+1}^{2}(\omega)=W_{k}^{2}(\omega) B_{k}^{e}(\omega) ;$

(xi) Determinar os coeficientes do filtro: $h(n)$.

Em relação aos parâmetros do filtro, a vantagem do S-WLSC, em relação ao filtro S-WLS, ocorre em ele permitir que o $\delta$ (valor do parâmetro associado às larguras das bandas de transição) seja reduzido sem prejudicar o máximo erro de aproximação (ripple) na banda passante. Com isso, o compromisso entre a banda de transição e a banda passante é mantido.

Esse filtro tem os mesmos parâmetros do filtro S-WLS: $\alpha, \delta, w_{0}, M$ e $N$. Tais parâmetros estão definidos na Subseção 4.2. Além desses, o S-WLSC possui o parâmetro $\Gamma$, que determina a razão entre a atenuação na banda de rejeição (WLS) e o ripple na banda passante.

Para testar o filtro S-WLSC, e compará-lo ao filtro S-WLS, foi necessário definir a melhor configuração de parâmetros para cada tamanho $(N)$ do filtro - para as séries mensais e trimestrais.

A escolha da melhor configuração de parâmetros e os resultados desse novo filtro, em comparação com o filtro S-WLS, são apresentados neste capítulo.

\section{2 . Metodologia para seleção do filtro S-WLSC para as séries mensais e trimestrais}

Para selecionar a melhor configuração de parâmetros para o filtro S-WLSC, utilizou-se como métrica a SNR desse filtro, em relação à SNR do filtro S-WLS. O objetivo era escolher os valores dos parâmetros que fizessem com que o filtro SWLSC tivesse desempenho superior ao filtro S-WLS. 
No geral, o filtro S-WLSC apresenta $\gamma_{0}$ e $\left|1-\gamma_{1}\right|$ menores do que o filtro SWLS, favorecendo a uma SNR maior. Porém, o filtro S-WLSC é sensível ao tamanho $(N)$, e à combinação entre $\delta, \mathrm{w}_{0}$ e $\Gamma$.

No que tange aos parâmetros do filtro S-WLSC, notou-se que quanto maior for o valor de $\Gamma$, melhor. No entanto, é necessário analisar se houve convergência para a função objetivo.

Em relação aos demais parâmetros, observou-se que quanto menor for o $\delta \mathrm{e}$ o $\mathrm{w}_{0}$, melhor. Porém, para cada $\delta$, existe um valor mínimo de $\mathrm{w}_{0}$ a ser aceito pelo filtro, além dele possuir uma relação delicada com $\Gamma$, que afeta a possibilidade de convergência na implementação utilizada no MATLAB.

Destaca-se que, na seleção dos parâmetros do filtro, foi mantido o valor de $\alpha$ igual a $\frac{1}{3}$, ou seja, a largura da banda passante será a mesma do filtro S-WLS.

Diferentemente do procedimento utilizado para a seleção do filtro S-WLS, buscou-se aqui uma combinação diferente de parâmetros para cada tamanho $(N)$, visando à SNR máxima. Essa decisão foi tomada uma vez que o algoritmo de projeto do filtro S-WLSC, implementado no MATLAB, não convergiu para todos os tamanhos $(N)$.

$\mathrm{Na}$ busca de um melhor filtro para cada ' $N$ ', procurou-se um filtro cujo o valor do $\delta$ fosse o menor possível, assim como o $\mathrm{w}_{0}$; e o $\Gamma$, o maior possível. Vale citar que os filtros com valores de $\delta>\frac{1}{500}$ resultam nos mesmos valores de $S Q, \gamma_{0}$ e $\left|1-\gamma_{1}\right|$.

Nessa tentativa de encontrar um filtro adequado, foram gerados filtros com diversas combinações de parâmetros, para cada ' $N$ ', a saber:

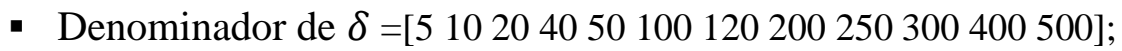

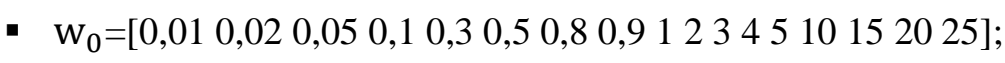

- $\Gamma=[0,0050,0040,0030,0020,0010,010,020,030,040,050,080,10,150,300,50,811,2]$.

Quanto aos demais parâmetros, foi mantido o valor $\alpha=\frac{1}{3}$, assim como o fator de superamostragem $\mathrm{M}=401$.

Esses filtros tiveram a SNR calculada, e aqueles que geraram as maiores SNRs foram selecionados. Após isso, para verificar a adequação da combinação de parâmetros, foi construído o gráfico da resposta em magnitude de cada filtro, sendo considerados apenas os filtros onde houve convergência para a função objetivo. 
A seguir é especificada a seleção da combinação de parâmetros para cada tamanho $(N)$ de filtro, sendo eles mensais e trimestrais. Além disso, são apresentados os resultados das comparações com o filtro S-WLS obtidos para as séries com decomposição aditiva e multiplicativa.

\section{3.}

\section{Série mensal aditiva - filtro S-WLSC}

Esta Subseção 6.3 apresenta os filtros selecionados para as séries mensais, e a comparação com o filtro S-WLS. Está subseção está organizada da seguinte forma: a Subseção 6.3.1 mostra a seleção da combinação de parâmetros para cada tamanho $(N)$ de filtro da séries mensais; a Subseção 6.3.2 apresenta o resumo dos filtros selecionados; e a Subseção 6.2.3 apresenta a comparação do filtro S-WLSC com o filtro S-WLS, para séries mensais com decomposição aditiva. A comparação entre os coeficientes do filtro S-WLSC com o filtro S-WLS está apresentada no Apêndice J.

\subsection{1.}

\section{Seleção do melhor filtro S-WLSC para cada ' $N$ ': filtro mensal}

A decisão em relação ao melhor filtro foi tomada com base em sinais artificiais, com vários valores para ' $A / s$ ', ' $b$ ' e ' $k$ ', definidos na Equação (5-1). Os resultados são apresentados a seguir - para cada ' $N$ ' definido no Quadro 5.4 (ver Subseção 5.3.1). Nas comparações, foi utilizado o filtro S-WLS. 


\subsubsection{1.}

\section{Resultado: filtro S-WLSC mensal com $N=117$}

O filtro de tamanho 117 que obteve os melhores resultados, e convergência para a função objetivo, teve a seguinte configuração:

$$
\alpha=\frac{1}{3} ; \delta=\frac{1}{10} ; w_{0}=0,9 ; \Gamma=0,5 .
$$

A resposta em magnitude desse filtro está apresentada no Gráfico 6.1 e a comparação da resposta em magnitude dele com o filtro S-WLS de mesmo tamanho está apresentada no Gráfico 6.2.

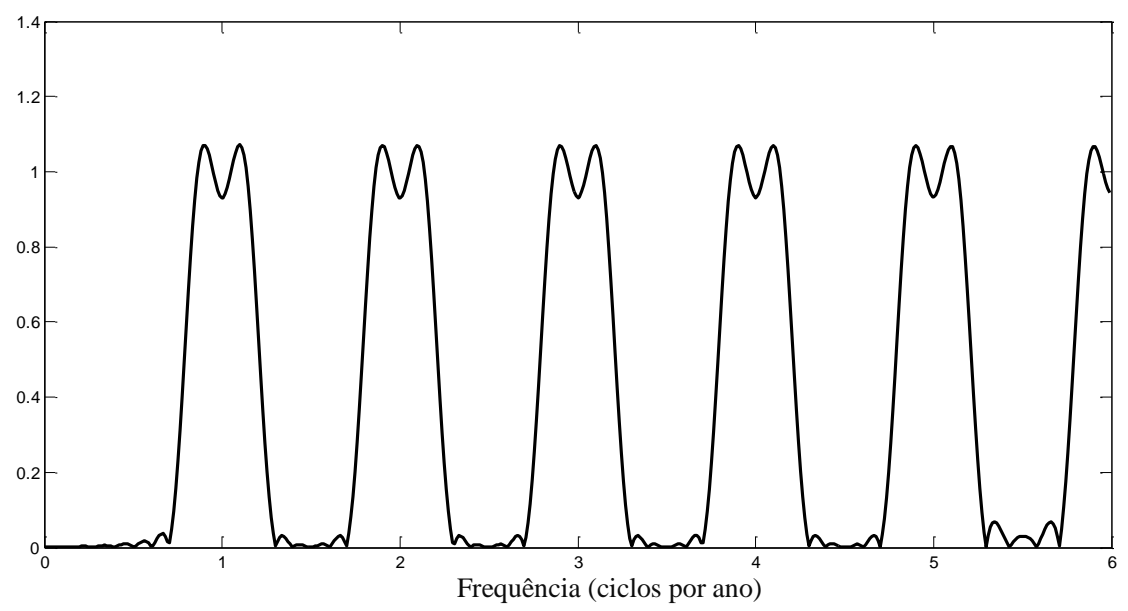

Gráfico 6.1 Resposta em magnitude do filtro S-WLSC mensal (N = 117)

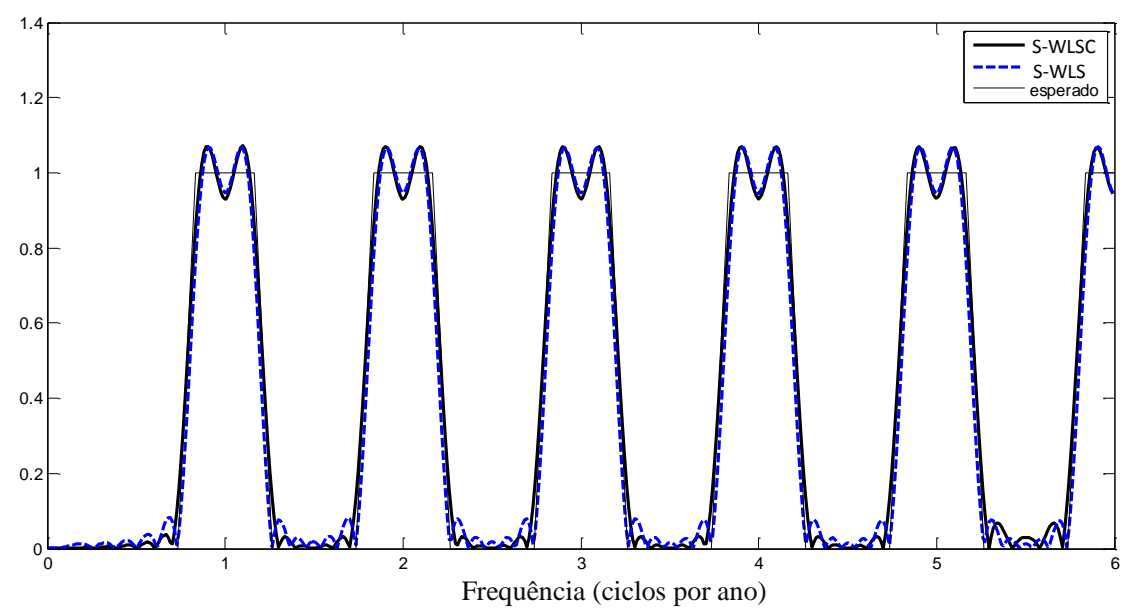

Gráfico 6.2 Resposta em magnitude do filtro S-WLSC, do S-WLS e o esperado $(N=117)$ 
No Gráfico 6.2 é possível notar que a atenuação da banda de rejeição do filtro S-WLSC é maior do que a do filtro S-WLS. Porém, na banda passante, o filtro SWLS apresentou melhor desempenho (menor ripple na banda passante), como pode ser observado no Gráfico 6.3.

Esse filtro de tamanho 117 apresentou problemas de instabilidade numérica, o que fez com que ele não fosse competitivo em relação ao filtro S-WLS.

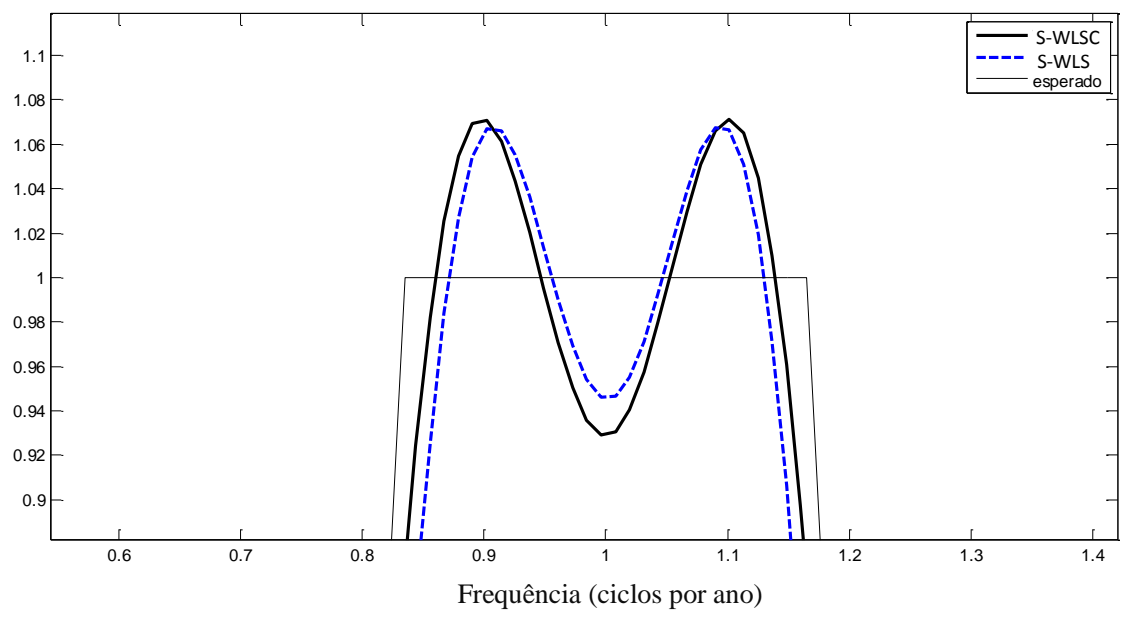

Gráfico 6.3 Banda passante dos filtros S-WLSC e S-WLS ( $N=117)$ 


\subsubsection{2.}

\section{Resultado: filtro S-WLSC mensal com $N=121$}

O filtro S-WLSC que gerou SNR maior do que o filtro S-WLS para o tamanho $N=121$ foi o que apresentou a seguinte configuração:

$$
\alpha=\frac{1}{3}, \delta=\frac{1}{250}, w_{0}=2, \Gamma=0,01 .
$$

A resposta em magnitude deste filtro S-WLSC está apresentada no Gráfico 6.4. No Gráfico 6.5 é apresentada a comparação entre as respostas em magnitude dos filtros S-WLSC e S-WLS, juntamente com o filtro esperado.

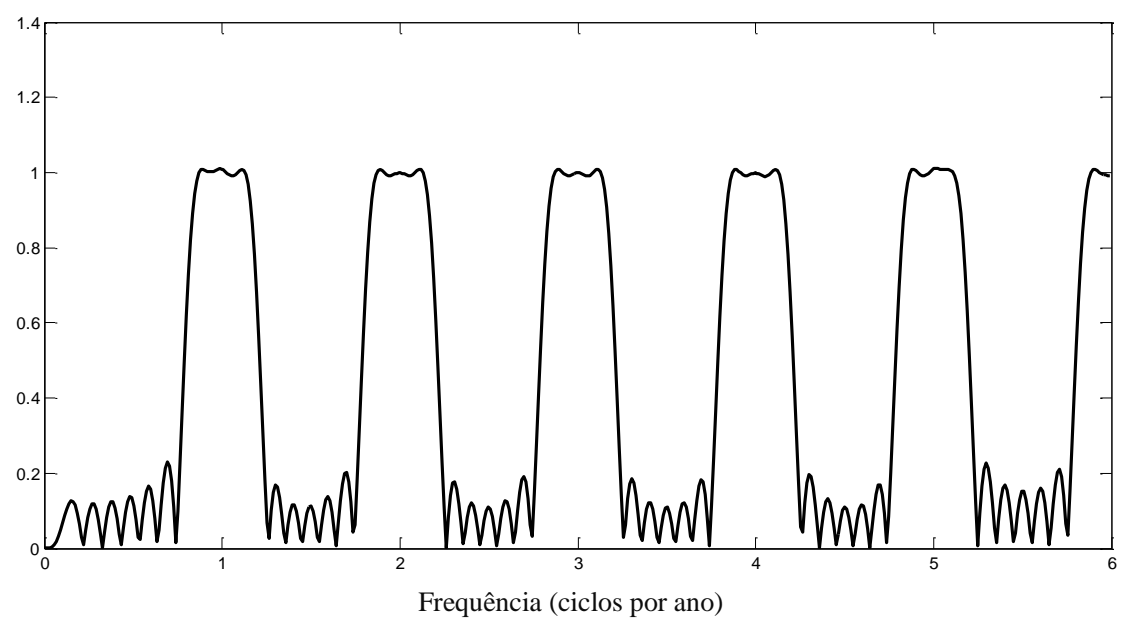

Gráfico 6.4 Resposta em magnitude do filtro S-WLSC mensal $(\mathrm{N}=121)$

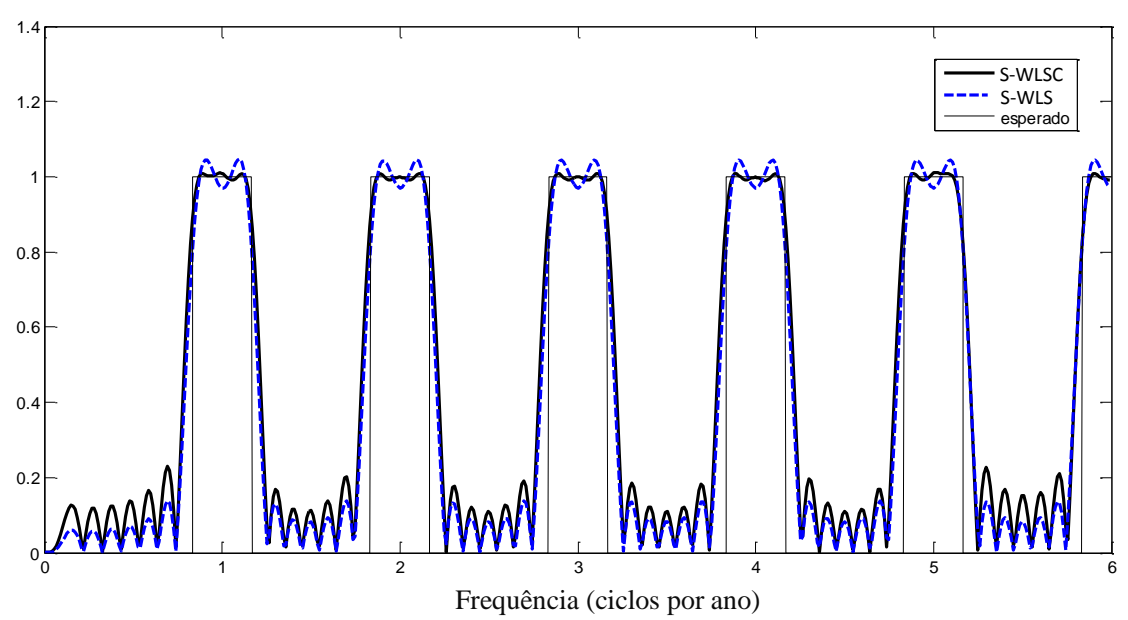

Gráfico 6.5 Resposta em magnitude do filtro S-WLSC, do S-WLS e o esperado $(N=121)$

No Gráfico 6.6 é exibida a ampliação da banda passante, onde nota-se que o S-WLSC apresenta um menor ripple na banda passante, estando mais próximo do esperado. 
Capítulo 6. Filtro sazonal-WLS-Chebyshev

208

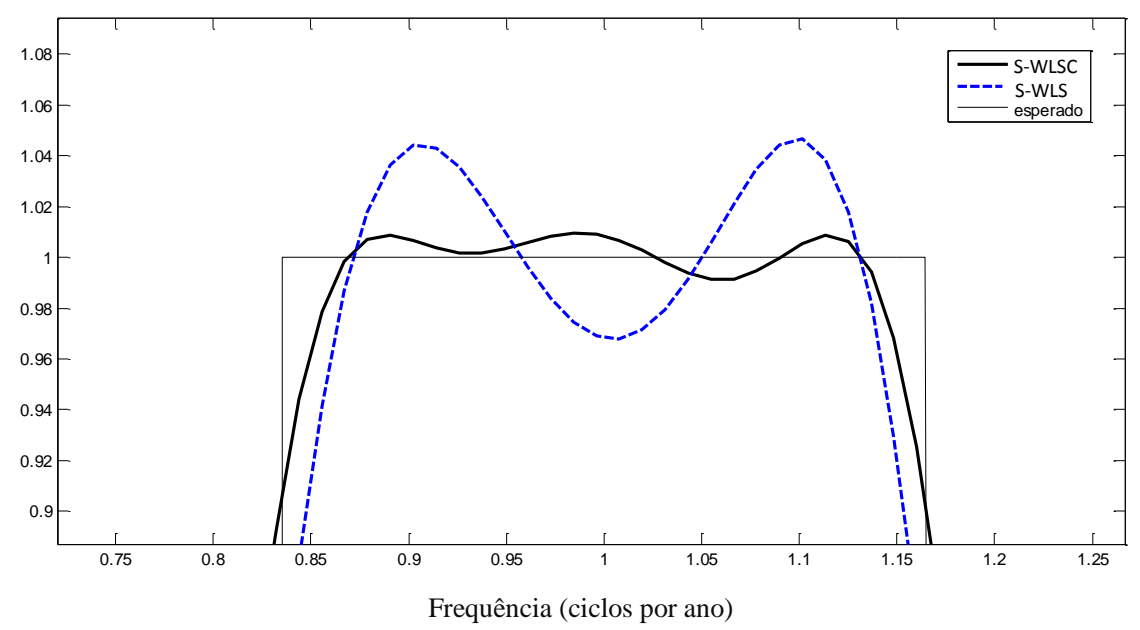

Gráfico 6.6 Banda passante dos filtros S-WLSC e S-WLS $(N=121)$

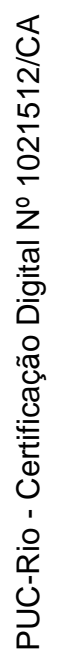




\subsubsection{3.}

\section{Resultado: filtro S-WLSC mensal com $N=131$}

Em relação ao filtro de tamanho 131, os melhores resultados foram obtidos com a seguinte configuração:

$$
\alpha=\frac{1}{3} ; \delta=\frac{1}{250} ; w_{0}=2 ; \Gamma=0,01 .
$$

Esta configuração é a mesma do filtro 121. A resposta em magnitude do filtro está apresentada no Gráfico 6.7; já a comparação da resposta em magnitude dele com o filtro S-WLS de mesmo tamanho está apresentada no Gráfico 6.8; e a ampliação da banda passante é apresentada no Gráfico 6.9.

Analisando os gráficos é possível notar o menor ripple na banda passante, da resposta em magnitude do filtro S-WLSC (Gráfico 6.9). Por outro lado, a atenuação na banda de rejeição foi melhor no filtro S-WLS (Gráfico 6.8).

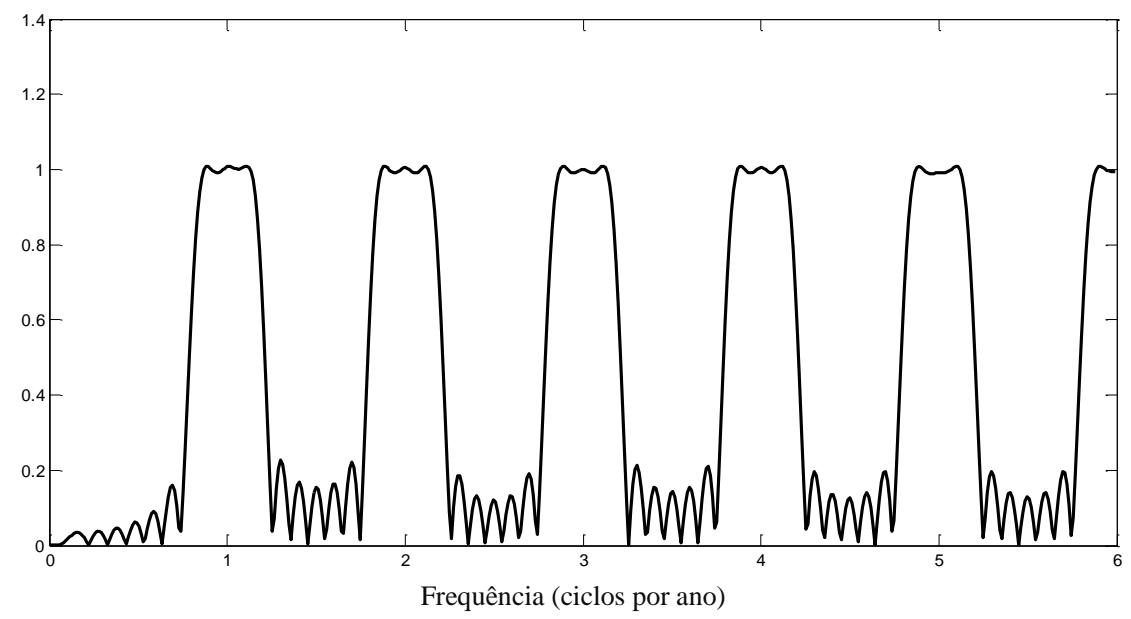

Gráfico 6.7 Resposta em magnitude do filtro S-WLSC mensal $(\mathrm{N}=131)$

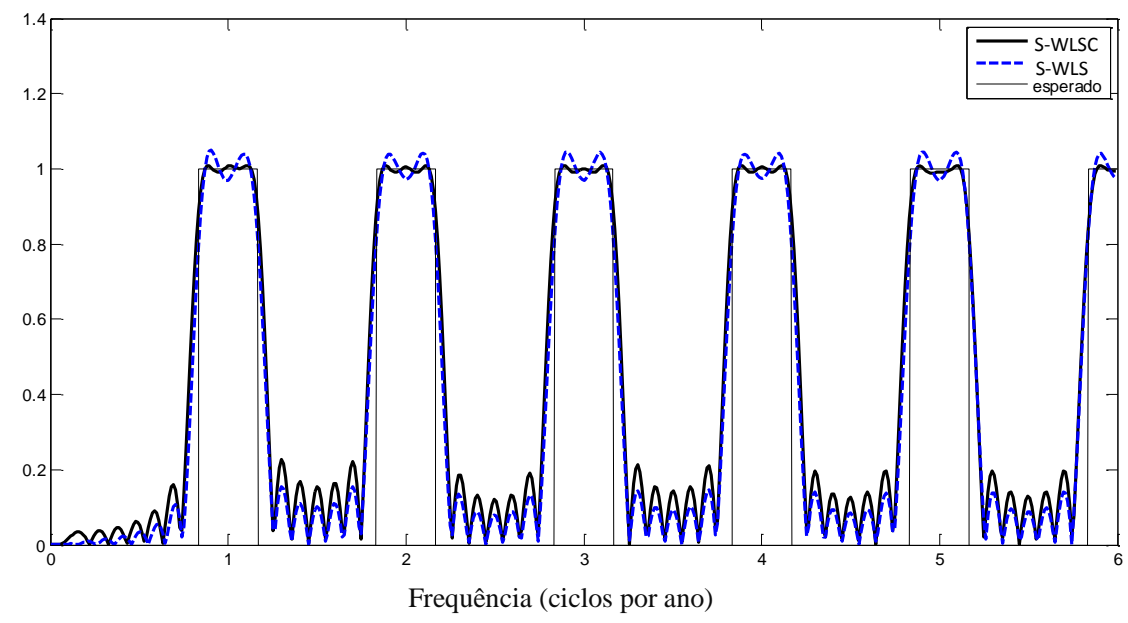

Gráfico 6.8 Resposta em magnitude do filtro S-WLSC, do S-WLS e o esperado $(N=131)$ 
Capítulo 6. Filtro sazonal-WLS-Chebyshev

210

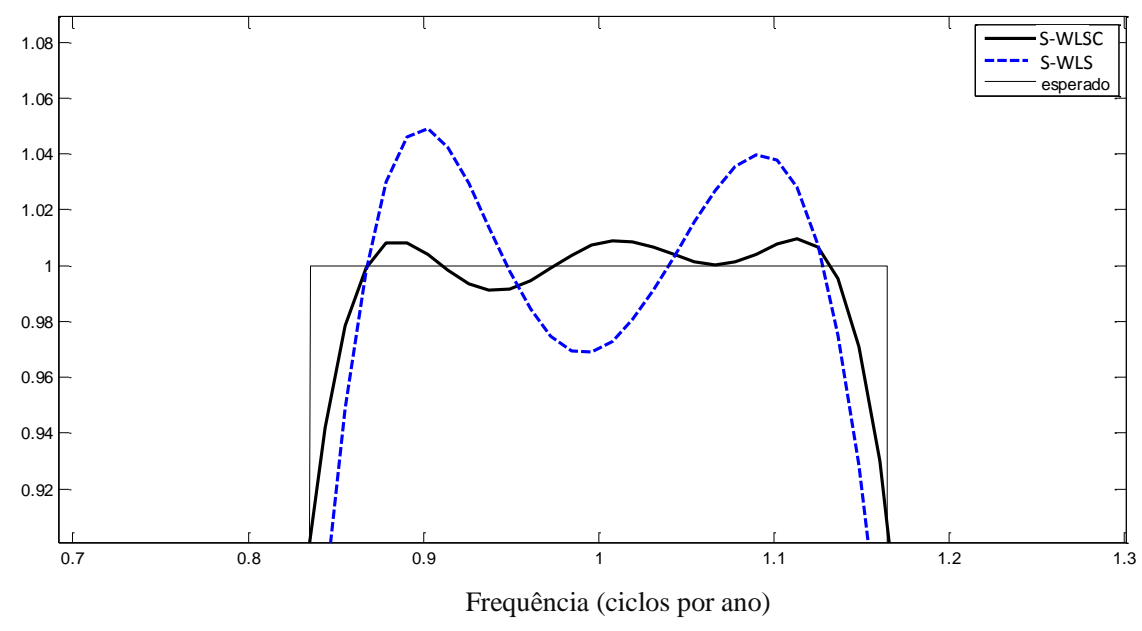

Gráfico 6.9 Banda passante dos filtros S-WLSC e S-WLS $(N=131)$

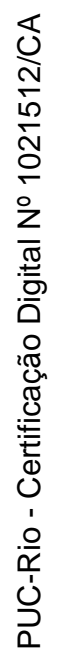




\subsubsection{4.}

\section{Resultado: filtro S-WLSC mensal com $N=141$}

Quanto ao filtro de tamanho 141, os melhores resultados foram obtidos com a configuração:

$$
\alpha=\frac{1}{3} ; \delta=\frac{1}{120} ; w_{0}=2 ; \Gamma=0,008
$$

A resposta em magnitude deste filtro está apresentada no Gráfico 6.10; a comparação da resposta em magnitude dele com a do filtro S-WLS de mesmo tamanho está apresentada no Gráfico 6.11; e a ampliação da banda passante se encontra no Gráfico 6.12.

Assim como no casos dos filtros de tamanho 121 e 131, observa-se que o filtro S-WLSC apresentou um menor ripple na banda passante (Gráfico 6.12), porém, a atenuação na banda de rejeição foi um pouco melhor no filtro S-WLS (Gráfico 6.11).

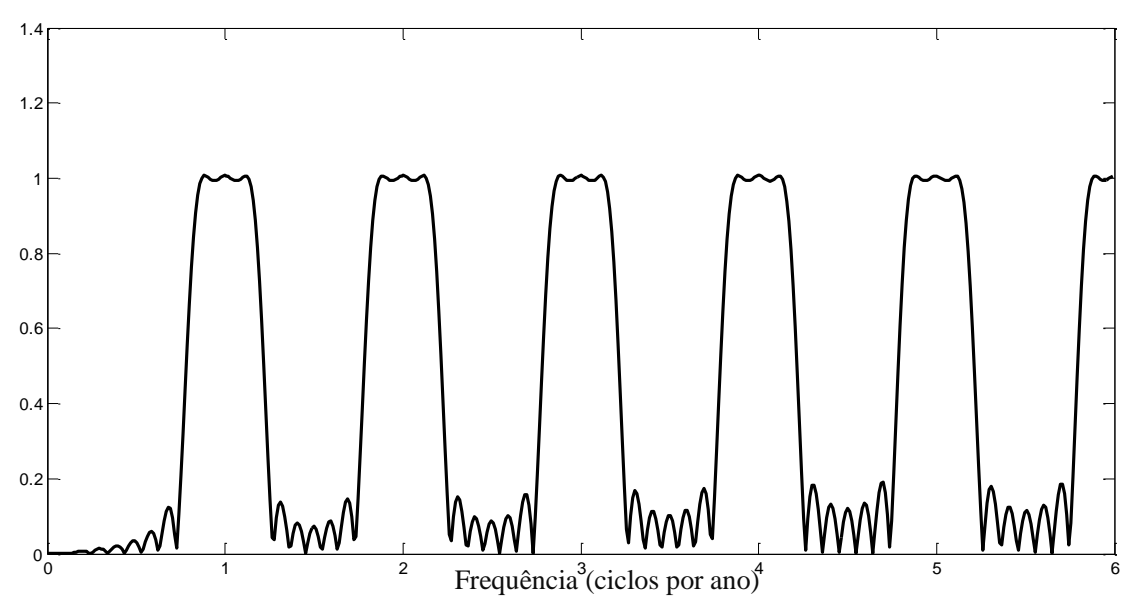

Gráfico 6.10 Resposta em magnitude do filtro S-WLSC mensal ( $N=141)$

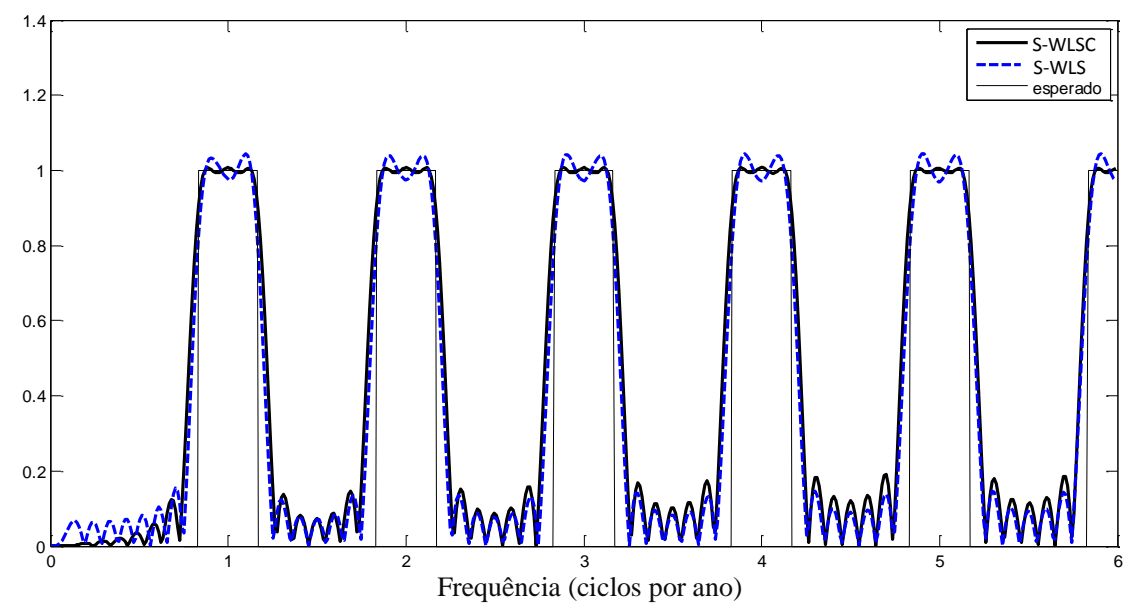

Gráfico 6.11 Resposta em magnitude do filtro S-WLSC, do S-WLS e o esperado $(N=141)$ 
Capítulo 6. Filtro sazonal-WLS-Chebyshev

212

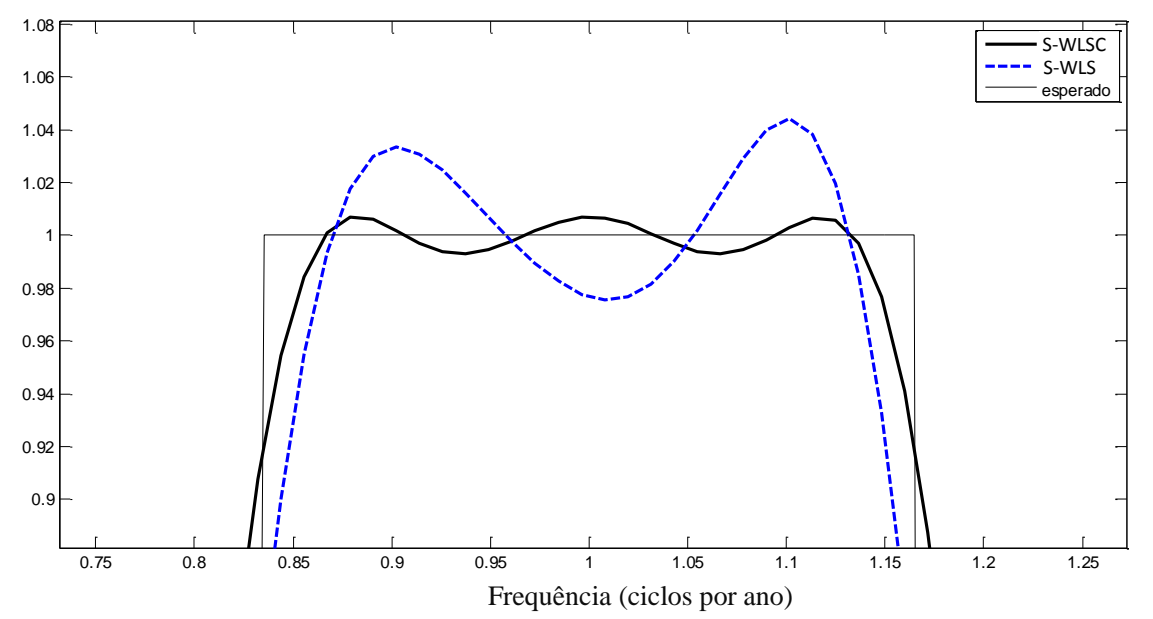

Gráfico 6.12 Banda passante dos filtros S-WLSC e S-WLS $(N=141)$

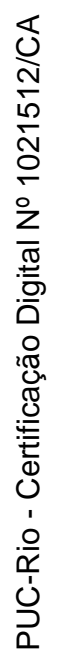




\subsubsection{5.}

\section{Resultado: filtro S-WLSC mensal com $N=145$}

Os melhores resultados para o filtro de tamanho 145 foram obtidos com a seguinte configuração:

$$
\alpha=\frac{1}{3} ; \delta=\frac{1}{250} ; w_{0}=1 ; \Gamma=0,01 .
$$

A resposta em magnitude desse filtro está apresentada no Gráfico 6.13; a comparação da resposta em magnitude dele com a do filtro S-WLS de mesmo tamanho está apresentada no Gráfico 6.14; e a ampliação da banda passante está apresentada no Gráfico 6.15.

Com base nos gráficos observa-se que o filtro S-WLSC gerou um menor ripple na banda passante e uma maior atenuação na banda de rejeição, se comparado ao filtro S-WLS. Dos outros filtros já definidos, esse foi o que apresentou melhor desempenho em relação à atenuação.

Como os filtros de tamanho 155 e 189 ficaram com resultados da SNR inferiores ao filtro de tamanho 145, nas comparações com o filtro S-WLS será usado este filtro de tamanho 145.

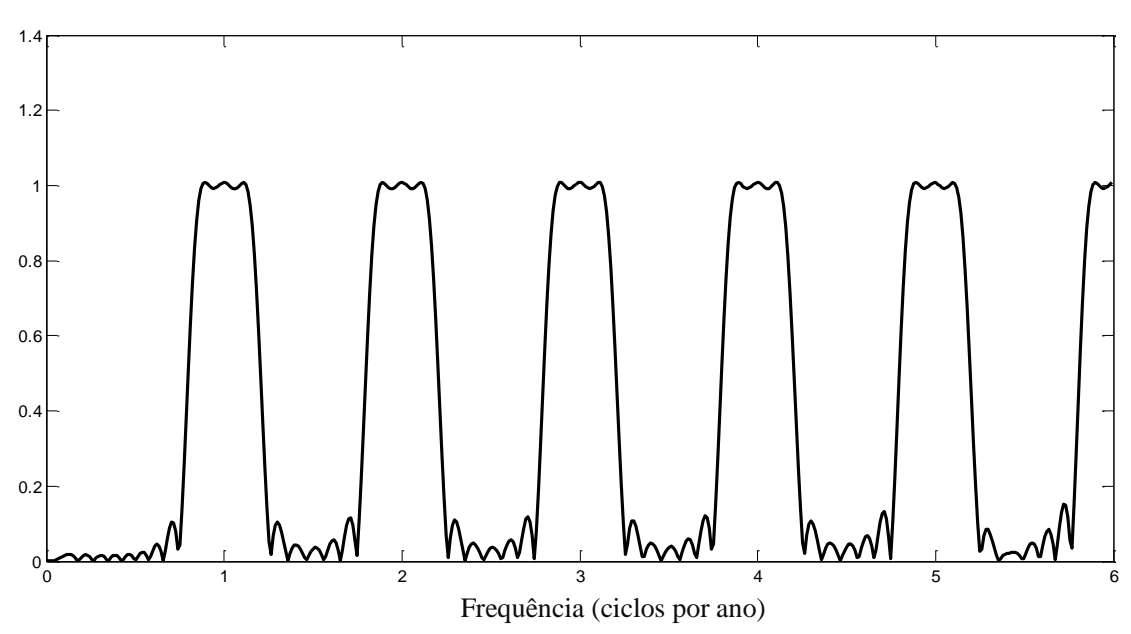

Gráfico 6.13 Resposta em magnitude do filtro S-WLSC mensal ( $=145)$ 


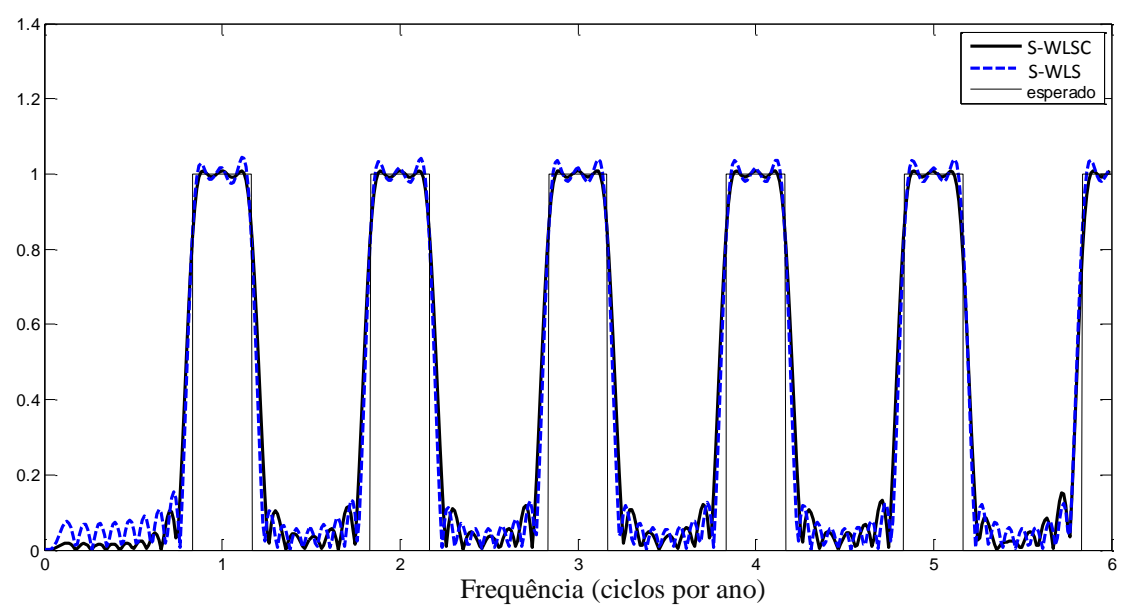

Gráfico 6.14 Resposta em magnitude do filtro S-WLSC, do S-WLS e o esperado $(N=145)$

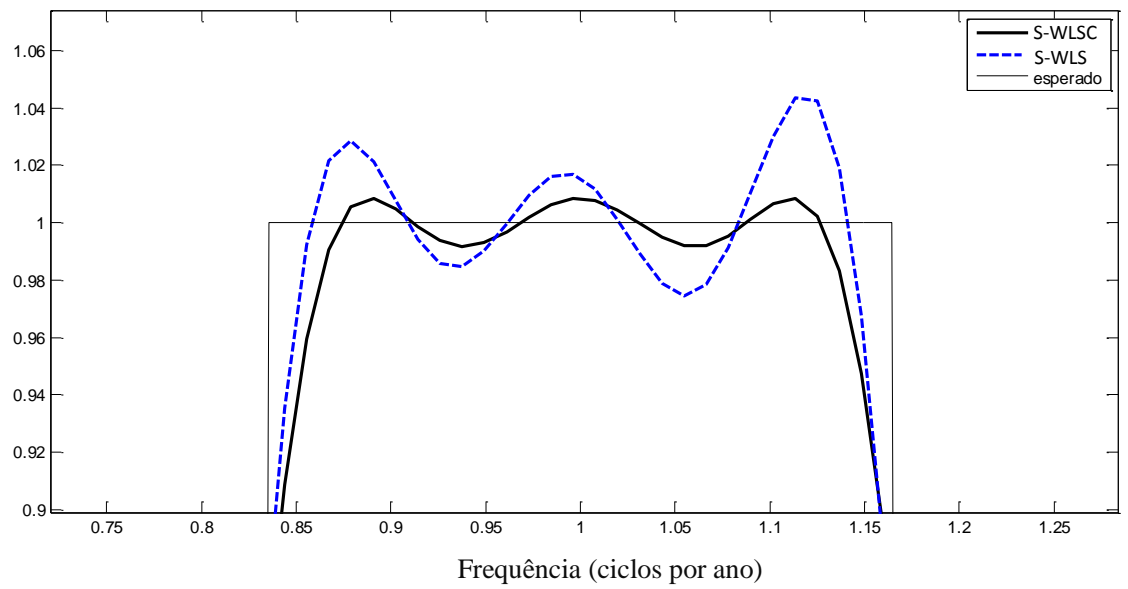

Gráfico 6.15 Banda passante dos filtros S-WLSC e S-WLS ( $N=145)$ 


\subsubsection{6.}

\section{Resultado: filtro S-WLSC mensal com $N=155$}

No que tange ao filtro de tamanho 155, não foi possível obter uma combinação de parâmetros que gerasse bons resultados, em termos de SNR, e que ao mesmo tempo apresentasse convergência para a função objetivo. Sendo assim, optou-se por utilizar o filtro de tamanho 145, apresentado na Subseção 6.3.1.5.

A comparação entre a resposta em magnitude do filtro S-WLSC 145 e a do filtro S-WLS de tamanho 155 está apresentada no Gráfico 6.16. E a ampliação da banda passante desses dois filtros é apresentada no Gráfico 6.17.

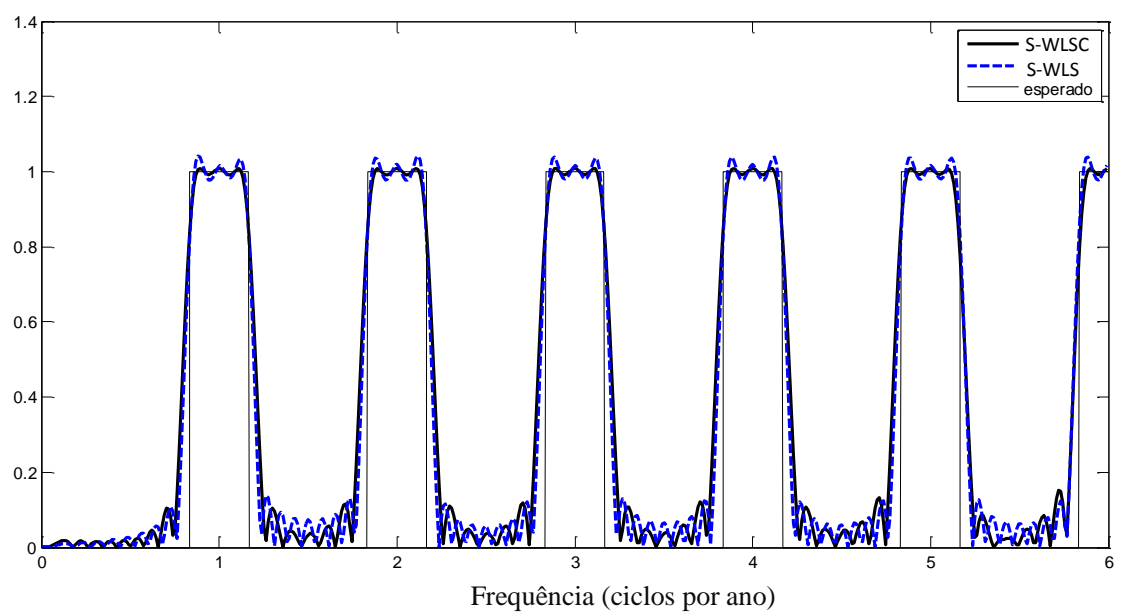

Gráfico 6.16 Resposta em magnitude do filtro S-WLSC (N=145), do S-WLS $(N=155)$ e o esperado

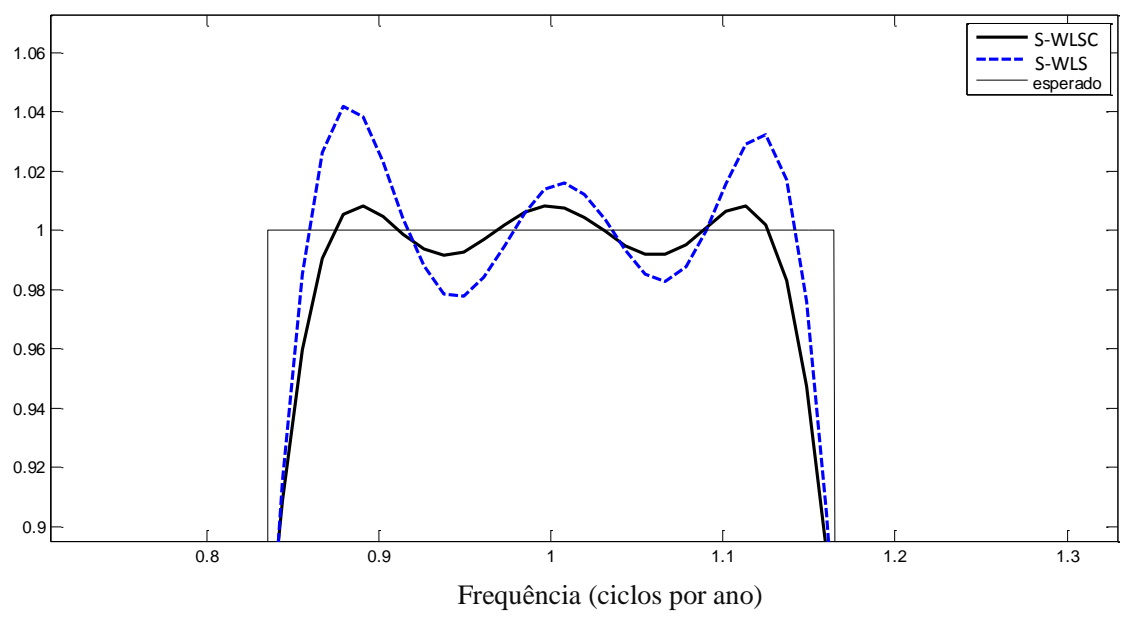

Gráfico 6.17 Banda passante dos filtros S-WLSC (N=145) e S-WLS ( $N=155)$ 


\subsubsection{7.}

\section{Resultado: filtro S-WLSC mensal com $N=189$}

Com o filtro de tamanho 189, também não foi possível obter uma combinação de parâmetros que gerasse SNR elevada com convergência para a função objetivo. Sendo assim, optou-se por utilizar o filtro de tamanho 145, apresentado na Subseção 6.3.1.5.

No Gráfico 6.18 é apresentada a comparação entre a resposta em magnitude do filtro S-WLSC 145 e a resposta em magnitude do filtro S-WLS de tamanho 189. A ampliação da banda passante dos filtros é apresentada no Gráfico 6.19.

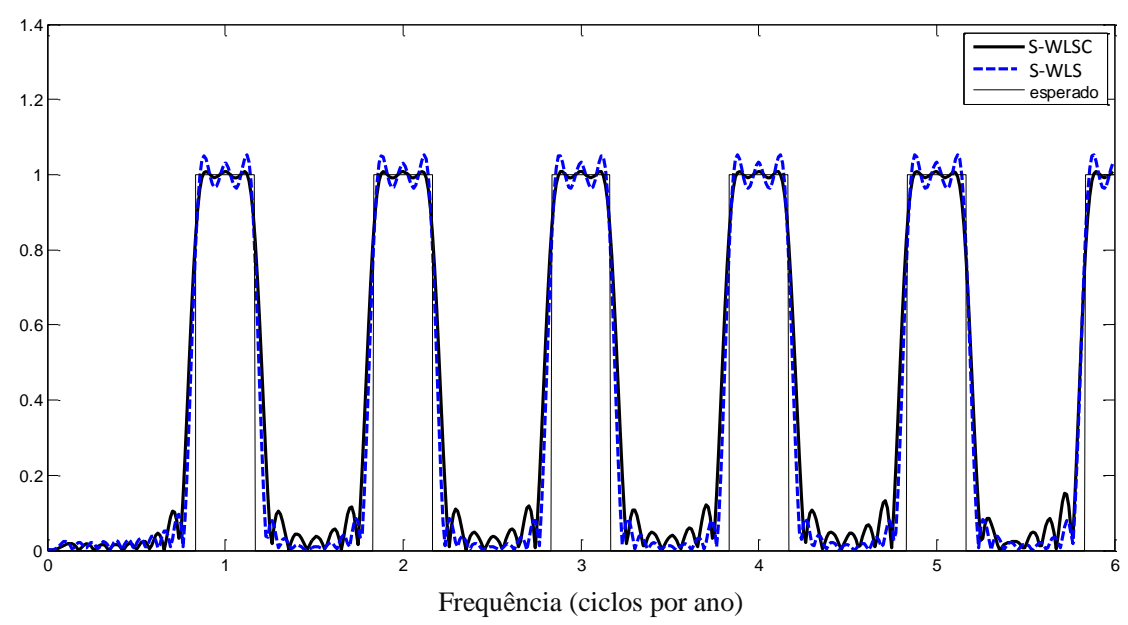

Gráfico 6.18 Resposta em magnitude do filtro S-WLSC (N=145), do S-WLS $(N=189)$ e o esperado

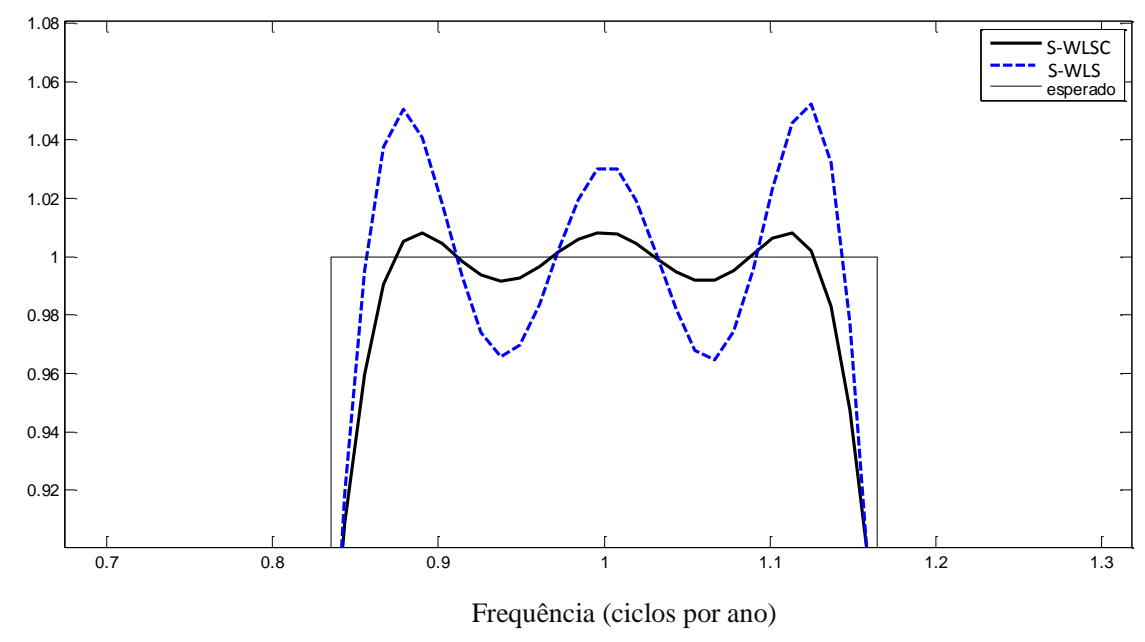

Gráfico 6.19 Banda passante dos filtros S-WLSC $(\mathrm{N}=145)$ e S-WLS (N=189) 


\subsubsection{8.}

\section{Resultado: filtro S-WLSC mensal com $N=193$}

Em relação ao filtro S-WLSC de tamanho 193, a melhor configuração obtida foi a seguinte:

$$
\alpha=\frac{1}{3} ; \delta=\frac{1}{150} ; w_{0}=1 ; \Gamma=0,01 .
$$

A resposta em magnitude deste filtro está apresentada no Gráfico 6.20; a comparação da resposta em magnitude dele com a do filtro S-WLS de mesmo tamanho está apresentada no Gráfico 6.21; e a ampliação da banda passante está apresentada no Gráfico 6.22 .

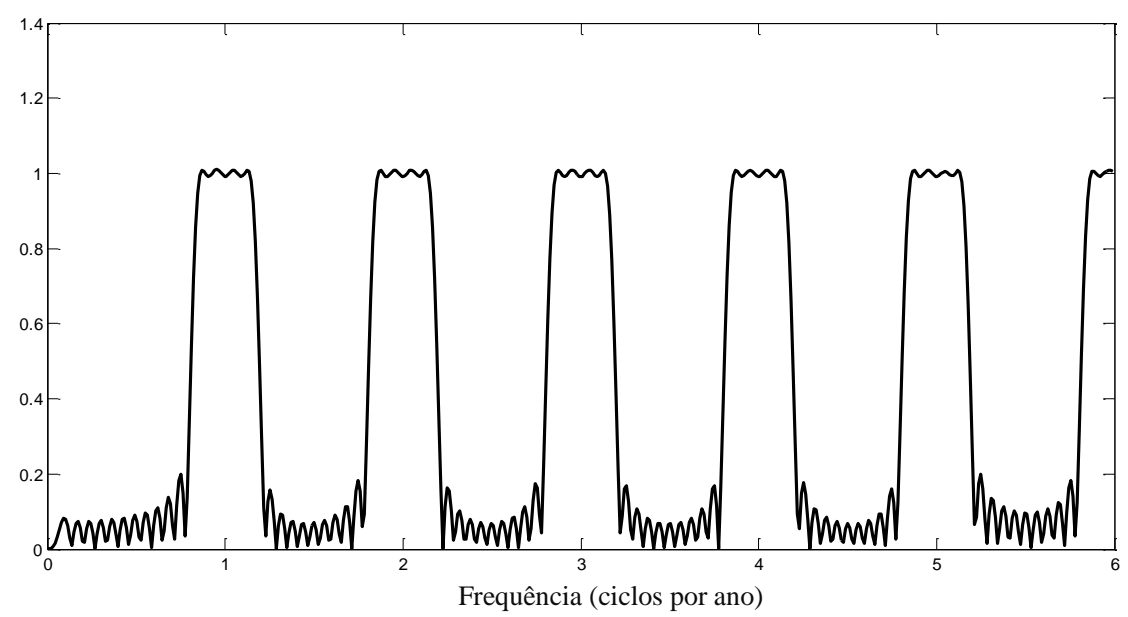

Gráfico 6.20 Resposta em magnitude do filtro S-WLSC mensal ( $\mathrm{N}=193)$

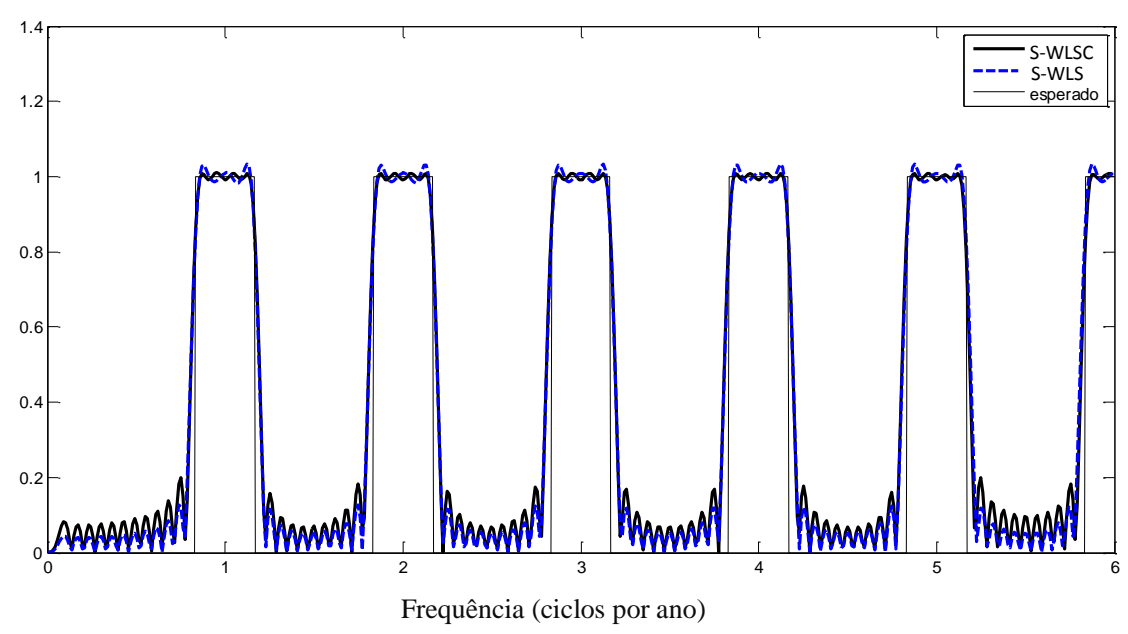

Gráfico 6.21 Resposta em magnitude do filtro S-WLSC, do S-WLS e o esperado $(N=193)$ 
Capítulo 6. Filtro sazonal-WLS-Chebyshev

218

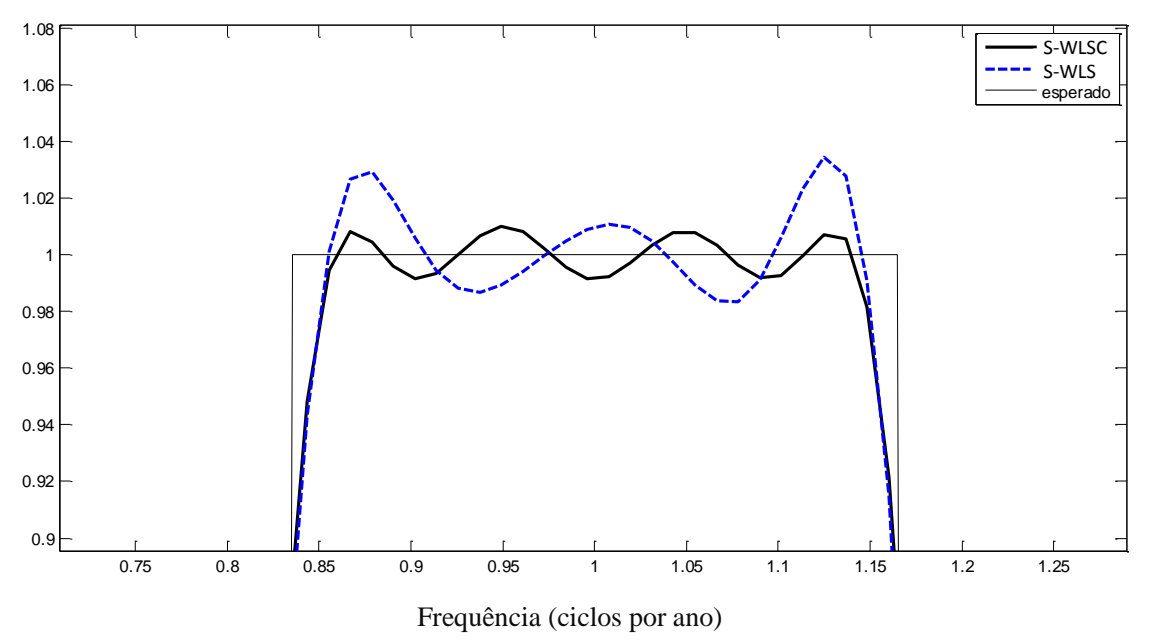

Gráfico 6.22 Banda passante dos filtros S-WLSC e S-WLS ( $N=193)$

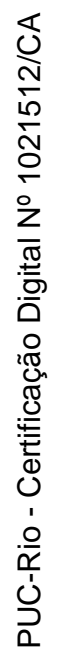




\subsubsection{9.}

\section{Resultado: filtro S-WLSC mensal com $N=203$}

Para o filtro de tamanho 203, não foi possível obter uma combinação de parâmetros que gerasse bons resultados. Com isso, decidiu-se utilizar o filtro de tamanho 193, apresentado na Subseção 6.3.1.8.

A comparação entre a resposta em magnitude do filtro S-WLSC de tamanho 193 e a do filtro S-WLS de tamanho 203 está apresentada no Gráfico 6.23; e a ampliação da banda passante desses filtros é apresentada no Gráfico 6.24.

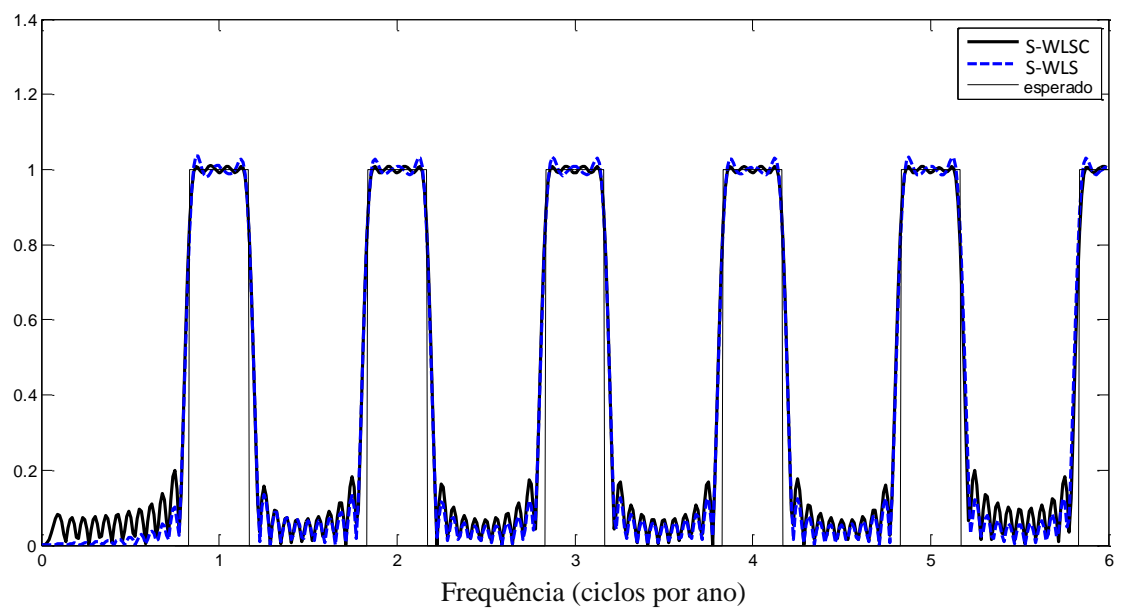

Gráfico 6.23 Resposta em magnitude do filtro S-WLSC (N=193), do S-WLS $(N=203)$ e o esperado

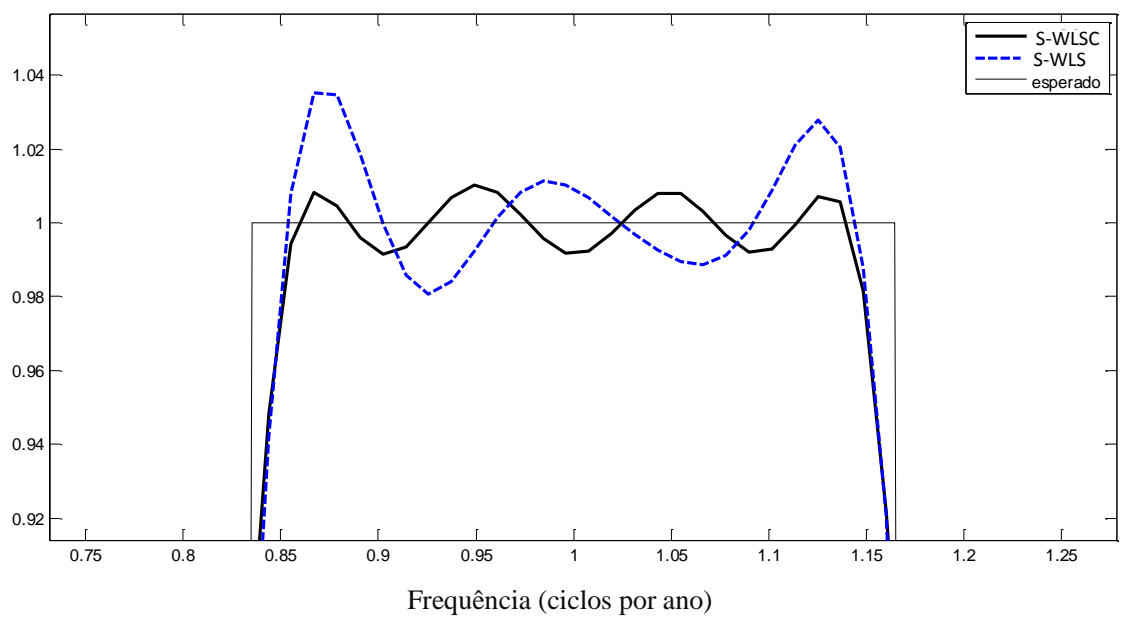

Gráfico 6.24 Banda passante dos filtros S-WLSC $(\mathrm{N}=193)$ e S-WLS $(N=203)$ 


\subsection{2.}

\section{Parâmetros do filtro S-WLSC: séries mensais}

A Tabela 6.1 apresenta os valores dos parâmetros selecionados na Subseção 6.3.1, para cada tamanho do filtro S-WLSC.

\begin{tabular}{c|c|c|c|c}
\hline $\boldsymbol{N}$ & $\boldsymbol{\alpha}$ & $\boldsymbol{\delta}$ & $\boldsymbol{\Gamma}$ & $\boldsymbol{w}_{\mathbf{0}}$ \\
\hline $\mathbf{1 1 7}$ & $1 / 3$ & $1 / 10$ & 0,5 & 0,9 \\
\hline $\mathbf{1 2 1}$ & $1 / 3$ & $1 / 250$ & 0,01 & 2 \\
\hline $\mathbf{1 3 1}$ & $1 / 3$ & $1 / 250$ & 0,01 & 2 \\
\hline $\mathbf{1 4 1}$ & $1 / 3$ & $1 / 120$ & 0,008 & 2 \\
\hline $\mathbf{1 4 5}$ & $1 / 3$ & $1 / 250$ & 0,01 & 1 \\
\hline $\mathbf{1 5 5} *$ & $1 / 3$ & $1 / 250$ & 0,01 & 1 \\
\hline $\mathbf{1 8 9} *$ & $1 / 3$ & $1 / 250$ & 0,01 & 1 \\
\hline $\mathbf{1 9 3}$ & $1 / 3$ & $1 / 150$ & 0,01 & 1 \\
\hline $\mathbf{2 0 3} * *$ & $1 / 3$ & $1 / 150$ & 0,01 & 1 \\
\hline 0 \\
Nota $\left.{ }^{*}\right)$ Para $\mathrm{N}=155$ e 189, usar o filtro de tamanho 145. \\
Nota $(* *)$ Para N $=203$, usar o filtro de tamanho 193.
\end{tabular}

Tabela 6.1 Filtros S-WLSC mensais para cada $\mathbf{N}$

Os valores relacionados às medidas necessárias para o cálculo da $\mathrm{SNR}$, ou seja, $S Q, \gamma_{0}$ e $\left|1-\gamma_{1}\right|$ de cada filtro, estão apresentados na Tabela 6.2.

\begin{tabular}{c|c|c|c}
\hline $\boldsymbol{N}$ & $\boldsymbol{S} \boldsymbol{Q}$ & $\boldsymbol{\gamma}_{\mathbf{0}}$ & $\left|\mathbf{1}-\boldsymbol{\gamma}_{\mathbf{1}}\right|$ \\
\hline $\mathbf{1 1 7}$ & 0,358 & 0,929 & 0,1256 \\
\hline $\mathbf{1 2 1}$ & 0,3566 & 1,0092 & 0,1595 \\
\hline $\mathbf{1 3 1}$ & 0,3559 & 1,0072 & 0,1480 \\
\hline $\mathbf{1 4 1}$ & 0,3626 & 1,0069 & 0,1141 \\
\hline $\mathbf{1 4 5}$ & 0,3381 & 1,0083 & 0,2009 \\
\hline $\mathbf{1 5 5}$ & 0,3381 & 1,0083 & 0,2009 \\
\hline $\mathbf{1 8 9}$ & 0,3381 & 1,0083 & 0,2009 \\
\hline $\mathbf{1 9 3}$ & 0,3337 & 0,9917 & 0,1662 \\
\hline $\mathbf{2 0 3}$ & 0,3337 & 0,9917 & 0,1662 \\
\hline
\end{tabular}

Tabela 6.2 Valores de SQ e $\gamma_{s}$ para os filtros S-WLSC mensais, para $k=72$

Os programas, desenvolvidos em MATLAB, estão apresentados no Apêndice $\mathrm{K}$. 


\subsection{3.}

\section{Comparação do desempenho do filtro S-WLSC com o filtro S-WLS, para séries mensais com decomposição aditiva}

O objetivo desta Subseção 6.3.3 é comparar a SNR do filtro S-WLSC com a SNR do filtro S-WLS para identificar as situações em que o S-WLSC é superior ao filtro S-WLS, e, nesses casos, mensurar o ganho ao se utilizar o filtro S-WLSC.

Nessa comparação, foram considerados os seguintes valores em relação às características sazonais das séries: $k=96, b=40 \%$ e $A / s=6$. A SNR dos filtros foi calculada, assim como a razão $\frac{\text { SNR S-WLSC }}{\text { SNR S-WLS }}$. Os valores estão apresentados na tabela a seguir, onde observa-se que a SNR do S-WLSC é superior à SNR do filtro S-WLS em todos os casos, exceto quando $\mathrm{N}=117$ e $\mathrm{N}=141$.

\begin{tabular}{c|c|c|c}
\hline $\boldsymbol{N}$ & SNR S-WLSC & SNR S-WLS & $\frac{\text { SNR S-WLSC }}{\text { SNR S-WLS }}$ \\
\hline $\mathbf{1 1 7}$ & 42,38 & 49,28 & 0,860 \\
\hline $\mathbf{1 2 1}$ & 54,25 & 53,62 & 1,012 \\
\hline $\mathbf{1 3 1}$ & 54,45 & 53,54 & 1,017 \\
\hline $\mathbf{1 4 1}$ & 54,22 & 54,88 & 0,988 \\
\hline $\mathbf{1 4 5}$ & 57,28 & 56,45 & 1,015 \\
\hline $\mathbf{1 5 5}$ & 57,28 & 56,83 & 1,008 \\
\hline $\mathbf{1 8 9}$ & 57,28 & 55,34 & 1,035 \\
\hline $\mathbf{1 9 3}$ & 58,01 & 57,61 & 1,007 \\
\hline $\mathbf{2 0 3}$ & 58,01 & 57,47 & 1,010 \\
\hline
\end{tabular}

Tabela 6.3 SNRs e razão entre as SNRs, para $A / s=6, b=40 \% k=96$ (Eq. 5-1), considerando todos os filtros mensais: comparação entre S-WLSC e S-WLS

Para a comparação mais detalhada dos resultados entre o filtro S-WLSC e o filtro S-WLS, foram utilizados os tamanhos $N=121$ e $N=189$. A escolha do filtro de tamanho 121 foi devida à SNR do filtro S-WLS, que é a máxima dentre os filtros com tamanho de média móvel sazonal $3 \times 3$. Em relação ao filtro $N=189$, a escolha foi feita uma vez que ele é o que apresenta a maior razão entre as SNRs. Vale mencionar que, como não foi possível obter um filtro S-WLSC competitivo para $N=117$ e $N=141$, eles não foram utilizados nas comparações.

O Gráfico 6.25, a seguir, apresenta a razão entre as SNRs dos filtros S-WLSC e S-WLS de tamanhos 121 e 189, para diferentes valores da taxa de variação da amplitude sazonal $(b)$. Quanto maior é o valor de ' $b$ ' mais alta é a razão entre a 
SNR do S-WLSC e a SNR do S-WLS, significando uma melhor adequação do SWLSC, tanto para $N=121$, quanto para $N=189$, para altos graus de sazonalidade móvel.

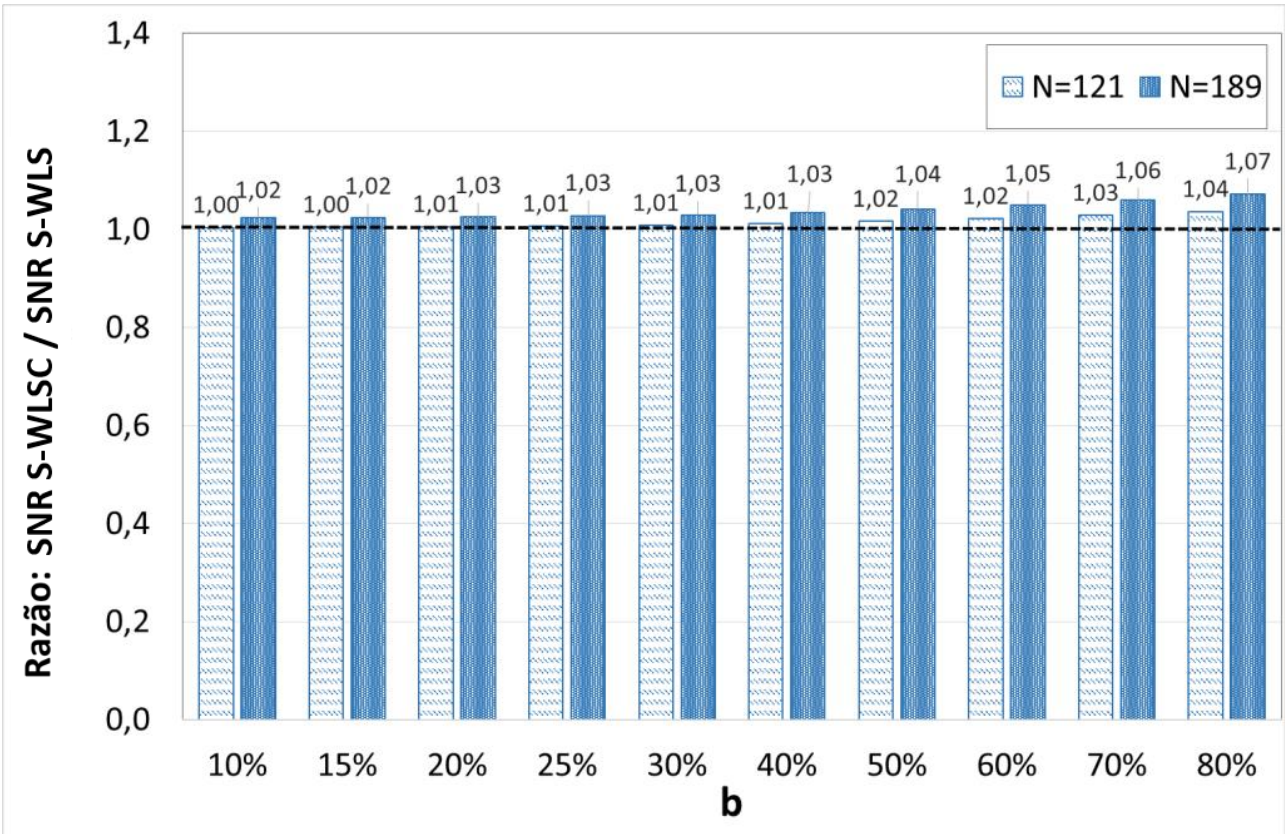

\section{Gráfico 6.25 Razão entre a SNR do S-WLSC e a SNR do S-WLS}

considerando $A / s=6$ e $k=96$, para diversos valores de $b$ : comparação entre $\mathrm{N}=121$ e $\mathrm{N}=189$

Os Gráficos 6.26 e 6.27 mostram as SNRs dos filtros S-WLSC e S-WLS, para cada valor de ' $b$ ', considerando os tamanhos 121 e 189, separadamente. Na Tabela 6.4, nota-se que, à medida que ' $b$ ' aumenta, o distanciamento entre as SNRs dos filtros de tamanho 189 é mais acentuado, se comparado aos filtros de tamanho 121. Destaca-se, também, que mesmo para valores baixos de ' $b$ ', como 10\%, a SNR do filtro S-WLSC é maior do que a SNR do filtro S-WLS.

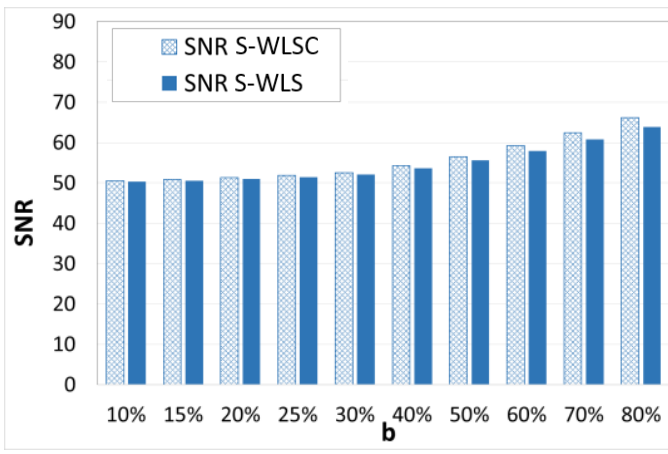

Gráfico 6.26 SNR S-WLSC e SNR $S$-WLS considerando $A / s=6$ e $k=96$, para diversos valores de $b(\mathrm{~N}=121)$

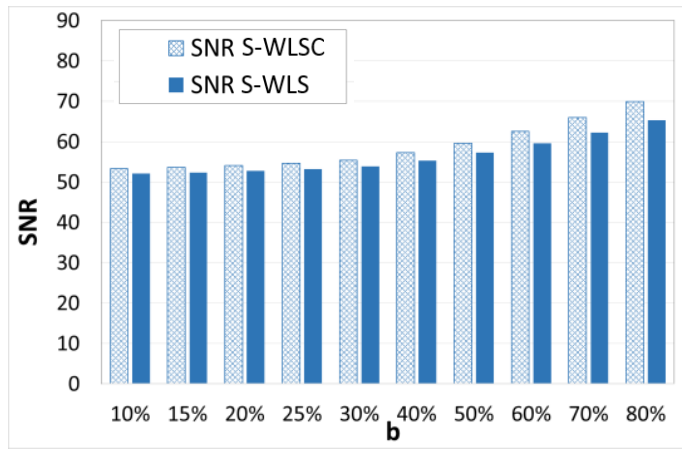

Gráfico 6.27 SNR S-WLSC e SNR $S-W L S$ considerando $A / s=6$ e $k=$ 96, para diversos valores de 


\begin{tabular}{|c|c|c|c|c|c|c|}
\hline \multirow[b]{2}{*}{$\boldsymbol{b}$} & \multicolumn{3}{|c|}{$N=121$} & \multicolumn{3}{|c|}{$N=189$} \\
\hline & $\begin{array}{c}\text { SNR } \\
\text { S-WLSC }\end{array}$ & $\begin{array}{c}\text { SNR } \\
\text { S-WLS }\end{array}$ & $\begin{array}{c}\text { SNR } \\
\text { S-WLSC / } \\
\text { SNR } \\
\text { S-WLS }\end{array}$ & $\begin{array}{c}\text { SNR } \\
\text { S-WLSC }\end{array}$ & $\begin{array}{c}\text { SNR } \\
\text { S-WLS }\end{array}$ & $\begin{array}{c}\text { SNR } \\
\text { S-WLSC } \\
\text { SNR } \\
\text { S-WLS }\end{array}$ \\
\hline $10 \%$ & 50,5 & 50,3 & 1,004 & 53,3 & 52,1 & 1,023 \\
\hline $15 \%$ & 50,8 & 50,6 & 1,005 & 53,6 & 52,4 & 1,024 \\
\hline $20 \%$ & 51,3 & 51,0 & 1,006 & 54,1 & 52,7 & 1,026 \\
\hline $25 \%$ & 51,8 & 51,5 & 1,007 & 54,7 & 53,2 & 1,027 \\
\hline $30 \%$ & 52,5 & 52,1 & 1,008 & 55,4 & 53,8 & 1,030 \\
\hline $40 \%$ & 54,3 & 53,6 & 1,012 & 57,3 & 55,3 & 1,035 \\
\hline $50 \%$ & 56,5 & 55,6 & 1,017 & 59,7 & 57,3 & 1,042 \\
\hline $60 \%$ & 59,2 & 58,0 & 1,022 & 62,6 & 59,6 & 1,050 \\
\hline $70 \%$ & 62,5 & 60,7 & 1,029 & 66,0 & 62,3 & 1,060 \\
\hline $80 \%$ & 66,2 & 63,9 & 1,037 & 69,9 & 65,3 & 1,071 \\
\hline
\end{tabular}

Tabela 6.4 SNR S-WLSC e SNR S-WLS, variando $b$ para $A / s=6, k=96$ e $N=$ 121 e 189

O Gráfico 6.28, a seguir, apresenta a razão entre as SNRs dos filtros S-WLSC e S-WLS, de tamanho 121 e 189 , para diferentes valores da taxa de ' $k$ '. Vale lembrar que o parâmetro ' $k$ ' indica o tempo (em meses) necessário para o padrão de sazonalidade móvel se repetir. Diferente do que ocorre com a variação de ' $b$ ', à medida que ' $k$ ' aumenta, a razão entre as SNRs se mantém constante.

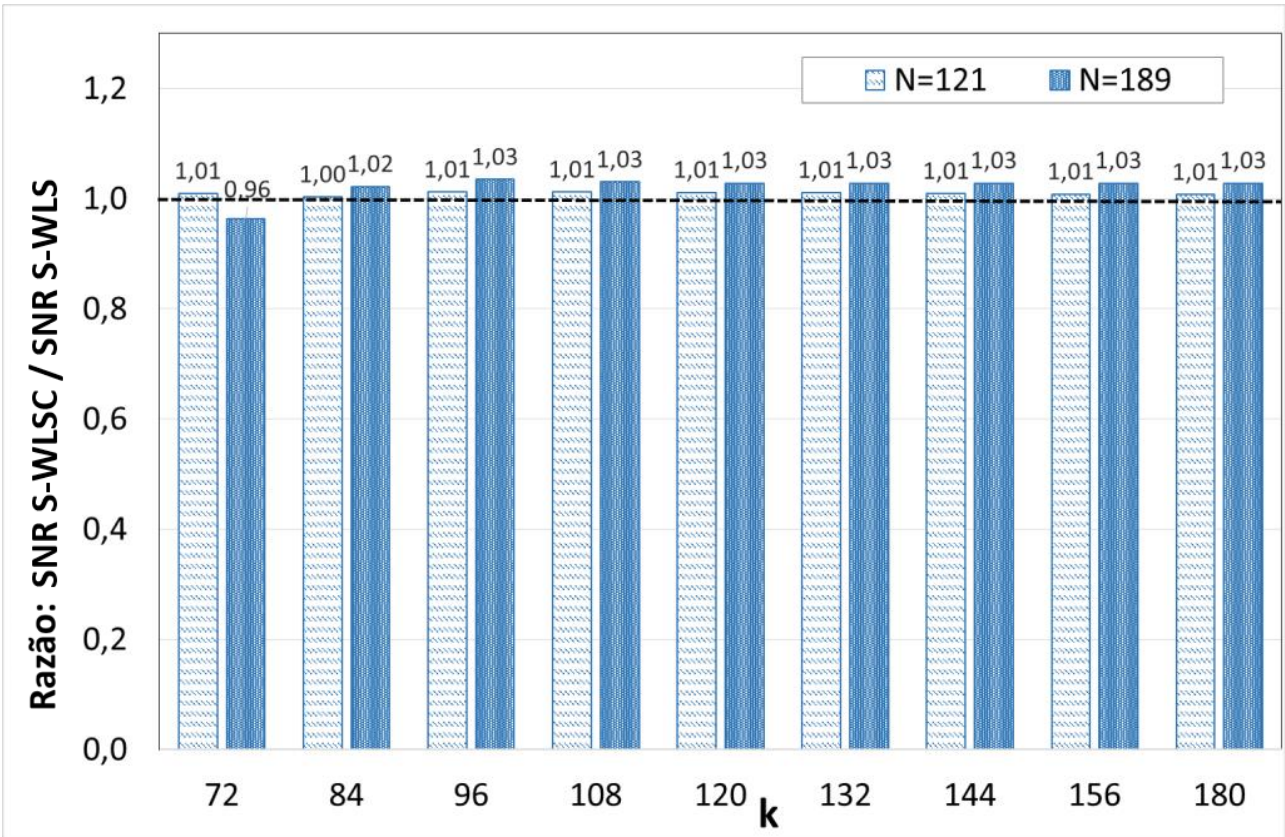

Gráfico 6.28 Razão entre a SNR do S-WLSC e a do SNR S-WLS considerando $A / s=6$ e $b=40 \%$, para diversos valores de $k$ : comparação entre $\mathrm{N}=121$ e $\mathrm{N}=189$ 
Os Gráficos 6.29 e 6.30 mostram as SNRs individuais (dos filtros S-WLSC e S-WLS), com a variação de ' $k$ ', para cada $N$, separadamente. E a Tabela 6.5 apresenta os valores usados para a construção desses gráficos.

Observando os resultados, nota-se que, para $k \geq 96$, a SNR do filtro S-WLSC permanece constante. Além disso, vale citar que o único caso em que o filtro $S$ WLSC apresenta SNR menor do que o filtro S-WLS, ocorre quando $k=72$, no filtro de tamanho 189. Como o filtro foi definido $\operatorname{com} \alpha=1 / 3$, o que equivale a $k=72$, ele pode apresentar instabilidade no ajuste de séries que apresentem esse valor de $k$.

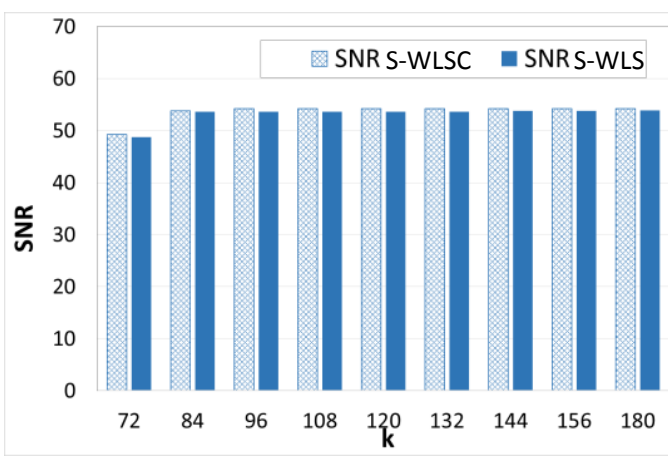

Gráfico 6.29 SNR S-WLSC e SNR S-WLS considerando $A / s=6$ e $b=$ $40 \%$, para diversos valores de $k$ ( $N=121)$

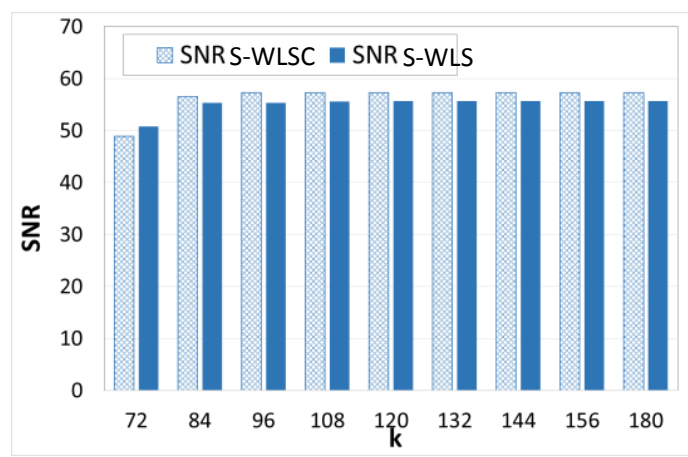

Gráfico 6.30 SNR S-WLSC e SNR S-WLS considerando $A / s=6$ e $b=$ $40 \%$, para diversos valores de $k(\mathbf{N}=189)$

\begin{tabular}{c|ccc|ccc}
\hline & & $N=\mathbf{1 2 1}$ & & & \\
& & & & $\mathbf{1 8 9}$ & \\
& $\begin{array}{c}\text { SNR } \\
\text { S-WLSC }\end{array}$ & $\begin{array}{c}\text { SNR } \\
\text { S-WLS }\end{array}$ & $\begin{array}{c}\text { SNR } \\
\text { S-WLSC } / \\
\text { SNR } \\
\text { S-WLS }\end{array}$ & $\begin{array}{c}\text { SNR } \\
\text { S-WLSC }\end{array}$ & $\begin{array}{c}\text { SNR } \\
\text { S-WLS }\end{array}$ & $\begin{array}{c}\text { SNR } \\
\text { S-WLS } / \\
\text { SNR } \\
\text { S-WLS }\end{array}$ \\
\hline $\mathbf{7 2}$ & 49,2 & 48,8 & 1,009 & 48,9 & 50,8 & 0,963 \\
$\mathbf{8 4}$ & 53,8 & 53,6 & 1,003 & 56,6 & 55,3 & 1,022 \\
$\mathbf{9 6}$ & 54,3 & 53,6 & 1,012 & 57,3 & 55,3 & 1,035 \\
$\mathbf{1 0 8}$ & 54,3 & 53,6 & 1,012 & 57,3 & 55,6 & 1,030 \\
$\mathbf{1 2 0}$ & 54,3 & 53,6 & 1,011 & 57,3 & 55,7 & 1,028 \\
$\mathbf{1 3 2}$ & 54,3 & 53,7 & 1,011 & 57,3 & 55,7 & 1,028 \\
$\mathbf{1 4 4}$ & 54,3 & 53,8 & 1,009 & 57,3 & 55,7 & 1,028 \\
$\mathbf{1 5 6}$ & 54,3 & 53,8 & 1,008 & 57,3 & 55,7 & 1,028 \\
$\mathbf{1 8 0}$ & 54,3 & 53,9 & 1,007 & 57,3 & 55,7 & 1,028 \\
\hline
\end{tabular}

Tabela 6.5 SNR S-WLSC e SNR S-WLS, variando $k$ para $A / s=6, b=40 \%$ e $N=121$ e 189 
Em relação à razão entre as SNRs dos filtros S-WLSC e S-WLS, para diferentes valores da razão ' $A / s$ ', é apresentado o Gráfico 6.31 , com os resultados encontrados.

A razão ' $A / s$ ' indica a amplitude do sinal sazonal em relação ao ruído. Notase que se ' $A / s$ ' apresentar valor baixo (1, 2 ou 3), a SNR do S-WLSC é menor do que a SNR do S-WLS. Isso ocorre porque a atenuação na banda de rejeição é maior no filtro S-WLS. A partir de $A / s=4$, a SNR do S-WLSC supera a SNR do S-WLS no filtro de tamanho 189. Além disso, destaca-se que quanto maior for a razão ' $A / s$ ', mais adequado é o ajuste sazonal realizado pelo S-WLSC se comparado ao ajuste do filtro S-WLS.

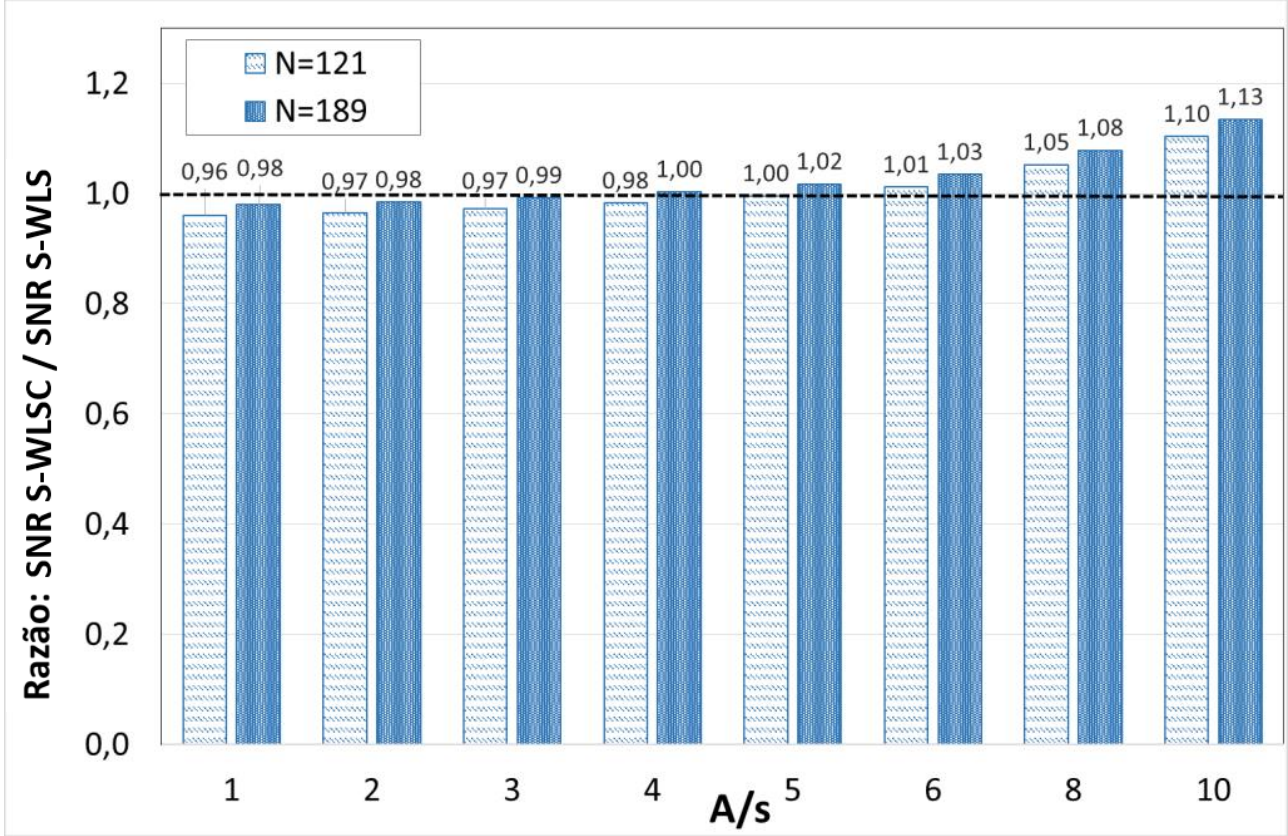

Gráfico 6.31 Razão entre a SNR do S-WLSC e a SNR do S-WLS considerando $k=96, b=40 \%$, para diversos valores de $A / s$ : comparação entre $\mathrm{N}=121$ e $\mathrm{N}=189$

Os Gráficos 6.32 e 6.33 mostram as SNRs individuais dos filtros segundo os valores de ' $A / s$ ', considerando $N=121$ e $N=189$, respectivamente. Nesses gráficos, nota-se o distanciamento entre as SNRs dos filtros à medida que a razão 'A/s' aumenta. 


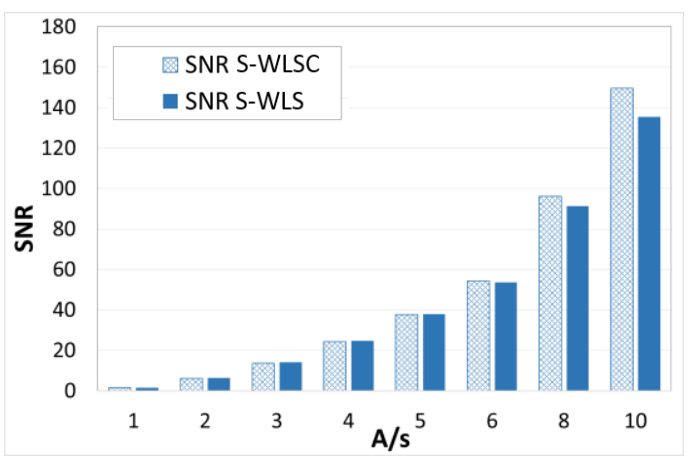

Gráfico 6.32 SNR S-WLSC e SNR S-WLS considerando $k=96, b=40 \%$, para diversos valores de $A / s$ $(\mathrm{N}=121)$

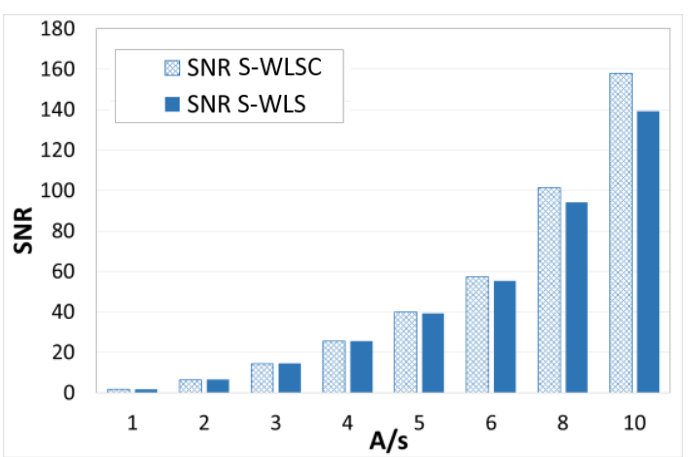

Gráfico 6.33 SNR S-WLSC e SNR $S$-WLS considerando $k=96, b=$ $40 \%$, para diversos valores de $A / s$ $(\mathbf{N}=189)$

A Tabela 6.6, a seguir, apresenta os valores usados para a construção dos Gráficos 6.31 a 6.33 .

\begin{tabular}{c|ccc|ccc}
\hline \multirow{3}{*}{$\boldsymbol{A} / \boldsymbol{s}$} & \multicolumn{3}{|c|}{$\boldsymbol{N = 1 2 1}$} & \multicolumn{3}{c}{$\boldsymbol{N}=\mathbf{1 8 9}$} \\
\cline { 2 - 7 } & SNR & SNR & SNR WLSC / & SNR & SNR & SNR WLSC/ \\
& WLSC & S-WLS & SNR S-WLS & WLSC & S-WLS & SNR S-WLS \\
\hline $\mathbf{1}$ & 1,5 & 1,6 & 0,961 & 1,6 & 1,6 & 0,980 \\
$\mathbf{2}$ & 6,1 & 6,3 & 0,965 & 6,4 & 6,5 & 0,984 \\
$\mathbf{3}$ & 13,6 & 14,0 & 0,973 & 14,4 & 14,5 & 0,992 \\
$\mathbf{4}$ & 24,2 & 24,6 & 0,983 & 25,5 & 25,4 & 1,003 \\
$\mathbf{5}$ & 37,7 & 37,9 & 0,996 & 39,8 & 39,1 & 1,018 \\
$\mathbf{6}$ & 54,3 & 53,6 & 1,012 & 57,3 & 55,3 & 1,035 \\
$\mathbf{8}$ & 96,1 & 91,3 & 1,052 & 101,5 & 94,1 & 1,079 \\
$\mathbf{1 0}$ & 149,4 & 135,4 & 1,104 & 158,0 & 139,2 & 1,135 \\
\hline
\end{tabular}

Tabela 6.6 SNR S-WLSC e SNR S-WLS, variando $A / s$, para $k=96, b=40 \%$ e

$$
N=121 \text { e } 189
$$




\section{4 .}

\section{Série mensal multiplicativa - filtro S-WLSC vs filtro S-WLS}

Com a configuração de parâmetros do filtro mensal já definida na Subseção 6.3.1, testou-se o desempenho do S-WLSC em relação ao filtro S-WLS para as séries mensais com decomposição multiplicativa. Nesta subseção são comparados os resultados, utilizando-se a estatística MSE.

Nos testes, foram utilizados os filtros de tamanho 121 e 189. Como padrão para a componente de tendência, utilizou-se a série X6; e para os coeficientes da componente sazonal artificial, foi usado o padrão da série Y157.

Para cada combinação de valores dos parâmetros do sinal artificial (Eq. 5-3), geraram-se 100 séries referentes à componente irregular, cada uma com distribuição $\mathrm{N}\left(0, \sigma^{2}\right)$, utilizando a função randn do MATLAB, com base no desvio-padrão da série Y157. Isso permitiu o cálculo da média e do desvio-padrão da MSE. Os valores utilizados, foram os seguintes:

\begin{tabular}{c|c|c|c|c|c}
\hline $\boldsymbol{A}$ & $\boldsymbol{s}$ & $\mathbf{b}_{\mathbf{0}}$ & $\mathbf{b}_{\mathbf{1}}$ & $\mathbf{b}_{\mathbf{2}}$ & $\mathbf{b}_{\mathbf{3}}$ \\
\hline 0,015 & 0,0025 & 76,3 & 0,3114 & 0,0028 & 0,00001 \\
\hline
\end{tabular}

Tabela 6.7 Valores da componente de tendência, desvio-padrão da irregular e amplitude da componente sazonal

Os Gráficos 6.34 e 6.35 ilustram a MSE do filtro S-WLSC e do filtro S-WLS para diversos valores de ' $b$ ', quando $N=121$ e $N=189$, respectivamente. E a Tabela 6.8 apresenta os valores da MSE de cada filtro, para os valores de ' $b$ ', utilizados nos gráficos.

Analisando a Tabela 6.8, nota-se uma melhora significativa na MSE do filtro S-WLSC, em comparação com o filtro S-WLS, para o caso em que $N=189$.

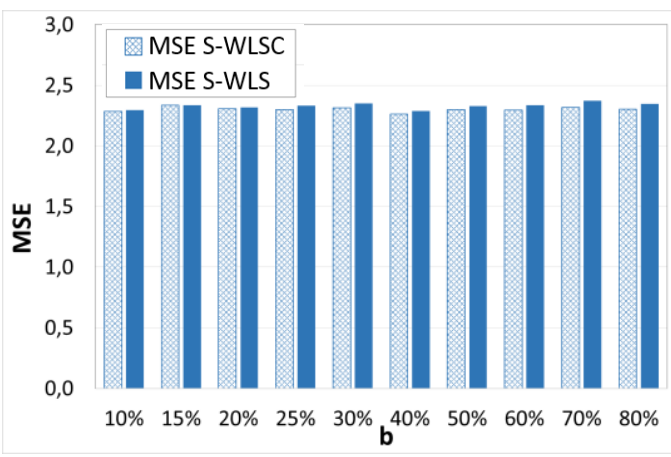

Gráfico 6.34 MSE S-WLSC e MSE S-WLS $\left({\left.\mathrm{x} 10^{6}\right)}^{6}\right.$ considerando $k=96$, $A / s=6$, para diversos valores de $b$ : $\mathrm{N}=121$

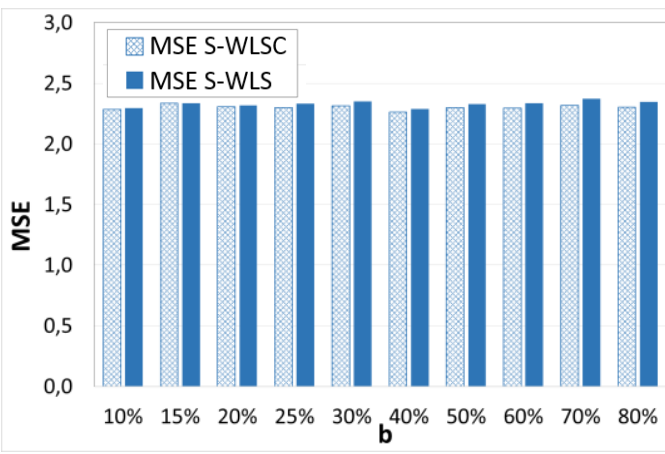

Gráfico 6.35 MSE S-WLSC e MSE S-WLS $\left(\mathrm{x}^{6} 0^{6}\right)$ considerando $k=96$, $A / s=6$, para diversos valores de $b$ : $\mathrm{N}=189$ 


\begin{tabular}{c|ccc|ccc}
\hline \multirow{2}{*}{$\boldsymbol{b}$} & \multicolumn{3}{|c|}{$\boldsymbol{N = 1 2 1}$} & \multicolumn{3}{c}{$\boldsymbol{N = 1 8 9}$} \\
\cline { 2 - 7 } & $\begin{array}{c}\text { MSE } \\
\text { S-WLSC }\end{array}$ & $\begin{array}{c}\text { MSE } \\
\text { S-WLS }\end{array}$ & valor-p & $\begin{array}{c}\text { MSE } \\
\text { s-WLSC }\end{array}$ & $\begin{array}{c}\text { MSE } \\
\text { S-WLS }\end{array}$ & valor-p \\
\hline $\mathbf{1 0 \%}$ & 2,28 & 2,29 & 0,386 & 2,16 & 2,28 & $0,001^{*}$ \\
$\mathbf{1 5 \%}$ & 2,34 & 2,33 & 0,528 & 2,23 & 2,35 & $0,001^{*}$ \\
$\mathbf{2 0 \%}$ & 2,31 & 2,32 & 0,375 & 2,16 & 2,26 & $0,002^{*}$ \\
$\mathbf{2 5 \%}$ & 2,30 & 2,33 & 0,227 & 2,16 & 2,30 & $0,000^{*}$ \\
$\mathbf{3 0 \%}$ & 2,31 & 2,35 & 0,146 & 2,16 & 2,31 & $0,000^{*}$ \\
$\mathbf{4 0 \%}$ & 2,26 & 2,29 & 0,262 & 2,19 & 2,38 & $0,000^{*}$ \\
$\mathbf{5 0 \%}$ & 2,30 & 2,33 & 0,199 & 2,14 & 2,34 & $0,000^{*}$ \\
$\mathbf{6 0 \%}$ & 2,29 & 2,33 & 0,108 & 2,16 & 2,39 & $0,000^{*}$ \\
$\mathbf{7 0 \%}$ & 2,32 & 2,37 & 0,079 & 2,19 & 2,44 & $0,000^{*}$ \\
$\mathbf{8 0 \%}$ & 2,30 & 2,35 & 0,121 & 2,15 & 2,46 & $0,000^{*}$ \\
\hline Nota: $\left.{ }^{*}{ }^{*}\right)$ valor-p $\left.<1 \% ;{ }^{* *}\right)$ valor-p $<5 \%$. & \multicolumn{3}{|c}{} \\
\hline
\end{tabular}

Tabela 6.8 MSE S-WLSC e MSE S-WLS, $\left(\mathrm{x}^{10}{ }^{6}\right)$, variando ' $b$ ' $\operatorname{para} A / s=6, k=$ $96(N=121$ e 189). Valores baseados no teste $t$, unilateral, de comparação de médias 
A MSE dos filtros, segundo os diversos valores de ' $k$ ', está ilustrada nos Gráficos 6.36 e 6.37, para os tamanhos 121 e 189. Considerando $N=189$, o S-WLSC é novamente melhor em todos os casos, ou seja, para todos os valores de ' $k$ '. No entanto, para $N=121$, o S-WLSC é significativamente superior ao filtro S-WLS somente para $k=72$.

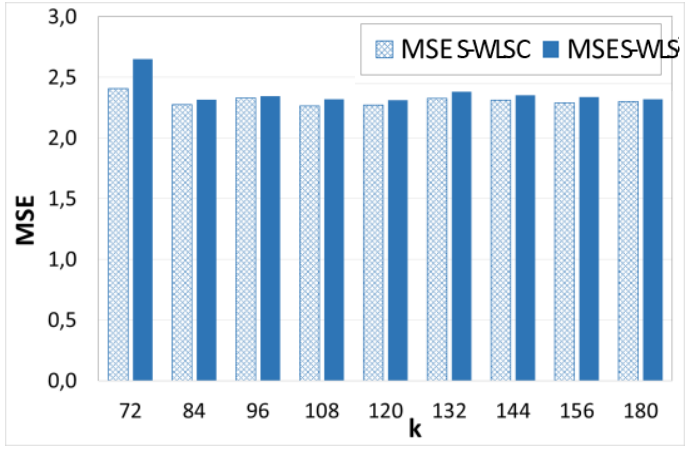

Gráfico 6.36 MSE S-WLSC e MSE SWLS $\left(\mathrm{x}^{6} 0^{6}\right)$ considerando $A / s=6$ e $b=$ $40 \%$, para diversos valores de $k$ $(\mathrm{N}=121)$

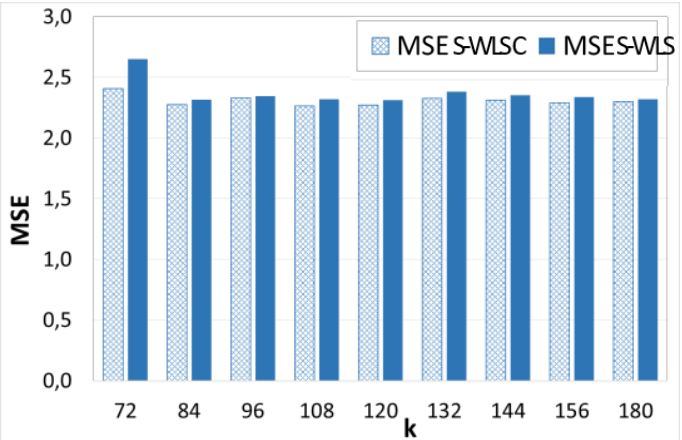

Gráfico 6.37 MSE S-WLSC e MSE S-WLS $\left(\mathrm{x10}^{6}\right)$ considerando $A / s=6$ e $b=40 \%$, para diversos valores de $\boldsymbol{k}(\mathbf{N}=\mathbf{1 8 9})$

A Tabela 6.9, a seguir, apresenta os valores da MSE de cada filtro, com o valor-p para os diversos valores de ' $k$ '.

\begin{tabular}{c|ccc|ccc}
\hline \multirow{2}{*}{$\boldsymbol{k}$} & \multicolumn{3}{|c|}{$\mathbf{N = 1 2 1}$} & \multicolumn{3}{c}{$\boldsymbol{N = 1 8 9}$} \\
\cline { 2 - 7 } & $\begin{array}{c}\text { MSE } \\
\text { S-WLSC }\end{array}$ & $\begin{array}{c}\text { MSE } \\
\text { S-WLS }\end{array}$ & valor-p & $\begin{array}{c}\text { MSE } \\
\text { S-WLSC }\end{array}$ & $\begin{array}{c}\text { MSE } \\
\text { S-WLS }\end{array}$ & valor-p \\
\hline $\mathbf{7 2}$ & 2,41 & 2,65 & $0,000^{*}$ & 2,40 & 2,69 & $0,000^{*}$ \\
$\mathbf{8 4}$ & 2,27 & 2,32 & 0,149 & 2,19 & 2,33 & $0,000^{*}$ \\
$\mathbf{9 6}$ & 2,33 & 2,35 & 0,322 & 2,20 & 2,37 & $0,000^{*}$ \\
$\mathbf{1 0 8}$ & 2,26 & 2,32 & 0,072 & 2,18 & 2,32 & $0,000^{*}$ \\
$\mathbf{1 2 0}$ & 2,27 & 2,31 & 0,140 & 2,14 & 2,24 & $0,006^{*}$ \\
$\mathbf{1 3 2}$ & 2,33 & 2,38 & 0,077 & 2,20 & 2,34 & $0,000^{*}$ \\
$\mathbf{1 4 4}$ & 2,31 & 2,35 & 0,152 & 2,17 & 2,29 & $0,002^{*}$ \\
$\mathbf{1 5 6}$ & 2,29 & 2,34 & 0,080 & 2,16 & 2,29 & $0,001^{*}$ \\
$\mathbf{1 8 0}$ & 2,30 & 2,32 & 0,327 & 2,18 & 2,33 & $0,000^{*}$ \\
\hline Nota: $\left({ }^{*}\right)$ valor-p $<1 \% ;\left({ }^{* *}\right)$ valor-p $<5 \%$. & \multicolumn{3}{|c}{}
\end{tabular}

Tabela 6.9 MSE do S-WLSC e MSE do S-WLS, $\left(\mathrm{x}^{10^{6}}\right)$, variando ' $k$ ' para $A / s$ $=6, b=40 \%(N=121$ e 189). Valores baseados no teste $t$, unilateral, de comparação de médias 
Quanto aos valores da razão ' $A / s$ ', os Gráficos 6.38 e 6.39 mostram a MSE do S-WLSC e do filtro S-WLS, para $N=121$ e $N=189$, respectivamente.

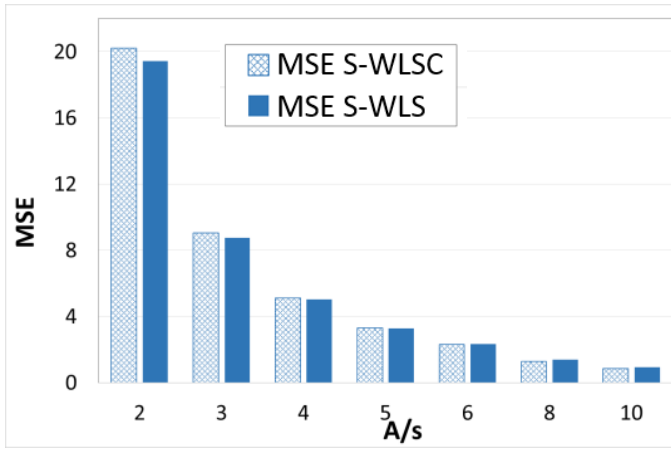

Gráfico 6.38 MSE S-WLSC e MSE SWLS $\left(\mathrm{x10}^{6}\right)$ considerando $k=96, b=40 \%$, para diversos valores de $A / s(\mathrm{~N}=121)$

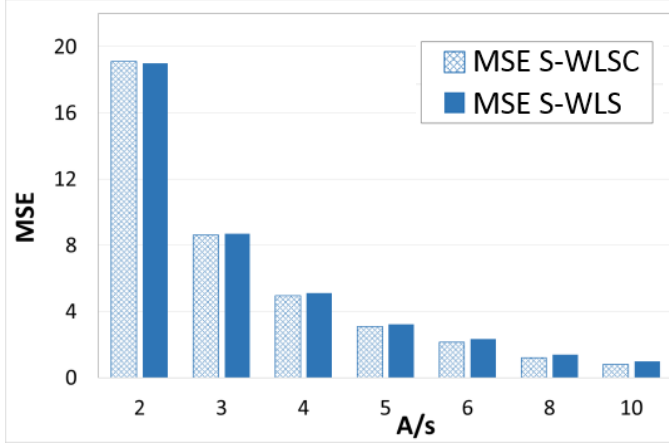

Gráfico 6.39 MSE S-WLSC e MSE SWLS ( $\left(10^{6}\right)$ considerando $k=96, b=40 \%$, para diversos valores de $A / s(\mathrm{~N}=189)$

A Tabela 6.10 apresenta os valores da MSE de cada filtro, para os diversos valores de ' $A / s$ ', utilizados nos Gráficos 6.38 e 6.39 .

\begin{tabular}{c|ccc|ccc}
\hline \multirow{2}{*}{$\boldsymbol{A} / \boldsymbol{s}$} & \multicolumn{3}{|c|}{$\mathbf{N = 1 2 1}$} & \multicolumn{3}{c}{$\mathbf{N = 1 8 9}$} \\
\cline { 2 - 7 } & $\begin{array}{c}\text { MSE } \\
\text { S-WLSC }\end{array}$ & $\begin{array}{c}\text { MSE } \\
\text { S-WLS }\end{array}$ & valor-p & $\begin{array}{c}\text { MSE } \\
\text { S-WLSC }\end{array}$ & $\begin{array}{c}\text { MSE } \\
\text { S-WLS }\end{array}$ & valor-p \\
\hline $\mathbf{1}$ & 81,69 & 78,04 & 0,998 & 78,42 & 78,04 & 0,603 \\
$\mathbf{2}$ & 20,22 & 19,44 & 0,987 & 19,11 & 19,00 & 0,637 \\
$\mathbf{3}$ & 9,05 & 8,76 & 0,984 & 8,62 & 8,70 & 0,299 \\
$\mathbf{4}$ & 5,11 & 5,04 & 0,798 & 4,95 & 5,10 & 0,059 \\
$\mathbf{5}$ & 3,30 & 3,29 & 0,571 & 3,10 & 3,22 & $0,016^{* *}$ \\
$\mathbf{6}$ & 2,32 & 2,34 & 0,286 & 2,16 & 2,33 & $0,000^{*}$ \\
$\mathbf{8}$ & 1,30 & 1,38 & $0,000^{*}$ & 1,20 & 1,39 & $0,000^{*}$ \\
$\mathbf{1 0}$ & 0,84 & 0,94 & $0,000^{*}$ & 0,80 & 0,99 & $0,000^{*}$ \\
\hline
\end{tabular}

Nota: $\left({ }^{*}\right)$ valor-p $\left.<1 \% ;{ }^{* *}\right)$ valor-p $<5 \%$.

Tabela 6.10 MSE S-WLSC e MSE S-WLS, $\left(x 10^{6}\right)$, variando ' $A / s$ ', para $k$ $=96, b=40 \%(N=121$ e 189). Valores baseados no teste $t$, unilateral, de comparação de médias

Observa-se na Tabela 6.10 que, em se tratando de $N=189$, o S-WLSC é significativamente superior ao filtro $S$-WLS para $A / s \geq 5$, enquanto que para o filtro de tamanho 121 , isso ocorre somente quando $A / s \geq 8$. 
Por fim, para ilustrar a qualidade do ajuste sazonal realizado pelo filtro SWLSC, no caso das séries mensais multiplicativas, foram gerados os espectros da componente irregular, considerando séries com $k=72$ e $k=96$, para $A / s=6,5$ e $b=40 \%$ e $b=10 \%$. Nesse momento, foi utilizado o filtro S-WLSC de tamanho 121.

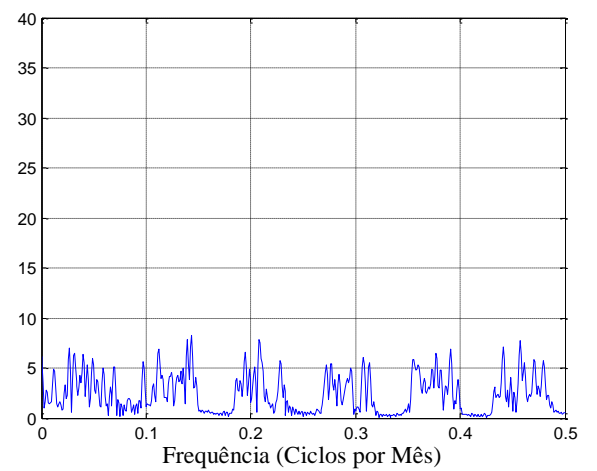

Gráfico 6.40 Espectro da irregular da série dessazonalizada pelo filtro $\mathrm{S}$ -

WLSC, sem tendência, e: $A / s=6,5 ; k=$ $72 ; b=40 \%(N=121)$

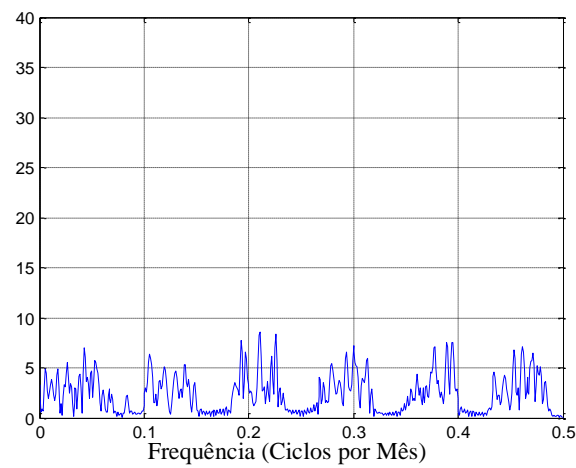

Gráfico 6.42 Espectro da irregular da série dessazonalizada pelo filtro $S$ WLSC, sem tendência, e: $A / s=6,5$; $k=72 ; b=10 \%(\mathrm{~N}=121)$

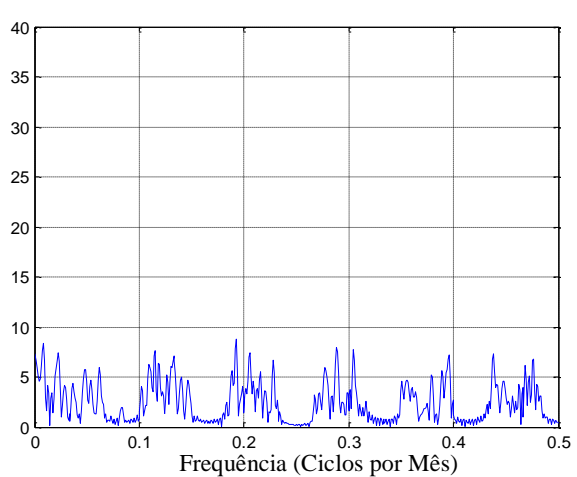

Gráfico 6.41 Espectro da irregular da série dessazonalizada pelo filtro S-WLSC, sem tendência, e: $A / s$ $=6,5 ; k=96 ; b=40 \%(N=121)$

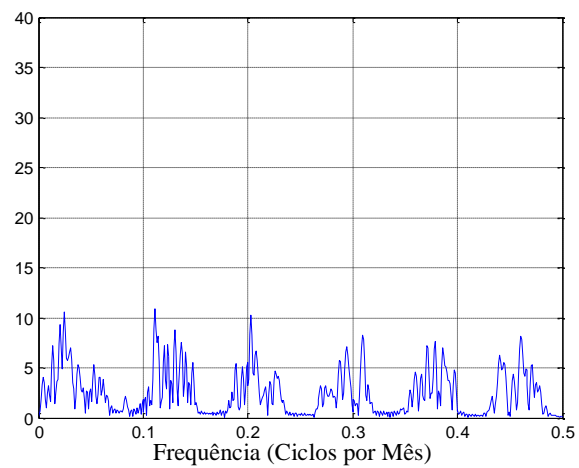

Gráfico 6.43 Espectro da irregular da série dessazonalizada pelo filtro S-WLSC, sem tendência, e: $A / s$ $=6,5 ; k=96 ; b=10 \%(N=121)$

Nos Gráficos 6.40 a 6.43, observa-se que o filtro S-WLSC realiza um ajuste sazonal adequado, pois não se notam picos nas frequências sazonais, ou próximos a elas. Vale citar que nesses gráficos não foi incluída a componente de tendência. 
No caso de haver componente de tendência na série, os espectros da componente irregular, resultantes da aplicação do filtro de dessazonalização SWLSC, seriam os seguintes:

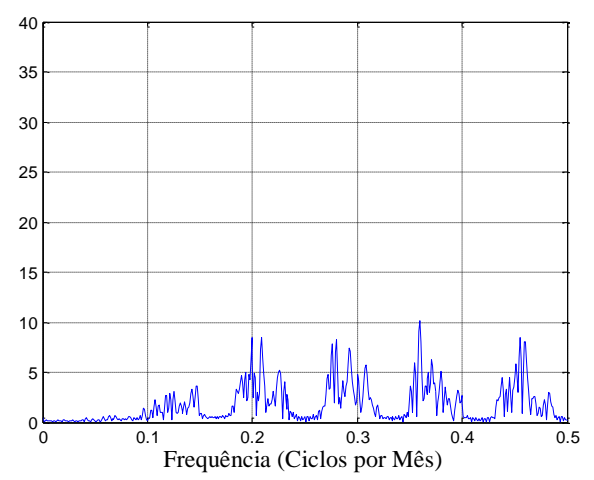

Gráfico 6.44 Espectro da irregular da série dessazonalizada pelo filtro $\mathrm{S}$ WLSC, com tendência, e: $A / s=6,5$; $k=72 ; b=40 \%(N=121)$

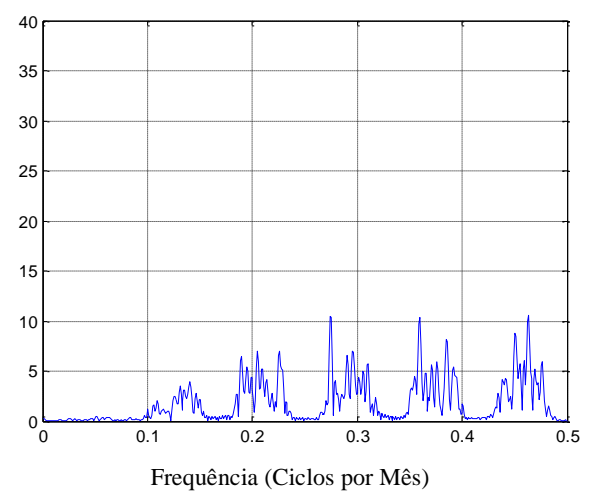

Gráfico 6.46 Espectro da irregular da série dessazonalizada pelo filtro S-WLSC, com tendência, e: $A / s$ $=6,5 ; k=72 ; b=10 \%(\mathrm{~N}=121)$

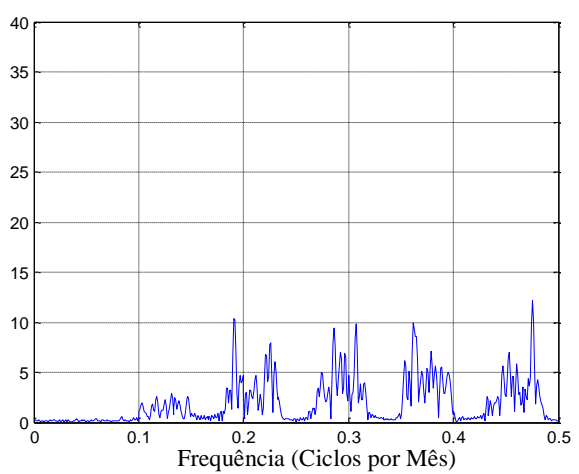

Gráfico 6.45 Espectro da irregular da série dessazonalizada pelo filtro $\mathrm{S}$ WLSC, com tendência, e: $A / s=6,5$; $k=96 ; b=40 \%(\mathrm{~N}=121)$

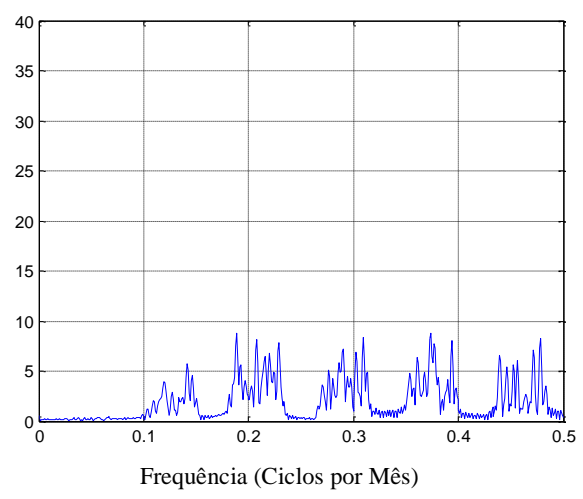

Gráfico 6.47 Espectro da irregular da série dessazonalizada pelo filtro S-WLSC, com tendência, e: $A / s$ $=6,5 ; k=96 ; b=10 \%(\mathrm{~N}=121)$

Observando os Gráficos 6.44 a 6.47, nota-se que não há picos nas frequências sazonais, ou próximos a elas, o que indica uma correta dessazonalização. 


\section{5 .}

\section{Série trimestral aditiva - filtro S-WLSC}

A seguir, na Subseção 6.5.1, é especificada a seleção da combinação de parâmetros para cada tamanho $(N)$ de filtro da séries trimestrais, juntamente com a resposta em magnitude de cada filtro. Na Subseção 6.5.2 é apresentado o resumo dos filtros selecionados; e na Subseção 6.5.3 é apresentada a comparação do filtro S-WLSC com o filtro S-WLS, para séries trimestrais com decomposição aditiva.

\subsection{1.}

\section{Seleção do melhor filtro para cada ' $N$ ': filtro trimestral}

A decisão em relação ao melhor filtro foi tomada com base em sinais artificiais, com vários valores para ' $A / s$ ', ' $b$ ' e ' $k$ ', definidos na Equação (5-2). Os resultados são apresentados separadamente - para cada ' $N$ '. Nas comparações, foi utilizado o filtro S-WLS.

\subsubsection{1.}

Resultado: filtro S-WLSC trimestral $\operatorname{com} N=41$

Nos filtros S-WLSC, quanto maior o $\Gamma$, menor é a $S Q$, mas o $\left|1-\gamma_{1}\right|$ piora, ou seja, aumenta. Então, foram necessárias algumas comparações para verificar qual das combinações de valores dos parâmetros gerava resultados com maior SNR. Após as verificações, o filtro escolhido foi o que apresentou a seguinte estrutura:

$$
\alpha=\frac{1}{3}, \delta=\frac{1}{500}, w_{0}=2, \Gamma=0,006 .
$$

A resposta em magnitude do filtro S-WLSC de tamanho 41 está apresentada a seguir, no Gráfico 6.48. No gráfico ao lado, está a comparação dela com a resposta em magnitude do filtro S-WLS, juntamente com o filtro desejado (esperado). E, na sequência (Gráfico 6.50), é apresentada uma ampliação da banda passante, mostrando ripple menor na resposta em magnitude do filtro S-WLSC. 


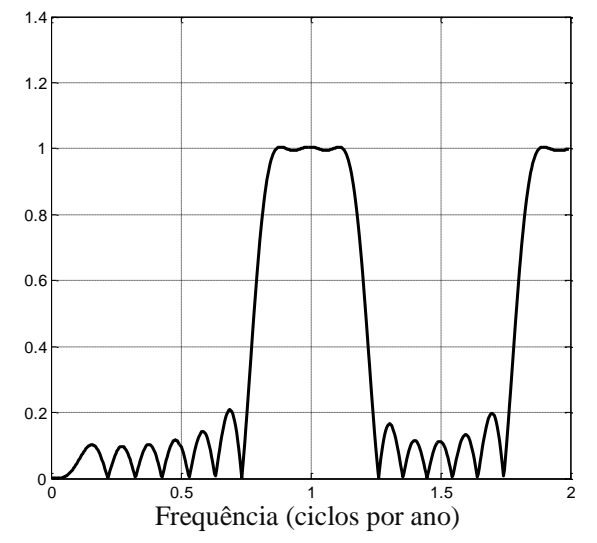

Gráfico 6.48 Resposta em magnitude do filtro S-WLSC $(N=41)$

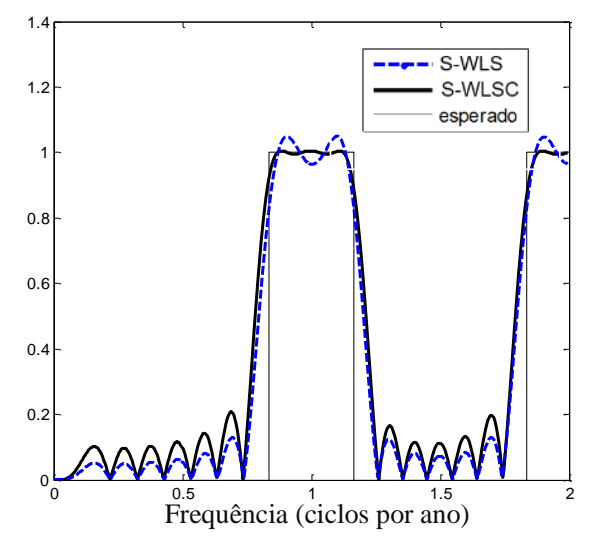

Gráfico 6.49 Resposta em magnitude do filtro S-WLSC, do S-WLS e o esperado $(N=41)$

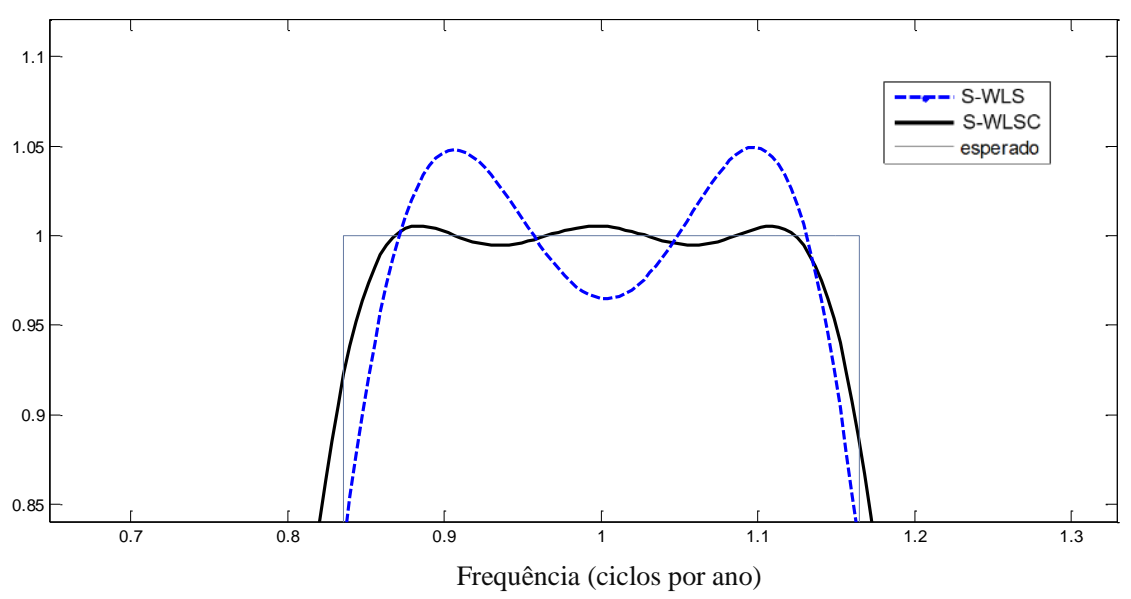

Gráfico 6.50 Banda passante dos filtros S-WLSC e S-WLS ( $N=41)$

\subsubsection{2.}

\section{Resultado: filtro S-WLSC trimestral com $N=43$ e 65}

O filtro que gerou SNR maior do que a do filtro S-WLS, para tamanhos $N=43$ e $N=65$, foi o que apresentava a seguinte configuração:

$$
\alpha=\frac{1}{3}, \delta=\frac{1}{500}, w_{0}=2, \Gamma=0,01 .
$$

As respostas em magnitude dos filtros S-WLSC de tamanho 43 e 65 estão apresentadas a seguir, nos Gráficos 6.51 e 6.54, respectivamente. Ao lado de cada 
uma, está a comparação com a resposta em magnitude do filtro S-WLS, juntamente com a função do filtro desejado (esperado). E, em seguida uma ampliação da banda passante, mostrando que o ripple da resposta em magnitude do filtro S-WLSC é menor do que o do filtro S-WLS.

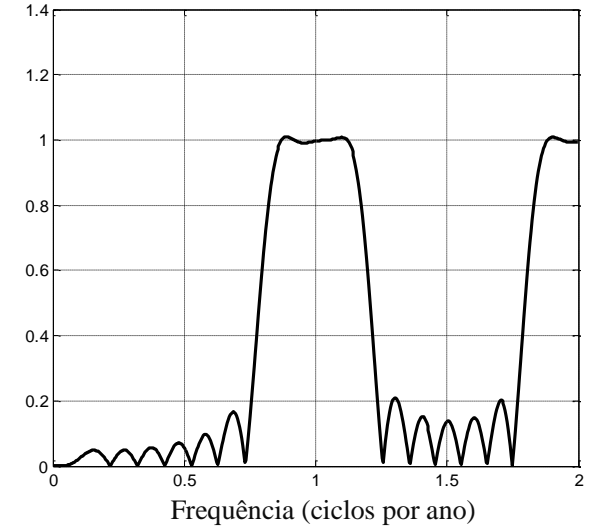

Gráfico 6.51 Resposta em magnitude do filtro S-WLSC $(\mathrm{N}=43)$

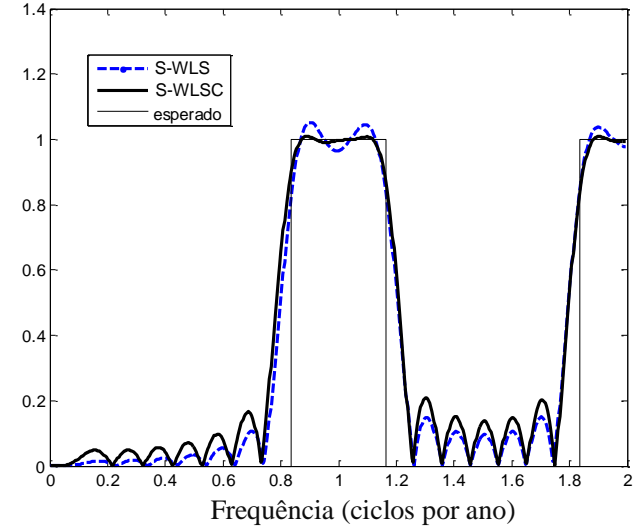

Gráfico 6.52 Resposta em magnitude do filtro S-WLSC, do S-WLS e o esperado, para $(N=43)$

No Gráfico 6.53, a seguir, observa-se um menor ripple na banda passante do filtro S-WLSC, quando comparado com o filtro S-WLS.

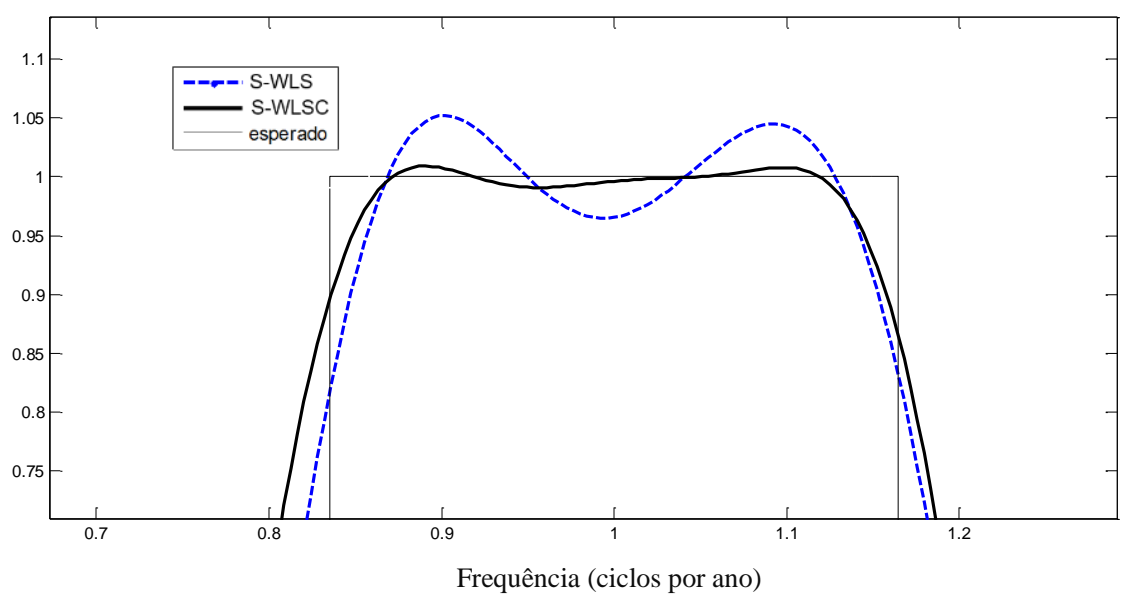

Gráfico 6.53 Banda passante dos filtros S-WLSC e S-WLS (N=43)

Em relação ao filtro de tamanho 65 (Gráfico 6.55), notam-se as bandas de transição sobrepostas, mas uma boa redução do ripple na banda passante do SWLSC. Nota-se também que a atenuação da banda de rejeição do filtro S-WLSC é 
menor. Entretanto, espera-se que o compromisso entre o erro de estimativa da sazonalidade (ripple na banda passante) e a atenuação da banda de rejeição seja melhor no filtro S-WLSC.

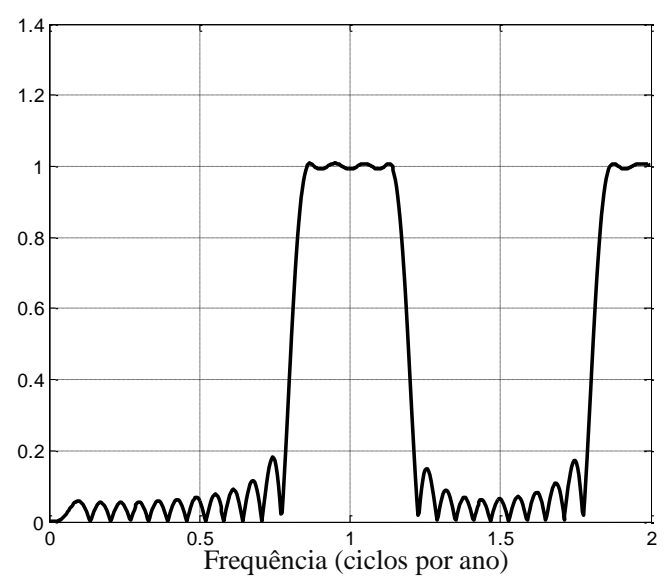

Gráfico 6.54 Resposta em magnitude do filtro $S$-WLSC $(N=65)$

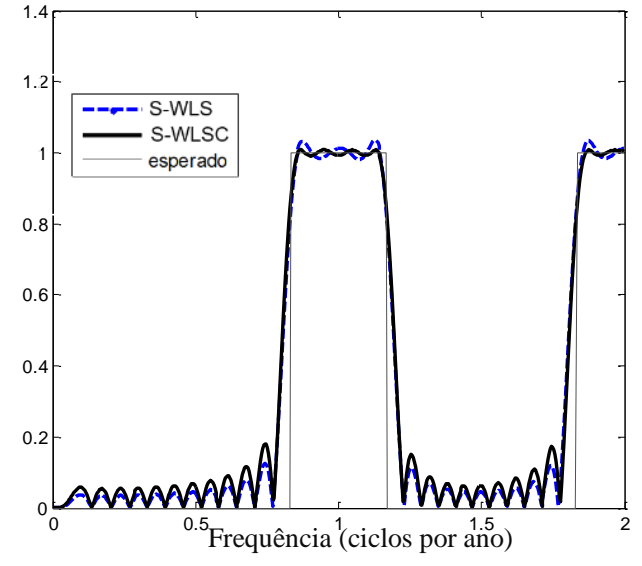

Gráfico 6.55 Resposta em magnitude do filtro S-WLSC, do S-WLS e o esperado $(\mathbf{N}=65)$

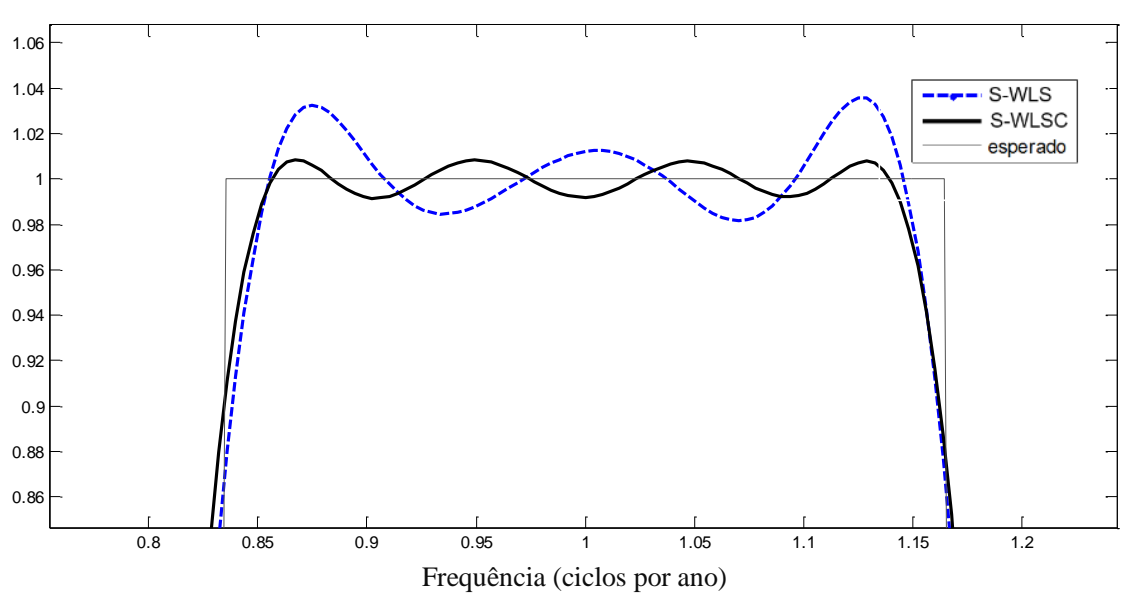

Gráfico 6.56 Banda passante dos filtros S-WLSC e S-WLS (N=65)

\subsubsection{3.}

Resultado: filtro S-WLSC trimestral $\operatorname{com} N=49$

Para $N=49$, o filtro escolhido foi:

$$
\alpha=\frac{1}{3}, \delta=\frac{1}{120}, w_{0}=1, \Gamma=0,02 .
$$


A resposta em magnitude do filtro S-WLSC, de tamanho 49, está apresentada a seguir, no Gráfico 6.57. Ao lado dela, está a comparação com a função do filtro S-WLS, juntamente com o filtro desejado (esperado). E, em seguida, no Gráfico 6.59 , tem-se uma ampliação da banda passante, mostrando um ripple menor do $\mathrm{S}$ WLSC, se comparado ao filtro S-WLS.

Nesse filtro de tamanho 49, observa-se que a atenuação na banda de rejeição do filtro S-WLSC é muito parecida com atenuação no filtro S-WLS. Nota-se, também, que a banda de transição dele é um pouco menor do que a do filtro S-WLS, o que é positivo para a SNR do S-WLSC, pois isso reduz a SQ.

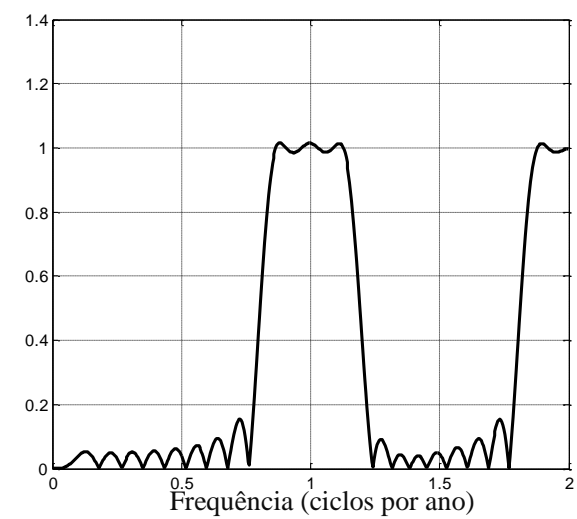

Gráfico 6.57 Resposta em magnitude do filtro S-WLSC $(\mathrm{N}=49)$

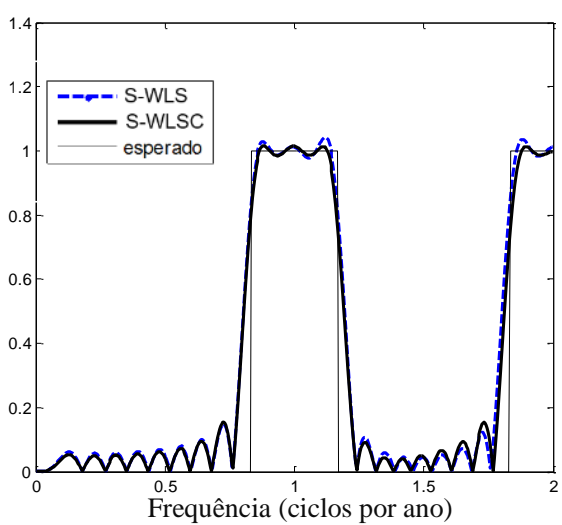

Gráfico 6.58 Resposta em magnitude do filtro S-WLSC, do S-WLS e o esperado $(\mathbf{N}=49)$

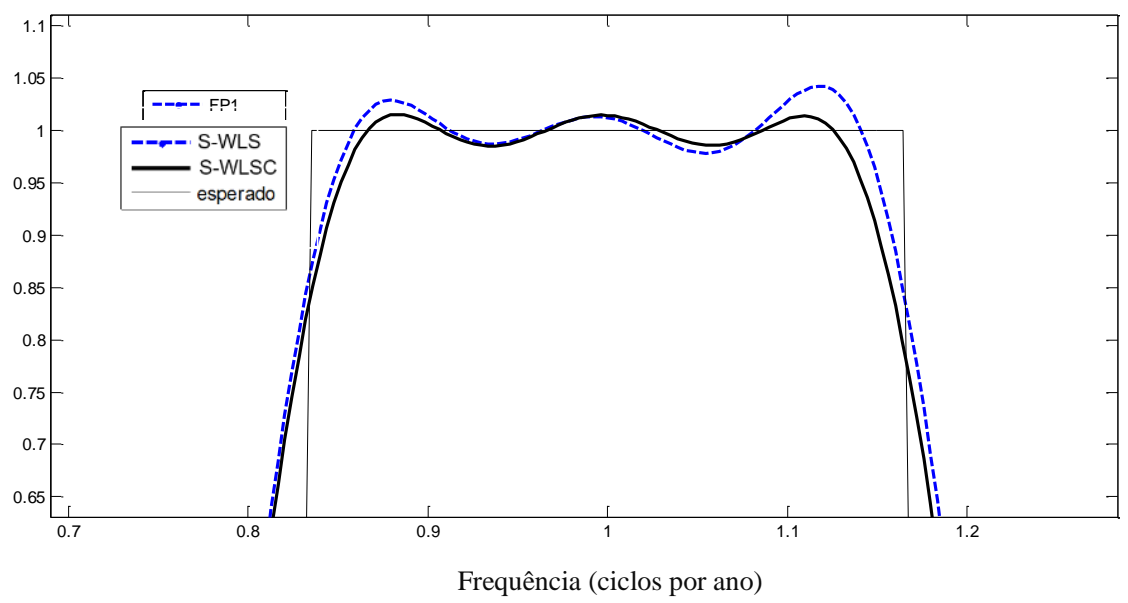

Gráfico 6.59 Banda passante dos filtros S-WLSC e S-WLS (N=49) 


\subsubsection{4.}

\section{Resultado: filtro S-WLSC trimestral $\operatorname{com} N=67$}

Para a seleção do filtro de tamanho 67, o filtro escolhido com base nos resultados da SNR foi:

$$
\alpha=\frac{1}{3}, \delta=\frac{1}{250}, w_{0}=1, \Gamma=0,01 .
$$

A resposta em magnitude do filtro S-WLSC, de tamanho 67, está apresentada no Gráfico 6.60. Ao lado dele, está a comparação com a função do filtro S-WLS, juntamente com o filtro desejado (esperado). E, em seguida, é ilustrada a banda passante, ampliada, mostrando o ripple no filtro S-WLSC menor do que o do filtro S-WLS.

Nota-se que a atenuação na banda de rejeição do filtro S-WLSC é menor do que a atenuação no filtro S-WLS, mas por outro lado o filtro S-WLSC possui menor ripple na banda passante. Essas características atuam na SNR da seguinte forma: a menor atenuação na banda de rejeição aumenta a $S Q$, o que é ruim para a SNR do filtro; mas um menor ripple na banda passante, diminui o valor de $\left|1-\gamma_{1}\right|$, contribuindo para uma SNR maior.

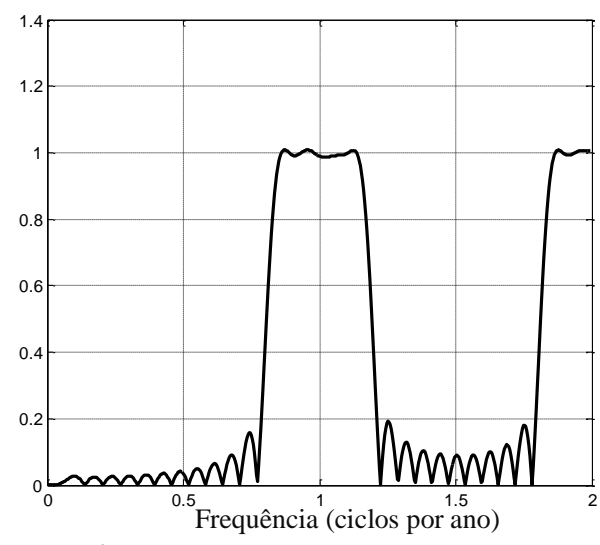

Gráfico 6.60 Resposta em magnitude do filtro $\mathrm{S}$-WLSC $(\mathrm{N}=67)$

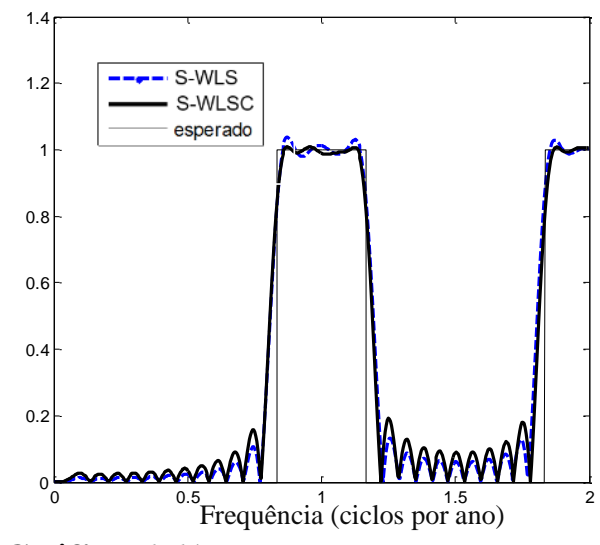

Gráfico 6.61 Resposta em magnitude do S-WLSC, do S-WLS e esperado $(\mathrm{N}=67)$

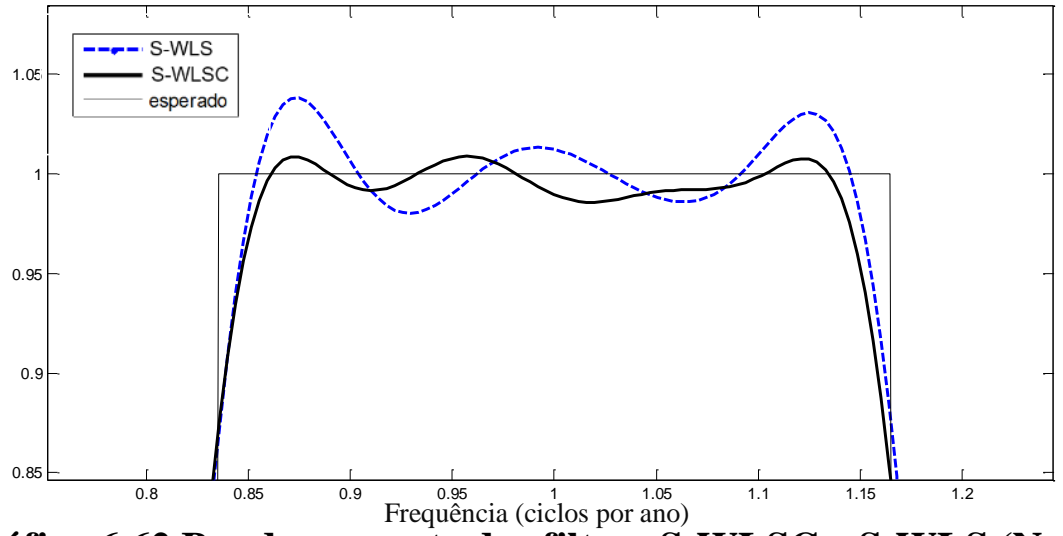

Gráfico 6.62 Banda passante dos filtros S-WLSC e S-WLS (N=67) 


\subsubsection{5.}

\section{Resultado: filtro S-WLSC trimestral $\operatorname{com} N=51$}

Para o filtro trimestral de tamanho 51, de todas as combinações testadas de valores para os parâmetros do filtro, nenhuma obteve bons resultados. A convergência do filtro foi difícil de ser atingida e quando acontecia, o resultado da SNR não era competitivo frente ao filtro S-WLS. Com isso, decidiu-se adotar o filtro de menor tamanho (49), na comparação com o filtro S-WLS de tamanho 51.

A resposta em magnitude do filtro S-WLSC de tamanho 49 está apresentada no Gráfico 6.63. Ao lado dela, está a comparação com a função do filtro S-WLS de tamanho 51, juntamente com o filtro desejado (esperado). E em seguida (Gráfico 6.65), é apresentada uma ampliação da banda passante, mostrando que o ripple é menor no filtro S-WLSC.

É interessante observar que, nesse caso, a atenuação na banda de rejeição do filtro S-WLSC é maior se comparada ao filtro S-WLS.

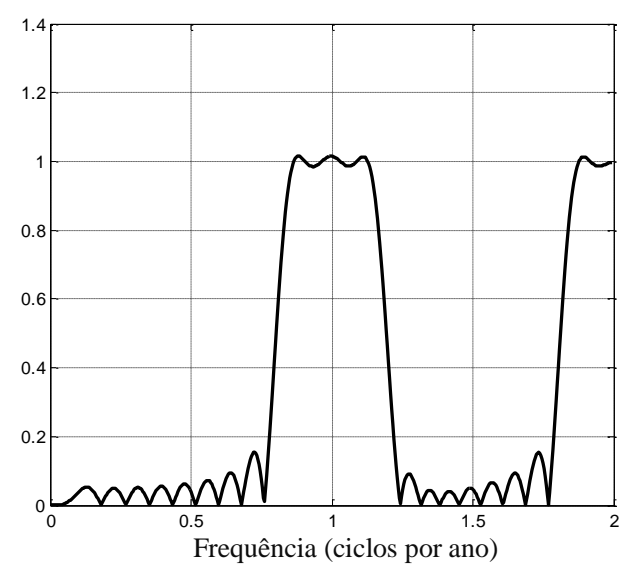

Gráfico 6.63 Resposta em magnitude do filtro $\mathrm{S}-\mathrm{WLSC}(\mathrm{N}=49)$

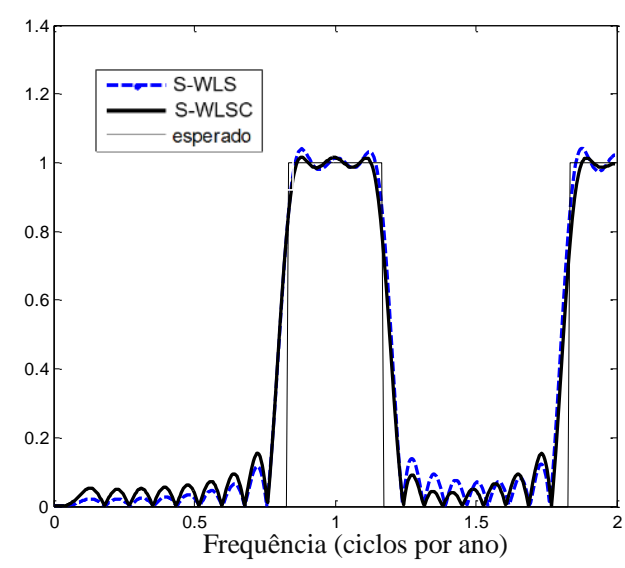

Gráfico 6.64 Resposta em magnitude do filtro S-WLSC $(\mathrm{N}=49)$, do S-WLS $(\mathrm{N}=51)$ e o esperado

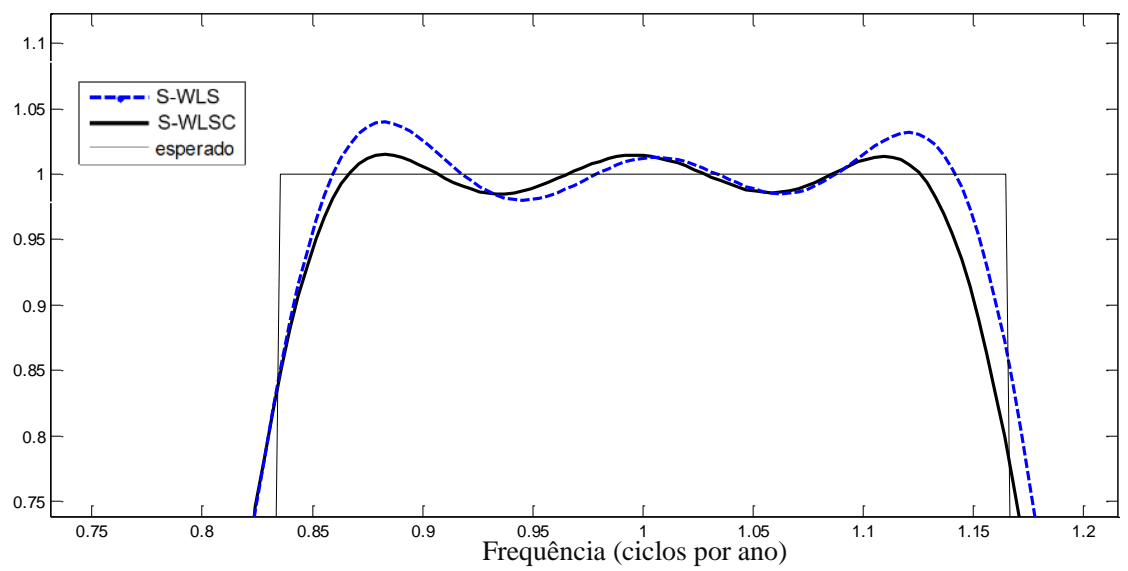

Gráfico 6.65 Banda passante dos filtros S-WLSC e S-WLS (N=51) 


\subsection{2.}

\section{Parâmetros do filtro S-WLSC para séries trimestrais}

A Tabela 6.11 apresenta os valores dos parâmetros selecionados na Subseção 6.5.1, para cada tamanho de filtro S-WLSC.

\begin{tabular}{c|c|c|c|c}
\hline $\mathbf{N}$ & $\boldsymbol{\alpha}$ & $\boldsymbol{\delta}$ & $\boldsymbol{\Gamma}$ & $\boldsymbol{w}_{\mathbf{0}}$ \\
\hline $\mathbf{4 1}$ & $1 / 3$ & $1 / 500$ & 0,006 & 2 \\
\hline $\mathbf{4 3}$ & $1 / 3$ & $1 / 500$ & 0,01 & 2 \\
\hline $\mathbf{6 5}$ & $1 / 3$ & $1 / 500$ & 0,01 & 2 \\
\hline $\mathbf{6 7}$ & $1 / 3$ & $1 / 250$ & 0,01 & 1 \\
\hline $\mathbf{4 9}$ & $1 / 3$ & $1 / 120$ & 0,02 & 1 \\
\hline $\mathbf{5 1} *$ & $1 / 3$ & $1 / 120$ & 0,02 & 1 \\
\hline
\end{tabular}

Tabela 6.11 Filtros S-WLSC trimestrais para cada $N$

Os valores relacionados às medidas necessárias para o cálculo da SNR, ou seja, $S Q, \gamma_{0}$ e $\left|1-\gamma_{1}\right|$ de cada filtro, estão apresentados na Tabela 6.12.

\begin{tabular}{c|c|c|c}
\hline $\mathbf{N}$ & $\boldsymbol{S Q}$ & $\boldsymbol{\gamma}_{\mathbf{0}}$ & $\left|\mathbf{1}-\boldsymbol{\gamma}_{\mathbf{1}}\right|$ \\
\hline $\mathbf{4 1}$ & 0,2919 & 1,0052 & 0,1296 \\
\hline $\mathbf{4 3}$ & 0,2849 & 0,9963 & 0,1626 \\
\hline $\mathbf{6 5}$ & 0,2718 & 0,9994 & 0,1658 \\
\hline $\mathbf{6 7}$ & 0,2675 & 0,9895 & 0,1781 \\
\hline $\mathbf{4 9}$ & 0,2644 & 1,0144 & 0,2331 \\
\hline $\mathbf{5 1}$ & 0,2644 & 1,0144 & 0,2331 \\
\hline
\end{tabular}

Tabela 6.12 Valores de SQ e $\gamma_{s}$ para os filtros S-WLSC trimestrais, para $k=24$

Para ilustrar a qualidade do ajuste sazonal realizado pelo filtro S-WLSC, foram gerados espectros da componente irregular, considerando séries com $k=24$ e $k=32$, para $A / s=6$ e $b=40 \%$, apresentados nos Gráficos 6.66 e 6.67. Observase que, tanto para $k=24$ quanto para $k=32$, o filtro S-WLSC realiza um ajuste sazonal adequado, pois não se notam picos nas frequências sazonais, ou próximos a elas. Nessa análise, foi utilizado o filtro de tamanho 43. 


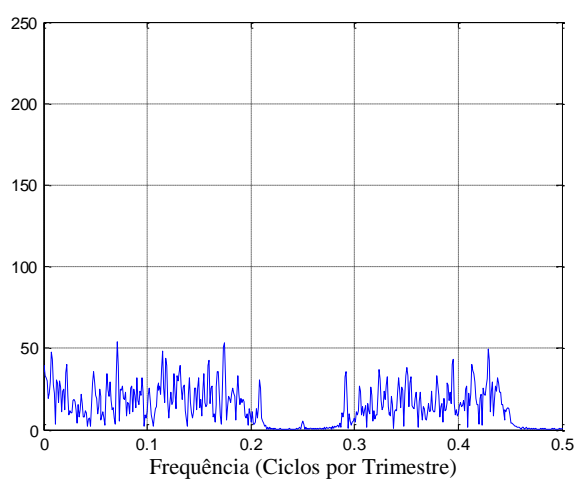

Gráfico 6.66 Espectro da irregular da série dessazonalizada pelo S-WLSC $(\mathrm{N}=43$ ), com: $A / s=6, k=24$ e $b=40 \%$ (Eq. 5-2)

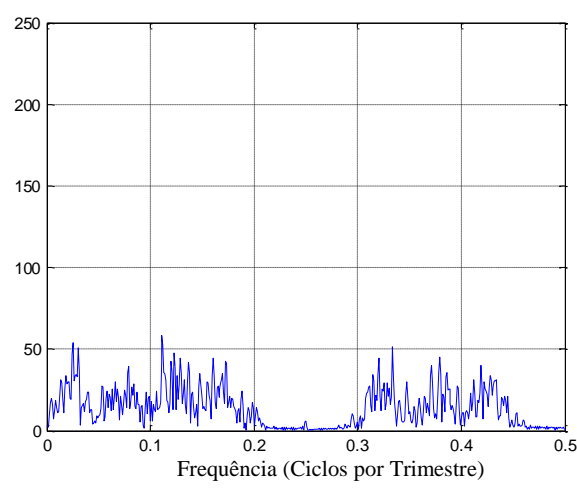

Gráfico 6.67 Espectro da irregular da série dessazonalizada pelo $S$ WLSC ( $\mathrm{N}=43$ ), com: $A / s=6, k=32 \mathrm{e}$ $b=40 \%($ Eq. 5-2)

\subsection{3.}

\section{Comparação do filtro S-WLSC com o filtro S-WLS, para séries trimestrais com decomposição aditiva}

O objetivo desta subseção é comparar a SNR do filtro S-WLSC com a SNR do filtro S-WLS, para identificar as situações em que o S-WLSC é superior ao filtro S-WLS e, nesses casos, mensurar o ganho ao se utilizar o filtro S-WLSC.

Nessa comparação, foram considerados os seguintes valores em relação às características sazonais das séries: $k=32, b=40 \%$ e $A / s=6$. A SNR dos filtros foi calculada, assim como a razão $\frac{\text { SNR S-WLSC }}{\text { SNR S-WLS }}$. Os valores estão apresentados na tabela a seguir.

\begin{tabular}{c|c|c|c}
\hline $\mathbf{N}$ & $\begin{array}{c}\text { SNR } \\
\text { S-WLSC }\end{array}$ & $\begin{array}{c}\text { SNR } \\
\text { S-WLS }\end{array}$ & $\begin{array}{c}\text { SNR S-WLSC } \\
\text { SNR S-WLS }\end{array}$ \\
\hline 41 & 66,5 & 63,8 & 1,042 \\
43 & 68,1 & 63,7 & 1,069 \\
49 & 72,4 & 69,1 & 1,048 \\
51 & 72,4 & 69,3 & 1,045 \\
65 & 71,5 & 69,9 & 1,022 \\
67 & 72,1 & 69,9 & 1,031 \\
\hline
\end{tabular}

Tabela 6.13 SNRs e razão entre as SNRs, para $A / s=6, b=40 \% k=32$ (Eq. 52), considerando todos os filtros trimestrais: comparação entre S-WLSC e SWLS

Na Tabela 6.13, observa-se que o melhor tamanho de filtro para o S-WLSC, em comparação com o filtro S-WLS, é o 43, por possuir maior razão entre as SNRs. Já quando $N=65$, a razão entre as SNRs é a menor de todas, indicando ser esse o 
melhor caso para o filtro S-WLS, em relação ao S-WLSC. Em razão disso, nas comparações futuras, serão usados apenas os tamanhos de filtro 43 e 65 .

O Gráfico 6.68, a seguir, apresenta a razão entre as SNRs dos filtros S-WLSC e S-WLS de tamanho 43 e 65, para diferentes valores da taxa de variação da amplitude sazonal $(b)$. Quanto maior é o valor de ' $b$ ' mais alta é a razão entre a SNR do S-WLSC e a SNR do S-WLS, significando uma melhor adequação do SWLSC, tanto para $N=43$, quanto para $N=65$.

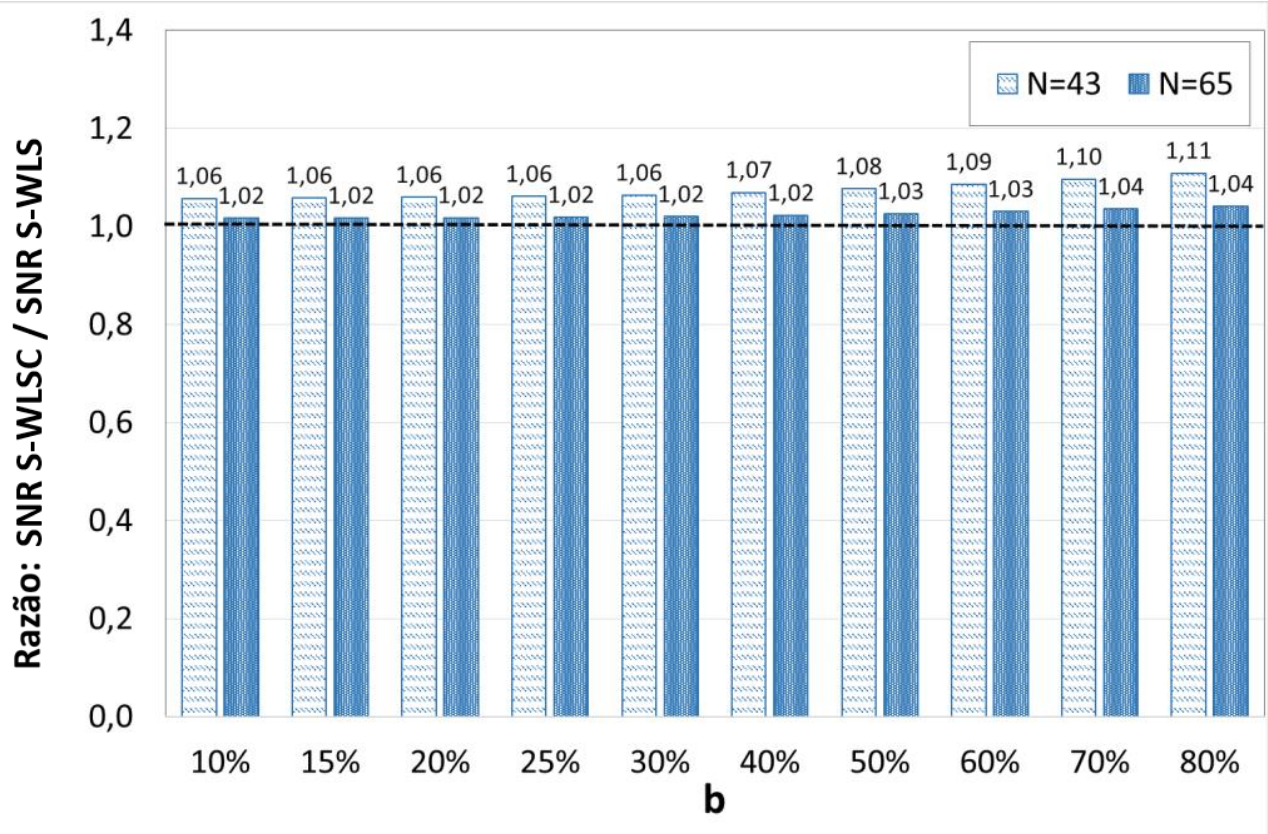

Gráfico 6.68 Razão entre a SNR S-WLSC e a SNR S-WLS considerando $A / s$ $=6$ e $k=32$, para diversos valores de $b$ : comparação entre $N=43$ e $N=65$

Os Gráficos 6.69 e 6.70 mostram as SNRs dos filtros S-WLSC e S-WLS, para cada valor de ' $b$ ', considerando os tamanhos 43 e 65, separadamente. Nota-se que, à medida que ' $b$ ' aumenta, o distanciamento entre as SNRs dos filtros de tamanho 43 é mais acentuado, se comparado aos filtros de tamanho 65 .

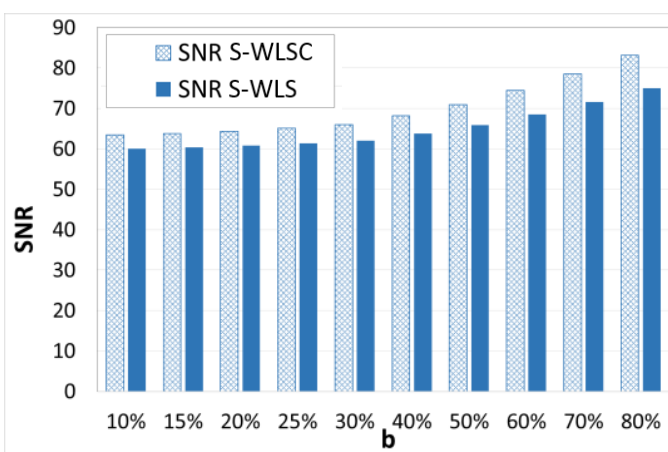

Gráfico 6.69 SNR S-WLSC e SNR SWLS considerando $A / s=6$ e $k=32$, para diversos valores de $b(\mathrm{~N}=43)$

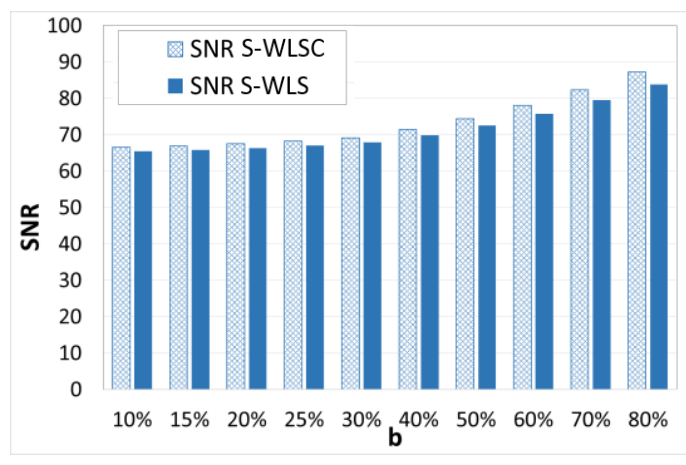

Gráfico 6.70 SNR S-WLSC e SNR SWLS considerando $A / s=6$ e $k=32$, para diversos valores de $b(\mathrm{~N}=65)$ 
Os valores apresentados nos Gráficos 6.69 e 6.70 estão dispostos na Tabela 6.14:

\begin{tabular}{c|ccc|ccc}
\hline & \multicolumn{3}{|c|}{$\boldsymbol{N = 4 3}$} & \multicolumn{3}{c}{$\boldsymbol{N = 6 5}$} \\
\cline { 2 - 7 } $\boldsymbol{b}$ & $\begin{array}{c}\text { SNR } \\
\text { S-WLSC }\end{array}$ & $\begin{array}{c}\text { SNR } \\
\text { S-WLS }\end{array}$ & $\begin{array}{c}\text { SNR } \\
\text { S-WLSC / } \\
\text { SNR } \\
\text { S-WLS }\end{array}$ & $\begin{array}{c}\text { SNR } \\
\text { S-WLSC }\end{array}$ & $\begin{array}{c}\text { SNR S- } \\
\text { WLS }\end{array}$ & $\begin{array}{c}\text { SNR } \\
\text { S-WLSC / } \\
\text { SNR } \\
\text { S-WLS }\end{array}$ \\
\hline $\mathbf{1 0 \%}$ & 63,4 & 60,0 & 1,057 & 66,6 & 65,5 & 1,017 \\
$\mathbf{1 5 \%}$ & 63,8 & 60,3 & 1,058 & 67,0 & 65,8 & 1,017 \\
$\mathbf{2 0 \%}$ & 64,4 & 60,8 & 1,059 & 67,5 & 66,4 & 1,018 \\
$\mathbf{2 5 \%}$ & 65,1 & 61,3 & 1,061 & 68,3 & 67,0 & 1,019 \\
$\mathbf{3 0 \%}$ & 65,9 & 62,0 & 1,063 & 69,2 & 67,9 & 1,020 \\
$\mathbf{4 0 \%}$ & 68,1 & 63,7 & 1,069 & 71,5 & 69,9 & 1,022 \\
$\mathbf{5 0 \%}$ & 71,0 & 65,9 & 1,077 & 74,4 & 72,6 & 1,026 \\
$\mathbf{6 0 \%}$ & 74,4 & 68,5 & 1,086 & 78,0 & 75,8 & 1,030 \\
$\mathbf{7 0 \%}$ & 78,5 & 71,6 & 1,096 & 82,3 & 79,5 & 1,035 \\
$\mathbf{8 0 \%}$ & 83,2 & 75,0 & 1,108 & 87,2 & 83,8 & 1,041 \\
\hline
\end{tabular}

Tabela 6.14 SNR S-WLSC e SNR S-WLS, variando $b$ para $A / s=6, k=32$ e $N=$ 43 e 65

O Gráfico 6.71, a seguir, apresenta a razão entre as SNRs dos filtros S-WLSC e S-WLS, de tamanho 43 e 65 , para diferentes valores da taxa de ' $k$ '. Vale lembrar que o parâmetro ' $k$ ' indica o tempo (em trimestres) necessário para o padrão de sazonalidade móvel se repetir. Diferente do que ocorre com a variação de ' $b$ ', à medida que ' $k$ ' aumenta, a razão entre as SNRs se mantém constante.

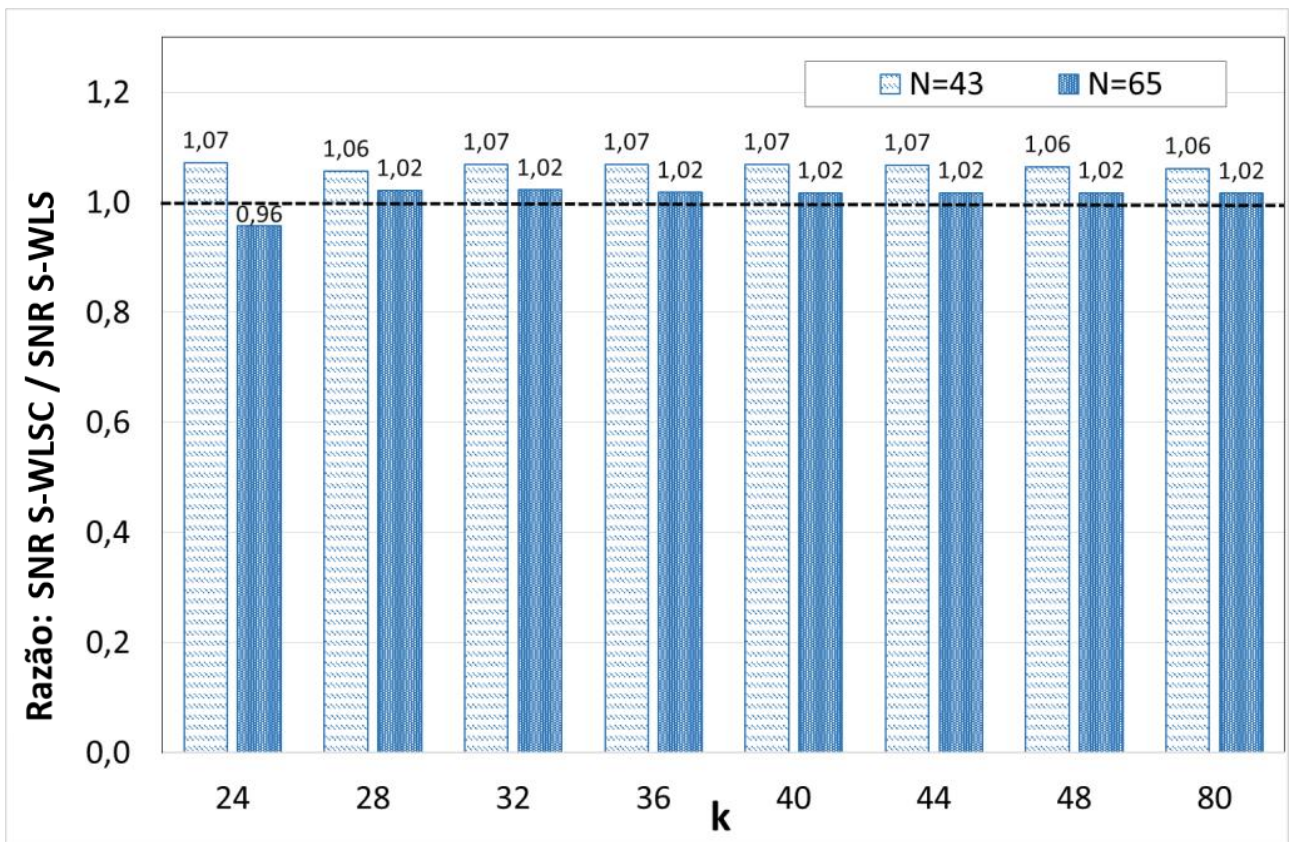

Gráfico 6.71 Razão entre a SNR do S-WLSC e a SNR S-WLS considerando $A / s=6$ e $b=40 \%$, para diversos valores de $k$ : comparação entre $N=43$ e $N=65$ 
Os Gráficos 6.72 e 6.73 mostram as SNRs individuais (dos filtros S-WLSC e S-WLS), com a variação de ' $k$ ', para cada $N$, separadamente. E a Tabela 6.15 apresenta os valores usados para a construção desses gráficos.

Observando os resultados, nota-se que, para $k \geq 32$, a SNR do filtro S-WLSC permanece constante. Além disso, vale citar que o único caso em que o filtro $S$ WLSC apresenta SNR menor do que o filtro S-WLS, ocorre quando $k=24$, no filtro de tamanho 65. Como o filtro foi definido $\operatorname{com} \alpha=1 / 3$, o que equivale a $k=24$, ele pode apresentar instabilidade no ajuste de séries com esse valor de $k$.

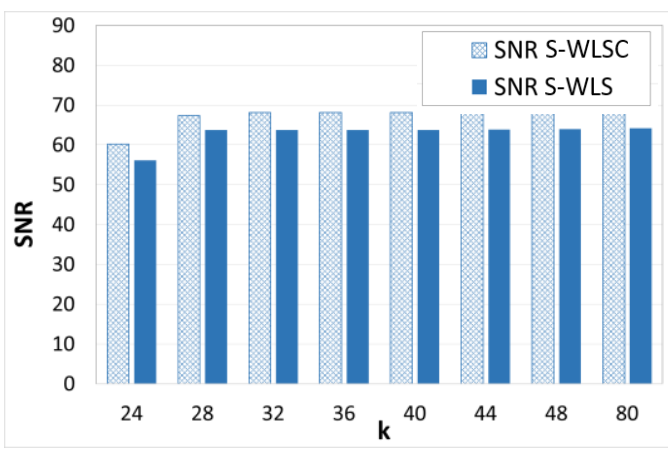

Gráfico 6.72 SNR S-WLSC e SNR SWLS considerando $A / s=6$ e $b=40 \%$, para diversos valores de $k(\mathrm{~N}=43)$

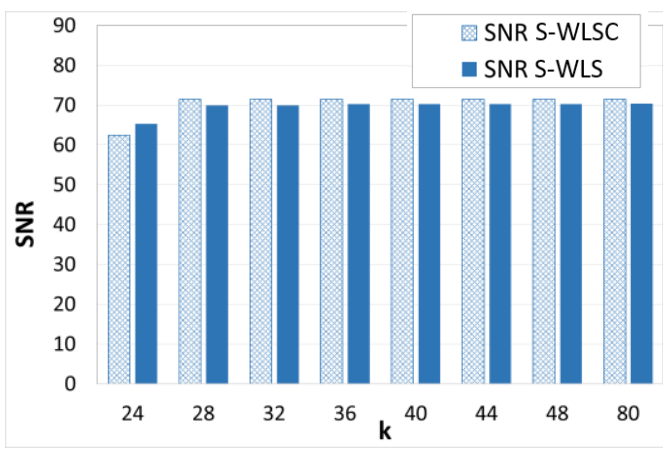

Gráfico 6.73 SNR S-WLSC e SNR S-WLS considerando $A / s=6$ e $b=$ $40 \%$, para diversos valores de $\boldsymbol{k}(\mathrm{N}=\mathbf{6 5})$

\begin{tabular}{c|ccc|ccc}
\hline & \multicolumn{3}{|c|}{$\boldsymbol{N = 4 3}$} & \multicolumn{3}{c}{$\boldsymbol{N = 6 5}$} \\
\cline { 2 - 7 } $\boldsymbol{k}$ & $\begin{array}{c}\text { SNR } \\
\text { S-WLSC }\end{array}$ & $\begin{array}{c}\text { SNR } \\
\text { S-WLS }\end{array}$ & $\begin{array}{c}\text { SNR } \\
\text { S-WLSC } / \\
\text { SNR } \\
\text { S-WLS }\end{array}$ & $\begin{array}{c}\text { SNR } \\
\text { S-WLSC }\end{array}$ & $\begin{array}{c}\text { SNR } \\
\text { S-WLS }\end{array}$ & $\begin{array}{c}\text { SNR } \\
\text { S-WLSC } / \\
\text { SNR } \\
\text { S-WLS }\end{array}$ \\
\hline $\mathbf{2 4}$ & 60,1 & 56,1 & 1,072 & 62,4 & 65,3 & 0,957 \\
$\mathbf{2 8}$ & 67,3 & 63,7 & 1,057 & 71,4 & 69,9 & 1,022 \\
$\mathbf{3 2}$ & 68,1 & 63,7 & 1,069 & 71,5 & 69,9 & 1,022 \\
$\mathbf{3 6}$ & 68,1 & 63,7 & 1,069 & 71,5 & 70,2 & 1,018 \\
$\mathbf{4 0}$ & 68,1 & 63,7 & 1,069 & 71,5 & 70,3 & 1,017 \\
$\mathbf{4 4}$ & 68,1 & 63,8 & 1,068 & 71,5 & 70,3 & 1,017 \\
$\mathbf{4 8}$ & 68,1 & 64,0 & 1,065 & 71,5 & 70,3 & 1,017 \\
$\mathbf{8 0}$ & 68,1 & 64,2 & 1,062 & 71,5 & 70,3 & 1,016 \\
\hline
\end{tabular}

Tabela 6.15 SNR S-WLSC e SNR S-WLS, variando $\mathrm{k}$ para $A / s=6, b=40 \%$ e $N=43$ e 65 
Em relação à razão entre as SNRs dos filtros S-WLSC e S-WLS, para diferentes valores da razão ' $A / s$ ', é apresentado o Gráfico 6.74, com os resultados encontrados.

A razão ' $A / s$ ' indica a amplitude do sinal sazonal em relação ao ruído. Notase que se ' $A / s$ ' apresentar valor baixo (1 ou 2), a SNR do S-WLSC é menor do que a do filtro S-WLS, para $N=43$. Isso ocorre porque a atenuação na banda de rejeição do S-WLSC é menor do que no filtro S-WLS. A partir de $A / s=3$, a SNR do SWLSC supera a SNR do filtro S-WLS para os dois valores de $N$ testados. Além disso, observa-se que quanto maior for a razão ' $A / s$ ', mais adequado é o ajuste sazonal realizado pelo S-WLSC se comparado ao ajuste do filtro S-WLS.

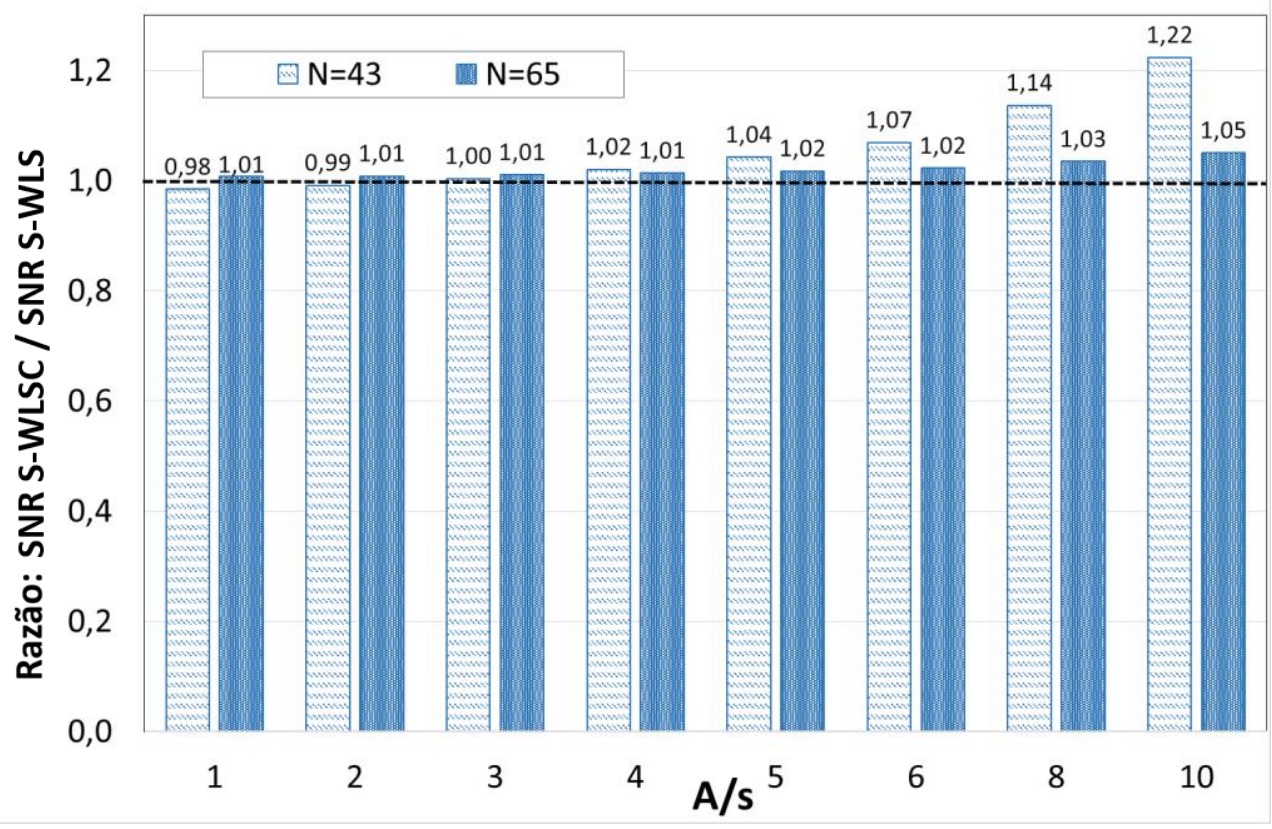

Gráfico 6.74 Razão entre a SNR do S-WLSC e a do S-WLS considerando $k=32, b=40 \%$, para diversos valores de $A / s$ : comparação entre $N=43$ e $N=65$

Os Gráficos 6.75 e 6.76 mostram as SNRs individuais dos filtros segundo os valores de ' $A / s$ ', considerando $N=43$ e $N=65$, respectivamente.

Nesses gráficos, nota-se o distanciamento entre as SNRs dos filtros à medida que a razão ' $A / s$ ' aumenta. Para $N=43$ (Gráfico 6.75), o distanciamento entre as SNRs é mais acentuado, se comparado aos filtros de tamanho 65. 


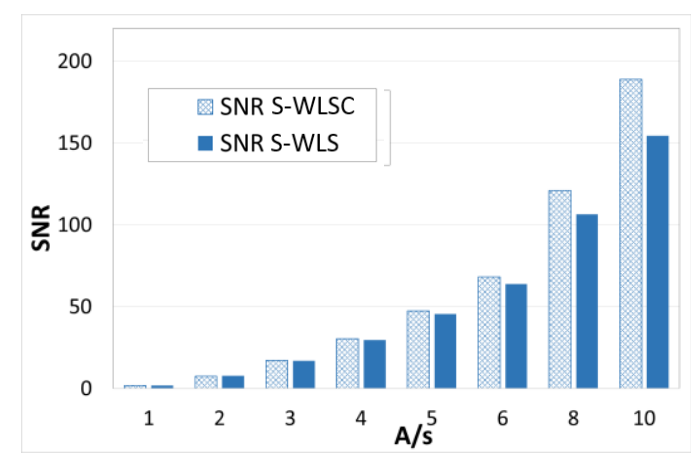

Gráfico 6.75 SNR S-WLSC e SNR

S-WLS considerando $k=32, b=40 \%$, para diversos valores de $A / s(\mathrm{~N}=43)$

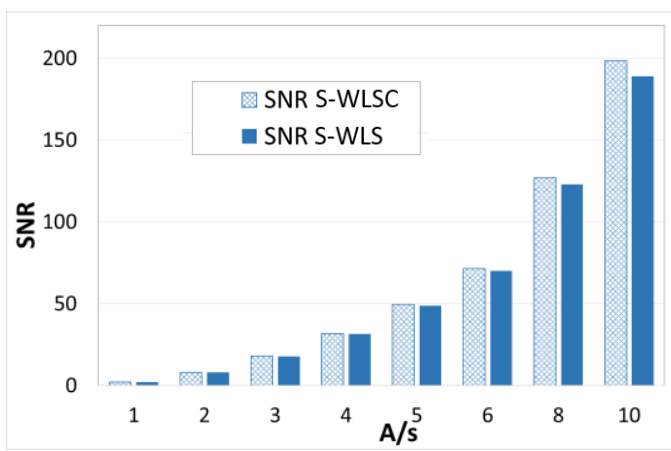

Gráfico 6.76 SNR S-WLSC e SNR SWLS considerando $k=32, b=40 \%$, para diversos valores de $A / s(\mathrm{~N}=65)$

A tabela a seguir, apresenta os valores usados para a construção dos Gráficos 6.74 a $6.76:$

\begin{tabular}{c|ccc|ccc}
\hline & \multicolumn{3}{|c|}{$\boldsymbol{N = 4 3}$} & \multicolumn{3}{c}{$\boldsymbol{N = 6 5}$} \\
\cline { 2 - 7 } $\boldsymbol{A} / \boldsymbol{s}$ & $\begin{array}{c}\text { SNR } \\
\text { S-WLSC }\end{array}$ & $\begin{array}{c}\text { SNR } \\
\text { S-WLS }\end{array}$ & $\begin{array}{c}\text { SNR } \\
\text { S-WLSC } / \\
\text { SNR } \\
\text { S-WLS }\end{array}$ & $\begin{array}{c}\text { SNR } \\
\text { S-WLSC }\end{array}$ & $\begin{array}{c}\text { SNR } \\
\text { S-WLS }\end{array}$ & $\begin{array}{c}\text { S-WLSC } \\
\text { SNR } \\
\text { S-WLS }\end{array}$ \\
\hline $\mathbf{1}$ & 1,9 & 1,9 & 0,984 & 2,0 & 2,0 & 1,007 \\
$\mathbf{2}$ & 7,6 & 7,6 & 0,992 & 7,9 & 7,9 & 1,008 \\
$\mathbf{3}$ & 17,1 & 17,0 & 1,004 & 17,9 & 17,7 & 1,011 \\
$\mathbf{4}$ & 30,3 & 29,7 & 1,021 & 31,8 & 31,4 & 1,014 \\
$\mathbf{5}$ & 47,3 & 45,4 & 1,043 & 49,6 & 48,8 & 1,018 \\
$\mathbf{6}$ & 68,1 & 63,7 & 1,069 & 71,5 & 69,9 & 1,022 \\
$\mathbf{8}$ & 121,0 & 106,5 & 1,137 & 127,0 & 122,8 & 1,035 \\
$\mathbf{1 0}$ & 188,8 & 154,3 & 1,224 & 198,4 & 188,8 & 1,050 \\
\hline
\end{tabular}

Tabela 6.16 SNR S-WLSC e SNR S-WLS, variando $A / s$, para $k=32, b=40 \%$ e $N=43$ e 65 


\section{6.}

\section{Série trimestral multiplicativa - filtro S-WLSC vs filtro S-WLS}

Com o filtro trimestral já definido, testou-se o desempenho do S-WLSC em relação ao filtro S-WLS para as séries trimestrais com decomposição multiplicativa. Nesta subseção são comparados os resultados, utilizando-se a estatística MSE.

Nos testes, foram utilizados os filtros de tamanho 43 e 65 . Como padrão para a componente de tendência, utilizou-se a série Y91; e para os coeficientes da componente sazonal artificial, foi usado o padrão da série Y99.

Para a componente irregular, foram geradas 100 séries, com distribuição $\mathrm{N}\left(0, \sigma^{2}\right)$, com base no desvio-padrão da irregular das séries reais. Com isso, calculou-se a média e o desvio-padrão da MSE.

Os valores utilizados foram os seguintes:

\begin{tabular}{c|c|c|c|c|c}
\hline $\boldsymbol{A}$ & $\boldsymbol{s}$ & $\mathbf{b}_{\mathbf{0}}$ & $\mathbf{b}_{\mathbf{1}}$ & $\mathbf{b}_{\mathbf{2}}$ & $\mathbf{b}_{\mathbf{3}}$ \\
\hline 0,03 & 0,0046 & 1504 & $-23,41$ & 3,8097 & 0,004 \\
\hline
\end{tabular}

Tabela 6.17 Valores da componente de tendência, desvio-padrão da irregular e amplitude da componente sazonal

Os Gráficos 6.77 e 6.78 ilustram a MSE do filtro S-WLSC e do filtro S-WLS para diversos valores de ' $b$ ', quando $N=43$ e $N=65$, respectivamente. E a Tabela 6.18 apresenta os valores da MSE de cada filtro, para os valores de ' $b$ ', utilizados nos gráficos.

Analisando os gráficos e a tabela, nota-se uma melhora significativa na MSE do filtro S-WLSC, em comparação com o filtro S-WLS, para o caso em que $N=$ 43. Já para o caso em que $N=65$, o S-WLSC só é significativamente melhor quando $b \geq 40 \%$.

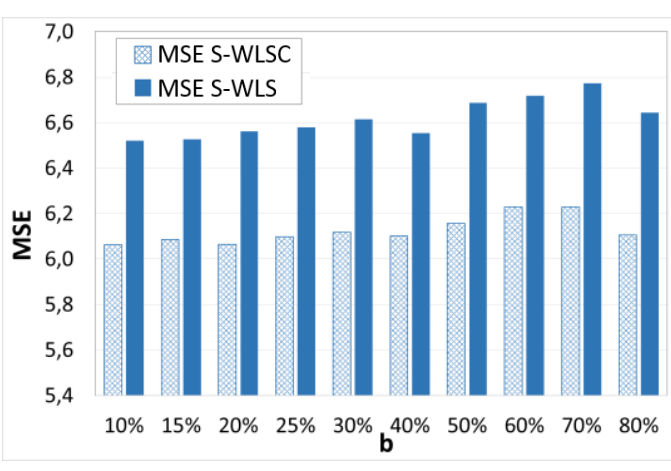

Gráfico 6.77 MSE S-WLSC e MSE S-WLS $\left(\mathrm{x10}^{6}\right)$ considerando $k=32$, $A / s=6$, para diversos valores de $b: N$ $=43$

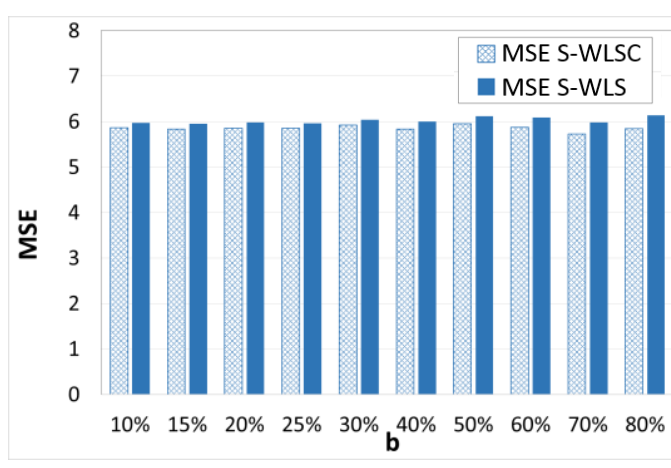

Gráfico 6.78 MSE S-WLSC e MSE S-WLS $\left(\mathrm{x10}^{6}\right)$ considerando $k=32$, $A / s=6$, para diversos valores de $b$ : $N=65$ 


\begin{tabular}{c|ccl|ccc}
\hline \multirow{2}{*}{$\boldsymbol{b}$} & \multicolumn{3}{|c|}{$\boldsymbol{N = 4 3}$} & \multicolumn{3}{c}{$\boldsymbol{N = 6 5}$} \\
\cline { 2 - 7 } & $\begin{array}{c}\text { MSE } \\
\text { S-WLSC }\end{array}$ & $\begin{array}{c}\text { MSE } \\
\text { S-WLS }\end{array}$ & valor-p & $\begin{array}{c}\text { MSE } \\
\text { s-WLSC }\end{array}$ & $\begin{array}{c}\text { MSE } \\
\text { S-WLS }\end{array}$ & valor-p \\
\hline $\mathbf{1 0 \%}$ & 6,06 & 6,52 & $0,000^{*}$ & 5,86 & 5,97 & 0,143 \\
$\mathbf{1 5 \%}$ & 6,09 & 6,53 & $0,000^{*}$ & 5,84 & 5,95 & 0,146 \\
$\mathbf{2 0 \%}$ & 6,06 & 6,56 & $0,000^{*}$ & 5,85 & 5,98 & 0,108 \\
$\mathbf{2 5 \%}$ & 6,10 & 6,58 & $0,000^{*}$ & 5,85 & 5,96 & 0,166 \\
$\mathbf{3 0 \%}$ & 6,12 & 6,61 & $0,000^{*}$ & 5,92 & 6,04 & 0,138 \\
$\mathbf{4 0 \%}$ & 6,10 & 6,55 & $0,000^{*}$ & 5,84 & 6,00 & $0,044^{* *}$ \\
$\mathbf{5 0 \%}$ & 6,16 & 6,69 & $0,000^{*}$ & 5,95 & 6,11 & 0,065 \\
$\mathbf{6 0 \%}$ & 6,23 & 6,72 & $0,000^{*}$ & 5,87 & 6,09 & $0,012^{* *}$ \\
$\mathbf{7 0 \%}$ & 6,23 & 6,77 & $0,000^{*}$ & 5,73 & 5,98 & $0,011^{* *}$ \\
$\mathbf{8 0 \%}$ & 6,11 & 6,64 & $0,000^{*}$ & 5,85 & 6,14 & $0,003^{*}$ \\
\hline Nota: ( $\left.^{*}\right)$ valor-p $\left.<1 \% ;{ }^{* *}\right)$ valor-p $<5 \%$. & \multicolumn{3}{|c}{} \\
\hline
\end{tabular}

Tabela 6.18 SNR S-WLSC e SNR S-WLS, $\left(\mathrm{x}^{10^{6}}\right)$, variando ' $b$ ' $\operatorname{para} A / s=6$, $k=32(N=43$ e 65) 
A MSE dos filtros, segundo os diversos valores de ' $k$ ', está ilustrada nos Gráficos 6.79 e 6.80, para os tamanhos 43 e 65, e na Tabela 6.19. Nota-se que, considerando $N=43$, o S-WLSC é novamente melhor em todos os casos, ou seja, para todos os valores de ' $k$ '. No entanto, quando $N=65$, o S-WLSC não é significativamente superior ao filtro S-WLS em nenhum dos casos.

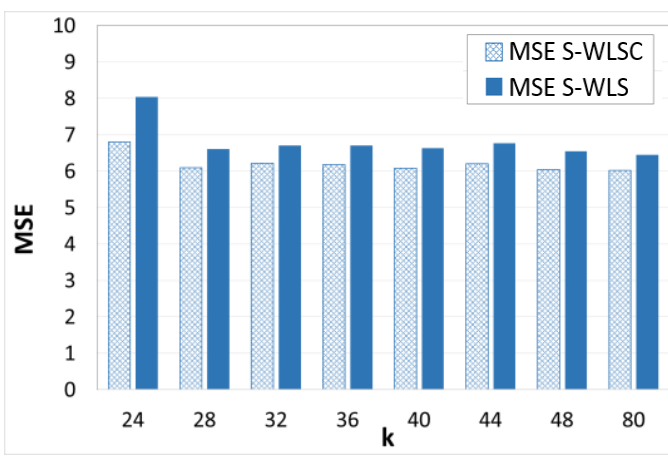

Gráfico 6.79 MSE S-WLSC e MSE S-WLS $\left(\mathrm{x10}^{6}\right)$ considerando $A / s=6,5$ e $b=40 \%$, para diversos valores de $k$ $(\mathrm{N}=43)$

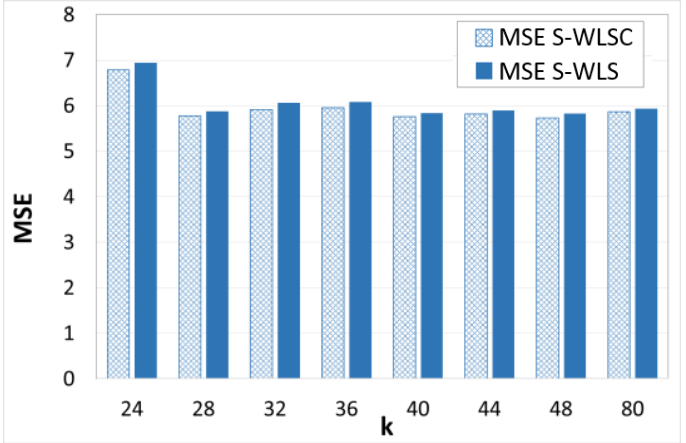

Gráfico 6.80 MSE S-WLSC e MSE S-WLS $\left(\mathrm{x}^{6} 0^{6}\right)$ considerando $A / s=6,5$ e $b=40 \%$, para diversos valores de $k(\mathrm{~N}=65)$

\begin{tabular}{c|ccl|ccc}
\hline \multirow{2}{*}{$\boldsymbol{k}$} & \multicolumn{3}{|c|}{$\mathbf{N = 4 3}$} & \multicolumn{3}{c}{$\boldsymbol{N = 6 5}$} \\
\cline { 2 - 7 } & $\begin{array}{c}\text { MSE } \\
\text { S-WLSC }\end{array}$ & $\begin{array}{c}\text { MSE } \\
\text { S-WLS }\end{array}$ & valor-p & $\begin{array}{c}\text { MSE } \\
\text { S-WLSC }\end{array}$ & $\begin{array}{c}\text { MSE } \\
\text { S-WLS }\end{array}$ & \multirow{2}{*}{ valor-p } \\
\hline $\mathbf{1 6}$ & 35,6 & 37,9 & $0,000^{*}$ & 56,8 & 51,4 & 1,000 \\
$\mathbf{2 0}$ & 11,6 & 15,0 & $0,000^{*}$ & 20,1 & 19,8 & 0,990 \\
$\mathbf{2 4}$ & 6,8 & 8,0 & $0,000^{*}$ & 6,8 & 6,9 & 0,085 \\
$\mathbf{2 8}$ & 6,1 & 6,6 & $0,000^{*}$ & 5,8 & 5,9 & 0,171 \\
$\mathbf{3 2}$ & 6,2 & 6,7 & $0,000^{*}$ & 5,9 & 6,1 & 0,087 \\
$\mathbf{3 6}$ & 6,2 & 6,7 & $0,000^{*}$ & 5,9 & 6,1 & 0,116 \\
$\mathbf{4 0}$ & 6,1 & 6,6 & $0,000^{*}$ & 5,8 & 5,8 & 0,237 \\
$\mathbf{4 4}$ & 6,2 & 6,8 & $0,000^{*}$ & 5,8 & 5,9 & 0,191 \\
$\mathbf{4 8}$ & 6,1 & 6,5 & $0,000^{*}$ & 5,7 & 5,8 & 0,163 \\
$\mathbf{8 0}$ & 6,0 & 6,4 & $0,000^{*}$ & 5,9 & 5,9 & 0,242 \\
\hline
\end{tabular}

Nota: $\left({ }^{*}\right)$ valor-p $<1 \%$; $\left.{ }^{* *}\right)$ valor-p $<5 \%$.

Tabela 6.19 MSE do S-WLSC e MSE do S-WLS, $\left(\mathrm{x}^{10}{ }^{6}\right)$, variando ' $k$ ' para $A / s$ $=6, b=40 \%(N=43$ e 65$)$ 
Quanto aos valores da razão ' $A / s$ ', os Gráficos 6.81 e 6.82 e a Tabela 6.20 mostram a MSE do S-WLSC e do filtro S-WLS, para $N=43$ e $N=65$, respectivamente. Com base nisso, pode-se observar que, em se tratando de $N=43$, o S-WLSC é significativamente superior ao filtro S-WLS para $A / s \geq 5$; enquanto que para o filtro de tamanho 65 , isso ocorre somente quando $A / s \geq 8$.

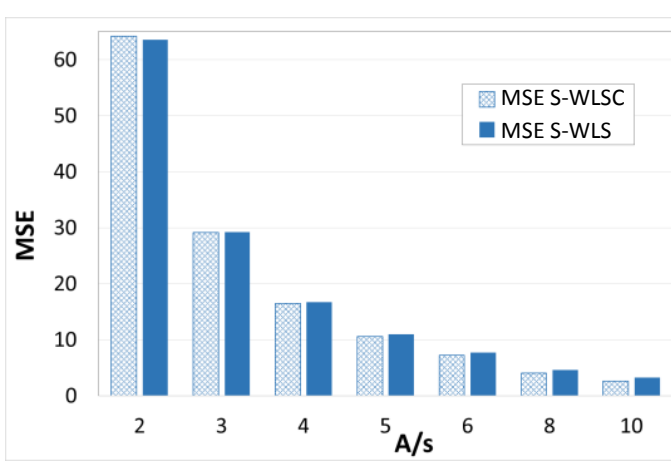

Gráfico 6.81 MSE S-WLSC e MSE S-WLS $\left(\mathrm{x}^{10}{ }^{6}\right)$ considerando $k=32$, $b=40 \%$, para diversos valores de $A / s(\mathrm{~N}=43)$

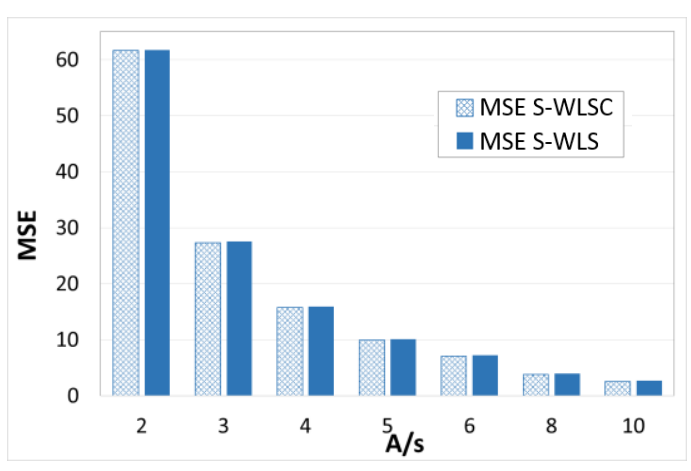

Gráfico 6.82 MSE S-WLSC e MSE S-WLS $\left(\mathrm{x} 10^{6}\right)$ considerando $k=32$, $b=40 \%$, para diversos valores de $A / s(\mathbf{N}=65)$

A tabela a seguir apresenta os valores da MSE de cada filtro, para os diversos valores de ' $A / s$ ', utilizados nos Gráficos 6.81 e 6.82.

\begin{tabular}{c|ccc|ccc}
\hline \multirow{2}{*}{$\boldsymbol{A} / \boldsymbol{s}$} & \multicolumn{3}{|c|}{$\boldsymbol{N = 4 3}$} & \multicolumn{3}{c}{$\boldsymbol{N = 6 5}$} \\
\cline { 2 - 7 } & $\begin{array}{c}\text { MSE } \\
\text { S-WLSC }\end{array}$ & $\begin{array}{c}\text { MSE } \\
\text { S-WLS }\end{array}$ & valor-p & MSE & MSE & valor-p \\
\hline $\mathbf{1}$ & 261,49 & 256,50 & 0,896 & 251,59 & 252,46 & 0,417 \\
$\mathbf{2}$ & 64,16 & 63,57 & 0,708 & 61,61 & 61,68 & 0,471 \\
$\mathbf{3}$ & 29,16 & 29,24 & 0,436 & 27,28 & 27,51 & 0,290 \\
$\mathbf{4}$ & 16,47 & 16,72 & 0,186 & 15,74 & 15,92 & 0,238 \\
$\mathbf{5}$ & 10,59 & 10,96 & $0,027^{* *}$ & 9,94 & 10,08 & 0,204 \\
$\mathbf{6}$ & 7,20 & 7,66 & $0,000^{*}$ & 7,10 & 7,26 & 0,116 \\
$\mathbf{8}$ & 4,04 & 4,56 & $0,000^{*}$ & 3,83 & 3,97 & $0,042^{* *}$ \\
$\mathbf{1 0}$ & 2,63 & 3,23 & $0,000^{*}$ & 2,51 & 2,65 & $0,002^{*}$ \\
\hline Nota: ( $\left.{ }^{*}\right)$ valor-p $<1 \% ;\left(^{* *}\right)$ valor-p $<5 \%$. & & & &
\end{tabular}

Nota: $\left({ }^{*}\right)$ valor-p $<1 \% ;\left({ }^{* *}\right)$ valor-p $<5 \%$

Tabela 6.20 MSE S-WLSC e MSE S-WLS, $\left(\times 10^{6}\right)$, variando ' $A / s$ ', para $k$ $=32, b=40 \%(N=43$ e 65$)$. Valores baseados no teste $t$, unilateral, de comparação de médias 
Por fim, para ilustrar a qualidade do ajuste sazonal realizado pelo filtro SWLSC, no caso das séries trimestrais multiplicativas, foram gerados os espectros da irregular, considerando séries com $k=24$ e $k=32$, para $A / s=6,5$ e $b=40 \%$. Nesse momento, foi utilizado o filtro de tamanho 43. Eles estão apresentados nos Gráficos 6.83 e 6.84 .

Observa-se que tanto para $k=24$, quanto para $k=32$, o filtro S-WLSC realiza um ajuste sazonal adequado, pois não se notam picos nas frequências sazonais, ou próximos a elas (Gráficos 6.83 e 6.84, respectivamente).

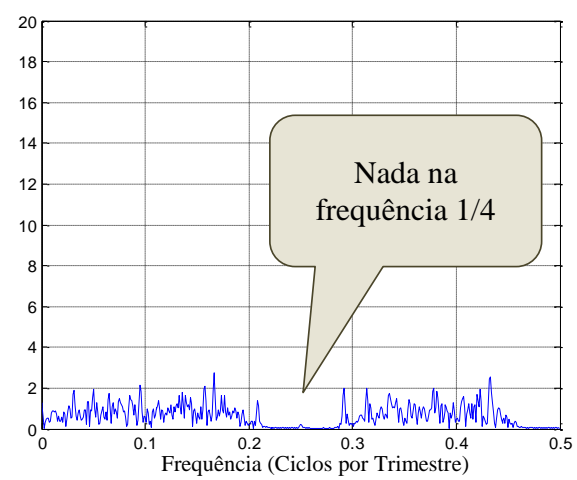

Gráfico 6.83 Espectro da irregular da série dessazonalizada pelo filtro $S$ WLSC, com: $A / s=6,5 ; k=24 ; b=40 \%$ $(N=43)$

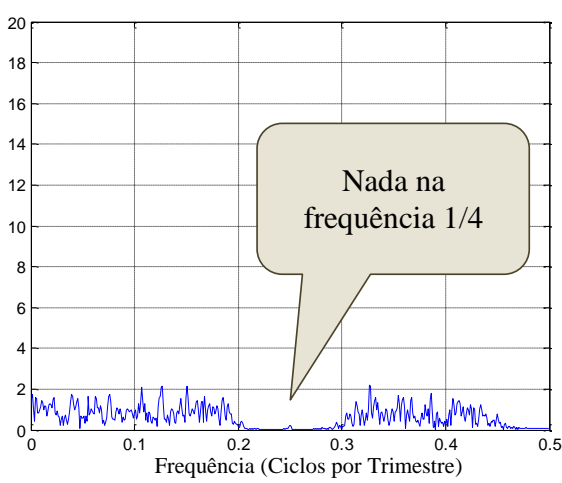

Gráfico 6.84 Espectro da irregular da série dessazonalizada pelo filtro S-WLSC, com: $A / s=6,5 ; k=32 ; b=$ $40 \%(N=43)$

Ao comparar o ajuste realizado pelo filtro S-WLSC (Gráficos 6.83 e 6.84), com o ajuste do filtro S-WLS (Gráficos 6.85 e 6.86), percebe-se um melhor desempenho do filtro S-WLSC, pois na frequência fundamental $\left(\frac{1}{4}\right)$, o espectro não possui qualquer pico. Vale citar que nesses gráficos não foi incluída a componente de tendência.

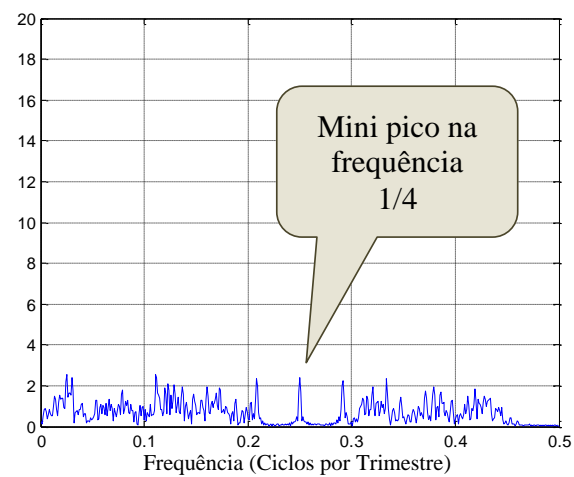

Gráfico 6.85 Espectro da irregular da série dessazonalizada pelo filtro S-WLS, com: $A / s=6,5 ; k=24 ; b=$ $40 \%(N=43)$

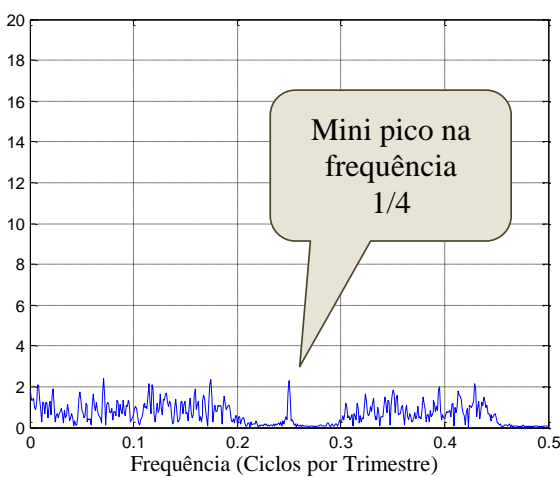

Gráfico 6.86 Espectro da irregular da série dessazonalizada pelo filtro S-WLS, com: $A / s=6,5 ; k=32 ; b=$ $40 \%(N=43)$ 
Caso houvesse a componente de tendência na série, e ela fosse dessazonalizada pelo filtro S-WLSC, os espectros da componente irregular para $k$ $=24$ e $32, b=40 \%$ e $10 \%$, e $A / s=6,5$, seriam os seguintes (Gráficos 6.87 a 6.90):

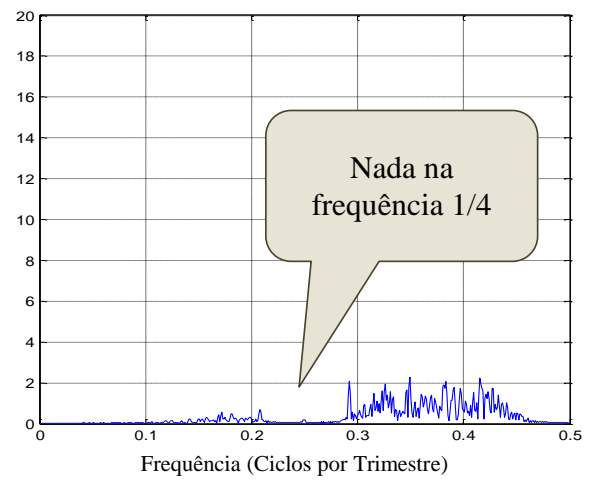

Gráfico 6.87 Espectro da irregular da série dessazonalizada pelo filtro $\mathrm{S}$ -

WLSC, com tendência, e: $A / s=6,5 ; k=$ $24 ; b=40 \%(N=43)$

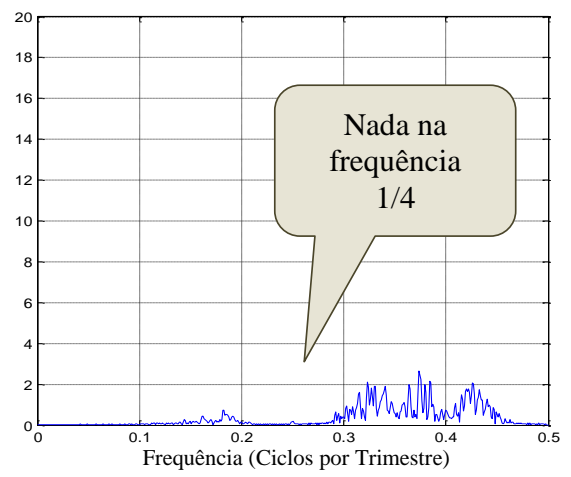

Gráfico 6.89 Espectro da irregular da série dessazonalizada pelo filtro $S$ WLSC, com tendência, e: $A / s=6,5$; $k=24 ; \quad b=10 \%(N=43)$

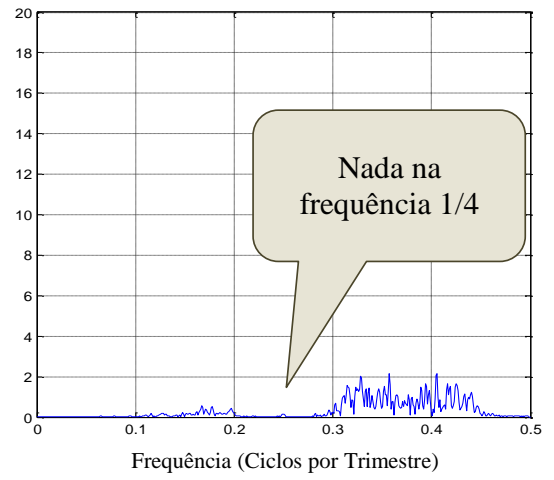

Gráfico 6.88 Espectro da irregular da série dessazonalizada pelo filtro S-WLSC, com tendência, e: $A / s$ $=6,5 ; k=32 ; \quad b=40 \%(N=43)$

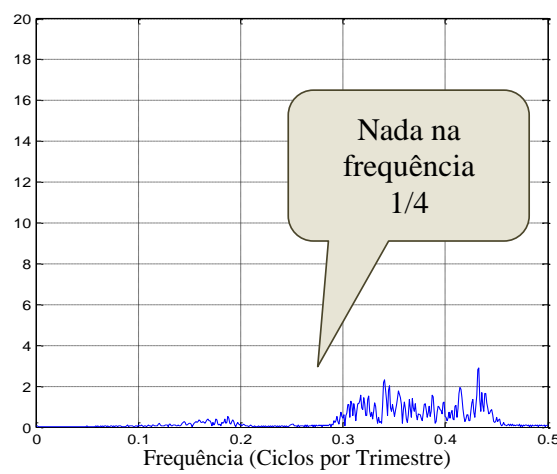

Gráfico 6.90 Espectro da irregular da série dessazonalizada pelo filtro S-WLSC, com tendência, e: $A / s$ $=6,5 ; k=32 ; \quad b=10 \%(N=43)$

Com base nesses gráficos, nota-se que o filtro S-WLSC realiza uma dessazonalização adequada, também nas séries com tendência, pois não existem picos nas frequências sazonais, ou próximos a elas.

Este capítulo apresentou o segundo filtro para a extração da sazonalidade, aqui chamado de S-WLSC, e comparou o seu desempenho com o filtro proposto anteriormente (S-WLS). 


\section{7 \\ Considerações Finais e Sugestões}

Neste capítulo são apresentadas as considerações finais quanto ao estudo realizado nesta tese. O capítulo está organizado em duas seções: a Seção 7.1 apresenta as conclusões a respeito dos filtros propostos para o ajuste sazonal; e a Seção 7.2 fornece recomendações para futuros estudos relacionadas ao assunto.

\section{1.}

\section{Considerações Finais}

Esta tese apresentou dois projetos de filtros de extração da componente sazonal, robustos a variações na sazonalidade. O primeiro projeto utilizou critérios baseados em mínimos quadrados, enquanto que o segundo combinou a abordagem de mínimos quadrados com as características dos filtros de Chebyshev. Ao primeiro projeto intitulou-se 'filtro sazonal-WLS' - S-WLS e ao segundo projeto, 'filtro sazonal-WLS-Chebyshev' - S-WLSC.

O desempenho do filtro S-WLS foi comparado com o desempenho do método X-11. A decisão de comparar os resultados do filtro S-WLS com o X-11 se deve ao fato do X-11 ser o método utilizado por muitas agências governamentais. Além disso, trata-se de um filtro ad hoc, assim como o S-WLS. Os resultados das comparações indicaram que quanto maior for o grau de sazonalidade móvel - tanto em relação à taxa de variação da amplitude sazonal, quanto em relação ao tempo que o padrão sazonal volta a se repetir - melhor é o ajuste do filtro S-WLS em relação ao do X-11. Essas comparações foram realizadas no domínio do tempo, com base nas estatísticas MSE e MAD, e no domínio da frequência, utilizando-se a razão sinal ruído e o espectro da componente irregular.

Nas comparações realizadas entre o filtro S-WLS e o X-11, foram utilizadas séries artificiais. Para a obtenção dos valores desses séries, foram analisadas 250 séries macroeconômicas, brasileiras e estrangeiras, sendo 144 mensais e 106 trimestrais. Utilizando-se o programa X-13A-S, realizou-se uma análise sobre a 
presença de sazonalidade móvel nessas séries. Ressalta-se que como foram utilizadas séries de diferentes periodicidades, foi necessário desenvolver filtros mensais e trimestrais. Os resultados da aplicação dos filtros, nas séries artificiais, indicaram um desempenho semelhante, apresentando uma boa adequação para o uso em séries com essas periodicidades.

No que se refere aos modelos de decomposição das componentes não observáveis, foram considerados neste estudo os modelos aditivo e multiplicativo. Os filtros sazonais utilizados para as séries com modelo aditivo foram os mesmos utilizados para as séries com modelo multiplicativo, porém a forma de aplicação deles foi diferente. Isso ocorreu porque não foi utilizada a transformação logarítmica nas séries multiplicativas, o que seria equivalente a utilizar o modelo aditivo. Recorda-se ainda que a transformação logarítmica introduz distorções nas características espectrais da componente sazonal, sendo esse o principal motivo da decisão de utilizar os dados originais. Quanto ao desempenho do filtro S-WLS, nos modelos aditivo e multiplicativo, os resultados foram semelhantes, indicando que o filtro é robusto, também, ao modelo de decomposição das componentes.

Acresce dizer que nas análises realizadas, foram consideradas séries com, e sem, componente de tendência. As características da componente de tendência foram obtidas nas séries reais. Os resultados encontrados com a aplicação do filtro S-WLS indicaram que a extração da sazonalidade nas séries com componente de tendência foi equivalente à extração nas séries sem essa componente, o que também representa um resultado satisfatório para o filtro.

Posteriormente, foi desenvolvido o projeto do segundo filtro: S-WLSC. O projeto desse filtro tem como principal característica minimizar simultaneamente o erro na estimativa da sazonalidade e a variância da componente irregular. O objetivo era deixar a resposta em magnitude o mais retangular possível, como mencionado por Nettheim (1965), mantendo o compromisso entre o máximo de erro na banda passante e a atenuação na banda de rejeição, e analisar o impacto no ajuste sazonal. Os resultados do ajuste sazonal com base no S-WLSC foram comparados aos obtidos com o sazonal-WLS, utilizando-se séries artificiais, mensais e trimestrais, com modelo de decomposição aditivo e multiplicativo. Os resultados indicaram que o filtro S-WLSC apresenta melhor desempenho nos casos em que o grau de sazonalidade móvel é elevado. 
Vale ressaltar que nesses projetos de filtros foi especificada uma largura para a banda passante, que permite uma dessazonalização adequada nos casos de instabilidade da componente sazonal. Tal característica havia sido mencionada por Nettheim (1965), como sendo de difícil solução para um filtro que se proponha a tratar a sazonalidade na presença de mudança do padrão sazonal.

Após analisar criteriosamente as situações nas quais o filtro S-WLS e o SWLSC se destacam, conclui-se que na presença de sazonalidade móvel o filtro SWLS obtém melhores resultados se comparado ao método X-11. E nas situações em que o grau de sazonalidade móvel é alto - tanto em relação à taxa de variação da amplitude da componente sazonal, quanto em relação ao tempo com que o padrão de sazonalidade móvel volta a se repetir - o desempenho do filtro S-WLSC tende a ser superior ao do filtro S-WLS.

\section{2. \\ Sugestões para trabalhos futuros}

Esta tese considerou especificamente a questão da sazonalidade móvel e como ela é tratada no método X-11. No entanto, por não fazer parte do escopo deste estudo, não foram abordados outros métodos de ajuste sazonal, como o SEATS, por exemplo. Surge assim a necessidade de estudos futuros que abordem outros métodos, verificando o desempenho do filtro, aqui proposto, em comparação com os demais métodos utilizados para a dessazonalização.

Devido ao foco deste estudo estar na escolha automática dos filtros de média móvel do X-11, não houve a comparação com o filtro de 3-termos, também utilizado pelo X-11, uma vez que esta opção não faz parte da seleção automática. Sendo assim, novos estudos podem comparar os resultados com este filtro.

Cabe ainda uma análise a respeito dos testes para sazonalidade móvel, comparando o teste usado no X-13A-S com os demais. Tal análise seria interessante para investigar a partir de que nível de sazonalidade, apontado nesses testes, o filtro S-WLS é mais indicado do que o X-11 para o ajuste sazonal.

Além disso, como o objetivo foi utilizar séries macroeconômicas, pois são as utilizadas pelas agências governamentais, as periodicidades consideradas foram apenas a mensal e a trimestral. Porém, os projetos de filtro apresentados podem ser 
adaptados para diferentes periodicidades, como semanal, diária, dentre outras. Portanto, análises em relação às séries com outras periodicidades podem ser testadas.

Acredita-se que as análises ora apresentadas, bem como os estudos futuros aqui propostos, poderão contribuir para o melhoramento dos métodos de ajuste sazonal. 


\section{8 Referências bibliográficas}

AKAIKE, Hirotugu. Seasonal adjustment by a Bayesian modeling. In: Selected Papers of Hirotugu Akaike. Springer New York, 1998. p. 333-345.

ATUK, O.; URAL, B. Seasonal Adjustment in Economic Time Series. Central Bank of the Republic of Turkey, Statistics Department, Discussion Paper, $n$. 2002/1, 2002.

AZEVEDO, J. A Multivariate Band-Pass Filter for Economic Time Series. Journal of the Royal Statistics Society - Series C Applied Statistics, v. 60, n. 1, p. 1, 2011.

BAXTER, M.; KING, R. Measuring business cycles: approximate band-pass filters for economic time series. Review of Economics and Statistics, v. 81, n. 4, p. 57593, 1999.

BELL, W. Signal Extraction for Nonstationary Time Series. The Annals of Statistics, v. 12, , p. 646-664, 1984.

BELL, W.; PUGH, M. Alternative approaches to the analysis of time series components. In: Proceedings of the Statistics Canada Symposium on Analysis of Data in Time. Ottawa: Statistics. 1989.

BELL, W.; MONSELL, B. X-11 Symmetric Linear Filters and their Transfer Functions. Bureau of the Census, Research Report n. RR 92, v. 15, 1992.

BELL, W.; HILLMER, S. Issues involved with the seasonal adjustment of economic time series. Journal of Business \& Economic Statistics, v. 20, n. 1, p. 98-127, 2002.

BELL, W.; MARTIN, D. Computation of asymmetric signal extraction filters and mean squared error for ARIMA component models. Journal of Time Series Analysis, v. 25, n. 4, p. 603-623, 2004.

BLOEM, A.; DIPPELSMAN, R.; MÆHLE, N. Quarterly national accounts manual: concepts, data sources, and compilation. International Monetary Fund, 2001. 
BONGARD, J. Some remarks on moving averages. In: Seasonal Adjustment on Electronic Computers, Proceedings of Conference of November. OECD, pp. 361-390, 1960.

BOX, G; HILLMER, C.; TIAO, G. Analysis and modeling of seasonal time series. In: Seasonal Analysis of Economic Time Series. NBER, 1978. p. 309-344.

BRUCE, A.; JURKE, S. Empirical Comparison of Two Methods for NonGaussian Seasonal Adjustment: a book of plots. Technical report, Victoria University, Wellington, New Zealand, 1992.

BURMAN, J. Moving Seasonal Adjustment of Economic Time Series. Journal of the Royal Statistical Society. Series A, n. 128, p. 534-558 1965.

BURRIDGE, P.; WALLIS, K. Unobserved-components models for seasonal adjustment filters. Journal of Business \& Economic Statistics, v. 2, n. 4, p. 350359, 1984.

BUSETTI, F.; HARVEY, A. Seasonality tests. Journal of Business \& Economic Statistics, v. 21, n. 3, p. 420-436, 2003.

CANER, M. A locally optimal seasonal unit-root test. Journal of Business \& Economic Statistics, v. 16, n. 3, p. 349-356, 1998.

CANOVA, F. Forecasting time series with common seasonal patterns. Journal of Econometrics, v. 55, n. 1, p. 173-200, 1993.

CANOVA, F.; GHYSELS, E. Changes in seasonal patterns: are they cyclical?. Journal of Economic Dynamics and Control, v. 18, n. 6, p. 1143-1171, 1994.

CANOVA, F.; HANSEN, B. Are seasonal patterns constant over time? A test for seasonal stability. Journal of Business \& Economic Statistics, v. 13, n. 3, p. 237$252,1995$.

CAYTON, P.; BERSALES, L. Median-based seasonal adjustment in the presence of seasonal volatility. School of Statistic: University of the Philippines Diliman. UPSS Working Paper n. 2012-03, 2012.

CHEN, Q. et al. Singular spectrum analysis for modeling seasonal signals from GPS time series. Journal of Geodynamics, v. 72, p. 25-35, 2013.

CHRISTIANO, L.; FITZGERALD, T. The band-pass filter. International Economic Review, v. 44, n. 2, p. 435-65, 2003.

CLENDENIN, J. Measurement of variations in seasonal distribution. Journal of the American Statistical Association, v. 22, n. 158, p. 213-220, 1927. 
CLEVELAND, W.; TIAO, G. Decomposition of seasonal time series: A model for the Census X-11 program. Journal of the American Statistical Association, v. 71, n. 355, p. 581-587, 1976.

CLEVELAND, W.; TERPENNING, I. Graphical methods for seasonal adjustment. Journal of the American Statistical Association, v. 77, n. 377, p. 52-62, 1982.

DAGUM, E. Seasonal Factor Forecast from ARIMA Models. In: Proceedings of the International Institute of Statistics. 1975. p. 206-219.

Modelling, Forecasting and Seasonally Adjusting Economic Time Series with the X-11 ARIMA Method. The Statistician, p. 203-216, 1978.

The X-11-ARIMA/88 Seasonal Adjustment Method: Foundations and User's Manual. Ottawa: Statistics Canada, 1988.

X11ARIMA version 2000: Foundations and User's Manual. Ottawa: Statistics Canada, 1999.

DE GOOIJER, J.; HYNDMAN, R. 25 years of time series forecasting. International Journal of Forecasting, v. 22, n. 3, p. 443-473, 2006.

DE LIVERA, A.; HYNDMAN, R.; SNYDER, R. Forecasting time series with complex seasonal patterns using exponential smoothing. Journal of the American Statistical Association, v. 106, n. 496, p. 1513-1527, 2011.

DICKEY, D.; HASZA, D.; FULLER, W. Testing for unit roots in seasonal time series. Journal of the American Statistical Association, v. 79, n. 386, p. 355-367, 1984.

DILLER, S. The seasonal variation of interest rates. In: Essays on Interest Rates, v. 2, p. 35-133, 1971.

DINIZ, P.; SILVA, E.; NETTO, S. Digital Signal Processing: System Analysis and Design. 2.ed. Cambridge University Press, 2010. 912p.

ENGLE, R.; GRANGER, C.; HALLMAN, J. Merging short-and long-run forecasts: An application of seasonal cointegration to monthly electricity sales forecasting. Journal of Econometrics, v. 40, n. 1, p. 45-62, 1989.

ENGLE, R. et al. The Japanese consumption function. Journal of Econometrics, v. 55, n. 1 , p. $275-298,1993$.

EUROPEAN COMMISSION GRANT. Seasonal Adjustment Methods and Practices. Hungarian Central Statistical Office. Budapest, 2007. Disponível em: 
<http://epp.eurostat.ec.europa.eu/SEASONAL_ADJUSTMENT_METHODS_PR ACTICES.pdf>. Acesso em: 04 abr. 2013.

EUROSTAT. ESS Guidelines on Seasonal Adjustment. Luxembourg, European Communities, 2009.

FINDLEY, D. et al. New Capabilities and Methods of the X-12-ARIMA SeasonalAdjustment Program. Journal of Business and Economic Statistics, v. 16, p.127$177,1998$.

FINDLEY, D. Some recent developments and directions in seasonal adjustment. Journal of Official Statistics-Stockholm, v. 21, n. 2, p. 343, 2005.

FINDLEY, D.; WILLS, K.; MONSELL, B. Seasonal adjustment perspectives on "Damping seasonal factors: shrinkage estimators for the X-12-ARIMA program". International Journal of Forecasting, v. 20, n. 4, p. 551-556, 2004.

FINDLEY, D.; MARTIN, D. Frequency domain analyses of SEATS and X-11/12ARIMA seasonal adjustment filters for short and moderate-length time series. Journal of Official Statistics-Stockholm, v. 22, n. 1, p. 1, 2006.

FIORENTINI, G.; PLANAS, C. Non Admissibility and the Specification of Unobserved Component Models. Eurostat Working Papers n. 9, 1997.

FISCHER, B. Decomposition of Time Series: Comparing Different Methods in Theory and Practice. Eurostat, 1995.

FOLDESI, E. et al. Seasonal Adjustment Methods and Practices. Hungarian Central Statistical Office, Budapest, 2007.

FRANSES, P. A multivariate approach to modeling univariate seasonal time series. Journal of Econometrics, v. 63, n. 1, p. 133-151, 1994.

Recent advances in modelling seasonality. Journal of Economic Surveys, v. 10, n. 3 , p. $299-345,1996$.

FRANSES, P.; HOBIJN, B. Critical values for unit root tests in seasonal time series. Journal of Applied Statistics, v. 24, n. 1, p. 25-48, 1997.

FRANSES, P.; KOEHLER, A. A model selection strategy for time series with increasing seasonal variation. International Journal of Forecasting, v. 14, n. 3, p. 405-414, 1998.

FRANSES, P.; VAN DIJK, D. The forecasting performance of various models for seasonality and nonlinearity for quarterly industrial production. International Journal of Forecasting, v. 21, n. 1, p. 87-102, 2005. 
FRANZINI, L.; HARVEY, A. C. Testing for deterministic trend and seasonal components in time series models. Biometrika, v. 70, n. 3, p. 673-682, 1983.

GARDNER, E. Exponential Smoothing: The State of the Art. Journal of Forecasting, v. 4, n. 1, p. 1-28, 1985.

GASMI, A. Seasonal Adjustment versus Seasonality Modelling: Effect on Tourism Demand Forecasting. Advances in Management \& Applied Economics, v. 3, n. 4, p. 119-132, 2013.

GEWEKE, J. The revision of seasonally adjusted time series. In: Proceedings of the Business and Economic Statistics Section, American Statistical Association, 1978. p. 320-325.

GHYSELS, E.; LEE, H.; NOH, J. Testing for unit roots in seasonal time series: some theoretical extensions and a Monte Carlo investigation. Journal of Econometrics, v. 62, n. 2, p. 415-442, 1994.

GHYSELS, E. Unit-root tests and the statistical pitfalls of seasonal adjustment: the case of US postwar real gross national product. Journal of Business \& Economic Statistics, v. 8, n. 2, p. 145-152, 1990.

. [New Capabilities and Methods of the X-12-ARIMA Seasonal-Adjustment Program]: Comment. Journal of Business \& Economic Statistics, p. 165-167, 1998.

GODFREY, M.; KARREMAN, H. A spectrum analysis of seasonal adjustment. Princeton University, 1964.

GÓMEZ, V.; MARAVALL, A. Program TRAMO and SEATS: Instructions for the User. Bank of Spain, Working Paper n. 9628, 1996.

Seasonal adjustment and signal extraction in economic time series. In:

Peña, D. et al. Chapter 8 in a course in time series analysis. New York: John Wiley and Sons, p. 202-246, 2001.

GOULD, P. et al. Forecasting time series with multiple seasonal patterns. European Journal of Operational Research, v. 191, n. 1, p. 207-222, 2008.

GRETHER, D.; NERLOVE, M. Some Properties of "Optimal" Seasonal Adjustment. Econometrica, v. 38, n. 5, p. 682-703, 1970.

HAMILTON, J. Time Series Analysis. Princeton University Press, 1994.

HANNAN, E. The estimation of seasonal variation in economic time series. Journal of the American Statistical Association, v. 58, n. 301, p. 31-44, 1963. 
The estimation of a changing seasonal pattern. Journal of the American Statistical Association, v. 59, n. 308, p. 1063-1077, 1964.

HAYWOOD, J.; WILSON, G. Selection and estimation of component models for seasonal time series. Journal of Forecasting, v. 19, n. 5, p. 393-417, 2000.

HIGGINSON, J. An F Test for the Presence of Moving Seasonality when using Census Method X-11 Variant. Statistics Canada, 1975.

HILlMER, S; TIAO, G. An ARIMA-Model Based Approach to Seasonal Adjustment. Journal of the American Statistical Association, v. 77, n. 377, p. 63$70,1982$.

HILLMER, S. Measures of Variability for Model-Based Seasonal Adjustment Procedures. Journal of Business and Economic Statistics, v. 3, n. 1, p. 60-68, 1985.

HOOD, C.; ASHLEY, J.; FINDLEY, D. An empirical evaluation of the performance of TRAMO/SEATS on simulated series. Proceedings of the Business and Economics Section, 2000.

HOOD, C. Comparison of time series characteristics for seasonal adjustments from SEATS and X-12-ARIMA. Proceedings, Business and Economic Statistics Section, Alexandria, VA: ASA, 2002.

HUSSAIN, F; MEADER, R. Seasonal adjustment: 2014 update. Bank of England, 2014. Disponível em: <http://www.bankofengland.co.uk/statistics/Documents/ms/ articles/art3may14.pdf >. Acesso em: 02 jul. 2014.

HYLLEBERG, S. et al. Seasonal integration and cointegration. Journal of Econometrics, v. 44, n. 1, p. 215-238, 1990.

HYLLEBERG, S. Modeling Seasonality. Oxford University Press, 1992.

HYLLEBERG， S.; JØRGENSEN， C.; SØRENSEN, N. Seasonality in macroeconomic time series. Empirical Economics, v. 18, n. 2, p. 321-335, 1993.

HYLLEBERG, Svend; PAGAN, Adrian R. Seasonal integration and the evolving seasonals model. International Journal of Forecasting, v. 13, n. 3, p. 329-340, 1997.

INTERNATIONAL MONETARY FUND. Monetary and Financial Statistics Compilation Guide. Washington, D.C., 2008.

JAIN, R. State Space Model-Based Method of Seasonal Adjustment. Monthly Labor Review, v. 124, p. 37, 2001. 
The Quality of Seasonal Adjustment: An Empirical Comparison of Various Seasonal Adjustment Methods. Bureau of Labor Statistics. Business and Economic Statistics Section. October, 2012.

JOINT EUROPEAN COMMISSION - OECD. Performance of Seasonal Adjustment Procedures: Simulation and empirical results. Econometric Institute Research Papers, 2005.

KAISER, R. Detection and estimation of structural changes and Outliers in unobserved components. Madrid: Universidad Carlos III,1998.

KAISER, R.; MARAVALL, A. An application of TRAMO-SEATS: changes in seasonality and current trend-cycle assessment: the German retail trade turnover series. Madrid: Universidad Carlos III, 2000.

KENNY, P.; DURBIN, J. Local trend estimation and seasonal adjustment of economic and social time series. Journal of the Royal Statistical Society, series A (General), p. 1-41, 1982.

KING, W. Willford I. An improved method for measuring the seasonal factor. Journal of the American Statistical Association, v. 19, n. 147, p. 301-313, 1924.

KRANE, S.; WASCHER, W. The cyclical sensitivity of seasonality in US employment. Journal of Monetary Economics, v. 44, n. 3, p. 523-553, 1999.

KUIPER, J. Spectral Properties of Various Methods of Seasonally Adjusting Dutch Unemployment Series. Amsterdam: Conference on Seasonal Adjustment, 1976.

KUZNETS, S. Seasonal pattern and seasonal amplitude: measurement of their short-time variations. Journal of the American Statistical Association, v. 27, n. 177, p. 9-20, 1932.

Seasonal Variations in Industry and Trade. National Bureau of Economic Research. New York, 1933.

LEE, L.; STEEHOWER, H. A Zero Phase Shift Band Pass Filter. OFRC Working Paper Series. Ortec Finance Research Center, Rotterdam, 2012.

LI, T.; HINICH, M. A filter bank approach for modeling and forecasting seasonal patterns. Technometrics, v. 44, n. 1, p. 1-14, 2002.

LOPES, A. Sazonalidade em Séries Temporais Econômicas: uma introdução e duas contribuições. Centre for Applied Mathematics and Economics (CEMAPRE), School of Economics and Management (ISEG), Technical University of Lisbon, 2010. 
LOTHIAN, J.; MORRY, M. A test for the presence of identifiable seasonality when using the X-11 program. Seasonal Adjustment and Time Series Analysis Staff, Statistics Canada, 1978.

LOTHIAN, J. The Identification and Treatment of Moving Seasonality in X-11ARIMA. In: Proceedings of the Business and Economic. Statistics Section of the of the American Statistical Association, 1984. p. 166-171.

MACAULAY, F. The Smoothing of Time Series. New York: National Bureau of Economic Research, 1931.

MARAVALL, A. On Minimum Mean Squared Error Estimation of the Noise in Unobserved Component Models. Journal of Business and Economic Statistics, v. 5, n. 1, p. 115-120, 1987.

Unobserved Components in Economic Time Series. The Handbook of Applied Econometrics. Oxford: Basil Blackwell, 1995.

Press, 1999.

Short-term analysis of macroeconomic time series. Oxford University A class of diagnostics in the ARIMA-model-based decomposition of a time series. Seasonal Adjustment, p. 23-36, 2003.

MARAVALL, A.; PIERCE, D. A Prototypical Seasonal Adjustment Model. Journal of Time Series Analysis, v. 8, n. 2, p. 177-193, 1987.

MCELROY, T. A nonparametric method for asymmetrically extending signal extraction filters. Journal of Forecasting, v. 30, n. 7, p. 597-621, 2011.

MELNICK, E.; MOUSSOURAKIS, J. Filter design for the seasonal adjustment of a time series. Communications in Statistics-Theory and Methods, v. 3, n. 12, p. 1171-1186, 1974.

MENDERSHAUSEN, H. Annual survey of statistical technique: Methods of computing and eliminating changing seasonal fluctuations. Journal of the Econometric Society, p. 234-262, 1937.

MILLER, D.; WILLIAMS, D. Damping seasonal factors: Shrinkage estimators for the X-12-ARIMA program. International Journal of Forecasting, v. 20, n. 4, p. 529-549, 2004.

MONSELL, B.; ASTON, J.; KOOPMAN, S. Toward x-13. ASA Proceedings, Business and Economic Statistics Section, Alexandria, VA: ASA, 2003. 
NERLOVE, M. Spectral analysis of seasonal adjustment procedures. Econometrica: Journal of the Econometric Society, p. 241-286, 1964.

A Comparison of Modified 'Hannan' and the BLS Seasonal Adjustment Filters. Journal of the American Statistical Association, v. 60, p. 442-491, 1965.

NERLOVE, M.; GRETHER, D.; CARAVALLO, J. Analysis of economic time series: a synthesis. Economic theory, econometrics, and mathematical economics (USA), 1979.

NETTHEIM, N. A spectral study of "overadjustment" for seasonality. U.S. Department of Commerce, Bureau of the Census. Technical report 1, 1964.

Fourier methods for evolving seasonal patterns. Journal of the American Statistical Association, v. 60, n. 310, p. 492-502, 1965.

OSBORN, D. A survey of seasonality in UK macroeconomic variables. International Journal of Forecasting, v. 6, n. 3, p. 327-336, 1990.

PASANEN, L.; LAUNONEN, I.; HOLMSTRÖM, L. A scale space multiresolution method for extraction of time series features. Stat, v. 2, n. 1, p. 273-291, 2013.

PEDERSEN, M.; FÆSTE, C. Seasonal adjustment of Danish financial time series using the X-12-ARIMA procedure. Danmarks National Bank Working Papers, n. 44, 2006.

PEREIRA, B.; PAIS, M.; SALES, P. Análise Espectral de Séries Temporais: uma introdução para engenharia, economia e estatística. Rio de Janeiro: Arte Final Leasing Editora, 1986. 101p.

PEZZULLI, S.; STEPHENSON, D.; HANNACHI, A. The variability of seasonality. Journal of Climate, v. 18, n. 1, p. 71-88, 2005.

PFEFFERMANN, D. A General Method for Estimating the Variances of X-11 Seasonally Adjusted Estimators. Journal of Time Series Analysis, v. 15, n. 1, p. 85-116, 1994.

PIERCE, D. Seasonal Adjustment when Both Deterministic and Stochastic Seasonality are Present. Seasonal Analysis of Economic Time Series. NBER, p. 242-280, 1979.

Signal Extraction Error in Nonstationary Time Series. Annals of Statistics, v. 7, n. 6, p. 1303-1320, 1979.

Data Revisions in Moving Average Seasonal Adjustment Procedures.

Journal of Econometrics, v. 14, n. 1, p. 95-114, 1980. 
PLANAS, C. The analysis of seasonality in economic statistics: a survey of recent developments. Questiio 22.1, 1998. Disponível em: 〈http://eudml.org/doc/40241〉. Acesso em: 20 mar. 2013.

POLLOCK, D. A Review of TSW: The Windows Version of the TRAMO-SEATS Program. Journal of Applied Econometrics, v. 17, n. 3, p. 291-299, 2002.

RUFINO, C. Signal Extraction from the Philippine National Accounts Statistics using ARIMA Model-based Methodology. DLSU Business \& Economics Review, v. 21, n. 2, 2012.

SCOTT, S. An Extended Review of the X11ARIMA Seasonal Adjustment Package. International Journal of Forecasting, v. 8, n. 4, p. 627-633, 1992.

SCOTT, S.; TILLER R.; CHOW, D. Empirical Evaluation of X-11 and Modelbased Seasonal Adjustment Methods. Business and Economic Statistics Section. Bureau of Labor Statistics, 2007.

SHISKIN, J.; YOUNG, A.; MUSGRAVE, J. The X-11 variant of the census method II seasonal adjustment program. Technical Paper 15, US Department of Commerce, Bureau of the Census, 1967.

SOUZA, R.; CAMARGO, M. Análise e previsão de séries temporais: os modelos ARIMA. 2.ed. Rio de Janeiro: Gráfica e Editora Regional, 2004. 187p.

SUTCLIFFE, A. X11 Time Series Decomposition and Sampling Errors. Australian Bureau of Statistics, Working Papers, n. 93/2, 1993.

SUTRADHAR, B.; MACNEIL, I.; DAGUM, E. A simple test for stable seasonality. Journal of Statistical Planning and Inference, v. 43, n. 1, p. 157167, 1995.

SUTRADHAR, B.; DAGUM, E. Bartlett-type modified test for moving seasonality with applications. Journal of the Royal Statistical Society: Series D (The Statistician), v. 47, n. 1, p. 191-206, 1998.

TAYLOR, A. Locally optimal tests against unit roots in seasonal time series processes. Journal of Time Series Analysis, v. 24, n. 5, p. 591-612, 2003.

THURY, G. Seasonal adjustment by signal extraction. Empirica, v. 12, n. 2, p. 191-207, 1985.

TILLER, R.; CHOW, D.; SCOTT, S. Empirical Evaluation of X-11 and Modelbased Seasonal Adjustment Methods. U. S. Bureau of Labor Statistics, 2007. Disponível em: <http://www.bls.gov/osmr/abstract/st/st070120.htm>. Acesso em: 12 mai. 2013. 
UNITED NATIONS. Report on Seasonal Adjustment Survey to the EECCA and Western Balkan countries. Economic Commission for Europe, Economic Statistics Section. 2009.

U.S. CENSUS BUREAU. X-13ARIMA-SEATS Reference Manual. Statistical Research Division. Washington, DC, 2012

VAN DIJK, D.; STRIKHOLM, B.; TERÄSVIRTA, T. The effects of institutional and technological change and business cycle fluctuations on seasonal patterns in quarterly industrial production series. The Econometrics Journal, v. 6, n. 1, p. 7998, 2003.

WAllgren, A.; WALlgrEN, B. Consistent Seasonal Adjustment and Trendcycle Estimation. Labour and Education Statistics, v. 3, 2013.

WALLIS, K.; THOMAS J. Seasonal Variation in Regression Analysis. Journal of the Royal Statistical Society, v. 134, p. 57-72, 1971.

WALLIS, K. Seasonal Adjustment and Relations Between Variables. Journal of the American Statistical Association, v. 69, p. 18-32, 1974.

Seasonal adjustment and revision of current data: Linear filters for the X11 method. Journal of the Royal Statistical Society, Series A, p. 74-85, 1982.

Models for X-11 and X-11 Forecast Procedures for Preliminary and Revised Seasonal Adjustments. Time Series Analysis and Macroeconometric Modelling: The Collected Papers of Kenneth F. Wallis, p. 147-158, 1995.

WELLS, J. Modelling seasonal patterns and long-run trends in US time series. International Journal of Forecasting, v. 13, n. 3, p. 407-420, 1997.

WHITTLE, P. Prediction and Regulation. English Universities Press, 1963.

WILCOX, V. Seasonal Adjustment of QGDP: The Implications of Outliers. Statistics New Zealand, 2000.

WILCOX, D.; CECCHETTI, S.; KASHYAP, A. Interactions between the seasonal and business cycles in production and inventories. The American Economic Review, p. 884-892, 1997.

WINTERS, P. Forecasting sales by exponentially weighted moving averages. Management Science, v. 6, n. 3, p. 324-342, 1960.

ZANI, S. Filtros Ótimos que Conservam Qualquer Ordem de Tendência. Rio de Janeiro, 2008. 246p. Tese (Doutorado em Engenharia Elétrica) - Pontifícia Universidade Católica do Rio de Janeiro. 
ZELLNER, A. Seasonal Analysis of Economic Time Series. National Bureau of Economic Research, 1978. Disponível em: 〈http://www.nber.org/books/zell78-1>. Acesso em: 16 nov. 2012.

ZHANG, G. Peter; QI, Min. Neural network forecasting for seasonal and trend time series. European Journal of Operational Research, v. 160, n. 2, p. 501-514, 2005 . 


\section{APÊNDICE A - Teste para sazonalidade estável}

Será apresentado o teste para sazonalidade estável de uma série mensal, com modelo aditivo. Este texto foi retirado de Pedersen \& Fæste (2006).

O teste paramétrico para a presença de sazonalidade estável tem como hipótese nula, $\mathbf{H}_{\mathbf{0}}$, o fato de que não há sazonalidade estável presente na série. Para testar essa hipótese é realizado o teste $F$, de uma ANOVA de 1 fator, cuja estatística de teste é:

$$
F_{S}=\frac{\sigma_{m}^{2}}{\sigma_{r}^{2}}
$$

- $\sigma_{m}^{2}=\frac{N}{11} \sum_{j=1}^{12}\left(\bar{X}_{j}-\bar{X}\right)^{2}$ é a variância 'entre meses'. É a variância explicada pela sazonalidade estável

- $\sigma_{r}^{2}=\frac{1}{(N-12)} \sum_{i j}\left(X_{i j}+\bar{X}_{j}\right)^{2}$ é a variância residual.

O número de graus de liberdade do numerador e do denominador são (12-1) e $(\mathrm{N}-12)$, respectivamente.

Segundo Dagum (1999), a série utilizada nesse teste é a série original corrigida pela primeira estimativa de tendência.

Como algumas premissas do teste $F$ são, provavelmente, violadas, o valor da estatística $F$, é testado ao nível de significância de $0,1 \%$. 


\section{APÊNDICE B - Teste para sazonalidade móvel}

Este teste é baseado em Higginson (1975). Trata-se de um teste $F$ de Análise da Variância (ANOVA) para a presença de sazonalidade móvel, caracterizada por mudanças graduais na amplitude da razão, ou diferença, da componente sazonalirregular. Será apresentado o teste para os modelos aditivos mensais.

Seja $(S+I)_{i j}$ a componente sazonal-irregular correspondente ao ano i, e ao mês j, j=1,2,..,12. Chama-se, aqui, $(S+I)_{i j}$ de $S I$. O teste substitui cada valor de SI pelo seu valor absoluto, e realiza uma ANOVA de 2 fatores nos dados transformados.

O modelo teórico adotado para este estudo é dado pela seguinte equação:

$$
(S+I)_{i j}=a_{i}+b_{j}+e_{i j}
$$

onde: $a_{i}$ representa a contribuição devido ao efeito do i-ésimo ano;

$b_{j}$ representa a contribuição devido ao efeito do j-ésimo mês;

$e_{i j}$ é o erro, conhecido como componente irregular, com distribuição normal com média zero e variância constante.

A análise da variância é baseada na decomposição da variância total das observações com a soma das variâncias parciais: $\sigma_{m}^{2}, \sigma_{y}^{2}$ e $\sigma_{r}^{2}$.

- $\sigma_{m}^{2}=\frac{N}{11} \sum_{j}\left(\bar{X}_{. j}-\overline{\bar{X}}\right)^{2}$ é a variância 'entre meses'. Ela mede a magnitude da sazonalidade, onde $\bar{X}_{. j}=\frac{1}{N} \sum_{i=1}^{n}|S+I|_{i j}^{2}$ e $\quad \overline{\bar{X}}=\frac{1}{12 N} \sum_{i j}|S+I|_{i j} ;$

- $\sigma_{y}^{2}=\frac{11}{N-1} \sum_{i}\left(\bar{X}_{i .}-\overline{\bar{X}}\right)^{2}$ é a variância ‘entre anos’. Ela mede a variação da sazonalidade, onde $\bar{X}_{i .}=\frac{1}{12} \sum_{j}|S+I|_{i j}$;

- $\sigma_{r}^{2}=\frac{1}{11(N-1)} \sum_{i j}\left(|S+I|_{i j}-\bar{X}_{. j}-\bar{X}_{i .}+\overline{\bar{X}}\right)^{2}$ é a variância residual.

A hipótese nula é de que não há efeito do fator 'ano', e com isso não há alteração no padrão sazonal.

$$
\mathrm{H}_{0}: a_{1}=a_{2}=\cdots=a_{n}
$$

Esta hipótese pode ser testada pela razão: $F_{M}=\frac{\sigma_{y}^{2}}{\sigma_{r}^{2}}$. Esta estatística de teste é comparada com o valor da distribuição $F$-Snedecor com 11 e $11(\mathrm{~N}-1)$ graus de liberdade.

Para os modelos multiplicativos mensais, o teste é análogo ao anterior, sendo que agora os valores de $S I$ são substituídos por desvios absolutos em relação ao valor 100 , ou seja, $S I=|S I-100|$. 


\section{APÊNDICE C - Cálculo do tamanho do filtro equivalente ao $\mathrm{X}-11$ para séries mensais e trimestrais}

Na Subseção 2.3.2 são apresentadas as etapas do método X-11. Em cada uma são aplicadas diferentes médias móveis. A partir delas, é definido o tamanho do filtro. O número de observações necessárias em cada uma das etapas, para a seleção padrão (default) do filtro, está especificado nos quadros a seguir, para séries mensais e trimestrais, respectivamente.

\begin{tabular}{|c|c|c|c|c|}
\hline \multicolumn{2}{|l|}{ Etapa } & Filtro utilizado & $\begin{array}{c}\text { No de obs. } \\
\text { necessárias } \\
\text { em cada } \\
\text { extremidade }\end{array}$ & Total \\
\hline \multirow[t]{5}{*}{ Estágio 1} & $\mathbf{i}$ & Média Móvel 2x12 & 6 & 12 \\
\hline & ii & - & - & - \\
\hline & iii & Média Móvel Sazonal 3x3 & 24 & 48 \\
\hline & iv & - & - & - \\
\hline & $\mathbf{v}$ & - & - & - \\
\hline \multirow[t]{5}{*}{ Estágio 2} & $\mathbf{i}$ & Filtro de Henderson de 13 termos ${ }^{*}$ & 6 & 12 \\
\hline & ii & - & - & - \\
\hline & iii & Média Móvel Sazonal 3x5 ${ }^{* *}$ & 36 & 72 \\
\hline & iv & - & - & - \\
\hline & $\mathbf{v}$ & - & - & - \\
\hline \multicolumn{3}{|l|}{ Total } & 72 & $\begin{array}{c}144+1= \\
145\end{array}$ \\
\hline
\end{tabular}

(*) Podem ser usados os filtros de tamanho 9 ou 23

$\left(^{* *}\right)$ Podem ser usados os filtros de MMs 3x3 (48 obs. necessárias) ou 3x9 (120 obs. necessárias) (Findley et al., 1998)

Quadro 1 Tamanho do filtro equivalente para séries mensais

\begin{tabular}{|c|c|c|c|c|}
\hline \multicolumn{2}{|l|}{ Etapa } & Filtro utilizado & $\begin{array}{c}\mathbf{N}^{0} \text { de obs. } \\
\text { necessárias } \\
\text { em cada } \\
\text { extremidade }\end{array}$ & Total \\
\hline \multirow[t]{5}{*}{ Estágio 1} & $\mathbf{i}$ & Média Móvel 2x4 & 2 & 4 \\
\hline & iii & - & - & - \\
\hline & iii & Média Móvel Sazonal 3x3 & 8 & 16 \\
\hline & iv & - & - & - \\
\hline & $\mathbf{v}$ & - & - & - \\
\hline \multirow[t]{5}{*}{ Estágio 2} & $\mathbf{i}$ & Filtro de Henderson de 5 termos ${ }^{*}$ & 2 & 4 \\
\hline & ii & - & - & - \\
\hline & iii & Média Móvel Sazonal 3x5 $5^{* *}$ & 12 & 24 \\
\hline & iv & - & - & - \\
\hline & $\mathbf{v}$ & - & - & - \\
\hline \multicolumn{3}{|l|}{ Total } & 24 & $48+1=49$ \\
\hline
\end{tabular}

$\left(^{*}\right)$ Podem ser usados o filtro de tamanho 7

(**) Podem ser usados os filtros de MMs 3x3 (16 obs. necessárias) ou 3x9 (40 obs. necessárias)

Quadro 2 Tamanho do filtro equivalente para séries trimestrais 


\section{APÊNDICE D - Dedução das expressões da potência do sinal e da potência do ruído}

\section{(1) Potência do ruído do filtro}

Para demonstrar a potência do ruído de um filtro, considera-se a figura abaixo, que representa a resposta do filtro ao impulso.

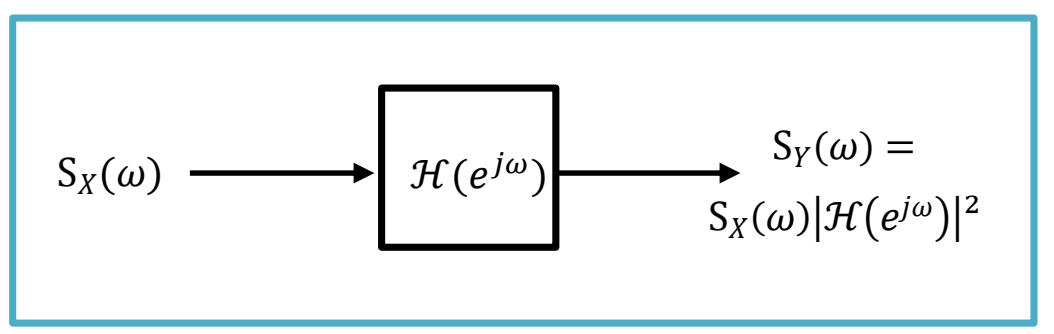

Onde $S_{X}(\omega)$ é a densidade espectral de potência do sinal de entrada, $\mathcal{H}\left(e^{j \omega}\right)$ é a transformada de Fourier da resposta em magnitude do sistema linear e $S_{Y}(\omega)$ é a densidade espectral de potência do sinal de saída.

Com base na figura acima, a potência do ruído é dada por:

$$
\begin{aligned}
\frac{1}{2 \pi} \int_{-\pi}^{\pi} S_{Y}(\omega) d \omega & =\frac{1}{2 \pi} \int_{-\pi}^{\pi} S_{X}(\omega)\left|\mathcal{H}\left(e^{j \omega}\right)\right|^{2} d \omega=\int_{-\pi}^{\pi} \sigma^{2}\left|\mathcal{H}\left(e^{j \omega}\right)\right|^{2} d \omega \\
= & \sigma^{2} \sum h(n)^{2}=\sigma^{2} S Q
\end{aligned}
$$

onde $h(n)$ são os coeficientes do filtro; $S Q=$ soma dos quadrados dos coeficientes do filtro, também chamada de SQE (soma dos quadrados dos erros); $\sigma^{2}$ é a variância da irregular.

\section{(1.1) Potência do ruído do filtro sazonal-WLS}

Considere a figura a seguir, que apresenta os valores referentes à resposta em frequência na banda passante. Tais valores são $\left(\gamma_{0}\right)$, quando se referir à resposta na frequência fundamental, e $\left(\gamma_{1}\right)$, que é a resposta de frequência que mais se desvia do valor 1 , no intervalo equivalente à largura de banda. 


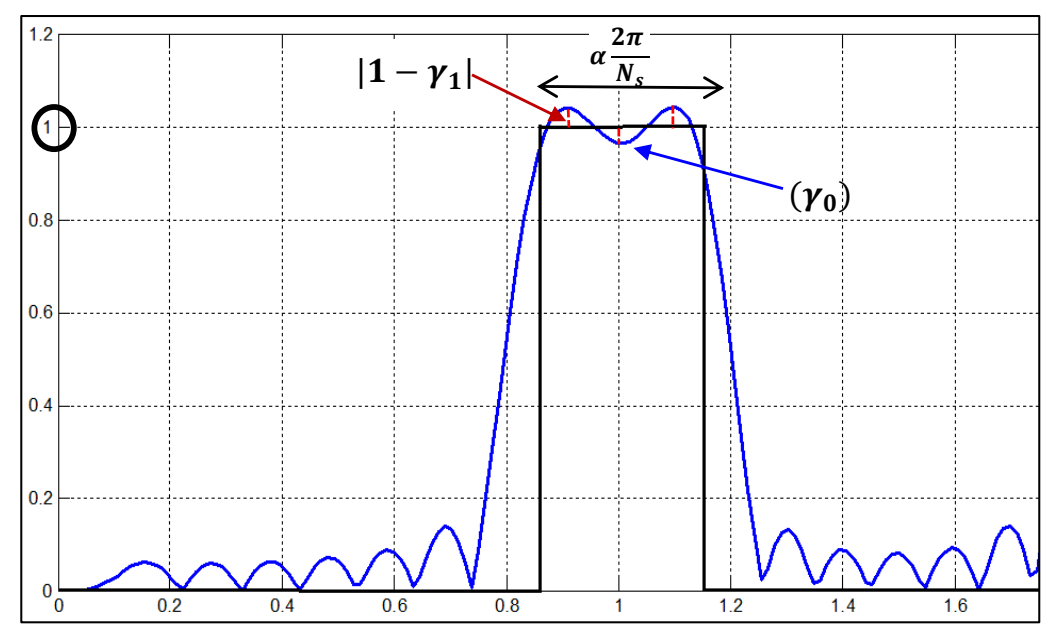

No caso dos filtros propostos (S-WLS e S-WLSC), quando $\gamma_{0}=1 \mathrm{e}$ $\left|1-\gamma_{1}\right|=0$, a potência do erro é igual a $\sigma^{2} S Q$. Caso contrário, será igual a:

$$
\sigma^{2} S Q+\left(1-\gamma_{0}\right)^{2} \frac{A^{2}}{2}+\left(1-\gamma_{1}\right)^{2} \frac{A^{2} b^{2}}{4}
$$

\section{(1.2) Potência do ruído do filtro X-11}

Para isso, considere a figura abaixo, onde a linha tracejada representa o espectro do filtro equivalente ao $\mathrm{X}-11$, e a linha contínua representa a magnitude do espectro do filtro ideal para um determinado grau de sazonalidade móvel, dado pelo valor de $\alpha$ :

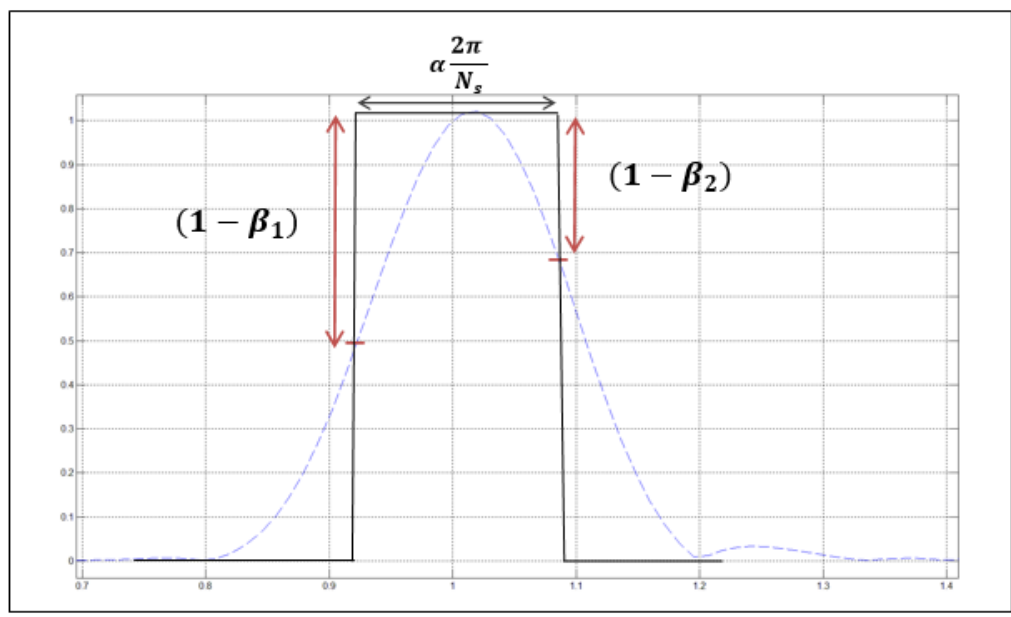

No filtro $X-11$, se $\beta_{1}=1$ e $\beta_{2}=1$, a potência do erro é igual a $\left(\sigma^{2} S Q\right)$. Como isso não ocorre, a potência será igual a:

$$
\begin{gathered}
\sigma^{2} S Q+\frac{\left[\left(1-\beta_{1}\right)^{2} \frac{A^{2} b^{2}}{4}+\left(1-\beta_{2}\right)^{2} \frac{A^{2} b^{2}}{4}\right]}{2}= \\
\sigma^{2} S Q+\frac{A^{2} b^{2}}{4}\left[\left(1-\beta_{1}\right)^{2}+\left(1-\beta_{2}\right)^{2}\right]
\end{gathered}
$$


Para diferenciar em relação à potência do erro dos filtros propostos (S-WLS e S-WLSC), chama-se a soma dos quadrados dos coeficientes do filtro X-11 de $S$, e a variância da componente irregular de $N_{0}$.

Sendo assim, a potência do ruído do X-11 é dada por:

$$
S N_{0}+\frac{A^{2} b^{2}}{4}\left[\left(1-\beta_{1}\right)^{2}+\left(1-\beta_{2}\right)^{2}\right] \text {. }
$$

\section{(2) Potência do sinal}

Define-se o sinal sazonal artificial como:

$$
A\left(1+b \operatorname{sen} \omega_{2} t\right) \cos \omega_{1} t
$$

onde: $\omega_{2}=\frac{2 \pi}{k}$ e $\omega_{1}=\frac{2 \pi}{12}$

A figura abaixo, que representa o espectro desse sinal sazonal artificial, na frequência:

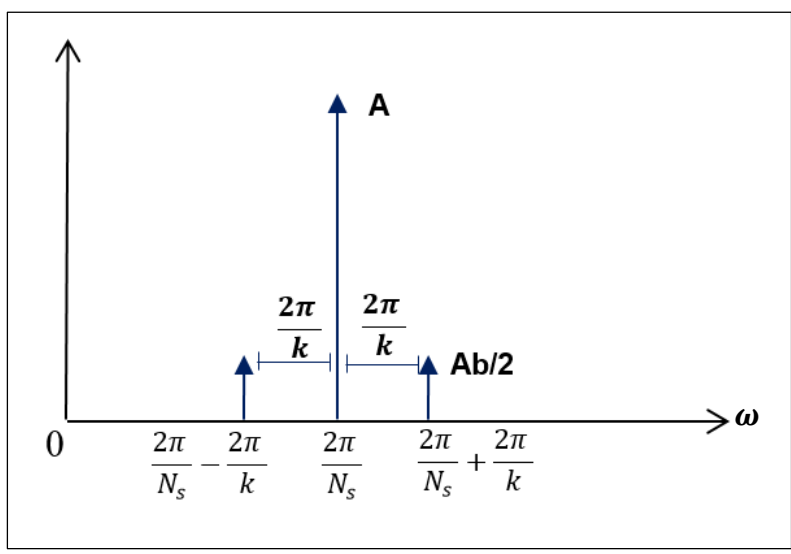

A potência do sinal é dada pela amplitude quadrática dele, sendo assim, temse:

$$
\begin{gathered}
\left\{A \cos \omega_{1} t+\frac{A b}{2} \cos \left(\omega_{1}-\omega_{2}\right) t+\frac{A b}{2} \cos \left(\omega_{1}+\omega_{2}\right) t\right\}^{2}= \\
A^{2} \frac{1}{2}+\frac{A^{2} b^{2}}{4} \frac{1}{2}+\frac{A^{2} b^{2}}{4} \frac{1}{2}= \\
\frac{A^{2}}{2}+\frac{A^{2} b^{2}}{4}
\end{gathered}
$$




\section{APÊNDICE E - Séries históricas mensais brasileiras}

\begin{tabular}{|c|c|c|}
\hline ID & Série & Fonte \\
\hline $\mathbf{X} 1$ & $\begin{array}{l}\text { Produção física industrial. Índice de base fixa mensal (Base: média de } \\
2002=100 \text { ) - Indústria geral Brasil }\end{array}$ & \multirow{11}{*}{$\begin{array}{l}\text { Instituto Brasileiro de } \\
\text { Geografia e Estatística }\end{array}$} \\
\hline $\mathbf{X} 2$ & Produção física industrial. Indústria geral Ceará & \\
\hline $\mathbf{X 3}$ & Produção física industrial. Indústria geral Pernambuco & \\
\hline $\mathbf{X} 4$ & Produção física industrial. Indústria geral Bahia & \\
\hline X5 & Produção física industrial. Indústria geral Minas Gerais & \\
\hline X6 & Produção física industrial. Indústria geral Espírito Santo & \\
\hline $\mathbf{X 7}$ & Produção física industrial. Indústria geral Rio de Janeiro & \\
\hline $\mathbf{x} 8$ & Produção física industrial. Indústria geral São Paulo & \\
\hline $\mathbf{X 9}$ & Produção física industrial. Indústria geral Paraná & \\
\hline $\mathbf{X 1 0}$ & Produção física industrial. Indústria geral Santa Catarina & \\
\hline $\mathrm{X} 11$ & Produção física industrial. Indústria geral Rio Grande do Sul & \\
\hline $\mathrm{X} 12$ & $\begin{array}{l}\text { Comércio - automotivo e construção - faturamento - índice (média } 1998 \\
=100)- \text { RMSP }\end{array}$ & \multirow{17}{*}{$\begin{array}{c}\text { Federação do Comércio } \\
\text { do Estado de São } \\
\text { Paulo, Pesquisa } \\
\text { Conjuntural do } \\
\text { Comércio Varejista da } \\
\text { Região Metropolitana de } \\
\text { São Paulo (Fecomercio } \\
\text { SP) }\end{array}$} \\
\hline $\mathrm{X} 13$ & Comércio - autopeças - faturamento - índice (média 1998 = 100) - RMSP & \\
\hline $\mathrm{X} 14$ & Comércio - calçados - faturamento - índice (média $1998=100$ ) - RMSP & \\
\hline $\mathbf{X} 15$ & $\begin{array}{l}\text { Comércio - cine, foto, som e ótica - faturamento - índice (média } 1998 \text { = } \\
\text { 100) - RMSP }\end{array}$ & \\
\hline $\mathbf{X} 16$ & $\begin{array}{l}\text { Comércio - concessionárias de veículos - faturamento - índice (média } \\
1998=100 \text { ) - RMSP }\end{array}$ & \\
\hline $\mathrm{X} 17$ & $\begin{array}{l}\text { Comércio - bens duráveis - faturamento - índice (média } 1998=100 \text { ) - } \\
\text { RMSP }\end{array}$ & \\
\hline $\mathrm{X} 18$ & $\begin{array}{l}\text { Comércio - bens não duráveis - faturamento - índice (média } 1998 \text { = 100) } \\
\text { - RMSP }\end{array}$ & \\
\hline X19 & $\begin{array}{l}\text { Comércio - bens semi-duráveis - faturamento - índice (média } 1998=100 \text { ) } \\
\text { - RMSP }\end{array}$ & \\
\hline X20 & $\begin{array}{l}\text { Comércio - bens de consumo - faturamento - índice (média } 1998=100 \text { ) } \\
\text { - RMSP }\end{array}$ & \\
\hline X21 & $\begin{array}{l}\text { Comércio - cine, foto, som, ótica e CD - faturamento - índice (média } 1998 \\
=100)- \text { RMSP }\end{array}$ & \\
\hline X22 & $\begin{array}{l}\text { Comércio - automotivo e construção - faturamento real - índice (média } \\
1998 \text { = 100) - RMSP }\end{array}$ & \\
\hline $\mathrm{X} 23$ & $\begin{array}{l}\text { Comércio - autopeças - faturamento real - índice (média } 1998=100 \text { ) - } \\
\text { RMSP }\end{array}$ & \\
\hline X24 & $\begin{array}{l}\text { Comércio - calçados - faturamento real - índice (média } 1998=100 \text { ) - } \\
\text { RMSP }\end{array}$ & \\
\hline X25 & $\begin{array}{l}\text { Comércio - cine, foto, som e ótica - faturamento real - índice (média } 1998 \\
=100)- \text { RMSP }\end{array}$ & \\
\hline X26 & $\begin{array}{l}\text { Comércio - cine, foto, som, ótica e CD - faturamento real - índice (média } \\
1998=100 \text { ) - RMSP }\end{array}$ & \\
\hline X27 & $\begin{array}{l}\text { Comércio - bens duráveis - faturamento real - índice (média } 1998=100 \text { ) } \\
\text { - RMSP }\end{array}$ & \\
\hline X28 & $\begin{array}{l}\text { Comércio - bens não duráveis - faturamento real - índice (média } 1998= \\
\text { 100) - RMSP }\end{array}$ & \\
\hline
\end{tabular}




\begin{tabular}{|c|c|c|}
\hline ID & Série & Fonte \\
\hline X29 & $\begin{array}{l}\text { Comércio - bens semi-duráveis - faturamento real - índice (média } 1998 \\
=100)-\mathrm{RMSP}\end{array}$ & \\
\hline $\mathbf{X} 30$ & $\begin{array}{l}\text { Comércio - bens de consumo - faturamento real - índice (média } 1998= \\
\text { 100) - RMSP }\end{array}$ & \\
\hline X31 & Cheques sem fundo - (\%) & Serasa \\
\hline X32 & Consumo aparente - álcool carburante - média - qde./dia - Barril (mil) & $\begin{array}{l}\text { Agência Nacional do } \\
\text { Petróleo (ANP) }\end{array}$ \\
\hline X33 & Consumo - energia elétrica - Região Centro-Oeste (CO) - qde. - GWh & \multirow{5}{*}{ Eletrobras } \\
\hline X34 & Consumo - energia elétrica - Região Nordeste (NE) - qde. - GWh & \\
\hline X35 & Consumo - energia elétrica - Região Norte (N) - qde. - GWh & \\
\hline X36 & Consumo - energia elétrica - Região Sudeste (SE) - qde. - GWh & \\
\hline X37 & Consumo - energia elétrica - Região Sul (S) - qde. - GWh & \\
\hline X38 & Consumo aparente - gasolina - média - qde./dia - Barril (mil) & \multirow{3}{*}{$\begin{array}{l}\text { Agência Nacional do } \\
\text { Petróleo (ANP) }\end{array}$} \\
\hline X39 & $\begin{array}{l}\text { Consumo aparente - derivados de petróleo - outros - média - qde./dia - } \\
\text { Barril (mil) }\end{array}$ & \\
\hline $\mathrm{X} 40$ & Consumo aparente - óleo combustível - média - qde./dia - Barril (mil) & \\
\hline $\mathrm{X} 41$ & Consumo - energia elétrica - comércio - qde. - GWh & \multirow{7}{*}{ Eletrobras } \\
\hline $\mathrm{X} 42$ & Consumo - energia elétrica - indústria - qde. - GWh & \\
\hline $\mathrm{X} 43$ & Consumo - energia elétrica - residência - qde. - GWh & \\
\hline X44 & Consumo - energia elétrica - qde. - GWh & \\
\hline $\mathrm{X} 45$ & Consumo - energia elétrica - comércio - tarifa média por $\mathrm{MWh}$ - $\mathrm{R} \$$ & \\
\hline X46 & Consumo - energia elétrica - indústria - tarifa média por $M W h$ - $R \$$ & \\
\hline $\mathrm{X} 47$ & Consumo - energia elétrica - residência - tarifa média por $M W h$ - $R \$$ & \\
\hline X48 & Consumo aparente - gás GLP - média - qde./dia - Barril (mil) & $\begin{array}{l}\text { Agência Nacional do } \\
\text { Petróleo (ANP) }\end{array}$ \\
\hline X49 & Consumo - energia elétrica - outros setores - qde. - GWh & Eletrobras \\
\hline $\mathrm{X} 50$ & Faturamento nominal - indústria - índice (média 2006 = 100) & \multirow{2}{*}{$\begin{array}{l}\text { Confederação Nacional } \\
\text { da Indústria }\end{array}$} \\
\hline $\mathrm{X} 51$ & Faturamento real - indústria - índice (média $2006=100$ ) & \\
\hline $\mathrm{X} 52$ & Usecheque - número de consultas - Unidade & \multirow{2}{*}{$\begin{array}{l}\text { Associação Comercial } \\
\text { de São Paulo }\end{array}$} \\
\hline $\mathrm{X} 53$ & SPC - número de registros recebidos - Unidade & \\
\hline $\mathrm{X} 54$ & Índice de condições econômicas atuais (ICEA) & $\begin{array}{l}\text { Federação do Comércio } \\
\text { do Estado de São Paulo }\end{array}$ \\
\hline X55 & Venda - fertilizantes - Tonelada & $\begin{array}{l}\text { Associação Nacional } \\
\text { para Difusão de Adubos }\end{array}$ \\
\hline
\end{tabular}




\begin{tabular}{|c|c|c|}
\hline ID & Série & Fonte \\
\hline $\mathrm{X} 56$ & Vendas nominais - indústria - índice (média $2006=100$ ) - SP & $\begin{array}{l}\text { Federação e Centro das } \\
\text { Indústrias do Estado de São } \\
\text { Paulo }\end{array}$ \\
\hline $\mathrm{X} 57$ & Vendas reais - varejo - índice (média $2011=100$ ) & \multirow{9}{*}{$\begin{array}{l}\text { Instituto Brasileiro de } \\
\text { Geografia e Estatística, } \\
\text { Pesquisa Mensal de } \\
\text { Comércio (IBGE/PMC) }\end{array}$} \\
\hline $\mathrm{X} 58$ & Vendas nominais - varejo - índice (média $2011=100$ ) & \\
\hline X59 & $\begin{array}{l}\text { Vendas reais - varejo - combustíveis e lubrificantes - índice (média } 2011 \\
=100)\end{array}$ & \\
\hline $\mathrm{X} 60$ & $\begin{array}{l}\text { Vendas reais - varejo - artigos farma., méd., ortoped. e perfum. - índice } \\
\text { (média } 2011=100 \text { ) }\end{array}$ & \\
\hline $\mathrm{X} 61$ & $\begin{array}{l}\text { Vendas nominais - varejo - hipermercados e superm. - índice (média } \\
2011=100 \text { ) }\end{array}$ & \\
\hline $\mathrm{X} 62$ & $\begin{array}{l}\text { Vendas nominais - varejo - móveis e eletrodomésticos índice (média } \\
2011=100 \text { ) }\end{array}$ & \\
\hline $\mathrm{X63}$ & $\begin{array}{l}\text { Vendas nominais - varejo - veículos, motos, partes e peças - índice } \\
\text { (média } 2011=100 \text { ) }\end{array}$ & \\
\hline X64 & $\begin{array}{l}\text { Vendas nominais - varejo - materiais de construção - índice (média } 2011 \\
=100)\end{array}$ & \\
\hline $\mathrm{X} 65$ & Vendas reais - varejo ampliado - índice (média $2011=100$ ) & \\
\hline $\mathrm{X} 66$ & Vendas reais - indústria - índice - RJ - (média $2006=100)$ - $(\%)$ & $\begin{array}{l}\text { Federação das Indústrias } \\
\text { do Estado do Rio de Janeiro } \\
\text { (Firjan) }\end{array}$ \\
\hline $\mathrm{X} 67$ & $\begin{array}{l}\text { Produção industrial - bens de consumo - quantum - índice (média } 2002 \\
=100 \text { ) }\end{array}$ & \multirow{8}{*}{$\begin{array}{c}\text { Instituto Brasileiro de } \\
\text { Geografia e Estatística, } \\
\text { Pesquisa Industrial } \\
\text { Mensal - Produção } \\
\text { Física }\end{array}$} \\
\hline $\mathrm{X} 68$ & $\begin{array}{l}\text { Produção industrial - bens de consumo duráveis - quantum - índice } \\
\text { (média } 2002=100 \text { ) }\end{array}$ & \\
\hline $\mathrm{X} 69$ & $\begin{array}{l}\text { Produção industrial - bens de consumo não duráveis - quantum - índice } \\
\text { (média } 2002=100 \text { ) }\end{array}$ & \\
\hline $\mathrm{X} 70$ & $\begin{array}{l}\text { Produção industrial - indústria geral - quantum - índice (média } 2002 \text { = } \\
\text { 100) }\end{array}$ & \\
\hline $\mathrm{X} 71$ & $\begin{array}{l}\text { Produção industrial - indústria de transformação - quantum - índice } \\
\text { (média } 2002=100 \text { ) }\end{array}$ & \\
\hline $\mathrm{X} 72$ & Produção industrial - alimentos - quantum - índice (média 2002 = 100) & \\
\hline $\mathrm{X} 73$ & Produção industrial - bebidas - quantum - índice (média $2002=100$ ) & \\
\hline $\mathrm{X} 74$ & Produção industrial - têxtil - quantum - índice (média 2002 = 100) & \\
\hline $\mathrm{X} 75$ & Horas trabalhadas - indústria - índice (média $2006=100$ ) & \multirow{3}{*}{$\begin{array}{l}\text { Confederação Nacional } \\
\text { da Indústria - CNI }\end{array}$} \\
\hline$x 76$ & Utilização da capacidade instalada - indústria - (\%) & \\
\hline $\mathrm{X} 77$ & Pessoal empregado - indústria - índice (média $2006=100$ ) & \\
\hline $\mathrm{X78}$ & $\begin{array}{l}\text { Produção industrial - bens de capital - quantum - índice (média } 2002 \text { = } \\
\text { 100) }\end{array}$ & $\begin{array}{l}\text { Instituto Brasileiro de } \\
\text { Geografia e Estatística, } \\
\text { (IBGE/PIM-PF) }\end{array}$ \\
\hline
\end{tabular}




\section{APÊNDICE F - Séries históricas mensais estrangeiras}

\begin{tabular}{|c|c|c|}
\hline ID & Série & Fonte \\
\hline Y122 & Employment Level; USA; Number in thousands & BLS, USA \\
\hline Y128 & 452: General Merchandise Stores; USA; millions of dollars & \multirow{10}{*}{ Census, USA } \\
\hline Y132 & 447: Gasoline Stations; USA; millions of dollars & \\
\hline Y133 & 448: Clothing and Clothing Access. Stores; USA; millions of dollars & \\
\hline Y138 & Nonmetallic Mineral Products; U.S.; millions of dollars & \\
\hline Y154 & Dairy Product Manufacturing; U.S.; millions of dollars & \\
\hline Y140 & Construction Machinery Manufacturing; U.S.; millions of dollars & \\
\hline Y145 & Plastics and Rubber Products; U.S.; millions of dollars & \\
\hline Y146 & Construction Materials and Supplies; U.S.; millions of dollars & \\
\hline Y149 & Food Products; U.S.; millions of dollars & \\
\hline Y156 & Beverage and Tobacco Products; U.S.; millions of dollars & \\
\hline Y166 & Relative consumer price indices, $2010=100 ;$ Iceland & \multirow{8}{*}{ OECD } \\
\hline Y170 & Relative consumer price indices, $2010=100$; Korea & \\
\hline Y162 & Relative consumer price indices, $2010=100 ;$ Finland & \\
\hline Y180 & Relative consumer price indices, $2010=100$; Turkey & \\
\hline Y181 & Relative consumer price indices, $2010=100$; United Kingdom & \\
\hline Y187 & Relative consumer price indices, $2010=100$; Indonesia & \\
\hline Y169 & Relative consumer price indices, $2010=100$; Japan & \\
\hline Y175 & Relative consumer price indices, $2010=100$; Norway & \\
\hline Y124 & 44Y72: Retail Trade and Food Services, ex Auto; USA; millions of dollars & Census, USA \\
\hline Y178 & Relative consumer price indices, $2010=100 ;$ Sweden & OECD \\
\hline Y127 & 4451: Grocery Stores; USA; millions of dollars & Census, USA \\
\hline Y182 & Relative consumer price indices, $2010=100$; United States & OECD \\
\hline Y150 & Consumer Goods; U.S.; millions of dollars & Census, USA \\
\hline Y165 & Relative consumer price indices, $2010=100$; Greece & OECD \\
\hline Y153 & Grain and Oilseed Milling; U.S.; millions of dollars & Census, USA \\
\hline Y179 & Relative consumer price indices, $2010=100$; Switzerland & OECD \\
\hline Y168 & Relative consumer price indices, $2010=100$; Italy & OECD \\
\hline Y152 & Consumer Nondurable Goods; U.S.; millions of dollars & Census, USA \\
\hline Y137 & Wood Products; U.S.; millions of dollars & Census, USA \\
\hline Y160 & Relative consumer price indices, $2010=100$; Canada & OECD \\
\hline Y130 & 445: Food and Beverage Stores; USA; millions of dollars & Census, USA \\
\hline Y164 & Relative consumer price indices, $2010=100$; Germany & \multirow{6}{*}{ OECD } \\
\hline Y167 & Relative consumer price indices, $2010=100$; Ireland & \\
\hline Y174 & Relative consumer price indices, $2010=100 ;$ New Zealand & \\
\hline Y184 & Relative consumer price indices, $2010=100$; Brazil & \\
\hline Y173 & Relative consumer price indices, $2010=100$; Netherlands & \\
\hline Y172 & Relative consumer price indices, $2010=100$; Mexico & \\
\hline Y143 & Audio and Video Equipment; U.S.; millions of dollars & Census, USA \\
\hline Y183 & Relative consumer price indices, $2010=100$; Euro area ( 17 countries) & \multirow{8}{*}{ OECD } \\
\hline Y185 & Relative consumer price indices, $2010=100$; China & \\
\hline Y159 & Relative consumer price indices, $2010=100$; Belgium & \\
\hline Y157 & Relative consumer price indices, $2010=100$; Australia & \\
\hline Y158 & Relative consumer price indices, $2010=100$; Austria & \\
\hline Y163 & Relative consumer price indices, $2010=100$; France & \\
\hline Y177 & Relative consumer price indices, $2010=100$; Spain & \\
\hline Y176 & Relative consumer price indices, $2010=100$; Portugal & \\
\hline
\end{tabular}




\begin{tabular}{|c|c|c|}
\hline ID & Série & Fonte \\
\hline Y148 & Computers and Related Products; U.S.; millions of dollars & Census, USA \\
\hline Y171 & Relative consumer price indices, $2010=100$; Luxembourg & \multirow{2}{*}{ OECD } \\
\hline Y186 & Relative consumer price indices, $2010=100$; India & \\
\hline Y139 & Machinery; U.S.; millions of dollars & Census, USA \\
\hline Y141 & Industrial Machinery Manufacturing; U.S.; millions of dollars & Census, USA \\
\hline Y161 & Relative consumer price indices, $2010=100$; Denmark & OECD \\
\hline Y123 & 44X72: Retail Trade and Food Services; USA; millions of dollars & \multirow{13}{*}{ Census, USA } \\
\hline Y129 & 441: Motor Vehicle and Parts Dealers; USA; millions of dollars & \\
\hline Y125 & 44000: Retail Trade; USA; millions of dollars & \\
\hline Y155 & Meat, Poultry, and Seafood Product Processing; U.S.; millions of dollars & \\
\hline Y147 & Information Technology Industries; U.S.; millions of dollars & \\
\hline Y131 & 4411,4412: Auto and Other Motor Vehicles; USA; millions of dollars & \\
\hline Y151 & Consumer Durable Goods; U.S.; millions of dollars & \\
\hline Y144 & Electrical Equipment Manufacturing; U.S.; millions of dollars & \\
\hline Y135 & Total Manufacturing; U.S.; millions of dollars & \\
\hline Y142 & Electronic Computer Manufacturing; U.S.; millions of dollars & \\
\hline Y134 & 442: Furniture and Home Furnishings Stores; USA; millions of dollars & \\
\hline Y136 & Durable Goods; U.S.; millions of dollars & \\
\hline Y126 & 722: Food Services and Drinking Places; USA; millions of dollars & \\
\hline
\end{tabular}




\section{APÊNDICE G - Séries históricas trimestrais estrangeiras}

\begin{tabular}{|c|c|c|}
\hline ID & Série & Fonte \\
\hline Y1 & B1_GE: Gross domestic product - expenditure approach; Australia; & \multirow{52}{*}{ OECD } \\
\hline Y2 & B1_GE: Gross domestic product - expenditure approach; Austria; & \\
\hline Y3 & B1_GE: Gross domestic product - expenditure approach; Belgium; & \\
\hline Y4 & B1_GE: Gross domestic product - expenditure approach; Czech Republic; & \\
\hline Y5 & B1_GE: Gross domestic product - expenditure approach; Denmark; & \\
\hline Y6 & B1_GE: Gross domestic product - expenditure approach; Estonia; & \\
\hline Y7 & B1_GE: Gross domestic product - expenditure approach; Finland; & \\
\hline Y8 & B1_GE: Gross domestic product - expenditure approach; France; & \\
\hline Y9 & B1_GE: Gross domestic product - expenditure approach; Germany; & \\
\hline Y10 & B1_GE: Gross domestic product - expenditure approach; Greece; & \\
\hline Y11 & B1_GE: Gross domestic product - expenditure approach; Hungary; & \\
\hline Y12 & B1_GE: Gross domestic product - expenditure approach; Italy; & \\
\hline Y13 & B1_GE: Gross domestic product - expenditure approach; Japan; & \\
\hline Y14 & B1_GE: Gross domestic product - expenditure approach; Korea; & \\
\hline Y15 & B1_GE: Gross domestic product - expenditure approach; Luxembourg; & \\
\hline Y16 & B1_GE: Gross domestic product - expenditure approach; Mexico; & \\
\hline Y17 & B1_GE: Gross domestic product - expenditure approach; Netherlands; & \\
\hline Y18 & B1_GE: Gross domestic product - expenditure approach; New Zealand; & \\
\hline Y19 & B1_GE: Gross domestic product - expenditure approach; Norway; & \\
\hline Y20 & B1_GE: Gross domestic product - expenditure approach; Poland; & \\
\hline Y21 & B1_GE: Gross domestic product - expenditure approach; Slovak Republic; & \\
\hline Y22 & B1_GE: Gross domestic product - expenditure approach; Slovenia; & \\
\hline Y23 & B1_GE: Gross domestic product - expenditure approach; Sweden; & \\
\hline Y24 & B1_GE: Gross domestic product - expenditure approach; Switzerland; & \\
\hline Y25 & B1_GE:Gross domestic product - expenditure approach; Euro area (17 countries); & \\
\hline Y26 & B1_GE: Gross domestic product - expenditure approach; European Union; & \\
\hline Y27 & B1_GE: Gross domestic product - expenditure approach; Brazil; & \\
\hline Y28 & B1_GE: Gross domestic product - expenditure approach; South Africa; & \\
\hline Y29 & P6: Exports of goods and services; Australia; & \\
\hline Y30 & P6: Exports of goods and services; Austria; & \\
\hline Y31 & P6: Exports of goods and services; Belgium; & \\
\hline Y32 & P6: Exports of goods and services; Czech Republic; & \\
\hline Y33 & P6: Exports of goods and services; Denmark; & \\
\hline Y34 & P6: Exports of goods and services; Estonia; & \\
\hline Y35 & P6: Exports of goods and services; Finland; & \\
\hline Y36 & P6: Exports of goods and services; France; & \\
\hline Y37 & P6: Exports of goods and services; Germany; & \\
\hline Y38 & P6: Exports of goods and services; Hungary; & \\
\hline Y39 & P6: Exports of goods and services; Italy; & \\
\hline Y40 & P6: Exports of goods and services; Japan; & \\
\hline Y41 & P6: Exports of goods and services; Korea; & \\
\hline Y42 & P6: Exports of goods and services; Luxembourg; & \\
\hline Y43 & P6: Exports of goods and services; Mexico; & \\
\hline Y44 & P6: Exports of goods and services; Netherlands; & \\
\hline Y45 & P6: Exports of goods and services; New Zealand; & \\
\hline Y46 & P6: Exports of goods and services; Norway; & \\
\hline Y47 & P6: Exports of goods and services; Poland; & \\
\hline Y48 & P6: Exports of goods and services; Slovak Republic; & \\
\hline Y49 & P6: Exports of goods and services; Slovenia; & \\
\hline Y50 & P6: Exports of goods and services; Sweden; & \\
\hline Y51 & P6: Exports of goods and services; Switzerland; & \\
\hline Y52 & P6: Exports of goods and services; Euro area (17 countries); & \\
\hline
\end{tabular}




\begin{tabular}{|c|c|c|}
\hline ID & Série & Fonte \\
\hline Y53 & P6: Exports of goods and services; European Union (27 countries); & \multirow{52}{*}{ OECD } \\
\hline Y54 & P6: Exports of goods and services; Brazil; & \\
\hline Y55 & P6: Exports of goods and services; South Africa; & \\
\hline Y71 & P7: Imports of goods and services; Australia; & \\
\hline Y72 & P7: Imports of goods and services; Austria; & \\
\hline Y73 & P7: Imports of goods and services; Belgium; & \\
\hline Y74 & P7: Imports of goods and services; Czech Republic; & \\
\hline Y75 & P7: Imports of goods and services; Denmark; & \\
\hline Y76 & P7: Imports of goods and services; Estonia; & \\
\hline Y77 & P7: Imports of goods and services; Finland; & \\
\hline Y78 & P7: Imports of goods and services; France; & \\
\hline Y79 & P7: Imports of goods and services; Germany; & \\
\hline Y80 & P7: Imports of goods and services; Hungary; & \\
\hline Y81 & P7: Imports of goods and services; Italy; & \\
\hline Y82 & P7: Imports of goods and services; Japan; & \\
\hline Y83 & P7: Imports of goods and services; Korea; & \\
\hline Y84 & P7: Imports of goods and services; Luxembourg; & \\
\hline Y85 & P7: Imports of goods and services; Mexico; & \\
\hline Y86 & P7: Imports of goods and services; Netherlands; & \\
\hline Y87 & P7: Imports of goods and services; New Zealand; & \\
\hline Y88 & P7: Imports of goods and services; Norway; & \\
\hline Y89 & P7: Imports of goods and services; Poland; & \\
\hline Y90 & P7: Imports of goods and services; Slovak Republic; & \\
\hline Y91 & P7: Imports of goods and services; Slovenia; & \\
\hline Y92 & P7: Imports of goods and services; Sweden; & \\
\hline Y93 & P7: Imports of goods and services; Switzerland; & \\
\hline Y94 & P7: Imports of goods and services; Euro area (17 countries); & \\
\hline Y95 & P7: Imports of goods and services; European Union (27 countries); & \\
\hline Y96 & P7: Imports of goods and services; Brazil; & \\
\hline Y97 & P7: Imports of goods and services; South Africa; & \\
\hline Y98 & SAFGD: Total gross debt; Australia; & \\
\hline Y99 & SAFGD: Total gross debt; Austria; & \\
\hline Y100 & SAFGD: Total gross debt; Belgium; & \\
\hline Y101 & SAFGD: Total gross debt; Canada; & \\
\hline Y102 & SAFGD: Total gross debt; Czech Republic; & \\
\hline Y103 & SAFGD: Total gross debt; Denmark; & \\
\hline Y104 & SAFGD: Total gross debt; Estonia; & \\
\hline Y105 & SAFGD: Total gross debt; Finland; & \\
\hline Y106 & SAFGD: Total gross debt; France; & \\
\hline Y107 & SAFGD: Total gross debt; Germany; & \\
\hline Y108 & SAFGD: Total gross debt; Hungary; & \\
\hline Y109 & SAFGD: Total gross debt; Ireland; & \\
\hline Y110 & SAFGD: Total gross debt; Italy; & \\
\hline Y111 & SAFGD: Total gross debt; Japan; & \\
\hline $\mathrm{Y} 112$ & SAFGD: Total gross debt; Luxembourg; & \\
\hline Y113 & SAFGD: Total gross debt; Netherlands; & \\
\hline Y114 & SAFGD: Total gross debt; Norway; & \\
\hline $\mathrm{Y} 115$ & SAFGD: Total gross debt; Portugal; & \\
\hline Y116 & SAFGD: Total gross debt; Spain; & \\
\hline Y117 & SAFGD: Total gross debt; Sweden; & \\
\hline $\mathrm{Y} 118$ & SAFGD: Total gross debt; United Kingdom; & \\
\hline Y119 & SAFGD: Total gross debt; United States; & \\
\hline $\mathrm{Y120}$ & Retail Sales; Total; (millions of dollars) & Census, USA \\
\hline Y121 & Retail Sales; E-commerce; (millions of dollars) & \\
\hline
\end{tabular}

Nota: Séries em preços correntes; moeda nacional; milhões. 
APÊNDICE H - Coeficientes dos filtros mensais: S-WLS e $X-11$

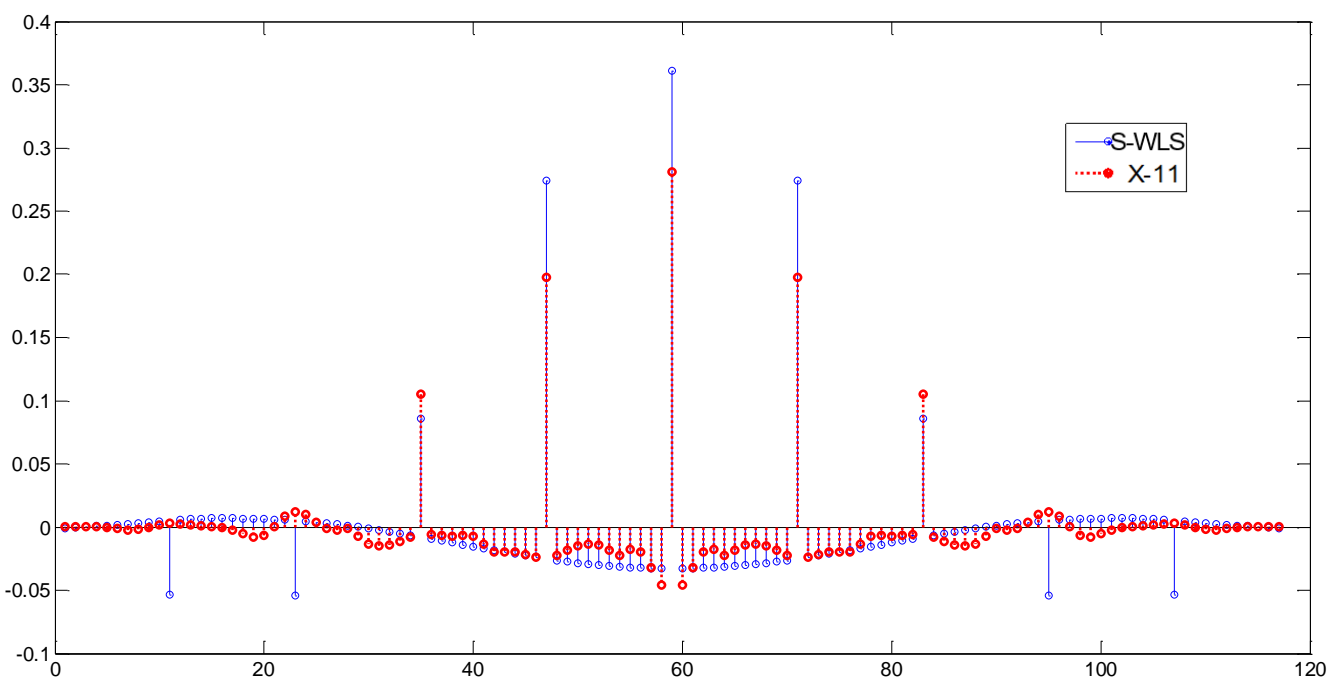

Filtro mensal de tamanho 117

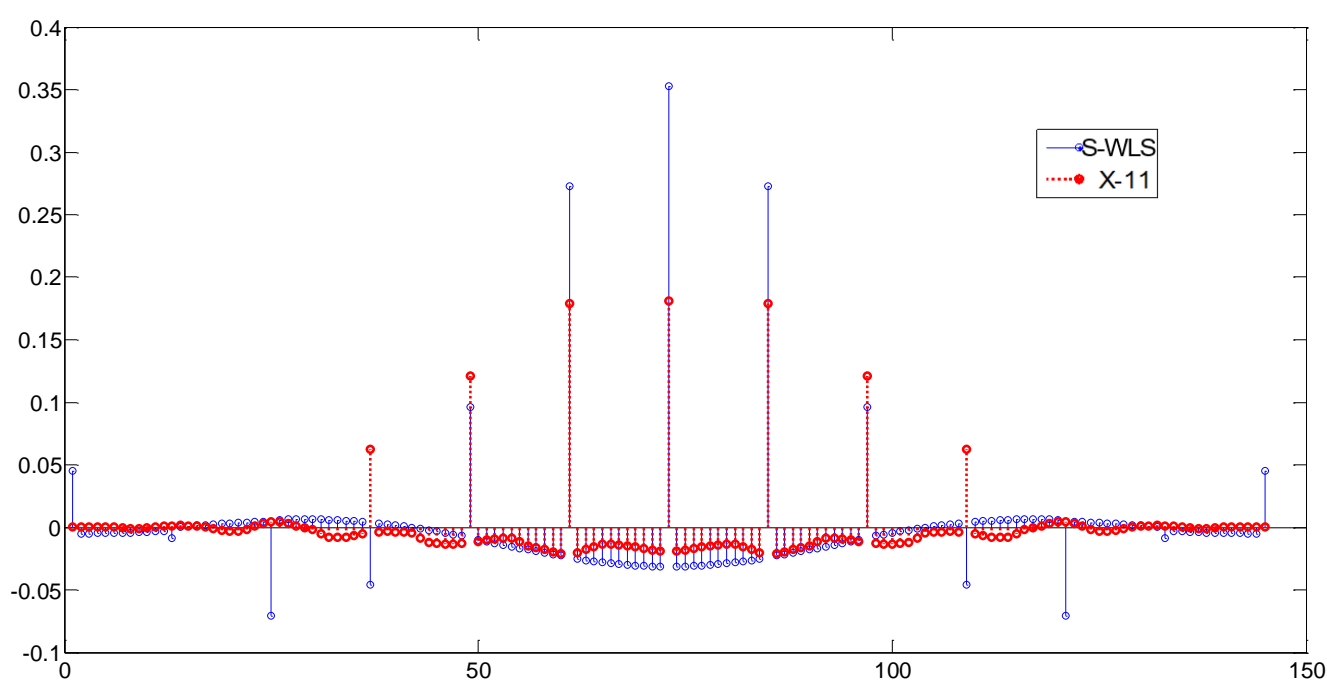

Filtro mensal de tamanho 145

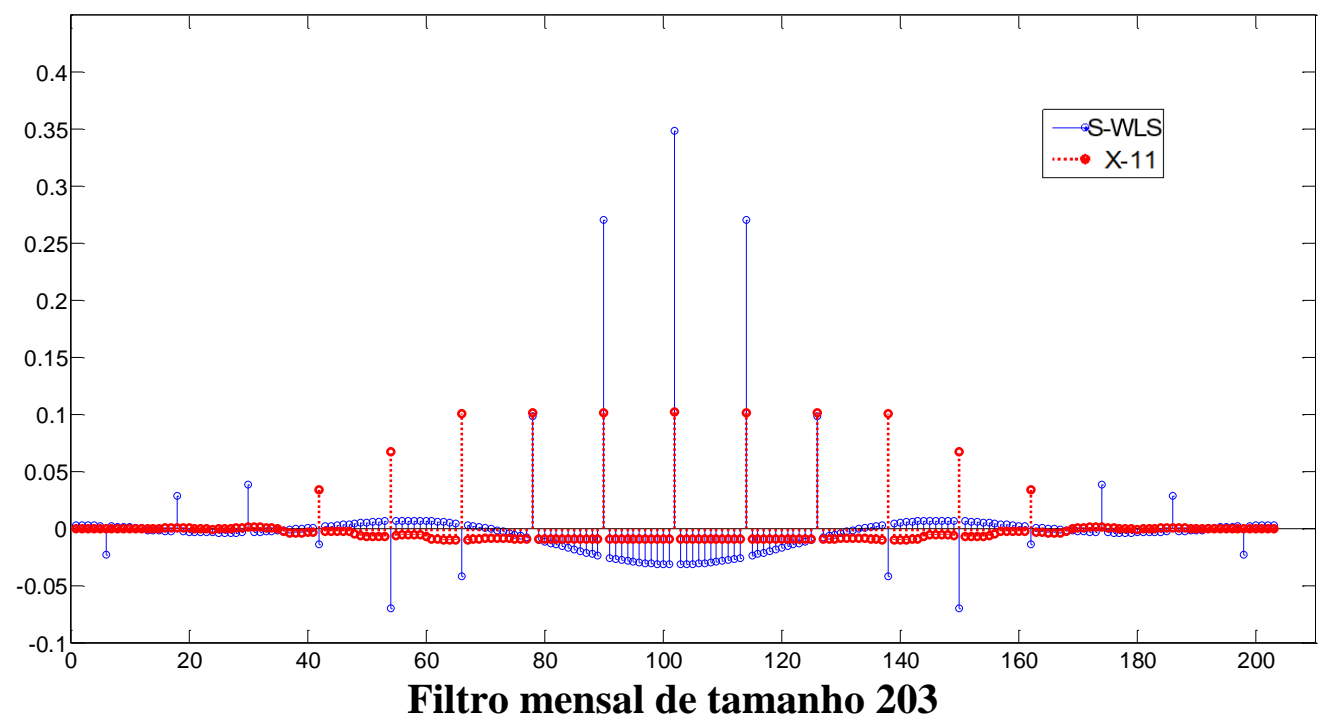


APÊNDICE I - Coeficientes dos filtros trimestrais: S-WLS e $X-11$

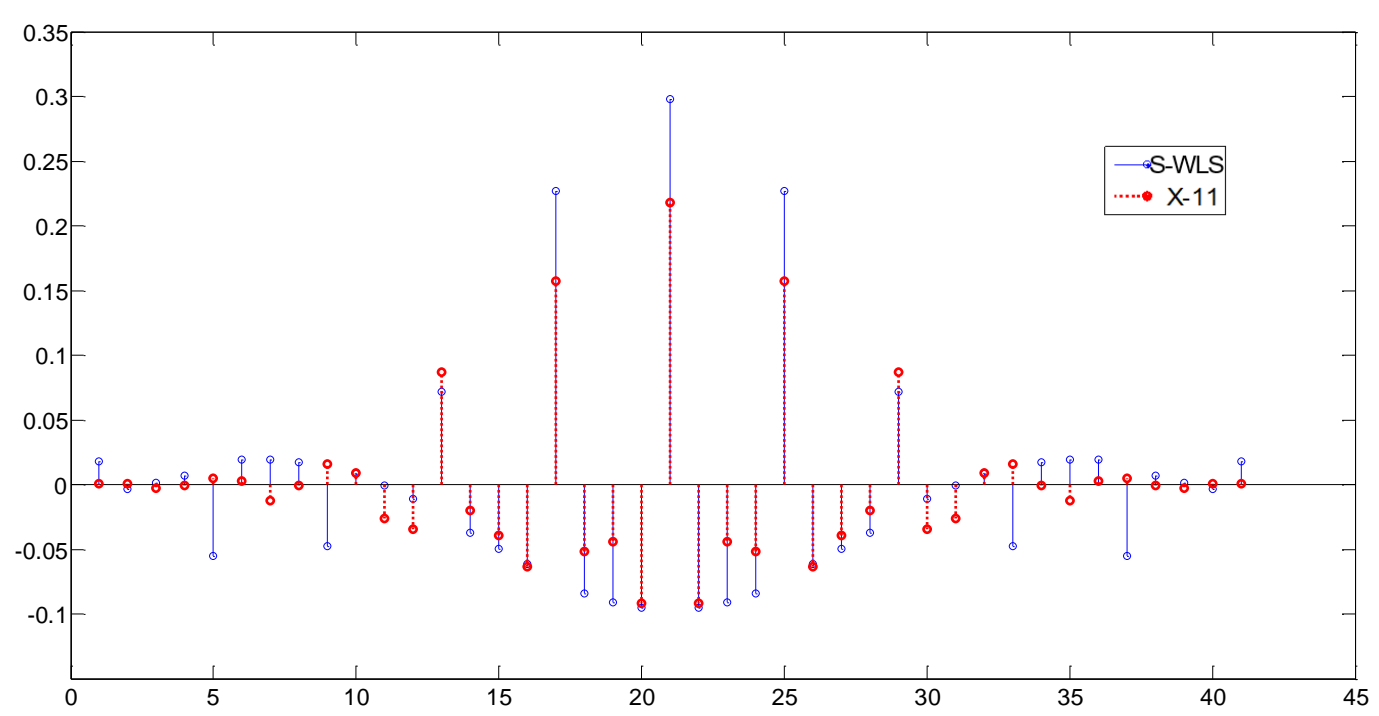

Filtro trimestral de tamanho 41
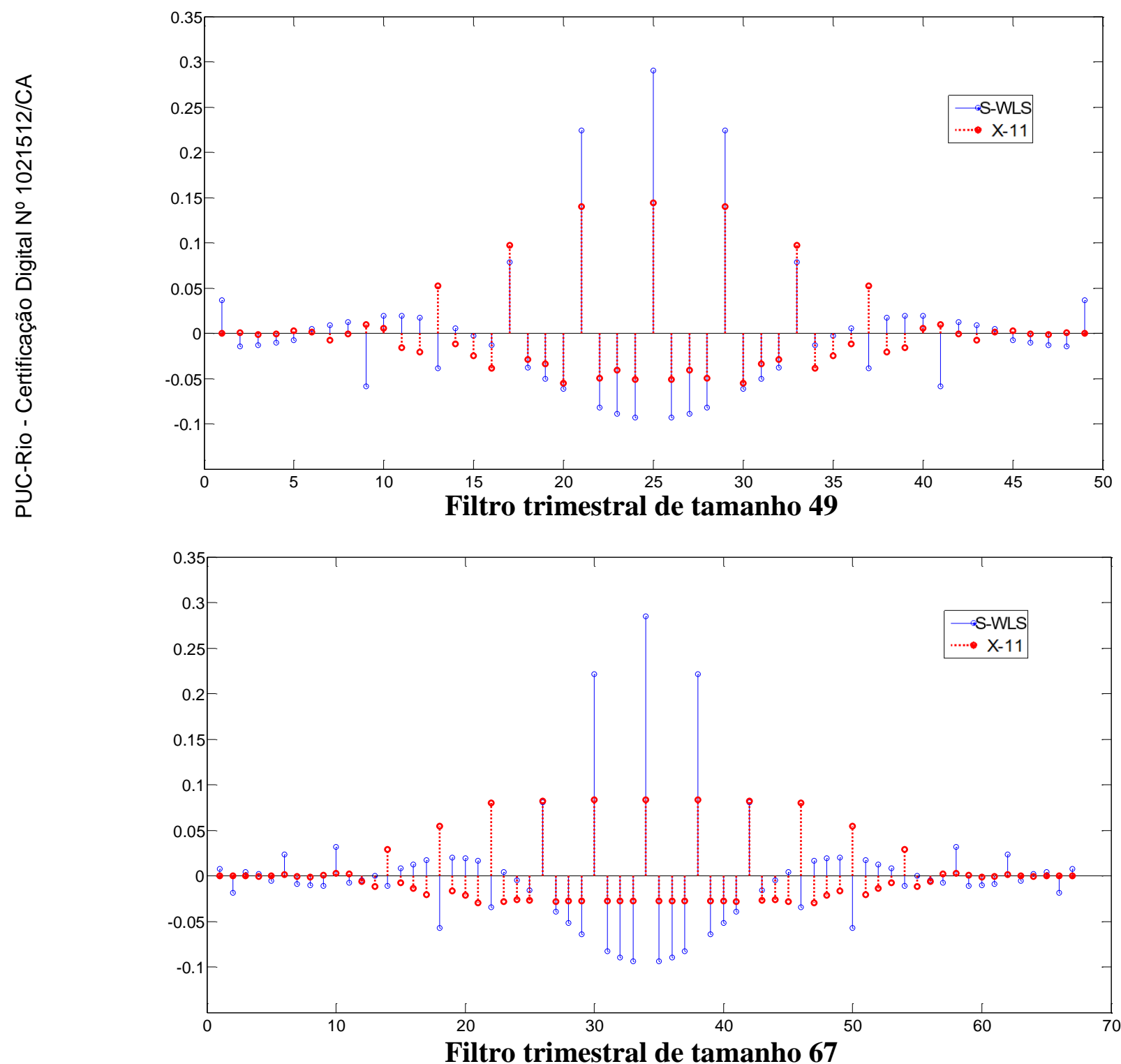


\section{APÊNDICE J - Coeficientes dos filtros mensais: S-WLS e S-WLSC}

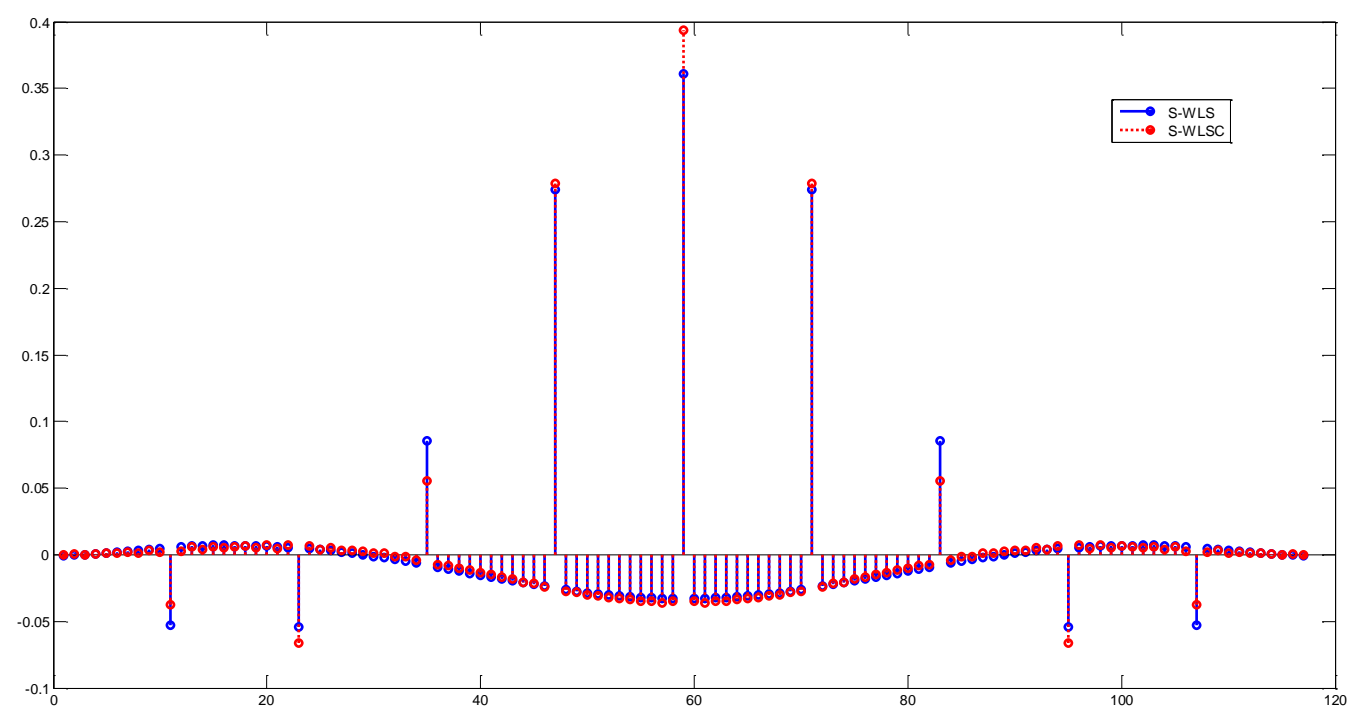

Filtro mensal de tamanho 117

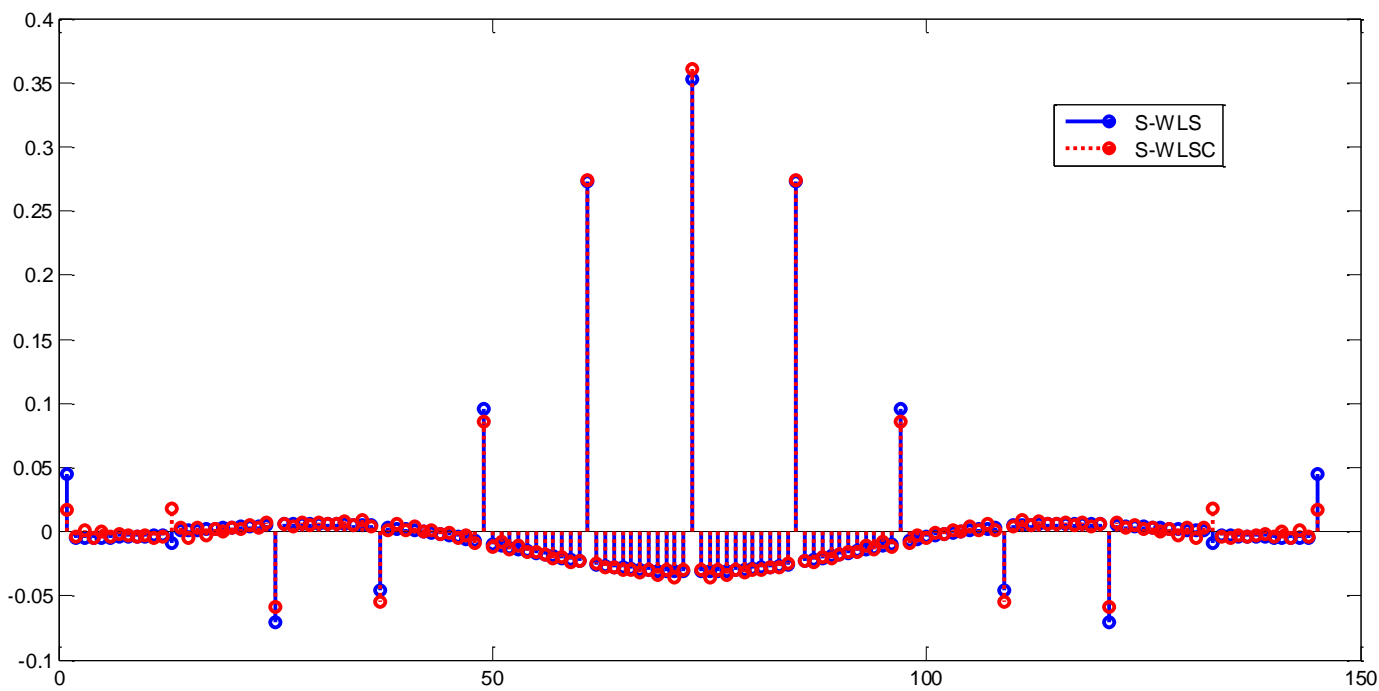

Filtro mensal de tamanho 145

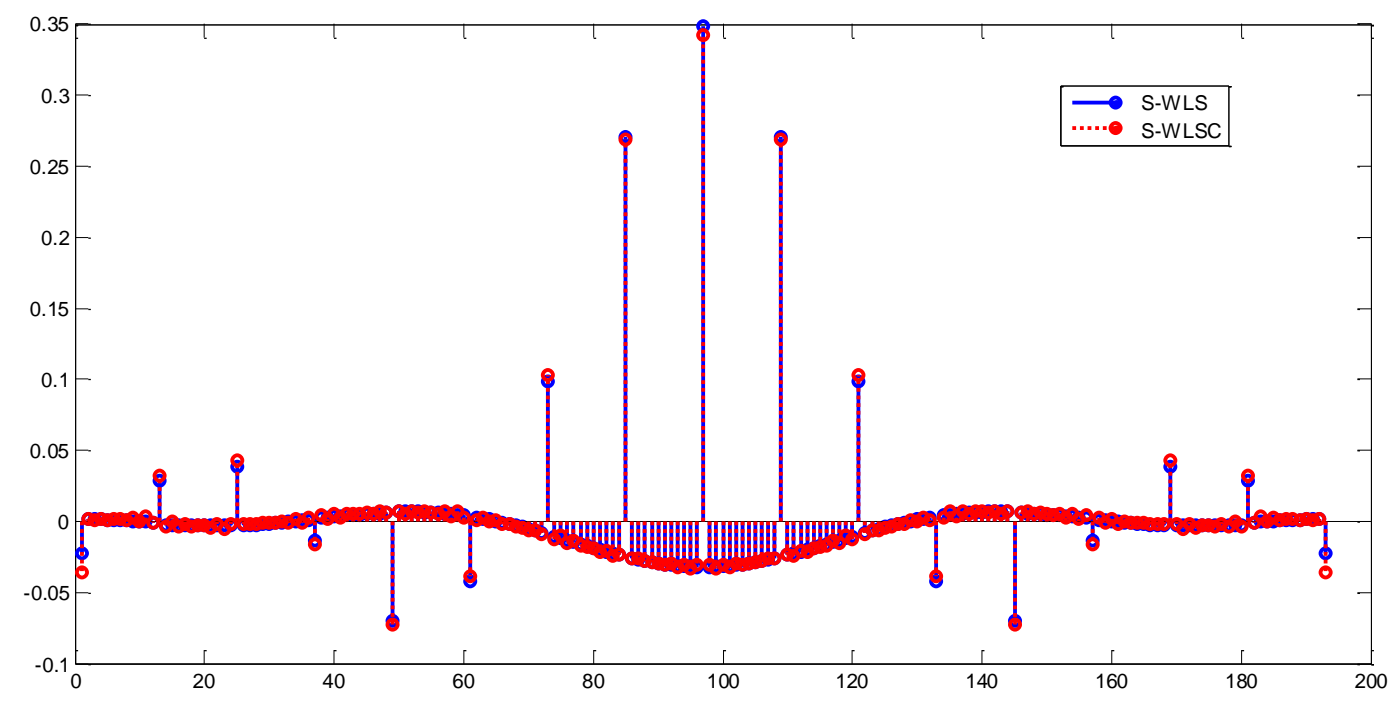

Filtro mensal de tamanho 193

Nota: assim como os coeficientes dos filtros mensais, os coeficientes dos filtros trimestrais (S-WLS e S-WLSC) são muito parecidos. Portanto, não são apresentados. 


\section{APÊNDICE K - Programas MATLAB: filtro sazonal-WLS e filtro sazonal-WLS-Chebyshev}

\section{(a) Filtro S-WLS mensal}

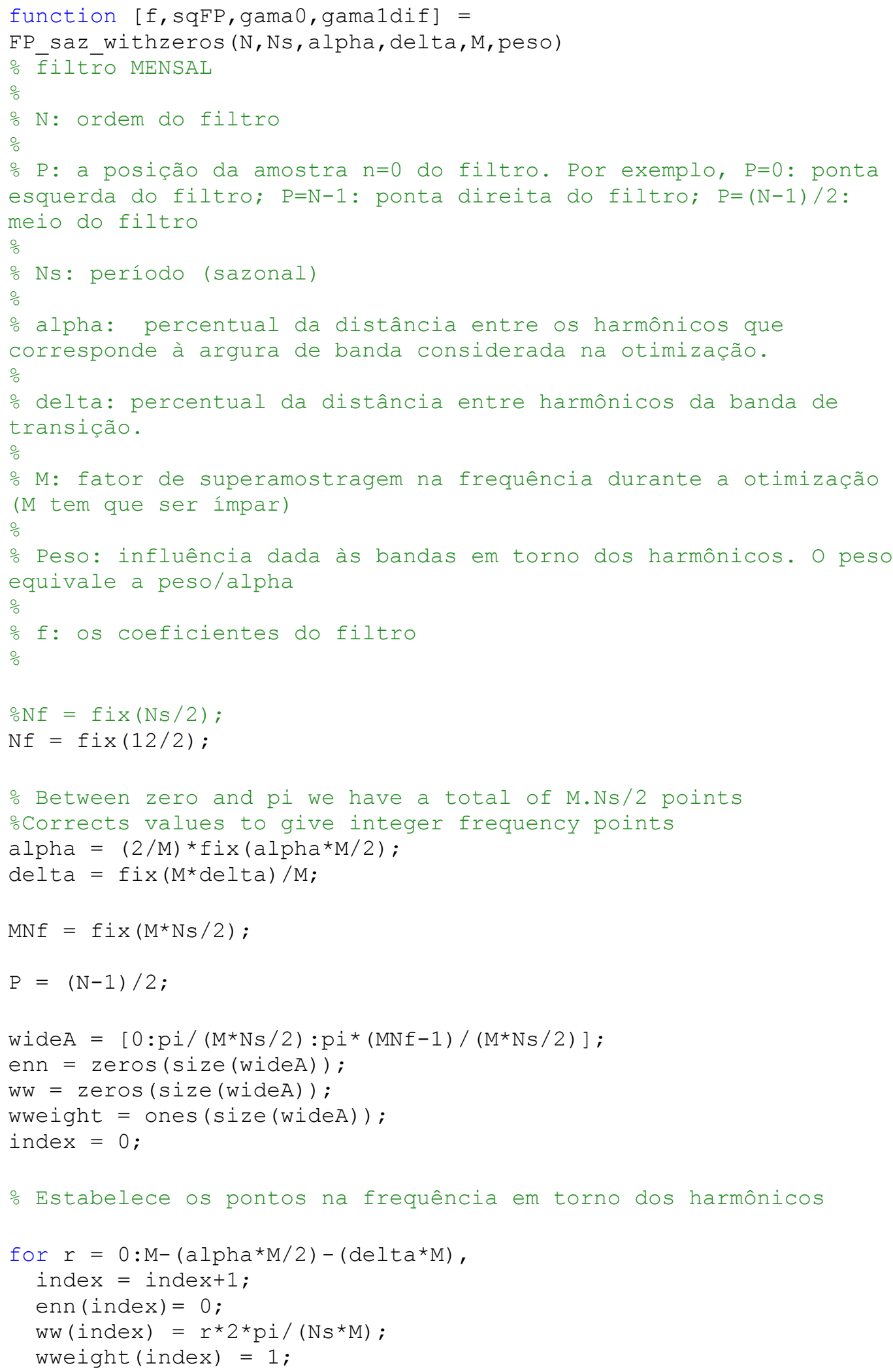




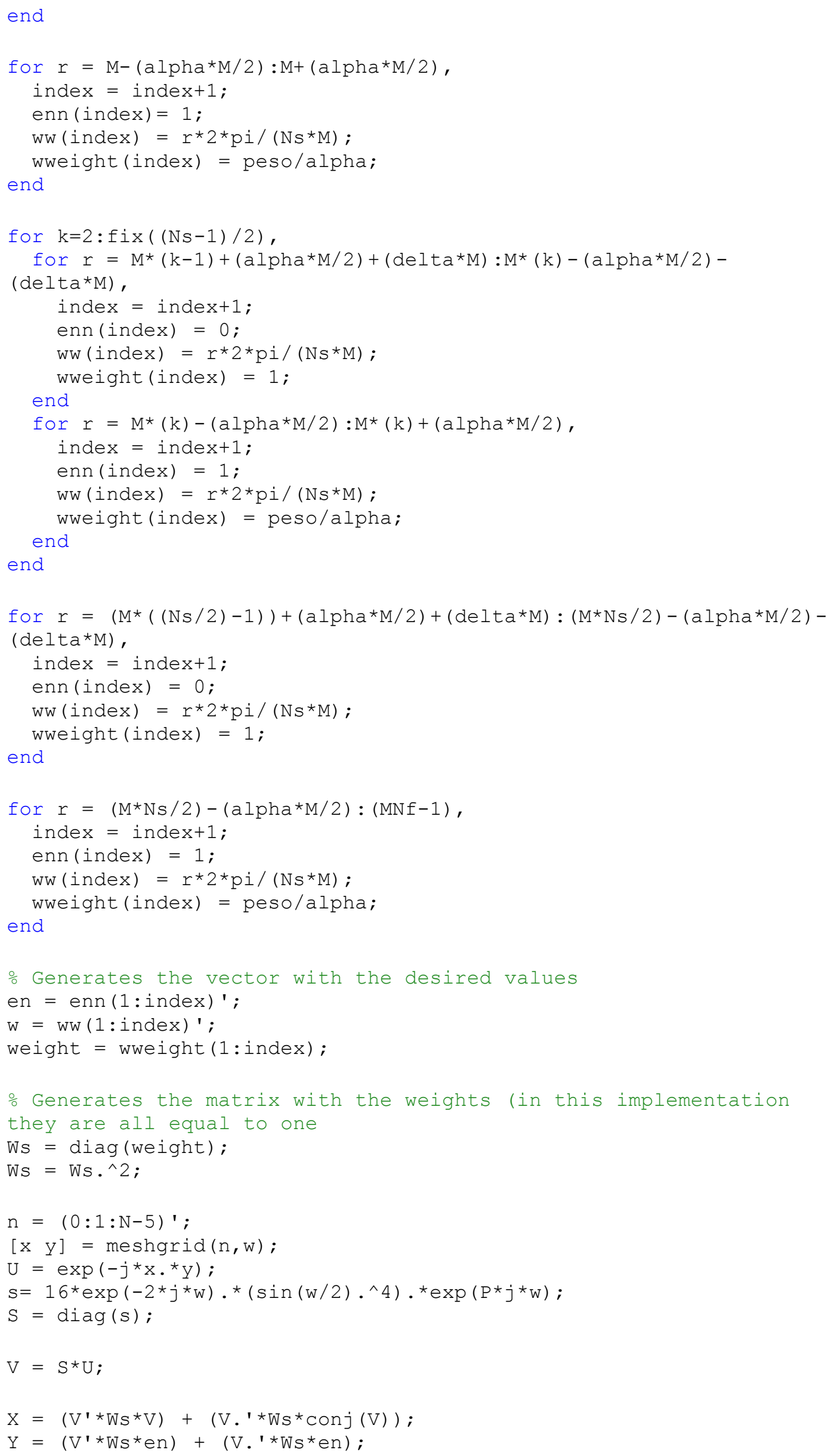




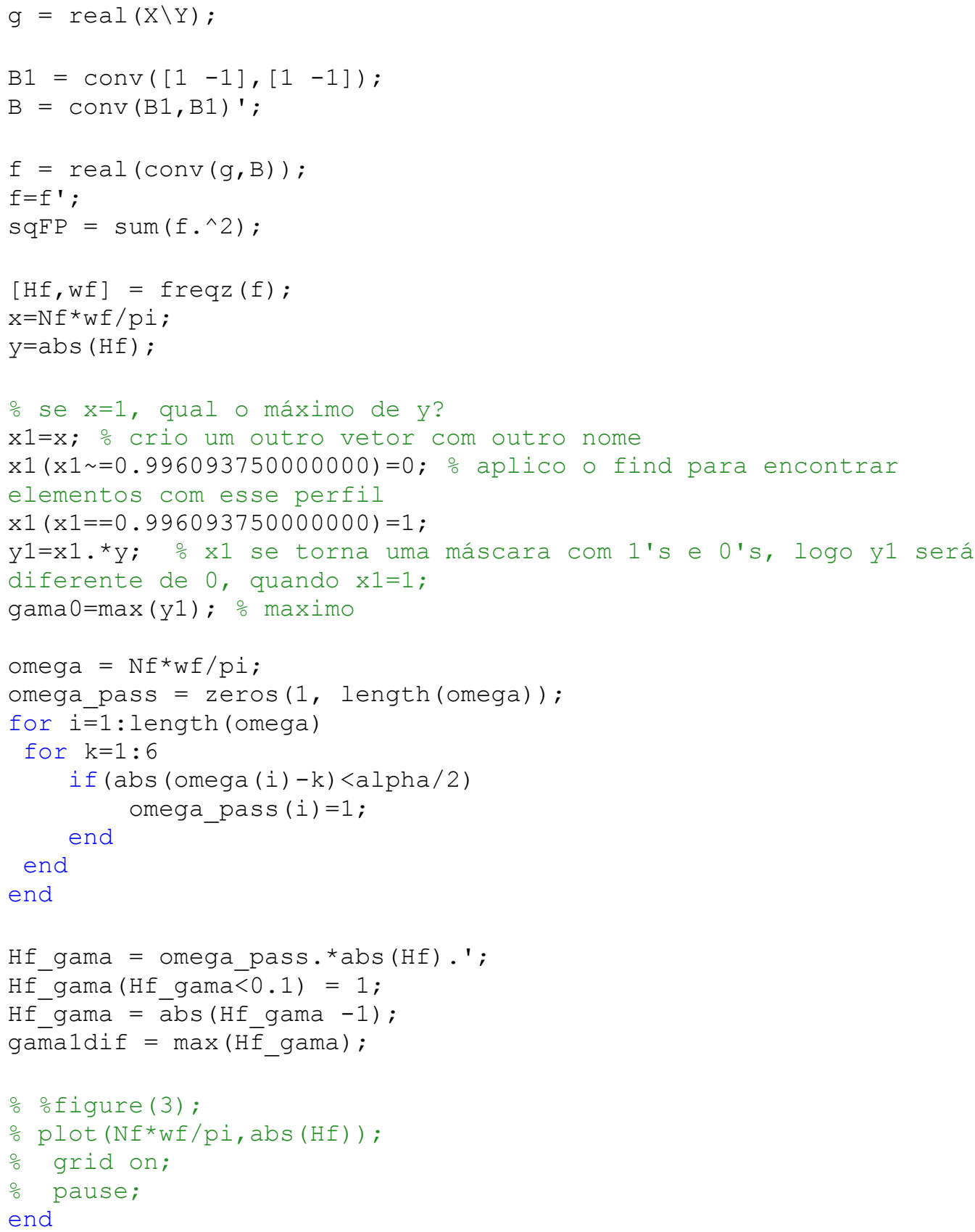




\section{(b) Filtro S-WLS trimestral}

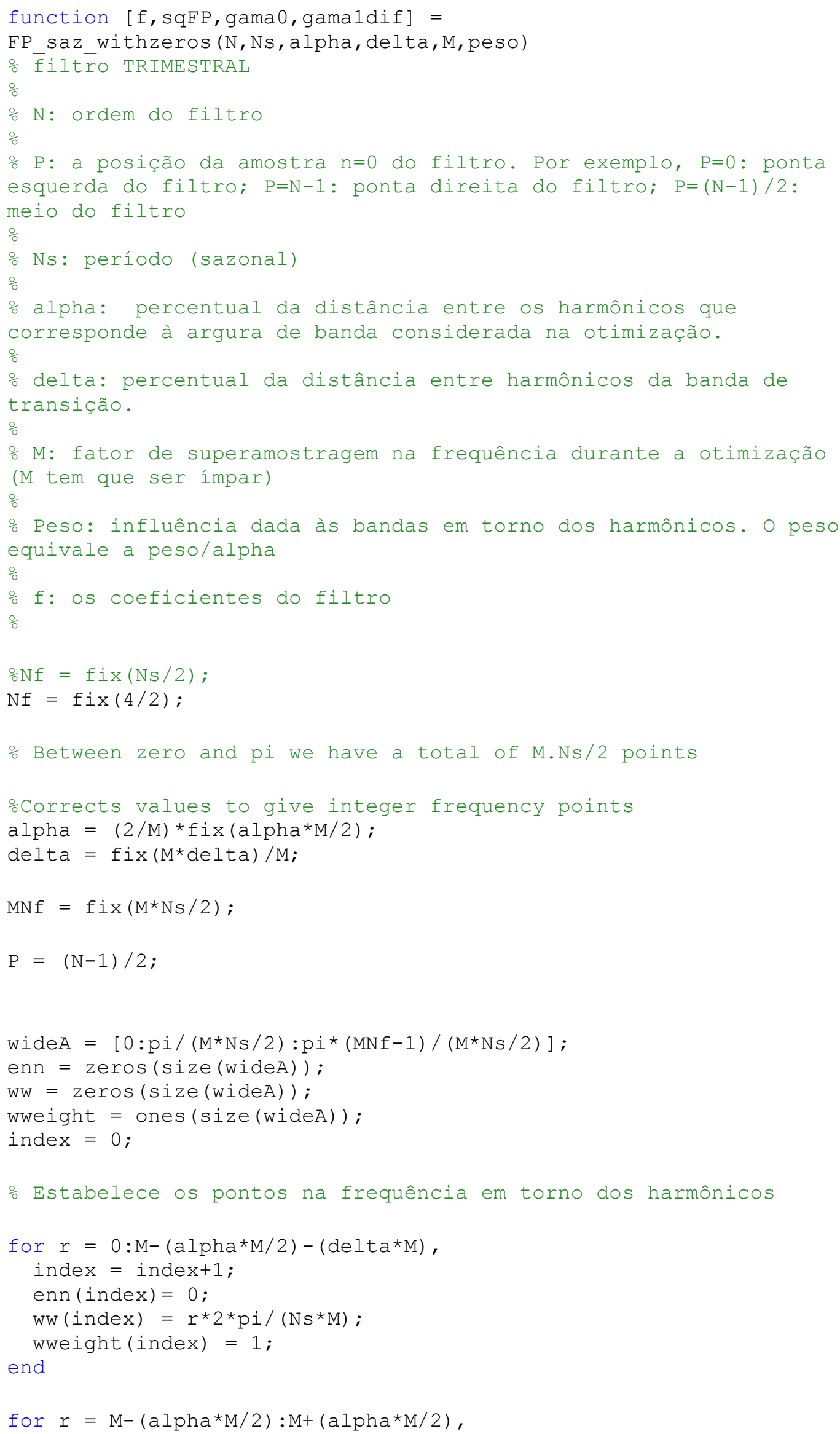




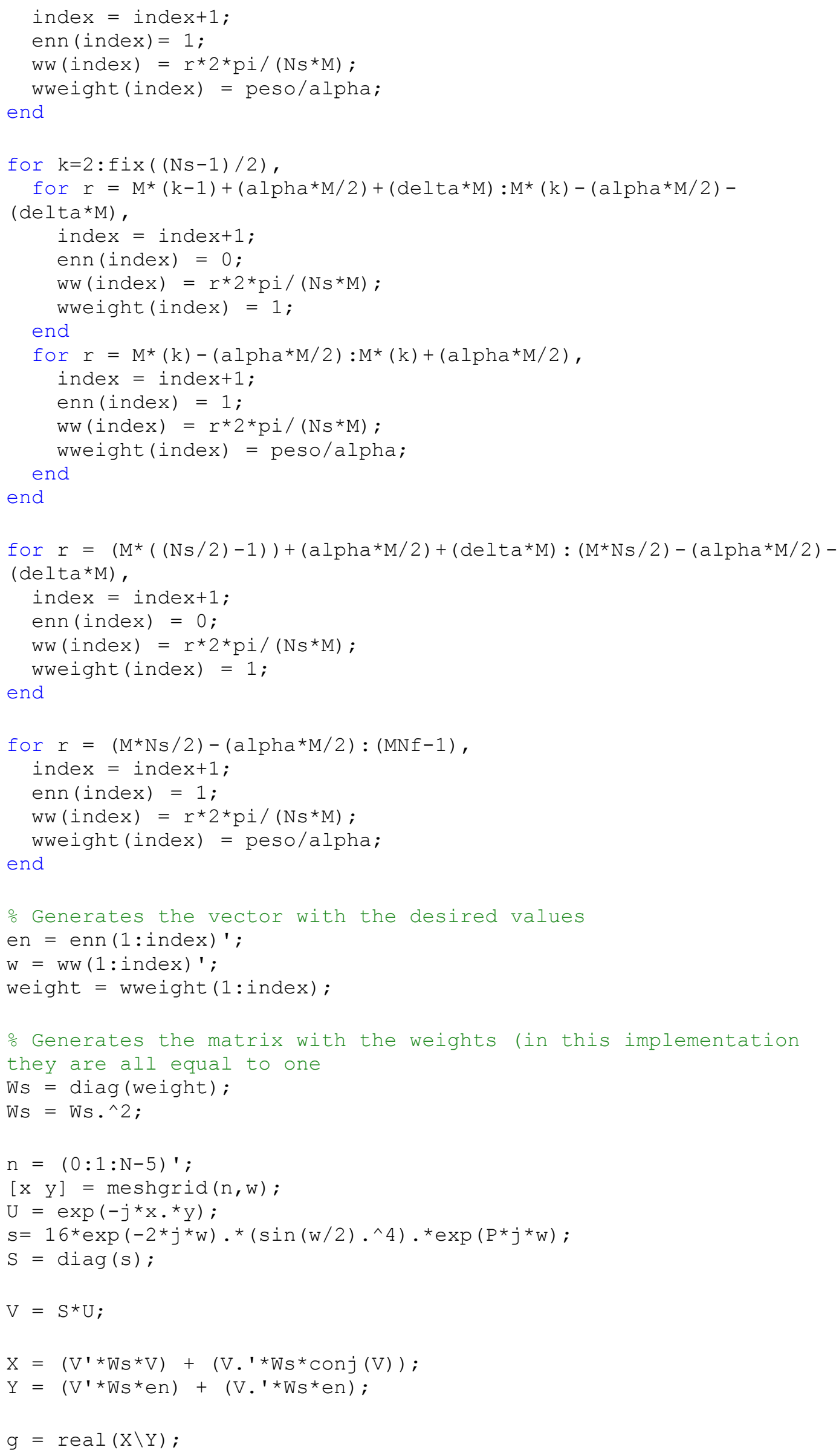




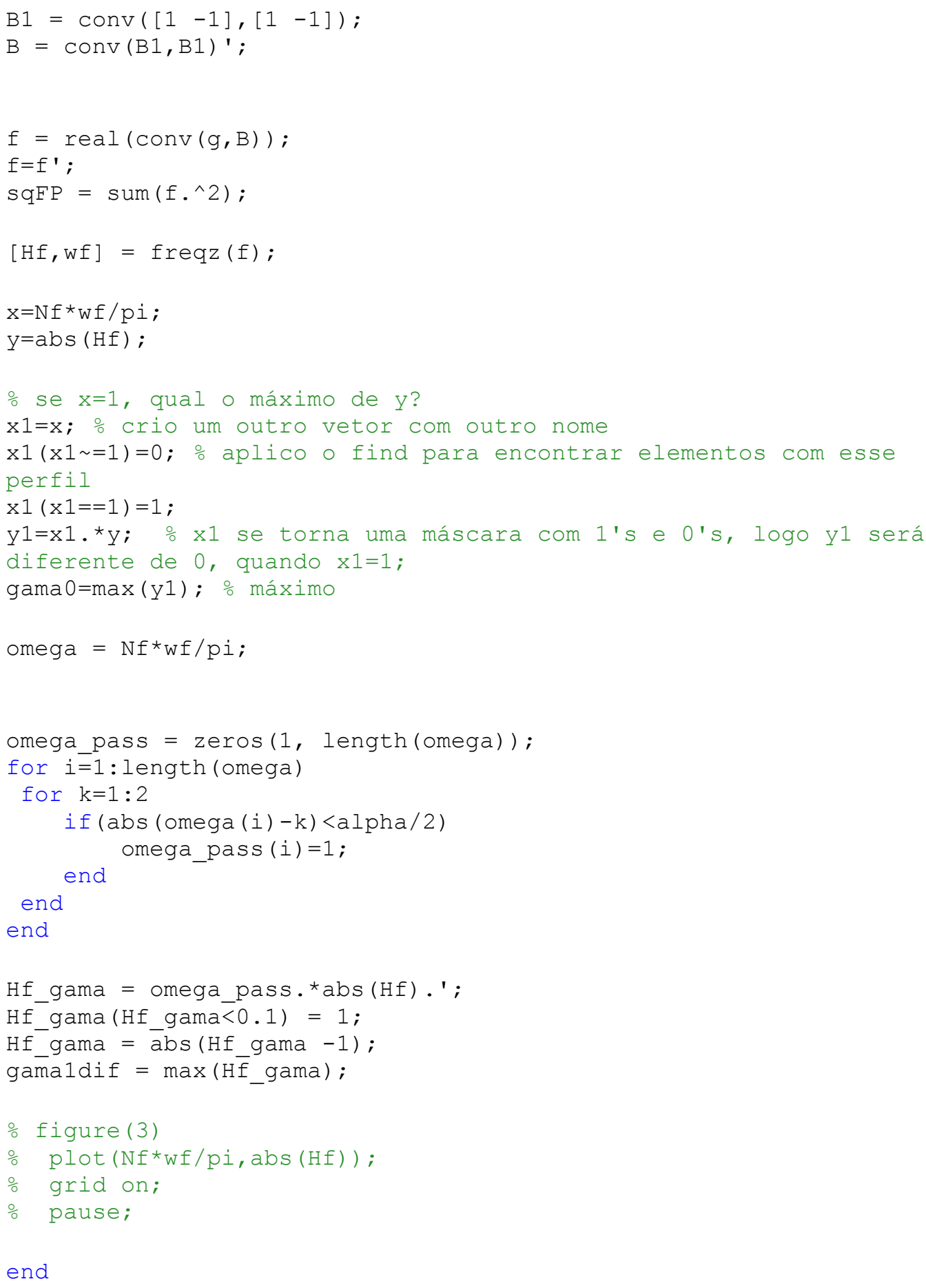

end 


\section{(c) Filtro S-WLSC mensal}

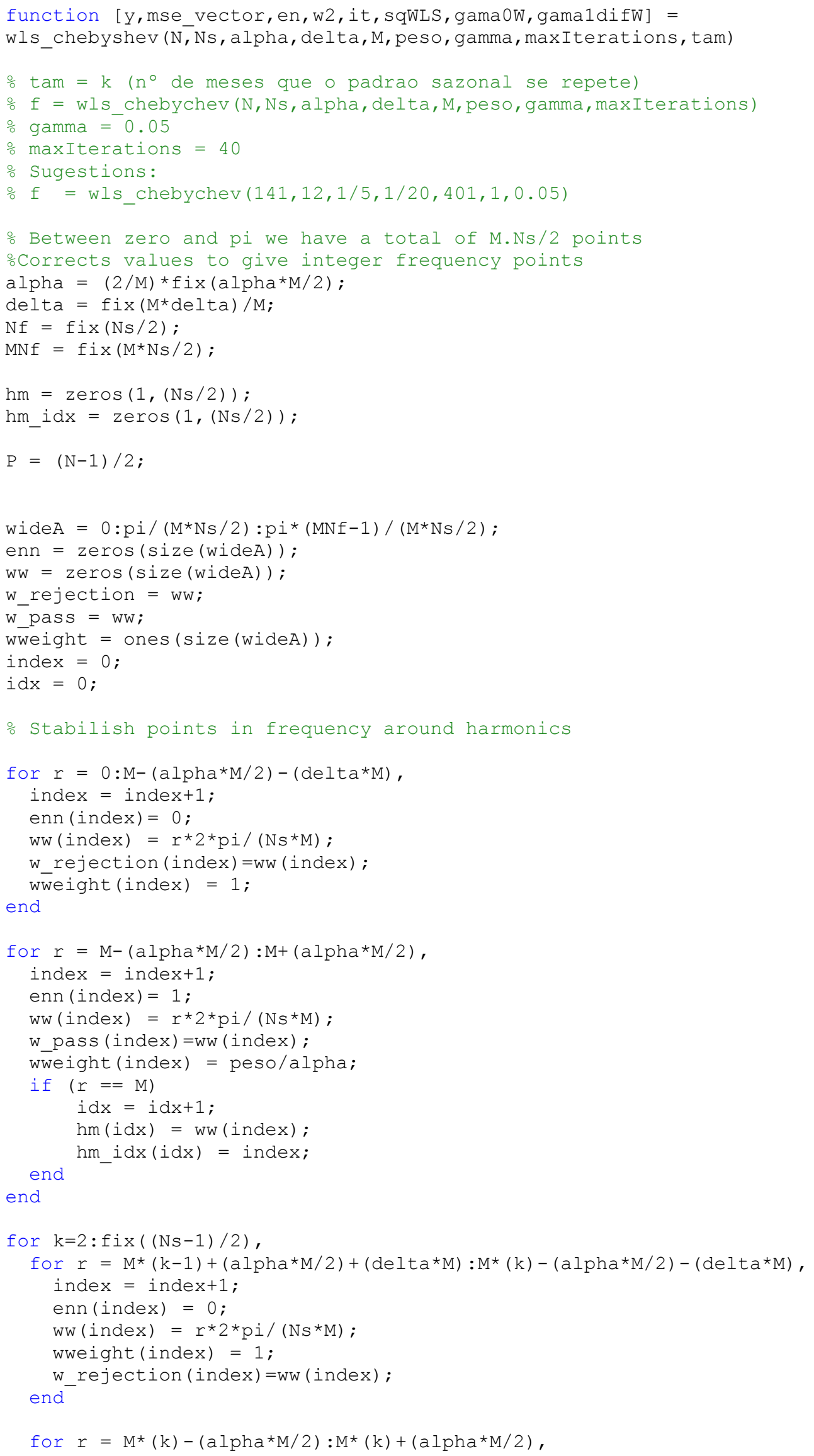




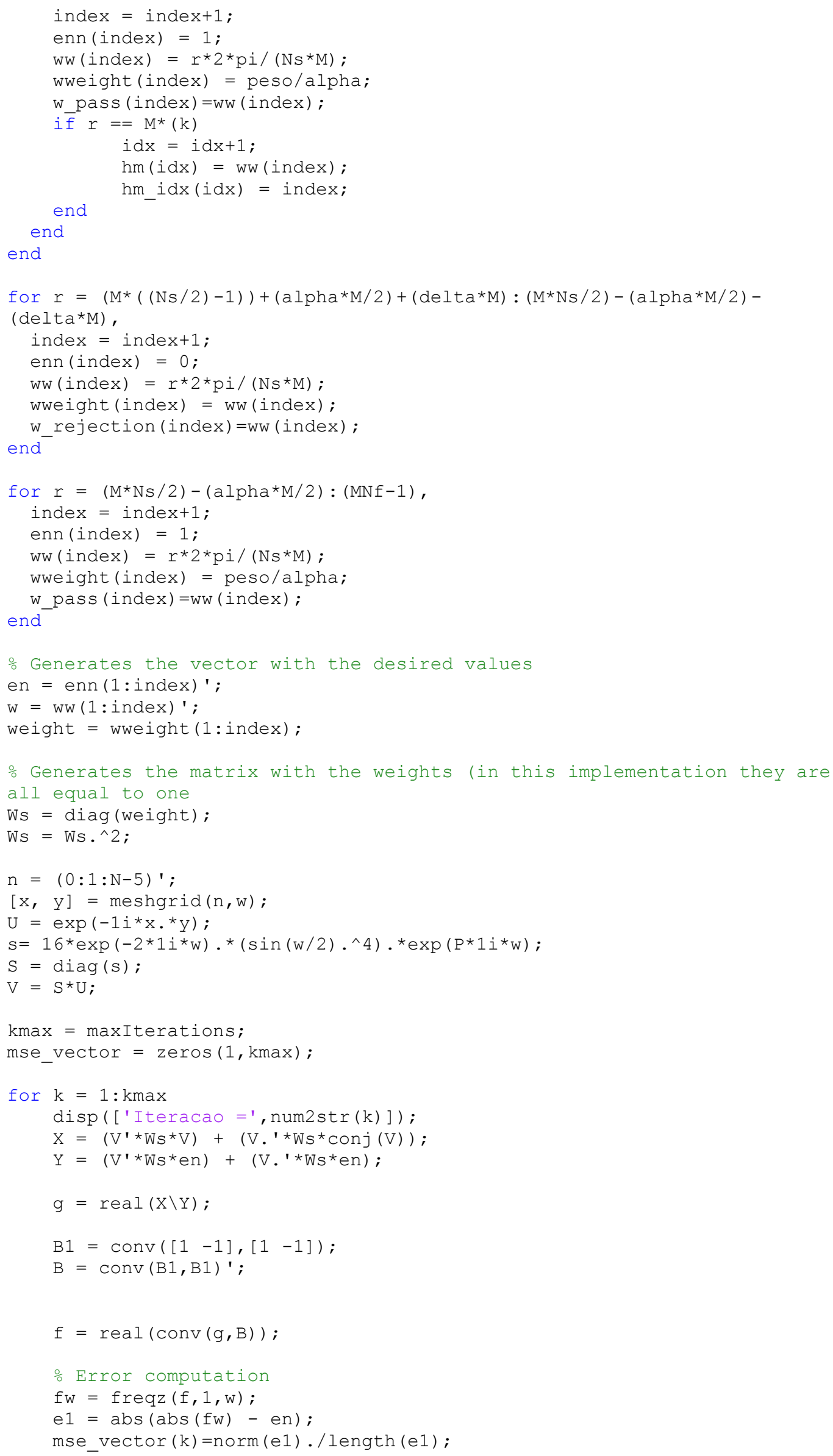




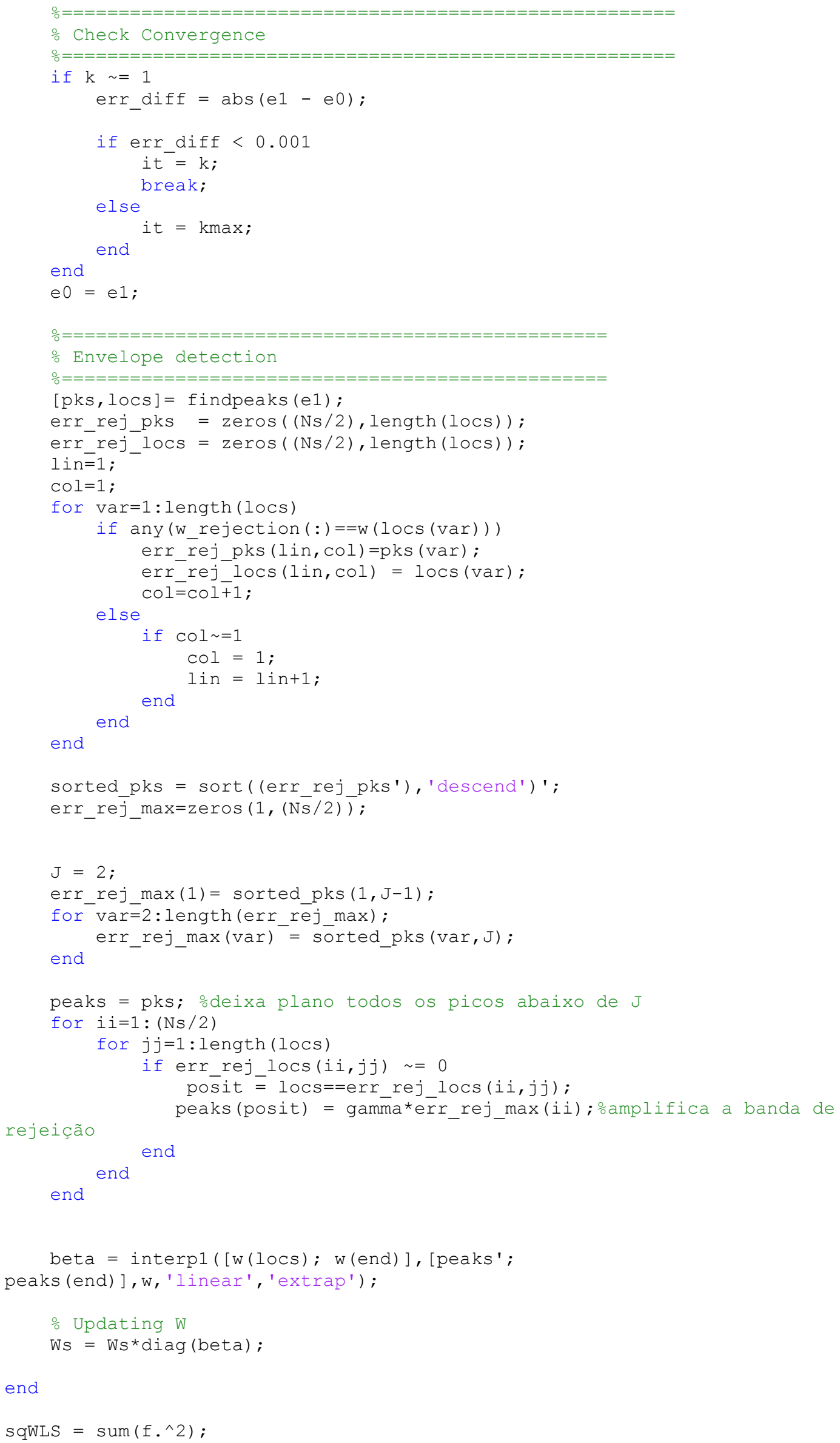




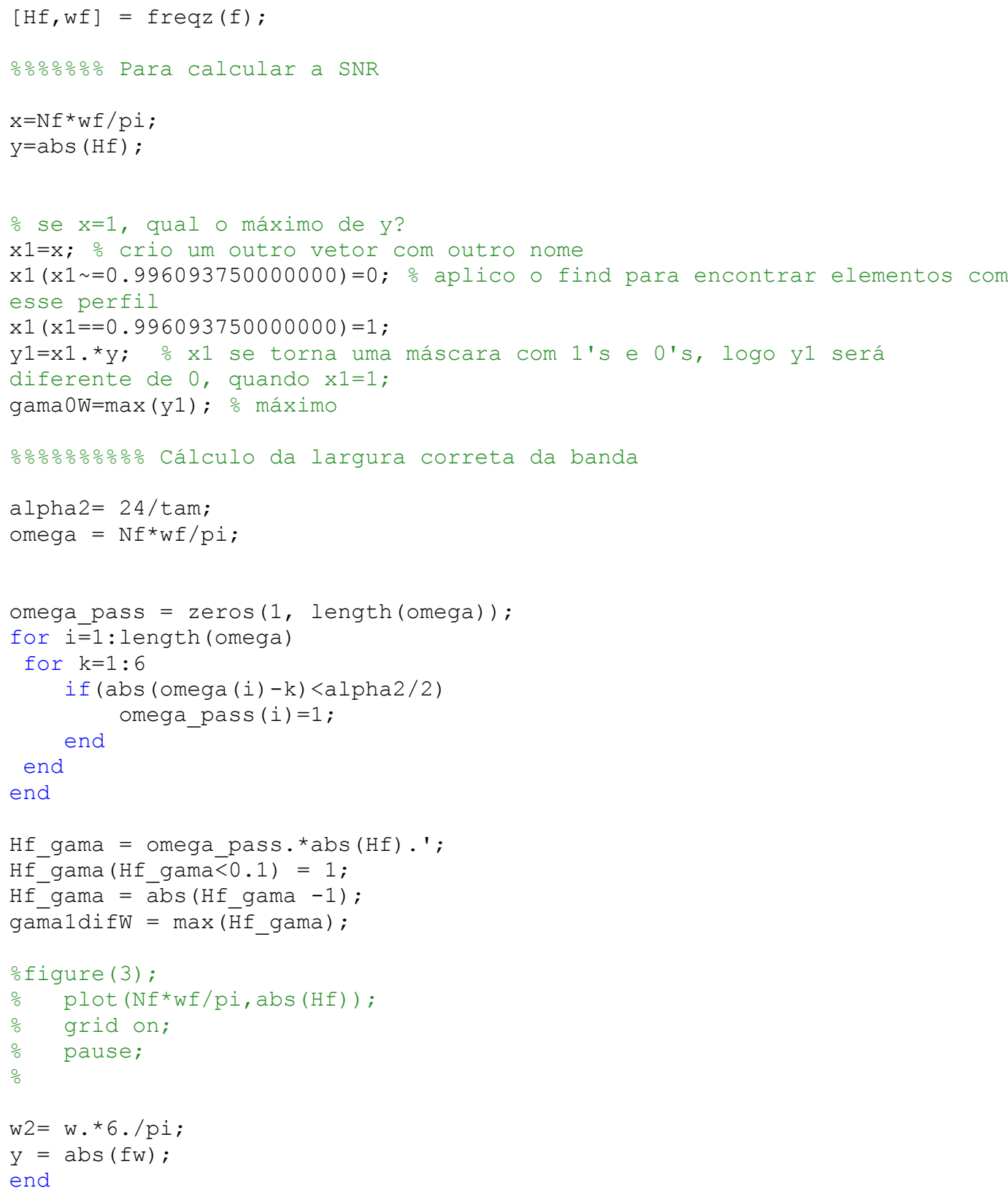




\section{(d) Filtro S-WLSC trimestral}

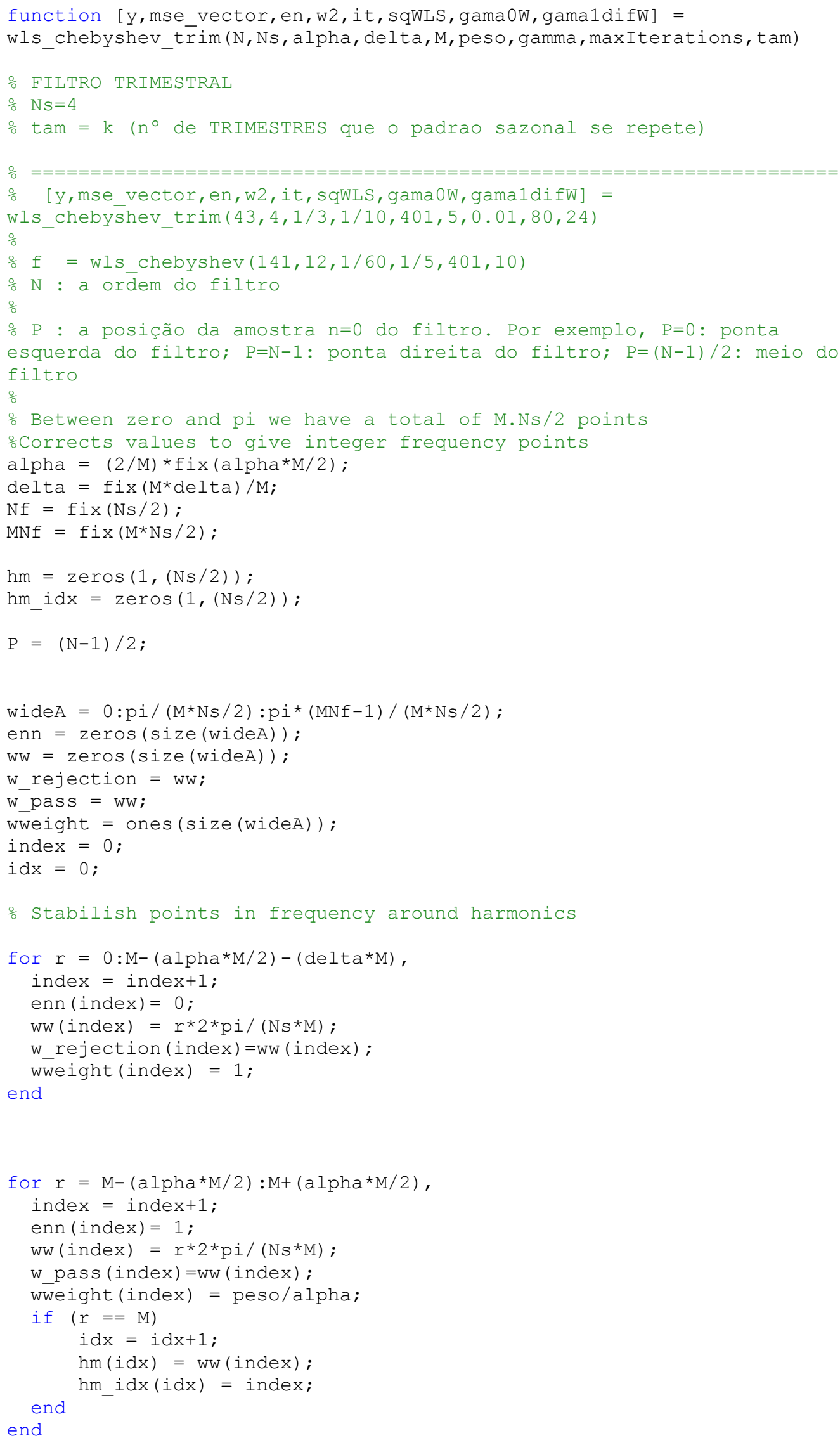




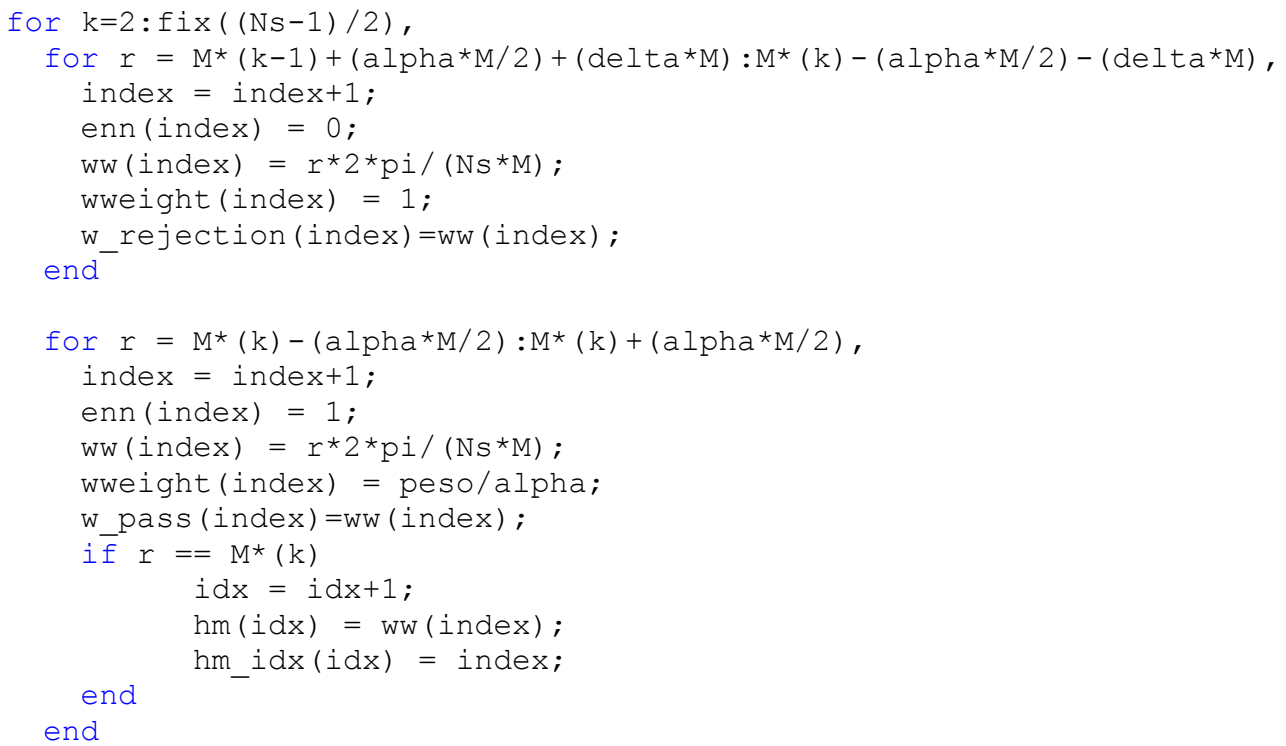




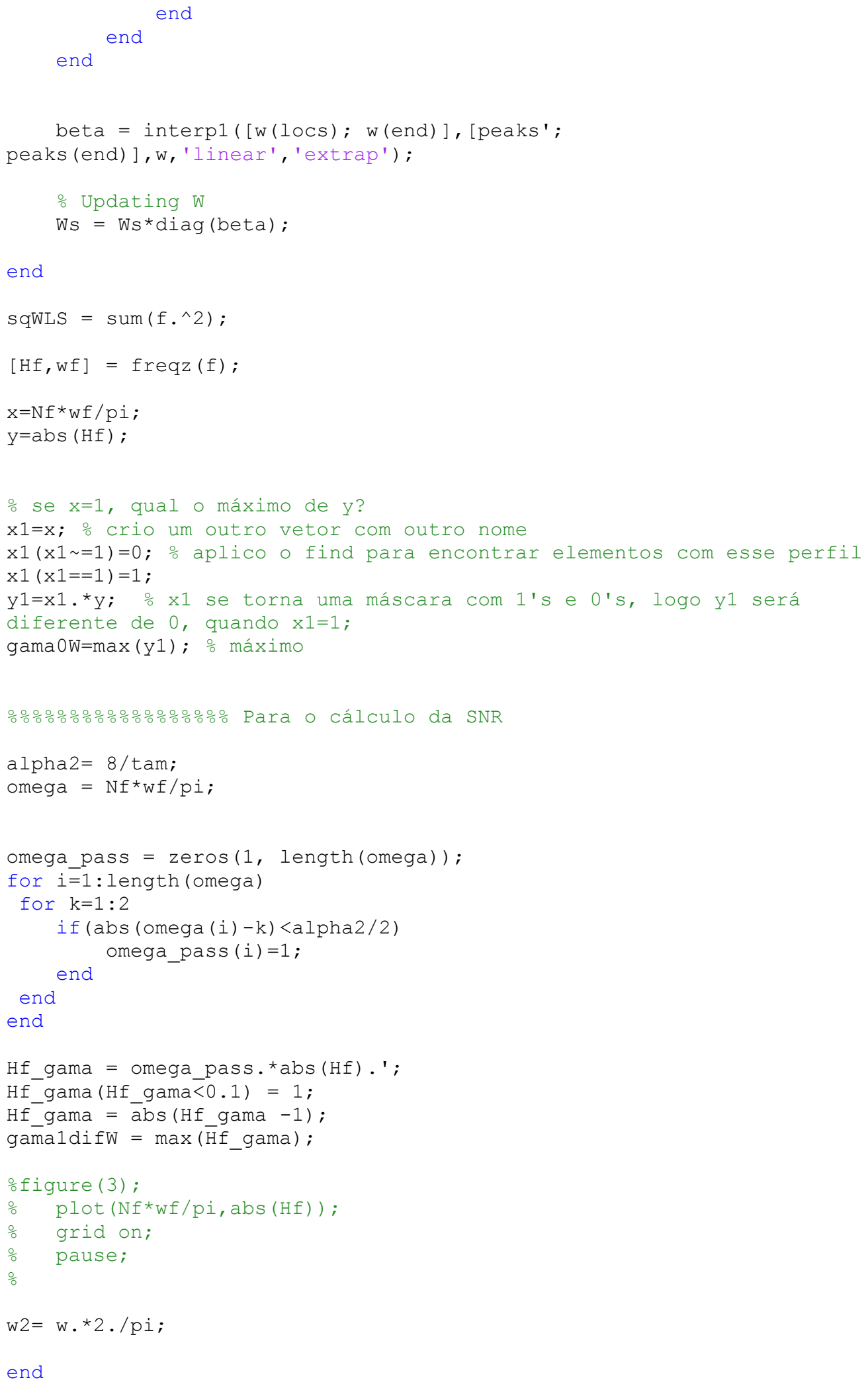

